


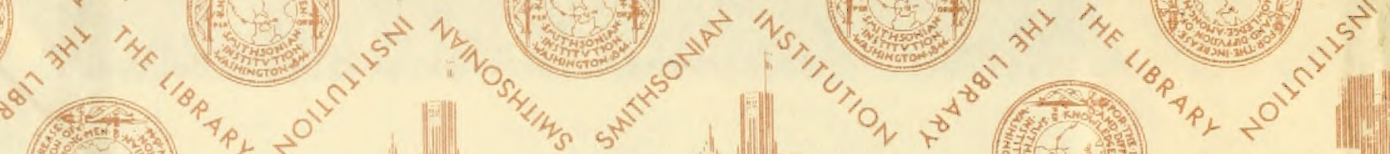

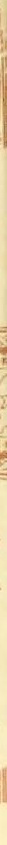

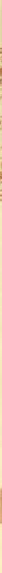

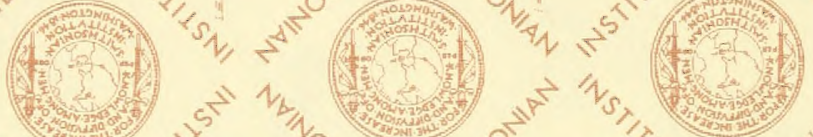

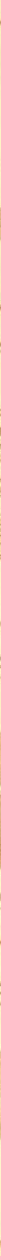




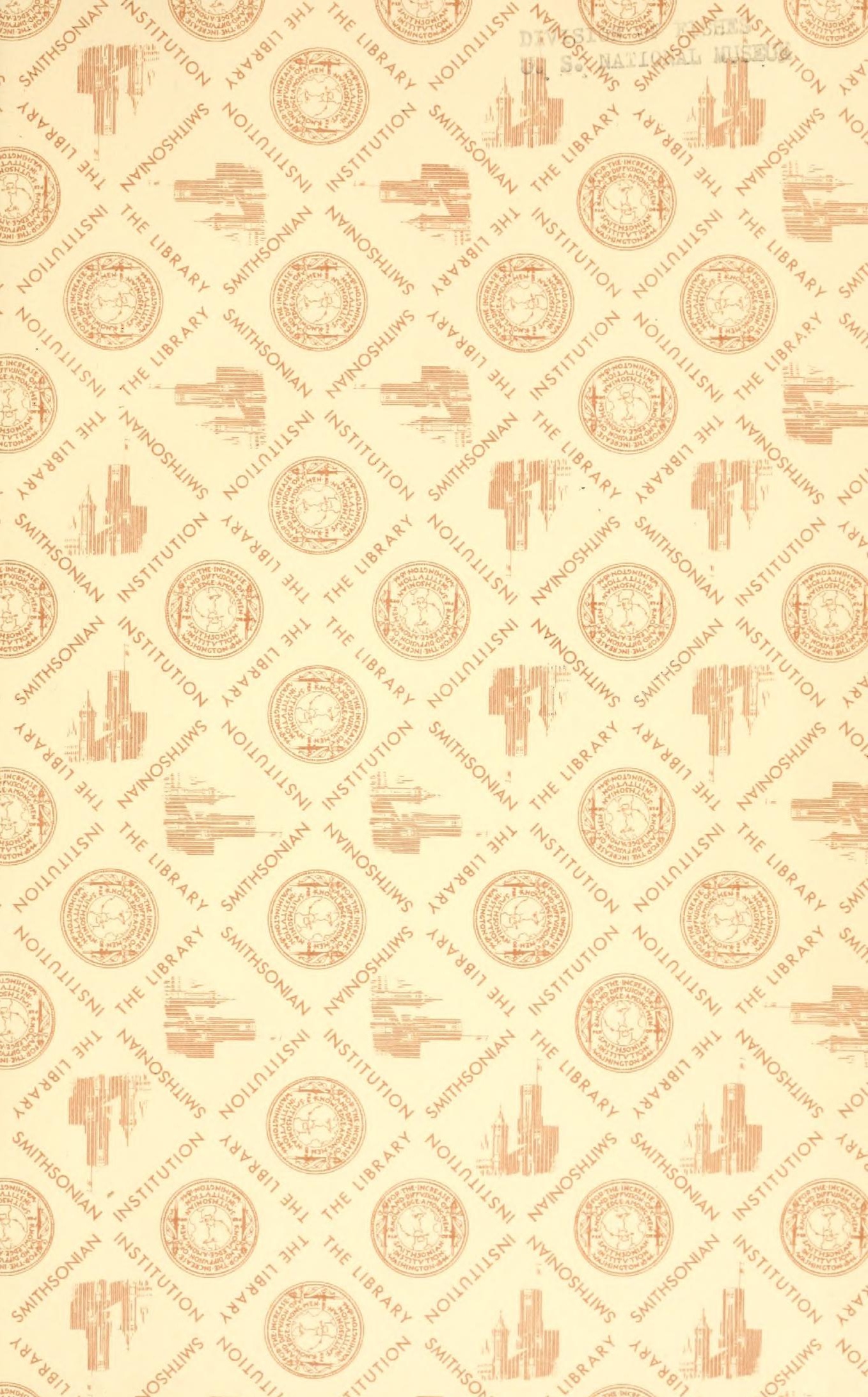






1.678130
8 mith
46

THE FISHES

OF THE

INDO-AUSTRALIAN ARCHIPELAGO 




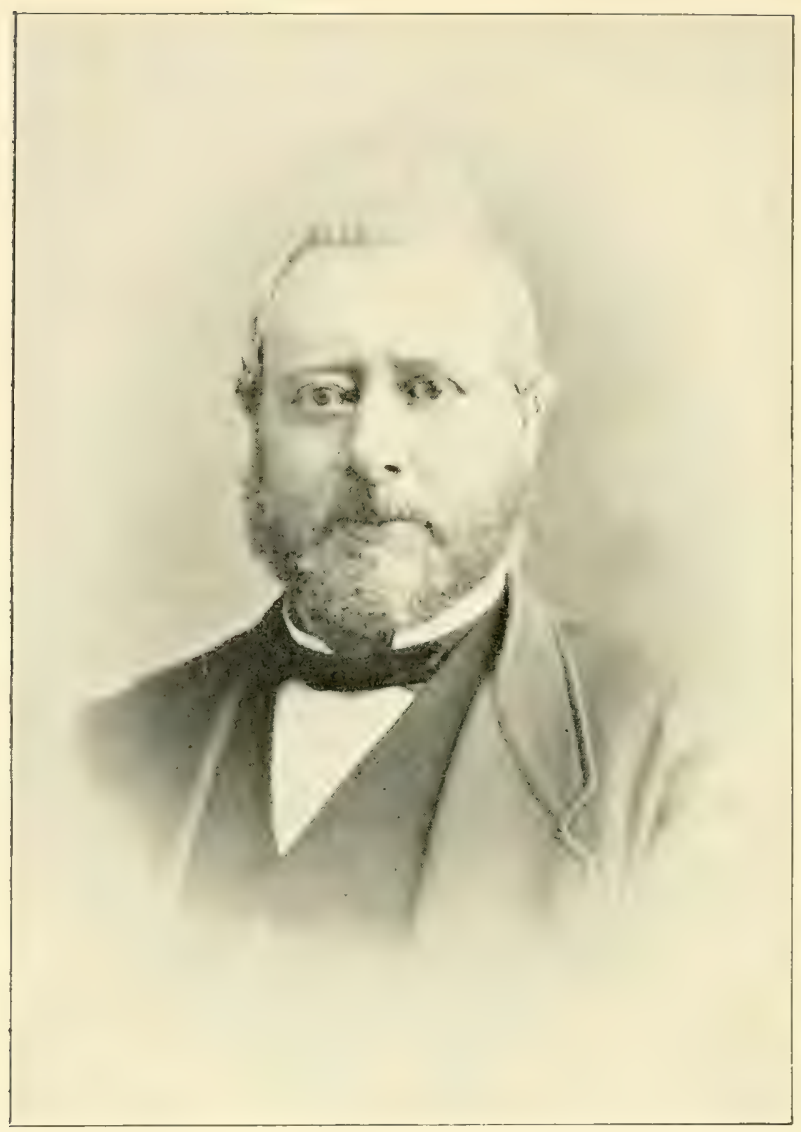

P. BLEEKER.

I $8 \mathrm{r} 9$, July Io $-\mathrm{I}_{7} 8$, January 24. 


\title{
THE FISHES
}

OF THE

\section{INDO-AUSTRALIAN ARCHIPELAGO}

\author{
I
}

INDEX OF THE ICHTHYOLOGICAL PAPERS

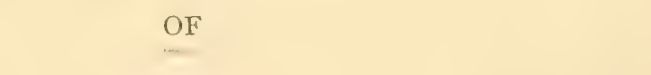

P. BLEEKER

BY

Dr. MAX WEBER

Professor at the University of Amsterdam

AND

Dr. L. F. DE BEAUFORT 
Printed by E. J. BRILL, LEYDEN (Holland). 


\section{PREFACE}

The numerous writings of Dr. P. Bleeker are necessary to everyone who studies fishes, not only of the indo-australian Archipelago but also those of the great indo-pacific region. No less than 500 of his writings are about fishes of the indoaustralian Archipelago, fishes from Japan, China, Australia, New Zealand, British India, Guiana, Cape of Good Hope, Surinam and Europe - whilst but few treat exclusively of systematic problems of fishes and it is still rarer that they contain biological or anatomical questions.

The most important of his writings are the 432 articles about the fish fauna of the indo-australian Archipelago, in which new species are described. They also contain a critical study of the known species and generally give an account of their distribution among the islands of the Archipelago. The importance of these writings for the study of the fish fauna of the indo-pacific region is obvious and it is greatly to be regretted that it is exceedingly difficult to consult them, partly on account of their having appeared in various periodicals, sometimes difficult to obtain, and partly on account of their character and the way in which Bleeker chose to publish them. This is in a way due to the circumstances in which they originated, which will best be explained by a short sketch of Bleeker's life, derived from his autobiography ').

1) Levensbericht van Picter Bleeker door hemzelven. Jaarboek der Kon. Akademie van Wetenschappen $18_{77}$, Amsterdam IS 78 . Again published in Natuturkundig Tijdschrift voor Nederlandsch-Indië XL (serie S, deel I) Batavia ISSI. 
But not only for this reason do we consider that in this volume, which is a monument of Bleeker's ichthyological work, a short biographical notice is indispensible about this remarkable man. For, besides being a great ichthyologist, he knew how to make the most of his manysided talents, of his great love of work and his inexhaustable zeal in other directions also. His enthusiasm for science was of incalculable advantage to scientific life in Dutch East India and as he mounted higher and became Privy Councillor, he was a great champion for more liberal ideas in the government of the colony.

$\checkmark$ Pieter Bleeker was born at Zaandam on Io July, I\&I9. His parents were simple folk and not rich, so after having passed through the primary school, he had to content himself with a very simple training for apothecary and to that end was apprenticed for 3 years in Amsterdam. This awakened his love of scientific work, especially of anatomy, physiology and zoology, and decided him to become qualified as a physician. Once more he was forced to manage in the cheapest way and so he attended the clinical school at Haarlem, then still in existence. By diligent private study, especially in the well-provided library of Teyler's Institution in Haarlem, Bleeker managed to fill the gaps which the mediocrity of the abovementioned school left in his training, and so in 1840 he was qualified as surgeon and country practitioner. His youth and especially his youthful appearance stood in the way of his success as a physician, so after having vainly sollicited a post at the Museum of Natural History at Leyden he went to Paris, where his very slender means enabled him to remain for a little more than 6 months. Here he spent his morning hours in the hospitals, his afternoons in the institutions of the Jardin des Plantes.

After his return to Holland in the spring of $\mathrm{I} 8_{4} \mathrm{I}$ he tried again for an appointment at the Leyden Museum, but being once more unsuccessful he passed the necessary examination for army surgeon. In May IS4I he received his commission 
as army surgeon $3^{\text {rd }}$ class of the Dutch East Indian army. On IO March, I $\$ 42$ Bleeker arrived in Batavia and remained there till Io September, IS60, when he returned to Holland.

All this time, the I 8 best years of his life, he spent exclusively at Batavia with just two exceptions. That is to say he spent two years as a physician at Samarang, Sourabaya and Ambarawa (Java) and he accompanied Mr. Duymaer van Twist, then governor-general of Dutch East India, in $1 S_{5} 6$ on a voyage to Celebes and the Moluccos.

Soon after his arrival in Java, Blecker began to investigate the fauna of his surroundings, doing which he encountered several unknown species of fishes, which drove him to study this group of animals. He encountered many difficulties in this entirely new field of study, not the least of which was at first the lack of really good literary resources, but his undaunted perseverance and great power of work triumphed and no later than ${ }^{8} 44$ he could communicate his first notes on the study of the material he had in the meanwhile collected. Then already he first planned to publish an atlas of the indian fishes and so the question was how to collect the necessary material. In this he succeeded beyond measure during the $\mathrm{I} S$ years he spent in India. For rarely, if ever, did one man procure such extensive fish collections. As he was but rarely out of Batavia, and as all his journeys were principally in Java, it was only possible te collect study material from the other parts of the Archipelago by the assistance of army surgeons and officials of the civil service who were quartered in different islands and who regularly sent him collections. As a rule he published the result of his investigations as soon as possible, usually giving lists with descriptions of the new species. Between the years 1852 and 1873 for instance he published 26 papers on the fish fauna of Amboina, from $I_{5} 2$ to I 6424 lists of fishes of Sumatra and between I 444 and I 86544 papers on fishes of Java. Though this way of publishing caused endless repetitions, yet it gave him an opportunity to revise the descriptions of species formerly clescribed 
on insufficient material as well as to make remarks on synonymy and nomenclature. 1 His nomenclature and often also the systematic part of his work (besides differing from our present day conception) were liable to fluctuations, which adds new difficulties to the use of his papers.

In I 857 Bleeker published an "Index descriptionum specierum piscium Bleekerianarum in voluminibus I ad XIX Diarii Socictatis Scientiarum Indo-Batavae", in I 858 an "Index specierum piscium in Vol. I-III Actorum Societatis Scient. Indoneerl. descriptarum", in the year I 859 his "Enumeratio specierum piscium hucusque in Archipelago indico observatarum, adjectis habitationibus citationibusque ubi descriptiones earum recentiores reperiuntur, nec non speciebus Musei Bleekeriani Bengalensibus, Japonicis, Capensibus Tasmanicisque". But useful though these works are they don't give later information than the year 1858 , while Bleeker's last paper appeared in 1880. Moreover his "Enumeratio" is arranged systematically and although the synonyms are given they don't appear in the Index, so it is difficult to find a given species without knowing Bleeker's nomenclature.

Finally we mention the "Atlas ichthyologique des Indes orientales", which is the chief work of Bleeker's life and intended to be a repertorium of everything he had collected about the fishes of the indo-australian Archipelago. It has, notwithstanding its great and lasting merits, peculiar difficulties for practical use, first and foremost being the regrettable fact that owing to its author's premature death on 24 January, I 878 this standard work was never completed (vide page 26 of this volume). Another difficulty arises from the fact that none of the 9 volumes has an index of synonyms, besides which one must remember that the first volume appeared in $\mathbf{I} 862$, many years before the end of Bleeker's ichthyological career. $\$$

Under these circumstances it appeared necessary to facilitate the use of Bleeker's papers by publishing a key to them. It would hardly suffice to give a mere bibliographical list of Bleeker's ichthyological works as he did himself in his auto- 
biography. For instance titles as "Nalezingen op de ichthyologische Fauna van het eiland Banka". or "Douzième notice sur la faune ichthyologique de l'île de Ternate" do not tell us anything about the contents of these papers.

When the first undersigned contemplated the publishing of a work on the fishes of the indo-australian Archipelago these difficulties became more pronounced, and induced to the composition of this "Index".

It is needless to give an account of the reasons which led to the projects for the above-named faunistical work: sufficient to state that the first undersigned mapped out elaborate plans, to which Prof. M. Treub, then head of the Department of Agriculture of Dutch East India, gave his full consent. The first undersigned is anxious to have this mentioned, as well as the fact that the recommendation of these plans to the colonial and home government was the last official act of his never to be forgotten friend, since then alas! deceased. The world of science is conscious of what thanks are due to this great botanist and splendid organizator and it is now nearly a year since her voice was heard in all the civilized nations lamenting his loss. Zoologists too knew how to appreciate him, for Treub ever had a keen eye to the growth and development of every branch of science, which he proved again by being very much interested in this projected work.

From the beginning. Dr. J. C. Koningsberger, Prof. Treub's successor as Director of 's Lands Plantentuin at Buitenzorg, has shown the same interest and it is with his help that this first volume of the fish fauna of the indo-australian Archipelago is published. The Colonial Department granted a sum for the publication, for which we express our gratitude to the Minister of the Colonies.

Here may follow some remarks on the way in which this Index was prepared. It was only by very carefully excerpting Bleeker's papers that it was possible to indicate the exact places where certain genera and species are mentioned. To lessen this immense labour we excluded the "Atlas ichthyo- 
logique" as this work stands by itself; besides the synonyms of the species mentioned therein can be found in the other papers. The "Enumeratio" and the two "Conspectus" mentioned above, were also excluded as they already consist of lists. But notwithstanding these restrictions, about 22000 labels had to be written, on each of them being noted the name of the genera and species, the paper and the page where the name was to be found. These notes were afterwards combined in so far as they contained identical genera and species.

That this enormous task was brought to a succesful close is mainly due to the accuracy, perseverance and patience of Miss C. H. W. Met, who accomplished this work under our supervision and to whom every one must be grateful who takes any interest in Bleeker's papers.

The arrangement of the Index is as follows: it is opened by an "Index Bibliographicus" of Bleeker's ichthyological papers. This bibliography is slightly different to that given by Bleeker in his autobiography, some papers being added that Bleeker himself overlooked, besides being arranged according to the date of their publication, whereas Bleeker arranged them according to the date on which he finished his manuscript, which two dates in many instances lie far apart. It often was difficult and sometimes even impossible to ascertain the exact date of publication, moreover some of the periodicals in which he published were - from a bibliograpical point of view - sometimes rather unusually edited. For instance the five papers (mentioned in our Index as $n^{\circ}$. $13,14,15,16$ and I7) which appeard in Vol. 22 of "Verhandelingen van het Bataviaasch Genootschap van Kunsten en Wetenschappen" bear the signature "23. deel I 849 " as they were really intended for that volume when being printed, but one of the authors failing to send his manuscript, promised for vol. 22, the 5 papers were after all included in vol. 22.

The "Index Bibliographicus" is followed by a list of the 500 papers arranged according to localities. 
The "Index generum et specierum" which comes after this, contains all the genera and species and their synonyms mentioned by Bleeker in his papers.

One remark on account of this list will be necessary. Bleeker's papers are far from being without misprints, which are principally due to the primitive state of the printing-offices at Batavia in his time. We have for the greater part corrected these slips, presuming that anyone who wants to know where a certain species is mentioned, will look for it under its correct name. Other slips however had to be taken up without alteration to prevent worse misunderstandings. For instance an ichthyologist consulting "Contribution à la faune ichthyologique de l'île Maurice" will find on page $\delta$ the name "Tristius brasiliensis Gill." Only by entering this name in our Index was it possible to draw attention to the fact that this name is a misprint for "Isistius brasiliensis Gill".

We left Bleeker's often peculiar orthography unaltered, as our intention is only to give quotations and no criticism.

Finally we are glad of this opportunity to express a warm word of thanks to the publishing and printing firm E. J. Brill Ltd. of Leyden, whose accuracy in this difficult typographical task greatly facilitated our work. But what helped us through this work was the conviction of its usefulness in rendering the access to Bleeker's ichthyological work more easy.

MAX WEBer.

L. F. DE BEAUfort.

Eerbeek, Holland, September I9II. 


$$
\begin{aligned}
& \text { Nin- Peprine is Webt de T3. No } 3 \text {. }
\end{aligned}
$$

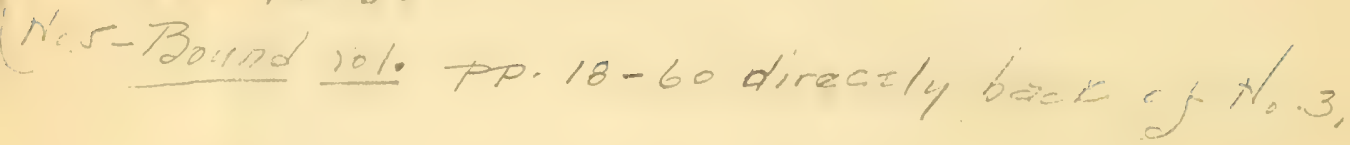




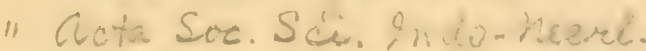

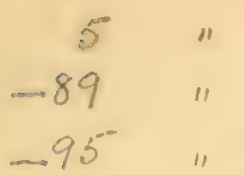

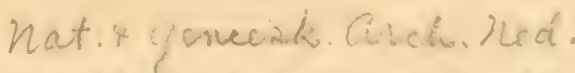

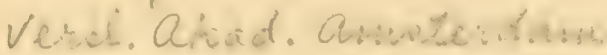

red. Tijdscher. Somu ü:

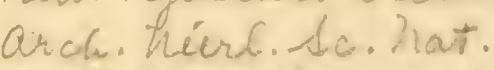

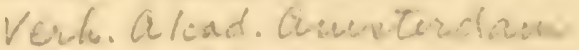

\section{Index Bibliographicus.}

I Bijdragen tot de geneeskundige Topographic van Batavia. Generisch overzicht der Fauna.

Nat. \& Geneesk. Arch. Ned. Ind. I, I844, p. 551--553.

2 Idem, continuatio.

Nat. \& Geneesk. Arch. Ned. Ind. II, r845, p. 505-528.

a1 3 Overzicht der Siluroïlen welke te Batavia voorkomen.

Nat. \& Geneesk. Arch. Ned. Ind. III (2), I846, p. I35-i84.

4 Siluroideorum bataviensium species nuperrime detectae.

Nat. \& Geneesk. Arch. Ned. Ind. III (2), r846, p. 284-293.

5 Siluroideorum bataviensium conspectus diagnosticus.

Verh. Bat. Gen XXI, I, I847, p. I-6o.

6 Labroideorum ctenoideorum bataviensium diagnoses et adumbrationes.

Verh. Bat. Gen. XXI, I, I847, p. I-33.

7 Nieuwe bijdrage tot de kennis der Siluroïden van Java.

Verh. Bat. Gen. XXI, I, I847, P. I -I2.

8 Pharyngognathorum Siluroideorumque species norae Javanenses.

Nat. \& Geneesk. Arch. Ned. Ind. IV (2), 1847, p. I55-169.

9 A contribution to the Ichthyology of Sumbawa.

Journ. Ind. Arch. II, No IX, I848, p. 632-639.

Io A contribution to the knowledge of the ichthyological fauna of Celebes.

Journ. Ind. Arch. III, I 849, p. $65-74$.

I I Overzicht der te Batavia voorkomende Gladschubbige Labrö̈den, met beschrijving van I I nieuwe soorten.

Verh. Bat. Gen. XXII, I849, p. I-64.

12 Idem Opus, Verh. Bat. Gen. XXII.

Vers. Gallice in Moniteur des Indes Orientales et occidentales.

Part. I, I 849, p. 4 I -65 et $97-$ Ir 2.

INDEX BLEEKER. 
13 Bijdrage tot de kennis der Percoïden van den MalayoMolukschen Archipel, met beschrijving van 22 nieuwe soorten.

Verh. Bat. Gen. XXII, I849, p. $\mathbf{1} \div 64$.

I + Bijdrage tot de kennis der Scleroparei van den SoendaMolukschen Archipel.

Verh. Bat. Gen. XXII, I849, p. I-ro.

I 5 Bijdrage tot de kennis der Blenniö̈den en Gobioiden van den Soenda-Molukschen Archipel, met beschrijving van 42 nieuwe soorten.

Verh. Bat. Gen. XXII, I849, p. I-40.

I6 Bijdrage tot de kennis der ichthyologische fauna van het eiland Madura, met beschrijving van eenige nieuwe species.

Verh. Bat. Gen. XXII, 1849, p. I-I6.

I 7 Bijdrage tot de kennis der ichthyologische fauna van het eiland Bali, met beschrijving van eenige nieuwe species.

Verh. Bat. Gen. XXII, I849, p. I-II.

is Bijdrage tot de kennis der Sciaenoiden van den Soenda-Molukschen Archipel, met beschrijving van 7 nieuwe soorten.

Verh. Bat. Gen. XXIII, I850, p. I-3I.

19 Bijdrage tot de kennis der Sparoïden van den SoendaMolukschen Archipel.

Verh. Bat. Gen. XXIII, r850, p. I-I6.

20 Bijdrage tot de kennis der Maenoïden van den SoendaMolukschen Archipel.

Verh. Bat. Gen. XXIII, I850, p. I-I3.

2 I Bijdrage tot de kennis der Visschen met doolhofvormige kieuwen van den Soenda-Molukschen Archipel.

Verh. Bat. Gen. XXIII, I850, p. I-I5.

22 Bijdrage tot de kennis der Chaetodontoïden van den Soenda-Molukschen Archipel.

Verh. Bat. Gen. XXIII, 1850, p. r-31.

23 Bijdrage tot de kennis der Teuthiden van den SoendaMolukschen Archipel.

Verh. Bat. Gen. XXIII, 1850 , P. I - I3. 
1324 Bijdrage tot de kennis der Notacanthini van den Soenda-Molukschen Archipel.

Verh. Bat. Gen. XXIII, I850, p. I-6.

25 Bijdrage tot de kennis der ichthyologische fauna van Midden- en Oost-Java, met beschrijving van eenige nieuwe species.

Verh. Bat. Gen. XXIII, I $85^{\circ}$, p. I-23.

26 Bijdrage tot de kennis der ichthyologische fauna van Borneo, met beschrijving van i 6 nieuwe soorten van zoetwatervisschen.

Nat. Tijdschr. Ned. Ind. I, I851, p. I-I6.

27 Over eenige nieuwe soorten van Scleroparei van den Indischen Archipel.

Nat. Tijdschr. Ned. Ind. I, I85I, p. I7-27.

28 Over twee nieuwe soorten van Callionymus van den Indischen Archipel.

Nat. Tijdschr. Ned. Ind. I, I85 r, p. 28-32.

i 29 Over eenige nieuwe soorten van Belone en Hemiramphus van Java.

Nat. Tijdschr. Ned. Ind. I, I85 I, p. 93-95.

30 Over drie nieuwe soorten van Tetraodon van den Indischen Archipel.

Nat. Tijdschr. Ned. Ind. I, I 85 I, p. 96-97.

3 I Faunae ichthyologicae Javae insularumque adjacentium genera et species novae.

Nat. Tijdschr. Ned. Ind. I, 185 I, p. 98-108.

32 Visschen van Banka.

Nat. Tijdschr. Ned. Ind. I, I8 5 I, p. I59-I 6 I.

33 Visschen van Borneo.

Nat. Tijdschr. Ned. Ind. I, I851, p. I6I-I62.

34 Over eenige nieuwe soorten van Blennioiden en Gobioiden van den Indischen Archipel.

Nat. Tijdschr. Ned. Ind. I, r85 r, p. $236-25^{8}$.

35 Nieuwe bijdrage tot de kennis der ichthyologische fauna van Borneo, met beschrijving van eenige nieuwe soorten van zoetwatervisschen.

Nat. 'Tijdschr. Ned. Ind, I, 185 I, p. 259-275. 
36 Oxybeles Brandesii Blkr, een nieuwe soort van Ophidini van Banda Neira.

Nat. Tijdschr. Ned. Ind. I, I851, p. $276-278$.

37 Over eenige nieuwe geslachten en soorten van Makreelachtige visschen van den Indischen Archipel.

Nat. Tijdschr. Ned. Ind, I, r 85 r, p. $34 \mathrm{I}-372$.

38 Over eenige nieuwe soorten van Pleuronectöiden van den Indischen Archipel.

Nat. Tijdschr. Ned. Ind. I, I85I, p. 40I-4I6.

39 Over eenige nieuwe soorten van Megalops, Dussumieria, Notopterus en Astronesthes.

Nat. Tijdschr. Ned. Ind. I, I85I, p. 4I7-424.

40 Visschen van Biliton.

Nat. Tijdschr. Ned. Ind. I, I85I, p. 478-479.

4I Visschen van Straat Malakka, Prince of Wales Island en Singapore.

Nat. Tijdschr. Ned. Ind. I, I 85 I, p. $480-48$ I.

42 Derde bijdrage tot de kennis der ichthyologische fauna van Borneo, met beschrijving van eenige nieuwe soorten van zoetwatervisschen.

Nat. Tijdschr. Ned. Ind. II, I85I, p. 57-70.

43 Cheilinoïdes een nieuw geslacht van gladschubbige Labroïden van Batavia.

Nat. Tijdschr. Ned. Ind. II, I85 I, p. 7x-72.

44 Nieuwe bijdrage tot de kennis der Percoidei, Scleroparei, Sciaenoidei, Maenoidei, Chaetodontoidei en Scomberoidei van den Soenda-Molukschen Archipel.

Nat. Tijdschr. Ned. Ind. II, I85x, p. 163-179.

45 Vierde bijdrage tot de kennis der ichthyologische fauna van Borneo, met beschrijving van eenige nieuwe soorten van zoetwatervisschen.

Nat. Tijdschr. Ned. Ind. II, I851, p. 193-208.

46 Nieuwe bijdrage tot de kennis der ichthyologische fauna van Celebes.

Nat. Tijdschr. Ned. Ind. II, I85I, p. 209-224.

47 Bijdrage tot de kennis der ichthyologische fauna van de Banda-eilanden.

Nat. Tijdschr. Ned. Ind. II, r851, p. 225-261. 


\section{${ }_{4} S$ Visschen van Solor.}

Nat. 'Tijdschr. Ned. Ind. II, I 85 I, p. 347-348.

\$49 Vijfde bijdrage tot de kennis der ichthyologische fauna van Borneo, met beschrijving van eenige nieuwe soorten van zoetwatervisschen.

Nat. Tijdschr. Ned. Ind. II, I85 I, p. 4I5-442.

Ii 50 Bijdrage tot de kennis der ichthyologische fauna van Riouw.

Nat. Tijdschr. Ned. Ind. II, I85 I, p. 469-497.

5 I Bijdrage tot de kennis der ichthyologische fauna van Singapore.

Nat. Tijdschr. Ned. Ind. III, I852, p. 5 I -86.

52 Bijdrage tot de kennis der ichthyologische fauna ran Blitong (Billiton), met beschrijving van cenige nieuwe soorten van zoetwatervisschen.

Nat. Tijdschr. Ned. Ind. III, 1852, p. 87-roo.

53 Bijdrage tot de kennis der ichthyologische fauna van Timor.

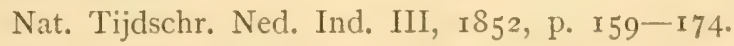

p -54 Bijdrage tot de kennis der ichthyologische fauna van de Moluksche eilanden. Visschen van Amboina en Ceram.

Nat. Tijdschr. Ned. Ind. III, I852, p. 229-309.

55 Zesde bijdrage tot de kennis der ichthyologische fauna van Borneo. Visschen van Pamangkat, Bandjermassing, Braboekarta en Sampit.

Nat. Tijdschr. Ned. Ind. III, I 852, p. $407-442$.

56 Bijdrage tot de kennis der ichthyologische fauna van het eiland Banka.

Nat. 'Tïjdschr. Ned. Ind. III, r852, p. 443-460.

57 Bijdrage tot de kennis der Makreelachtige visschen van den Soenda-Molukschen Archipel.

Verh. Bat. Gen. XXIV, I 852 , p. I- 93 .

$5 \&$ Bijdrage tot de kennis der Snoekachtige visschen van den Soenda-Molukschen Archipel.

Verh. Bat. Gen. XXIV, $\mathrm{I}_{52}$, p. $\mathrm{I}-2 \mathrm{~S}$.

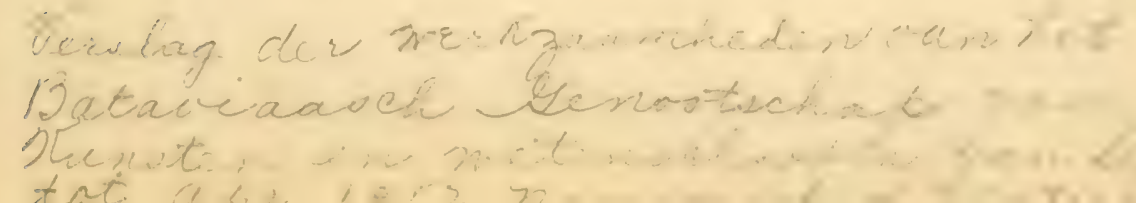


$\mathrm{ZJ}^{2} 59$ Bijdrage tot de kennis der Chirocentroidei, Lutodeiri, Butirini, Elopes, Notopteri, Salmones, Echeneoidei en Ophidini van den Soenda-Molukschen Archipel. Verh. Bat. Gen. XXIV, 1852 , p. I-32.

60 Bijdrage tot de kennis der Haringachtige visschen van den Soenda-Molukschen Archipel.

Verh. Bat. Gen. XVIV, I852, p. I- 52 .

6I Bijdrage tot de kennis der Pleuronecteoidei van den Soenda-Molukschen Archipel.

Verh. Bat. Gen. XXIV, I852, p. I-32.

62 Bijdrage tot de kennis der Blootkakige visschen van den Soenda-Molukschen Archipel.

Verh. Bat. Gen. XXIV, 1852 , p. $I-26$.

63 Bijdrage tot de kennis der Balistini en Ostraciones van den Indischen Archipel.

Verh. Bat. Gen. XXIV, I852, p. I- 38 .

6+ Bijdrage tot de kennis der Plagiostomen van den Indischen Archipel.

Verh. Bat. Gen. XXIV, r852, p. I-92.

-65 Visschen van Solor.

Nat. Tijdschr. Ned. Ind. III, I852, p. 490-49r.

66 Nieuwe bijdrage tot de kennis der ichthyologische fauna van Amboina.

Nat. Tijdschr. Ned. Ind. III, I852, p. $545-568$.

. 67 Diagnostische beschrijvingen van nieuwe of weinig bekende vischsoorten van Sumatra. Tiental I-IV. Nat. Tijdschr. Ned. Ind. III, I852, p. 569-608.

68 Nieuwe visschen van Banda-Neira.

Nat. Tijdschr. Ned. Ind. III, r852, p. 643-646.

69 Nieuwe bijdrage tot de kennis der ichthyologische fauna van Ceram.

Nat. Tijdschr. Ned. Ind. III, I852, p. 689-7r4.

70 Nieuwe bijdrage tot de kennis der ichthyologische fauna van het eiland Banka.

Nat. Tijdschr. Ned. Ind. III, 1852, p. 715-738. 
Q -7 I Derde bijdrage tot de kennis der ichthyologische fauna van Celebes.

Nat. Tijdschr. Ned. Ind. III, I852, p. $739-782$.

72 Derde bijdrage tot de kennis der ichthyologische fauna van Amboina.

Nat. Tijdschr. Ned. Ind. IV, r853, p. 9I-r 30.

73 Bijdrage tot de kennis der ichthyologische fauna van Ternate.

Nat. Tijdsch. Ned. Ind. IV, 1853, p. I3I-I40,

$\pi 74$ Over eenige nieuwe soorten van Homaloptera v. Hass (Balitora Gr.) van Java en Sumatra.

Nat. Tijdschr. Ned. Ind. IV, I853, p. 155-I64.

- 75 Exocoetus hexagona, eene nieuwe soort van Banka. Nat. Tijdschr. Ned. Ind. IV, I853, p. 206-207.

76 Diagnostische beschrijvingen van nieuwe of weinig bekende vischsoorten van Sumatra. Tiental V-X.

Nat. Tijdschr. Ned. Ind. IV, I853, p. 243-302.

$p-77$ Sicydium Parvei, een nieuwe soort van de PreangerRegentschappen.

Nat. Tijdschr. Ned. Ind. IV, I853, p. 426-427.

7778 Diagnostische beschrijvingen van nieuwe of weinig bekende vischsoorten van Batavia. Tiental I-VI.

Nat. Tijdschr. Ned. Ind. IV, I 853 , p. 451-516.

79 Nieuwe bijdrage tot de kennis der ichthyologische fauna van Ternate en Halmaheira (Gilolo).

Nat. Tijdschr. Ned. Ind. IV, I853, p. 595-61о.

p-80 Bijdrage tot de kennis der ichthyologische fauna van Solor.

Nat. Tijdschr. Ned. Ind. V, 1853, p. $67-96$.

₹ 8 I Vierde bijdrage tot de kennis der ichthyologische fauna van Celebes.

Nat. Tijdschr. Ned. Ind. V, I853, p. I53-I 74.

P-S2 Nalezingen op de ichthyologische fauna van het eiland Banka.

Nat. Tijdschr. Ned. Ind. V, I853, p. I75-r94. 
$S_{3}$ Derde bijdrage tot de kennis der ichthyologische fauna van Ceram.

Nat. Tijdschr. Ned. Ind. V, 1853, p. $233-248$.

$8+$ Vierde bijdrage tot de kennis der ichthyologische fauna van Amboina.

Nat. Tijdschr. Ned. Ind. V, 1853, p. 317-352.

$S_{5}$ Zevende bijdrage tot de kennis der ichthyologische fauna van Borneo. Zoetwatervisschen van Sambas, Pontianak en Pengaron.

Nat. Tijdschr. Ned. Ind. V, I 853, p. $427-462$.

86 Nieuwe tientallen diagnostische beschrijvingen van nieuwe of weinig bekende vischsoorten van Sumatra.

Nat. Tijdschr. Ned. Ind. V, I853, p. 495-534.

- 87 Antennarius notophthalmus, eene nieuwe soort van de Meeuwenbaai.

Nat. Tijdschr. Ned. Ind. V, 1853, p. 543-545.

88 Bijdrage tot de kennis der Muraenoïden en Symbranchoïden van den Indischen Archipel. Verh. Bat. Gen. XXV, I853, p.. -76 .

So Bijdrage tot de kennis der Troskieuwige visschen van den Indischen Archipel.

Verh. Bat. Gen. XXV, I853, p. $\mathrm{r}-3$.

90 Nalezingen op de ichthyologie van Japan.

Verh. Bat. Gen. XXV, 1853 , p. I- 56 .

9 I Nalezingen op de ichthyologische fauna van Bengalen en Hindostan.

Verh. Bat. Gen. XXV, I853, p. I-I64 cum r Tab. et 6 Tab. in T. XXVI. Aanhangsel. Maart 1854, p. I65-r66.

92 Over eenige nieuwe soorten van Notopterus van den Indischen Archipel.

Nova Acta Academ. Caes. Leop. Carol. Naturae Curios. XXIV, P. I, I854, P. 49-62.

93 Bijdrage tot de kennis der ichthyologische fauna van Japan.

Verh. Akad. Amsterdam I, I854, p. I-I6.

94 Bijdrage tot de kennis der ichthyologische fauna van Halmaheira (Gilolo).

Nat. Tijdschr. Ned. Ind. VI, I854, p. 49-62. 
F -95 Dercle bijdrage tot de kennis der ichthyologische fauna van de Banda-eilanden.

Nat. Tijdschr. Ned. Ind. VI, 1854, p. 89-Ir4.

$p-96$ Diagramma haematochir, eene nieuwe soort van Ternate.

Nat. 'Tijdschr. Ned. Ind. VI, I854, p. I75-I76.

T 97 Species piscium bataviensium novae vel minus cognitae.

Nat. Tijdschr. Ned. Ind. VI, I 854 , p. I9I-202.

$R^{2} 98$ Nieuwe bijdrage tot de kennis der ichthyologische fauna van Timor.

Nat. Tijdschr. Ned. Ind. VI, I854, p. 203-2т4.

$\pi \quad$ Bijdrage tot de kennis der ichthyologische fauna van het eiland Flores.

Nat. Tijdschr. Ned. Ind. VI, I854, p. $3 \mathrm{Ir}-338$.

Q-ioo Syngnathus tapeinosoma, eene nieuwe zeenaald van Anjer.

Nat. Tijdschr. Ned. Ind. VI, I854, p. $375-376$.

\& - IO I Diagramma polytaenioides, eene nieuwe soort van Solor.

Nat. Tijdschr. Ned. Ind. VI, I854, p. $376-378$.

F. 102 Faunae ichthyologicae japonicae species novae.

Nat. Tijdschr. Ned. Ind. VI, x 854, p. 395-426.

I03 Vijfde bijdrage tot de kennis der ichthyologische fauna van Amboina.

Nat. Tijdschr. Ned. Ind. VI, I854, p. 455-508.

f -104 Eleotris Tolsoni, eene nieuwe soort van Java's Westhoek nabij de Meeuwenbaai.

Nat. Tijdschr. Ned. Ind. VI, I 854 , p. $542-543$.

P I05 Bijdrage tot de kennis der ichthyologische fauna van de Kokos-eilanden.

Nat. 'Tijdschr. Ned. Ind. VII, I854, p. $37-48$.

- Io6 Overzicht der ichthyologische fauna van Sumatra, met beschrijving van eenige nieuwe soorten.

Nat. 'Tijdschr. Ned. Ind. VII, I854, p. 49-108.

107 Iets over visschen levende in zeesterren en over eene nieuwe soort van Oxybeles.

Nat. Tijdschr. Ned. Ind. VII, I854, p. I62-163. 
Ios Visschen van de Natoena-eilanden.

Nat. Tijdschr. Ned. Ind. VII, I854, p. I63-164.

- 109 Vijfde bijdrage tot de kennis der ichthyologische fauna van Celebes.

Nat. Tijdschr. Ned. Ind. VII, I854, p. 225-260.

I I Ichthyologische waarnemingen gedaan op verschillende reizen in de residentie Bantam.

Nat. Tijdschr. Ned. Ind. VII, I 854, p. 309-326.

I I I Over eenige nieuwe visschen van de Kokos-eilanden.

Nat. Tijdschr. Ned. Ind. VII, I854, p. 353-358.

- I 2 Bijdrage tot de kennis der ichthyologische fauna van Batjan.

Nat. 'Tijdschr. Ned. Ind. VII, I854, p. 359-378.

I 3 Specierum piscium javanensium novarum vel minus cognitarum diagnoses adumbratae.

Nat. Tijdschr. Ned. Ind. VII, I854, p. 415-448.

I + Zesde bijdrage tot de kennis der ichthyologische fauna van Celebes.

Nat. Tijdschr. Ned. Ind. VII, I854, p. 449-452.

- I 5 Dactylopterus cheirophthalmus van de Bandaeilanden.

Nat. Tijdschr. Ned. Ind. VII, I854, p. 494-495.

- I 6 Nog iets over visschen levende in Echinodermen.

Nat. Tijdschr. Ned. Ind. VII, I854, p. 495-496.

I 7 Bijdrage tot de kennis der Sphyraenoiden van den Indischen Archipel.

Verh. Bat. Gen. XXVI, $1854-57$, p. I-22.

I 8 Nieuwe nalezingen op de ichthyologie van Japan.

Verh. Bat. Gen. XXVI, I854-57, p. I- 132 .

I I 9 Over eenige visschen van van Diemensland.

Verh. Akad. Amsterdam, II, I855, p. I-30.

I 20 Index Specierum Piscium in vol. XXI-XXVI Actorum Societatis Artium et Scient. Batav. descriptarum adjectis citationibus ubi descriptiones Bleekerianae recentiores emendataeque reperiuntur.

Verh. Bat. Gen. XXVI, I $854-57$, p. I -24 . 
ז. I I Achtste bijdrage tot de kennis der ichthyologische fauna van Borneo. Zoetwatervisschen van Bandjermasin.

Nat. Tijdschr. Ned. Ind. VIII, I855, p. r 5 I-r68.

-I22 Derde bijdrage tot de kennis der ichthyologische fauna van de Kokos-eilanden.

Nat: 'Tijdschr. Ned. Ind. VIII, I855, p. I69-I80.

$\tau^{*}$. 123 Antennarius Lindgreeni, eene nieuwe soort van Banka.

Nat. 'Tijdschr. Ned. Ind. VIII, I855, p. I92--I 93.

$\pi^{2}$ I 24 Vijfde bijdrage tot de kennis der ichthyologische fauna van Ternate.

Nat. Tijdschr. Ned. Ind. VIII, I855, p. 295-304.

- I25 Bijdrage tot de kennis der ichthyologische fauna van de Batoe-eilanden.

Nat. Tijdschr. Ned. Ind. VIII, 1855, p. 305-328.

- I 26 Visschen van de Duizend-eilanden.

Nat. Tijdschr. Ned. Ind. VIII, I855, p. 344.

- I27 Visschen van Tikoe, Sumatra's Westkust.

Nat. 'Tijdschr. Ned. Ind. VIII, I855, p. 345 .

$\pi$ - I 28 Zesde bijdrage tot de kennis der ichthyologische fauna van Amboina.

Nat. 'Tijdschr. Ned. Ind. VIII, I855, p. 39r-434.

T2 $\quad$ I29 Zevende bijdrage tot de kennis der ichthyologische fauna van Celebes.

Nat. Tijdschr. Ned. Ind. VIII, I855, p. 435--444.

$T=130$ Vierde bijdrage tot de kennis der ichthyologische fauna van de Kokos-eilanden.

Nat. Tijdschr. Ned. Ind. VIII, I855, p. 445-460.

$\pi=$ I3I Tweede bijdrage tot de kennis der ichthyologische fauna van de Batoe-eilanden.

Nat. Tijdschr. Ned. Ind. IX, I855, p. 65-72.

I32 Tweede bijdrage tot de kennis der ichthyologische fauna van Halmaheira (Gilolo).

Nat. 'Tijdschr. Ned. Ind. IX, I855, P. I05-II2. 
-I 33 Nieuwe bijdrage tot de kennis der ichthyologische fauna van Sumbawa.

Nat. Tijdschr. Ned. Ind. IX, I855, p. II3-II5.

I 34 Over eenige nieuwe visschen van Ternate.

Nat. Tijdschr. Ned. Ind. IX, I855, p. I55.

I 35 Tweede bijdrage tot de kennis der ichthyologische fauna van Batjan.

Nat. Tijdschr. Ned. Ind. IX, I855, p. I9I-202.

I 36 Nalezingen op de vischfauna van Sumatra. Visschen van Lahat en Sibogha.

Nat. Tijdschr. Ned. Ind. IX, I855, p. 257-280.

I 37 Achtste bijdrage tot de kennis der ichthyologische faüna van Celebes.

Nat. Tijdschr. Ned. Ind. IX, I855, p. 28I-3I4.

I 38 Verslag van eenige vischverzamelingen van OostJava.

Nat. Tijdschr. Ned. Ind. IX, 1855, p. 391-4I4.

I 39 Negende bijdrage tot de kennis der ichthyologische fauna van Borneo. Zoetwatervisschen van Pontianak en Bandjermasin.

Nat. Tijdschr. Ned. Ind. IX, I855, p. 415-330.

I 40 Bijdrage tot de kennis der ichthyologische fauna van het eiland Groot-Obi.

Nat. Tijdschr. Ned. Ind. IX, I 855 , p. $43 \mathrm{I}-438$.

I 4 I Derde bijdrage tot de kennis der ichthyologische fauna van Batjan.

Nat. Tijdschr. Ned. Ind. IX, I855, p. 491-504.

I 42 Beschrijvingen van nieuwe en weinig bekende vischsoorten van Amboina, verzameld op eene reis door den Molukschen Archipel, gedaan in het gevolg van den Gouverneur-Generaal Duymaer van Twist in September en October 1855 .

142 a. Act. Soc. Sc. Indo-Neerl. I, I8 8 , p. I-76.

I 43 Beschrijvingen van nieuwe of weinig bekende vischsoorten van Menado en Makassar grootendeels verzameld op eene reis naar den Molukschen Archipel 
in het gevolg van den Gouverneur-Generaal Duymaer van Twist.

Act. Soc. Sc. Indo-Neerl. I, 1856 , p. I-8o.

$\pi^{2}$ I 44 Tweede bijdrage tot de kennis der ichthyologische fauna van het eiland Bintang.

Nat. Tijdschr. Ned. Ind. X, I\$56, p. 345-356.

$\pi \quad$ I 45 Zevende bijdrage tot de kennis der ichthyologische fauna van Ternate.

Nat. Tijdschr. Ned. Ind. X, 1856, p. $357-386$.

$\pi^{2}$ I 46 Carcharias (Prionodon) amblyrhynchus, eene nieuwe haaisoort gevangen nabij het eiland Solombo.

Nat. Tijdschr. Ned. Ind. X, I856, p. 467-468.

I 47 Visschen van Saparoea.

Nat. Tijdschr. Ned. Ind. X, r8 86 , p. 469 .

${ }_{1} 4$ Verslag omtrent eenige vischsoorten gevangen aan de Zuidkust van Malang in Oost-Java.

Nat. Tijdschr. Ned. Ind. XI, 1856 , p. $81-92$.

I 49 Vijfde bijdrage tot de kennis der ichthyologische fauna van de Banda-eilanden.

Nat. Tijdschr. Ned. Ind. XI, I856, p. 93-r ז0.

- I jo Visschen waargenomen te Laboeha, eiland Batjan. Nat. Tijdschr. Ned. Ind. XI, I856, p. 253-254.

$F=15$ I Bijdrage tot de kennis der ichthyologische fauna van het eiland Boero.

Nat. Tijdschr. Ned. Ind. XI, I856, p. 383-4I4.

- I5 2 Verslag van eenige verzamelingen van zee- en zoetwatervisschen van het eiland Banka.

Nat. Tijdschr. Ned. Ind. XI, 1856, p. 4I5-420.

- 53 Vischsoorten nieuw voor de kennis der fauna van het eiland Ceram.

Nat. Tijdschr. Ned. Ind. XI, I856, p. $486-487$.

154 Reis door de Minahassa en den Molukschen Archipel gedaan in de maanden September en October 1855 in het gevolg van den Gouverneur-Generaal A. J. Duymaer van Twist. 2 Deelen; Batavia, I 856. 
I 55 Achtste bijdrage tot de kennis der ichthyologische fauna van Ternate.

Nat. Tijdschr. Ned. Ind. XII, I856-I857, p. I9I-210.

- I56 Bijdrage tot de kennis der ichthyologische fauna van Nias.

Nat. Tijdschr. Ned. Ind. XII, $1856-1857$, p. $211-228$.

I 57 Derde Bijdrage tot de kennis der ichthyologische fauna van de Batoe-eilanden.

Nat. Tijdschr. Ned. Ind. XII, $1856-1857$, p. 229-242.

I5S Beright omtrent eenige vischsoorten van Tobali, eiland Banka.

Nat. Tïjdschr. Ned. Ind. XII, I856-I857, p. 273-275.

- I59 Nieuwe bijdrage tot de kennis der ichthyologische fauna van Bali.

Nat. Tijdschr. Ned. Ind. XII, $1856-1857$, p. 29I-302.

160 Beright omtrent eenige vischsoorten nieuw voor de kennis van het eiland Ceram.

Nat. 'Tijdschr. Ned. Ind. XII, $185_{5} 6-x 857$, p. 508.

I6 I Conspectus specierum piscium moluccensium hucusque cognitarum.

Act. Soc. Sc. Indo-Neerl. II, 1857, p. $x-23$.

I62 Tiende bijdrage tot de kennis der ichthyologische fauna van Borneo. Visschen van de rivieren Barito, Kahajan en Kapoeas.

Act. Soc. Sc. Indo-Neerl. II, I857, p. I-2 т.

7. $\quad 163$ Achtste bijdrage tot de kennis der vischfauna van Amboina.

Act. Soc. Sc. Indo-Neerl. II, I857, p. I-102.

I64 Tweede bijdrage tot de kennis der ichthyologische fauna van Boeroe.

Nat. Tijdschr. Ned. Ind. XIII, I857, p. 55-82.

165 Over eenige vischsoorten nieuw voor de kennis der fauna van Biliton.

Nat. Tijdschr. Ned. Ind. XIII, I857, p. 283-287.

I66 Descriptiones specierum piscium javanensium novarum vel minus cognitarum diagnosticae.

Nat. Tijdschr. Ned. Ind. XIII, I857, p. 323-368. 
R 67 Bijdrage tot de kennis der ichthyologische fauna van de Sangi-eilanden.

Nat. Tijdschr. Ned. Ind. XIII, I857, p. 369-380.

$\pi=168$ Verslag van eene nieuwe verzameling visschen van Batjan.

Nat. Tijdschr. Ned. Ind. XIII, 1857, p. 38 r -386 .

$\pi^{2}{ }^{2} 69$ Verslag omtrent eenige vischsoorten van TimorKoepang en Timor-Delhi.

Nat. Tijdschr. Ned. Ind. XIII, 1857, p. 387-390.

- I 0 Over eenige vischverzamelingen van verschillende gedeelten van Java.

Nat. Tijdschr. Ned. Ind. XIII, I857, p. 475-480.

很 I I Over eenige vischsoorten gevangen bij Prigi aan Java's Zuidkust.

Nat. Tijdschr. Ned. Ind. XIV, 1857, p. 244-246.

$P$ - I 72 Index descriptionum specierum piscium Bleekerianarum in voluminibus I ad XIX Diarii Societatis Scientiarum Indo-Batavae.

Nat. Tijdschr. Ned. Ind. XIV, I857, p. 447-486.

I 73 Über das Vorkommen von Fischen in Echinodermen und über eine neue Species von Oxybeles (Oxybeles Brandesii).

Arch. f. d. Holländisch. Beiträge z. Natur- u, Heilk. I Bd. 1858, p. $255-257$.

B2 174 Zesde bijdrage tot de kennis der vischfauna van Sumatra. Visschen van Padang, Troessan, Priaman, Siboga en Palembang.

Act. Soc. Sc. Indo-Neerl. III, I858, p. I-50.

B I75 Vierde bijdrage tot de kennis der ichthyologische fauna van Japan.

Act. Soc. Sc. Indo-Neerl. III, 1858 , p. $x-46$.

$B^{2}$ I 76 Tiende bijdrage tot de kennis der vischfauna van Celebes.

Act. Soc. Sc. Indo-Neerl. III, I858, p. I-I6.

$5=177$ Elfde bijdrage tot de kennis der vischfauna van Celebes. Visschen van Makassar.

Act. Soc. Sc. Indo-Necrl. III, I 858, p. I-2. 
I $\mathrm{S}$ Elfde bijdrage tot de kennis der ichthyologische fauna van Borneo. Visschen van Sinkawang.

Act. Soc. Sc. Indo-Neerl. III, I $85^{8}$, p. I-4.

I 79 Negende bijdrage tot de kennis der vischfauna van Amboina.

Act. Soc. Sc. Indo-Neerl. III, I858, p. I-6.

I 80 Tiende bijdrage tot de kennis der vischfauna van Amboina.

Act. Soc. Sc. Indo-Neerl. III, I858, p. I-4.

I 8 I Index specierum piscium in Vol. I-III Actorum Societatis Scient. Indo-Neerl. descriptarum.

Act. Soc. Sc. Indo-Neerl. III, $185^{8}$, p. $\mathrm{I}-6$.

I 82 Visschen van Java's Zuidkust.

Nat. Tijdschr. Ned. Ind. XV, I858, p. ז59-I62.

I 83 Holacanthus pseudannularis, eene nieuwe soort van Batavia.

Nat. Tijdschr. Ned. Ind. XV, I858, p. r69-17r.

I 84 Bijdrage tot de kennis der vischfauna van den Goram-Archipel.

Nat. Tijdschr. Ned. Ind. XV, 1858, p. 197-218.

I 85 Vierde bijdrage tot de kennis der vischfauna van Biliton.

Nat. Tijdschr. Ned. Ind. XV, I 858 , p. 219-240.

186 Tweede bijdrage tot de kennis der vischfauna van Singapore.

Nat. 'Tijdschr. Ned. Ind. XV, I858, p. $24 \mathrm{I}-254$.

P $\quad-187$ Enumeratio specierum piscium javanensium hucusque cognitarum.

Nat. Tijdschr. Ned. Ind. XV. 1858, p. $359-456$.

I $S S$ Vijfde bijdrage tot de kennis der ichthyologische fauna van de Kokos-eilanden.

Nat. Tijdschr. Ned. Ind. XV, I858, p. 457-468.

I 99 De visschen van den Indischen Archipel. Siluri. Act. Soc. Sc. Indo-Neerl. IV, 1858 .

I $89 a$ Ichthyologiae Archipelagi Indici Prodomus Vol. I. Siluri. Batavia, Lange \& Co. I 85 \&. [Idem opus]. 
$\dot{B}$ I 90 Zevende bijdrage tot de kennis der vischfauna van Sumatra. Visschen van Palembang.

Act. Soc. Sc. Indo-Neerl. V, I858-1859, p. I-I 2.

B I9 I Twaalfde bijdrage tot de kennis der vischfauna van Borneo. Visschen van Sinkawang.

Act. Soc. Sc. Indo-Neerl. V, I858-r859, p. I-ro.

B 192 Twaalfde Bijdrage tot de kennis der vischfauna van Celebes. Visschen van Manado.

Act. Soc. Sc. Indo-Neerl. V, $185^{8-1859, ~ p . ~ I-4 . ~}$

B I93 Vijfde bijdrage tot de kennis der ichthyologische fauna van Japan.

Act. Soc. Sc. Indo-Neerl. V, I858-1859, p. I-I2.

P-194 Visschen van Tikoe verzameld door E. A. Ludeking.

Nat. 'Tijdschr. Ned. Ind. XVI, I858-I859, p. 26.

P-I95 Visschen van Amboina verzameld door den heer Schröder.

Nat. Tijdschr. Ned. Ind. XVI, I858-1859, p. 28-30

P - 196 Vischsoorten van Tjilatjap, verzameld door $H$. Tievez.

Nat. Tijdschr. Ned. Ind. XVI, I858-1859, p. 37.

- 197 Systema Silurorum.

Nat. Tijdschr. Ned. Ind. XVI, I858-1859, p. 38-41.

$P-198$ Vischsoorten van Anjer, verzameld door F. $H$. Eysinger.

Nat. 'Tijdschr. Ned. Ind. XVI, I858-1859, p. 45.

- 199 Vischsoorten van Riouw en Lingga, verzameld door E. Netscher, E. F. Meyer en H. Raat.

Nat. Tijdschr. Ned. Ind. XVI, I858-I859, p. 45-47.

P -200 Vischsoorten van Banjoewangi en Buitenzorg, verzameld door $\mathrm{H}$. Zollinger, $\mathrm{H}$. von Rosenberg en O. J. U. F. Huguenin.

Nat. 'Tijdschr. Ned. Ind. XVI, I858-I859, p. $47-48$.

P_20I Vischsoorten van Sinkawang, verzameld door J. H. A. B. Sonnemann Rebentisch.

Nat. 'Tijdschr. Ned. Ind. XVI, I858-1859, p. 195-196. INDEX BLEEKER. 
202 Visschen uit de omstreken van Montrado verzameld door G. J. Filet.

Nat. Tijdschr. Ned. Ind. XVI, I858-I859, p. I96-I 97.

203 Visschen van de Kokos-eilanden, verzameld door J. G. C. Ross en Dr. A. J. Anderson.

Nat. Tijdschr. Ned. Ind. XVI, r858-r859, p. 205-206.

204 Visschen van Koetei, verzameld door J. Wolff.

Nat. Tijdschr. Ned. Ind. XVI, I858-I859, p. 206.

205 Vischsoorten van Batjan, verzameld door J. G. F. Bernelot Moens.

Nat. Tijdschr. Ned. Ind. XVI, I858-I 859, p. 208-2 го.

206 Vischsoorten uit de zoete wateren van Benkoelen, verzameld door $\mathrm{J}$. A. W. van Ophuysen.

Nat. Tijdschr. Ned. Ind. XVI, I858-I859, p. 2 I0-2II.

207 Eenige vischjes uit de rivier van Priaman en eenige van de Zuidkust van Java, verzameld door G. F. James.

Nat. Tijdschr. Ned. Ind. XVI, I858-I859, p. $21 \mathrm{I}-2$ I 2.

208 Zeevisschen gevangen in de nabijheid van Benkoelen, verzameld door J. A. W. van Ophuysen.

Nat. Tijdschr. Ned. Ind. XVI, I858-r859, p. 239.

209 Vischsoorten van de Kokos-eilanden, verzameld door Dr. Anderson.

Nat. Tijdschr. Ned. Ind. XVI, I858-r859, p. 240.

2 I O Ophiocephalus micropeltes K. v. H. gevangen in de rivieren van westelijk Borneo en aangeboden door den heer C. M. H. Kroesen.

Nat. Tijdschr. Ned. Ind. XVI, I858-1859, p. 24 r.

2 I I Visschen uit de Serayoe, verzameld door G. C. Schonck.

Nat. Tijdschr. Ned. Ind. XVI, I858-I859, p. 24I-242.

2 I 2 Vischsoorten gevangen bij Benkoelen en aangeboden door. J. A. W. van Ophuysen.

Nat. Tijdschr. Ned. Ind. XVI, I858-1859, p. 243-244.

- 2 I 3 Vischsoorten van Muntok, verzameld door F. W. Dolge.

Nat. Tijdschr. Ned. Ind. XVI, $185^{8}-1859$, p. $25 \mathrm{I}-252$. 
2 I 4 Nog iets over het geslacht Heterophthalmus.

Nat. 'Tijdschr. Ned. Ind. XVI, r858-1859, p. 253-255.

2 I 5 Visschen van Biliton, verzameld door A. Hendriks. Nat. Tijdschr. Ned. Ind. XVI, I858-I859, p. 26 I-262.

-2 I6 Vischsoorten van Palembang, verzameld door E. A. Lange en F. J. P. Storm van 's Gravensande. Nat. Tijdschr. Ned. Ind. XVI, I858-1859, p. 263-266.

-217 Over eene- nieuwe verzameling vischsoorten van Benkoelen van A. J. W. van Ophuysen.

Nat. Tijdschr. Ned. Ind. XVI, I $858-1859$, p. $273-275$.

-2 I 8 Conspectus specierum Mugilis Archipelagi indici analyticus.

Nat. Tijdschr. Ned. Ind. XVI, $1858-1859$, p. $275-280$.

2 I 9 Visschen van Wonosobo.

Nat. Tijdschr. Ned. Ind. XVI, I858-I859, p. 302.

220 Over de geslachten der Cobitinen.

Nat. Tijdschr. Ned. Ind. XVI, I858-1859, p. 302-304.

22 I Exocoetus speculiger van Banda.

Nat. Tijdschr. Ned. Ind. XVI, $1858-1859$, p. 3 I4.

222 Vischsoorten van Tanara.

Nat. Tijdschr. Ned. Ind. XVI, I858-1859, p. 316-3г 7 .

223 Eene verzameling zee- en zoetwatervisschen, gevangen bij Priaman, verzameld door $\mathrm{H}$. Diepenhorst.

Nat. Tijdschr. Ned. Ind. XVI, $1858-1859$, p. 336-338.

224 Vischsoorten van Palembang, verzameld door $R$. V. Heyliger.

Nat. Tijdschr. Ned. Ind. XVI, I858-I859, p. 338-34I.

225 Zoetwatervisschen uit de omstreken van Ngawi, verzameld door $\mathrm{J}$. T. van Bloemen Waanders.

Nat. 'Tijdschr. Ned. Ind. XVI, I858-I 859 , p. 357-358.

226 Vischsoorten uit de Moessi, Saleh, Padang en Kommering, verzameld door E. A. Lange en G. A. van Delden.

Nat. 'Tijdschr. Ned. Ind, XVI, IS $58-I 859$, p. $3 S_{4}-388$.

227 Vischsoorten gevangen bij Japara, verzameld door S. A. Thurkow.

Nat. Tijdschr. Ned. Ind. XVI, I8 $88-$ I 859, p. 406-409. 
228 Visschen van Anjer, verzameld door J. G. F. Bernelot Moens.

Nat. Tijdschr. Ned Ind. XVI, I858-I859, p. 424-425.

229 Verslag van eene verzameling visschen van Westelijk Borneo, aangeboden door J. H. A. Sonneman Rebentisch.

Nat. Tijdschr. Ned. Ind. XVI, I858-1859, p. 433-436.

230 Vierde bijdrage tot de kennis der ichthyologische fauna van Timor. Visschen van Atapoepoe.

Nat. Tijdschr. Ned. Ind. XVII, I858-I859, p. I29-I40.

23I Derde bijdrage tot de kennis der ichthyologische fauna van Bali.

Nat. Tijdschr. Ned. Ind. XVII, I858-I859, p. I4I-I75.

232 Bijdrage tot de kennis der vischfauna van NieuwGuinea.

Act. Soc. Sc. Indo-Neerl. VI, I859, p. x-24.

233 Enumeratio specierum piscium hucusque in Archipelago indico observatarum, adjectis habitationibus citationibusque ubi descriptiones earum recentiores reperiuntur, nec non speciebus Musei Bleekeriani Bengalensibus, Japonicis, Capensibus Tasmanicisque.

Act. Soc. Sc. Indo-Neerl. VI, I859, p. I-XXXVI et p. I-276.

234 Bijdrage tot de kennis der vischfauna van Bawean. Nat. Tijdschr. Ned. Ind. XVIII, I859, p. 35I-358.

235 Negende bijdrage tot de kennis der vischfauna van Banka.

Nat. Tijdschr. Ned. Ind. XVIII, 1859, p. 359-378.

236 Over eenige vischsoorten van de Zuidkust-wateren van Java.

Nat. Tijdschr. Ned. Ind. XIX, I859, p. 329-352.

237 Derde bijdrage tot de kennis der vischfauna van Soembawa.

Nat. Tijdschr. Ned. Ind. XIX, I859, p. 434-440.

238 Vischsoorten van Bintang, verzameld door E. F. Meyer.

Nat. Tijdschr. Ned. Ind. XX, I859-I860, p. 87-88. 
339 Vischsoorten van Siam, verzameld door Fr. de Castelnau.

Nat. Tijdschr. Ned. Ind. XX, I859-r860, p. 10I-102.

240 Vischsoorten van Badjoa, verzameld door B. Schreuders. Nat. Tijdschr. Ned. Ind, XX, I859-I860, p. 129.

24 I Vischsoorten van Badjoa, verzameld door li. Netscher.

Nat. 'Tijdschr. Ned. Ind. XX, I859-1860, p. I40- 442.

242 Tiental vischsoorten van de Kokos-eilanden, verzameld door A. J. Anderson.

Nat. Tijdschr. Ned. Ind. XX, I859-I860, p. I42-I43.

$2+3$ Soorten van visschen van Badjoa, verzameld door E. Netscher.

Nat. Tijdschr. Ned. Ind. XX, I859-I860, p. I97-I98.

244 Vischsoorten van Priaman, verzameld door $H$. Diepenhorst.

Nat. 'Tijdschr. Ned. Ind. XX, I859-I860, p. I98-I99.

245 Zoetwatervisschen van Sintang, verzameld door A. H. Thepas.

Nat. Tijdschr. Ned. Ind. XX, $1859-$ I860, p. 199-200.

246 Vischsoorten van Biliton, verzameld door den heer Kruymel.

Nat. Tijdschr. Ned. Ind. XX, I859-r86o, p. $20 \mathrm{I}-202$.

247 Vischsoorten van de Kokos-eilanden, verzameld door A. J. Anderson.

Nat. Tijdschr. Ned. Ind. XX, I859-1860, p. 202.

$24 S$ Vischsoorten van Karangbollong, verzameld door F. J. Schultze.

Nat. Tijdschr. Ned. Ind. XX, 1859-1860, p. 202-204.

249 Vischsoorten van Amboina, verzameld door D. S. Hoedt.

Nat. 'Tijdschr. Ned. Ind. XX, 1859-1860, p. 204.

250 Vischsoorten van Bali, verzameld door P. L. van Bloemen Waanders.

Nat. 'Tijdschr.' Ned. Ind. XX, I859-I860, p. 205-207.

251 Vischsoorten van Singapore, verzameld door Fr. Graaf de Castelnau.

Nat. 'Tijdschr. Ned. Ind. XX, I859-I860, p. 2I6-2I7. 
252 Visschen uit de omstreken van Tandjong aan de Samangka-baai, verzameld door den heer Hunnius.

Nat. Tijdschr. Ned. Ind. XX, I859-r860, p. 219-220.

253 Vischsoorten van Japan, verzameld te Desima, door Jhr. J. L. C. Pompe van Meerdervoort.

Nat. Tijdschr. Ned. Ind. XX, I859-1860, p. 234-236.

254 Nieuwe vischsoorten van Singapore, verzameld door Fr. Graaf de Castelnau.

Nat. Tijdschr. Ned. Ind. XX, I859-I860, p. 236-239.

255 Visschen van Anjer en uit het meer Dano, verzameld door J. G. F. Bernelot Moens.

Nat. 'Tijdschr. Ned. Ind, XX, I859-r860, p. 240-24I.

256 Visschen gevangen in de baai van Amboina en aangeboden door $\mathrm{O}$. Mohnike.

Nat. Tijdschr. Ned. Ind. XX, I859-1860, p. 33I-332.

257 Vischsoorten van de Aroe-eilanden, aangeboden door O. Mohnike.

Nat. Tijdschr. Ned. Ind. XX, $1859-1860$, p. 332.

258 Vischsoorten van Bonthain, verzameld door J. L. de Zeeger.

Nat. Tijdschr. Ned. Ind. XX, 1859-1860, p. 332.

259 Over de plaatsing in het stelsel van de Luciocephaloïden.

Nat. Tijdschr. Ned. Ind. XX, I859-I860, p. 395-397.

260 Hemiramphus Buffonis $V$ al. en Mugil vaigiensis $Q G$. van Batjan, verzameld door O. J. U. F. Huguenin.

Nat. Tijdschr. Ned. Ind. XX, r859-1860, p. 4I 7.

26 I Conspectus systematis Cyprinorum.

Nat. Tijdschr. Ned. Ind. XX, I859-1860, p. 42I-44I.

262 Vijfde bijdrage tot de kennis der vischfauna van Timor. Vischsoorten van Atapoepoe.

Nat. Tijdschr. Ned. Ind. XX, I859-1860, p. 442-445.

263 Derde bijdrage tot de kennis der vischfauna van Singapore.

Nat. Tijdschr. Ned. Ind. XX, I859-r860, p. 446-456.

$26+$ Over het natuurlijk stelsel der visschen.

Nat. 'Tijdschr. Ned. Ind. XX, I859-1860, p. 46I-462. 
265 Ordo Cyprini, Karpers.

Act. Soc. Sc. Indo-Neerl. VII, (1. 5. deel II), 1860, p. I-492.

265a Ichthyologiae Archipelagi indici prodomi. vol. II.

Cyprini. Batavia, Lange \& Co. I 860. [Idem opus].

2 is 266 Over eenige vischsoorten van de Kaap de Goede Hoop.

Nat. Tijdschr. Ned. Ind. XXI, I860, p. 49-80.

13. -267 Tiende bijdrage tot de kennis der vischfauna van Banka.

Nat. Tijdschr. Ned. Ind. XXI, r860, p. I35-142.

268 Zoetwatervisschen van Singapoera, verzameld door F. Graaf de Castelnau.

Nat. 'Tijdschr. Ned. Ind. XXI, r860, p. 334.

P- 269 Zesde bijdrage tot de kennis der vischfauna van Japan.

Act. Soc. Sc: Indo-Neerl. VIII, I860, p. I-I04.

270 Achtste bijdrage tot de kennis der vischfauna van Sumatra. Visschen van Benkoelen, Priaman, Tandjong, Palembang. en Djambi.

Act. Soc. Sc. Indo-Neerl. VIII, I86o, p. I-88.

27 I Negende bijdrage tot de kennis der vischfauna van Sumatra. Visschen uit de Lematang-Enim en van Benkoelen.

Act. Soc. Sc. Indo-Neerl. VIII, I860, p. I-r2.

2 2 Dertiende bijdrage tot de kennis der vischfauna van Borneo.

Act. Soc. Sc. Indo-Neerl. VIII, I860, p. I-64.

273 Elfde bijdrage tot de kennis der vischfauna van Amboina.

Act. Soc. Sc. Indo-Neerl. VIII, I860, p, r-r4.

$27+$ Twaalfde bijdrage tot de kennis der vischfauna van Amboina.

Act. Soc. Sc. Indo-Neerl. VIII, I860, p. I-4.

275 Dertiende bijdrage tot de kennis der vischfauna van Celebes. Visschen van Bonthain, Badjoa, Sindjai, Lagoeși en Pompenoea.

Act. Soc. Sc. Indo-Neerl. VIII, I860, p. I-6o. 
276 Over eenige vischsoorten van Benkoelen, verzameld door A. J. W. van Ophuysen.

Nat. Tijdschr. Ned. Ind. XXII, r86r, p. 65-66.

277 Vischsoorten gevangen bij Prigi, verzameld door C. G. C. Greiner.

Nat. Tijdschr. Ned. Ind. XXII, I86r, p. 88-89.

278 Vischsoorten van Nieuw-Guinea, verzameld door F. G. Beckman.

Nat. Tijdschr. Ned. Ind. XXII, r86r, p. 98-roo.

279 Zoetwatervisschen van Gombong in zuidelijk MiddenJava, verzameld door P. Bruyn van Rozenburg.

Nat. Tijdschr. Ned. Ind, XXII, I86r, p. roo-ror.

280 Vischsoorten, nieuw voor de kennis der fauna van Singapoera, verzameld door Fr. Graaf de Castelnau.

Nat. Tijdschr. Ned. Ind. XXII, I86I, p. IOI-IO2.

28 I Elfde bijdrage tot de kennis der vischfauna van Banka.

Nat. Tijdschr. Ned. Ind. XXII, I86r, p. Iо7-ro8.

$2 S_{2}$ Derde bijdrage tot de kennis der ichthyologische fauna van Boeroe.

Nat. Tijdschr. Ned. Ind. XXIr, I86r, p. ro9-iı4.

$28_{3}$ Zesde bijdrage tot de kennis der vischfauna van Ceram.

Nat. Tijdschr. Ned. Ind. XXII, I86r, p. 228-238.

284 Vierde bijdrage tot de kennis der vischfauna van Bali.

Nat. Tijdschr. Ned. Ind. XXII, I86x, p. 239-242.

F. $\quad 285$ Tweede bijdrage tot de kennis der vischfauna van Bawean.

Nat. Tijdschr. Ned. Ind, XXII, I86r, p. 243-246.

286 Zesde bijdrage tot de kennis der vischfauna van Timor.

Nat. Tijdschr. Ned. Ind. XXII, I86r, p. 247-26r.

$2 S 7$ Mededeeling omtrent vischsoorten, nieuw voor de kennis der fauna van Singapoera.

Versl. Akad. Amsterdam XII, I86r, p. 28-63.

288 Iets over de vischfauna van het eiland Pinang.

Versl. Akad. Amsterdam XII, r86I, p. 64-80. 
1. 289 Aanteekening van den Heer P. Bleeker [Synonymia et prioritas nominum piscium].

Versl. Akad. Amsterdam XII, I86r, p. 81-82,

Te 290 Iets over de geslachten der Scaroïden en hunne Indisch-archipelagische soorten.

Versl. Akad. Amsterdam XII, I86I, p. 228-244.

$\pi$ 29I Notice sur le genre Trachinus et ses espèces.

Ann. d. Sc. nat. 4. Sér. Zool. T. XVI, I86r, p. 375-382. idem: Versl. Akad. Amsterdam XIV, I862, P. II3-I22.

$\mathbb{F}^{2} 292$ Conspectus generum Labroideorum analyticus.

Versl. Akad. Amsterdam XIII, I862, p. 94-ro9.

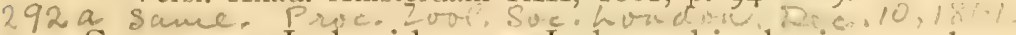

293 Synonyma Labroideorum Indo-archipelagicorum hucusque observatorum revisa, adjectis specierum novarum descriptionibus.

Versl. Akad. Amsterdam XIII, 1862, p. 274-308.

$2 \cdot 294$ Sur quelques genres de la famille des Pleuronectö̈des.

Versl. Akad. Amsterdam XIII, I862, p. 422-429.

te 295 Neuvième article sur la faune ichthyologique de l'île de Ternate.

Versl. Akad. Amsterdam XIV, I862, p. 95--98.

296 Sixième mémoire sur la faune ichthyologique de l'île Batjan.

Versl. Akad. Amsterdam XIV, I862, p. 99-Ir2.

297 Notices ichthyologiques (I-X).

Versl. Akad. Amsterdam XIV, r862, p. r23-r4I.

I. Sur le genre Eleutheronema Blkr. p. I23-I24.

2. Sur le genre Pseudetroplus Blkr. p. r25-r26.

3. Sur deux nouvelles espèces de Scarus p. I26-I 28 .

4. Sur une nouvelle espèce de Cossyphus (oxycephalus) p. $\mathrm{I} 28$ - I 30 .

5. Sur une nouvelle espèce de Pseudolabre (Güntheri) de la Nouvelle Hollande p. I30-r33.

6. Sur un nouveau genre de la famille des Mulloïdes (Pseudupeneus) p. I33-I34.

7. Sur le genre Porthmeus C. V. p. I34-I35.

8. Sur le genre Hemicaranx Blkr. p. I35-I40.

9. Sur le genre Micropus Gr. p. I40-I4I.

I0. Sur les genres Ditrema Schl. et Gerres Cuv. p. I4I.

298 Description de quelques espices nouvelles de Silures de Suriname.

Versl. Akad. Amsterdam XIV, r862, p. $37 \mathrm{I}-389$. 
299 Notice sur les genres Parasilurus, Eutropichthys, Pseudeutropius et Pseudopangasius.

Versl. Akad. Amsterdam XIV, I862, p. 390-399.

300 Notice sur les genres Tracheliopterichthys, Hemicetopsis et Pseudocetopsis.

Versl. Akad. Amsterdam XIV, 1862, p. 400-103.

30 I Atlas ichthyologique des Indes Orientales Néerlandaises, publié sous les auspices du Gouvernement colonial néerlandais. T. I- $[\mathrm{IX}]$, Livrais. I-36. Amsterdam. Fréd. Müller, I 862-77.

T. I. Scaroides et Labroides, I862. PI. $\mathrm{I}-48$.

"II. Siluroides, Chacoides et Hétérobranchoides, I862. Pl. 49-ior.

" III. Cyprins, I863. Pl. I02-I44.

"IV. Murènes, Symbranches, Leptocéphales, I864. Pl. I 45-I 93 .

" V. Baudroies, Ostracions, Gymnodontes, Balistes, 1865. Pl. I94-23I.

"VI. Pleuronectes, Scombrésoces, Clupées, Clupésoces, Chauliodontes, Saurides, I866-72. Pl. 232-278.

" VII. Percoides I, Priacanthiformes, Serraniformes, Grammisteiformes, Percaeformes, Datniaeformes I $873-76$. Pl. 279-320.

" VIII. Percoides II, (Spariformes), Bogodoides, Cirrhitéoides I876-I $877, \mathrm{Pl} .32 \mathrm{I}-354,36 \mathrm{I}-362$.

" [IX.] Livr. 35 et 36 tantum: Toxotoidei, Pempheridoidei, Chaetodontoidei, Nandoidei (p. I-80); Pl. 355-360 Trachichthyoidei, $363-382$ Chaetodontoidei, $38{ }_{3} \mathrm{Pem}$ pheridoidei, $384-388$ Sciaenoidei, 389 Sillaginoidei, 390 Pseurochromidoidei, 39 - 394 Nandoidei, Mulloidei, 395-396 Osphromenoidei, 397-399 Ophiocephaloidei, 400-4IO Pomacentroidei, 4II-4I7 Scorpaenoidei, 418-420 Platycephaloidei.

302 Mémoire sur les poissons de la côte de Guinée.

Nat. Verh. Holl. Maatsch. Wetensch., 2. Verz. Deel XVIII, I 863 , P. I-I36.

303 Sur quelques genres nouveaux du groupe des Doras. Ned. Tijdschr. Dierk. I, I863, p. 10-I8.

304 Sur quelques nouveaux genres du groupe des Synodontis.

Ned. Tijdschr. Dierk. I, I863, p. 52-55.

305 Over eenige vischsoorten van het eiland Banka, 
het Rijks Museum van natuurlijke historie te Leiden aangeboden door J. F. R. S. van den Bossche.

Ned. 'Tijdschr. Dierk. I, I863, p. 72-73.

306 Systema Silurorum revisum.

Ned. Tijdschr. Dierk. I, I863, p. 77-I 22.

T 307 Description de quelques espèces de poissons nouvelles ou peu connues de Chine envoyées au Musée de Leide par M. G. Schlegel.

Ned. Tijdschr. Dierk. I, I863, p. I35-150.

308 Septième notice sur la faune ichthyologique de l'île de Batjan.

Ned. Tijdschr. Dierk. I, I863, p. I5I-I52.

309 Troisième mémoire sur la faune ichthyologique de l'île de Halmahera.

Ned. Tijdschr. Dierk. I, r863, p. I53-I59.

3 IO Notice sur quelques poissons de l'île de Morotai. Ned. Tijdschr. Dierk. I, I863, p. I60.

3 I I Notice sur deux espèces inédites du genre Aphthalmichthys Kp.

Ned. 'Tijdschr.' Dierk. I, I863, p. I63-166.

3 I 2 Sur quelques espèces nouvelles ou peu connues de Gymnothorax de l'Inde archipélagique.

Ned. Tijdschr. Dierk. I, r863, p. r67-r7r.

3 I 3 Description de quelques espèces nouvelles ou peu connues d'Ophisuroides de l'Inde archipélagique.

Ned. Tijdschr. Dierk. I, I863, p. I79-I86.

3 I 4 Systema Cyprinoideorum revisum.

Ned. Tijdschr. Dierk. I, I863, p. I87-218.

315 Onzième notice sur la faune ichthyologique de l'île de Ternate.

Ned. Tijdschr. Dierk. I, I 863, p. $228-238$.

316 Douxième notice sur la faune ichthyologique de l'île d'Obi.

Ned. Tijdschr. Dierk. I, r863, p. 239-245.

3 I 7 Sur une nouvelle espèce d'Echidne de l'île de Rotti.

Ned. 'Tijdschr. Dierk. I, IS63, p. 246-247. 
3 Is Deuxième notice sur la faune ichthyologique de l'île de Flores.

Ned. Tijdschr. Dierk. I, I 863 , p. $248-252$.

3 I 9 Septième notice sur la faune ichthyologique de l'île de Céram.

Ned. Tijdschr. Dierk. I, I863, p. 253-26r.

320 Septième mémoire sur la faune ichthyologique de l'île Timor.

Ned. Tijdschr. Dierk, I, I863, p. 262-276.

32 I Treizième mémoire sur la faune ichthyologique de l'île d'Amboine.

Versl. Akad. Amsterdam XV, I863, p. 19-29.

Idem: Ned. Tijdschr. Dierk. I, I863, p. 355-360.

322 Glyphidodon Westermani, une nouvelle espèce de Java.

Versl. Akad. Amsterdam XV, I863, p. 30-3I.

Idem: Ned. Tijdschr. Dierk. I, I863, p. $360-36$ r.

323 Sur les genres de la famille des Cobitioïdes.

Versl. Akad. Amsterdam XV, I863, p. 32-44.

Idem: Ned. Tijdschr. Dierk. I, I863, p. $36 \mathrm{I}-368$.

324 Description de trois espèces nouvelles de Siluroïdes de l'Inde archipélagique.

Versl. Akad. Amsterdam XV, I863, p. 70-76.

Idem: Ned. Tijdschr. Dierk. I, I863, p. 368-37I.

325 Beschrijving en afbeelding van eene nieuwe soort van Brama (Abramis) uit de omstreken van Leiden.

Versl. Akad. Amsterdam XV, r862, p. 235-238.

Idem: Ned. Tijdschr. Dierk. I, I863, p. 37I-373.

326 Description de quelques espèces nouvelles de Cyprinoïdes de Ceylon.

Versl. Akad. Amsterdam XV, r863, p. 239-253.

Idem: Ned. Tijdschr. Dierk. I, I863, p. 373-380.

327 Description d'une nouvelle espèce du genre Nemacheilus.

Versl. Akad. Amsterdam XV, I 863, p. $254-256$.

Idem: Ned. Tijdschr. Dierk. I, I863, p. $380-38 \mathrm{r}$.

328 Sur une nouvelle espèce de poisson du Japon appartenant à un nouveau genre.

Versl. Akad. Amsterdam XV, r863, p. 257-260.

Idem: Ned. 'Tijdschr. Dierk, I, I863, p. $382-383$. 
329 Notice sur les noms de quelques genres de la famille des Cyprinoides.

Versl. Akad. Amsterdam XV, 1863, p. $26 \mathrm{I}-264$.

Idem: Ned. Tijdschr. Dierk. I, I 865, p. $65-67$.

R 330 Dixième notice sur la faune ichthyologique de l'île de Ternate.

Versl. Akad. Amsterdam XV, I863, p. 265-266.

Idem: Ned. Tijdschr. Dierk. II, I865, p. 67-68.

$\pi 33$ I Notices sur une collection de poissons de la Nouvelle Hollande faite à Port Jackson.

Versl. Akad. Amsterdam XV, I863, p. 442-45I.

Idem: Ned. Tijdschr. Dierk. II, 1865, p. 68-73.

$\pi 32$ Sur deux nouvelles espèces de Citharichthys de Suriname et de Guatimala.

Versl. Akad. Amsterdam XV, I863, p. 452-455.

Idem: Ned. Tijdschr. Dierk. II, r865, p. 73-74.

$\pi^{2} 333$ Sur une nouvelle espèce de Synaptura du Cap de Bonne Espérance.

Versl. Akad. Amsterdam XV, r863, p. 456-459.

Idem: Ned. Tijdschr. Dierk. II, I865, p. $75-76$.

334 Notice sur le genre Ichthyophis Less. et sur l'Ichthyophis tigrinus et l'Ichthyophis pantherinus de Lesson.

Versl. Akad. Amsterdam XV, I863, p. 460-466.

Idem: Ned. Tijdschr. Dierk. II, r 865, p. $77-79$.

B. 7335 Description de quelques espèces de Cobitioides et de Cyprinoides de Ceylon.

Nat. Verh. Holl. Maatsch. Wetensch. 2. Verz. XX, I864, p. I-23.

BR 336 Description des espèces de Silures de Suriname conservées aux Musées de Leide et d'Amsterdam.

Nat. Verh. Holl. Maatsch. Wetensch.2. Verz.XX, I864, p. I-104.

$\downarrow 337$ Notice sur la faune ichthyologique de Siam.

Versl. Akad. Amsterdam XVI, 1864, p. 352-358.

Idem: Ned. Tijdschr. Dierk. II, r865, p. $9^{6-99}$.

$33^{8}$ Deuxième notice sur la faune ichthyologique de l'île de Saparoea.

Versl. Akad. Amsterdam XVI, I864, p. 359-36r.

Idem: Ned. Tijdschr. Dierk. II, I 865, p. 99 - - 00.

339 Notice sur quelques poissons de l'île Grand-Key.

Versl. Akad. Amsterdam XVI, 1864, p. $362-363$.

Idem: Ned. Tijdschr. Dierk. II, r 865 , p. 100-Ior. 
340 Notice sur quelques poissons de l'île de Noussalaut. Versl. Akad. Amsterdam XVI, I864, p. 364-365.

Idem: Ned. Tijdschr. Dierk. II, I865, p. I98-I99.

34 I Notice sur la faune ichthyologique des îles Arou.

Versl. Akad. Amsterdam XVI, 1864, p. 366-368.

Idem: Ned. 'Tijdschr. Dierk. II, I865, p. IоI-102.

342 Rhinobagrus et Pelteobagrus, deux genres nouveaux de Siluroïdes de Chine.

Ned. 'Tijdschr. Dierk. II, I865, p. 7-Io.

343 Description de deux espèces inédites de Cobitioïdes. Ned. Tïjdschr. Dierk. II, r865, p. II-г4.

344 Paralaubuca, un genre nouveau de Cyprinoides de Siam.

Ned. Tijdschr. Dierk. II, ז865, p. 15-17.

345 Notices sur quelques genres et espèces de Cyprinoïdes de Chine.

Ned. Tijdschr. Dierk. II, I865, p. 28-29.

346 Notice sur quelques poissons de la baie de Manille.

Ned. Tijdschr. Dierk. II, I865, p. $30-3^{2}$.

347 Nouvelle notice sur la faune ichthyologique de Siam.

Ned. Tijdschr. Dierk. II, 1865, p. 33-37:

348 Poissons inédits indo-archipélagiques de l'ordre des Murènes.

Ned. Tijdschr. Dierk. II, 1865, p. $3^{8}-54$.

349 Notice sur les poissons envoyés de Chine au Musée de Leide par M. G. Schlegel.

Ned. Tijdschr. Dierk. II, I865, p. 55-62.

350 Notice sur quelques poissons de l'île de Harouko.

Ned. Tijdschr. Dierk. II, I $86_{5}$, p $63-64$.

35 I Systema Muraenorum revisum.

Ned. Tijdschr. Dierk. II, I865, p. II3-122.

352 Synonyma Muraenorum indo-archipelagicorum hucusque observatorum revisa, adjectis habitationibus citationibusque, ubi descriptiones figuraeque eorum recentiores reperiuntur.

Ned. Tijdschr. Dierk. II, г865, p. 123-136. 
353 Description de deux espèces inédites de Gnathopogon et de Rasbora de l'île de Java.

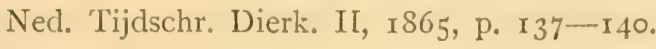

$35+$ Quatrième notice sur la faune ichthyologique de l'île de Bouro.

Ned. Tijdschr. Dierk. II, I865, p. $x_{41}$ - 15 r.

355 Notice sur le genre Paraploactis et description de son espèce type.

Ned. Tijdschr. Dierk. II, г865, p. I68-r 70.

356 Sixième notice sur la faune ichthyologique de Siam.

Ned. Tijdschr. Dierk. II, I865, p. г7г-176.

357 Description de quelques espèces inédites de poissons de l'Archipel des Moluques.

Ned. Tijdschr. Dierk. II, I865, p. I77-I8r.

$35^{S}$ Enumération des espèces de poissons actuellement connues de l'île de Céram.

Ned. Tijdschr. Dierk. II, I865, p. I82-193.

359 Notice sur une nouvelle espèce de Xiphasia V.

Versl. Akad. Amsterdam XVII, r865, p. r93-r97.

Idem: Ned. Tijdschr. Dierk. II, I865, p. I94-I96.

360 Sur une nouvelle espèce de Puntius à épine anale dentelée.

Versl. Akad. Amsterdam XVII, r865, p. 198-202.

Idem: Ned. Tijdschr. Dierk. II, I865, p. I96-Ig8.

36 I Description de deux espèces inédites des genres Callechelys et Pisoodonophis.

Ned. Tijdschr. Dierk. II, I865, p. 213-2x6.

362 Description de quelques espèces de Murènes de Suriname.

Ned. Tijdschr. Dierk. II, I 865, p. 233-249.

363 Description de quelques espèces de poissons du Japon, du Cap de Bonne Espérance et de Suriname, conservées au Musée de Leide.

Ned. Tijdschr. Dierk. II, r 865 , p. $250-269$.

B 364 Enumération des espèces de poissons actuellement connues de l'île d'Amboine.

Ned. Tijdschr. Dierk, II, x865, p. 270-293. 
365 Notice sur les Ostracions, confondus sous le nom d'Ostracion quadricornis L. et description des Ostracion notacanthus et guineensis.

Ned. Tijdschr. Dierk. II, I865, p. 298-305.

366 Notice sur le genre Apionichthys Kp. (Soleotalpa Gthr.).

Ned. Tijdschr. Dierk. II, r865; p. 306-308.

$3^{6} 7$ Systema Balistidorum, Ostracionidorum, Gymnodontidorumque revisum.

Ned. Tijdschr. Dierk. III, r866, p. 8-ig.

368 Synonyma Balistidorum, Ostracionidorum, Gymnodontidorumque Indo-archipelagicorum hucusque observatorum revisa, adjectis habitationibus citationibusque, ubi descriptiones figuraeque eorum recentiores reperiuntur.

Ned. Tijdschr. Dierk. III, 1866, p. 20-40.

369 Deuxième notice sur la faune ichthyologique de l'île de Harouko.

Ned. Tijdschr. Dierk. III, I866, p. 4t-42.

370 Description de quelques espèces inédites des genres Pseudorhombus et Platophrys de l'Inde archipélagique.

Ned. Tïjdschr. Dierk. III, I866, p. 43-50.

37 I Description d'une espèce inédite de Triacanthus de l'Inde archipélagique.

Ned. Tijdschr. Dierk. III, r866, p. 5i-52.

372 Sur les espèces d'Exocet de l'Inde archipélagique.

Ned. Tijdschr. Dierk. III, I866, p. I05-I29.

373 Description d'une espèce inédite d'Exocet découverte par M. Francois Pollen.

Ned. Tijdschr. Dierk. III, I866, p. ז30-133.

374 Description d'une espèce inédite de Cheilinus.

Ned. Tijdschr. Dierk. III, I866, p. I34-I35.

375 Révision des Hémirhamphes de l'Inde archipélagique.

Ned. Tijdschr. Dierk. III, I866, p. I36-I70. 
376 Description du Narcacion Polleni, espèce inédite des mers de l'île de la Réunion.

Ned. Tijdschr. Dierk. III, I866, p. 171-173.

377 Notice sur le Cirrhites punctatus CV.

Ned. Tijdschr. Dierk. III, I866, p. I74-177.

378 Description d'une espèce inédite de Stolephorus de Suriname.

Ned. Tijdschr. Dierk. III, 1866, p. I78-I80.

379 Révision des espèces de Mastacembelus (Belone Cuv.) de l'Inde archipélagique.

Ned. Tijdschr. Dierk. III, I866, p. 214-236.

3 So Sur la pluralité des espèces Indo-archipélagiques du genre Megalops.

Ned. Tijdschr. Dierk. III, I866, p. 278-292.

${ }_{3} S$ I Description de quelques espèces inédites ou peu connues de Clupéoides de l'Inde archipélagique.

Ned. Tijdschr. Dierk. III, I866, p. 293-308.

T3 382 Quatrième notice sur la faune ichthyologique de l'île de Halmahéra.

Arch. Néerl. Sc. Nat. II, I867, p. 397-399.

${ }_{3} S_{3}$ Description et figure d'une espèce inédite de Crossorhinus de l'archipel des Moluques.

Arch. Néerl. Sc. Nat. II, I867, p. 400-402.

$3 S_{4}$ Description de quelques espèces nouvelles de Gobius de Madagascar.

Arch. Néerl. Sc. Nat. II, I867, p. $403-420$.

${ }_{3} S_{5}$ Description de deux espèces inédites de Choerops.

Arch. Néerl. Sc. Nat. III, I868, p. 273-277.

386 Description d'une espèce de Rhombodites de l'île de la Réunion.

Arch. Néerl. Sc. Nat. III, I868, p. $277-278$.

${ }_{3} 87$ Description et figure d'une nouvelle espèce de Trachypterus de l'île d'Amboine.

Arch. Néerl. Sc. Nat. III, I868, p. 279-280.

388 Notice sur la faune ichthyologique de l'île de Guébé.

Versl. Akad. Amsterdam (2) II, I868, p. 27 I-272.

INDEX BLEEKER. 
389 Douzième notice sur la faune ichthyologique de l'île de Ternate.

Versl. Akad. Amsterdam (2) II, I868, p. 273-274.

390 Troisième notice sur la faune ichthyologique de l'île d'Obi.

Versl. Akad. Amsterdam (2) II, I868, p. 275.

39x Huitième notice sur la faune ichthyologique de l'île de Batjan.

Versl. Akad. Amsterdam (2) II, r868, p. 276-277.

392 Description de deux espèces nouvelles de Blennioïdes de l'Inde archipélagique.

Versl. Akad. Amsterdam (2) II, I868, p. 278-280.

393 Troisième notice sur la faune ichthyologique de la Nouvelle-Guineé.

Versl. Akad. Amsterdam (2) II, I868, p. 28I-282.

394 Cinquième notice sur la faune ichthyologique de l'île de Solor.

Versl. Akad. Amsterdam (2) II, I868, p. 283-288.

395 Sixième notice sur la faune ichthyologique de l'île de Bintang.

Versl. Akad. Amsterdam (2) II, I868, p. 289-294.

396 Notice sur la faune ichthyologique de l'île de Waigiou.

Versi. Akad. Amsterdam (2) II, I868, p. 295-30I.

397 Deuxième notice sur la faune ichthyologique des îles Sangir.

Versl. Akad. Amsterdam (2) II, I868, p. 302-304.

398 Deuxième notice sur la faune ichthyologique des îles Arou.

Versl. Akad. Amsterdam (2) II, I868, p. 305-306.

399 Description de trois espèces inédites de Chromidoïdes de Madagascar.

Versl. Akad. Amsterdam (2) II, I868, p. 307-314.

400 Description de trois espèces inédites de poissons des îles d'Amboine et de Waigiou.

Versl. Akad. Amsterdam (2) II, I868, p. 331-335. 
B 40 I Description de deux espèces inédites d'Epinephelus rapportées de l'île de la Réunion par M.M. Pollen et van Dam.

Versl. Akad. Amsterdam (2) II, I868, p. 336-34I.

402 Notice sur le Parupeneus bifasciatus (Mullus bifasciatus Lac.) de l'île de la Réunion.

Versl. Akad. Amsterdam (2) II, I868, p. $342-348$.

e 403 Sur les espèces confondues sous le nom de Genyoroge bengalensis Gthr.

Versl. Akad. Amsterdam (2) III, I869, p. 64-77.

Is 404 Description d'une espèce inédite de Caesio de l'île de Nossibé.

Versl. Akad. Amsterdam (2) III, I869, p. 78-79.

405 Description d'une espèce inédite de Chaetopterus de l'île d'Amboine.

Versl. Akad. Amsterdam (2) III, I869, p. 80-85.

406 Description d'une espèce inédite de Glyphidodón de l'île de la Réunion.

Versl. Akad. Amsterdam (2) III, I869, p. 23r-233.

407 Description de deux espèces inédites d'Alticus de Madagascar.

Versl. Akad. Amsterdam (2) III, I869, p. 234-236.

408 Neuvième notice sur la faune ichthyologique du Japon.

Versl. Akad. Amsterdam (2) III, I869, p. 237-252.

409 Description et figure d'une espèce inédite de Platycèphale.

Versl. Akad. Amsterdam (2) III, I869, p. 253-254.

4 IO Description et figure d'une espèce inédite de Rhynchobdella de Chine.

Versl. Akad. Amsterdam (2) IV, I870, p. 249-250.

4I I Mededeeling omtrent eenige nieuwe vischsoorten van China.

Versl. Akad. Amsterdam (2) IV, IS70, p. 25I-253.

4 I 2 Description d'une espèce inédite de Botia de Chine et figures du Botia elongata et du Botia modesta.

Versl. Akad. Amsterdam (2) IV, 1870, p. 254-256. 
4 I 3 Description et figure d'une espèce inédite de Hemibagrus de Chine.

Versl. Akad. Amsterdam (2) IV, 1870, 3. 257-258.

414 Description de deux espèces inédites de Labroïdes d'Amboine.

Arch. Néerl. Sc. Nat. VI, ז87 I, p. 326-328.

4 5 Mémoire sur les Cyprinoïdes de Chine.

Verh. Akad. Amsterdam XII, I87I, p. I-9I. Quoque separatim: Amsterdam, C. G. van der Post, I87 I.

4r6 Notice sur les peintures chinoises de Cyprinoïdes déposées au Muséum de l'université de Groningue par M. J. Senn van Basel.

Versi. Akad. Amsterdam (2) VI, I872, p. II7-I2I.

4 I 7 Sur le genre Moronopsis Gill (Paradules Blkr.) et ses espèces indo-archipélagiques.

Arch. Néerl. Sc. Nat. VII, 1872, p. 373-380.

4 I $S$ Description de quelques espèces inédites de Xenocypris.

Ned. Tijdschr. Dierk. IV, I873, p. 62-69:

4 I9 Description de trois espèces inédites du genre Acanthorhodeus Blkr.

Ned. Tijdschr. Dierk. IV, I873, p. $70-76$.

420 Sur les espèces du genre Culter Basil.

Ned. Tijdschr. Dierk. IV, I873, p. 77-88.

42 I Notice sur le Synodus macrocephalus Lac. (Luciobrama typus Blkr.).

Ned. Tijdschr. Dierk. IV, 1873, p. 89-91.

422 Description de quelques espèces de poissons de l'île de la Réunion et de Madagascar.

Ned. Tijdschr. Dierk. IV, 1873, p. 92-105.

423 Notice sur les Grammistes punctatus et ocellatus. Ned. Tijdschr. Dierk. IV, I873, p. ro6-110.

424 Description d'une espèce inédite de Heliases d'Amboine.

Ned. Tijdschr. Dierk. IV, 1873 , p. тाI-II2.

425 Mémoire sur la faune ichthyologique de Chine.

Ned. Tijdschr. Dierk. IV, I873, p. II3-I54. 
426 Révision des espèces inclo-archipélagiques du groupe des Anthianini.

Ned. Tijdschr. Dierk. IV, 1873, p. I55-169.

13 427 Révision des espèces indo-archipélasiques du groupe des Priacanthini.

Ned. Tijdschr. Dierk. IV, IS73, p. I70-I77.

428 Révision des espèces indo-archipélagriques du genre Myripristis.

Ned. Tijdschr. Dierk. IV, I873, p. I78-i 97 .

429 Révision des espèces indo-archipélagiques du genre Holocentrum.

Ned. Tijdschr. Dierk. IV, I873, p. 198-232.

430 Addition au Mémoire sur la faune ichthyologique de Chine.

Ned. Tijdschr. Dierk. IV, I873, p. 233-234.

43 I Sur les espèces indo-archipélagiques d'Odontanthias et de Pseudopriacanthus.

Ned. Tijdschr. Dierk. IV, I873, p. 235-240.

B 432 Révision des espèces indo-archipélagiques des genres Plectorhynchus et Pristipoma.

Ned. Tijdschr. Dierk. IV, I873, p. 273-317.

+33 Révision des espèces indo-archipélagiquès du genre Lethrinus.

Ned. Tijdschr. Dierk. IV, I873, p. 318-344.

434 Sur les espèces de Scolopsis Cuv. de l'Inde archipélagique.

Ned. Tijdschr. Dierk. IV, I873, p. 345-37I.

435 Révision des espèces insulindiennes du genre Therapon.

Ned. 'Tijdschr. Dierk. IV, I873, p. 372-393.

436 Description et figure du Cichla temensis Humb.

Versl. Akad. Amsterdam (2) VII, I873, p. 32-34.

437 Troisième notice sur la faune ichthyologique des îles Arou.

Versl. Akad. Amsterdam (2) VII, I873, p. 35-39.

+3S Mededeelingen omtrent eene herzicning der Indisch- 
Archipelagische soorten van Epinephelus, Lutjanus, Dentex en verwante geslachten.

Versl. Akad. Amsterdam (2) VII, I873, p. 40-46.

439 Description et figure d'une espèce insulindienne d'Orthagoriscus.

Versl. Akad. Amsterdam (2) VII, I873, p. I5I-I 53.

440 Révision des espèces insulindiennes des genres Diapterus et Pentaprion.

Versl. Akad. Amsterdam (2) VII, I873, p. 233-255.

44I Révision des espèces de Dentex, Synagris, Gymnocranius, Gnathodentex et Pentapus de l'Inde archipélagique et du Japon.

Verh. Akad. Amsterdam XIII, 1873, p. x-64.

442 Révision des espèces indo-archipélagiques des genres Lutjanus et Aprion.

Verh. Akad. Amsterdam XIII, I873, p. I-I02,

443 Sur le genre Parapristipoma et sur l'identité spécifique des Perca trilineata Thunb., Pristipoma japonicum $\mathrm{Cv}_{\mathrm{v}}$. et Diagramma japonicum Blkr.

Arch. Néerl. Sc. Nat. VIII, I873, p. I9-24.

444 Description et figure du Lethrinus Güntheri Blkr.

Arch. Néerl. Sc. Nat. VIII, I873, p. I53-I54.

445 Révision des espèces Indo-archipélagiques du genre Caesio et de quelques genres voisins.

Arch. Néerl. Sc. Nat. VIII, I873, p. 155-182.

446 Description de deux espèces nouvelles de Sciénoïdes de Surinam.

Arch. Néerl. Sc. Nat. VIII, 1873, p. 456-461.

447 Description d'une espèce inédite de Gadus (Boreogadus) des mers d'Islande.

Arch. Néerl. Sc. Nat. VIII, I873, p. 462-463.

448 Mededeeling omtrent den stand der uitgave van den Atlas ichthyologique des Indes orientales.

Versl. Akad. Amsterdam (2) VIII, 1874, p. I23-r26.

449 Typi nonnulli generici piscium neglecti.

Versl. Akad. Amsterdam (2) VIII, I874, p. 367-37I. 
t 450 Notice sur les genres Amblyeleotris, Valenciennesia et Brachyeleotris.

Versl. Akad. Amsterdam (2) VIII, I874, P. 372-376.

451 Révision des especes indo-archipélagiques du groupe des Epinephelini et de quelques genres voisins.

Verh. Akad. Amsterdam XIV, I874, p. I-I 34.

$45^{2}$ Mémoire sur les Sciénoïdes et les Sillaginoïdes de l'Inde archipélagique.

Verh. Akad. Amsterdam XIV, I 874 , p. I -76 .

B 453 Esquisse d'un système naturel des Gobioïdes.

Arch. Néer. Sc. Nat. IX, I874, p. 289-331.

B 454 Sur les espèces insulindiennes de la famille des Nandoïdes.

Arch. Néerl. Sc. Nat. IX, I874, p. 455-465.

455 Sur les espèces insulindiennes de la famille des Opisthognathoïdes.

Arch. Néerl. Sc. Nat. IX, I874, p. 466-476.

$45^{6}$ Révision des espèces indo-archipélagiques du groupe des Apogonini.

Nat. Verh. Holl. Maatsch. Wetensch. 3. verz. II, No . I, I874, p. $x-82$.

Te B 457 Rérision des espèces d'Ambassis et de Parambassis de l'Inde archipélagique.

Nat. Verh. Holl. Maatsch. Wetensch. 3. verz. II, N ${ }^{0}$. 2, 1874, p. 83 - ro6.

${ }_{45}$ S Révision des espèces insulindiennes de la famille des Synanceoïdes.

Nat. Verh. Holl. Maatsch. Wetensch. 3. verz. II, $\mathrm{N}^{0} .3, \mathrm{I} 874$, p. I-22.

$\checkmark 459$ Recherches sur la faune de Madagascar et de ses dépendances d'après les découvertes de François P. L. Pollen et D. C. van Dam. $4^{\text {e Partie. Pois- }}$ sons de Madagascar et de l'île de la Réunion. Leide, I 875 .

Continet: Enumération des espèces de poissons actuellement connues du groupe géographique de Madagascar.

R B 460 Notice sur les Eleotriformes et description de trois espèces nouvelles.

Arch. Néerl. Sc. Nat X, I875, p. IOI-II2. 
46I Gobioideorum species insulindicae novae.

Arch. Néerl. Sc. Nat. X, I875, p. I I3-I34.

462 Description du genre Parascorpis et de son espèce type. Arch. Néerl. Sc. Nat. X, I875, p. $380-382$.

463 Sur les espèces insulindiennes de la famille des Cirrhitéoïdes.

Verh. Akad. Amsterdam XV, I875, p. I-20.

464 Révision des espèces insulindiennes de la famille des Mulloïdes.

Verh. Akad. Amsterdam XV, I875, p. I-40.

13465 Sur la famille des Pseudochromidoïdes et révision de ses espèces insulindiennes.

Verh. Akad. Amsterdam XV, 1875, p. I-32.

466 Notice sur l'Elopichthys dahuricus.

Proc. Zool. Soc. London I 875, p. 534-536.

467 Notice sur le genre Chaetodon Art. (Pomacanthus Lac. Cuv.) et sur la pluralité de ses espèces vivantes. Arch. Néerl. Sc. Nat. XI, ז876, p. $178-185$.

468 Systema Percarum revisum.

Arch. Néerl. Sc. Nat. XI, 1876, Pars I. p. 247-288; Pars II. p. $289-340$.

469 Description de quelques espèces insulindiennès inédites des genres Oxyurichthys, Paroxyurichthys et Cryptocentrus.

Verh. Akad. Amsterdam (2) IX, I876, p. ${ }_{3} 8-$ I48.

470 Notice sur les genres Gymnocaesio, Pterocaesio, Paracaesio et Liocaesio.

Versl. Akad. Amsterdam (2) IX, I876, p. I49-154.

47 I Sur la pluralité des espèces insulindiennes de Toxotes.

Versl. Akad. Amsterdam (2) IX, 1876, p. 155-167.

472 Révision des Sicydiini et Latrunculini de l'Insulinde.

Versl. Akad. Amsterdam (2) IX, 1876, p. 27 I-293.

473 Genera familiae Scorpaenoideorum conspectus analyticus.

Versl, Akad. Amsterdam (2) IX, I876, p. 294-300. 
R B 474 Notice sur les genres et sur les espèces des Chétodontoïdes de la sousfamille des Taurichthyiformes.

Versl. Akad. Amsterdam (2) X, r876, p. 308-320.

Th B. 475 Mémoire sur les espèces insulindiennes de la famille des Scorpénoïdes.

Verh. Akad. Amsterdam XVI, I876, p. г-гоo.

T B 476 Description de quelques espèces inédites de Pomacentroïdes de l'Inde archipélagique.

Versl. Akad. Amsterdam (2) X, I876, p. 384-39r.

B 477 Sur les espèces confondues sous les noms de Chrysophrys hasta, berda, calamara et Schlegeli.

Versl. Akad. Amsterdam (2) XI, I877, p. I-I2.

B 478 Révision des espèces insulindiennes de la sousfamille des Eleotriformes.

Versl. Akad. Amsterdam (2) XI, I877, p. I3-r ro.

B 479 Notice sur l'identité des genres Gnathanacanthus Blkr. et Holoxenus Gthr.

Versl. Akad. Amsterdam (2) XI, I877, p. r32-r34.

4So Description de deux espèces inédites du genre Prochilus Klein (Amphiprion Bl. Schn.).

Versl. Akad. Amsterdam (2) XI, I877, p. I35-r37.

R 3 48 Révision des espèces insulindiennes de la famille des Chétodontoïdes.

Verh. Akad. Amsterdam XVII, I877, p. I-I74.

$\bar{E}+82$ Notice sur la sousfamille des Holacanthiformes et description de quelques espèces insuffisamment connues.

Arch. Néerl. Sc. Nat. XII, I877, p. I7-37.

Z $34_{3} 8_{3}$ Notice sur les espéces nominales de Pomacentrö̈des de l'Inde archipélagique.

Arch. Néerl. Sc. Nat. XII, I877, p. 38-4I.

+84 Révision des espèces de Pempheris de l'Inde archipélagique.

Arch. Néerl. Sc. Nat. XII, I 877 , p. 42-54.

T: 485 Mémoire sur les Chromides marins ou Pomacentroïdes de l'Inde archipélagique.

Nat. Verh. Holl. Maatsch. Wetensch. 3e Verz. II, No.6, 1877 , p. $x-166$. 
- 486 Vischsoorten van Nieuw-Guinea, van Singapore, China, Japan en Mauritius.

Versl. Akad. Amsterdam, Proc.-Verb. 27 Oct. 1877.

$48_{7}$ Over slokdarm en maag van Caprodon Schlegeli.

Versl. Akad. Amsterdam, Proc.-Verb. 24 Nov. 1877.

488 Sur deux espèces inédites de Cichloïdes de Madagascar.

Versl. Akad. Amsterdam (2) XII, I878, p. I92-I98.

489 Description des espèces insulindiennes du genre Stigmatogobius.

Versl. Akad. Amsterdam (2) XII, r878, p. 199-208.

490 Sur les espèces du genre Hypophthalmichthys Blkr. (Cephalus Basil. nec Bl. nec Al.).

Versl. Akad. Amsterdam (2) XII, I878, p. 209-2I8.

v49 I Quatrième mémoire sur la faune ichthyologique de la Nouvelle-Guinée.

Arch. Neerl. Sc. Nat. XIII, I878, p. 35-66.

492 Notice sur le Sparus Cuvieri (Chrysophrys Cuvieri Day).

Versl. Akad. Amsterdam (2) XIII, I878, p. 43-46.

493 Révision des espèces insulindiennes du genre Uranoscopus L.

Versl. Akad. Amsterdam (2) XIII, I878, p. 47-59.

494 Révision des espèces insulindiennes de la famille des Callionymoïdes.

Versl. Akad. Amsterdam (2) XIV, I879, p. 79-107.

495 Contribution à la faune ichthyologique de l'île Maurice.

Verh. Akad. Amsterdam XVIII, I879, p. I-23.

496 Sur quelques espèces inédites ou peu connues de Poissons de Chine appartenant au Muséum de Hambourg.

Verh. Akad. Amsterdam XVIII, 1879, p. I-I7.

497 Enumération des espèces de poissons actuellement connues du Japon et description de trois espèces inédites.

Verh. Akad. Amsterdam XVIII, I879, p. I-33. 
${ }_{49} 8$ Mémoire sur les Poissons à pharyngiens labyrinthiformes de l'Inde archipélagique.

Verh. Akad. Amsterdam XIX, 1879, p. I-56.

499 Révision des espèces insulindiennes du genre Platycephalus.

Verh. Akad. Amsterdam XIX, I879, p. I-3I.

500 Musei Hamburgensis species piscium novas minusque cognitas descripsit et depingi curavit P. Bleeker. Pisces novae-guinaici novi.

Abh. naturw. Ver. Hamburg VII, I. Abth. I880, p. 25-30.

\section{Papers arranged according to localities.}

\section{Ambon.}

54. 66. 72. 84. I03. I 28.142 . 163. I79. 180. 195. 249.256. 273. 274. $321.361 \cdot 364 \cdot 374 \cdot 387 \cdot 400$. 405. 409. 414. 423. 424 .

\section{Aru Islands.}

257. 34I. 398. 437 .

\section{Australia.}

I I 9. 233. $297 \cdot 331 \cdot 355$.

Bali.

I7. 159. 23I. $250,284$.

Banda Islands.

36. 47. 68. 95. I I5. I 49, $22 \mathrm{r}$.

\section{Banka.}

32. $56.70,75.82,123.152$. I 58.213 .235 .267 .281 .305$.

\section{Batjan.}

II 2. I35. I4I. I50, I68, 205. 260. 296. 308. 39I.

\section{Batu Island.}

I25. I3I. I 57 .

\section{Bawean.}

234. 285 .

\section{Bengal.}

91. $233 \cdot 466.477 \cdot 492$.

\section{Biliton.}

40. 52, I65, I85, $215,246$.

\section{Bintang.}

144. 238.395 .

\section{Borneo.}

26. 33. 35. 42. $45 \cdot 49 \cdot 55 \cdot \delta_{5}$. I 2 I. I39. I62. I78. I9I. 201. 202. 204. 210.229 .245 .272$.

\section{Buru.}

I 5 I. I64. 282.354 .

Cape of Good Hope.

$233,266,333,363.462$.

Celebes.

I0. 46. 7I. 8I. I09. II4. I29. I37. I 43. I 54. I76. 177. 192. 240. 24I. 243. 258.275 .

\section{Ceram.} $35^{8}$.

54. 69. $83.153,160,283.3$ I9.

\section{Ceylon.}

326. $327 \cdot 335$.

\section{China.}

307. 342. 343. 345. 349. 4 IO. 4II. 4I2. 4I3. 4I5. 4I6. 418. 4I9. 420. 42 I. $425 \cdot 430.443 \cdot 486.490$. 496.

\section{Cocos Islands.}

105. II I. 122, I30. I88, 203. 209. 242.247.

\section{Duizend Isles.} 126 .

Europo.

325. 447 . 
Flores.

99. 318.

Goram Archipelago. I 84 .

Gébé.

388.

\section{Guinea.}

291. $297 \cdot 302 \cdot 3^{6} 5$.

\section{Halmahera.}

79. 94. I32. 309. 382 .

Haruku.

350. 369 .

Indo-Australian Archipelago.

I3. I4. I 5. I8, I9, 20, $21,22$. 23. 24. 27. 28. 30. 34. 37. 38. 39 . 44. 57. 58. 59. 60. 61. 62. 63. 64. 88. 89. 92. II7. I89. 214.218. 220. 233. $265 \cdot 290.293 \cdot 301.3 I 1$. 312. 313. 324. 334. 348. 352.368. 370. 37 I. $372.375 \cdot 379 \cdot 380.38$ r. 385. 392. 403. 4I7. 426. 427. 428. 429. 43 I. 432 . 433. 434. 435.438 . 439. 440. 44 I. 442 . 445.448 .450$. 45 I. 452 . $454.455 \cdot 456.457 \cdot 45^{8}$. 460. 46r. 463. 464. 465. 469. $47 \mathrm{r}$. 472. $475.476 .477 \cdot 478,480.481$. 482. $483.484 .485 \cdot 489.493 \cdot 494$. 498. 499 .

\section{Japan.}

90. 93. IO2. II8. I75. I 93233. 253. 269. 297. 328. 363. 408. 44 I. 443. 444. 477.486 .497$.

Java.

I. 2. 3. 4. 5. 6. 7. 8. I I. I 2. 25. 29. 31. $43 \cdot 74 \cdot 77 \cdot 78.87 \cdot 97 \cdot 100$. 104. I IO. I I3. I38. I 48. I66. I 70. I71. I82. I83. I87. I96. I98. 200. 207. 2 II. 2I9. 222. 225. 227. 228. 236. $248.255 \cdot 277 \cdot 279 \cdot 322.353$.

Kei Islands.

339. 385 .

Madagascar.

384. 399. 407. 422. 459. 488 .

Madura. I6.

Mauritius. 486. 495 .
Morotai. 310.

Natuna Islands. I08.

New Guinea. 232. 278 . 393. 486. 49I. 500.

Nias. I 56 .

Nossibé. 404.

Nusa Laut. 340.

Philippine Islands. 346.

\section{Ponang.} 288 .

Obi major. I40. 316.390 .

\section{Réunion.}

376. 377.385 .386 .401 .402 . 403. 406. 422 . 423. 459.

\section{Riouw.}

50. 199 .

Rotti.

3 I 7 .

Sangi Islands.

I67. 397 .

\section{Saparua.}

I 47.338 .

Siam.

$239 \cdot 337 \cdot 343 \cdot 344 \cdot 347 \cdot 356 \cdot 360$.

\section{Singapore.}

4I. 5I. I86. 25 I. 254.263. 268 . 280. 287.486 .

\section{Solombo. 146.}

Solor. 48. 65. 80. 101. 394.

Sumatra.

67. 74. 76. 86. 106. 127. 136. I 74. I90. I94. 206. 207. 208. 2 I 2. $216.217,223,224,226,244,252$. 270. 271.276 .

\section{Sumbawa.}

9. 133.237 . 
Surinam.

297. 298. 303. 332. 336. 362. $363 \cdot 366.378 .446 .467$.

\section{Ternate.}

73. 79. 96. I 24. I 34.145. I 55 . 295. 3I 5. 330. 389 .

\section{Timor.}

53. 98. 169. 230. 262. 286.320.

\section{Varia.}

107. I 6 . I20. 172. $173.18 \mathrm{I}$. 197. 259. 26 r. 264. 289. 29r. 292. 294. 297. 299. 300. 304. 306. 314. 323. 329. 351. 359. 365. 367.373. 403. $436.449 \cdot 453 \cdot 460 \cdot 467 \cdot 468$. 470. 473. 474. 479. 482. 487 .

Waigeu.

396. 400 . 

INDEX.

GENERUM ET SPECIERUM. 

The numbers in heavy type refer to the numbers of the papers quoted in the Index bibliographicus, the numbers in small type to the pages.

Abeona Gir. 468, 290.

Abramis Cuv. 261, 436. 314, 2 11. 329, 263.

Abramis bramula Rich. cf. Parabramis bramula Blkr.

Abramis Buggenhagii 325, 237.

Abramis erythropterus 325, 237.

Abramis Leuckartii 325, 237.

Abramis mantschuricus Bas. 425, I46. cf. Parabramis bramula Blkr.

Abramis pekinensis Bas. cf. Parabramis pekinensis Blkr.

Abramis persa 325, 237.

Abramis rhomboidalis Rich. 425, 146. cf. Parabramis bramula Blkr.

Abramis tenellus $325,237$.

Abramis terminalis Rich. 425, I46. cf. Parabramis bramula Blkr.

Abramocephalus microlepis Stivit. cf. Hypophthalmichthys microlepis Blkr.

Abrostomus Smith 261, 424. 314, I93.

Abrostomus capensis Smit/2 266, 56 .

Abrostomus umbratus Snith 266, 56 .

Acanthaluterus Blkr. 367, r $3 .^{3}$

Acantharchus Gill. 468, 249.

Acantharchus pomotis Gill. 468, 249.

Acanthias Blainvillei Risso. 266, 80.

Acanthias vulgaris Risso. 119, I8. 266, 79. 459, 6S. cf. Spinax acanthias Flem.

Acanthicus Spix. 306, 79.

Acanthicus hystrix Spix. 306, 7.9.

Acanthistius Gill. 468, $25^{6 .}$

Acanthistius leopardinus Blkr. 425, I38. cf. Paracanthistius leopardinus Blkr.

Acanthistius maculatus Blkr. 315, 232. cf. Paracanthistius maculatus Blkr.

Acanthistius oligacanthus Blkr. 364, 281. cf. Paracanthistius oligacanthus Blkr.

Acanthistius serratus Gill. 331, 445. 468, 256.

Acanthobrama Heck.261, 432. 314, 2 10. 411, 253.

Acanthobrama pekinensis Blkr. cf. Parabramis pekinensis Blkr.

Acanthobrama Simoni Blkr. 345, 25. cf. Xenocypris Simoni Blkr. 
Acanthocepola Blkr.449, 369 .

Acanthocepola Krusensterni Blkr. 497, I8.

Acanthocepola mesoprion Blkr. 497, I8.

Acanthocepola oxylepis Blkr. 487, 35. 496, 8.

Acanthochaetodon Blkr. 468, 308 .

Acanthochaetodon alternans Blkr. 482, 27.

Acanthochaetodon annularis Blkr. 481, I52. 482, 2 I.

Acanthochaetodon asfur Blkr. 482, 29.

Acanthochaetodon chrysurus Blkr. 482, 2 I.

Acanthochaetodon imperator Blkr. 481, I 50. 482, 2 1.491, 45.495, I4.

Acanthochaetodon lepidolepis Blkr. 481, I43. 482, 2 I.

Acanthochaetodon maculosus Blkr. 482, 32.

Acanthochaetodon mokhella Blkr. 482, $2 \mathrm{I}$.

Acanthochaetodon nicobariensis Blkr. 481, 148. 482, 2 I. 495, r4.

Acanthochaetodon semicirculatus $B l k r .481$, I46. 482, 2 I. 491, 45 .

Acanthochaetodon striatus Blkr. 481, r 45. 482, 21.

Acanthochromis polyacanthus Gill. 485, I5I.

Acanthoclinus fuscus Jen. 119, I 2.

Acanthocobitis Pet. 323, 37 .

Acanthocobitis bilturio Blkr. 323, 40.

Acanthocobitis longipinnis Pet. 323, 40.

Acanthocobitis monoceros Blkr. 323, 40.

Acanthocobitis mooreh Blkr. 323, 40.

Acanthocobitis pavonacea Blkr. 323, 40.

Acanthocobitis rubridipinnis Blkr. 323, 4 o.

Acanthocobitis Rüppelli Blkr. 323, 40.

Acanthocobitis semizonata Blkr. 323, 40.

Acanthocybium sara Gill. 497, I5.

Acanthodoras Blkr. 306, 86.

Acanthodoras cataphractus Blkr. 303, 17. 306, 86. 336, 40.

Acanthodoras Hancocki Blkr. 303, I 7 .

Acanthogobius Gill. 453, 320.

Acanthogobius eleotris Blkr. 425, I 28 .

Acanthogobius flavimanus Gill. 408, 238. 497, I9.

Acanthogobius ommaturus Blkr. 425, I28.

Acanthogobius stigmothonus Blkr. 425, I28.

Acanthogobius tannao Blkr. 425, $\mathbf{2} 28$.

Acantholabrus $V a l .292$, ro7.

Acantholatris Gill. 468, 315.

Acanthonotus Gray 306, I I7. cf. Ailia Gray.

Acanthonotus Hardwickii Gray cf. Ailia coila Blkr.

Acanthinion orbicularis Lac. cf. Platax teira Cuv.

Acanthophthalmus $v$. Hass. 220, 304. 261, 422. 323, 38.

Acanthophthalmus fasciatus $v$. Hass. 271, 2. 323, 42. cf. Cobitis

Kuhlii C.V. 
Acanthophthalmus javanicus $K$. $\%$. H. 270, 50. 323, 42. cf. Cobitis oblonga K. v. H.

Acanthophthalmus pangia Blkr. 323, 42.

Acanthopodus Boddaerti Lac. cf. Holacanthus diacanthus Gthr.

Acanthopsis $\%$. Hass. 220, 303. 261, 421. 323, 38.

Acanthopsis choirorhynchos Blkr. 270, 50. 323, 42.

Acanthopsis dialyzona v. Hass. 2, 513. 272, 21. 323, 42. cf. Cobitis macrorhynchos Blkr.

Acanthopsis spectabilis Blkr. 323, 42 .

Acanthorhinus moluccensis Blkr. 364, 270.

Acanthorhodeus 411, 253 .

Acanthorhodeus Guichenoti Blkr. 415, 4r. 419, 73. 425, I 44 .

Acanthorhodeus hypselonotus Blkr. 415, 43. 419, 74. 425, I 44.

Acanthorhodeus macropterus Blkr.411, 252.415, 40.419, 7 I.425, I 44 .

Acanthorhodeus taenialis Gïnth. 496, I2.

Acanthostracion Blkr. 367, I 5 .

Acanthurus 1, 553 .

Acanthurus achilles $C$. $V$. cf. Rhombotides achilles Blkr.

Acanthurus ali ala Less. cf. Acanthurus glaucopareius $C . V$.

Acanthurus altivelis $C$. $V$. cf. Harpurus rhombeus Blkr.

Acanthurus annularis $C . V$. cf. Rhombotides matoides Blkr.

Acanthurus bariene Less. cf. Rhombotides nummifer Blkr.

Acanthurus bipunctatus Gthr. cf. Rhombotides bipunctatus Blkr.

Acanthurus Bleekeri Gthr. 309, I56.

Acanthurus Blochii $C$. $V$. cf. Chaetodon nigricans $\mathrm{Bl}$., Rhombotides matoides Blkr.

Acanthurus celebicus Blkr. 71, 76r. 275, 36. cf. Rhombotides celebicus Blkr.

Acanthurus chrysosoma Blkr. 164, 67. 250, 206.

Acanthurus ctenodon $C . V$. 309, I56. 315, 235. 318, 252. 320, $27 \pi$. 354 , I 49. 358, г9о. 364, 288. 394, 288. 437, 37. 491, 51.

Acanthurus Delisiani $C . V .91,48$. cf. Acanthurus leucosternon Benn.

Acanthurus Delisianus $C$. $V$. cf. Rhombotides leucosternon Blkr.

Acanthurus Desjardinii Benn. cf. Harpurus Desjardini Blkr.

Acanthurus doreënsis $C$. $V$. cf. Rhombotides doreënsis Blkr.

Acanthurus Dussumieri $C$. $V$. cf. Rhombotides Dussumieri Blkr.

Acanthurus eparai Less. cf. Acanthurus humeralis C. $V$.

Acanthurus flavescens Benn. cf. Harpurus rhombeus Blkr.

Acanthurus fuliginosus Less. 71, 762 .

Acanthurus gahm (Ehr.) C. $V$. 176, 8. 275, 36. cf. Rhombotides gahm Blkr.

Acanthurus gahmoides Guich. cf. Rhombotides gahmoides Blkr.

Acanthurus gemmatus $C . V$. cf. Harpurus gemmatus $B l k r$.

Acanthurus glaucopareius $C: V .142,47$.

Acanthurus goramensis Blkr.184, 208. 
Acanthurus grammoptilus Rich. 119, I I.

Acanthurus guttatus Bl. Schn. cf. Rhombotides guttatus Blkr.

Acanthurus harpurus Shaze. cf. Naseus lituratus $C . V$.

Acanthurus hepatus Bl. Schn. 99, 325. 270, 32. 275, 36. cf. Paracanthurus hepatus Blkr.

Acanthurus hirudo Benn. cf. Acanthurus triostegus Bl. Schn.

Acanthurus humeralis $C . V .71,762$. 184, 200. 275, 36. cf, Rhombotides olivaceus Blkr.

Acanthurus hypselopterus Blkr. 99, 327.

Acanthurus Lamarrii $C$. $V$. cf. Rhombotides Lamarrii Blkr.

Acanthurus leucosternon Benn. 157, 237. cf. Rombotides leucosternon Blkr.

Acanthurus lineatus Lac. 76, 263. 91, 48. 236, 331, 333. 270, 32. 275, 37. 330, 266. cf. Rhombotides lineatus Blkr.

Acanthurus lineolatus $C . V$. 95, ror.

Acanthurus lituratus $B l$. Schn. cf. Naseus lituratus $C$. $V$.

Acanthurus lunulatus Lien. cf. Harpurus lunulatus, Rhombotides lunulatus.

Acanthurus mata $C . V .91,48.113,432.231,147.275,37.296,108$. Acanthurus matoides $C . V$. 23. 12. 53, I6r. 113, 434. 167, 372. 171, 245. 184, 200. 188, 459. 231, 147. 234, 354. 236, 33 I, 333. 270, 32. 275,37 .

Acanthurus melanurus C. $V .54,271.91,48.157,233.170,478$. 231, 147. 236, 331. 275, 37. cf. Acronurus melanurus Gthr.

Acanthurus melas C. $V .91,48$.

Acanthurus nigricans Blkr. 113, 434, 296, ro8.

Acanthurus nigrofuscus $C$. $V$. cf. Rhombotides nigrofuscus $B l k r$.

Acanthurus nummifer $C$. $V$. cf. Rhombotides nummifer $B / k r$.

Acanthurus olivaceus $B l$. Schn. cf. Acanthurus humeralis $C . V$.

Acanthurus orbicularis $Q$. G. cf. Acronurus orbicularis Gthr.

Acanthurus pentazona Blkr. 23, 13. 31, 107.

Acanthurus plagiatus Pet. 495, I9.

Acanthurus rasi $C . V .91,48$. cf. Acanthurus matoides $C$. $V$.

Acanthurus rhombeus $K$ ittl. 309, I56. cf. Harpurus rhombeus $B l k \%$.

Acanthurus Rüppelli Benn.(Blkr. Gthr.). 125, 3r6. 130, 45 I. 275,

37. 330, 266. cf. Harpurus Rüppelli Blkr.

Acanthurus scalprum Langsd. ap. C. $V$. cf. Naseus scalprum Blkr.

Acanthurus scopas $C . V .48,348.157,233$. cf. Harpurus rhombeus Blkr.

Acanthurus strigosus Benn。 76, 264. 95, 102. 113, 433. 157, 233. 167, 372. 170, 478.182, х 59. 184, 200. 231, 147. 270, 32. 275, 37. 459, 96. 491, 51. 495, I9.

Acanthurus suillus $C . V$. cf. Harpurus suillus Blkr.

Acanthurus triostegus Bl. Schn. (C.V., Stens.) 23, I3. 54, 237. 91, 48. 119, Iт. 167, 372. 171, 245. 184, 200. 188, 459. 236, 33r, 
333. 270, 32. 275, 37. 285, 245. cf. Rhombotides triostegus Blkr. Acanthurus theuthis Lac. cf. Acanthurus hepatus Bl. Schn.

Acanthurus velifer Bl. Schn. (C.V., Rüpp). 91, 166. 105, 42. 119, I1. 125, 315. 157, 233. cf. Acanthurus Rüppellii Blkr., Harpurus Rüppelli Blkr.

Acanthurus vittatus Benn. cf. Acanthurus lineatus Lac.

Acanthurus xanthopterus $C$. V.283, 73. cf. Rhombotides matoides Blkr.

Acanthurus xanthurus Blyth. 91, 165.

Acanthurus zebra Lac. cf. Acanthurus triostegus Bl. Schn.

Acentrachme pachyacanthus Blkr. 364, 274.

Acentrachme scutata Blkr. 354, I44. 358, r84. 364, 274. 369, 42. cf. Centriscus scutatus $L$.

Acentrogobius Blkr. 453, 321 .

Acentrogobius leptochilus Blkr. 461, r3I.

Acentrogobius oligactis Blkr. 461, I32.

Acentrogobius ornatus Blkr. 491, 54 .

Acentrogobius Pflaumi Blkr. 497, I9.

Acentrogobius Yokohamae Blkr. 497, I9.

Acentronura gracillima $K p .497$, I3.

Acerina Cuv. 468, 266.

Acerina cernua Cuv. 468, 266.

Acerinaeformes. 468, 266.

Acestra Kner. 306, 82.

Acestra acus Kner. 306, 82 .

Acheilognathus Blkr. 261, 427. 269, 91. 314, 213.

Acheilognathus intermedius Blkr. 497, 23.

Acheilognathus lanceolatus Blkr. 497, 23. cf. Acheilognathus melanogaster Blkr.

Acheilognathus limbatus Blkr. 497, 23.

Acheilognathus melanogaster Blkr. 269, 92. 497, 23.

Achilognathus 411, 253.

Achilognathus imberbis Gthr. cf. Parachilognathus imberbis Blkr.

Achiroïdes leucorhynchos Blkr. 38, 4r I. 61, 20. 225, $35^{\text {S. }}$

Achiroïdes melanorhynchos Blkr. 38, 402. 45, 197. 49, 42I. 55, 4 I 4 . $61,19$.

Achiroïdes melanorhynchus $B l k r .35,262.42,60$.

Achirophichthys Blkr. 351, II8.

Achirophichthys typus Blkr. 348, 42. 352, I 26.

Achirus 1, 552 .

Achirus bilineatus Lai. cf. Plagusia quadrilineata $K . v . H$.

Achirus cynoglossus 91, 153 .

Achirus Hartzfeldii Blkr. 72, 123. 318, 250. 320, 265. 358, I 84. $364,274.491,55$.

Achirus japonicus Schl. 497, 22.

Achirus maculatus $K . v . H .2,509.16,6.25,12$. 
Achirus marmoratus Lac. 459, 77. 495, 21.

Achiroides melanorhynchos Blkr. 270, 62. 272, S.

Achirus melanospilos Blkr. 109, 257. 275, I4. 287, 4I. 296, 103.

Achirus melanospilus Blkr. 364, 274.

Achirus Mogki Blkr. 280, ror.

Achirus pavoninus Lac. 38, 402. 54, 238. 56, 446. 61, I8. 156, 2 I7. 270, 62. 275, I4. 287, 41. 288, 69. 296, Іо3. 358, т 84.437, 37. 496,3 .

Achirus plagusia Basil. cf. Cynoglossus trigrammicus Gthr. 425, I3I. Achirus poropterus Blkr. 38, 410. 61, I 9. 156, 2 I 7. 270, 62. 364, 274. Achirus Thepassii Blkr. 103, 500. 275, I4. 396, 298.

Acipenser mantschuricus Basil cf. Acipenser sinensis $G r$.

Acipenser sinensis $G r .425$, I 20.

Acomus Gir. 261, 428. 314, I89.

Acra Blkr. 314, 197.

Acrocheilus Ag. 261, 425. 314, 206.

Acrochordonichthys Blkr. 306, 105.

Acrochordonichthys melanogaster Blkr. 270, 47.

Acrochordonichthys platycephalus Blkr. 270, 47. 306, I05.

Acrochordonichthys pleurostigma Blkr. 270, 47.

Acrochordonichthys rugosus Blkr. 270, 47 .

Acrochordonichthys zonatus Blkr. 225, 357.

Acronurus anginosus Blkr. 459, 97.

Acronurus melanurus Gthr. 320, 27̣1. 358, I90. 364, 288. 491, 5 I.

Acronurus orbicularis Gthr. 425, I 34. 459, 97.

Acropoma Schl. 468, 260.

Acropoma apogonides Blkr. 468, 260.

Acropoma apogonoides Blkr. 497, 6 .

Actenolepis Dyboroski 468, 265 .

Actenolepis Ditmarii Dybozoski 468, 265.

Actinogobius Blkr. 453, 319.

Actinogobius ommaturus Blkr. 496, 3 .

Aesopia japonica Blkr. 269, 7 I. cf. Brachirus japonicus Blkr.

Aesopia zebra Kf. 267, I39. 270, 62. 272, S. 287, 4r. 288, 69. cf. Brachirus zebra Stons.

Aëtobatis flagellum $M . H .10,68.46,214.91,82.275$, Iо.

Aëtobatis indica Stons. cf. Aëtobatis narinari $M$. $H$.

Aëtobatis narinari $M . H .16,6.25,13.51,60.64,87.91,82.270$,

68. 275, го. $288,67.316,24$ г. 354, I 43. 425 , г $20.459,69.495,8$.

Aëtoplatea tentaculata $V a l .91,82$.

Aëtoplatea zonurus Blkr. 64, 79.

Ageneiosus Lac. 306, ro8.

Ageneiosus armatus Lac. cf. Ageneiosus militaris $\mathrm{Bl}$.

Ageneiosus brevifilis $V a l$. cf. Pseudageneiosus brevifilis $B l k r$.

Ageneiosus dentatus Kner. cf. Pseudageneiosus dentatus Blkr. 
Ageneiosus inermis Lac. cf. Pseudageneiosus inermis Blkr.

Ageneiosus militaris Blkr. 306, ro8. 336, 8r.

Ageneiosus quadrifilis $K n \in r$. cf. Tetranematichthys quadrifilis $B l k r$.

Aglyptosternon Blkr. 306, го3.

Aglyptosternon cous Blkr. 306, I04.

Agonostoma dobuloides Gthr. 459, 79. 495, I8.

Agonostoma Forsteri Gthr. 331, 448.

Agonostoma oxyrhynchus Gthr. 296, Iro. 358, I9I. 364, 29 r.

Agonostoma plicatile Gthr. 425, I43.

Agonostoma Telfairi Benn.459, 79. 495, r8.

Agonus laevigatus Tiles. 497, I3.

Agonus rostratus Tiles. cf. Brachyopsis rostratus Gill.

Agosia Gir. 261, 434. 314, 209.

Agrammus Schlegelii Gïnth.497, rr.

Agriopes spinifer Smith. 266, 52.

Agriopus C. $V .473,300$.

Agriopus leucopoecilus Rich. 119, 8. 487.

Agriopus melanosoma Blkr. 495, 6 .

Agriopus torvus C. $V .266,53.473,300$.

Agriopus verrucosus $C . V .266,53$.

Ailia Gray. 306, I I 7.

Ailia bengalensis Gr. cf. Ailia coila Blkr.

Ailia coila Blkr. 91, I I2. 306, I I 7.

Ailurichthys B. Gir. 306, 92 .

Akysis Blkr. 306, 105.

Akysis makronema Blkr. 271, I I.

Akysis variegatus Blkr. 271, I. 306, 105.

Alausa brachysoma Blkr. 86, 527. 270, 57 .

Alausa brevis Blkr. 60, 35. 237, 438 .

Alausa champil Cant. 91, 74. 288, 79.

Alausa chapra $C . V .91,74$.

Alausa ctenolepis Blkr. 32, I60. 51, 74. 56, 445. 60, 32. 270, 57.

Alausa ilisha Blkr. 60, 33. 91, 74. 270, 57.

Alausa kanagurta Blkr. 32, r6o. 56, 445. 60, 34. 91, 74. 227, 408. $270,57.356$, I 76.

Alausa macrurus Blkr. 60, 3I. 78, 501. 270, 57. 272, 26. 287, 6r.

288, 79. cf. Alosa macrurus Blkr.

Alausa melanosticta $C v .119,15$.

Alausa melanurus $C . V .17$, I0. $54,238.60,32.231$, I 50. 270, 57.

275, 49. 358, I92. 364, 295. cf. Clupea (Harengula) melanurus Blkr.

Alausa microlepis C. $V .91,146$.

Alausa palasah $C . V .25$, I . 91, 74 .

Alausa platycephalus Blkr. 302, 123.

Alausa Schrammii Blkr. 17, I r. cf. Meletta Schrammii Blkr.

Alausa scombrina $C . V .91,74$. 
Alausa toli $C . V .42,60.45$, I97. 49, 42 I. 51, 59. 55, 4I 4. 91, 74. 270, 57. 272, 26. 288, 79 .

Albula bananus $C . V$. ( $\mathrm{Val}$.). 16, 6. 25, I I. 56, 445. 59, II. 147, 469 . 185, 226. 231, I 49. 270, 56. 275, 47. 338, 36 I. cf. Conorhynchus glossodon $B l k r$.

Albula erythrocheilos Val., Cant. 288, 79.

Albula glossodonta Riipp. 316, 243. 364, 294. cf. Conorhynchus glossodon Blkr.

Albula neoguinaica, $C$. $V$. cf. Conorhynchus glossodon $B l k r$.

Albula seminuda $C$. $V$. cf. Conorhynchus glossodon $B l k r$.

Albulichthys Blkr. 261, 430. 314, 200.

Albulichthys albuloides Blkr. 270, 53. 272, 23.

Alburnellus Gir. 261, 437. 314, $211.329,263$.

Alburnops Gir. 261, 438. 314, 2 I2. 329, 264.

Alburnus Heck. 261, 437. 314, 2 I I. 329, 263.

Alepidogobius Blkr. 453, 3 ro.

Alepisaurus Richardsonii Blkr. 119, ro.

Aleuteres 10, 70.

Aleuteres laevis Cuv. 9, 639. cf. Aluteres scriptus Blkr.

Aleuteres laevis Rich. cf. Alutarius laevis Cuv.

Aleuteres nasicornis Schl. cf. Pseudaluteres nasicornis Blkr.

Aleuteres scriptus Blkr. 388, $27 \mathrm{I}$.

Aleuterius variabilis Richd, 331, 443 .

Aleuterus personatus Less. 396, 296.

Algansea Gir. 261, 426. 314, 207.

Algoma Gir. 261, 426. 314, 206.

Alosa 2, 509 .

Alosa argyrochloris $C . V .459$, I03. 495, 22.

Alosa brevis Blkr. 9, 638 .

Alosa elongata Raffl. cf. Pellona Grayana $C . V$.

Alosa ilisha Blkr. 425, I48. cf. Alosa malayana Blkr. 425, 148 .

Alosa macrurus Blkr. 395, 294.

Alosa malayana Blkr. 381, 294.

Alosa melanura $C . V .459$, 103 .

Alosa palasah Rich. cf. Alosa Reevesii Blkr.

Alosa Reevesii Blkr. 425, 148 .

Alosa Reevesii Rich. cf. Alosa Reevesii Blkr.

Alosa scombrina $C . V .459,103$.

Alosa venenosa $C . V .459$, го3.

Alopecias vulpes $B p .459,67.495,8$.

Alopias vulpes $R p . \mathbf{2 6 6}, 58$.

Alphestes gembra $B l$. Schn. cf. Lutjanus argentimaculatus $B l k r$.

Alphestes sambra Bl. Schn. cf. Lutjanus argentimaculatus Blkr.

Alutarius amphacanthoïdës Blkr. 63, 23. 296, 103. 364, 273.

Alutarius barbatus Cant. cf. Pogonognathus barbatus Blkr. 
Alutarius Baueri Rich. 119, r6.

Alutarius Berardi Less. cf. Aluteres monoceros Cuv.

Alutarius Brownii Rich. 119, r6.

Alutarius laevis Cuv. 54, 304. 63, 21. 91, So. 188, 462. 237, 439. 275, I 2. 288, 68. 309, I 54. 358, I83. 364, 273. cf. Aluteres scriptus Blkr.

Alutarius macracanthus Blkr. 63, 22. 235, 374. 270, 65.

Alutarius maculosus Rich. 119, r6.

Alutarius nasicornis T. Schl. 84, 352. 275, I2. 354, I43.

Alutarius obliteratus Cant. 288, 68.

Alutarius paragaudatus Rich. 119, 16.

Alutarius prionurus Blkr. 47, 260. 63, 20. 364, 273. cf. Paraluteres prionurus Blk\%.

Alutarius serraticornis Blkr. 364, 273.

Alutarius trossulus Rich. 119, r6.

Alutarius variabilis Rich. 119, I 7 .

Alutarius velutinus Jen. 119, I7.

Alutera cinerea Schl. cf. Aluteres monoceros Cuv.

Alutera nasicornis Schl. cf. Pseudaluteres nasicornis Blkr.

Aluteres Cuv. 2, 506. 367, I4.

Aluteres Berardi Less. cf. Aluteres monoceros Blkr.

Aluteres cryptacanthus Cuv. 10, 70.

Aluteres laevis Cuv. cf. Aluteres scriptus Blkr.

Aluteres monoceros (de Bl.) Culv. Blkr. 368, 28. 425, I22. 491, 53. 497, I 7 .

Aluteres personatus Less. 368, 29. 491, 53.

Aluteres rhinoceros Holl. cf. Pseudaluteres nasicornis Blkr.

Aluteres scriptus Blkr. 368, 28. 394, 285. 425, I22. 459, 7 r. 491, 53.

Aluthera barbata S. Mïll. 10, 68, 70, 74.

Alticus aspilus Blkr. 407, 235. 459, 6I, 74 .

Alticus monochrous Blkr. 459, 74 .

Alticus monochrus Blkr. 407, 234. 459, 60.

Alticus saltatorius Comm. 459, 74 .

Amanses Gray. 367, I2.

Amanses scopas Blkr. 364, 273. 368, 27. 459, 7 1. 495, 20.

Ambassis C. $V .1,553.2,526.468,292$.

Ambassis alta $C$. $V$. cf. Ambassis lala $C . V$.

Ambassis apogonides Blkr. 270, 23. 272, I I. cf. Parambassis apogonides $B l k r$.

Ambassis apogonoïdes Blkr. 45, 200. 49, 4I8. 55, 4II.

Ambassis baculis C.V. 91,30 .

Ambassis batjanensis Blkr. 135, I96. 170, 478. 237, 436. 275, $3^{\text {I }}$. $284,239.287,48.296,107.317,246.354,147.364,284.457,17$.

Ambassis Barlovi Sykes. 91, 30.

Ambassis bogoda C. $V$. cf. Bogoda nama Blkr. 
Ambassis buröensis Blkr. 231, I44. 270, 23. 354, I47. 457, I4.

Ambassis buruensis Blkr. 151, 396.

Ambassis Commersonii C. $V$., Kner. 2, 526. 13, 30. 309, I55. 457, II. 459, 94. 468, 292. 495, I3. cf. Ambassis nalua $C . V$.

Ambassis Dussumierii $C . V .2,526.13,30.16,4.25,7.10,66.46$, 2т . 53, г6о. 91, 3о. 231, I 44. 235, 363. 270, 23. 275, 3 г. 287, 48. 288, 72. 296, Iо7. 320, 269. 354, г 47. 358, I88, 364, 284. 395, 292. 425, I37. cf. Ambassis gymnocephalus Blkr.

Ambassis gymnocephalus Blkr. 457, I5. 459, 94. 495, r3.

Ambassis indica Mac. Cl. 91, 30.

Ambassis interrupta Blkr. 69, 696. 272, I I. 275, 3 I. 296, 107. 354, I 47.358, I $88.364,284.457$, I 3 .

Ambassis Kopsii Blkr. 186, 253. 267, I 40. 457, 7 .

Ambassis lala C. $V .91,88.468,292$.

Ambassis macracanthus Blkr. 13, 30. 78, 455. 156, 2 I2. 270, 24. 275, 31. 287, 49. 364, 284. cf. Ambassis Commersonii C. $V$.

Ambassis macranthus Blkr. cf. Ambassis Commersoni C. $V$.

Ambassis macrolepis Gthr. cf. Parambassis microlepis Blkr.

Ambassis nama $C . V .91,30$. cf. Bogoda nama Blkr.

Ambassis nalua $C . V .9,632.13,29,30.16,4.25,7.55,4$ II. 56, 444. 78, 453. 91, 30. 158, 273. 270, 24. 272, Ix. 275, 31. 287, 49. 288, 72. 296, 107. 457, го.

Ambassis oblonga $C$. $V$. cf. Bogoda nama Blkr.

Ambassis phula C. $V$. cf. Bogoda namía Blkr.

Ambassis productus Guich. 459, 94 .

Ambassis ranga $C . V .468,292$. cf. Ambassis lala $C . V$.

Ambassis robustus Schl. cf. Ambassis Wolffi Blkr.

Ambassis safgha Blkr. 364, 284 .

Ambassis thermalis C. $V .91,30$.

Ambassis urotaenia Blkr. 54, 257. 56, 444. 156, 2 I2. 167, 370. 270, 24. 275, 3І. 287, 49. 296, Іо7. 315, 233. 354, I 47. 358, I88. $364,284.457,8.459,94$.

Ambassis Vachelli Rich.425, 137.

Ambassis Vachelli Peter's cf. Ambassis gymnocephalus Blkr.

Ambassis Wolffi Blkr. 26, 9. 35, 26 r. 42, 58. 45, r95. 49, 418. 55, 4II. 239, IO2. 270, 24. 272, II. 356, I 73. 457, 5.

Ambassus Szons. 468, 292.

Amblodon Raf. 468, 324 .

Ambloplites Raf. 468, 249.

Ambloplites aeneus 468, 249.

Amblyapistus Blkr. 473, 297.

Amblyapistus macracanthus Blkr. 475, 73.

Amblyapistus taenianotus Blkr., Gthr. 459, 87. 475, 7 I. 495, I6. 497, х 2.

Amblyceps Blyth. 306, I05. 
Amblyceps coecutiens Blyth. 306, I05.

Amblychaeturichthys Blkr. 453, 324.

Amblychaeturichthys hexanema Blkr. 497, 19.

Amblycirrhites amblycephalus Blkr. cf. Paracirrhites amblycephalus Blkr.

Amblycirrhites arcatus Blkr. cf. Paracirrhites arcatus Blkr.

Amblycirrhites fasciatus Gill. 468, 314.

Amblycirrhites Forsteri Blkr. 389, 273. cf. Paracirrhites Forsteri Blkr.

Amblycirrhites Forsteri Blkr. 396, 389 .

Amblycirrhitus Gill. 468, 3 I4.

Amblydoras Blkr. 306, 86.

Amblydoras affinis Blkr. 303, I8. 306, 86.

Amblydoras truncatus Blkr. 303, i8.

Amblydoras Weddelli Blkr. 303, I8.

Amblyeleotrii 460, I04.

Amblyeleotris Blkr. 450, 373. 453, 307. 460, I04, Iо6.

Amblyeleotris periophthalmus Blkr. 478, гог.

Amblygaster 10, 70 .

Amblygaster clupeoides Blkr. 10, 73. cf. Sardinella clupeöides Blkr.

Amblygobius Blkr. 453, 322.

Amblygobius semicinctus $B l k r .495$, I 7 .

Amblygobius sphynx Blkr. 491, 54 .

Amblyopodiformes 453, 328 .

Amblyopodini 453, 329 .

Amblyopus Val. 453, 330.

Amblyopus anguillaris Rich. cf. Gobioides Hermannianus Blkr.

Amblyopus brachysoma Blkr. 86, 510. 270, 42. 453,329. cf. Gobioïdes brachysoma Blkr.

Amblyopus coeculus $C . V$. 91, 50. cf. Gobioïdes coeculus Blkr.

Amblyopus eruptionis Blkr. 15, 38. 25, 10. 364, 292.

Amblyopus gracilis $C . V .15,38.25,10.91,50$.

Amblyopus Hermannianus C. V. 91, Iо3. 270, 42. 287, 57. 288, 77. $364,292$.

Amblyopus Hermannianus Blk\%. (nce C. V.) cf. Taenioides anguillaris Blkr.

Amblyopus Lacepedii Schl. cf. Odontamblyopus Lacepedii Blkr.

Amblyopus mayenna $C$. $V$. cf. Amblyopus Hermannianus $C$. $V$.

Amblyopus melanurus Rich. cf. Gobioides melanurus Blkr.

Amblyopus roseus $C . V .91,5 \circ$.

Amblyopus rugosus Rich. cf. Gobioides rugosus Blkr.

Amblyopus (Tyntlastes) sagitta Gthr. 453, 329.

Amblyopus urolepis Blkr. 67, 581. 270, 42 .

Amblypharyngodon Blkr. 261, 433. 314, 202.

Amblypomacentrus breviceps Blkr. 483, 39. 485, 69.

Amblypomacentrus ovoides Blkr. 485, 70.

Amblyrhynchichthys Blkr. 261, 430. 314, 200. 
Amblyrhynchichthys truncatus Blkr. 239, го2. 270, 53.272, 23.356, г 76. Amblyrhynchotus oblongus $B i b r$. Tetrodon oblongus $B l$.

Amblyscion Gill. 468, 328.

Amblyscion argenteus Gill. 468, 328.

Amblytoxotes Blkr. 468, 3 II.

Amia Gron. 468, 257.

Amia amblyuropterus Blkr. 456, 68.

Amia amboinensis Blkr. 354, r47. 358, r88. 364, 284. 456, 44.

Amia annularis Blkr. 425, 137 .

Amia apogonides Blkr. 456, 58 .

Amia arenata Blkr. 456, 47 .

Amia aurea Blkr. 318, 25I. 354, I 47. 364, 284. 394, 287. 456, 48. $491,42$.

Amia aurita Blkr. 459, 93. 495, I I.

Amia bandanensis. Blkr. 315, 233. 316, 242. 318, 25 I. 320, 269.

354, I 47. 358, I88. 364, 284. 437, 38. 456, $27.491,42$.

Amia bifasciata Blkr. 425, I37.

Amia buroënsis Blkr. 354, 147. 456, 70.

Amia Cantori Blkr. 395, 292. 456, 25.

Amia carinata Blkr. 497, 6.

Amia ceramensis Blkr. 354, I47. 358, I88. 364, 284. 456, 45.491, 42.

Amia chrysopomus Blkr. 456, 34 .

Amia chrysotaenia Blkr. 456, 30.

Amia cyanosoma Blkr. 394, 287. 456, 37. 459, 93.

Amia cyanotaenia Blkr. 364, 284. 394, 287. 437, 38. 456, 33.

Amia endekataenia Blkr. 456, 3 I.

Amia fasciata Blkr.; Gill. 315, 233. 320, 269. 331, 447. 354, I47. 358, I88. 364, 284. 394, 287. 425, I $37.437,38.456,36.459,93$. $491,42.497,6$.

Amia frenata Blkr. 315, 233. 354, 147. 364, 284. 456, 42. 459, 93. $491,42.495$, II.

Amia glaga Blkr. 456, 66. 497, 6.

Amia Godini Blkr. 456, I7.

Amia gracilis Blkr. 456, 69 .

Amia Hartzfeldi Blkr. 315, 233. 354, I 47. 364, 284. 456, 35.491, 42.

Amia Hoevenii Blkr. 315, 233. 364, 284. 456, 49.

Amia hyalosoma Blkr. 364, 284. 456, 57. 459, 93.

Amia hypselonotus Blkr. 315, 233. 320, 269. 358, I88. 364, 284. 437, 38. 456, 53 .

Amia kallopterus Blkr.456, 41, 491, 42.

Amia kallosoma Blkr. 456, I6.

Amia koilomatodon Blkr. 315, 233. 364, 284. 456, 23.

Amia lateralis Blkr. 459, 93.

Amia leptacanthus Blkr. 315, 233. 456, 59.

Amia lineata Blkr. 497, 6. 
Amia macrophthalmus Blkr. 456, 63.

Amia macropteroides Blkr. 364, 284. cf. Amia macropterus Blkr.

Amia macropterus Blkr. 315, 233. 364, 284. 456, 72.

Amia margaritophora Blkr. 456, 46.

Amia melanorhynchus Blkr. 315, 233. 354, 147. 364, 284. 456, 43.

Amia melanurus Blkr. 456, 28.

Amia melas Blkr. 315, 233. 354, I47. 358, 188. 364, 284. 456, 52.

Amia modesta $B l k k$ r. 456, 6r.

Amia moluccensis Blkr. 320, 269. 358, I88. 364, 284. 456, 50, 5 I. $468,259$.

Amia monochrous Blkr. 364, 284. 425, I37. cf. Amia moluccensis Blkr.

Amia multilineata $B l k r .358$, I $88.364,284.456,29$.

Amia multitaeniata Blkr. 315, 233. 318, 251. 320, 269. cf. Amia multilineata Blkr.

Amia nematopterus Blkr. 456, I8.

Amia nigripinnis Blkr. 425, I37. 456, 64. 497, 6.

Amia nigromaculata Blkr. 456, 20. 491, 42.

Amia Noordzieki Blkr. 456, 15.

Amia Novae-Guineae Blkr. 456, 60. 491, 42.

Amia orbicularis Blkr. 358, I88. 364, 284. 456, I9.

Amia percaeformis Gron. 468, 259. cf. Amia moluccensis Blkr.

Amia perdix Blkr. 456, 65 .

Amia poecilopterus Blkr. 456, 54 .

Amia polystigma Blkr. 456, 67 .

Amia quadrifasciata Blkr. 425, 137. 456, 39. 459, 93.497, 6.

Amia rhodopterus Blkr. 456, 22.

Amia sangiensis Blkr. 456, 56 .

Amia Schlegeli Blkr. 497, 6.

Amia semilineata Blkr. 497, 6.

Amia semiornata Blkr. 495, I r.

Amia singapurensis Blkr. 456, $2 S$.

Amia taeniata Blkr. 456, 24.

Amia taeniopterus Blkr. 456, 63. 459, 93. 495, I I.

Amia timorensis Blkr. 320, 269. 456, 26.

Amia trimaculata Blkr. 354, I47.456, 2 I.

Amia trivittata Blkr. 425, I37, I50.

Amia truncata Blkr. 456, 62 .

Ania urostigma Blkr. 456, $5 \mathrm{r}$.

Amia variegata Blkr. 459, 93. 495, II.

Amia vittigera Blkr. 358 , I 88 .

Amia Wassinki Blkr. 320, 269. 456, 38 .

Amia zosterophora Blkr. 456, 7x.

Amiurus Raf. 306, 102.

Amiurus cantonensis Gthr. 425, I25. 
Amiurus catus Gill. 306, ro2.

Amiurus guttatus Gthr. 425, I25.

Ammopleurops brachysoma Blkr. 425, r30, I53.

Amora tentaculata $G r .494,107$.

Amphacanthus 1, 553.

Amphacanthus Abhortani C. $V$. cf. Teuthis Abhortani Gthr.

Amphacanthus albopunctatus $T$. Schl. cf. Amph. margaritiferus $C . V$. Amphacanthus albopunctatus Schl. cf. Teuthis albopunctata Blkr.

Amphacanthus aurantiacus Schl. cf. Teuthis aurantiaca Blkr.

Amphacanthus bifasciatus cf. Amphacanthus virgatus $C . V$.

Amphacanthus canaliculatus $B l ., B l k r .67,580.270,3$ I.

Amphacanthus chrysospilos Blkr. 51, 66. 113, 432.

Amphacanthus concatenatus $C . V .142,46.288,73$.

Amphacanthus corallinus C. V. 23, I I. 157, 233. 275, 36. cf. Teuthis corallina Gthr.

Amphacanthus cyanotaenia Blkr. 79, 606.

Amphacanthus doliatus C. $V .79,605$. cf. Teuthis doliata Gthr.

Amphacanthus dorsalis $C . V .2,515.23,9.25,9.53$, I6 1. 54, 235. 156, $215.157,233.166,332,335.234,354.235,367.237,437$.

$248,203.250,206.270,3$ I. $275,36.287,5$ I. $288,73$.

Amphacanthus firmamentum $C . V .270,31$.

Amphacanthus fuscescens $C . V$. 118, 106. cf. Teuthis fuscescens

Gthr. Blkr.

Amphacanthus fuscescens T. Schl. 253, 235.

Amphacanthus fuscescens Rich. cf. Teuthis albopunctata Gthr.

Amphacanthus guttatus Bl. Schn. (C. V.). 2, 5I5. 16, 5. 23, 10. 25,

9. 51, 58. 91, 48. 157, 233. 167, 372. 235, 367. 236, 333. 270,

31. 275, 36. cf. Teuthis guttata Gthr.

Amphacanthus gymnopareius Rich. 119, I I.

Amphacanthus hexagonatus Blkr. 105, 41. 237, 437. 287, 5 r.

Amphacanthus javaneus $K$. $v$. $H$. cf. Amphacanthus corallinus $C . V$.

Amphacanthus javus $L .\left(C . V_{0}\right) .2,5^{1} 5.16,5.23,9.25,9.50,471$.

51, 58. 91, I65. 156, 2 I5. 227, 408. 231, 147. 235, 367. 270, 3 I.

275, 36. 288, 73. cf. Teuthis java Gthr.

Amphacanthus Kopsii Blkr. 50, 483. 56, 445. 156, 2 I 5. cf. Teuthis

Iopsii Gthr.

Amphacanthus labyrinthodes Blkr. 78, $47 \mathrm{I}$.

Amphacanthus lineatus $C . V$. cf. Teuthis lineata Giüth.

Amphacanthus luridus Elr. cf. Teuthis lurida Gthr.

Amphacanthus maculatus $K$. $v$. $H$. cf. Amphacanthus dorsalis $C . V$.

Amphacanthus margaritifer $C . V$. cf. Teuthis margaritifera $G t h r$.

Amphacanthus margaritiferus $C . V .9,633.157,233.166,334.184$, 200. $185,223.231$, I $47.275,36.287,5$ I.

Amphacanthus margaritiferus Rich. cf. Teuthis albopunctata Gthr.

Amphacanthus marmoratus C. $V . ; Q$. G. 35, 26 r. 42, 59. 45, 195 . 
49, 4I9. 55, 4I2. 171, 245. 184, 200. 208, 239. 231, I 47. 236, $33 \mathrm{r}, 333.270,32.272$, I3. 275, 36. 285, 245. cf. Teuthis marmorata Blkr. Gthr.

Amphacanthus melanospilos Blkr. 113, 43 r.

Amphacanthus nebulosus Q. G. cf. Teuthis nebulosa Gthr.

Amphacanthus notostictus Rich. 119, Ir.

Amphacanthus nuchalis $C$. $V$. 113, 432.

Amphacanthus olivaceus $C$. $V$. cf. Teuthis nebulosa Gthr.

Amphacanthus puellus Schl. 275, 36. cf. Teuthis puella Gthr.

Amphacanthus Russelii $C$. $V$. 2, 5I5. 91, 48. cf. Amphac. javus $C . V$.

Amphacanthus scaroïdes Blkr. 76, 262.

Amphacanthus sutor $C . V .91,165$. cf. Teuthis sutor Gthr.

Amphacanthus tetrazona Blkr. 129, 441. 275, 36.

Amphacanthus tumifrons $C . V$. 119, Ir.

Amphacanthus vermicularis $M$. Schl. cf. Amph. vermiculatus $C . V$.

Amphacanthus vermiculatus $C . V .2,5$ I5. 23, II. 25, 9. 170, 479.

270, 32. 275, 36. 287, 5r. cf. Teuthis vermiculata Gthr.

Amphacanthus vermiculosus $K$. $v$. $H$. cf. Amph. vermiculatus $C$. $V$. Amphacanthus virgatus $C . V .2,5$ I5. 23, II. 51, 58. 126, 344. 185, 223. 234, 354. 235, 367. 270, 32. 275, 36. cf. Teuthis virgata Gthr. Amphacanthus vulpinus $M$. Schl. 10, 67, 68. 54, 236. 73, I35. 275, 36. cf. Teuthis vulpina Gthr.

Amphioxus Belcheri Gray, 35, 262. 42, 60. 45, I97. 49, 421. 55, 4I 5. 272, 28 .

Amphipnous J. Müll. 88, 57.

Amphipnous cuchia $J$. Mïll. 88, 61. 91, 78. 425, т24.

Amphiprion akallopisos Blkr. 76, 28r. 270, 35. cf. Prochilus akallopisus Blkr.

Amphiprion akalopisos 140, 438.

Amphiprion bicinctus Playf. cf. Prochilus polymnus Blkr.

Amphiprion bicolor Casteln. cf. Prochilus percula Blkr.

Amphiprion bifasciatus $B l$. Schn。 (C. $V$.). 6, I. 54, 282. 56, 445. 156, 2 I6. 158, 274. 184, 202. 250, 206. 275, I9. 287, 43. cf. Prochilus bifasciatus Blkr.

Amphiprion boholensis Cart. Prochilus polymnus Blkr.

Amphiprion chrysargurus Rich.68, 644. 184, 202. 275, I9. 287, 43. cf. Amphiprion xanthurus C. $V$. Prochilus Clarkii Blkr., Prochilus polymnus Blkr.

Amphiprion chrysargyrus Rich. cf. Prochilus polymnus Blk\%.

Amphiprion chrysogaster $C$. $V$. cf. Prochilus chrysogaster $B l k r$.

Amphiprion chrysopterus $C$. $V$. cf. Prochilus polymnus $B l k r$.

Amphiprion chrysurus $C$. $V$. cf. Prochilus polymnus Blkr.

Amphiprion Clarckii $C . V .81,52$. cf. Prochilus polymnus Blkr.

Amphiprion Clarkii Day. cf. Prochilus polymnus Blk\%.

Amphiprion ephippium Bl. Schn. (C. V.). 6, I. 10, 67, 68. 46, 2 13. 
91, 52. 125, 32I. 156, 2I6. 157, 234. 158, 274. 275, I9. cf. Prochilus ephippium Blkr.

Amphiprion frenatus Day (nec Brev.) cf. Prochilus ephippium Blkr. Amphiprion frenatus $B r e v$. cf. Prochilus frenatus $B l k r$.

Amphiprion fusciventer Benn. cf. Prochilus fusciventer Blkr.

Amphiprion intermedius M. Schl. 6, I. cf. Prochilus bifasciatus Blkr. Amphiprion japonicus Schl. cf. Prochilus polymnus Blkr.

Amphiprion laticlavius $C . V$. 6, r. cf. Amphiprion bifasciatus $B$. Schn., Prochilus bifasciatus Blkr.

Amphiprion melanopus Blkr. 66, 56r. 275, I9. 277, 88. 287, 43.

295, 97. cf. Prochilus melanopus Blkr.

Amphiprion melanostolus Rich. 119, I3.

Amphiprion melanurus $C . V .6$, I. 270, 35. cf. Prochilus percula Blkr.

Amphiprion Milii Thioll. cf. Prochilus polymnus Blkr.

Amphiprion monofasciatus Thioll. cf. Prochilus melanopus Blkr.

Amphiprion ocellaris $C . V .6$, r. 270, 35. cf. Prochilus percula Blkr.

Amphiprion percula $C . V .6$, I, 3. 10, 67, 68. 46, 21 3. 54, 287.

156, 216. 157, 234. 158, 274. 184, 202. 185, 224. 270, 35. 275,

19. 287, 43. 296, 104. cf. Prochilus percula Blkr.

Amphiprion perideraion Blkr. 140, 437. 184, 202. cf. Prochilus perideraion Blkr.

Amphiprion polymnus $B l$. Si hn. 6, 5. 91, I66. cf. Prochilus polymnus Blkr.

Amphiprion Rosenbergii Blkr. 232, I6. cf. Prochilus Rosenbergii Blk\%. Amphiprion rubrocinctus Rich. 119, I3.

Amphiprion Rüppelli Casteln. cf. Prochilus ephippium Blkr.

Amphiprion Sebae Blkr. 78, 478. 156, 2 I6. 270, 35. cf. Prochilus Sebae Blkr.

Amphiprion scansor Bl. Schn. cf. Anabas testudineus Cuv.

Amphiprion testudineus $B l$. Schn. cf. Anabas testudineus Cuv.

Amphiprion tricolor Gthr. cf. Prochilus ephippium Blkr.

Amphiprion trifasciatus $C . V .6$, I. 71, 767, 231, I48. 275, I9. cf. Prochilus bifasciatus, trifasciatus Blkr.

Amphiprion tunicatus $C$. $V$. cf. Prochilus percula Blkr.

Amphiprion xanthurus $C . V .6$, I. $66,560.68,644.78,480.126$, 344. 156, 216. 158, 274. 270, 35. 287, 43. cf. Prochilus polymnus, xanthurus Blkr.

Amphiprionichthys Blkr. 289, 8r. 297, I40.

Amphiprionichthys apistus Blkr. 122, I73. 289, 82. cf. Caracanthus apistus Blkr.

Amphisile punctulata Bianconi. cf. Centriscus punctulatus Blkr.

Amphisile scutata Cuv., Klein. 9, 633. 47, 245, 54, 235. 156, 2 I6.

157, 234. 167, 372. 184, 202. 235, 373. 270, 63. 275, x 5. 287, $4 \mathrm{r}$.

296, І03. 309, І 54. 320, 265. 425, г 33. cf. Centriscus scutatus. L. 
Amphisile strigata Gthr. 315, 230. 316, 241. 354, I44. 364, 274. 396, 298. 491, 49 .

Amphistichus $A g \cdot 468,29$ I.

Amphistichus argenteus $A g .468,29 \mathrm{I}$.

Anabas 1, 553 .

Anabas macrocephalus Blkr. 113, 430. 163, 59. 170, 476. 178, 2. 270, 34. 272, Iо. 275, 2 I. cf. A. testudineus Cuv.

Anabas microcephalus Blkr. 163, 58.498, то.

Anabas oligolepis Blkr. 121, I6r. 272, 10. 425, I36. 498, 5.

Anabas scandens $C . V .2,519.10,66.16,4.21,8.25,8.35,261$. 42, 59. 45, I95. 46, 2I2. 49, 4I9. 55, 4II. 91, 42. 166, 329. 170, 476. 191, 3. 225, 357. 228, 424. 231, I46. 235, 368. 237, 437. 239, Iог. 270, 34. 272, 1о. 275, 2 1. 279, го0. 287, 44. 288, 70. 356, I73. 395, 29 1. 425, I36. cf. A. testudineus Cuv.

Anabas sennal $C . V$. cf. Anabas scandens $C . V$.

Anabas spinosus Gray. cf. Anabas scandens $C . V$.

Anabas testudineus $C u v .498,7$. cf. Anabas scandens $C u v$.

Anabas trifoliatus Kaup. cf. Anabas testudineus $C u v$.

Anabas variegatus Blkr. 46, 220. 275, 21. cf. A. microcephalus Blkr. Anableps Art. 261, 44 r.

Anacanthus 2, 507 .

Anacanthus africanus $M$. H. cf. Rhachinotus africanus Cant.

Anacanthus barbatus Gray. 10, 70. cf. Pogonognathus barbatus Blkr. Anampses Cuv. 1, 552. 292, 98 .

Anampses amboinensis Blkr. 163, 80. 293, 282 .

Anampses coeruleopunctatus Rilpp. 236, 342, 293, 282.364, 276. $459,82.495,9$.

Anampses Cuvieri Val. 459, 82 .

Anampses diadematus Rüpp. 459, 82. 495, 9.

Anampses geographicus $C . V .142,58.293,282.358,1 S_{4} .495,9$.

Anampses lineolatus Benn. cf. Anampses diadematus Rüpp.

Anampses meleagrides $C . V .142,57.293,283$.

Anampses meleagris $C . V .459,82$.

Anampses melanurus Blkr. 163, 79. 293, 283.

Anampses (Anampses) neoguinaicus Blkr. 491, 57. 500, 27.

Anampses pterophthalmus Blkr. 163, 81. 293, 2S3. 320, 266. 358, IS.4.

Anampses Twistii Blkr. 142, 56. 293, 283.

Anampses viridis C. $V .459,82.495,9$.

Anampsodax Lienardi Blkr. 459, 83. 495, Io.

Anarrhichas fasciatus $B l k r .425$, I29, I5I.

Anchois taty $C . V$. cf. Engraulis taty $C . V$.

Ancistrus Kner. 306, 78 .

Ancistrus cirrhosus Kner. 306, 78 .

Ancistrus medians Kuner. cf. Hemiancistrus medians Blkr.

Ancistrus Temmincki Blkr. 336, II. 
Ancylodon Cu\%. 468, 33 $\mathrm{I}$.

Ancylodon jaculidens $C . V .363,255.468,33 \mathrm{I}$.

Ancyclodon parvipinnis $C . V .468,335$.

Anematichthys 261, 43I.

Anguilla Blkr. 1, 552. 351, II3.

Anguilla amblodon Gthr. cf. Muraena amblodon Blkr.

Anguilla Aucklandii Rich. 119, I5.

Anguilla australis Rich.88, I7. 119, I5. 169, 389. cf. Muraena australis Rich.

Anguilla avisotis Rich. cf. Muraena avisotis Blkr.

Anguilla bicolor $M C C l .88,17.91,76$.

Anguilla Bleekeri $K p$. cf. Muraena sidat $B l k r$.

Anguilla bostoniensis Les. cf. Muraena japonica Blkr.

Anguilla brevirostris $M c C l .91,76$.

Anguilla celebensis $K p$. cf. Muraena sidat Blkr.

Anguilla clathrata Rich. cf. Muraena clathrata Blkr.

Anguilla Conger Jen., Shaw. cf. Conger vulgaris Cuv.

Anguilla Delalandei $K p .266,56$.

Anguilla Dieffenbachii Gray, Rich. 88, r7. 119, I5.

Anguilla Dussumieri $K p$. cf. Muraena Dussumieri Blkr.

Anguilla Elphinstonei Sykes, 78, 504. 88, I5, 65. 91, 76. cf. Muraena maculata Ham. Buch.

Anguilla guttata Cuv. cf. Muraena maculata Ham. Buch.

Anguilla japonica Temm. Schl. 90, 5I. cf. Muraena bostoniensis, japonica Blkr.

Anguilla Johannae Gthr. cf. Muraena Johannae, mauritiana Blkr.

Anguilla labiata Pet. cf. Muraena labiata Blir.

Anguilla labrosa Rich. 88, I6.

Anguilla macroptera $\mathrm{MC} \mathrm{Cl.} \mathrm{90,52.} \mathrm{cf.} \mathrm{Muraena} \mathrm{latirostris} \mathrm{Blkr.}$

Anguilla malcumora Rüpp. cf. Muraena sidat Blkr.

Anguilla malgumora Schl. Kp. cf. Muraena malgumora Blkr.

Anguilla marmorata $Q$. G. 231, I50. 270, 59. 275, 50. 296, I I2. cf.

Muraena maculata Ham. Buch.

Anguilla mauritiana $B c n n$. cf. Muraena mauritiana Blkr.

Anguilla moa Blkr. 25, 22. cf. Muraena moa Blkr.

Anguilla moa Blkr. (ex parte) cf. Muraena malgumora Blkr.

Anguilla mowa Blkr. 88, 16. 270, 59.

Anguilla myriaster $B r e v$. cf. Ophisoma myriaster Blkr.

Anguilla nebulosa $M C$ Cl. 91, I 53 .

Anguilla nigropunctata Cuv. cf. Muraena australis Rich.

Anguilla (Moringua) raitaborua Cant. cf. Moringua raitaborua Cant.

Anguilla sidat Blkr. 88, I7. 156, 2 I7. 170, 476. 198, 45. 231, I50. 270, 59. 279, гог. cf. Muraena sidat Blkr.

Anguilla sinensis $M c C l$. cf. Anguilla japonica Temm. \& Schl., Muraena latirostris Blkr. 
Anguilla vamos Blkr.91, 76 .

Anguilla variegata $J . M C C l$. cf. Anguilla Elphinstonei Sykes, Muraena maculata Ham. Buch.

Anguilla virescens Gt/hr. cf. Muraena virescens Blkr.

Anguisurus $K p .351$, I20.

Anguisurus punctulatus $K p$. 207, 2 Ir. cf. Sphagebranchus polyophthalmus $K p$.

Anisotremus Gill. 468, 27 r.

Anodontostoma Hasseltii Blkr. 16, I5. 25, 12. cf. Chatoessus chacunda $C . V$.

Anomalops katoptron Blkr. 491, 48 .

Anomiolepis Gill. 468, 330.

Anoplocapros $K p$. 367, I6.

Anoplus Schl. 468, 277.

Anoplus banjos Rich. 425, I39. 468, 277. cf. Banjos typus Blkr.

Anosmius Bennetti Blkr. 167, 374. 184, 204. 231, I5I.

Anosmitus mappa Blkr. cf. Crayracion mappa Blkr.

Anosmius striolatus Blkr. 237, $43^{8}$.

Anostoma pictum v. Hass. cf. Betta picta Blkr.

Antennarius bigibbus Gthr. Comm. 358, I 84. 364, 274. 459, 7 I. 495, 20.

Antennarius biocellatus Blkr. 273, 4. 425, 123 .

Antennarius campylacanthus Blkr. 302, 28.

Antennarius caudimaculatus Blkr., Gthr. 119, I 2. 395, 290.

Antennarius chironectes Comm. 95, 104. 364, 274.

Antennarius coccineus Gthr. 358, r84. 364, 275. 459, 7r. 495, 20.

Antennarius Commersonii Cant., Gthr. 51, 58. 358, I84. 364, 275. 459, 7 I. 495, 20.

Antennarius dorehensis Blkr. 232, 2 1. 491, 55.

Antennarius goramensis Blkr. 357, I77.

Antennarius Güntheri Blkr. 364, 275.

Antennarius hispidus Cant. 51, 58. 54, 280. 91, 52. 288, 70. 354,

I 44. 358, I $84.364,275.459,7$ I. $495,20$.

Antennarius histrio Gthr. 425, I23. 459, 7 I. 495, 20.

Antennarius horridus Blkr. 80, 83. 242, r42. 275, I5. 318, 250.

354, I 44. 358, I $84.364,275.394,286$.

Antennarius Lacepedii Blkr. 142, 50.

Antennarius laevigatus Blkr. 266, 75 .

Antenmarius leprosus Blkr. 163, 68 .

Antennarius leucosoma Blkr. 99, 328. 318, 250. 364, 275.

Antennarius Lindgreeni Blkr. 123, 192.

Antennarius lioderma $B l k r .357$, I 78. 364, 275.

Antennarius maculatus Desjard. 459, 7 1. 495, 20.

Antennarius marmoratus Gthr. 358, I $84.364,275.394,285.396$, 298. 425, I23. 459, 72. 495, 20. 497, 20.

Antennarius melas Blkr. 163, 70. 
Antennarius moluccensis $B l k \%$. 128, 424. 184, 202.

Antennarius multiocellatus Gthr. 459, го3.

Antennarius nesogallicus Blkr. 80, 84. 318, 250.

Antennarius nesogallicus $\mathrm{Val}$. cf. marmoratus Gthr.

Antennarius nitidus E. T. Benn. cf. Antennarius laevigatus Blkr.

Antennarius notophthalmus Blkr. 87, 544. 156, 215. 196, 37. 275, I5.

308, I $52.320,265.358,184.364,275$.

Antennarius nummifer Blkr. 91, 52. 103, 497. 156, $216.167,372$.

184, 202. 188, 460. 236, 33 I. 287, 42. 354, I44. 358, r84. 491, 55.

Antennarius oligospilos Blkr. 163, 70.

Antennarius oligospilus Blkr. 358, I84. 491, 55 .

Antennarius phymatodes Blkr. 163, 69.

Antennarius pinniceps Comm. 142, 49. 159, 302. 459, 72. 495, 20.

cf. Antennarius hispidus Cant.

Antennarius polyophthalmus Blkr. 68, 644. 184, 202.

Antennarius raninus Cant. 69, 707.91, 52. 176, 12. 184, 202. 235, 370. 275, I 5. 288, 70. 302, 29. 315, 230. 339, 362. 491, 55. cf.

Antennarius marmoratus Gthr.

Antennarius tridens Gthr. 90, 47. 364, 275. 425, I23. 497, 20.

Antennarius trisignatus $B l k r .119, \mathbf{1} 2$.

Antennarius tuberosus Blkr. 188, 463 .

Antennarius unicornis Benn. cf. Antennarius bigibbus Gthr., Antennarius tuberosus Blkr.

Antennarius urophthalmus Blkr. 50, 488. 123, I92. 185, 237. 287, 42. cf. Antennarius caudimaculatus Gthr.

Anthianini. 468, $25 \mathrm{I}$.

Anthias $B l .468,25 \mathrm{I}$.

Anthias aplodactylus Blkr. 179, 3. cf. Dactylanthias aplodactylus Blkr. Anthias argus $B l$. cf. Epinephelus Hoevenii $B l k r$.

Anthias bifasciatus Bl.cf. Amphiprion bifasciatus Bl. Schn., cf. Prochilus bifasciatus Blkr.

Anthias bilineatus $B$ l. cf. Scolopsides bilineatus $C$. $V$.

Anthias borbonius $C$. $V$. cf. Odontanthias borbonicus Blkr.

Anthias borbonius Gthr. 422, 92 .

Anthias cheirospilos Blkr. 163, 36. cf. Pseudanthias chirospilus Blkr. Anthias chirospilus Blkr. 364, 280.

Anthias chrysostictus Gthr. cf. Odontanthias chrysostictus Blkr.

Anthias cichlops Blkr. 270, r8. cf. Pseudanthias cichlops Blkr.

Anthias Clarckii Benn. cf. Amphiprion xanthurus $C$. $V$.

Anthias Clarckii J. Whitch. cf. Prochilus polymnus Blkr.

Anthias filamentosus Gthr. cf. Serranus filamentosus $C$. $V$.

Anthias grunniens $B l$. Si $h n$. cf. Pristipoma guoraca $C . V$.

Anthias hamrur $B l$. Schn. cf. Priacanthus hamrur $C$. $V$.

Anthias Huchtii Blkr. 163, 38. 318, 25 1. cf. Pseudanthias Huchtii Blkr. Anthias Johnii Bl. cf. Lutjanus Johni Lac. 
Anthias lepidolepis Blkr. 163, 37. cf. Pseudanthias lepidolepis Blkr.

Anthias macropthalmus Bl. cf. Priacanthus Blochii Blkr., Priacanthus hamrur $C . V$.

Anthias maculatus $B l$. cf. Pristipoma caripa $C$. $V$., Pristipoma maculatum Gthr.

Anthias manadensis Blkr. 143, 39. 163, 34. 275, 24. cf. Pseudanthias manadensis Blkr.

Anthias orientalis $B l$. cf. Diagramma orientale $C . V$., Plectorhynchus orientalis Szons.

Anthias (Hemanthias) peruanus Steind. 468, $25^{2}$.

Anthias pleurotaenia Blkr. 163, 34. cf. Pseudanthias pleurotaenia Blkr.

Anthias polymna $B l$. cf. Amphiprion percula $C$. $V$.

Anthias polymnus $B l$. cf. Prochilus polymnus $B l k r$.

Anthias polymnus var. $B l$. cf. Prochilus percula $B l k r$.

Anthias rhodopeplus Gthr.468, 252.cf. Odontanthias rhodopeplus Blkr.

Anthias sacer $B l .468,252$.

Anthias Schlegelii Gthr. cf. Caprodon Schlegeli Blkr.

Anthias testudineus $B l$. cf. Anabas scandens $C$. $V$., Anabas testudineus Cur.

Anthias Vosmeri Bl.cf. Scolopsides Vosmeri $C . V$., Scolopsis Vosmaeri $C . V$.

Anthias zonatus Gthr. 549, 88 .

Antigonia Lozve. 468, 310.

Anyperodon Gthr. 468, 256.

Anyperodon leucogrammicus Gt/ur. 451, 22. 459, 88.468, 256.491, 42 .

Anyperodon urophthalmus Blkr. 438, 45. 451, 23.

Aperioptus pictorius Rich. 45, I96. 49, 2 I. 55, 4I 4. 272, 25. 323, 43.

Aphareini. 468, 277 .

Aphareus C. $V .468,278$.

Aphareus coerulescens $C$. $V$. cf. Aphareus furcatus Gthr.

Aphareus furcatus Gthr. 442, 99. 459, 9x. 468, 278. 495, I2.

Aphareus rutilans $C . V .163,52.275,28$. cf. Aphareus furcatus Gthr. Aphoristia orientalis Blkr. 487. 497, $3 \mathbf{I}$.

Aphritis Urvillei $C . V .119,6$.

Aphthalmichthys $K p .351$, II4.

Aphthalmichthys abbreviatus Blkr. 311, ז63.320, 272. 352, I24.358, x93. 364, 295. 397, 302.

Aphthalmichthys javanicus $K p .311$, I64. 320, 272. 352, I24.

Aphthalmichthys macrocephalus Blkr. 311, I65. 320, 272. 352, I24.

Aphya Risso. 453, 312.

Aphyocypris chinensis Gthr. 415, 12. 425, 145.

Apionichthys Dumerili $K p .366,307$.

Apisistus trachinoides $C . V .9,636$.

Apistus Cuv. 473, 296.

Apistus alatus C. $V .44,174.90$, 4. $91,36.253,235.425$, I 4 I. 473 , $296.475,59.497$, I 2 . 
Apistus amblycephaloides $B l k r .76,250.156,213.231$, I 45. 270, 25 . 275, 32. cf. Gymnaspistus niger Blkr.

Apistus amblycephalus Blkr. 27, 27. 156, 2 r3. 270, 25. cf. Gymnapistus niger Blkr.

Apistus australis $C . V .119,8.473,297$.

Apistus barbatus $C . V .14,4.142,36.270,25$. cf. Gymnapistus barbatus Srens.

Apistus Belengerii C. $V .91,36$.

Apistus binotatus Pet. cf. Amblyapistus taenianotus Blkr.

Apistus binotopterus Blkr. 27, 26. cf. Prosopodasys depressifrons Gthr. Apistus carinatus $C . V .91,36$. cf. Apistus alatus $C . V$.

Apistus cottoïdes $C . V$. 49, 4I8. 55, 4II. 78, 459. 272, I1. 473, 298. cf. Cottapistus cottoides Blkr.

Apistus cottoïdes Rich. cf. Prosopodasys (Prosopodasys) cottoides Gthr.

Apistus depressifrons Rich. 71, 753. 156, 213. 194, 26. 270, 25. cf. Prosopodasys depressifrons Gthr., Blkr.

Apistus dermacanthus Blkr. 54, 268. 90, 29. cf. Cocotropus dermacanthus Blkr.

Apistus dracaena $C . V .91,36$.

Apistus fusco-virens $Q$. G. 14, 4. 54, 269. 68, 643. 275, 32. 287, 49. cf. Paracentropogon longispinis $B ! k r$.

Apistus hypselopterus Blkr. 47, 238. 54, 270. cf. Paracentropogon longispinis Blkr.

Apistus Jenynsii Blkr. 119, 8.

Apistus lencogaster Rich. 72, Irr. cf. Gymnapistus leucogaster Blkr., Prosopodasys (Prosopodasys) leucogaster Gthr.

Apistus leucoprosopon Blkr. cf. Paracentropogon leucoprosopon Blkr. Apistus leucoprosopos Blkr. 142, 35 .

Apistus longispinis $C . V .14,4.47,229.473,297$. cf. Paracentropogon longispinis $B l k r$.

Apistus macracanthus Blkr. 54, 267. 296, 107. cf. Amblyapistus macracanthus Blkr.

Apistus marmoratus $C . V .14,4.119,8.473,299$. cf. Gymnapistus marmoratus Swns.

Apistus melas Blkr. 27, 26. 54, 270. cf. Apistus barbatus C.V., Gymnapistus barbatus Stens.

Apistus minous Cur. cf. Minous monodactylus woora $C . V$. Apistus monodactylus $C$. $V$. cf. Minous monodactylus $C . V$.

Apistus multicolor Rich. 54, 270. cf. Paracentropogon longispinis Blkr., Prosopodasys (Tetraroge) longispinis Blkr.

Apistus niger C. V. 27, 26. 91, 36. cf. Gymnapistus niger Blkr.

Apistus panduratus Rich. 119, 8. 473, 297.

Apistus plagiometopon Blkr. 71, 753. 275, 32. 296, 10\%. cf. Prosopodasys depressifrons Gthr.

Apistus rubripinnis T. Schl. 193, 5. cf. Gymnapistus rubripinnis Gill. 
Apistus Russellii Stons. cf. Minous monodactylus, woora C. $V$.

Apistus taenianotus $C . V .66,557.275,32.473,298$. cf. Amblyapistus taenianotus Blkr. Gthr.

Apistus taenianotus Rich. (nec C. V.) cf. Prosopodasys (Tetraroge) crista galli Blkr.

Apistus trachinoïles C. $V$. 14, 8. 16, 4. 25, 7. 51, 56. 71, 754. 194, 26. 288, 72. 473, 298. cf. Prosopodasys trachinoïdes Cant. Gthr.

Apistus Zollingeri Blkr. 9, 636. 14, S. 71, 754. cf. Prosopodasys Zollingeri Gthr.

Aploactis Schl. 473, 300.

Aploactis aspera Blkr., Rich. 90, 29. 425, I4I. 473, 300.497, I2. Aploactis dermacanthus Blkr. 358, r89. cf. Cocotropus dermacanthus $B l k r$.

Aploactis echinatus Blkr. 288, 72. cf. Cocotropus echinatus $K p$.

Aploactis Milesii Rich. 119, 8.

Aploactis pusillus Blkr. 193, 6. cf. Minous pusillus Schl.

Aploactis trachycephalus Blkr. 114, 451. 156, 2I3. 275, 32. cf. Minous trachycephalus Gthr.

Aploactisoma Cast. 473, 300.

Aplocheilichthys typus Blkr. 302, I 6 .

Aplocheilos latipes Blkr. 269, 99.

Aplocheilus $M C$ Cl. 261, 44I.

Aplocheilus chrysostigmus $M C C l$. cf. Panchax Buchanani $C$. $V$.

Aplocheilus javanicus Blkr. 110, 323. 170, 477.

Aplocheilus latipes Blkr. 497, 24.

Aplocheilus melastigmus $M C C$. cf. Panchax Buchanani $C$. $V$.

Aplocheilus panchax $M C C$. cf. Panchax Buchanani C. $V$.

Aplochilus latipes Blkr. 408, 238.

Aplodactyliformes. 468, $3 \mathbf{I} 6$.

Aplodactylus $C . V .468,3$ I 7 .

Aplodactylus arctidens Rich. 119, 6.

Aplodactylus punctatus C. $V .468,3$ I 7 .

Aplodinoti. 468, 324 .

Aplodinotus Raf. 468, 324 .

Aplodinotus grunniens Raf. 468, 324.

Apocryptei. 453, 327.

Apocrypteini. 453, 327 .

Apocryptes $V a l .1,553.453,327$.

Apocryptes bato C. $V$. 91, 103. 453, 327.

Apocryptes borneënsis Blkr. 139, 42 I. 272, I7.

Apocryptes brachypterus Blkr. 138, 40I. 453, 3Ir. cf. Gobiopterus brachypterus Blkr.

Apocryptes cantonensis Osbcck cf. Periophthalmus Koelreuteri C. $V$. Apocryptes changua $C . V .15,36.16,5.25$, ro. 26, 3. 35, 261. 
42, 59. 45, I95. 49, 419. 51, 58. 55, 412. 91, 50. 170, 476. 191, 2. 198,45 .

Apocryptes dentatus $C . V .91,50$.

Apocryptes glyphisodon Blkr. 15, 36. 234, 356. 287, 56.

Apocryptes Henlei Blkr. 15, 37. 16, 5. 25, 10.

Apocryptes lanceolatus Cint. 272, I7. 287, 56. 288, 77. 356, I74. $453,328$.

Apocryptes macrolepis Blkr. 42, 66. 45, I95. 49, 419. 55, 412. 272,

17. $287,56.453,327$.

Apocryptes madurensis Blkr. 15, 35. 16, 5. 25, I0. 453, 327.

Apocryptes nexipinnis Cant. 288, 77 .

Apocryptes polyophthalmus Gthr. 425, I29.

Apocryptes rictuosus C.V. 91, 50.

Apocryptes serperaster Rich.425, I 29.

Apocryptes variegatus Ptrs. cf. Gobiopterus variegatus Blkr.

Apocryptodon Blkr.453, 327.

Apogon Lac. 1, 553. 2, 526. 10, 70. 468, 259.

Apogon amblyuropterus Blkr. 69, 695. cf. Amia amblyuropterus Blkr.

Apogon amboinensis Blkr. 84, 329. 184, I99. 185, 223. 234, 352 . 287, 48. cf. Amia amboinensis $B l k r$.

Apogon annularis $C$. $V$. cf. Amia annularis $B l k r$.

Apogon annularis Rüpp. cf. Amia aurea Blkr.

Apogon arenatus Cast. 263, 455. cf. Amia arenata Blkr.

Apogon argenteus $\mathrm{Val}$. cf. Amia macropterus Blkr.

Apogon aroubiensis Hombr. Jacq. cf. Amia fasciata Blkr., Gill.

Apogon aureus Blkr. 296, ro7. cf. Amia aurea Blkr.

Apogon auritus $C$. $V$. cf. Amia aurita $B l k r$.

Apogon balinensis Blkr. 13, 28. 17, 5. cf. Amia fasciata Gill. Apogon novemfasciatus $C$. $V$.

Apogon bandanensis Blkr. 95, 95. 167, 370. 184, 199. 185, 223. 188, 458. 234, 352. 270, I6. 275, 30. 287, 48. 296, го7. cf. Amia bandanensis Blkr.

Apogon bifasciatus Rüpp. cf. Amia taeniata Blkr.

Apogon Bleekeri Gthr. cf. Amia macropterus Blkr.

Apogon buroënsis Blkr. cf. Amia buroënsis Blkr.

Apogon buruënsis Blkr. 151, 394.

Apogon Cantoris Blkr. 50, 479. cf. Amia Cantori Blkr.

Apogon carinatus Schl. cf. Amia carinata Blkr.

Apogon carinatus $C$. $V$. cf. Apogonichthys carinatus $B l k r$.

Apogon ceramensis Blkr. 54, 256. 234, 352. 270, I6. 275, 30. 287, 48. cf. Amia ceramensis Blkr.

Apogon ceylonicus $C$. $V$. cf. Amia macropterus $B l k r$.

Apogon chrysopomus Blkr. 109, 239. 275, 30. 285, 245. 287, 48. cf. Amia chrysopomus Blkr. 
Apogon chrysosoma Blkr. 54, 256. cf. Amia moluccensis Blkr., Apogon moluccensis $\mathrm{Val}$.

Apogon chrysotaenia Blkr. 44, I68. cf. Amia chrysotaenia Blkr.

Apogon chrysotaenia Day cf. Amia Wassinki Blkr.

Apogon cupreus Ehr. cf. Amia moluccensis Blkr.

Apogon cyanosoma Blkr. 80, $7 \mathrm{r}$. cf. Amia cyanosoma Blkr.

Apogon cyanotaenia Blkr. 80, 7I. cf. Amia cyanotaenia Blkr.

Apogon endekataenia Blkr. 56, 449. 158, 273. 185, 223. 285, 245.

287, 48. cf. Amia endekataenia Blkr.

Apogon fasciatus Gthr. cf. Amia endekataenia Blkr.

Apogon fasciatus Q. G. cf. Amia fasciata Blkr., Gill.

Apogon frenatus $C . V$. 142, 25. 184, I99. 275, 30, 31. 296, 107. cf. Amia frenata $B l k r$.

Apogon (Pristiapogon) frenatus Klunz. cf. Amia melanorhynchus Blkr.

Apogon fucatus Cant. 288, 72. cf. Amia macropterus Blkr.

Apogon glaca Blkr. 2, 526. 10, 7 1. 13, 29. 25, 7. 51, 55. 90, 3. cf. Amia glaga Blkr., Apogonichthys glaga Blkr.

Apogon Godini Blkr. 86, 496. 270, I6. cf. Amia Godini Blkr.

Apogon Hartzfeldii Blkr. 54, 254. 103, 482. 184, 199. cf. Amia Hartzfeldi $B l k r$.

Apogon Hoevenii Blkr. 103, 483. 184, I99. 275, 30. cf. Amia Hoevenii Blkr.

Apogon hyalosoma Blkr. 84, 329. 156, 212. 185, 223. 237, 436. 270, I6. 275, 30. 287, 48. cf. Amia hyalosoma Blkr.

Apogon hypselonotus Blkr. 125, 309. 156, 212. 157, 231. 182, I59. 184, I99. 185, 223. 231, I44. 235, 303. 236, 330. 275, 30. cf. Amia hypselonotus Blkr.

Apogon kallopterus Blkr. 143, 33. 167, 370. 275, 30. cf. Amia kallopterus $B l k r$.

Apogon kallosoma Blkr. 56, 448. 185, 223.

Apogon kalosoma Blkr. cf. Amia kallosoma Blkr.

Apogon koilomatodon Blkr. 73, 134. 167, 370. 227, 406. 287, 48. 296, ro7. cf. Amia koilomatodon Blkr.

Apogon lateralis $\mathrm{Val}$. cf. Amia lateralis Blkr.

Apogon leptacanthus Blkr. 155, 204. cf. Amia leptacanthus Blkr.

Apogon lineatus T. Schl. 118, 54. 253, 234.

Apogon lineatus Schl. cf. Amia lineata Blkr.

Apogon macropteroïdes Blkr. 70, 724. 275, 30. 287, 48. cf. Amia macropterus Blkr.

Apogon macropterus K.v.H., BlKr. 13, 3. 44, 168. 70, 724. 231, 1 44. 234, 352. $235,363.270$, э6. $275,30.287,48.296,107$. 309, 155. cf. Amia macropterus Blkr.

Apogon margaritophorus Blkr. 112, 363. 285, 245. 287, 48. cf. Amia margaritophora Blkr.

Apogon melanorhynchos B!kr. 54, 255. 142, 26.275, 30. 285, 245. 287, 
48. cf. Amia frenata, melanorhynchus, Blkr., Apogon frenatus $\mathrm{Val}$. Apogon melanurus Cast. 263, 454. cf. Amia melanurus Blkr.

Apogon melas Blkr. 9, 635. 13,' 29. 142, 27. 156, 212. 157, 23 I. 275, 30. 287, 48. cf. Amia melas Blkr.

Apogon modestus Blkr. 110, 3r5. 113, 4I6. 287, 48. cf. Amia modesta Blkr.

Apogon moluccensis $V a l .184$, I99. 275, 30. cf. Amia moluccensis Blkr. Apogon monochrous Blkr. 143, 34. 167, 370. 231, I44. 242, I42. 267, I 39. 270, I6. 275, 30. 285, 245. 287, 48. cf. Amia moluccensis, monochrous Blkr.

Apogon multitaeniatus Blkr. cf. multilineata Blkr.

Apogon multitaeniatus Ehr. 9, 635. 13, 28. 184, 199. 275, 3 o.

Apogon nematopterus Blkr. 143, 35. 275, 30. cf. Amia nematopterus Blkr.

Apogon nigripinnis $C . V .10,66.13,3.46,2$ II. 91, $30275,30$. cf. Amia nigripinnis Blkr., Apogon monochrous Blkr.

Apogon nigripinnis Schl. cf. Amia nigripinnis Blkr.

Apogon nigromaculatus Hombr. Jacq. cf. Amia nigromaculata Blkr. Apogon Noordzieki Blkr. 236, 336. cf. Amia Noordzieki Blkr.

Apogon Novae-Guineae $V a l . ~ 110,316.113,416$. cf. Amia NovaeGuineae Blkr.

Apogon Novae-Hollandiae Val. 119, I9. 125, 3 10.

Apogon novemfasciatus $C . V .10,66,68.13,3.46,2$ I . 53, r63. 157, 232. 167, 370. 171, 245. 184, I 99. 188, 458. 231, I 44. 234, 352. 235, 363. 236, 330, 333. 270, 16. 275, 30. cf. Amia fasciata Blkr., Gill.

Apogon novemfasciatus Schl. (nec. C. V.) cf. Amia endekataenia, Schlegeli Blkr.

Apogon novemstriatus Rüpp. cf. Cheilodipterus quinquelineatus $C . V$, Paramia quinquelineata $B l k r$.

Apogon notata Day. cf. Amia macropterus Blkr.

Apogon orbicularis K.v.H. 13, 3. 54, 254. 142, 28. 156, 212. 167, 370. 184, I99. 185, 223. 270, I6. 287, 48. cf. Amia orbicularis Blkr.

Apogon poecilopterus K.v.H. 13, 3. 51, 55. 288, 72. cf. Amia poecilopterus Blkr.

Apogon punctulatus Blkr. cf. Amia polystigma Blkr. Apogonichthys polystigma Blkr.

Apogon punctulatus Riupp. 69, 696 .

Apogon quadrifasciatus $C . V .13,28.51,55.56,444.91,30.119$, 5. 227, 406. 275, 31. 288, 72. cf. Amia quadrifasciata Blkr.

Apogon quinquevittatus Blyth. cf. Glyphidodon (Glyph.) coelestinus (Sol.) C. $V$.

Apogon rhodopterus Blkr. 51, 62. cf. Amia rhodopterus Blkr.

Apogon roseipinnis $C . V .13,3.54,253.91,30.275,31$. cf. Amia aurea Blkr. 
Apogon sangiensis Blkr. 167, 375. cf. Amia sangiensis Blkr.

Apogon Schlegelii Blkr. 118, 55. cf. Amia endekataenia Blkr.

Apogon semilineatus Temm. Schl. 118, 55. cf. Amia semilineata Blkr. Apogon semiornatus Pct. cf. Amia semiornata Blkr.

Apogon singapurensis Castcln. 263, 454. cf. Amia singapurensis Blkr. Apogon taeniatus Ellr. 56, r49. cf. Amia taeniata Blkr.

Apogon taeniopterus Benn. cf. Amia taeniopterus Blkr.

Apogon thermalis $C . V .2,526.9,632,635.13,27.25,7.91,30$.

Apogon thermalis Blkr. cf. Apog. hyalosoma Blkr.

Apogon timorensis Blkr. 98, 207. cf. Amia timorensis Blkr.

Apogon trimaculatus $C . V$. 13, 3. cf. Amia trimaculata Blkr.

Apogon trimaculatus Rich. (nec. C. V.) cf. Amia bifasciata, taeniata Blkr. Apogon truncatus Blkr. 113, 415. Amia truncata Blkr.

Apogon variegatus $\mathrm{Val}$. cf. Amia variegata Blkr., Apogonichthys polystigma Blkr.

Apogon vittiger Benn. cf. Amia frenata Blkr., Apogon frenatus $\mathrm{Val}$. Apogon Wassinki Blkr. 286, 257. cf. Amia Wassinki Blkr.

Apogon zeylonicus $C . V .91,30$.

Apogon zosterophorus Blk\%. 143, 36. 275, 31. cf. Amia zosterophorus Blkr.

Apogonichthys Blkr. 118, 56. 468, 259.

Apogonichthys amblyuropterus Blkr. 358, I88. cf. Amia amblyuropterus Blkr.

Apogonichthys auritus Day. zie Amia polystigma Blkr.

Apogonichthys carinatus Blkr. 118, 56. 253, 234. 425, 137. cf. Amia carinata Blkr.

Apogonichthys glaga Blkr. 118, 57. 267, r40. 287, 48. cf. Amia glaga Blkr.

Apogonichthys gracilis Blkr. 145, 37 r. 315, 233.cf. Amia gracilis Blkr. Apogonichthys macropthalmus Cast. 263, 455. cf. Amia macrophthalmus Blkr.

Apogonichthys perdix Blkr. 99, 321. 318, 25r. 354, 147. cf. Amia perdix Blkr.

Apogonichthys polystigma Blkr. 103, 484. 145, 372. 167, 370. 270, I6. 275,3 I. $287,48.315,234.354$, I47. 358, I88. 364, 284. 437, 38. cf. Amia polystigma Blkr.

Apogonichthys punctulatus Blkr. 437, 38. cf. Amia polystigma Blkr. Apogonichthys taeniopterus Cast. 263, 455. cf. Amia taeniopterus Blkr. Apogonini. 468, 334.

Apogonoïdes 10, 70 .

Apogonoides macassariensis Blkr. 10, 7r.

Apolectus niger Cuv.425, I33.497, г6.

Apolectus stromateus $C$. $V$. cf. Stromateus niger $B l$.

Apomotis Raf. 468, 336.

Apostata Heck. 468, 336. 
Aprion C. $V .438,44.468,276,336$.

Aprion brevirostris $C . V .459,90$.

Aprion (Aprion) microlepis Blkr. 442, 94. 459, 26, 90.

Aprion (Aprion) pristipoma Blkr.442, 96. 491, 43.

Aprion Sieboldi Blkr. 497, 7 .

Aprion (Aprion) virescens C. $V .438,44.442,92.459,90.468,276$.

Apseudobranchus Gill. 468, 329.

Apseudobranchus toeroe Gill. cf. Otolithus cayennensis Blkr.

Apsilus C. $V .468,276$.

Apsilus fuscus C. $V .459,90$.

Apterichthys Dum. 88, 38. 351, I 20.

Apterichthys quadratus Rich. 425, 124.

Apterigia immaculata Basil. cf. Monopterus javanensis Lac.

Apterigia nigromaculata Basil. cf. Monopterus javanensis Lac.

Apterigia saccogularis Basil. cf. Monopterus javanensis Lac.

Apterioptus pictorius Rich. 33, I62. 35, 262, 42, 60.

Apua Blyth. 323, 39.

Apua fusca Blyth. 323, 43.

Aracana Gr. 367, I5.

Aracana aculeata Gïnth. 497, 27.

Aracana (Kentrocapros) aculeata Blkr. 408, 238.

Aracana hexagonus $K p$. cf. A. (Kentrocapros) aculeata $B l k r$.

Aracana lineata $G r$. cf. Ostracion (Aracana) auritus Shaw.

Archamia Gill. 468, 259.

Archamia Bleekeri Gill. cf. Amia macropterus Blkr.

Archoplites Gill. 468, 249.

Archoscion Gill. 468, 330.

Arelia bornëensis Blkr. 272, 9.

Arelia brachyrhynchos Blkr. 275, I4. 287, 41. 296, 103.

Arelia Feldmanni Blkr. 270, 62. 272, 9.

Arelia javanica $K p .235,373.272,9.287,4 \mathrm{r}$.

Arelia Kaupii Blkr. 270, 73.

Arelia Kopsi Blkr. 270, 63. 287, 4I.

Arelia lida Blkr. 275, I5.

Arelia macrolepidota $K p$., Blkr. 235, 373. 270, 63 .

Arelia melanopterus Blkr. 270, 63. 272, 9.

Arelia microlepis Blkr. 272, 9.

Arelia oxyrhynchos Blkr. 272, 9.

Arelia polytaenia Blkr. 270, 63. 287, 41.

Arelia potous $K p$. 267, I39. 272, 9. 287, 41. 288, 69.

Arelia quadrilineata Blkr., Kp. 235, 373. 270, 63. 272, 9. 275, I 5 .

287, $4 \mathrm{r}$.

Arelia sumatrana Blkr. 270,63.

Arelia Waandersi Blkr. 270, 63 .

Argentina bonuk Lac. cf. Albula bananus $C . V$. 
Argentina carolina $L$. cf. Elops saurus $L$.

Argentina glossodonta Forsk. cf. Albula bananus $C . V$.

Argentina machnata Forsk. cf. Elops saurus $L$.

Argentina retropinna Kich. 119, I5.

Arges $\mathrm{Val}$. 306, IIo.

Arges sabalo Val. 306, тाо.

Argyreiosus Lac. 297, 137.

Argyreiosus setipinnis Gthr. cf. Vomer setipinnis Ayr.

Argyreus Heck. 261, 434. 314, 209.

Argyropelecus Olfersii C. $V .266,{ }_{5}^{6}$.

Argyrops Stons. 468, 28 I.

Ariodes Miull. Trosch. 306, 9г.

Ariodes acutus Blkr. 270, 44. 287, 58.

Ariodes arenarius $M$. Trosch. 306, 9I.

Aroides goniaspis Blkr. 270, 44.

Ariodes leiocephalus Blkr. 275, 46. 287, 58. 288, 77.

Ariodes polystaphylodon $B l k r .270,45$.

Ariodes tonggol Blkr. 235, 370. 270, 45. 287, 58 .

Ariosoma Stons. 351, Ir6.

Arius Val., Blkr. 2, 5I I. 306, 9г.

Arius acutivelis $\mathrm{Val}$. cf. Melanodactylus nigrodigitatus Blkr.

Arius acutus Blkr. 3, I67. 4, 287. 5, 33. 7, 6. 25, Iо.

Arius aequibarbis $C . V .91,56$.

Arius angulatus Blkr. 3, I70. 4, 287. 5, 36. 91, г20.

Arius arenatus $V$ al. 336, 53.

Arius argyropleuron $V a l .3$, I36. 5, 2.

Arius arioïdes Blkr. 91, 56 .

Arius arius $C . V ., \quad V a l .51,58.91,56.270,44.272$, IS. 281, 108.

288, 77. cf. Ar. ocellatus Blkr., Pseudarius arius Blkr.

Arius Belangerii $C . V .91,56$.

Arius bilineatus $B l k r .91,5^{6}$.

Arius bornëensis Blkr. 42, 67. 45, 196. 49, 420. 55, 413.

Arius caelatoides Blkr. 3, I59. 4, 286. 5, 25. 25, Iо.

Arius caelatus $V a l ., C . V .3,136.5,2.25$, ro. 91, 56.

Arius chinta Blkr.91, 56.

Arius chondropterygioides Blkr. 3, I70. 4, 287. 5, 36. 91, I20.

Arius chondropterygius $B l k r .3,162.4,286.5,28.25$, го.

Arius clypeaster Blkr. 3, I6I. 4, 286. 5, 27.

Arius clypeastroides Blkr. 3, I6I. 4, 286. 5, 27.

Arius coelatus $\mathrm{Val} .356,175.411,25^{2}$.

Arius crossocheilos Blkr. 3, I72. 4, 287. 5, 38.

Arius Dieperinki Blkr. 298, 375. 336, 50.

Arius doroïdes Blkr. 91, 56 .

Arius Dussumierii C. $V .91,56$.

Arius (Pseudarius) falcarius Blkr. 425, I25. 
Arius falcarius Rich. 459, I03. cf. Ar. (Pseudarius) falcarius Blkr.

Arius fissus $\mathrm{Val}$. 336, 52 .

Arius gagora $C . V .91$, I 19 .

Arius gagorides $B l k r .91,56$.

Arius gagoroides Blkr. 3, т68. 4, 287. 5, 34. 7, 6. 25, то. 91, г2о.

Arius goniaspis Blkr. 174, 44.

Arius grandicossis $V a l$. 306, 91 .

Arius granosus $C . V$. 91, 56 .

Arius Hamiltonis Blkr. 4, 29I, 5, 58.

Arius hastatus $C . V .91,56$.

Arius Heckelii Blk\%. 3, 169. 4, 287. 5, 35. 91, 120.

Arius laeviceps Blkr. 3, I65. 5, 31. 16, 5. 25, го.

Arius leiotetocephalus Blkr. 4, 292. 5, 59. 25, 10. 51, $5^{8 .}$

Arius (Hexanematichthys) leptaspis Blkr. 491, 55.

Arius leptonotacanthus Blkr. 16, I I. 25, Iо.

Arius longibarbis Cast. cf. Leiarius longibarbis Blkr.

Arius macrocephalus Blkr. 3, r67. 4, 287. 5, 33 .

Arius macronotacanthus Blkr. cf. Bagrus sumatranus Raffl. 3, I59. 4, 2S6. 5, 25 .

Arius macruropterygius Blkr. 3, I64. 4, 286. 5, 30. 32, г6о. 50, 472.

$51,58.56,445$.

Arius majong $B l k r$. cf. Arius (Arius) venosus $\mathrm{Val}$.

Arius manjong Blkr. 3, I63. 4, 286. 5, 29.

Arius melanochir Blkr. 67, 590.

Arius melanopterygius Blkr. 16, 10. 25, ro.

Arius microcephalus Blkr. 139, 423. 272, I8.

Arius microgastropterygius Blkr. 3, 160. 4, 286. 5, 26.

Arius micronotacanthus Blkr. 3, I62. 4, 286. 5, 28.

Arius micruropterygius Blkr. 3, I64, 4, 286. 5, 30.

Arius militaris $C, V$. cf. Osteogeneiosus militaris $B l k r$.

Arius nasutus $V$ al. $C . V$. 3, I 58. 4, 286. 5, 24. 7, 6. 25, 10. 127, 345 .

156, 217 . cf. Arius (Netuma) thalassinus Blkr.

Arius nenga Blkr. 91, 56 .

Arius netuma Blkr. 81, 56 .

Arius nodosus C. $V$., Val. 91, 56. cf. Pseudauchenipterus nodosus Blkr.

Arius ocellatus Blkr. 497, 22.

Arius oetik Blkr. 3, I66. 4, 286. 5, 32 .

Arius papillosus $V a l$. cf. Diplomystes papillosus $D u m$.

Arius pavimentatus $C . V .91,56$.

Arius pidada Blkr. 3, I69. 4, 287. 5, 35. 16, 5, 25, I 0. 91, І 20. 270, 44 .

Arius polystaphylodon Blkr. 3, I72. 4, 287.5, 38 .

Arius pumilus $V a l .91,56$.

Arius quadriscutis $\mathrm{Val}$. cf. Netuma quadriscutis Blkr.

Arius rita $C . V$. cf. Rita Buchanani Blkr.

Arius ritoïdes $C . V$. cf. Rita Buchanani Blkr. 
Arius rostratus $C . V .91,56$.

Arius sagor Blkr. 91, 56.

Arius Schlegeli Blkr. 307, r46. cf. Ar. (Pseudarius) falcarius Blkr.

Arius (Arius) sinensis $V a l .425,125$.

Arius stricticassis $\mathrm{Val} .336,49$.

Arius subrostratus $C . V .91,5^{8}$.

Arius sundaicus Blkr. 178, 2.

Arius (Netuma) thalassinus Blkr. 395, 293.

Arius (Ariodes) tonggol Blkr. 395, 293.

Arius tonggol Blkr. 4, 292. 5, 59. 50, 472. 56, 445. cf. Ar. (Ariodes)

tonggol $B l k r$.

Arius trachipomus Blkr.91, 58 .

Arius truncatus $\mathrm{Val} . \mathrm{C} . \mathrm{V}$. 3, I36. 5, 2. 55, 426. 178, 2. 356, I75.

Arius (Arius) venosus $\mathrm{Val} .3,165.4,286.5,3$ I. 395, 293.

Arius viviparus Blkr. 3, I71. 4, 287. 5, 37. 91, I20.

Arnoglossus Will. 294, 427 .

Arothron J. Mïll. 54, 300.

Arothron bondarus Blkr. 288, 68.

Arothron calamara $J$. Mïll., Blkr. 270, 65. 287, 39. 358, r83. cf.

Tetraödon calamara Russ.

Arothron calamaroïdes Blkr. 157, 235.

Arothron carduus Blkr. 288, 68.

Arothron erythrotaenia $B l k r .275$, I2.

Arothron firmamentum Blkr. 118, I24.

Arothron hypselogeneion Blkr. 231, I5I. 275, I2. 296, I03. 358, I 83. Arothron immaculatus Blkr. 288, 68 .

Arothron kappa Blkr. 156, 218. 231, I5I. 255, 24т. 270, 66. 275,

I 2. 281 , 108. $285,243.287,39.288,69 . \mathrm{cf}$. Leiodon patoca Blkr. Arothron laterna Blkr., Rich. 257, 332. 270, 66. 271, 2. 276, 65.

296, I03. cf. Crayracion implutus Blkr.

Arothron leiurus Blkr. 200, 48. 239, го2. 270, 66. 272, 7. 356, I72.

Arothron lineatus Blkr. 142, 67. 250, 207. 275, 12. 287, 39.

Arothron mappa Blkr. 287, 39. 356, I72. 358, I 8 .

Arothron melanorhynchos Blkr. 132, III. 156, 2 I8.

Arothron melanorhynchus Blkr. 309, I54.

Arothron palembangensis Blkr. 270, 66. 272, 7 .

Arothron potamophilus $B l k r .178 .2 .235,374$. 270, 66. 272, 7. 287, 39 .

Arothron reticularis Blkr. 119, 16.

Arothron scaber Blkr. 156, 2 I8. 157, 235. 270, 66. 275, I2. 287,

39. 296, 103. cf. Crayracion immaculatus $B l k r$.

Arothron simulans Blkr. 287, 40. 288, 69.

Arothron testudineus J. Mïll. 234, 357. 235, 374. 270, 66. 275, I3.

287, 40. 288, 69. 354, 143. cf. Crayracion testudineus Blkr.,

Tetraödon testudineus $B l$.

Arothron trichoderma Blkr. 156, 218. 157, 236. 270, 66. 
Arothron trichodermatoides Blkr. $310, \mathrm{x} 60$.

Arothron virgatus Blkr. 119, r6. 257, 332. 275, r3. 296, Іо3. 346, 3. 354, I43. 358, I83. cf. Tetraodon virgatus Rich.

Arothron Waandersii Blkr. 235, 374.

Arripis Jen. 468, 275 .

Arripis georgianus Jen. 331, 446. 468, 275 .

Asphareus coerulescens Swons. cf. Aphareus furcatus Gthr.

Aspidobagrus Blkr. 306, 96.

Aspidobagrus gulio Blkr. 306, 96. 425, 125.

Aspidoparia Heck. 261, 427. 314, I97.

Aspidophorus acipenserinus C. $V .425, \mathrm{I} 25$.

Aspidophorus laevigatus $C . V$. cf. Agonus laevigatus Tiles.

Aspidophorus pusillus Langsd. cf. Hoplichthys Langsdorfii C. $V$., pusillus Blkr.

Aspisurus 137, 306 .

Aspisurus elegans Rüpp. cf. Naseus lituratus C. $V$.

Aspisurus lituratus Rïpp. cf. Naseus lituratus $C$. $V$.

Aspius $A g .261,436.314,2$ I3. 329, 264. 411, 253.

Aspius spilurus Gthr. 415, Ir. 425, I44.

Aspredinichthys Blkr. 306, II8.

Aspredinichthys tibicen Blkr. 306, II8. 336, 98 .

Aspredo L. 306, II 7 .

Aspredo batrachus $L$. 306, Ir8. 336, 93 .

Aspredo laevis $V a l$. cf. Aspr. batrachus $L$.

Aspredo octocirrhus Guér. cf. Aspr. batrachus $L$.

Aspredo sexcirrhis Val. cf. Aspr. batrachus L., Platystacus cotylephorus $B l$.

Aspredo spectrum Gron. cf. Platystacus cotylephorus $B l$.

Aspredo tibicen Temm. cf. Aspredinichthys tibicen Blkr.

Astemomycterus Guich. 306, II3.

Asterophysus Kner. 306, 89.

Asterophysus batrachus Kner. 306, 89 .

Asterropteryx Rüpp. 453, 305.460, I03, го6.

Asterropteryx cyprinoides Blkr. 459, 78 .

Asterropteryx leuciscus Blkr. 478, 79 .

Asterropteryx modestus Blkr. 460, III. 478, SI.

Asterropteryx semipunctatus Rüpp. 453, 306.

Asterropteryx taenionotopterus Blkr. 478, So.

Astrape capensis $M . H$. 266, $58.459,68$.

Astrape dipterygia $M . H .51,60.91,82.288,67.425$, г20.

Astrape japonica: $T$. Schl. 175, 42. 497, 4. cf. Astr. dipterygia $M$. $H$.

Astroblepus Humb. 306, г го.

Astroblepus Grixalvi Humb. 306, I 10.

Astrodoras Blkr. 306, 86.

Astrodoras asterifrons $B l k \%$. 303, г \%. 306, 86 . 
Astrodoras Heckeli Blkr. 303, 17.

Astronesthes chrysophekadion Blkr. 39, 424. 59, 2r. 231, I 49.

Astronesthes nigra Rich. 425, I47.

Ateleopus japonicus Blkr. 497, 2 I.

Atherina 1, 553. 90, 40 .

Atherina affinis Benn. cf. Atherina pinguis Lac.

Atherina afra Peters. 459, I04.

Atherina argyrotaenia Blkr. 231, I47. 275, 4I.

Atherina argyrotaeniata Blkr. 10, 72. 46, 213.

Atherina australis $\int$. White. cf. Engraulis Brownii C. $V$.

Atherina bimanensis Blkr. 9, 637 .

Atherina Bleekeri Gthr. 425, I42. 497, I 7.

Atherina bonasicosi $C . V .90,40$.

Atherina brachypterus Blkr. 47, 243.

Atherina breviceps $C . V .266,68$.

Atherina Brownii $G m$. cf. Engraulis Brownii $C$. $V$.

Atherina Commersonii Shawo cf. Engraulis Brownii C. $V$.

Atherina cylindrica C. V. 364, 29I. 396, 297.

Atherina duodecimalis $C . V .50,485.56,445.91,48.108,164.147$, 469. 184, 201. 185, 223. 208, 239. 231, 147. 234, 354. 237, 438 . 270, 32. 275,4 I. 287, 55. 296, г го. $315,236.320,27$ г. 338,36 г. 358, 191. 364, 291. 395, 293, 491, 53.

Atherina endrachtensis $Q . G ., C . V .119$, I 1. 491, 53.

Atherina Forskilii Rüpp. 10, 72. 25, 9. 288, 76 .

Atherina hepsetus Forsk. cf. Atherina pinguis Lac.

Atherina Humboldtiana C. V. 90, 40.

Atherina jacksoniana $C . V . \mathbf{1 1 9}$, I $\mathrm{t}$.

Atherina japonica Blkr. (nec. Houtt.) 90, 40. 425, I42. cf. Atherina Bleekeri Gthr.

Atherina japonica Houtt. cf. Engraulis Brownii $C$. $V$.

Atherina lacunosa Forst., Forsk., Val., 86, 504. 184, 20r. 231, 147. 234, 354. 270, 32. 275, 4r. 287, 55. 315, 236. 320, 27r. 364, 2 r. $396,297.491,53$.

Atherina Lichtensteinii C. $V .90,40$.

Atherina nigrans Rich. 119, x $\mathrm{I}$.

Atherina parvipinnis $C, V .266,54$.

Atherina pectoralis $C . V .2,5^{14} .81,48.119, \mathrm{Ir} . c f$. Atherina pinguis $L a c$. Atherina pellucida Comm. cf. Atherina pinguis Lac.

Atherina pinguis Lac. 270, 84. 459, 79. 495, I 8.

Atherina presbyteroïdes Rich. 119, I I.

Atherina punctata Benn. 459, 79. 495, I8.

Atherina regia Humb. 90, 40.

Atherina sihama Forsk. cf. Sillago sihama Rüpp.

Atherina 'Temmincki Blkr.86, 506. 270, 32. 275, 4I. 315, 236. 320, 27 I.

Atherina vaigiensis Q.G. cf. A. cylindrica $C$. V., A. lacunosa Forst. INDEX BI.EEKLR. 
Atherina Valenciennei Blkr. 86, 507.

Atherina Valenciennesi Blkr. 248, 203. 270, 32. 287, 55 .

Atherina vomerina $C . V .90,4 \mathrm{I}$.

Atherina waigiensis Q.G. cf. Ath. lacunosa Forst.

Atherinoïdes 90,40 .

Atheronichthys 90, 4I.

Atimostoma capensis Smith. 266, 53.

Atopomycterus Verr. 367, I7.

Atractoperca Gill 468, 255.

Atractoscion Gill 468, 329.

Atractoscion aequidens C. $V .468,329$.

Atypichthys Gthr. 468, 298.

Atypichthys strigatus Gthr.468, 298.497, 8.

Atypus Gthr. 468, 298.

Atypus strigatus Gthr. 468, 298.

Auchenaspis Blkr. 306, гох.

Auchenaspis biscutatus Blkr.306, Icr.

Auchenipterichthys Blkr. 306, 89.

Auchenipterichthys thoracatus Blkr. 306, 89.

Auchenipterus $\mathrm{Val}$. 306, I09.

Auchenipterus colymbetes Blkr. 336, 4.

Auchenipterus dentatus $V$ al. 336, 86.

Auchenipterus furcatus $\mathrm{Val}$. cf. Pseudauchenipterus nodosus $\mathrm{Blkr}$.

Auchenipterus immaculatus $V a l$. cf. Parauchenipterus galeatus Blkr.

Auchenipterus maculosus $\mathrm{Val}$. cf. Parauchenipterus galeatus $\mathrm{Blkr}$.

Auchenipterus nodosus $M$. Trosch. cf. Pseudauchenipterus nodosus Blkr.

Auchenipterus nuchalis $\mathrm{Val}$. 306, I09.

Auchenipterus thoracatus Kner cf. Auchenipterichthys thoracatus. Blkr.

Auchenipterus trachycorystes $\mathrm{Val}$. cf. Trachycorystes typus $B l k r$.

Aulacocephalus Schl. 468, 254.

Aulacocephalus Schlegelii Blkr. 459, 88. 495, го.

Aulacocephalus Temmincki Blkr. 468, 254. 497, 5 .

Aulichthys japonicus Brev. 497, I4.

Aulopus elongatus Schl. cf. Saurida tumbil $\mathrm{Val}$.

Aulopus japonicus Gïnth.497, 24.

Aulopus Milesii Cथ. 119, I5.

Aulopyge Heck. 261, 432. 314, 207.

Aulostoma chinense Lac., Cuv., Schl. 315, 235. 354, I49. 358, I9I.

364, 290. 369, 42. 396, 300. 425, г26. 459, 74 491, 50.497, I 4.

Aurata Risso. 468, 336 .

Auxis tapeinosoma Blkr. 102, 408. 118, 98. 124, 302. 497, I 5 .

Auxis taso $C . V .2,516.25,8.124,302.491,50$.

Auxis taso Blkr. cf. Thynnus thunnina $C . V$.

Auxis thynnoïdes Blkr. 124, 30I. 315, 235. 364, 289.

Auxis vulgaris $C . V .124,301$. 
Awaous Val. 453, 320.

Awaous Commersoni Blkr. 495, 17.

Awaous Lienardi Blkr. 495, 17 .

Awaous ocellaris Blkr. 495, I 7 .

Awaous pallidus Blkr. 495, I 7 .

Awaous personatus $B l k r .495$, I 7 .

Axinurus 137, 305 .

Axinurus dipeltis Val. cf. Naseus unicolor Lien.

Axinurus leptopeltis $C$. $V$. cf. Naseus leptopeltis Blkr.

Axinurus thynnoides $C . V$. 137, 306. cf. Naseus thynnoïdes $B l k r$.

Aylopon Raf. 468, $25 \mathrm{I}$.

Badis Blkr. 468, 318 .

Badis Buchanani Blkr. 91, 106. 468, 3 I8.

Badis dario Blkr. 91, 52 .

Bagarius Blkr. 306, I04.

Bagarius Buchanani Blkr. 91, I21. 170, 477. 306, 104.

Bagarius cavia Blkr. 91, 58.

Bagrichthys Blkr. 306, 94.

Bagrichthys hypselopterus Blkr. 270, 45. 272, 18. 306, 94.

Bagroides Blkr. 306, 94.

Bagroïdes macracanthus Blkr. 106, 88. 270, 45 .

Bagroïdes macropterus Blkr. 86, 5I5. 270, 46. 356, I75. cf. Pseudobagrichthys macropterus Blkr.

Bagroïdes melanopterus Blkr. 55, 4I3. 270, 46. 272, I8. 306, 94.

Bàgroïdes melapterus Blkr. 45, 204. 49, 420.

Bagrus Cuv. 1, 552. 306, 96.

Bagrus abbreviatus $V a l .3$, I35. 5, I. cf. Bagrus gulio $C . V$.

Bagrus albilabris $C$. $V$. cf. Bagrus gulio $C$. $V$.

Bagrus angius $C . V .91,54$, I 2 .

Bagrus anisurus $V a l .3$, I35. 5, I.

Bagrus aor $C$. $V$. 91, I I 5 .

Bagrus aorinus $V a l .91,56$.

Bagrus atherinoïdes $C . V .91,56$.

Bagrus aurantiacus $T$. Schl., cf. Pseudobagrus aurantiacus Blkr.

Bagrus bagre Cuv. cf. Pimelodus filamentosus Blkr.

Bagrus bajad Cuv. 306, 96.

Bagrus batasis $C . V .91,56$.

Bagrus bonderius Rich. cf. Pseudobagrus Vachelli Gthr.

Bagrus capensis Smith cf. Galeichhthys feliceps $C . V$.

Bagrus carchariorhynchos Blkr. 4, 291. 5, 58 .

Bagrus carcio Blkr. 91, 56 .

Bagrus cavasius $V a l .3$, I35. 5, I. 91, II 3 .

Bagrus corsula $C$. $V .91,56$.

Bagrus crinalis Rich. cf. Arius (Pseudarius) falcarius $B l k \%$. 
Bagrus exodon $C . V . \mathbf{9 1}$, III.

Bagrus (Bagrus) feliceps Mïll. cf. Galeichthys feliceps $C$. $V$.

Bagrus flavus Blkr. 3, I 56. 4, 285. 5, 22.

Bagrus fuscus $C$. $V$. cf. Bagrus gulio $C$. $V$.

Bagrus genidens $\mathrm{Val}$. cf. Genidens Cuvieri Cast.

Bagrus goongwaree Blkr. 91, $5^{6}$.

Bagrus gulio $C . V .91$, г 6.121, I63. 227, 408. 270, 46. 272, г 8. 288, 78. cf. Aspidobagrus gulio Blkr.

Bagrus gulioides Blkr. 3, I 52. 4, 285. 5, I8. 7, 6. 16, 5. 25, го. cf. Bagrus gulio $C$. $V$.

Bagrus Herzbergii $\mathrm{Val}$. cf. Netuma Herzbergi Blkr.

Bagrus heterurus Blkr. 3, I5 1. 4, 285. 5, I7.

Bagrus Hoevenii Blkr. 3, 154. 4, 285. 5, 20. 55, 4r3. 170, 476. 191, 3. 235, 370. 270, 46. 271, I. 272, I8.

Bagrus hypselopterus Blkr. 67, 588 .

Bagrus itchkea Blkr. 91, 56 .

Bagrus javensis $V a l .3,136.5,2$.

Bagrus keletius $V a l .3$, I35. 5, I. 91, I I5.

Bagrus Lamarrei $V a l$. cf. Macrones Lamarrii C. Dum.

Bagrus Lamarrii C. V. 91, 56 .

Bagrus limbatus Rich. cf. Hemibagrus limbatus Blkr.

Bagrus lona Sykes 91, 56 .

Bagrus macronema Blkr. 225, 357. 270, 46. 272, I8. cf. Hypselobagrus macronema Blkr.

Bagrus macronemus Blkr. 3, I 50. 4, 285. 5, I6. 7, 6. 25, го.

Bagrus macronemus Ranz. cf. Pimelodus bagre Lac.

Bagrus melas Blkr. 3, I52. 4, 285. 5, r8. cf. Bagrus gulio $C$. $V$.

Bagrus micracanthus Blkr. 3, I5 I. 4, 285. 5, г7. 7, 6. 25, 10. 55, 4I3. 170, 476. 211, 242. 225, 357. 270, 46. 272, 18. 279, 100.

Bagrus micropogon Blkr. 52, 94. cf. Bagrus poecilopterus $K . v$. $H$.

Bagrus murius $C . V .91,56$, I 2.

Bagrus nemurus $C . V$. 3, I 55. 4, 285. 5, 21. 7, 6. 25, 1о. 35, 26 г. $42,59.45$, г96. $49,420.55,4$ I3. 56, 445. 170, 476, 477. 225, 357. 239, 102. 270, 46. 272, 18. cf. Hemibagrus nemurus Blkr.

Bagrus netuma Rüpp. 3, I39. 5, 5 .

Bagrus nigriceps $\mathrm{Val}$. 3, 135. 5, I.

Bagrus nigrita $V a l$. cf. Octonematichthys nigrita Blkr.

Bagrus oculatus $C . V .91,56$.

Bagrus passany $V a l$. cf. Netuma proops $B l k r$.

Bagrus pemecus $V a l$. cf. Netuma Herzbergi Blkr.

Bagrus piramuta Kner cf. Piramutana piramuta Blkr.

Bagrus planiceps C. V. 3, I54. 4, 285. 5, 20. 170, 477. 270, 46, $272,18$.

Bagrus poecilopterus Val. 3, 136. 5, 2. 85, 445. cf. Leiocassis poecilopterus Blkr. 
Bagrus proops $V a l$. cf. Netuma proops Blkr.

Bagrus punctulatus Kner cf. Platynematichthys punctulatus Blkr.

Bagrus reticulatus Kner cf. Piratinga reticulata $B l k r$.

Bagrus rhodonotus Blkr. 3, 157. 4, 286. 5, 23. 7, 6. 25, 1о. 50, 472.

Bagrus rhodopterygius Blkr. 3, I 53. 4, 285,5 , r9. cf. Bagrus gulio $C . V$.

Bagrus schilbeides $C . V .91$, I 2 .

Bagrus Schlegelii Blkr. 3, I53, 4, 285. 5, I9. cf. Bagrus gulio C. $V$.

Bagrus seenghala Blkr. 91, 56 .

Bagrus seenghtee Blkr. 91,56 .

Bagrus Sieboldii Blkr. 3, I 55. 4, $2 S_{5} .5,2 \mathrm{I}$.

Bagrus singaringan $B l k r .3,150.4,285.5,16$.

Bagrus sondaicus $C . V$. 3, I56. 4, 286. 5, 22. 7, 6. 16, 5. 25, 10. 56, 445. cf. Arius sundaicus Blkr., Hexanematichthys sundaicus Blkr. Bagrus stenomus $\mathrm{Val}$. 3, I35. 5, I.

Bagrus sumatranus Raffl. 3, I42. 5, 8 . cf. Arius macronotacanthus Blkr. Bagrus taakree Blkr. 91, 56.

Bagrus tengana $C . V$. 91, 56.

Bagrus tengara $C . V .91$, I 4 .

Bagrus trachacanthus $C . V .91,5^{6 .}$

Bagrus urua $C . V .91,56$, I 12 .

Bagrus vacha $C . V .91,56$, I 2 .

Bagrus Vachelli Rich. cf. Pseudobagrus Vachelli Gthr.

Bagrus venaticus Rich. 119, I4.

Bagrus vertagus Rich. 119, 14.

Bagrus vittatus $C . V .91,56$.

Bagrus Wolffii Blkr. 45, 205. 49, 420. 55, 4r3. 270, 46. 272, r.

Bagrus Yarelli Sykes 91, 56.

Bairdiella Gill 468, 329.

Balantiocheilos Blkr. 261. 430 .

Balantiocheilos melanopterus Blkr. 239, I62. 270, 52. 272, 22.

Balantiocheilus Blkr. 314, r98.

Balantiochilus melanopterus Blkr. 356, I76.

Balistapus Til. 367, r 1 .

Balistes Art. 1, 552. 2, 506. 367, 10.

Balistes aculeatus L. (Bl. Schn。Blkr.) 9, 63S 47, 22S. 49, 421.55, 415. 63, I5. 119, I6. 156, 218. 184, 204. 188, 462. 270, 64. 272, 7. 275 , II. $315,230.318,249.338,360.354$, I 43.358, I $S_{3}$. cf. Balistes (Balistapus) aculeatus Blkr.

Balistes aculeatus major Forsk. cf. Balistes viridescens Comm.

Balistes aculeatus minor Forsk. cf. Balistes lineatus B!. Schn.

Balistes (Balistapus) aculeatus Bl. (Blkr.) 364, 272. 368, 24. 369, 42. 394, 285. 425, I22. 459, 70. 491, 52. 495, 20.

Balistes albicaudatus Rüpp. cf. Balistes armatus Lac.

Balistes americanus Lac. cf. Balistes conspicillum Bl. Schn.

Balistes angulatus Sol. cf. Balistes cinctus Lac. 
Balistes angulosus Q. G. cf. Balistes (Canthidermis) maculatus Blkr. Balistes arcuatus Bl. Schn. cf. Balistes (Balistapus) cinereus Blkr.

Balistes armatus L. (Lac.) 46, 224. 63, I6. 91, I66. 156, 218. 167, $374,270,64.275$, II. 316, 24 I. 320, 265. 338, 360. cf. Balistes (Balistapus) armatus, niger $B l k r$.

Balistes armatus $C . V$. cf. Balistes aculeatus $B l$.

Balistes (Balistapus) armatus Blkr. 364, 272. 368, 23. 394, 285. 491,52 .

Balistes assasi Rüpp. cf. Balistes (Balistapus) assasi Blkr.

Balistes (Balistapus) assasi Blkr. 459, 7 I.

Balistes auromarginatus Benn. cf. Balistes (Canthidermis) auromarginatus Blkr.

Balistes (Canthidermis) auromarginatus Blkr. 459, 70. 495, 20.

Balistes (Anacanthus) barbatus Gr. Hardw. cf. Pogonognathus barbatus Blkr.

Balistes Beeri Blkr. 275, 53.

Balistes biaculeatus $B l$. cf. Triacanthus Blochii Blkr.

Balistes biaculeatus Benn. cf. Triacanthus Russellii Blkr.

Balistes bicolor Shazo. cf. Balistes conspicillum Bl. Schn.

Balistes brevissimus Holl. cf. Balistes (Canthidermis) oculatus Swns.

Balistes bursa Schn. 84, 350. 315, 230. cf. Balistes (Balistapus) bursa Blkr.

Balistes bursa Lac. cf. Balistes (Balistapus) bursa Blkr.

Balistes (Balistapus) bursa Blkr. 364, 272. 368, 23. 459, 71. 491, 52. $495,20$.

Balistes calolepis Holl. cf. Balistes (Canthidermis) calolepis Blkr.

Balistes (Canthidermis) calolepis Blkr. 422, Iо3. 459, 3, 7 o.

Balistes (Balistes) capriscus L. Gm. 497, 17 .

Balistes castaneus Rich. cf. Balistes flavimarginatus Rüpp., Balistes viridescens Comm.

Balistes chinensis $B l$. cf. Monacanthus chinensis Cuv.

Balistes chrysospilos Blkr. 80, 94. 275, I I.

Balistes chrysospilus Blkr. cf. Balistes (Parabalistes) chrysospilus Blkr.

Balistes (Parabalistes) chrysospilus Blkr. 368, 22. 394, 285 .

Balistes (Pseudobalistes) chrysospilus Blkr. cf. Balistes (Parabalistes) fuscus Blkr.

Balistes cinctus Lac. 163, 96. 170, 480. 272, 7. cf. Balistes (Balistapus) cinctus Blkr.

Balistes (Balistapus) cinctus Blkr. Lac. 364, 272. 368, 24. 396, 296. 425, I22. 459, 71. 491, 52. 495, 2 .

Balistes cinereus Benn., Bonn. cf. Balistes (Balistapus) cinereus Blkr. Balistes (Balistapus) cinereus Blkr. 459, 71. 495, 20.

Balistes conspicillum Bl. Schn. Cuv. 51, 59. 65, 490. 71, 780. 108, I64. 275, I I. 288, 68. 320, 265. cf. Balistes (Balistapus) conspicillum Blkr. 
Balistes (Balistapus) conspicillum Blkr. 364, 272. 368, 23. 394, 285 . 425, I 22.459, 7I. 491, 52. 495, 20. 497, I7.

Balistes erythropteron Less. cf. Balistes cinctus Lac., Balistes (Balistapus) cinctus $L a c$.

Balistes fimbriatus J. G. Forst. cf. Balistes vidua Sol.

Balistes flavimarginatus Rüpp. 54, 303. cf. Balistes (Pseudobalistes) flavimarginatus Blkr.

Balistes (Pseudobalistes) flavimarginatus Rüpp., Blkr. 368, 22. 391, 276. 491,52 .

Balistes flavomarginatus Rüpp. 234, 357. 275, I I. 308, I 52. 358, I83.

Balistes (Pseudobalistes) flavomarginatus Blkr. 364, 272. 396, 298.

Balistes forcipatus Gm., Gr., Lac. 270, 64. 287, 39. 354, I43.

Balistes frenatus Lac. 143, 74. 270, 64. 275, Ir. 316, 241. cf. Balistes (Balistapus) frenatus Blkr.

Balistes frenatus Rich. cf. Balistes (Balistapus) frenatus Blkr.

Balistes (Balistapus) frenatus $B l k r .364,272.368,22.425$, I22. 459, 71. $491,52.495,20$.

Balistes Freycineti Cuv.cf. Monacanthus Freycineti Holl.

Balistes fuscus Lac. cf. Balistes (Parabalistes) fuscus Blkr.

Balistes (Parabalistes) fuscus Blkr. 459, 70. 495, 20.

Balistes geographicus Péron. cf. Monacanthus geographicus $C u v$.

Balistes griseus Comm. cf. Balistes stellatus Lac.

Balistes gutturosus Holl. 459, 70.

Balistes heteracanthus Blkr. 232, 22. cf. Balistes (Balistapus) heteracanthus Blkr.

Balistes (Balistapus) heteracanthus Blkr. 364, 272. 368, 23. 491, 52. Balistes hihpe Rich. cf. Balistes (Balistapus) frenatus $B(k)$..

Balistes hispidus $L$. cf. Monacanthus chrysospilos Blkr.

Balistes japonicus Tiles. cf. Monacanthus japonicus $C$.

Balistes laevis $B l$. cf. Aleuteres laevis Cuv., Alutarius laevis Cuv.

Balistes lima Benn. 459, 70. 495, 19.

Balistes lineatus Bl., Schn. 10, 68. 46, 214. 47, 260. 63, 14. 91, 7 S. 156, 2 I8. 157, 236. 167, 374. 182, I60.184, 204. 231, I 5. 236, 332. 270, 64. 275, II. 296, I02. 309, I 54. 315, 230. 318, 249. 320, 265. 354, I43. 358, I83. cf. Balistes (Balistapus) lineatus Blkr. Kp., undulatus Blkr.

Balistes (Balistapus) lineatus Blkr. Kp. 364, 272. 368, 24. 388, 27 I. $394,285.396,298.491,52$.

Balistes lineopunctatus Holl. cf. Balistes (Parabalistes) ringens Blkr.

Balistes macrophthalmus 91,78 .

Balistes maculatus /3/.296, 102. cf. Balistes (Canthiclermis) maculatus lilkr.

Balistes (Canthidermis) maculatus Blkr. 368, 25. 425, I22. 459, 70. 491,52 .

Balistes Medinilla Q. G. cf. Balistes (Balistapus) cinctus Lac.

Balistes melanopleura Blkr. 10, 7 I, 73. 17, 4. cf. Balistes praslinus Lac. 
Balistes mitis Benn. cf. Balistes (Balistapus) frenatus Blkr.

Balistes monoceros Sol. cf. Alutarius laevis Cuz'.

Balistes niger M. Park, cf. Balistes armatus Lac., Balistes (Balistapus) niger Blkr.

Balistes niger Lac. cf. Erythrodon niger Rüpp.

Balistes niger $O s b$. Gthr. cf. Melichthys ringens $B l k r$.

Balistes (Balistapus) niger Blkr. 425, I 22. 459, 7 I. 495, 20.

Balistes (Parabalistes) notatus Blkr. 368, 22.

Balistes occultator Hardzo. cf. Balistes stellatus Lac.

Balistes oculatus $G r$. cf. Balistes stellatus Lac.

Balistes (Canthidermis) oculatus Szons., Blkr. 368, 25. 388, 27 . 394, 285. 491, 52. 496, 2 .

Balistes penicilligerus Péron. cf. Monacanthus penicilligerus Cuv.

Balistes phaleratus Rich. cf. Seiurus stellatus Blkr.

Balistes praslinensis $Q . G$. cf. Balistes (Balistapus) verrucosus $K p$.

Balistes praslinoïdes Less. cf. praslinus Lac.

Balistes praslinus Lac. 46, 214. 54, 238. 63, I 4. 167, 374. 171, 245. 184, 204. 231, I5I. cf. Balistes (Balistapus) verrucosus Blkr.

Balistes rectangulus $B l$. Schn. 49, 42I. 55, 4I5. cf. Balistes (Balistapus) cinctus Blkr.

Balistes reticulatus Holl. cf. Balistes (Parabalistes) fuscus Blkr.

Balistes ringens L. 270, 69. cf. Balistes (Parabalistes) ringens Blkr.

Balistes ringens Osb. cf. Melichthys ringens Blkr.

Balistes (Parabalistes) ringens Blkr. 459, 70.

Balistes Schmittii Blkr. 63, 37. 86, 532.

Balistes scriptus Osbeck 54, 304. cf. Alutarius laevis Cuv.

Balistes senticosus Rich. 80, 93. cf. Balistes (Canthidermis) maculatus Blkr., oculatus Srons.

Balistes spinosissimus $Q$. $G$. cf. Monacanthus penicilligerus Cur.

Balistes stellaris Bl. Schn. cf. Balistes stellatus Lac.

Balistes stellatus Lac. 51, 59. 63, г3. 91, 78. 156, 2 I 8 . 275, г г. 318, 249. cf. Leiurus stellatus Blkr.

Balistes subarmatus $G r$. cf. Balistes armatus Lac.

Balistes tomentosus $L$. cf. Monacanthus tomentosus Cur.

Balistes triangularis $\mathbf{9 1}, 78$.

Balistes undulatus M. Park. cf. Balistes lineatus Bl. Schn.

Balistes (Balistapus) undulatus Blkr. 425, I22. 497, I 7.

Balistes unicornis Basil. cf. Aluteres monoceros Blkr.

Balistes Vachelli Rich. cf. Leiurus stellatus Blkr.

Balistes verrucosus $L .236,332.270,64.275$, I I. 296, го2. 315, 230. 316, 241. 320, 265. 354, 143. 358, 183. cf. Balistes (Balistapus) verrucosus Blkr.

Balistes (Balistapus) verrucosus $K p ., B l k r .364,272.368,25.388$, 27 I. 394, 285. 396, 296, 298. 425, I 22. 459, 7I. 491, 52. 495,20 . 
Balistes (Balistes) vetula $L .425$, r22. 459, 70.

Balistes vidua Sol. 66, 565. 188, 462. 309, 154. cf. Melichthys vidua $K p$.

Balistes vidua Rich. cf. Melichthys vidua Blk\%.

Balistes viredescens Comm. Lac. 112, 374. 156, 2 I 8. 170, 480. 184, 204. 196, 37. 234, 357. 275, I I. 358, i 83 . cf. Bal. (Pseudobalistes) viridescens Blkr.

Balistes (Pseudobalistes) viridescens Blkr. 364, 272. 368, 22. 459, 70, 491, 52. 495, 20.

Balistes viridis Bl. Schn。 cf. Balistes (Balistapus) verrucosus Blkr.

Balitora Brucei Gray cf. Homaloptera Brucei.

Balitora erythrorhina $C . V$. cf. Homaloptera erythrorhina $V$. Hass.

Balitora lineolata $C$. $V$. cf. Homaloptera lineolata.

Balitora maculata Gray cf. Homaloptera maculata.

Balitora nasuta $\mathrm{Mc}$. Cl. cf. Homaloptera nasuta.

Balitora ocellata $C . V$. cf. Homaloptera, ocellata $V$. Hass.

Balitora pavonina $C$. $V$. cf. Homaloptera pavonina.

Ballerus Heck. 261, 436. 314, 2 I I. 329, 263.

Bangana H.B. 314, I94. 329, 262.

Banjos Blkr. 468, 277.

Banjos typus Blkr. 468, 277. 497, 7 .

Barbichthys Blkr. 261, 424. 314, I93.

Barbichthys laevis Blkr. 270, 51. 272, 22.

Barbodes Blkr. 261, 43I. 314, 200.

Barbus Cuv. 1, 552. 261, 431. 314, I98.

Barbus amblycephalus $B 7 k \%$. 121, I66.

Barbus apogon $K u \hbar l$. cf. Systomus apogon $C$. $V$.

Barbus armatus $C$. $V$. cf. Barbus Valenciennesii Blkr. 25, I 7.

Barbus bilitonensis Blkr. 158, 274. 170, 477. 185, 226. 191, 3. 231, 149.

Barbus bphotatus $K u h l$. 56, 445. 138, 408. 170, 477. 215, 26 I.

Barbus blitonensis Blkr. 52, 96.

Barbus brachiatus Blkr. 91, 60.

Barbus brachynemus Blkr. 25, I8. cf. Barbus laevis C. $V$.

Barbus bramoides $C . V$. 25, I5. 225, 357.

Barbus bunter Blkr. 166, 350 .

Barbus capensis 266, 55 .

Barbus cetopsis Gthr. cf. Saurogobio cetopsis Blkr.

Barbus chagunio Blkr. 91, 60.

Barbus chelynoïdes $M C C l .91,60$.

Barbus chrysopoma C. V.91,6o. cf. Puntius (Barbodes) chrysopoma Blkr.

Barbus cocsa C. $V .91,60$.

Barbus deauratus $V a l$. cf. Puntius (Barbodes) deauratus Blkr.

Barbus deliciosus $\mathrm{MCC}$. 91, 6 o.

Barbus diplochilus Heck. 91, 60.

Barbus douronensis $C . V .106,91$.

Barbus Duvaucelii C. $V .91,60$. 
Barbus enoplos Blkr. 25, 16. 225, 357 .

Barbus erythropterus Blkr. 25, I5.

Barbus fasciatus Blkr. 82, I90.

Barbus fasciolatus Gthr. cf. Puntius (Capoeta) Güntheri Blkr.

Barbus gardonides $C . V$. 25, I6. 91, 126. cf. Barbus rubripinnis K. v. H.

Barbus gibbosus $C . V .25$, I $5.91,60$.

Barbus gobioïdes Blkr. 67, 592 .

Barbus gonionotus Blkr. 25, I 5 .

Barbus Hasseltii Blkr. 166, 355 .

Barbus heteronema Blkr. 85, 446.

Barbus hexastichus $M c C l$. cf. Labeobasbus tor Blkr.

Barbus Hoevenii Blkr. 45, 207. 49, 420. 55, 413.

Barbus Huguenini Blkr. 76, 294.

Barbus hypselonotus v. Hass. 166, 349 .

Barbus javanicus Blkr. 138, 403. 170, 477. 225, 357.

Barbus kadoon Blkr. 91, 60.

Barbus kakus $C . V .91,60$.

Barbus kalopterus Blkr. 26, I3. 35, 262. 42, 60. 45, 196. 49, 420.

55, 4I3. cf. Epalzeorhynchos kalopterus Blkr.

Barbus khudree Sykes 91, 60.

Barbus koilometopon Blkr. 166, 347.

Barbus kusanensis Blkr. 55, 429. 156, 2 r7. 185, 226.

Barbus laevis C. $V$. 25, I8. 85, 447. 170, 476.

Barbus lateristriga $C . V .52,95.56,445.185,226.191,3$.

Barbus macracanthus Blkr. 86, 516 .

Barbus macrocephalus $M c$ Cl. 91, 60.

Barbus macrophthalmus Blkr. 138, 404.

Barbus maculatus $K$. $\%$. H. cf. Barbus binotatus $K u h l$.

Barbus marequensis 266, 55 .

Barbus marginatus C. V. 86, 518. 170, 476, 477. 200, 48. 225, 357 .

Barbus melanopterus Blkr. 26, II. 35, 262. 42, 59. 45, I96. cf.

Systomus melanopterus Blkr.

Barbus microlepis Blkr. 26, I2. 35, 262, cf. Systomus microlepis Blkr.

Barbus micropogon $C . V .91,60$.

Barbus mosal C. V. 91, 60.

Barbus mussullah Sykes. 91, 6o.

Barbus nudicephalus $K$. v. H. 67, 593 .

Barbus obtusirostris $v$ Hass. 166, 353 .

Barbus oresigenes Blkr. 25, I7.

Barbus orphoides $C . V$. 138, 407 .

Barbus platysoma Blkr. 138, 404.

Barbus podonemus Blkr. 25, I8. cf. Luciosoma setigerum Blkr.

Barbus Polydori C. V. 91, 60.

Barbus polyspilos Blkr. 166, 35I. 200, 48 . 
Barbus putitora C. V. 91, 60.

Barbus repasson Blkr. 76, 295.

Barbus rhododactylus $M_{C} C l .91,60$.

Barbus roseipinnis C. $V .91,60$.

Barbus rubripinnis $K . v . H .2,5$ I2. 25, I I. 91, I 27. 138, 406.

Barbus sada $C . V .91,60$.

Barbus sarana $C . V$. 25, I6. 91, 60, 127.

Barbus sarananella Blkr. 25, 16. 211, 242.

Barbus schagra $C . V .91,60$.

Barbus Schwanenfeldii Blkr. 86, 5 r 7 .

Barbus setigerus C. $V$. 25, I9. cf. Luciosoma setigerum Blkr.

Barbus sophore $C . V$. cf. Systomus sophore $M C C l$.

Barbus soro C. V. 106, 90.

Barbus spilopholus $M_{C} C l .91,60$.

Barbus spinulosus $M C C l .91,60$.

Barbus subnasutus $C . V .91,60$.

Barbus tambra K. \%.H. 2, 512.

Barbus tetrazona Blkr. 162, I4.

Barbus tor Val. cf. Labeobarbus tor Blkr.

Barbus truncatus Blkr. 26, I3. 35, 262. cf. Systomus truncatus Blkr.

Barbus Valenciennesii Blkr. 25, I7.

Barbus wadon Blkr. 25, I4.

Barbus ypselonatus $K . v . H .2,5^{1} 3$.

Barilius Ham. Buch. 314, 203. 329, 263.

Barilius (Barilius) acutipinnis Blkr. Guich.415, 15.425, I46.

Barilius (Opsarius) bambusa Kner. cf. Elopichthys bambusa, dahuricus $B l k r$.

Barilius corëensis Blkr. 497, 23.

Barilius macropus Blkr. 497, 23.

Barilius minor Blkr. 497, 23 .

Barilius (Barilius) minor Blkr. 408, 248.

Barilius platypus Blkr. 497. 23 .

Barilius Sieboldi Blkr. 497. 23.

Barilius Temmincki Blkr. 497, 23.

Batasio Blyth.306, 94.

Batasio Buchanani Blyth.306, 94.

Bathygobius coalitus Blkr. 495, I 7 .

Bathygobius nebulopunctatus Blkr. 491, 54 .

Bathygobius petrophilus Blkr. 491, 54.

Bathygobius punctillatus Blkr. 495, I7.

Bathygobius soporator Blkr.495, I7.

Bathythryssa dorsalis Giunth.497, 25.

Batrachocephalus Blkr. 306, 93.

Batrachocephalus ageneiosus Blkr. 3, 176. 4, 288. 5, 42. 16, 5. 25, Iо.

Batrachocephalus micropogon Blkr. 270, 45. 306, 93. 
Batrachocephalus mino Blkr. Gthr. 91, 58. 425, I25.

Batrachoglanis Gill. 306. хог.

Batrachoïdes diemensis Lesueur. cf. Batrachus diemensis Rich.

Batrachus 1, 553 .

Batrachus apiatus $C . V .266,55$.

Batrachus barbatus $\mathrm{Val}$. cf. Batrachus didactylus Bl. Schn.

Batrachus didactylus Bl. Schn. 302, 99.

Batrachus diemensis Rich. 53, I68. 54, 237. 119, х2. 235, 370. 320, 27 I. 358, I9 I. 364, 292. 425, I26. $437,37$.

Batrachus dubius Rich. 119, I2

Batrachus Dussumieri C. $V .91,52$.

Batrachus elminensis Blkr. 302, 98.

Batrachus grunniens $C . V .50,487.51,58.55,4$ I2. 91, 52. 185, 224. 226, 387. 234, 356. 235, 370, 270, 43. 272, I6. 275, 43 . 288, 76. 356, I 75. 364, 292. 395, 293.

Batrachus Güntheri Blkr. 302, IоI.

Batrachus indicus $B l$. Schn. cf. Platycephalus indicus $B l k r$.

Batrachus indicus $B l$. cf. Platycephalus insidiator $B l$.

Batrachus pictus 2, 5 13 .

Batrachus quadrispinis $C$. $V$. cf. Batrachus diemensis Rich.

Batrachus uranoscopus Guich. 459, 76.

Bdellostoma Bürgeri Gir. 497, 28.

Bdellostoma Forsteri J. Mïll. 119, I9.

Bedula Gr. 468, 3 I 8 .

Bedula Hamiltonii Gr. cf. Nandus marmoratus $C$. $V$.

Bedula nebulosus $G r$. cf. Nandus nebulosus $B l k r$.

Belobranchus Blkr. 159, 300. 453, 304. 460, I03, I05.

Belobranchus Quoyi Blkr. 275, 45. 296, rir. 358, I92. 453, 304. 478, 53.

Belobranchus taeniopterus Blkr. 159, 3ог. 296, x II. cf. Belobranchus Quoyi Blkr.

Belodontichthys Blkr. 299, 394. 306, II 5 .

Belodontichthys macrochir Blkr. 226, 384. 270, 47. 272, I9. 306, I I 5. 356, I 75 .

Belone 1, $55^{2}$.

Belone anastomella $\mathrm{V} a l$. cf. Mastacembelus anastomella Blkr.

Belone annulata $C . V .10,67,68.46,214.91,72.275,47.288,78$. cf. Mastacembelus annulatus Blkr.

Belone brachyrhynchos Blkr. 94, 6r.

Belone brachyrhynchus Blkr. 309, I56. cf. Mastacembelus brachyrhynchus Blkr.

Belone cancila $C . V .91, \mathrm{r} 45$.

Belone canciloïdes Blkr. 85, 454. 270, 55. 271, 2. 272, 25. cf. Mastacembelus canciloïdes Blkr.

Belone carinata $C V$. cf Belone platura Rüpp. 
Belone caudimacula Cuv. 2, 5I2, 16, 5. 25, I I. 32, I60. 35, 262. $42,60.45$, г $96.49,42$ I. $50,472.51,59.55,4$ I $3.56,445.58$, г 2. 91, 72. 119, 14. 156, 217. 178, 2. 194, 26. 270, 55. 272, 25. 275, 47. 288, 78. cf. Mastacembelus strongylurus Blkr.

Belone caudimaculata $D_{a y}$. cf. Nastacembelus strongylurus Blkr.

Belone choram Rüpp. cf. Mastacembelus choram Blkr.

Belone ciconia Rich. cf. Mastacembelus anastomella, gracilis Blkr.

Belone coromandelica $C .2,5$ I2.

Belone coromandelica v. Hass. cf. Mastacembelus crocodilus Blkr.

Belone crocodila Less. 2, 5 i 2.

Belone crocodila Blkr. cf. Belone schismatorhynchus Blkr. 58, 15.

Belone crocodilus Lesueur 25, I . cf. Mastaccembelus choram Blkr., Mastacembelus crocodilus Blkr.

Belone cylindrica Blkr. 50, 472. 54, 235. 58, I3. cf. Belone gigantea T.Schl., Mastacembelus annulatus, choram Blkr.

Belone esocina Basil. cf. Mastacembelus esocinus Blkr.

Belone gigantea T.Schl. 175, 21. 200, 48. 231, I 49. 234, 356. 242, I42. 267, I $40.270,55.275,47.287,60.296$, i i I. cf. Mastacembelus anulatus Blkr., Mastacembelus choram Blkr.

Belone gracilis $T$. Schl. 118, I 6 . cf. Mastacembelus schismatorhynchus Blkr., Mastacembelus gracilis Blkr.

Belone Grayi Sykes 91, 72.

Belone leiuroïdes Blkr. 40, 479. 51, 59. 52, 89. 185, 226. cf. Mastacembelus leiuroides Blkr.

Belone leiurus Blkr. 29, 94. 58, I3. 170, 480. 231, 149. 270, 55. 296, III. cf. Mastacembelus leiurus Blkr.

Belone macrolepis Blkr. 156, 225. 167, 374. cf. Mastacembelus macrolepis Blkr.

Belone melanostigma $C$. $V$. cf. Mastaccembelus melanostigma Blkr.

Belone melanotus Blkr. 29, 94. 51, 59. 58, 14. 275, 47. cf. Mastacembelus crocodilus Blkr.

Belone melanurus Blkr. 16, II. 25, I I. cf. Belone cylindrica Blkr., Belone gigantea T.Schl., Mastacembelus choram Blkr.

Belone microstoma Basil. cf. Zenarchopterus microstoma Blkr.

Belone platura Rupp. 163, 85. cf. Mastaccembelus, Mastacembelus platurus Blkr.

Belone platurus Rüpp. 287, 60.

Belone schismatorhynchus Blkr. 29, 95. 58, I5. cf. Mastacembelus gracilis Blkr.

Belone strongulura $v$. Hass. cf. Mastacembelus strongylurus Blkr.

Belone tenuirostris Blyth. cf. Nastacembelus anastomella Blkr.

Belone timucoïdes S. Miül. 10, 67, 68. 46, 2 14. 275, 47.

Belone Urvillii $\mathrm{Val}$. cf. Mastacembelus macrolepis Blkr.

Belonopsis Brandt 351, 15 .

Bembras curtus Schl. 449, 370. 
Bembras curtus C. $V$. cf. Parabembras curtus Blkr.

Bembras japonicus $C . V$. 269, 82. 497, I2.

Bembrops caudimacula Stnd. 497, I3.

Bendilisis Blkr. 261, 436. 314, 203.

Benthophilus Eichw. 453, 309.

Benthophilus macrocephalus Eichw. 453, 309.

Beryx lineatus C. V. 119, 6. 459, 86. 495, I5.

Betta anabatoïdes Blkr. 35, 269. 42, 59. 45, I95. 49, 419. 52, 89.

55, 4II. 56, 445. 185, 223. 270. 34. 272, 10 287, 44. 288, 7 O.

cf. Betta picta Blkr.

Betta picta Blkr. 498, 26.

Betta pugnax Gthr. 356, I73. cf. Betta picta Blkr.

Betta trifasciata Blkr. 21, I2. 25, I 4. 31, I07. 56, 445. 170, 477.

185, 223. 200, 48. 270, 34. cf. Betta picta Blkr.

Blennechis cyprinoïdes $C . V .34,255$.

Blennechis Dussumieri $C$. $V$. cf. Petroskirtes Dussumieri Blkr.

Blennechis filamentosus $C . V .15,3$. cf. Petroskirtes rostratus Blkr.

Blennechis grammistes $C . V . \mathbf{1 5}, 3$.

Blennechis mitratus $C$. $V$. cf. Petroskirtes mitratus Rüpp.

Blennechis polyodon Blkr. 34, 254.

Blennius aurosplendidus Rich. 425, I 29.

Blennius capensis Forst $\mathrm{cf}$. Clinus superciliosus $C . V$.

Blennius capito $C . V .266,55$.

Blennius dolichogaster Pall. cf. Centronotus dolichogaster Gill.

Blennius edentulus $B l$. Schn. cf. Salarias Forsteri $C$. $V$.

Blennius fasciatus Bloch. cf. Salarias Forsterii $C . V$.

Blennius fasciolaticeps Rich. 425, 129.

Blennius mustelaris $L$. cf. Clinus superciliosus $C . V$.

Blennius ocellaris $L .425$, r29.

Blennius polyactocephalus Pall. cf. Clinus polyactocephalus Brev.

Blennius punctulatus $L a c$. cf. Clinus superciliosus $C . V$.

Blennius quadricornis $C . V . \mathbf{2 6 6}, 55$.

Blennius rostratus Sol. cf. Petroskirtes rostratus Blkr.

Blennius sinensis Gthr. 425, I29.

Blennius spadiceus Bl.Schn. cf. Clinus superciliosus $C . V$.

Blennius superciliosus $L_{\text {. }} \mathrm{cf}$. Clinus superciliosus $C . V$.

Blennius tasmanius Rich. 119, I 2.

Blennius truncatus Forst. cf. Salarias Forsteri $C$. $V$.

Blennius versicolor Pappe 266, 55 .

Blennophis anguillaris Swns. cf. Clinus anguillaris Szens.

Blepharis fasciatus Rüpp. cf. Carangoïdes blepharis Blkr.

Blepharis indicus $C$. $V$. cf. Carangoïdes blepharis $B l k r$.

Blepharis indicus Rich. cf. Citula ciliaris Blkr.

Blepharus indicus Cv. Schl. cf. Citula ciliaris Blkr.

Blicca Heck. 261, 436. 314, 2 I 1. 329, 263. 
Bliccopsis Heck. 261, 436. 314, 2 II. 329, 263.

Bodianus Gill. 468, 256.

Bodianus boenack Bl. cf. Epinephelus formosus Blkr., Serranus boenack $C . V$.

Bodianus boenak Bl. cf. Epinephelus boelang Blkr.

Bodianus fasciatus $B l$. Schn cf. Lutjanus argentimaculatus $B l k r$.

Bodianus guttatus Bl. cf. Epinephelus argus Bl. Schn.

Bodianus leutjan Lac. cf. Lethrinus leutjanus $C$. $V$.

Bodianus leutjanus Lac. cf. Lethrinus leutjanus $C$. $V$.

Bodianus louti Bl. Schn. cf. Variola louti Blkr.

Bodianus macrocephalus Lac. cf. Epinephelus flavocoeruleus Blkr.

Bodianus maculatus $B$ l. cf. Paracanthistius maculatus Blkr., Plectropoma maculatum $C$. $V$.

Bodianus mesothorax Blkr. 491, 4I.

Bodianus miniatus Bl.Schn.var. cf. Serranus urodelus $C$. $V$.

Bodianus palpebratus Lac. 214, 253. 273, 7 .

Bodianus Sebae Bl. Schn. cf. Percis cylindrica C. $V$.

Bodianus sexlineatus Lac. cf. Grammistes orientalis Bl. Schn.

Bodianus summana Bl.Schn. cf. Epinephelus summana Blkr.

Bodianus tetracanthus Lac. of. Percis cancellata $C$. $l$.

Bodianus undulosus Q. G. cf. Epinephelus undulosus Blkr.

Bogoda Blkr. 162, 13. 468, 292.

Bogoda macrolepis Blkr. 162, I3. 272, II. cf. Parambassis micro-

lepis Blkr.

Bogoda nama Blkr. 91, 89. 468, 292.

Bogodoidei 468, 29 I.

Bogota Blyth. 468, 336 .

Bola Ham. Buch. 468, 327 .

Bola coioides Ham. Buch. cf. Epinephelus pantherinus Blkr.

Boleophthalmi 453, 328 .

Boleophthalmus $\mathrm{Val}$. 453, 328 .

Boleophthalmus aucupatorius Rich. cf. Boleophthalmus viridis $V a l$.

Boleophthalmus Boddaerti C. $V$. 2, 514. 15, 40. 16, 5. 25, 10. 35, 26 I. 42, 59. 45, I95.49, 419. 55, 412.91, 50. 191, 2. 272, I 7. 275, 44. 281, то8. 287, 57. 288, 77. 425, I 29. 453, 328. 497, r9.

Boleophthalmus Boddaerti Schl. (nec $C . V$.) cf. Boleophthalmus pectinirostris Rich.

Boleophthalmus campylostoma Rich. 425, I29.

Boleophthalmus chinensis $\mathrm{Val}$. cf. Boleophthalmus viridis $\mathrm{Val}$.

Boleophthalmus dentatus $C . V .91,50$.

Boleophthalmus Dussumieri C. $V .91,50$.

Boleophthalmus pectinirostris Rich. 288, 77.425, г 29.496, 3.497, 9 .

Boleophthalmus Plinianus $C$. $I$. cf. Boleophthalmus Bodelaerti $C$. $I$ :

Boleophthalmus sinensis $\mathrm{Val}$. cf. Boleophthalmus viridis $\mathrm{Val}$.

Boleophthalmus viridis $C V .91,50.288,77.425$, r $29.453,328$. 
Boleops Gill. 453, 328 .

Boops Cuv. 468, 282.

Boops salpa $C$. $V .266,53.468,28_{3}$.

Boopsidea Cast. 468, $28 \mathrm{r}$.

Boopsidea inornata Cast. 468, $28 \mathrm{I}$.

Boreogobius Gill. 453, 3 Iо.

Boridia C. $V .468,280$.

Boridia grossidens C. $V .468,280$.

Boridianini 468, 280 .

Bostockia Cast. 468, 336.

Bostrichoides oculatus Lac. cf. Ophiocephalus ocellatus $C . V$.

Bostrichthys C. Dum, Gill. 453, 301. 460, го3, I04.

Bostrichthys sinensis Lac. 425, I 28.

Bostrichthys sinensis $C$. Dum. 453, 30r.

Bostrichthys sinensis Gill. cf. Bostrychus sinensis LaC.

Bostrictis Raf. 453, zoI.

Bostrychus Lac. 453, 301 .

Bostrychus maculatus Lac. cf. Ophiocephalus maculatus $C$. $V$.

Bostrychus sinensis Lac. 395, 293. 478, I6. 496, 2.

Bothus Raf. Bp. 294, 424, 426.

Botia Gr. 323, 36 .

Botia Berdmorei Blkr. 323, 39.

Botia Blythi Blkr. 323, 39 .

Botia curta Blkr., Gthr. 323, 39. 497, 22.

Botia dario Blyth 323, 39 .

Botia elongata Blkr. 412, 254. 425, I46.

Botia geto Blyth 323, 39 .

Botia grandis $G r .323,39$.

Botia histrionica Blyth 323, 39.

Botia hymenophysa Blkr. 323, 39. 356, I75.

Botia macracanthus Blkr. 323, 39.

Botia modesta Blkr. 343, I I. 356, I 75 .

Botia nebulosa Blyth 323, 39 .

Bovichthys variegatus Rich. 119, 7 .

Box C. V. 468, 282 .

Box tricuspidatus Q.\& G. 269, 77.

Boxaodon Guich. 468, 275. 470, I53.

Brachaluteres Blkr. 367, I3.

Brachiistius frenatus Gill. 468, 290.

Brachionichthys Blkr. 119, $2 \mathrm{I}$.

Brachionichthys hirsutus Blkr. 119, 22.

Brachionichthys politus Blkr. 119, I2.

Brachirus foliaceus Blkr. 425, I 30.

Brachirus heterolepis Blkr. 394, 286.

Brachirus japonicus Blkr. 497, 22. 
Brachirus pan Blkr., Srons. 395, 290. 425, I30.

Brachirus quagga Blkr. 425, т 30.

Brachirus Swinhonis Blkr. 430, 233.

Brachirus zebra Blkr., Swns. 395, 290. 425, 130. 497, 22.

Brachyamblyopus Blkr. 453, 329.

Brachyconger Blkr. 351, 116 .

Brachyconger savanna Blkr. 362, 233.

Brachydeuteurus Gill 468, 27 I.

Brachyeleotrii 460, 103 .

Brachyeleotris Blkr. 450, 374. 453, 306. 460, Iо3, Iо6.

Brachyeleotris cyanostigma Blkr. 478, 84 .

Brachyeleotris ensifera Blkr. 450, 375. 478, 86 .

Brachygenys Scudd. 468, 336.

Brachygobii 453, 3 I 4 .

Brachygobius Blkr. 453, 3I 5 .

Brachyistius Gill 468, 290.

Brachymulius Blkr. 468, 333 .

Brachyochirus Nardo. 453, 312.

Brachyopsis rostratus Gill 497, I3.

Brachyplatystoma Blkr. 306, 97.

Brachyplatystoma Vaillanti Blkr. 306, 97. 336, 70.

Brachyprosopon Blkr. 294, 428.

Brachyrhinus Gill 468, 253.

Brachyrhinus creolus Gill 468, 253 .

Brachyrhinus furcifer. 468, 253 .

Brachyrus Srons. 473, 296.

Brachyrus brachypterus Szens. cf. Pseudomonopterus (Dendrochirus) brachypterus Blkr.

Brachyrus zebra Siins. cf. Pseudomonopterus (Dendrochirus) zelora Rlkr.

Brachysomophis $K p .163,90.351$, r1 8 .

Brachysomophis cirrhocheilus $B l k r$. 352, I 26.

Brachysomophis cirrochilus Blkr. 364, 296.

Brachysomophis crocodilinus Blkr. 459, 72. 495, 22.

Brachysomophis horridus $K p .397,3 \circ 3$.

Brachysynodontis Blkr. 306. 87 .

Brachysynodontis batensoda Blkr. 304, 55. 306, 87 .

Brama Klein 314, 2 I1. 329, 263.

Brama Risso (nec Klein). 468, 299.

Brama atropus $B l$. Schn. cf. Carangoides atropus Blkr.

Brama Dussumieri $C$. $V$. cf. Tylometopon Dussumieri Blkr.

Brama isognathus Blkr. 325, 235.

Brama melampus $B l$. cf. Carangoïdes atropus Blkr.

Brama Raji Bl. Schn. 468, 299.

Branchiosteus Gill 306, I03.

Branchiosteus laticeps Gill 306, 103. 
Bregmaceros Mc Clellandi Thomps. 425, гзо.

Brontes $\mathrm{Val}$. 306, I Iо.

Brontes prenadilla $\mathrm{Val}$. 306, I Iо.

Brotula armata T.Schl., Kp. 175, 23. 497, 20.

Brotula armata Schl. cf. Brotella armata $K p$.

Brotula burbonensis Kp. 459. 74 .

Brotula imberbis T. Schl. 164, 77. cf. Sirembo imberbis Blkr.

Brotula multibarbata T. Schl. 164, 75. 175, 22. 275, 46. 320, 272. 354, r5o. 364, 294. 497, 20.

Bryttus C. $V .468,249$.

Bryttus chaetodon Baird. 468, $25^{\circ}$.

Bubalichthys $A g$. 261, 429. 314, I9I.

Bufichthys grossa Szons. cf. Synanceia horrida Bl. Schn.

Bufichthys horrida Stens. cf. Synanceia horrida Bl. Schn.

Bunocephalichthys Blkr. 306, i 8 .

Bunocephalichthys hypsiurus Blkr. 306, I 8 .

Bunocephalus Kner., Blkr. 306, I I 8.

Bunocephalus hypsiurus Kner. cf. Bunocephalichthys hypsiurus Blkr. Bunocephalus verrucosus $K$ ner. 306, I 8 . 336, 5 .

Butii 453, 304. 460, 103.

Butirinis 2, 509 .

Butirinus glossodontus Rïpp. cf. Albula bananus $C$. $V$., Conorhynchus glossodon Blkr.

Butis Blkr. 453, 304. 460, 103, I05.

Butis amboinensis Blkr. 275, 44. 354, I50. 364, 293. 478, 66.

Butis butis Blkr. 288, 77. 364, 293.425, I 28.453, 304. 459, 78.478, 62 .

Butis caperatus Blkr. 425, I28. cf. Prionobutis koilomatodon Blkr.

Butis gymnopomus Blkr. 156, 2I5. 235, 370. 270, 4I. 284, 240. $287,57.478,70$.

Butis koilomatodon Blkr. 275, 45. 287, 57. 288, 77. cf. Prionobutis koilomatodon $B l k r$.

Butis melanopterus Blkr. 275, 45. 287, 57. 358, 192. 364, 293. 395, 293. cf. Butis butis Blkr.

Butis melanostigma Blkr. 270, 4I. 272, I7. 356, I74. 478, 68.

Butis melanotopterus Blkr. 354, r 50.

Butis prismaticus Blkr. 478, 61.

Butis Wolffi Blkr. 272, 17. cf. Butis melanostigma Blkr.

Butyrinus bananus Lac. cf. Albula bananus $C . V$.

Cacodoxus Cant. 468, 302.

Cacodoxus argus Cant. 320, 269. 346, 31. 354, I48. 358, I88. 364, 284. 425, I40. cf. Ephippus, Scatophagus argus $C . V$.

Cacodoxus Bougainvillei Blkr. 425, I 40.

Cacodoxus ornatus Cant., Blkr. 354, I48. 364, 284. 425, I40.

Cacodoxus tetracanthus Blkr. 459, 94. 
Caeriosoma Sieboldi $K p .497,8$.

Caesio Comm., Lac. 1, 553. 468, 274. 470; I52.

Caesio argenteus $C . V$. 425, I39.

Caesio azuraureus Rüpp. cf. Caesio coerulaureus Lac.

Caesio azuror Lac. cf. Caesio coerulaureus Lac.

Caesio chrysozona K.v.H. 2, 521. 20, 9. 47, 227. 126, 344. 156, 2I 4. 234, 353. 275, 28. 315, 233. 318, 25 I. 364, 28 .445, I 73. $491,42$.

Caesio coerulaureus Lac. 2, 521. 20, 8. 48, 348. 51, 56. 54, 237. $91,40.156,2$ I $4.157,232.231$, I 45. 270, 22. 275, 29. 288, 7 I. 296, I05. 309, I 55. 315, 233. 354, I 47.358, I $88.364,283$. $394,287.425$, I 39.445 , I 7 I. $459,92.468,274.470$, I 53. 495, I $2,496,2$.

Caesio coerulaureus var. Cant. cf. Caesio coerulaureus Lac.

Caesio coeruleo-taeniatus Ehr. cf. Caesio coerulaureus Lac.

Caesio cuning $C$. $V$. cf. Caesio erythrogaster $K$. v. $H$.

Caesio cylindricus Gthr. 459, 92. 468, 274. 470, I53.

Caesio diagramma Blkr. 445, 175 .

Caesio digramma Blkr. 357, r8o. 364, 283.

Caesio erythrogaster $K . v . H .2,52 \mathrm{I} .9,633.10,66.20,9.46,2$ I2. 50, 47 I. 51, 56. 126, 344. 156, 214. 157, 232. 227, 407. 234, 353. 270, 22. $275,29.309$, I 55. 354, I 47. 358, I88. 364, 283. 395,29 I. 445, I62.

Caesio erythropterus $K . v$. H. 235, 365 .

Caesio erythropterus Blkr. cf. Caesio erythrogaster $K . v . H$.

Caesio gymnopterus Blkr. 145, 372. 315, 233. 318, 251. 364, 283 . 470, I52. cf. Smaris gymnopterus Blkr.

Caesio lunaris Ehr. 44, I 77. 445, I65.

Caesio maculatus $C . V .364,283.425$, I39. 445, I 70. 459, 92.

Caesio multiradiatus Stcind.445, I77. 468, 274.470 , I53.

Caesio pinjalo Blkr. 20, 10. 31, 102.46, 212. 270, 22. 275, 29. 287, 47. 470, ז52. cf. Pinjalo typus Blkr.

Caesio pisang Blkr. 72, II3. 275, 29. 315, 233. 364, 283. 445, I68.

Caesio striatus Riupp. 2, 521. 459, $9^{2}$.

Caesio striatus Blkr. cf. Caesio chrysozona $K$. v. $H$.

Caesio tile $C . V .10,7$.

Caesio xanthonotus Blkr. 78, 466. 445, I67.

Caesio xanthurus Blkr. 404, 78. 459, 37. 470, I52. cf. Paracaesio xanthurus Blkr.

Caesiomorus Bailloni Lac. cf. Trachinotus Baillonii C. $V$.

Caesiomorus Blochii Lac. cf. 'Trachinotus mookalee C. $V$.

Caesiomorus quadripunctatus Rüpt. cf. Trachinotus Baillonii C. $V$.

Caesioperca Cant. 468, 252.

Caesiosoma Kp. 468, 299.

Caesiosoma Sieboldi $K p .468,299$. 
Calamus Srens. 468, 28r.

Callanthias Lone 468, 253.

Callanthias paradiseus Lowe 468, 253 .

Callanthias peloritanus Gthr. 468, 253 .

Callechelys $K p .351$, r 20.

Callechelys marmoratus Blkr. 352, I29.

Callechelys melanotaenia Blkr. 361, 21 3. 364, 296. 397, 302.

Calleleotris Gill 450, 372. 453, 307 .

Calleleotris strigata Gill cf. Valenciennesia strigata Blkr.

Callichrous Ham. Buch. 299, 395. 306, I 5 .

Callichrous bimaculatus Szens., Blkr. 356, I75. 425, I 25.

Callichrous canio Gthr. 425, I25.

Callichrous pabda Ham. Buch. 306, i 15.

Callichthys L. 306, 82 .

Callichthys albidus $\mathrm{Val}$. cf. Hoplosternum thoracatum Gill.

Callichthys asper $V a l$. cf. Callichthys tamoata $L$.

Callichthys callichthys Cuz». cf. Callichthys tamoata $L$.

Callichthys coelatus $\mathrm{Val}$. c.f. tamoata $L$.

Callichthys exaratus M. Tr. cf. Hoplosternum longifile Gill.

Callichthys laeviceps $V a l$. cf. Callichthys tamoata $L$.

Callichthys laevigatus $\mathrm{Val}$. cf. Hoplosternum laevigatum Gill.

Callichthys longifilis Cuv. cf. Hoplosternum longifile Gill.

Callichthys loricatus Gron. cf. Callichthys tamoata $L$.

Callichthys personatus Ranz. cf. Hoplosternum longifile Gill.

Callichthys pictus $M$. Tr. cf. Hoplosternum iongifile Gill.

Callichthys punctatus $\mathrm{Val}$. cf. Corydoras punctatus Blkr.

Callichthys subulatus $\mathrm{Val}$. cf. Hoplosternum laevigatum Gill.

Callichthys sulcatus Kner cf. Hoplosternum longifile Gill.

Callichthys tamoata L. 306, 82, 336, 22.

Callichthys thoracatus $V a l$. cf. Hoplosternum thoracatum Gill.

Calliomorus chacca Ham. Buch. cf. Platycephalus indicus Blkr.

Calliomorus indicus Lac. cf. Platycephalus indicus Blkr., Platycephalus insidiator $\mathrm{Bl}$.

Callionymus 1,553 .

Callionymus altovelis Schl. 497, 18.

Callionymus Belcheri Rich.494, 85. cf. Callionymus Reevesii Rich.

Callionymus calauropomus Rich. 119, I2.

Callionymus curvicornis $\mathrm{Val} .425$, I27. 459, 76.

Callionymus dactylopus $E d$. E. Benn. 66, 559. 275, 42. 287, 55. cf.

Vulsus dactylopus Gthr.

Callionymus enneactis Blkr. 494, 95.

Callionymus filamentosus $C . V .10,67.15,3.46,2$ I 3. 54, 278. 56, 455. 275, 42. 296, I Iо. 364, 292. 494, 88 .

Callionymus fluviatilis Day cf. Callionymus melanotopterus Blkr.

Callionymus goramensis Blkr. 184, 2 r 4. cf. Synchiropus goramensis Blkr. 
Callionymus Hindsii Rich. 425, 127.

Callionymus Huguenini Blkr. 193, 7. 497, i 8.

Callionymus indicus $L$. cf. Platycephalus indicus $B l k r$.

Callionymus indicus $B l$. cf. Platycephalus insidiator $B l$.

Callionymus inframundus Gill 497. r8.

Callionymus japonicus Houtt. cf. Callionymus longicaudatus T. Schl, Callionymus Reevesii Rich.

Callionymus japonicus $C V$. cf. Callionymus Valenciennei $T$. Schl.

Callionymus lineolatus $C . V .91^{\circ}, 52.459,76$.

Callionymus longecaudatus Schl. 497. x8.

Callionymus longicaudatus $T$. Schl. 175, I7. 253, 235. cf. Callionymus Reevesii Rich.

Callionymus lunatus Schl. 425, I27. 497, is.

Callionymus melanopterus Blkr. 275, 42 .

Callionymus melanotopterus Blkr. 28, 31. 281, 108. 287, 55. 494, 92.

Callionymus ocellatus Pall. 15, 3. 128, 422. 184, 201. 275, 42. 315, 236. 320, 27 I. 494, 98 .

Callionymus opercularis $C . V .91,52$.

Callionymus opercularioïdes Blkr. 28, 32. 194, 26. 250, 206. 270, 40. 296, I Io. 358, I9I. 364, 292. 388, 272. cf. Eleutherochir opercularioïdes Blkr.

Callionymus orientalis $B l$. Schn. 91, 52.

Callionymus picturatus Peters, 494, 97 .

Callionymus punctatus Rich. cf. Callionymus curvicornis $\mathrm{Val}$.

Callionymus punctatus Langsd. cf. Callionymus Valenciennei $T$. Schl.

Callionymus punctulatus Lac. cf. Callionymus occellatus Pall.

Callionymus Reevesii Rich. 83, 244. 90, 44. 175, 19. 275, 42. 354, I 49. 358, I9I. 364, 292, 425, I27. 497, I8. cf. Callionymus curvicornis Val., Callionymus Richardsonii Blkr.

Callionymus Reevesii Blkr. cf. C. Belcheri Rich.

Callionymus Richardsonii Blkr. 102, 4I4. 118, III. 175, I9. 497, I8.

Callionymus sagitta Pall. 15, 3. 28, 3I. 55, 4I2. 56, 445. 91, 52 . 272, х6. 275, 43. 287, 55 364, 292. 459, 76. 494, 9 ०.

Callionymus Schaapii Blkr. 56, 455. 494, 94.

Callionymus serrato-spinosus Gr. cf. Callionymus sagitta Pall.

Callionymus valencienneri $S c h l .497$, I8.

Callionymus Valenciennei $T$. Schl. 90, 45.

Callionymus Valenciennesi Blkr. 408, 238.

Callionymus variegatus Schl. 497, I8.

Calliurus Raf. 468, 249.

Calliurus melanops Gir. 468, 250.

Callogobius Blkr. 453, 318.

Callophysus Müll. Trosch. 306, гог.

Callophysus macropterus Mïll. Trosch. 306, Iо2.

Calloptilum mirum Rich. cf. Bregmaceros Mc Clellandi Thomps. 
Callorhynchus antarctica Gron. 119, I9.

Callorhynchus antarcticus Cuv. 266, 78. 425, I20.

Callorhynchus Smythii Lay \& Benn. cf. Callorhynchus antarcticus Cuv.

Callorhynchus tasmanius Rich. 119, I9.

Callyodon Gron., Cur. 290, 229.

Callyodon brachysoma Blkr. 290, 244.

Callyodon carolinus $\mathrm{Val} .262,444.290,244.320,265.364,275.491,40$.

Callyodon chlorolepis Rich. cf. Scarichthys auritus Blkr.

Callyodon genistriatus $C . V$. Blkr. 95, I I I. 275, I 5. 290, 244. 364, 275 .

Callyodon hypselosoma Blkr. 128, 425. 167, 374. 231, I 49. 270, 39. cf. Callyodon spinidens $C u v$.

Callyodon japonicus $C$. $V$., Schl. 118, II 5. 497, 4 .

Callyodon moluccensis Blkr. 290, 243. 296, I04. 315, 230. 320, 265. 358, I $84.364,275$.

Callyodon mutabilis Gron. 290, 243.

Callyodon spinidens Cur., Suns. 290, 244. 315, 230. 320, 265. 364, 275. 396, 296.

Callyodon vaigiensis $V a l .11,7.12,42$.

Callyodon viridescens Rüpp. 459, 8I. 495, S.

Callyodon waigiensis $C . V .47,256.54,238.128,426.188,46$ I. 275, I5. cf. Callyodon moluccensis Blkr.

Callyodontichthys Blkr. 290, 230.

Camarina Ayr. 468, 295.

Campostoma $A g .261,425.314,205$.

Campylomormyrus Blkr. 449, 367 .

Cannorhynchus immaculatus Cant. 315, 236. 316, 243. 358, 192. 364, 294. cf. Cannorhynchus serratus Blkr., Fistularia immaculata Comm.

Cannorhynchus serratus Blkr. 425, I26. 437, 37. 459, 74.

Cannorhynchus tabaccaria Blkr. 459, 74 .

Canthagaster Valentyni Blkr. 495, 23.

Cantharus Cuv. 468, $28_{3}$.

Cantharus Blochii C. V. 266, 6o.

Cantharus Castelnaui Blkr. 266, 59.

Cantharus emarginatus $C . V .266,60.495$, I2.

Cantharus guliminda $C . V$. cf. Dentex tolu $C . V$.

Cantharus lineatus White $468,28_{3}$.

Canthidermis Stons. 367, II.

Canthogaster Szons. 367, I9.

Canthogaster amboinensis Blkr. 368, $3^{8 .}$

Canthogaster grammatocephalus Blkr. 497, 27.

Canthogaster janthinopterus Blkr. 368, 39.

Canthogaster margaritatus Blkr. 368, 39. 394, 2S5.425, I 2 I.

Canthogaster ocellatus Blkr. 368, 39. 394, 285. 397, 302.

Canthogaster rivulatus $B l k r .497,27$.

an thogaster striolatus Blkr. 368, 39. 382, 397. 397, 302. 
Canthogaster Valentyni Blkr. 368, 38. 394, 285. 459, 69.

Cantoria pinangensis $K$, 288, 69 .

Capoëta Val. 261, 431. 314, 200.

Capoeta ampalong Blkr. 67, 594.

Capoeta amphibia $C . V .91,62$.

Capoeta brevis Blkr. 25, $2 \mathrm{I}$.

Capoeta chola C. $V .91,62$.

Capoeta chrysosomus C. V.91, 62 .

Capoeta Deventeri Blkr. 138, 4I3.

Capoëta elongata Schl. cf. Gnathopogon elongatus Blkr.

Capoeta enoplos Blkr. 49, 43I. 55, 4I3.

Capoëta gracilis Schl. cf. Gnathopogon gracilis Blkr.

Capoëta intermedia Schl. cf. Acheilognathus intermedius Blkr.

Capoeta javanica Blkr. 138, 4 I 2.

Capoeta kolus Blkr. 91, 62.

Capoëta lanceolata Schl. cf. Acheilognathus lanceolatus Blkr.

Capoëta limbata Schl. cf. Acheilognathus limbatus Blkr.

Capoeta macrolepidota $C . V .(K . v . H) 2,5$ I2. 25, 20. 67, 595. 170, $476.200,48$.

Capoeta microlepis Blkr. 45, 206. 49, 420. 55, 4r3.

Capoeta oligolepis Blkr. 76, 296.

Capoeta padangensis Blkr. 67, 593.

Capöeta rhombea Schl. cf. Paracheilognathus rhombeus Blkr.

Capoeta tetrazona Blkr. 136, 262.

Caprodon Schl. 468, 252.

Caprodon Schlegeli Blkr. 468, 252. 487. 497, 28.

Caprophomus $M$. Tr. 468, 3 10.

Capropygia $K p .367$, I6.

Capros Lac. 468, 3 Iо.

Capros aper Lac. 468, 310

Capros australis Rich. 119, I $\mathbf{I}$.

Caracanthus Krojer 297, I40.

Caracanthus apistus Blkr. 475, 96. 491, 49.

Caracanthus madagascariensis Blkr. 459, 87 .

Caracanthus unipinna Blkr. 495. I6.

Carangichthys Blkr. 128, 4I8. 297, I37.

Carangichthys typus Blkr. 71, 760. 184, 200. 231, 146. 234, 354. 270, 3०. 275, 39. 296, I09. 354, I 48. 358, I9 I. 364, 290.

Carangoïdes Blkr. 128, 418. 297, I37.

Carangoïdes alexandrinus Blkr. 302, 15 .

Carangoïdes armatus Blkr. 234, 354. 270, 30. 275, 39. 287, 53. 288, 74. cf. Citula armata Rüpp.

Carangoïdes atropus Blkr. 37, 366. 51, 57.57, 66.91, 44. 227, 407 . $231,146.270,30.288,74$.

Carangoïdes aureoguttatus Blkr. 78, 470. 96, I75. 287, 53. 
Carangoïdes blepharis Blkr. 54, 235. 57, 67. 90, 4. 194, 26. 231, 147. 270, 30. 275, 39.

Carangoïdes chrysophryoïdes Blkr. 37, 366. 57, 63 .

Carangoïdes ciliaris Blkr. 296, I09. cf. Citula ciliaris Blkr.

Carangoïdes citula Blkr. 46, 213. 51, 57. 56, 445. 57, 65. 91, 44. 127, 345. 227, 407. 231, 147. 237, 437.

Carangoïdes dinema Blkr. 37, 365. 57, 63.

Carangoïdes equula Blkr. 118, I02.

Carangoïdes equula Schl. cf. Citula equula Blkr.

Carangoïdes fulvoguttatus Blkr. 44, I78, 57, 89.

Carangoïdes gallichthys Blkr. 50, 47 I. 51, 57. 57, 68. 91, 44. 156, 2I5. 231, I 47. 235, 367. 270, 30. 272, I 4. 275, 39. cf. Citula gallus Blkr.

Carangoïdes gallus Blkr. 287, 53. 288, 74. 296, го9.

Carangoïdes gymnostethoïdes Blkr. 37, 364. 57, 6r.

Carangoïdes hemigymnostethus Blkr. 37, 364. 57, 6r.

Carangoïdes malabaricus Blkr. 227, 407. 270, 30. 275, 40. 287, 53. 288, 74. 296, rog.

Carangoïdes oblongus Blkr. 57, 62. 270, 30. 287, 53. 288, 74

Carangoïdes ophthalmotaenia Blkr. 54, 270. 57, 90. 147, 469. 275, 39 .

Carangoïdes plagiotaenia Blkr. 163, 59 .

Carangoïdes praeustus $B l k r .37,363.50,47$ 1. 57, 60. 91, 46. 185, 223. 235, 367. 270, 30. 275, 39. 287, 53. cf. Citula praeusta Blkr.

Carangoïdes talamparah Blkr. 51, 57. 57, 64. 91, 46.

Carangoïdes talamparoïdes Blkr. 57, 91. 67, 579. 270, 30.

Carangus carangus Cuv., Blkr. 425, I3 I. 459, 99. 495, I8. 497, I4.

Carangus ekala $C . V$. 315, 235. 316, 242. 358, 191. 364, 290.

Carangus Forsteri $C . V$., Blkr. 315, 235. 316, 242. 320, 270. 346, 3I. 354, I48. 358, I9I. 364, 290.

Carangus hippos Blkr. 393, 282. 396, 297, 300. 425, I31. 459, 99. 491, 50. 497, 14.

Carangus janthinospilos Blkr. 320, 270.

Carangus janthinospilus Blkr. 364, 290.

Carangus jarra $C . V$. 364, 290.

Carangus melampygus C. $V$., Blkr. 354, I48. 364, 290. 396, 297. 459, 99. 495, г 8.

Carangus Peronii $C . V .364,290$.

Carangus Quoyi Blkr. 491, 50.

Carangus sansum Blkr. 495, r8.

Carangus sansun Blkr. 459, 99.

Carangus tapeinosoma Blkr. 364, 290.

Caranx Lac. 1, 553. 128, 417. 297, I37.

Caranx affinis $K . v . H$. cf. Selar Hasseltii Blkr.

Caranx armatus C. $V$. 2, 517. cf. Carangoïdes citula Blkr., Citula armata Rïpp. 
Caranx auroguttatus Ehr. cf. Carangoïdes aureoguttatus Blkr.

Caranx bayad Rüpp. cf. Carangoïdes fulvoguttatus Blkr.

Caranx Belengerii C. V. 91, 44 .

Caranx Blochii C. V. 37, 364. 57, 6r.

Caranx boöps C.V. 315, 235. 338, 360. 364, 289. 390, 275. cf. Selar boöps Blkr.

Caranx brevis Gthr. 411, 252.

Caranx calla $C . V .425$, I 3 r.

Caranx cambon $C . V$. 2, 517. 16, 4. 25, 8. cf. Selar para Blkr.

Caranx cancroides Rich. 425, I3I.

Caranx cestus Rich. 425, I3r.

Caranx chloris Cuv. cf. Micropteryx chrysurus Gthr.

Caranx chrysophrys $C . V$. 37, 366. 57, 64. cf. Citula chrysophrys Blkr.

Caranx ciliaris $C . V .2,5$ I 7 . cf. Carangoïdes citula $B l k r$.

Caranx ciliaris Rich., C. $V$., Schl. cf. Citula armata Rüpp.

Caranx cirrhosus Elırenb. cf. Carangoïdes citula Blkr.

Caranx citula $C . V$. 2, 5 I 7. cf. Carangoïdes citula Blkr.

Caranx coeruleo-pinnatus $C$. $V$. cf. Carangus Quoyi Blkr., Citula coeruleopinnata Blkr.

Caranx crumenophthalmus $C . V .$, Lac. 302, r4. 459, 99.495, 18.496, 2.

Caranx cynodon Blkr. 37, 362. 57, 57. 156, 214. 270, 29.

Caranx ekala C.V. 2, 517.54, 235. 57, 59. 91, 44. 170, 479. 237, 437. 270, 29. 275, 39. 287, 52.296, rog. cf. Carangus carangus Cuv.

Caranx equula $T$. Schl. cf. Carangoïdes equula Blkr.

Caranx equula Schl. cf. Citula equula Blkr.

Caranx flavocoeruleus Schl. cf Carangus hippos Blkr.

Caranx Forsteri C. V. 10, 67, 68. 46, 213. 53, 164. 54, 235. 55, 412. $57,57.91,4+.119$, 10. $127,345.156,214.163,60.167,372$. 170, 478. 184, 200. 188, 459. 191, 2. 231, I46. 235, 367. 237, 437. 270, 29. 272, I4. 275, 39. 287, 52. 288, 74. 296, го9. 309, I56. 393, 282. cf. Carangus hippos Blkr.

Caranx fulvoguttatus $R \ddot{u} p p$. cf. Carangoïdes aureoguttatus Blkr.

Caranx fulvoguttatus $C$. $V$. cf. Carangoïdes fulvoguttatus Blkr., Citula bajad Blkr.

Caranx glaucus Lac. cf. Glaucus Rondeletii Will.

Caranx gymnostethus C. $V .37,364.57,6$ r. cf. Citula gymnostethus Blkr.

Caranx Hasseltii Gthr., Blkr. 364, 289. 459, 99. 491, 5०.

Caranx Heberi C. $V$. 91, 44.

Caranx helvolus C. V. 128, 4 I9.

Caranx hippos Gthr. cf. Carangus hippos Blkr.

Caranx ire $C . V$. 2, 5 I7.

Caranx janthinospilos Blkr. 142, 45.

Caranx jarra C. $V .57,58.91,44.270,29$.

Caranx kiliche $C . V .10,72$. 
Caranx Kuhli Blkr. 491, 5 o.

Caranx kurra C. V. 10, 72. cf. Decapterus kurra Blkr.

Caranx leptolepis $K . v . H .2,5$ I 7. 10, 7 I, 72. cf. Leptaspis leptolepis Blkr., Selaroïdes leptolepis Blkr.

Caranx Lessonii C. V. 2, 517. 25, 8. 91, 44. 119, 10. 393, 282. cf. Carangus hippos Blkr.

Caranx Lessonii Blkr. cf. Caranx ekala $C . V$.

Caranx mala $C$. $V$. cf Carangus carangus $C$. $V$.

Caranx malabaricus $C . V$. 2, 517. cf. Carangoïdes talamparah Blkr., Citula malabarica Blkr.

Caranx malam Blkr. 356, I73. 395, 293. 425, I3 I.

Caranx margarita Rich. cf. Gnathanodon margarita Blkr.

Caranx maruadsi $T$. Schl. cf. Decapterus maruadsi Blkr.

Caranx mate $C$. $V$. cf. Selar Hasseltii Blkr.

Caranx mauritianus Q.G. 2, 5 I7. cf. Caranx crumenophthalmus Lac.

Caranx melampygus $C . V .91,44.94,58.156,2$ I 5. 231, 146. 275, 39. 308, I52. 309, I56. cf. Carangus melampygus Blkr.

Caranx mentalis Ehrenb. cf. Carangoïdes atropus Blkr.

Caranx Mertensii C. $V$. cf. Leptaspis leptolepis Blkr.

Caranx micropterus Rüpp. 128, 4I 9 .

Caranx muroadsi Schl. cf. Decapterus muroadsi Blkr.

Caranx nigripes $C . V .2,5$ I 7. 16, 4. 17, 3. 25, S. cf. Carangoïdes atropus Blkr., Citula atropus Blkr.

Caranx Novae-Guineae $C . V .491,50$.

Caranx oblongus $C . V .2,5$ I7. cf. Carangoïdes oblongus Blkr.

Caranx para $C . V .356$, I73. cf. Selar para Blkr.

Caranx parapistes Rich. 119, Iо. cf. Carangus hippos Blkr.

Caranx Peronii $C . V .2,517.25,8.91,44$.

Caranx Peronii Blkr. cf. Selar para Blkr.

Caranx petaurista Rüpp. cf. Caranx Rüppelli Gthr.

Caranx praeustus Raffl. cf. Carangoïdes praeustus Blkr.

Caranx pseudopterygius Blkr. 10, 7 I. cf. Decapterus kurra Blkr.

Caranx punctatus Q.G. 91, 44. cf. Carangus Quoyi Blkr.

Caranx Rottleri C. $V$. 10, 67. 17, 3. 25, 8. cf. Megalaspis Rottleri Blkr.

Caranx Rüppelli Gthr. 459, 99. cf. Gnathanodon petaurista Blkr.

Caranx Russellii Rupp. cf. Decapterus kurra Blkr.

Caranx sansum Rüpp. cf. Carangus sansum Blkr.

Caranx sansun Rüpp. cf. Carangus sansun Blkr.

Caranx sem C. V. 91, 44 .

Caranx speciosus $C . V ., L a c .2$, 5 I7. 16, 4. 25, 8. cf. Gnathanodon speciosus Blkr.

Caranx sexfasciatus Q. G. 2, 5I7. 163, 60. cf. Carangus hippos Blkr., Caranx Forsteri $C . V$.

Caranx tapeinosoma Blk\% 142, 44.

Caranx tille C. $V .91,44$. 
Caranx torvus $J c n .315,235.316,242.338,360.364,289.497$, т4. cf. Selar torvus Blkr.

Caranx trachurus Lac. 364, 289. 425, г 31. 497, I 4 .

Caranx trachurus japonicus T. Schl. cf. Caranx trachurus Lac., Selar japonicus Blkr., Selar trachurus (japonicus) Blkr.

Caranx venator Playf. 459, 99.

Caranx vomerinus Playf. 459, 99.

Caranx xanthopygus $C$. $V .2,5$ I 7. 9, 633. cf. Carangus carangus Blkr., Caranx ekala $C . V$.

Caranx xanthurus $K$. \%. $H_{.}, C . V .10,67.16,4.17,3.25,8.356$, I 74. 364, 290. 382, 398. 395, 293. 459, 99. cf. Selar Hasseltii Blkr.

Caranxomorus sacrestinus Lac. cf. Aphareus furcatus Gthr.

Carassius Nils. 261, 429. 314, x9т.

Carassius auratus Nilss., Blkr. 90, 48, 91, 60. 253, 235. 267, 140. 356, I75. 364, 294. 408, 238. 411, 252. 415, 7. 416, I20.425, I 43. 459 , Iо2. 495,2 I. $496,3.497,23$

Carassius Bürgeri $T$. Schl. 269, 96. 408, 238. cf. Carassius auratus Blkr.

Carassius coeruleus Basil. cf. Carassius auratus Blkr., Carassius Bürgeri $T$. Schl.

Carassius Cuvieri, Schl. cf. Carassius auratus Blkr.

Carassius discolor Basil. cf. Carassius auratus Blkr.

Carassius gibelioides Blkr. 345, I9. cf. Carassius auratus Blkr.

Carassius grandoculis Schl. cf Carassius auratus Blkr.

Carassius Langsdorfii Schl. cf. Carassius auratus Blkr.

Carassius pekinensis Basil. cf. Carassius auratus Blkr.

Carassius thoracatus $C$. $V$. cf. Carassius auratus $N i l s$.

Carassius thoractes $C . V$. cf. Carassius auratus Blkr.

Carcharias 1, 55 I.

Carcharias acutidens Rüpp. cf. Carcharinus (Aprion) acutidens Blkr.

Carcharias acutus Rüpp. cf. Carcharias (Scoliodon) acutus $M$. $H$.

Carcharias (Scoliodon) acutus Rüpp., M. H. 50, 473. 51, 6o. 56, 446. 64, 3०. 191, 9. 227, 409. 270, 67. 288, 66. cf. Carcharinus (Scol.) acutus Blkr., Cynocephalus (Scol.) acutus Blkr.

Carcharias (Prionodon) amblyrhynchos Blkr. 146, 467. 191, 9. 287, 36.

Carcharias (Prionodon) amboinensis M. H. 103, 507. 191, 9.

Carcharias (Prinodon) Bleekeri A. Dum. cf. Carcharinus (Prinodon) Bleekeri.

Carcharias (Prionodon) borneënsis Blkr. 191, 8. 272, 6.

Carcharias (Prionodon) brachyrhynchos $R ! l:$. i $, 1,10$.

Carcharias (Aprion) brevipinna M. H. cf. Carcharias (Prionodon) brevipinna Blkr.

Carcharias (Prionodon) brevipinna Blkr. 78, 509. 191, Io.

Carcharias (Scoliodon) chloronotus Blkr. 25, r2.

Carcharias Dumerilii Gthr. cf. Carcharinus (Scoliodon) Dumerilii Blkr.

Carcharias (Scoliodon) Dumerilii Blkr. 142, 70. 191, 9. 275, 9. 
Carcharias (Prionodon) Dussumieri M. H. cf. Carcharinus (Prionace) Dussumieri Blkr.

Carcharias (Prionodon) fasciatus Blkr. 64, 37. 78, 510. 191, ro.

Carcharias (Prionodon) Henlei Blkr. 78, 507.

Carcharias (Prionodon) japonicus Schl. cf. Cynocephalus (Prionace) japonicus Blkr.

Carcharias Javanicus 2, 508 .

Carcharias (Prionodon) javanicus Blkr. 64, 38. 178, 2. 191, 10. 270, 67. 272, 6. 275, 9. 287, 36.

Carcharias (Scoliodon) laticaudus M. H. cf. Carcharinus (Scoliodon) laticaudus Blkr.

Carcharias (Prionodon) limbatus M. H. cf. Carcharinus (Prionodon) limbatus Blkr.

Carcharias (Hypoprion) Mackloti $M$. H. cf. Cynocephalus (Hypoprion) Mackloti Blkr.

Carcharias (Scoliodon) macrorhynchos Blkr. 64, 31. 144, 354. 191, 9. 270, 67. 272, 6. 287, 36 .

Carcharias melanopterus Dum. 2, 508 .

Carcharias melanopterus $Q . G$. cf. Carcharinus (Prionace) melanopterus $B l k r$. Cynocephalus (Prionace) melanopterus Blkr.

Carcharias (Prionace) melanopterus Cant. cf. Carcharias (Prionodon) melanopterus $Q$. $G$.

Carcharias (Prionodon) melanopterus Q. G. 16, 6. 25, I2. 64, 33. 119, I 7. 191, Iо. 270, 67. 275, 9. 287, 36.

Carcharias (Prionodon) melanopterus $M$. $H$. cf. Carcharinus (Prionace) melanopterus Blkr., Cynocephalus (Prionace) melanopterus Blkr.

Carcharias (Prionodon) menisorrah Val. 25, I2. 32, I6o. 56, 446. 64, 35. 119, I8. 156, 2 18. 191, 10. 287, 36.

Carcharias (Prionodon) munsing Blkr. 16, r6. 25, I2. 64, 32. 191, 10.

Carcharias (Hypoprion) notatus Blkr. 25, I 2.

Carcharias (Prionodon) pleurotaenia Blkr. 64, 40. 191, ro. 287, 36. cf. Cynocephalus (Prionace) pleurotaenia Blkr.

Carcharias (Prionodon) sorrah $\mathrm{Val} .10,68.16,6.25,12.46,214$. 64, 39. 191, 10. 270, 67. 275, 9. 287, 36.

Carcharias (Prionodon) sorrah $M$. $H$. cf. Carcharinus (Prionodon) sorrah Blkr.

Carcharias (Prionodon) tjutjot Blkr. 64, 36. 191, ro.

Carcharias vulpes $C u v$. cf. Alopecias vulpes $B p$.

Carcharias (Scoliodon) Walbeehmi Blkr. 144, 353. 185, 226. 191, 9. cf. Carcharinus (Scoliodon) Walbeehmi Blkr.

Carcharinus (Aprion) acutidens Blkr. 459, 67 .

Carcharinus (Scoliodon) acutus Blkr. 356, I72. 364, 270. 391, 276. 395, 290. 425, II9. 459, 67 .

Carcharinus (Prionace) amboinensis Blk\%. 364, 270. 398, 306.437, 36 . Carcharinus (Prionodon) Bleekeri. 459, 67. 
Carcharinus (Scoliodon) Dumerili Blkr. 425, II9.

Carcharinus (Prionace) Dussumieri Blkr. 425, i i9.

Carcharinus (Prionace) javanicus Blkr. 354, r43.

Carcharinus (Scoliodon) laticaudus Blkr. 425, I 19.

Carcharinus (Prionodon) limbatus Blkr. 459, 67.

Carcharinus (Prionace) melanopterus Blkr. 316, 24I. 320, 264. 354, I43. 364, 270. 396, 296. 425, I I 9 .

Carcharinus (Prionodon) sorrah Blkr. 459, 67.

Carcharinus (Scoliodon) Walbeehmi Blkr. 320, 264. 395, 290.

Carcharius acutidens Rüpp. 2, 508.

Carcharius (Scoliodon) acutus Rüpp. 91, 80.

Carcharius (Prionodon) Dussumieri $V a l .91,80$.

Carcharius (Prionodon) gangeticus M.H. 91, 80.

Carcharius (Prionodon) glyphis $M . H .91,80$.

Carcharius (Hypoprion) hemiodon $V a l .91$, So.

Carcharius (Scoliodon) laticaudus $M . H .91$, So.

Carcharius (Physodon) Mülleri $V a l .91,80$.

Carcharius (Prionodon) palasorra Cuv. 91, 80.

Carcharius (Prionodon) sorrah $\mathrm{Val} .91,80$.

Carcharius (Prionodon) sorrah kowah Blkr. 91, 80.

Carcharius (Prionodon) Temminckii M.H. 91, So.

Carcharodon capensis Smith 266, 57.

Carcharodon Rondeletii M. H. 266, $5^{8 .}$

Carpio Heck. 261, 429. 314, I 9r. 329, 262.

Carpio flavipinnis Blkr. 408, 238.

Carpio fossicola Blkr. 415, 7. 425, I 43.

Carpio melanotus Blkr. 408, 238.

Carpio vulgaris Blkr., Rüpp., Rapp. 411, 252.415, 6. 416, I20. 425, I 43. 497, 23.

Carpiodes Raf. 261, 428. 314, 190.

Carpiodes asiaticus Blkr. 345, I9. 415, 6. 425, 143.

Carpionichthys Blkr. 329, 262.

Cataphractus americanus Bl. Schn. cf. Acanthodoras cataphractus Blkr.

Cataphractus callychthis $B l$. cf. Callichthys tamoata $L$.

Cataphractus carinatus Bl. Schn. cf. Doras carinatus Lac.

Cataphractus costatus $B l$. cf. Platydoras costatus Blkr.

Cataphractus depressus Szens. cf. Callichthys tamoata $L$.

Cataphractus punctatus Bl. cf. Corydoras punctatus Blkr.

Catla Val. 261, 433. 314, 201.

Catla Buchanani C. V. 91, I42.

Catochaenum Cant. 468, 285 .

Catochaenum filamentosum Cant. cf. Diapterus filamentosus Blkr.

Catochaenum limbatum Cant. cf. Diapterus limbatus Blkr.

Catopra Blkr. 468, 3 I8. 
Catopra fasciata Blkr. 42, 65. 45, I95. 49, 418. 55, 412. 56, 445 . $191,3.270,35.271,1.272,9.356$, I 73. 454, 461.

Catopra Grostii Blkr. 52, 90. 185, 224. 285, 368. 454, 464.

Catopra nandoïdes Blkr. 44, I72. 270, 35. cf. Catopra fasciata Blkr.

Catropra siamensis Gthr. cf. Catopra fasciata Blkr.

Catostoma nasutum $K$. v. H. cf. Arius nasutus $V a l$.

Catostomus Les. 261, 428. 314, 189.

Caulolatilus doliatus $B l k r .495,17$.

Centaurus $K p$. 367, r6.

Centracanthus $\operatorname{Raf} .468,275.470$, I 54 .

Centracanthus cirrus $R a f .468,275.470,154$.

Centrarchiformes 468, 248.

Centrarchus Cuv. 468. 249.

Centrarchus aeneus $C . V .468,249$.

Centrarchus hexacanthus $C . V .468,248$.

Centrarchus irideus $C . V .468,249$.

Centridermichthys ansatus Rich. cf. Centridermichthys fasciatus Gt

Centridermichthys fasciatus Gthr. 425, I4I.

Centridermichthys intermedius Gir. 497, II.

Centridermichthys japonicus Steind. 497, I I.

Centridermichthys uncinatus Ger. 497, I I.

Centriscus gracilis Lowe 425, I 34.

Centriscus humerosus Rich. 119, 13.

Centriscus japonicus Gïnth. 497, I4.

Centriscus niloticus $B l$. Schn. 449, 368 .

Centriscus punctulatus $B l k r .459$, го3.

Centriscus scolopax Cuv. 266, 55 .

Centriscus scutatus $L .396,298.459,75 . \mathrm{cf}$. Amphisile scutata C. $V$.

Centroblennioidei Blkr. 449, 368 .

Centrochir Ag. 306, 84.

Centrochir crocodili $A g .303$, I 5. 306, 85 .

Centrogaster fuscescens Houtt. cf. Amphacanthus fuscescens $C$. $V$., Teuthis fuscescens Blkr.

Centrogenys Rich.468, 257.

Centrogenys waigiensis $B l k r .468,257$.

Centrogobius Blkr. 453, $32 \mathrm{I}$.

Centrolabrus Gthr. 292, 107.

Centrolophus fasciolatus $L a c$. cf. Lampugus fasciolatus $C . V$.

Centromochlus Kner 306, 89.

Centromochlus megalops Kner 306, 89.

Centronotus binotatus Raf. cf. Glaucus Rondeletii Will.

Centronotus crassispinis Gill 497, 20.

Centronotus dolichogaster Gill 497, 20.

Centronotus glaycos Risso cf. Glaucus Rondeletii Will.

Centronotus nebulosus Gill 497, 20. 
Centronotus ovalis Lac. cf. Trachinotus mookalee C. V. 57, 48.

Centronotus subfrenatus Gill 497, 20.

Centrophorus foliaceus Gïnth.497, 3 .

Centrophorus moluccensis Blkr. 273, 3.

Centrophorus squamulosus Günth. 497, 3 .

Centropistes georgianus $C$. 119, 6 .

Centropistes salar Rich. 119, 6.

Centropistes truttaceus $C$ \%. 119, 6.

Centropogon Gthr. 473, 297.

Centropogon fusco-virens Gthr. 358, I88. 364, 286. 369, 42. cf. Paracentropogon longispinis Blkr.

Centropogon hypselopterus Gthr. 354, I48. 358, I88. 364, 286.

Centropogon leucoprosopon Gthr. 364, 286. cf. Paracentropogon leucoprosopon Blkr.

Centropomus Cuv. nec. Lac. 468, 264.

Centropomus Lac. 468, 265, 336.

Centropomus ambassis Lac. cf. Ambassis Commersonii C.V., Ambassis nalua $C . V$.

Centropomus aureus Lac. cf. Amia aurea Blkr., Apogon roseipinnis C. $V$.

Centropomus hober Lac. cf. Lutjanus fulviflamma $B l k r$.

Centropomus rupestris Lac. cf. Dules rupestris $C$. $V$., Moronopsis rupestris $C . V$., Moronopsis rupestris Gill.

Centropomus sandat Lac. 468, 265.

Centropomus sexlineatus Lac. cf. Grammistes orientalis Bl. Schn.

Centropomus undecimalis $C . V .468,264$.

Centropristes Cuv. 468, 2.54, 336.

Centropristes georgianus $C$. $V$. cf. Arripis georgianus Jen.

Centropristes hirundinaceus $C . V$. 253, 234. 269, 75. 497, 5.

Centropristes nigricans $C . V .468,254$.

Centropristes savonaceus $\mathrm{Val} .459,88.495$, Iо.

Centropristes scorpaenoides $C . V$. 13, 4. cf. Myriodon scorpaenoïdes Bris., Myriodon waigiensis Gthr.

Centropristes scorpaenoides Rich. 468, 257.

Centropus Kner 289, 8r. 297, I4I.

Centropus staurophorus Kner 289, 82. cf. Caracanthus apistus Blkr.

Centropyge Kaup 468, 307 .

Centropyge tibicen $K$ aup cf. Holacanthus tibicen $C . V$.

Centrurophis $K p .351$, I I9.

Centrurophis bangko $K p$. cf. Ophichthys bangko $B l k r$.

Centrurophis grandoculis $K p$. cf. Ophichthys grandoculis $B l k r$.

Centrurophis macrochir $K p$. cf. Ophichthys macrochir $B l k r$.

Centrurophis spadiceus $K F$. cf. Muraenopsis marginatus $B / k \%$ ( ) phichthys cephalozona Blkr.

Cephalacanthus spinarella Lac., C.V. 302, I 5. cf. Corystion volitans Blkr.

Cephalinus Gron. 473, 300. 
Cephalocassis Blkr. 306, 91 .

Cephalocassis coelatus Blkr. 239, I02. 270, 45. 272, I8. 287, 58.

Cephalocassis macronotacanthus Blkr. 270, 45 .

Cephalocassis melanochir Blkr. 270, 45. 272, I8. 306, 9г.

Cephalocassis Stormi Blkr. 270, 45. 306, 90.

Cephalocassis truncatus $B l k r .191,2.239,102.270,45.272, ~ 18.288,78$.

Cephalocassis venosus Blkr. 235, 370. 287, $5^{8}$.

Cephalogobius Blkr. 453, 315, 320.

Cephalopholis Bl. Schn. 468, 256.

Cephalopholis argus $B l$. Schn. cf. Epinephelus argus Bl. Schn.

Cephaloptera 1, $55^{2}$.

Cephaloptera ereegoodoo-tenbee Cuv. 288, 67.

Cephaloptera japonica $M$.H. cf. Dicerobatis japonica Blkr.

Cephaloptera Kuhlii Val., M. H. 179, 6. cf. Dicerobatis Kuhlii Gthr.

Cephalus hypophthalmus Steind. cf. Hypophthalmichthys nobilis Blkr.

Cephalus mantschuricus Basil. cf. Hypophthalmichthys molitrix Blkr.

Cepola abbreviata $C . V$. 174, 38. 270 44. 287, 58. 288, 77. 425, I34.

Cepola hungta Rich. cf. Cepola Krusensternii Schl. part. Blkr.

Cepola Krusensternii Schl., Blkr. 90, 39. 93, 8. 102, 4ri. 118, 108. 253, 235. 411, 252. 425, I34. 449, 369. cf. Acanthocepola Krusensterni Blkr., Cepola Schlegeli Blkr.

Cepola limbata C. $V$. 497, г8. cf. Cepola Krusensternii T. Schl.

Cepola marginata C.V. 497, I8. cf. Cepola Krusensternii T. Schl.

Cepola mesoprion Blkr. 102, 4r4. 118, 109. cf. Acanthocepola mesoprion Blkr.

Cepola Schlegelii Blkr. 102, 412. 118, т го, 497, r8.

Cepola variegata Szons. cf. Cepola abbreviata $C$. $V$.

Cepoloidei 449, 369 .

Ceratacanthus Gill 367, 14.

Ceratichthys Baird 261, 437. 314, 2 I 2.

Ceratorhynchus $A g$. 306, го8.

Ceris caudimacula Giunth. cf. Hemicoris caudimacula Blkr.

Cerna Bp. 468, 25.

Cernua Bidyana Mitch. 119, I9.

Cestracion malleus Gill 497, 3 .

Cestracion mokarran $A$. Dum. 425, iा9.

Cestracion Philippi $M$. H. cf. Heterodontus zebra Gray.

Cestracion tiburo $A$ Dum. 425, II9.

Cestracion zebra Gr. 275, 9. cf. Heterodontus Philippi Blv. Heterodontus zebra $G r$.

Cestracion zygaena Blkr., Gill 364, 270. 425, I19. 459, 67.

Cestraeus oxyrhynchos $C$. $V$. 137, 307. 270, 33. 275, 41. 276, 65 .

Cestraeus oxyrhynchus $C . V . \mathbf{2 7 1}, 9$.

Cestraeus plicatilis C. $V .10,67,68.46,2$ I $3.275,4$ I.

Cetopsis $A g .300,403.306$, I I I. 
Cetopsis candira $A g$. cf. Hemicetopsis candira Blkr.

Cetopsis evecutiens $A g .306$, I I r.

Cetopsis gobioides Kner cf. Pseudocetopsis gobioides Blkr.

Chaca $\mathrm{Val}$. 306, II9.

Chaca bankanensis Blkr. 56, 455. 121, I65. 270, 49. 272, 20.

Chaca Buchanani Blkr. 272, 20.491, 55 .

Chaca lophioïdes C. V. 56, 456.91, 60. 306, I I9. cf. Chaca Buchanani Blkr.

Chaenobryttus Gill 468, 250.

Chaenobryttus melanops Gill 468, 250.

Chaenogobius Gill 453, 322.

Chaenogobius annularis Gill 453, 322. 497, I 9.

Chaenopsetta olivacea Blkr. 425, I30. 497, 2 r.

Chaenopsetta Wolffi Blkr. 497, 2 I.

Chaetodermis Swns. 367, I2.

Chaetodermis penicilligerus Swns. 368, 26. cf. Monacanthus penicilligerus Cuv.

Chaetodipteriformes 468, 300 .

Chaetodipterus Lac. 468, 300.

Chaetodipterus Plumieri Lac. 468, 30 r.

Chaetodon Art., Cuv. 1, 553. 468, 305, 308.

Chaetodon Abhortani $C$. $V$. cf. Chaet. dorsalis Reinw., Tetragonoptrus (Chaetodontops) melanotus Blkr.

Chaetodon abudafur Forsk. cf. Tetradrachmum arcuatum Cant.

Chaetodon acuminatus $L$. cf. Heniochus macrolepidotus $C$. $V$., Taurichthys macrolepidotus $B l \mathrm{kr}$.

Chaetodon albicauda Gron. cf. Platax vespertilio Cuv.

Chaetodon annularis L., Bl. cf. Acanthochaetodon annularis Blkr., Holacanthus annularis $L a c$.

Chaetodon araneus J.W. Benn. cf. Tetradrachmum arcuatum Cant.

Chaetodon arcuanus $L$. $(G m$.) cf. Dascyllus aruanus C.V., Tetradrachmum arcuatum Cant.

Chaetodon arcuatus $L .467$, I83. 482, 2 r. cf. Chaetodon angulatus $B l k r$. Chaetodon paru $B l .$, Đascyllus aruanus $C . V$. , Tetradrachmum arcuatum Cant.

Chaetodon argus L. (Bodd.) cf. Ephippus argus C. $V$., Scatophagus argus $C . V$.

Chaetodon arthriticus Bell. cf. Platax teira Cur.

Chaetodon aruanus $L_{0}\left(B l_{0}\right)$ cf. Dascyllus aruanus $C$. $V$., Tetradrachmum arcuatum Cant.

Chaetodon arusa Bonn. cf. Acanthochaetodon maculosus Blkr.

Chaetodon asfur Forsk. cf. Acanthochaetodon asfur Blkr.

Chaetodon asfur var. cocrulescens Forsk. cf. Acanthochaetodon maculosus Blkr.

Chaetodon atratus Gron. cf. Chaetodontoplus mesoleucus Blkr. 
Chaetodon atromaculatus Benn. cf. Ephippus argus $C . V$.

Chaetodon aureus $B l$. (nec Schl.) 467, I85. 482, 2 I.

Chaetodon aureus Schl. 175, I5. cf. Tetragonopterus aureus Blkr.; Tetragonoptrus aureus Blkr.

Chaetodon auriga Forsk. 81, I64. 91, 40. 167, 371. 170, 478, 479. 184, 200. 188, 459. 234, 353. 236, 33 г. 275, 34. cf. Tetragonoptrus auriga Blkr., Tetragonoptrus (Linophora) auriga Blkr.

Chaetodon austriacus Rüpp. cf. Chaetodon vittatus $B l$. Schn., Tetragonoptrus (Rabdophorus) trifasciatus Blkr. 22, 18.

Chaetodon baronessa $C . V .47,239.54,237.156,2$ I 4. 157, 232. 270, 26. cf. Tetragonoptrus (Gonochaetodon) triangulum Blkr.

Chaetodon bengalensis Bl. cf. Glyphisodon (Glyph.) bengalensis $C$. $V$.

Chaetodon Bennetti $C . V .78,467.270,26$. cf. Tetragonoptrus (Rabdophorus) Bennetti Blkr.

Chaetodon biaculeatus Bl. cf. Premnas biaculeatus Blkr., Premnas trifasciatus $C, V$.

Chaetodon bicolor Bl. cf. Holacanthus bicolor C.V., Lac.

Chaetodon bifascialis $C$. $V$. cf. Chaetodon trifascialis Q. G., Megaprotodon strigangulus $B l k r$.

Chaetodon bifasciatus Shave cf. Taurichthys macrolepidotus Blkr.

Chaetodon bimaculatus $B l$. cf. Tetragonoptrus bimaculatus Blkr., Tetragonoptrus (Chaetodontops) ocellatus Blkr.

Chaetodon biocellatus C. $V$. 151, 403. 167, 37 I. 234, 353. 236, 331, 333. 275, 34. cf. Tetragonoptrus biocellatus Blkr., Tetragonoptrus (Chaetodontops) fasciatus Blkr.

Chaetodon Blackburni Dess. cf. Tetragonoptrus Blackburni Blkr., Tetragonoptrus (Citharoedus) Blackburni Blkr., Tetragonoptrus (Rabdophorus) Blackburni Blkr.

Chaetodon Blochii Blkr. 91, 166.

Chaetodon Boddaerti L. $(G m$.) cf. Holacanthus diacanthus Gthr., Holacanthus dux $L a i$.

Chaetodon Brownriggii Benn. cf. Glyphidodontops antjerius Blkr.

Chaetodon canaliculatus Mungo Park cf. Amphacanthus canaliculatus $B l$.

Chaetodon canescens $L$. cf. Zanclus canescens Gthr.

Chaetodon capistratus Bl. 468, 305. cf. Tetragonoptrus (Hemichaetodon) capistratus Blkr.

Chaetodon chinensis $B l$. cf. Polyacanthus opercularis Rich.

Chaetodon chrysostomus Park cf. Taurichthys chrysostomus Blkr.

Chaetodon chrysozona K.v. H. 275, 34 .

Chaetodon chrysozonus K.v.H. 22, I 7. 468, 304. cf. Coradion chrysozonus $K p$., Tetragonoptrus chrysozonus Blkr.

Chaetodon chrysurus Brouss. cf. Pomacentrus (Psendopom.) trilineatus Blkr.

Chaetodon chrysurus Lién. cf. Tetragonoptrus chrysurus Blkr. 
Chaetodon chrysurus Desj. cf. Tetragonoptrus (Linophora) Mertensi Blkr., Tetragonoptrus (Linophora) xanthurus Blkr.

Chaetodon ciliaris $L .(B l$.$) cf. Chaetodon arcuatus L$., Holacanthus ciliaris Lac.

Chaetodon cingulatus $B l k r .482,2 \mathrm{I}$.

Chaetodon citrinellus Brouss. 86, 501. 182, I59. 236, 331. 270, 26. 275, 34. 481, 72. cf. Tetragonoptrus (Tetragonoptrus) miliaris Blkr.

Chaetodon collare Bl. 22, 19. 156, $214.270,26.275,34.288,73$. cf. Tetragonoptrus (Chaetodontops) collaris Blkr.

Chatodon collaris Bl. 468, 306. cf. Tetragonoptrus collaris Blkr.

Chaetodon cornutus $L$. cf. Zanclus cornutus $C . V$.

Chaetodon conagga Lac. cf. Acanthurus triostegus Bl. Schn.

Chaetodon curaçao Bl. cf. Glyphidodon (Amblyglyph.) trifasciatus Blkr.

Chaetodon daakar Bonn. cf. Platax teira Cuv.

Chaetodon decussatus $C . V$. 22, I9. 91, 40. 166, 328. 170, 479. 236, 33I, 333. cf. Chaetodon pictus Forsk., Tetragonoptrus (Linophora) vagabundus $B l k r$.

Chaetodon diacanthus Bodd. cf. Holacanthus diacanthus Gthr.

Chaetodon dichrous Gthr. cf. Tetragonoptrus (Tetragonoptrus) dichrous Blkr.

Chaetodon dizoster $C . V$. cf. Tetragonoptrus dizoster Blkr., Tetragonoptrus (Oxychaetodon) falcula Blkr.

Chatodon dorsalis Rvudt. 47, 240. 157, 232. 184, 200. 275, 34. cf. Tetragonoptrus dorsalis Blkr., Tetragonoptrus (Chaetodontops) melanotus Blkr.

Chaetodon dux L. $(G m$.) cf. Holacanthus diacanthus Gthr., Holacanthus dux $L a c$.

Chaetodon enceladus Shazo. cf. Chelmon rostratus C. $V$.

Chaetodon enneacanthus $C$. $V$. cf. Chaetodon chrysozonus $K . v . H$., Coradion chrysozonus $K p$.

Chatodon ephippium $C . V .84,337.99,325.134$, I55. 184, 200. 188, 459. 275, 34. cf. Tetragonoptrus (Rabdophorus) ephippium Blkr.

Chaetodon falcatus Lac. cf. Drepane longimana C. V., Harpochirus punctatus Cant.

Chaetodon falcula $B l .91,40.125,311.157,232$. cf. Tetragonoptrus (Oxychaetodon) falcula Blkr.

Chaetodon fasciatus Bl. cf. Holacanthus diacanthus Gth\%., Holacanthus dux Lac.

Chatodon fasciatus Forsk. cf. Tetragonoptrus fasciatus Blkr. Tetr. (Chaetodontops) fasciatus Blkr.

Chaetodon festivus Desj. cf. Coradion melanopus Blkr., Coradion merlangus Blkr.

Chaetodon festivus Lién. cf. Tetragonoptrus festivus $B l k r$.

Chaetodon flavescens Bcmn. cf. Tetragonoptrus Kleini Blkr., Tetr. (Lepidochaetodon) kleini $B / k r$. 
Chaetodon flavirostris Gthr. cf. Tetragonoptrus (Tetragonoptrus) flavirostris $B l k r$.

Chaetodon flavus $B l$. Schn. cf. Tetragonoptrus (Chaetodontops) fasciatus Blkr.

Chaetodon Frehmlii C. $V$. cf. Tetragonoptrus (Rabdophorus) Fremblii Blkr.

Chaetodon Fremblii Benn. cf. Tetragonoptrus (Rabdophorus) Fremblii Blkr.

Chaetodon gahhm Forsk. cf. Acanthurus gahhm Eh\%.

Chaetodon Garnotii Less. cf. Chaetodon ephippium C.V., Tetragonoptrus (Rabdophorus) ephippium Blkr.

Chaetodon gonyphoron $K$. v. H. cf. Tetragonoptrus (Gonochaetodon) triangulum Blkr.

Chaetodon gracilis Gthr. cf. Tetragonoptrus (Tetragonoptrus) sedenterius $B l k r$.

Chaetodon guttatissimus Benn. 91, 40. cf. Tetragonoptrus (Tetr.) miliaris Blkr.

Chaetodon guttatissimus Klunz. cf. Tetragonoptrus Klunzingeri Blkr.

Chaetodon guttatus Bl. cf. Amphacanthus guttatus Bl. Schn.

Chaetodon guttatus Gron. cf. Coradion chrysozonus Kaup.

Chaetodon hadjan Bl. Schn. cf. 'Tetragonoptrus (Oxychaetodon) mesoleucus Blkr.

Chaetodon humeralis Gthr. cf. Tetragonoptrus (Tetragonoptrus) humeralis Blkr.

Chaetodon imperator $B l$. cf. Acanthochaetodon imperator $B l$., $B l k r$., Holacanthus imperator Lac.

Chaetodon karraf $C . V$. cf. Tetragonoptrus (Gonochaetodon) triangulum Blkr.

Chaetodon Kleinii $B l .(C . V$.$) 54, 236. 275, 34. cf. Tetragonoptrus$ Kleini Blkr. Tetragonoptrus (Lepidochaetodon) Kleini Blkr.

Chaetodon labiatus $K$. v. $H$. 2, 520. cf. Chaetodon chrysozonus $K$. v. $H$., Coradion chrysozonus $K p$.

Chaetodon larvatus Ehr. cf. Tetragonoptrus (Gonochaetodon) triangulum Blkr.

Chaetodon Layardi Blyth 91, I65. cf. Tetragonoptrus Layardi.

Chaetodon Leachii $C$. $V$. cf. Megaprotodon strigangulus Blkr.

Chaetodon leucopleura Playf. cf. Tetragonoptrus (Rabdophorus) leucopleura Blkr.

Chaetodon lineatus $L$. cf. Acanthurus lineatus $L a c$.

Chaetodon lineolatus $Q . G ., C . V .99,323.188,459.468,306$. cf. Tetragonoptrus lineolatus Blkr., Tetragonoptrus (Oxychaetodon) lineolatus $B l k r$.

Chaetodon littoricola Poey cf. Chaetodon arcuatus $L$.

Chaetodon lividus Forst. cf. Eupomacentrus (Eupomac.) lividus Blkr. 
Chaetodon longimanus $B l$. Schn. cf. Drepane longimana $C$. $V$., Blkr., Harpochirus punctatus Cant.

Chaetodon longirostris Brouss. cf. Chelmon longirostris $C$. $V$., Prognathodus longirostris Blkr.

Chaetodon luctuosus $C$. $V$. cf. Tetragonoptrus (Rabdophorus) luctuosus Blkr.

Chaetodon lunaris Gron. cf. Tetragonoptrus (Linophora) auriga Blkr.

Chaetodon lunatus $C$. V., Ehr. 236, 33 r. cf. Chaetodon lineolatus Q.G., Tetragonoptrus (Oxychaetodon) lineolatus Blkr.

Chaetodon lunula C. V. 94, 57. 119, 9. 134, 155. 167, 371. 237, 437. 275, 34. 277, 89. cf. Tetragonoptrus (Chaetodontops) fasciatus $B l k r$. Tetragonoptrus lunula Blkr.

Chaetodon lunulatus $Q$. G. cf. Tetragonoptrus lunulatus.

Chaetodon luteus Bonn. cf. Chaetodon aureus $B l$.

Chaetodon macrolepidotus $L$. $(B l$.) cf. Heniochus macrolepidotus C.V., Taurichthys macrolepidotus $B l k r$.

Chaetodon maculatus $L$. ( $B l$. $)$ 297, i 26 . cf. Tetragonoptrus maculatus $B l k r$.

Chaetodon maculosus Forsk. cf. Acanthochaetodon maculosus Blkr.

Chaetodon marginatus Ehr. cf. Tetragonoptrus (Chaetodontops) melanotus Blkr.

Chaetodon melammystax $B l$. Schn. cf. Tetragonoptrus (Lepidochaetodon) Kleini Blkr.

Chaetodon melanopoma Playf: cf. Tetragonoptrus (Chaetodontops) melanopoma Blkr.

Chaetodon melanopterus Guich. cf. Tetragonoptrus melanopterus Blkr. Tetragonoptrus (Rabdophorus) melanopterus Blkr.

Chaetodon melanopus $C . V$. 321, 25. cf. Chaetodon chrysozonus $K$. v. H., Coradion melanopus Blkr.

Chaetodon melanotus Reinw. cf. Coradion chrysozonus Kaup.

Chaetodon melanotus $B l$. Schn. cf. Tetragonoptrus (Chaetodontops) melanotus Blkr.

Chaetodon melapterus Guich. cf. Tetragonoptrus (Rabdophorus) trifasciatus Blkr.

Chatetodon melastomus Bl. Sihn. cf. Tetragonoptrus (Lepidochaetodon) Kleini Blkr.

Chaetodon Mertensii C. $V$. 163, 54. cf. Tetragonoptrus (Linophora) Mertensi, xanthurus $B l k r$.

Chatodon mesoleucus Forsk. (nec. Bl.) cf. Tetragonoptrus (Oxychaetodon) mesoleucus Blkr. 474, 320 .

Chaetodon mesoleucus $B l$. cf. Chaetodontoplus mesoleucus Blkr., Holacanthus mesoleucos Lac.

Chaetodon mesomelas $L$. $(G m$.) cf. Chaetodontoplus mesoleucus Blkr. cf. Holacanthus mesoleucos Lac.

Chaetodon Meyeri Bl. Schn. 142, 37. 275, 34. 468, 305. cf. Tetragonoptrus (Citharoedus) Meyeri Blkr. 
Chaetodon microlepis Blkr. 76, 257. cf. Acanthochaetodon semicirculatus Blkr., Holacanthus semicirculatus C. $V$.

Chaetodon miliaris Q.G. cf. Tetragonoptrus (Tetragonoptrus) miliaris $B l k r$.

Chaetodon mitratus Gthr. cf. Tetragonoptrus mitratus Blkr.

Chaetodon modestus T. Schl., C. V. 47, 230. 90, 34.

Chaetodon modestus Schl. 93, 8. cf. Tetragonoptrus modestus Blkr.

Chaetodon Mulsanti Thioll. cf. Tetragonoptrus (Rapdophorus) ephippium Blkr.

Chaetodon multicinctus Garr. cf. Tetragonoptrus (Tetrag.) punctatofasciatus Blkr.

Chaetodon mycteryzans Gron. cf. Taurichthys macrolepidotus Blkr.

Chaetodon nesogallicus C. V. 47, 240. 270, 26. 285, 245. cf. Tetragonoptrus (Linophora) auriga Blkr., Tetr. nesogallicus Blkr.

Chatodon nicobariensis $B l$. Schn. cf. Acanthochaetodon nicobariensis $B l k r$. Holacanthus nicobariensis Blkr.

Chaetodon nigricans $B$. 113, 434. cf. Acanthurus matoides $C$. $V$.

Chatodon nigripinnis Pet. cf. Tetragonoptrus (Oxychaetodon) nigripinnis Blkr.

Chaetodon nudus Gron. cf. Zanclus cornutus $C . V$.

Chaetodon ocellatus Bl. Schn., Bl. 91, 66.98, 212. cf. Tetragonoptrus (Chaetodontops) ocellatus Blkr.

Chaetodon ocellatus $B l k r$. cf. Chaetodon biocellatus C. $V$., Tetragonoptrus (Chaetodontops) fasciatus $B l k r$.

Chaetodon ocellicauda C. $V$. cf. Tetragonoptrus (Oxychaetodon) ocellicauda Blkr.

Chaetodon octofasciatus C. $V ., B l .2,520.9,633.22$, I6, 126, 344. 158, 274. 234, 353. 275, 34. 287, 50. cf. Tetragonoptrus (Tetr.) octofasciatus Blkr.

Chaetodon octolineatus Gron. cf. Tetragonoptrus (Tetragonoptrus) octofasciatus $B l k r$.

Chaetodon oligacanthus Blkr. 2, 520. 22, I6. 31, 105. 54, 235. 56, 445. 275, 34. 287, 50. 449, 37I. 468, 304. cf. Parachaetodon ocellatus Blkr., Tetragonopterus oligacanthus Blkr.

Chaetodon orbicularis Forsk. cf. Platax orbicularis $C . \quad V$. Pl. teira Cuv.

Chaetodon orbis $B l$. cf. Ephippus orbis $C . V$., Ilarches orbis Cant.

Chaetodon ornatissimus Sol. 163, 55. cf. Tetragonoptrus (Citharoedus) ornatissimus Blkr.

Chaetodon ornatus $G r$. cf. Tetragonoptrus (Citharoedus) ornatissimus Blkr.

Chaetodon ovalis Thioll. cf. Tetragonoptrus (Rabdophorus) trifasciatus Blkr.

Chatodon oxycephalus Blkr. 79, 603. cf. Tetragonoptrus (Oxychaetodon) lineolatus Blkr. 
Chaetodon pairatalis Ham. Buch. cf. Ephippus argus C. $V$., Scatophagus argus $C$. $V$.

Chaetodon parallelus Gron. cf. Tetragonoptrus (Chaetodontops) collaris Blkr.

Chaetodon paru L. (Bl.) 468, 308. 482, $2 \mathrm{I}$.

Chaetodon pavo Bl. cf. Pomacentrus pavo Lac., Pomac. (Pomac.) pavo Lac.

Chaetodon pelewensis Kner cf. Teteagonoptrus (Chaetadontops) pelewensis Blkr.

Chatodon pentacanthus Lac. cf. Platax orbicularis C. V., Platax vespertilio Cuv.

Chaetodon pepek Montrouz. cf. Tetragonoptrus (Rabdophorus) ephippium Blkr., Tetr. (Rabd.) trifasciatus Blkr.

Chaetodon pictus Forsk. 44, I77. 270, 26. cf. Tetragonoptrus (Linophora) vagabundus $B l k r$.

Chaetodon pinnatus L. (Bonn). cf. Platax Blochii C. V., Platax pinnatus Blkr., Platax teira Cuv., Platax vespertilio $C u v$.

Chaetodon plebejus L. $(G m$.) cf. Tetragonoptrus (Rabdophorus) plebejus $B l k r$.

Chaetodon plectorhynchus Shaw cf. Diagramma plectorhynchus C.V., Plectorhynchus chaetodontoides Lac.

Chaetodon polylepis Blkr. 163, 54. 468, 304. cf. Hemitaurichthys polylepis Blkr.

Chaetodon praetextatus Cant. cf. Tetragonoptrus (Chaetodontops) collaris Blkr.

Chaetodon princeps C. V. 22, 19. 54, 235. 170, 479. 184, 200. 234, 353. 237, 437. 270, 26. 275, 34. Tetragonoptrus Rafflesi Blkr., Tetragonoptrus (Linophora) Rafflesi Blkr.

Chaetodon principalis $C . V$. cf. Tetragonoptrus (Rabdophorus) ephippium Blkr.

Chaetodon punctato-fasciatus $C . V .47,238$. cf. Tetragonoptrus (Tetr.) punctato-fasciatus $B l \vec{k} r$.

Chaetodon punctato-lineatus Gron. cf. Tetragonoptrus (Tetr.) punctatofasciatus Blkr.

Chaetodon punctatus L. (Park) cf. Chaetodon citrinellus Brouss., Drepane longimana $C . V$, Harpochirus punctatus Cant.

Chaetodon quadrifasciatus Seuvast. 468, 272.

Chaetodon quadrimaculatus $G r$. cf. Tetragonoptrus (Tetragonoptrus) quadrimaculatus $B / k r$.

Chaetodon Rafflesii Benn. cf. Chaetodon princeps C.V., Tetragonoptrus (Linophora) Rafflesi Blkr.

Chaetodon resimus Gron. cf. Holacanthus sexstriatus $K$..$H$.

Chaetodon reticulatus $C . V$. cf. Tetragonoptrus (Chaetodontops) collaris Blkr.

Chaetodon rhombeus $B l$. Schn. cf. Psettus Sebae C. $V$. 
Chaetodon robustus Gthr. cf. Tetragonoptrus robustus Blkr., Tetr. (Tetr.) robustus Blkr.

Chaetodon rostratus $L$. cf. Chelmon rostratus $C$. $V$.

Chaetodon sanctae Helenae Gthr. cf. Tetragonoptrus (Tetragonoptrus) sanctae Helenae Blkr.

Chaetodon saxatilis Bl. cf. Glyphidodon (Glyphidodon) coelestinus (Sol.) C. $V$., Glyphisodon rahti $C . V$.

Chaetodon saxatilis Forsk. cf. Glyphisodon bengalensis $C . V$.

Chactodon (Abudefduf) saxatilis Forsk. cf. Glyphidodon (Glyphidodon) coelestinus (Sol.) $C . V$.

Chaetodon Sebae C. $V$. 9, 633. cf. Chaetodon princeps $C . V$., Tetragonoptrus (Linophora) Rafflesi Blkr.

Chaetodon Sebanus C. $V .91$, 165. cf. Chaetodon auriga Forsk, Tetragonoptrus (Linophora) auriga Blkr.

Chaetodon sedentarius Pocy cf. Tetragonoptrus (Tetragonoptrus) sedentarius Blkr.

Chaetodon selene Blkr. 80, 76. cf. Tetragonoptrus selene Blkr.

Chaetodon semeion Blkr. 130, 450. 184, 200. 275, 34. cf. Tetragonoptrus (Rabdophorus) semeion Blkr.

Chaetodon semilarvatus Ehr. cf. Tetragonoptrus (Oxychaetodon) semilarvatus Blkr.

Chaetodon setifer Bl., Rich. cf. Chaetodon auriga Forsk., Tetragonoptrus auriga Blkr., Tetragonoptrus (Linophora) auriga Blkr.

Chaetodon setosus Gron. cf. Platax pinnatus Blkr.

Chaetodon sexfasciatus Rich. 119, 9. cf. Hemitaurichththys sexfasciatus Blkr.

Chaetodon sordidus Forsk., Gm. cf. Glyphidodon (Glyphidodon) sordidus Rüpp., Glyphisodon sordidus $C . V$.

Chaetodon speculum $K$. v. H. 47, 242. 234, 353. cf. 'Tetragonoptrus (Rhabdophorus) speculum Blkr.

Chaetodon spilopleura Rudt. cf. Tetragonoptrus (Rabdophorus) speculum Blkr.

Chaetodon striatus L. 302, I4. 468, 305. cf. Sarothrodius striatus, Tetragonoptrus (Tetragonoptrus) striatus Blkr.

Chaetodon strigangulus Sol. 47, 239. 54, 235. 275, 34. cf. Megaprotodon strigangulus $B l k r$.

Chaetodon strigangulus Lac. cf. Tetragonoptrus strigangulus Blkr.

Chaetodon strigatus Langsd. Laupd. 118, 94. 468, 298. cf. Micro. canthus strigatus Szons., Therapaina strigata $K p$.

Chaetodon subarcuatus Blkr. 482, 21.

Chaetodon superbus Brouss. cf. Tetragonoptrus (Chaetodontops) collaris Blkr.

Chaetodon Tallii Blkr. 95, 97. cf. Tetragonoptrus (Oxychaetodon) lineolatus Blkr.

Chatodon Taunay Q. G. cf. Megaprotodon strigangulus Blkr. 
Chaetodon tau-nigrum $C$. $V$. cf. Tetragonoptrus (Rabdophorus) taunigrum Blkr., Tetragonoptrus (Rabdophorus) trifasciatus Blkr.

Chaetodon teira Forsl. cf. Platax teira $C$. $V$.

Chaetodon triangularis Rüff. cf. Chaetodon strigangulus Soland., Mega. protodon striangulus $B l k r$.

Chaetodon triangulum $K$..$H .468$, 306. cf. Tetragonoptrus (Gonochaetodon) triangulum Blkr.

Chatodon trichrous Gt/ur.cf. Tetragonoptrus (Tetragonoptrus) trichrous Blkr.

Chaetodon tricolor $B l$. cf. Holacanthus tricolor Lac.

Chaetodon trifascialis $Q . G$. 125. 313. 157, 233. cf., Megaprotodon strigangulus $B / k r$.

Chaetodon trifasciatus Mungo Park cf. Chaetodon vittatus Bl. Schn., Tetragonoptrus (Rabdophorus) trifasciatus Blkr., Tetr. vittatus Blkr.

Chaetodon triostegus L. cf. Acanthurus triostegus Bl. Schn.

Chaetodon truncatus $K$ ner 468, 304. cf. Chelmonops truncatus Blkr.

Chaetodon Tyrwhitti I. W. Benn. cf. Glyphidodon (Glyphidodon) coelestinus (Sol.) C. $V$.

Chaetodon ulietensis C. $V$. 142, 38. cf. Tetragonoptrus (Oxychaetodon) falcula Blkr.

Chaetodon unicornis Forsk. cf. Naseus fronticornis Comm.

Chaetodon unifasciatus Gron. cf. Tetragonoptrus (Chaetodontops) collaris Blkr.

Chaetodon unimaculatus Bl. 47, 24I. 48, 348. 468, 306. cf. Tetragonoptrus unimaculatus $B l k r$.

Chaetodon unimaculatus $B l k r$. cf. Tetragonopterus unimaculatus Blkr.

Chaetodon vagabundus $L .(B l ., C . V$.$) 9, 633. 22, r8. 54, 235. 91, 40$. 127, 345. 156, 21 4. 157, 233. 167, 37 I. 170, 479. 184, 200. 234, 353. 236, 33I, 333. 270, 26. 275, 34. 285, 245. 296, Iо8. cf. Tetragonoptrus vagabundus $B l k r$., Tetr. (Linophora) vagabundus $B l k r$.

Chaetodon verticosus Gron. cf. Acanthochaetodon annularis Blkr.

Chaetodon vespertilio $L$. $(B l$. $)$ cf. Platax Blochii C. V., Platax vespertilio $C u \varkappa$.

Chaetodon vinctus Benn. cf. Tetragonoptrus (Rabdophorus) Bennetti Blkr.

Chaetodon virescens $C . V$. 22, r7. 47, 227. 48, 348. 53, I6r. 54, 235. 167, 37 I. 184, 200. 275, 34. 296, I08. cf. Tetragonopterus Kleini $B l$. Schn. (Blkr.), Tetragonoptrus (Lepidochaetodon) Kleini Blkr.

Chatodon viridis Blkr. 2, 520. cf. Chaetodon collare Bl., Tetragonoptrus (Chaetodontops) collaris Blkr.

Chaetoden vittatus $B l$. Silh. (C. V.) 22, I8. 48, 348. 54, 235. 157, 233. 184, 200. 188, 459. 270, 26. 275, 35. cf. T'etragonopterus vittatus Blkr., Tetragonoptrus (Rabdophorus) trifasciatus Blkr.

Chaetodon vorticosus Gron. cf. Acanthochaetodon annularis Blkr. 
Chaetodon Wiebeli Kaup. cf. Tetragonoptrus (Chaetodontops) fasciatus Blkr.

Chaetodon xanthocephalus Bcm. 91, 40. cf. Tetragonoptrus ('Tetragonoptrus) xanthocephalus $B l k r$.

Chaetodon xanthurus Blkr. 163, 53. cf. Tetragonoptrus (Linophora) xanthurus $B l k r$.

Chaetodon zanzibarensis Playf. cf. Tetragonoptrus (Rabdophorus) speculum Blkr., Tetragonoptrus Zanzibarensis Blkr.

Chaetodon zonipectus Blkr.482, 2 I.

Chaetodon zoster Benn. cf. Hemitaurichthys polylepis Blkr., Hemit. zoster Blkr., Tetragonoptrus zoster Blkr.

Chaetodontoidei. 449, 37 I. 468, 293.

Chaetodontoplus Blkr. 468. 307.

Chaetodontoplus chrysocephalus Blkr. 481, II5. 482, 22.

Chaetodontoplus dimidiatus Blkr. 481, II $8,482,23$.

Chaetodontoplus Duboulayi Blkr. 482, 22.

Chaetodontopius melanosoma Blkr. 481, II 7. 482, 22.

Chaetodontoplus mesoleucus Blkr. 481, II4. 482, 22.

Chaetodontoplus septentrionalis Blkr. 482, 35. 497, 9.

Chaetodontops Blkr. 468, 305 .

Chaetopterus Schl. 438, 44. 468, 276.

Chaetopterus microchir Blkr. cf. Aprion (Aprion) virescens $C$. $V$.

Chaetopterus microlepis Blkr. 405, So. cf. Aprion microlepis Blkr., Aprion (Aprion) microlepis Blkr.

Chaetopterus pristipoma Blkr. 405, 83. cf. Aprion (Aprion) pristipoma Blkr.

Chaetopterus Sieboldi Blkr. cf. Aprion Sieboldi Blkr.

Chaetostomus Heck 306, 78 .

Chaetostomus loborhynchus Kner 306, 78 .

Chaeturichthyi 453, 324 .

Chaeturichthys Rich. 453, 324 .

Chaeturichthys hexanema Blkr. 90, 43. 453, 325. cf. Amblychaeturichthys hexanema Blkr.

Chaeturichthys polynema Blkr.90, 44. 253, 235.408, 238. 425, I28. 453, 325. cf. Parachaeturichthys polynema Blkr.

Chaeturichthys stigmatias Rich. 90, 44. 453, 324 .

Chanda bogoda Buch. Ham. cf. Bogoda nama Blkr.

Chanda Dussumieri Cant. cf. Ambassis gymnocephalus Blkr.

Chanda lala Buch. Ham. cf. Ambassis lala $C$. $V$.

Chanda nalua Buch. Ham. cf. Ambassis nalua $C$. $V$.

Chanda nama Buch. Ham. cf. Bogoda nama Blkr.

Chanda phula Buch. Ham. cf. Bogoda nama Blkr.

Chanda ranga Buch. Ham. cf. Ambassis lala C. $V$.

Chanda ruconius Buch. cf. Equula ruconius $C$. $V$.

Channomuraena Rich. 351, I22. 
Channomuraena vittata Rich.425, I24.

Chanodichthys Blkr. 261, 432. 314, 2 10. 345, 23.

Chanodichthys aethiops Blkr. cf. Leuciscus aethiops Basil.

Chanodichthys bramula Gthr. cf. Parabramis bramula Blkr.

Chanodichthys leucisculus Gthr. cf. Hemiculter leucisculus Blkr.

Chanodichthys mongolicus Blkr. 345, I9. 411, 252. 415, 62. 425,145 .

Chanodichthys pekinensis Gthr. cf. Parabramis pekinensis Blkr.

Chanodichthys terminalis Gthr. cf. Parabramis bramula Blkr.

Chanos arabicus $L a c$. cf. Chanos salmoneus $C$. $V$.

Chanos chloropterus C. V. 91, 72. 288, 78.

Chanos indicus Blkr. 275, 46.

Chanos lubina C. $V .459$, ro2. 495, 22.

Chanos mento $C$. $V$. cf. Chanos salmoneus $C$. $V$.

Chanos nuchalis $C . V .91,72.288,78$.

Chanos orientalis $C . V .16,6.25$, II. 59, Ir.

Chanos salmoneus C. V., Gthr. 119, I4. 459, го2. 491, 55. 495, 22.

Chatoessus altus $G r$. cf. Chatoessus nasus $C$. $V$.

Chatoessus aquosus Rich. cf. Dorosoma punctatum Blkr.

Chatoessus chacunda C. $V .32$, I60. 50, 472. 56, 446, 458. 60, 46.

91, 74. 178, 2. 227, 408. 270, 58. 272, 27. 275, 48. 284, 240.

287, 62. 288, 79. 309, т56. 364, 295. cf. Dorosoma chacunda Blkr.

Chatoessus chanpole $C . V .91,74$.

Chatoessus chrysopterus Rich. cf. Dorosoma chrysopterus Blkr.

Chatoessus come Rich. cf. Chatoesus nasus $C . V$.

Chatoessus cortius C. V. 91, 76 .

Chatoessus maculatus Rich. cf. Dorosoma maculatum Blkr.

Chatoessus manmina C. $V .91,76$.

Chatoessus nasus $C . V .46,223.60,5$ r. 91, 76. 235, 373. 275, 48. 287, 62. 296, II2. 316, 243. 358, I92. cf. Dorosoma nasus Blkr.

Chatoessus Osbecki Val. cf. Dorosoma Osbecki Blkr.

Chatoessus punctatus T. Schl., Kner 90, 50. cf. Dorosoma punctatum Blkr.

Chatoessus selangkat Blkr. 56, 458. 60, 47. 170, 480. 275, 48. $364,295$.

Chatoessus tampo C. V. 270, 58.

Chatoessus triza Rich. cf. Dorosoma triza Blkr.

Charax Risso $C . V .468,284$.

Charax puntazzo C. $V .468,284$.

Chedrus Swns. 261, 433. 314, 208.

Cheilichthys kappa Blkr. 296, Iо3.

Cheilinoïdes cyanopleura Blkr. 43, 72.

Cheilinus Lac. 1, 552. 292, 94.

Cheilinus arenatus $C . V .459, \delta_{4} .495$, IC.

Cheilinus bifasciatus Blkr. 414, 328 . 
Cheilinus celebicus Blkr. 81, I7I. 167, 374. 275, I6. 293, 299. 296, I $64.316,24$ I. 364, $275.491,40$.

Cheilinus ceramensis Blkr. 54, 290. 250, 207. 293, 274. 320, 265. 358, I $84.364,275.394,286.491,40$.

Cheilinus chlorurus Cuv., Blkr. 47, 230. 126, 344. 188, 460. 275, I6. $287,42.293,274.315,230.320,265.340,364.354$, I 44 . 358, I $84.364,275.437,37.459,84.495$, Iо.

Cheilinus Commersonii Benn. cf. Cheilinus radiatus Blkr.

Cheilinus decacanthus Blkr. 46, 2 I3. 47, 256. 156, 2 I 7. 157, 235. 167, 374. 184, 203. 185, 225. 228, 424. 234, 355. 236, 332. 267, I39. 275, r6. 287, 42. cf. Cheilinus chlorurus Blkr.

Cheilinus diagrammus $C . V .11,38.12,64$. cf. Cheilinus radiatus $B l k r$.

Cheilinus fasciatus $C . V .11,31.12,59.126,344.156,217.184$, 203. 270, 38. 275, I6. 287, 42. 293, 275. 308, I 52. 318, 250. 364, 275. 394, 286. 396, 299. 459, 84. 491, 40. 495, го.

Cheilinus guttatus Blkr. 8, г6г. 11, 36. 12, 63. 47, 230. cf. Cheilinus chlorurus Blkr.

Cheilinus hexataenia Blkr. 163, 84. 184, 203. 292, 95. cf. Pseudocheilinus hexataenia Blkr.

Cheilinus Hoevenii Blkr. 293, 297. 308, I52. 364, 275.

Cheilinus lacrymans $V a l .11,7.12,42.293,275$.

Cheilinus lunulatus Rüpp. 459, 84 .

Cheilinus macrocheilus $K . v, H$. cf. Tautoga melapterus $C . V$.

Cheilinus melanopleura Blkr. 364, 275. 374, I34.

Cheilinus mentalis Rüpp. 296, I04.

Cheilinus nebulosus Rich. cf. Cheilinus trilobatus Lac.

Cheilinus notophthalmus Blkr. 78, 493. 293, 275.

Cheilinus oxycephalus Blkr. 84, 349. 157, 235. 293, 275. 296, Iо4. $318,250$.

Cheilinus oxyrhynchus $B l k r .293,300.364,275$.

Cheilinus punctatus Benn. 459, 84. 495, Iо.

Cheilinus punctulatus C. $V .47,230$. cf. Cheilinus punctatus Benn.

Cheilinus quinquecinctus Rüpp. 2, 513.

Cheilinus radiatus $B l k r .188,46$ I. 250, 207. 270, 39. 275, 16. 293, 275. 296, 104. 309, I 54. 315, 230. 364, 275. 459, 84. 491, 4 O. 495 , Iо.

Cheilinus sinuosus $Q$. G. cf. Cheilinus trilobatus Lac.

Cheilinus tetrazona Blkr. 76, 293. 134, I 55. 188, 46I. 228, 424. $250,207.270,39.275$, I $6.285,243$.

Cheilinus trilobatus Lac. 2, 513. 11, 34. 12, 61. 270, 39. 275, r6. 287, 42. 293, 275. 309, 154. 315, 230.320, 266. 338, 360. 354, I 44.358, I $84.364,276.425$, I $35.459,84.495$, Iо. cf. Cheilinus ceramensis $B l k r$.

Cheilinus undulatus Rüpp. 78, 492. 293, 276.

Cheilio Comm. 292, 96. 
Cheilio auratus Comm., C. V., Lac. 46, 22 I, 275, i8. cf. Cheilio hemichrysos $C . V$. , Cheilio inermis Rich.

Cheilio cyanochloris C. V. cf. Cheilio inermis Rich.

Cheilio fuscus $C . V$. cf. Cheilio inermis Rich.

Cheilio hemichrysos $C . V .47,255.54,237.167,373.184,203$. 234, 355. 236, 33I, 334. 270, 37. 275, I9.

Cheilio inermis Rich. 293, 277.308, I 52. 315, 230. 318, 250. 320, 265. 354, I44. 358, I $84.364,276.396,299.425$, I $35.437,37$. $459,83.491,40.495$, 10. 497,5 .

Cheilio lineatus C. V. 119, I9.

Cheilio ramosus Jen. cf. Cheilio inermis Rich.

Cheiliopsis bivittatus Steind. 459, 83. 495, Iо.

Cheilobranchus dorsalis Rich. 119, I6.

Cheilodactylini 468, 3 I5.

Cheilodactylus Lac. 163, 40. 468, 3I5.

Cheilodactylus aspersus Rich. 119, 8.

Cheilodactylus brachydactylus $C . V . \mathbf{2 6 6}, 52$.

Cheilodactylus carponemus $C . V .119,8$.

Cheilodactylus ciliaris 119,8 .

Cheilodactylus fasciatus Lac. 266, 63. 468, 3 I 5 .

Cheilodactylus gibbosus Kich. 119, 8 .

Cheilodactylus hecateius Rich. 119, 8 .

Cheilodactylus lineatus Rich. 119, 8.

Cheilodactylus macropterus Rich. 119, 8.

Cheilodactylus nigricans Rich. 119, 8.

Cheilodactylus nigripes Rich. 119, 8 .

Cheilodactylus quadricornis Gïnth. cf. Cheilodactylus (Goniistius) quadricornis Blkr.

Cheilodactylus (Groniistius) quadricornis Blkr. 497, 9.

Cheilodactylus zonatus C. $V .118,83.119,8.468,315$. cf. Cheilodactylus (Goniistius) zonatus Blk\%.

Cheilodactylus (Goniistius) zonatus Blkr. 497, 9.

Cheilodipterus Ham. Buch. C. V. 1, 553. 2, 526. 453, 303.468, 26 I.

Cheilodipterus acoupa Lac. cf. Otolithus cayennensis Blkr.

Cheilodipterus amblyuropterus Blkr. 151, 395. cf. Pseudamia amblyuropterus Blkr.

Cheilodipterus apogonides Blkr. 143, 37. cf. Amia apogonides Blkr.

Cheilodipterus apogonoides $B l k r .275,30$.

Cheilodipterus arabicus $C . V$. cf. Paramia lineata $B l k r$.

Cheilodipterus butis Ham. Buch. cf. Butis butis Blkr.

Cheilodipterus culius $H$. B. cf. Culius fuscus $B l k r$., Eleotris nigra $Q . G$.

Cheilodipterus heptacanthus Lac. cf. Cheilodipterus saltator Blkr., 'emnodon saltator $C . V$.

Cheilodipterus heptazona Blkr. 2, 526. 13, 29. cf. Paramia macrodon Blkr. 
Cheilodipterus lineatus Lac. 234, 352. 275, 30. 287, 47. cf. Paramia macrodon Blkr.

Cheilodipterus lineatus Gthr. cf. Paramia lineata Blkr.

Cheilodipterus macrodon Lac. cf. Paramia macrodon Blkr.

Cheilodipterus octovittatus $C . V .13,29.157,23$ r. 263, 453. 296, 107. cf. Paramia macrodon Blkr.

Cheilodipterus polystigma Cast. 263, 454. cf. Pseudamia polystigma Blkr.

Cheilodipterus quinquelineatus $C . V .54,252.156,2$ I2. 157, 23 I. 158, 273. 167, 370. 184, I99. 185, 222. 188, 458. 270, I6. 275, 30. 287, 48. 296, 107. 309, I55. cf. Paramia quinquelineata $B l k r$.

Cheilodipterus saltator Blkr. 364, 289. 459, Iо0.

Cheilodipterus singapurensis Cast. 263, 452. cf. Paramia singapurensis Blkr.

Cheilonemus Baird 261, 437. 314, 2 I r. 329, 264.

Cheilotrema Tschudi 468, 324.

Chela H. B. 1, 552. 261, 439. 314, 2 I 5. 329, 264.

Chela anomalura Blkr. 270, 54 .

Chela anomalurus Blkr. 272, 24.

Chela Castelnaui Blkr. 356, х76.

Chela hypophthalmus Blkr. 270, 54.

Chela melanopus Blkr. 416, II9, I2I. 425, I46.

Chela oxygastroides Blkr. 270, 54. 271, 2. 272, 24.

Chela scalpella Blkr. 335, 3 .

Chelonodon Dumerili Blkr. 347, 37. 356. I72.

Chelonodon kappa Blkr., Russ. 346, 3I. 354, I43. 358, I83. cf. Leiodon patoca Blkr.

Chelmo Gthr. 468, 303 .

Chelmo aculeatus Poey cf. Prognathodus aculeatus Poey.

Chelmo longirostris C. $V$. , Gthr. 459, 95. cf. Prognathodus longirostris $B l k r .$, Chelmon longirostris $C . V$.

Chelmo pelta Gthr. 468, 303. cf. Prognathodus aculeatus Poey.

Chelmo pulcher Steind. cf. Tetragonoptrus (Chaetodontops) pulcher Blkr.

Chelmo rostratus C. V., Gthr. 459, 95. cf. Chelmon rostratus Cuv.

Chelmo truncatus Gthr. 468, 304. cf. Chelmonops truncatus Blkr.

Chelmon Cuv. 1, 553. 468, $3 \circ 3$.

Chelmon longirostris $C . V .22,20.47,227.340,365.358$, 190. 364, 287. 389, 273. cf. Prognathodus longirostris Blkr.

Chelmon marginalis Rich. 119, 9. cf. Chelmon rostratus Cuv.

Chelmon rostratus $C . V .2,520,22,20.50,47$ I. 51, 56. 56, 445 . 126, 344. 158, 274. 185, 223. 227, 407. 270, 26. 275, 35. 285, 245. 346, 31. 395, 292. 468, 303. 474, 3Г5. 481, 30. 495, r4. cf. Prognathodus longirostris $B l k r$.

Chelmonini 468, 303.

Chelmonops Blkr. 468, 304.

Chelmonops truncatus Blkr. 468, 304. 474, 3 I5. 
Cheonda Gir. 261, 436. 314, 2 12. 329, 264.

Chilodactylus Gthr. 468, 3 I5.

Chilodactylus zonatus C. $V .425$, I 40.

Chilomycterus Bibr. 367, г7.

Chilomycterus orbicularis Gthr. 425, I2I. cf. Diodon orbicularis Bl.

Chilomycterus reticulatus Bibr., Gthr. 368, 33. cf. Diodon reticulatus Blkr.

Chilomycterus tigrinus Gthr. cf. Diodon tigrinus Cuv.

Chiloscyllium Freycineti Blkr. 396, 296.

Chiloscyllium Hasseltii Blkr. 64, 19. 270, 66. 287, 36.

Chiloscyllium malayanum M. H. 296, то2. 364, 270. 396, 296.

Chiloscyllium margaritiferum Blkr. 316, 243. 425, Irg.

Chiloscyllium ocellatum M. H. 119, I7.

Chiloscyllium ornatum Blkr. 307, I36. 425, i I9.

Chiloscyllium phymatodes Blkr.64, 21. 235, 374. 287, 36.

Chiloscyllium plagiosum M. H. 64, I7. 91, 80. 156, 218. 266, 57. 270, 66. 275, 9. 287, 36. 288, 66. 425, I I9. cf. Chiloscyllium Freycineti Blkr., Chiloscyllium ornatum Blkr.

Chiloscyllium punctatum M.H. 10, 68. 46, 2 I4. 50, 473. 64, 22. $271,2.275,9.276,65.287,36.395,290$.

Chiloscyllium trispeculare 119 , I7.

Chiloscyllium tuberculatum M. H. 64, 20. 266, 57. 287, 36. 395, 290. 425 , II 9 .

Chimaera antarctica Cuv. cf. Callorhynchus antarcticus Cuv.

Chimaera australis Shaw cf. Callorhynchus antarcticus Cu\%.

Chimaera callorhynchus $L$. cf. Callorhynchus antarcticus Cuv.

Chimaera monstrosa L. 54, 309. 273, 4. 358, I83. 364, 271. 497, 4.

Chirocentrus dentex 2, 5 10.

Chirocentrus dorab C. $V$. 10, 67. 16, 6. 25, I1. 46, 2 14, 50, 472. $51,59.56,445.59$, 10. 91, 72. 178, 2. 227, 408. 270, 56. 272, 25. $275,48.288,79.354$, I 50. $364,295.395,294.396,300$. 425, I 48.459, 102. $491,55.495,22.497,25$.

Chirocentrus hypselosoma Blkr. 51, 71. 55, 414. 59, 25. 91, 72. 191, 2. 267, I40. $272,25.275,48.308$, I 52. $316,243$.

Chirodactylus Gill 468, 3 I5.

Chiroidei 449, 370.

Chironectes biocellatus Cuv. cf. Antennarius biocellatus Blkr.

Chironectes caudimaculatus Rich. cf. Antennarius urophthalmus Blkr.

Chironectes coccineus Less. Garm. cf. Antennarius coccineus Gthr.

Chironectes Commersonii C. $V$. cf. Antennarius Commersonii Cant.

Chironectes gibbus De Kiay cf. Antennarius laevigatus Blkr.

Chironectes hirsutus $C$. $V$. cf. Brachionichthys hirsutus Blkr.

Chironectes hispidus $C . V$. cf. Antennarius hispidus $C$ ant.

Chironectes laevigatus Cuv. cf. Antennarius laevigatus Blkr.

Chironectes leprosus Eyrd. Soul. cf. Antennarius leprosus Blkr. 
Chironectes lophotes Cuv. cf. Antennarius hispidus Cant.

Chironectes marmoratus $C$. cf. Antennarius marmoratus Gïnth., Antennarius raninus Cant.

Chironectes multiocellatus C. $V$. cf. Antennarius multiocellatus Gthr.

Chironectes nesogallicus C. $V$. cf. Antennarius marmoratus Gthr., Antennarius nesogallicus Cuv.

Chironectes nummifer Cuv. cf. Antennarius nummifer Blkr.

Chironectes pardalis C. $V .68,644$.

Chironectes pictus $C$. $V$. cf. Antennarius laevigatus $B l k r$.

Chironectes pictus var, vittatus Rich. cf. Antennarius marmoratus $G t h r$.

Chironectes punctatus Cuv. cf. Brachionichthys hirsutus Blkr.

Chironectes raninus Rich. cf. Antennarius raninus Cant.

Chironectes scaber Cuv. cf. Antennarius histrio Gthr.

Chironectes tridens T. Schl. cf. Antennarius tridens Blkr., Gthr.

Chironectes tuberosus Cur. cf. Antennarius bigibbus Gthr., Antennarius tuberosus Blkr.

Chironectes variegatus $C$. $V$. cf. Antennarius chironectes Comm.

Chironemus C. $V .468,315$.

Chironemus georgianus $C . V .468,3$ I 5 .

Chironemus marmoratus Gthr. 331, 447.

Chirus agrammus Rich. cf. Agrammus Schlegelii Giunth.

Chirus hexagrammus Stell., C. $V$. 118, So. 408, 238. 497, 1 I.

Chirus lagocephalus Pall. cf. Grammatopleurus lagocephalus Gill.

Chirus octogrammus Ginth. cf. Octogrammus Pallasi Blkr.

Chlorichthys pavo $\mathrm{Bp}$. cf. Julis pavo $\mathrm{Val}$.

Chloroscombrus caribbaeus Gir. cf. Micropteryx chrysurus Gthr.

Chloroscombrus cosmopolita Gir. cf. Micropteryx chrysurus Gthr.

Choerodon 2, 5 r 3 .

Choeroichthys brachysoma Blkr., A. Dum. 270, 72. cf. Doryichthys Valenciennii Gthr.

Choeroichthys Valenciennei $K p$. cf. Doryichthys brachysoma Blkr., Doryichthys Valenciennii Gthr.

Choerops Rüpp. 292, го6.

Choerops anchorago Pet. 497, 5.

Choerops cyanostolus Blkr. 425, I35.

Choerops dodecacanthus Blkr. 385, 275. 459, 4, 8I.

Choerops japonicus Gthr., Blkr. 425, I35. 497, 5 .

Choerops leucozona Blkr. 293, 297.

Choerops macrodon Blkr. 293, 297. 296, 104. 315, 231. 320, 267. 358, I85. 395, 29I. cf. Choerops anchorago Pet.

Choerops meleagrides Rupp. cf. Labrus macrodontus Lac.

Choerops oligacanthus Blkr. 293, 297. 395, $29 \mathrm{I}$.

Choerops robustus Blkr. 459, 8I. 495, 9.

Choerops Schoenleini Blkr. 293, 297. 296, I04. 425, I35.

Choerops zosterophorus Blkr. 385, 273. 
Chondrochilus Heck. 314, I97.

Chondrorhynchus Heck. 314, 197.

Chondrostoma $A g .261,427.314,197$.

Chondrostoma boggut Sykes 91, 70.

Chondrostoma Duvaucelii C. $V .91,70$.

Chondrostoma fulungee Sykes 91, 70.

Chondrostoma gangeticum C. V. 91, 70.

Chondrostoma kaurus Sykes 91, 70.

Chondrostoma mullya Sykes 91, 70.

Chondrostoma semivelatus $C . V .91,70$.

Chondrostoma wattanah Sykes $91,70$.

Chondrostomus Heck. 314, I97.

Chonephorus Poey 453, 320.

Chonerhinos modestus Blkr. 270, 65. 272, 7 .

Chonerhinos naritus Blkr. 191, 2. 226, 386. 270, 65. 272, S. 288, 68. 496,4 .

Chonerhinus Blkr. 367, I9.

Chonerhinus modestus Blkr. 368, 38 .

Chonerhinus naritus Blkr. 356, I 72. 368, 38. 425, I2 I.

Choridactylus Rich. 473, 298.

Choridactylus multibarbis Rich. 473, 298. cf. Chorismodactylus multibarbus Rich.

Chorinemus aculeatus $C: V .10,67,68.46,2$ I3. 91, 42. 275. 38.

Chorinemus Commersonianus $C . V .25,8.32,160.51,57.55,412$. 56, 445. 57, 44. 91, 42. 119, I0. 191, 2. cf. Scomberoïdes Commersonianus Blkr., Lac.

Chorinemus Commersonii C. V. 2, 5 I6, 16, 4 .

Chorinemus delicatulus Rich. cf. Scomberoides delicatulus Blkr.

Chorinemus Farkharii $C$. $V$. cf. Chorinemus Commersonianus $C$. $V$.

Chorinemus Forsteri Rich. cf. Scomberoides lysan Blk\%.

Chorinemus goriënsis C. V. 297, I34.

Chorinemus leucophthalmus Rich. cf. Scomberoides leucophthalmus Blkr.

Chorinemus lysan $C . V .270,28.272$, I3. 275, 38. 287, 52. 288, 74 . cf. Chorinemus Commersonianus $C . V$.

Chorinemus mauritianus $C . V .10,67,68$. cf. Chorinemus sancti Petri C. $V$., Scomberoides sancti Petri $C$. V., Blkr.

Chorinemus moadetta $C . V .2,5$ 16. cf. Chorinemus sancti Petri $C . V$, Scomberoides sancti Petri C. V., Blkr.

Chorinemus orientalis $T$. Schl. 90, 36. cf. Scomberoides orientalis Blkr., tol Blkr.

Chorinemus sancti Petri C. V. 2, 516. 25, 8. 46, 212.50, 47I. 51, 57. 54, 237. 57, 45. $91,44.147,469.227,407.231,146.235$, 366. 270, 28. 275, 38. 296, 109. 309, г56. cf. Scomberoides sancti Petri Blkr., C. $V$. 
Chorinemus tala C. V. 91, 44. cf. Scomberoides tala Blkr.

Chorinemus tol $C . V$. 51, 57. 54, 235. 56, 445. 57, 43. 90, 36. 91, 44. 127, 345. 156, 214. 227, 407. 237, 437. 270, 28. 275, 38. 288, 74. 296, 109. cf. Scomberoides tol Blkr.

Chorinemus toloo C. $V .2,516.57,45.91,44.127,345.156,2$ r 4. 267, I40. 270, 28. 275, 38. cf. Scomberoides toloo Blkr.

Chorismodactylus Gthr. 473, 298.

Chorismodactylus multibarbus Rich. 425, I4I.

Chorististium Gill 468, 262.

Chromichthys Guich. 302, 38 .

Chromis amboinensis Blkr. 485, I62.

Chromis analis Blkr. 483, 4I. 485, I 56.

Chromis axillaris Blkr. 459, 84. 495, I 5 .

Chromis cinctus Blkr. 459, 84 .

Chromis cinerascens Blkr. 459, 84. 483, 4I. 485, I 55.

Chromis insulindicus Blkr. 476, 402. 485, I 54.

Chromis lepidolepis Blkr. 476, 401. 485, I63.

Chromis lepisurus Blkr. 483, 4I. 485, I65. 491, 47.

Chromis madagascariensis Guich. cf. Tilapia madagascariensis Blkr.

Chromis mossambicus Pet. cf. Tilapia mossambica Blkr.

Chromis niloticus Playf. cf. Tilapia nilotica Blkr.

Chromis notatus Blkr. 497, Io.

Chromis ternatensis Blkr. 485, I6I. 491, 48 .

Chromis xanthochir Blkr. 485, $15^{S}$.

Chromis xanthuris Blkr. 485, 159.

Chrosomus Raf. 261, 434. 314, 209.

Chrysichthys Blkr. 306, 95 .

Chrysichthys auratus Blkr. 306, 95 .

Chrysiptera azurea Szons. cf. Glyphidodontops cyaneus Blkr.

Chrysiptera Gaimardi Szens. cf. Glyphidodontops uniocellatus Blkr.

Chrysoblephus Sions. 468, $2 S_{1}$.

Chrysoblephus gibbiceps Stons. cf. Chrysophrys gibbiceps $C . V$.

Chrysophekadion Blkr. 261, 424. 314, I95.

Chrysophekadion polyporos Blkr. 239, I02. 270, 5 I.

Chrysophrys Cuv. 1, 553. 468, 281 .

Chrysophrys aries T. Schl. 118, 87. cf. Sparus aries Blkr.

Chrysophrys auripes Rich. cf. Sparus datnia Blkr., Sparus hasta Bl. Schn.

Chrysophrys berda C. $V$., Rich. 2, 522. 16, 4. 91, 38. cf. Sparus hasta Bl. Schn.

Chrysophrys berda Blkr. cf. Chrysophrys calamara $C . V$.

Chrysophrys berda Day cf. Sparus Schlegeli Blkr.

Chrysophrys berda var. calamara Day cf. Sparus datnia Blkr.

Chrysophrys bifasciata $C$. $V$. cf. Sparus bifasciatus Blkr.

Chrysophrys calamara $C . V .9,633.19$, Iо. 25, 8. 50, 47 I. 91, 38. 
270, 22. 275, 28. 284, 239. 287, 47. 288, 71. 395, 291. cf. Sparus calamara Blkr., Sparus hasta Bl. Schn。

Chrysophrys cardinalis $C . V .90,3 \mathrm{r}$. cf. Pagrus cardinalis Gthr., Sparus cardinalis Blkr.

Chrysophrys chrysargya $C . V .91,38$. cf. Sparus sarba Forsk.

Chrysophrys coracinus $C$. $V$. cf. Sparus coracinus Blkr.

Chrysophrys cristiceps $C . V .266,62$.

Chrysophrys Cuvieri Day cf. Sparus Cuvieri Blkr., Sparus Schlegeli Blkr.

Chrysophrys gibbiceps $C . V .266,62$.

Chrysophrys globiceps $C . V .266,62$.

Chrysophrys hasta Gthr. cf. Sparus datnia Blk\%., Sparus hasta Bl. Schn.

Chrysophrys laticeps $C . V .266,52$. cf. Sparus laticeps $B l k r$.

Chrysophrys longispinis $C . V .$, Rich. 91, 93. cf. Chrysophrys Schlegelii Blkr., Sparus datnia Blkr., Sparus hasta Bl. Schn., Sparus Schlegeli Blkr.

Chrysophrys madagascariensis $C$. $V$. cf. Sparus madagascariensis Blkr.

Chrysophrys major Schl. cf. Pagrus major Gthr., Sparus major Blkr.

Chrysophrys sarba $C . V .91,38$. cf. Sparus sarba Forsk.

Chrysophrys Schlegelii Blkr. 102, 400. 118, 86. cf. Sparus datnia Blkr., Sparus Schlegeli Blkr.

Chrysophrys tumifrons Schl. cf. Sparus tumifrons Blkr.

Chrysophrys unicolor $Q . G$. cf. Pagrus unicolor $C . V$.

Chrysophrys xanthopoda Rich. cf. Sparus datnia Blkr.. Sparus hasta Bl., Sclun.

Chrysostomus luna Lac. cf. Lampris luna Risso.

Chyloscyllium malayanum $M$. H. 25, I2.

Chyloscyllium plagiosum $M$. H. 25, I2.

Chyloscyllium tuberculatum M. H. 25, I 2 .

Cibotion punctatus $K p$. cf. Ostracion punctatus Lac.

Cichla Bl. Schn. 468, 336. Sec also Ciechle,

Cichla argyrea $B l$. Schn. cf. Diapterus argyreus Blkr.

Cichla erythrurus Bl. Schn. cf. Diapterus oyena Blkr.

Cichla temensis Humb. 436, 32, 33 .

Cichla tucunare Heck. cf. Cichla temensis Humb.

Cichlops Mïll. Trosch. 468, 320.

Cichlops cyclophthalmus $M . T r .71,766.465,8.468,320.497$, 10.

Cichlops Hellmuthii Blkr. 99, 329. 316, 24 I. 318, 25 I. 396, 299. 465, I2.

Cichlops japonicus Gill cf. Cichlops cyclophthalmus $M$. T'r.

Cichlops melanotaenia Blkr. 71, 765. 158, 274. 167, 372. 185, 224. 275,2 г. 465,7 .

Cichlops spilopterus Blkr. 81, 168. 167, 372. 185, 224. 275, 21. 296, I05. $396,299.465$, I I.

Cichlops trispilos Blk\%. 132, т1о. 296, то5. 309, т 55. 315, 23 I.

Cichlops trispilus Blk\%. 388, 272. 465, Iо.

Cichlopsini 468, 320 . 
Cirrhibarbus capensis $C . V . \mathbf{2 6 6}, 55$.

Cirrhilabrus Schl. 292, 95.

Cirrhilabrus cyanopleura Blkr. 293, 276.

Cirrhilabrus heterodon Blkr. 414, 326.

Cirrhilabrus solorensis Blkr. 80, 88. 293, 277. 317, 246. 318, 250. 358, I84. 364, $276.369,42.394,286.491,40$.

Cirrhilabrus Temminckii Schl. $497,4$.

Cirrhimens Gill 468, 326.

Cirrhimuraena $K p .351$, II9.

Cirrhimuraena chelopogon Blkr. 352, i26.

Cirrhimuraena chinensis $K p .425$, I 24.

Cirrhimuraena polyodon Blkr. 352, I 26.

Cirrhimuraena tapeinopterus Blkr. 313, I83. 352, I26.

Cirrhina Cuv. 261, 434. 314, I97. 329, 262.

Cirrhina $V a l$. (nec Cuv.) 314, 202. 329, 263.

Cirrhina bengalensis Blkr. 91, 136 .

Cirrhina Blochii C. V. 91, 64.

Cirrhina brachypterus $C . V .91,64$.

Cirrhina Burnesiana Blkr. 91, 64 .

Cirrhina dero $C . V .91,64$.

Cirrhina Dussumieri C. V. 91, 64, 136.

Cirrhina gohama C. V. 91, 64.

Cirrhina latius $C . V .91,64$.

Cirrhina micropogon $V a l$. cf. Rohita Belangeri $C . V$.

Cirrhina mrigala $C . V .91,64$.

Cirrhina reba $C . V .91,64,136$.

Cirrhina rubripinnis $C . V .91,64$.

Cirrhina sinensis Gthr. cf. Mrigala sinensis Blkr.

Cirrhinichthys Blkr. 314, 202. 329, 263.

Cirrhinus calbasu $\mathrm{MC} C$. cf. Rohita calbosu $\mathrm{MC} \mathrm{Cl}$.

Cirrhinus rohita $M C C l$. cf. Rohita Buchanani $C$. $V$.

Cirrhiptera $K . v . H .468,322$.

Cirrhitei 468, 3 I 3 .

Cirrhiteiformes 468,3 I 3 .

Cirrhiteini 468, 3 I 3 .

Cirrhiteoidei 468, 3 I 3 .

Cirrhites Comm., Lac. 1, 553. 163, 39. 468, $3^{\top} 3,33^{6}$.

Cirrhites amblycephalus Blkr. 167, 378. cf. Paracirrhites amblycephalus Blkr.

Cirrhites aprinus C. V. 13, 4. 320, 269.468, 314. cf. Cirrhitichthys aprinus Blkr.

Cirrhites arcatus $C . V .143,4$ I. 266, 52. 275, 28. 315, 233. cf. Paracirrhites arcatus Blkr.

Cirrhites aureus Schl. cf. Cirrhitichthys aureus Gthr., Blkr.

Cirrhites cinctus Gthr. cf. Paracirrhites cinctus Blkr. 
Cirrhites fasciatus $C . V .91,32.468,3$ I 4 .

Cirrhites fasciatus Bcnn. (nec $C . V$.) cf. Paracirrhites cinctus Blkr.

Cirrhites Forsteri Gthr. 364, 283. 468, 3I4. cf. Amblycirrhites, Paracirrhites Forsteri Blkr.

Cirrhites graphidopterus Blkr. 72, I06. cf. Cirrhitichthys aprinus Blkr.

Cirrhites maculatus Lac. 468, 313. cf. Cirrhites marmoratus Gill, Cirrhitichthys marmoratus Blkr.

Cirrhites maculosus Benn. cf. Cirrhites marmoratus Blkr.

Cirrhites marmoratus Gill, Blkr. 459, 93. 463, 3. 495, 13.

Cirrhites oxycephalus Blkr. 128, 408. cf. Cirrhitichthys oxycephalus Blkr.

Cirrhites pantherinus $C . V .47,232.91,32$. 167, 370. 236, 330. 275, 28. cf. Paracirrhites Forsteri Blkr.

Cirrhites punctatus $C . V .377$, I76. 459, 38, 93. 495, I3.

Cirrhites vittatus $V a l$. cf. Paracirrhites arcatus Blkr.

Cirrhitus Gill 468, 3 I3.

Cirrhitus maculosus Rïpp. cf. Cirrhites marmoratus Gill, Chirrhitichthys marmoratus Blkr.

Cirrhitus marmoratus Gill 468, 3 I 3 .

Cirrhitichthys Blkr. 163. 39. 468, 3 I 4 .

Cirrhitichthys aprinus $B l k r .459,93.463$, I2. 468, 314.

Cirrhitichthys aureus Gthr., Blkr. 425, r40. 497, 9.

Cirrhitichthys graphidopterus Blkr. 275, 28. 318, 251. 354, 147. 358, I88. 364, 283. 468, 3I4. cf. Cirrhitichthys aprinus Blkr.

Cirrhitichthys maculatus Gthr. cf. Cirrhites marmoratus Blkr., Gill.

Cirrhitichthys marmoratus Blkr.270, 76. 364, 283. cf. Cirrhites marmoratus Gill.

Cirrhitichthys oxycephalus Blkr. 184, 299. 354, 147. 364, 283.459, 93. 463, 1 $5.491,43$.

Cirrhitichthys oxyrhynchos Blkr. 184, 205.

Cirrhitichthys oxyrhynchus Blkr. 463, 13.

Cirrhitichthys polyactis Blkr. 463, I6.

Cirrhitopsis Gill 468, 314.

Cirriptera corallicola v. Hass. cf. Plesiops nigricans Rüpp.

Citharichthys Blkr. 294, 427 .

Citharichthys guatimalensis Blkr. 332, $45^{2}$.

Citharichthys guianensis Blkr. 332, 453.

Citharichthys sordidus Gthr. 332, 455.

Citharichthys spilopterus Gthr. 332, 455.

Citharoedus $K p .468,305$.

Citharoedus austriacus $K p$. cf. 'Tetragonoptrus (Rabdophorus) trifasciatus Blkr.

Citharoedus collaris $K$ au $p$ cf. 'Tetragonoptrus (Chaetodontops) collaris Blkr.

Citharoedus luctuosus $K p$. cf. 'Tetragonoptrus (Rabdophorus) luctuosus Blkr. 
Citharoedus melastomus Kaup ef. Tetragonoptrus (Lepidochaetodon) Kleini Blkr.

Citharoedus Mertensii Kaup cf. Tetragonoptrus (Linophora) Mertensi Blkr.

Citharoedus Meyeri $K p .468,305$. cf. Tetragonoptrus (Citharoedus) Meyeri Blkr.

Citharoedus octofasciatus $K p$. cf. Tetragonoptrus (Tetragonoptrus) octofasciatus Blkr.

Citharoedus ornatissimus $K p$. cf. Tetragonoptrus (Citharoedus) ornatissimus Blkr.

Citharoedus punctato-fasciatus $K p$. cf. Tetragonoptrus (Tetragonoptrus) punctato-fasciatus $B l k r$.

Citharoedus. speculum $K p$. cf. Tetragonoptrus (Rabdophorus) speculum Blkr.

Citharoedus tau-nigrum $K p$. cf. Tetragonoptrus (Rhabdophorus) taunigrum Blkr.

Citharoedus unimaculatus $K \not$. cf. Tetragonoptrus (Lepidochaetodon) unimaculatus Blkr.

Citharoedus vittatus $K p$. cf. Tetragonoptrus (Rabdophorus) trifasciatus Blkr.

Citharus Rond. 294, 424.

Cithiarus macrolepidotus 294, 424.

Citula armata $C$. $V$. , Rüpp., Blkr. 316, 242. 356, I74. 358, 19т. 364, 290. 425, I3 I. 459, 99. 491, 50. 497, 14. cf. Carangoïdes citula Blkr.

Citula atropus Blkr. 356, I74. 425, г32.

Citula bajad Blkr.459, 99 .

Citula chrysophrys Blkr. 425, I32. 459, 99.

Citula ciliaria Rüpp. cf. Carangoïdes citula Blkr.

Citula ciliaris Rüpp., Blkr. 315, 235. 320, 270. 358, I91. 364, 290. 425, 132. 497, I4.

Citula coeruleopinnata Blkr. 459, 99.

Citula equula Blkr. 497, 14.

Citula gallus Blkr. 316, 242. 356, I 74. 358, т91. 364, 290. 395, 293. $396,300.425,132.459,99$.

Citula gymnostethus Blkr. 459, 99.

Citula hemigymnostethus Blkr. 315, 235.

Citula malabarica Blkr. 356, I74. 364, 290. 425, I32. 459, 99.

Citula nigripes $C$. $V$. cf. Carangoïdes atropus Blkr.

Citula ophthalmotaenia Blkr. 338, 360. 364, 290.

Citula plagoitaenia Blkr. 364, 290.

Citula praeusta Blkr. 395, 293.

Clara Gill 468, 285 .

Clarias Gron. 2, 5 Ir. 306, I 20.

Clarias abbreviatus $\mathrm{Val}$. 425, I24.

Clarias batrachus C. $V .91,58.185,226.200,48.219,302.225$, 
357. 231, I 49. 239, I02. 255, 24I. 270, 49. 272, 20. 279, ІоІ. 287, 59. 288, 78. 356, I 75. 425, I 24.

Clarias capensis $C . V .266,55$.

Clarias Dussumieri C. $V .91,58$.

Clarias fuscus $C . V$. , Gthr. 3, 136. 5, 2. 270, 49. 425, 125.

Clarias hexacicinnus Rich. 425, I 25.

Clarias jagur $C . V .91,58$.

Clarias leiacanthus Blkr. 49, 430. 55, 413. 156, 217. 191, 2. 235, 37 1. 270, 49. 271, 2. 272, 20. 356, I 75.

Clarias magur C. V. 91, I 24. 496, 3.

Clarias marpus $C . V .91,58.306$, I 20.

Clarias meladerma $B l k r .3,178.4,288.5,44$.

Clarias melanoderma Blkr. 190, I. 235, 371. 270, 49. 272, 20.

Clarias melanosoma Blkr. 190, I.

Clarias melasoma Blkr. 55, 427 .

Clarias Nieuhofii C.V. 3, т78. 4, 288. 5, 44. 185, 226. 235, 37 . 270, 49. 272, 20.

Clarias pentapterus Blkr. 45, 206. 49, 420. 55, 4 I3.

Clarias pulicaris Rich. cf. Clarias fuscus Gthr.

Clarias punctatus $C . V .3,177.4,288.5,43.7,7.16,5.25,11.26$, 4. $35,262.42,59.45$, 196. 49, 420. 52, 89. 55, 413. 170, 476, 477 .

Clarias Teysmanni Blkr. 166, 344. 170, 477. 272, 20.

Clarotes Kner 306, 83 .

Clarotes Heuglini Kncr 306, 83 .

Clepticus Cuv. 292, I89.

Clidoderma Blkr. 294, 425 .

Clidoderma asperrimum Blkr. 294, 425. 497, 2 I.

Clinostomus Gir. 261, 436. 314, 213. 329, 264.

Clinus acuminatus $C . V .266,54$.

Clinus anguillaris $C . V .266,7 \mathrm{I}$.

Clinus brachycephalus $C . V$. 266, 54 .

Clinus cottoides $C . V .266,54$.

Clinus despicillatus Rich. 119, I2.

Clinus dorsalis Castcln. 266, 72 .

Clinus Dubuis Castcln. 266, 7 I.

Clinus heterodon C. V. 266, 54 .

Clinus latipennis $C . V .266,54$.

Clinus littoreus $C . V .119,12$.

Clinus nematopterus Gthr. 425, I29.

Clinus perspicillatus $C . V .119, \mathbf{1} 2$.

Clinus polyactocephalus brev. 497, 20.

Clinus superciliosus $C . V$. 266, 70.

Clinus xanthosoma Blkr. 166, 340. 170, $47 \mathrm{~S}$.

Cliola Gir. 261, 426. 314, 207.

Clupalosa bulan Blkr. 16, I 2. 25, I I. 60, 30. 
Clupanodon africanus Lac. cf. Pellona africana Blkr.

Clupanodon chacunda Ham. Buch. cf. Chatoessus chacunda $C$. V.

Clupanodon chanpole Ham. Buch. 9, 638.

Clupanodon chinensis Lac. cf. Alosa ilisha Blkr.

Clupanodon ilisha Ham. Buch. Alausa ilisha Blkr.

Clupanodon thrissa Lac. cf. Clupea (Clupanodon) thrissa Blkr.

Clupea 1, 552. 10, 70.

Clupea aftinis $G r$. cf. Pellona Grayana $C . V$.

Clupea africana $B /$. cf. Pellona africana $B l k r$.

Clupea (Harengula) arabica Blkr. 459, Iо2.

Clupea argyrotaenia Blkr. cf. Spratelloïdes argyrotaenia Blkr.

Clupea argyrotaeniata Blkr. 10,72. cf. Spratelloïles argyrotaenia Blkr.

Clupea (Harengula) atricauda 459, 102.

Clupea coeruleovittata Rich. cf. Clupea (Amblygaster) melanosticta Blkr.

Clupea cyprinoides Brouss. cf. Megalops cyprinoides Blkr.

Clupea cyprinoides Lac. cf. Megalops filamentosus Lac.

Clupea cyprinoides $B l$. cf. Megalops giganteus $B l k r$.

Clupea cyprinoïdes Forst. cf. Megalops indicus $C$. $V$.

Clupea delicatula Benn. cf. Spratelloides delicatulus Gthr.

Clupea dentex Bl. Schn. cf. Chirocentrus dorab Cuv.

Clupea dorab Forsk. cf. Chirocentrus dorab Cur.

Clupea fasciata Lac. cf. Equula filigera $C . V$.

Clupea (Harengula) fasciata Blkr. 459, Iо2.

Clupea (Harengula) fimbriata Blkr. 496, 3 .

Clupea flosmaris Rich. 425, I 48 .

Clupea gibbosa Blkr. 10, 72. cf. Spratella tembang Blkr.

Clupea gigantea Shazo cf. Megalops giganteus Blkr.

Clupea gracilis T. Schl. 71, 775. cf. Spratelloides gracilis Blkr.

Clupea haumela Forsk. cf. Trichiurus haumela $C . V$.

Clupea indica $G r$. cf. Alausa microlepis $C$. $V$.

Clupea inermis Basil. 425, I48.

Clupea isingleena Rich. cf. Clupea (Harengula) kowala Blkr. 425, 147.

Clupea (Harengula) Jussieui Blkr. 459, го3. 495, 22.

Clupea kowal Gthr. cf. Clupea (Harengula) kowala Blkr.

Clupea kowal T. Schl. cf. Harengula zunasi Blkr.

Clupea (Harengula) kowala Blkr. 425, I47.

Clupea (Amblygaster) leiogaster Blkr. 425, r47.

Clupea libertatis Gthr. cf. Clupea (Clupanodon) libertatis Blkr.

Clupea (Clupanodon) libertatis Blkr. 425, I47.

Clupea macassariensis Blkr. 10, 72: cf. Clupeoïdes macassariensis Blkr.

Clupea mauritiana Benn. 459, 102. 495, 22.

Clupea melanosticta Schl. cf. Clupea (Harengula) melanosticta Blkr.

Clupea melanosticta T.Schl. cf. Harengula punctata $C$. $V$.

Clupea (Amblygaster) melanosticta Blkr. 425, I 47.

Clupea (Harengula) melanosticta Blkr. 497, 25 . 
Clupea (Harengula) melanurus Blkr. 491, 55 .

Clupea melastoma Schn. 2, 509.

Clupea melastoma Schl. cf. Ilisha elongata Blkr.

Clupea melastoma Blkr. cf. Pellona Grayana $C$. $V$.

Clupea melastoma T. Schl. cf. Pellona Schlegelii Blkr.

Clupea micropus T. Schl. cf. Etrumeus micropus Blkr.

Clupea mystax Bl. Schn. cf. Engraulis mystacoüdes Blkr.

Clupea nasus Bl. Schn., cf. Chatoessus nasus $C$. $V$.

Clupea nymphaea Rich. cf. Clupea (Harengula) nymphaea Blkr.

Clupea (Harengula) nymphaea Blkr. 425, I47.

Clupea (Harengula) obtusirostris Blkr. 459, го3.

Clupea ocellata Papple 266, 56.

Clupea perforata Gthr. cf. Clupea (Harengula) perforata Blkr.

Clupea (Harengula) perforata Blkr. 425, 147.

Clupea quadrimaculata Rüpp. cf. Clupea (Harengula) arabica Blkr.

Clupea Reevesii Gthr. cf. Alosa Reevesii Blkr.

Clupea scombrina Gthr. cf. Alosa scombrina $C . V$.

Clupea setipinna Forst. cf. Megalops indicus $C$. $V$.

Clupea setirostris Brouss. cf. Engraulis setirostris $C . V$.

Cluper (Harengula) spilurus Blkr. 459, ro3.

Clupea synura Bl. Schn. cf. Notopterus kapirat Lac.

Clupea telara Ham. Buch. 16, I3. cf. Engraulis telara $C . V$.

Clupea (Clupanodon) thrissa Blkr.425, I48.

Clupea thrissoides Bl. Schn. cf. Megalops cyprinoides, giganteus Blkr.

Clupea thryssa Russ. cf. Chatoessus nasus $C$. $V$.

Clupea triza L. cf. Dorosoma triza Blkr.

Clupea (Harengula) venenosa $B l k r .459$, го3.

Clupea vittargentea Lac. cf. Engraulis Brownii C. $V$.

Clupea (Harengula) zunasi Blkr. 497, 25.

Clupeichthys goniognathus Blkr. 136, 275. 270, 56.

Clupeoidei 10, 70.

Clupeoïdes bornëensis Blkr. 35, 275. 42, 60. 45, 196, 49, 421. 55, 4I 4. 60, I7. 272, 25.

Clupeoïdes hypselosoma Blkr. 381, 293.

Clupeoïdes macassariensis Blkr.46, 214. 60, I7.71, 772. 147, 469 . 235, 372. 275, 48. 287, 6r. 309, I 56. 315, 236. 338, 36r. 364, 295.

Clupeonia Blochii C. $V .91,74$.

Clupeonia Commersonii C. $V .91,74$. cf. Clupea (Harengula) atricauda.

Clupeonia fasciata $C$. $V$. cf. Clupea (Harengula) fasciata Blkr.

Clupeonia Jussieui $C$. $V$. cf. Clupea (Harengula) Jussieui Blkr.

Clupeonia perforata Cant. 51, 59. 270, 57. 288, 79.

Clupisoma Srons. 299, 393. 306, I I 3 .

Clupisoma garua Blkr. 306, I 4 .

Cnidon Müll. Trosch. 468, 264.

Cobitichthys Blkr. 220, 304. 261, 422. 
Cobitichthys, anguillicaudatus Blkr. cf. Misgurnus anguillicaudatus Blkr.

Cobitichthys barbatuloides Blkr. 272, 2 I:

Cobitichthys dichachrous Blkr. 269, 89. cf. Misgurnus dichachrous Gthr.

Cobitichthys enalios Blkr. 269, 88. cf. Misgurnus enalios Blkr.

Cobitichthys polynema Blkr. 269, 90. cf. Misgurnus polynema Gthr.

Cobitis Art., Blkr. 220, 303. 261, 422. 323, 37.

Cobitis aculeata $V a l .91,70.323,4 \mathrm{I}$.

Cobitis amnicola $\mathrm{Val} .91,70.323,4 \mathrm{I}$.

Cobitis anguillicaudata Cant., $M c C l$. cf. Misgurnus anguillicaudatus Blkr., Gthr.

Cobitis arenata $C . V .91,70$.

Cobitis armata $M c C l .323,4 \mathrm{I}$.

Cobitis armatus $M C C l .91,70$.

Cobitis balgara Buch. 91, 70.

Cobitis barbatuloïdes Blkr. 49, 435. 55, 4I3. 220, 304 .

Cobitis Berdmorei Blkr. 323, 42 .

Cobitis bifurcata $M c C l$. cf. Misgurnus anguillicaudatus Gthr.

Cobitis bilturio. Buch. 91, 70.

Cobitis botia Ham. Buch. 91, 70. 323, 42.

Cobitis boutanensis $M_{C} C l .91,70$.

Cobitis chlorosoma $\mathrm{MC} \mathrm{Cl.91,70.}$

Cobitis choirorhynchos Blkr. 106, 95. 220, 303.

Cobitis chrysolaimos $K . v . H$. cf. Cobitis fasciata $C . V$.

Cobitis corica Buch. 91, 70.

Cobitis cucura Buch., Blkr. 91, 70. 323, 41.

Cobitis curta Schl. cf. Botia curta Gïnth.

Cobitis dario Buch. Ham. 91, 143 .

Cobitis decemcirrhosus Basil. cf. Misgurnus anguillicaudatus Gthr.

Cobitis elongata Heck. 323, $4 \mathrm{I}$.

Cobitis fasciata $C . V .106,96.157,235.170,476,477.211,242$. 220, 303. 270, 50. 279, IоI.

Cobitis fasciatus $V a l .271,2$.

Cobitis geto Buch. 91, 70.

Cobitis gongota Buch. 91, 70.

Cobitis grandis $C . V .91,70$.

Cobitis guntea Buch. Ham. 91, 143. 323, 41.

Cobitis guttata $M C$. Cl. 91, 70.

Cobitis Hasseltii C. V. 166, 365. 200, 48. 220, 303.

Cobitis hymenophysa $B l k r .67,602.220,303$.

Cobitis Jaklesii Blkr. 67, 604. 220, 303. 270, 50.

Cobitis Kuhlii C. V. 25, I . 170, 470, 477. 220, 304. 364, г66.

Cobitis linea Blkr. 323, $4 \mathrm{r}$.

Cobitis macracanthus Blkr. 67, 603. 220, 303 .

Cobitis macrochir Blkr. 106, 97. 220, 303 . 
Cobitis macrorhynchos Blkr. 162, 20. 220, 303.

Cobitis maculata Schl. cf. Misgurnus anguillicaudatus Gthr.

Cobitis marmorata Heck. 91, 70.

Cobitis maya Sykes 91, 70. 323, $4 \mathrm{I}$.

Cobitis micropogon Blkr.323, 42 .

Cobitis monoceros $\mathrm{MC} \mathrm{Cl.91,70.}$

Cobitis montana $C . V .91,70$.

Cobitis mooreh Sykes 91, 70.

Cobitis oblonga $K$. v. H., C. V. 170, 477. 174, 4S. 200, 48. 220, 304.

Cobitis pacifica I. R. Forst cf. Culius fuscus Blkr., Eleotris nigra $Q . G$.

Cobitis pangia Buch. 91, 70.

Cobitis pavonacea $M C C l .91,70$.

Cobitis pectoralis $M C C$. cf. Misgurnus anguillicaudatus $G t / 2$.

Cobitis Pfeifferi Blkr. 76, 298.

Cobitis phoxocheila $M C C l .91,70$.

Cobitis psammismus Rich. cf. Misgurnus anguillicandatus Gthr.

Cobitis punctifer Cast. 323, 43 .

Cobitis rubripinnis $T$. Schl. 90, 48. 93, 12. cf. Misgurnus anguillicatldatus Giunth.

Cobitis rubripinnis Blkr. cf. Cobitichthys enalios Blkr.

Cobitis rupecula $C . V .91,70$.

Cobitis Rüppellii Sykes 91, 70.

Cobitis savona Buch. 91, 70.

Cobitis scaturigina Buch. 91, 70.

Cobitis Schlegeli Blkr. 323, 4I.

Cobitis subfusca $C . V .91,70$.

Cobitis taenia L. 323, 41. 497, 22.

Cobitis taenia japonica Schl. cf. Cobitis taenia $L$.

Cobitis thermalis $C . V .91,70.327,256$. cf. Lepidocephalichthys thermalis Blkr.

Cobitis turio Buch. 91, 70.

Cobitis vittata Heck. 91, 72 .

Cobitis zebrata Cast. 323, 43.

Cobitis zonata $C . V .91,72$.

Cochlognathus Baird, Gir. 261, 425. 314, 204.

Cocotropus $K p .473,299$.

Cocotropus dermacanthus Blkr. 475, 92.

Cocotropus echinatus $K p .473,299.475$, 9x.

Codoma Gir. 261, 438. 314, 212. 329, 264.

Coecilia Lac. 351, I 20.

Coecilophis $K p$. 351, II9.

Coecilophis compar Kp. cf. Ophichthys compar blkr.

Coelonotus argulus Peters 459, 75.

Coelonotus liaspis Gthr. 459, 75 . 
Coïlia borneënsis Blkr. 42, 60. 45, r97. 49, 421. 55, 437. 60, 45. 91, I 49. 239, го2. 270, 58. 272, 27. 356, I 76.

Coilia Cantoris Blkr. 91, 148.

Coilia clupeoides Giunth. 496, 3 .

Coilia Dussumieri C. V. 60, 45. 91, 74. 267, 140. 287, 62.

Coilia Grayi Rich., Kner 91, I 49. cf. Coilia (Chaetomus) Grayi Blkr., Coilia (Chaetomus) nasus Blkr.

Coilia (Chaetomus) Grayi Blkr. 425, I 48.

Coilia Hamiltonii C. $V .91,74,149$.

Coilio Lindmani Blkr. 174, 48. 270, 58. 356, I 76.

Coilia macrognathos Blkr. 55, 436. 60, 50. 91, 149. 178, 2. 272, 27.

Coilia nasus Gïnth. 497, 25.

Coilia (Chaetomus) nasus Blkr. 425, 148.

Coilia Playfairii Rich. 91, I49. cf. Coilia (Chaetomus) Playfairi Blkr.

Coilia (Chaetomus) Playfairi Blkr. 425, I48.

Coilia quadragesimalis $C . V .91,74$.

Coilia Rebentischii Blkr. 191, 5. 272, 27.

Coilia Reynaldi C. V. 51, 59. 91, 148. 288, 79.

Coius H.B. 468, $33^{6}$.

Coius binotatus $G r$. cf. Datnioïdes polota Blkr.

Coius catus Ham. Buch. cf. Lutjanus Johni Lac.

Coius chatareus Ham. Buch. cf. Toxotes jaculator C. $V$.

Coius cobojius Ham. Buch. cf. Anabas scandens $C . V$.

Coius datnia Ham. Buch. cf. Datnia argentea $C$. V., Sparus datnia Blkr.

Coius nandus Ham, Buch. cf. Nandus marmoratus $C . V$.

Coius polota Buch. cf. Datniö̈des polota Blkr.

Coius trivittatus Ham. Buch. cf. Therapon (Datnia) trivittatus Blkr.

Coius vacti Ham. Buch. cf. Lates nobilis $C$. $V$.

Cojus chatareus Ham. Buch. cf. Toxotes chatareus Blkr.

Cojus cobojius Ham. Buch. cf. Anabas testudineus Cuv.

Colisa 1, 553 .

Colisa bejeus $C . V$. cf. Colisa vulgaris $C . V$.

Colisa chuna $C . V$. 91, 42 .

Colisa cotra $C$. $V$. cf Colisa vulgaris $C$. $V$.

Colisa lali $C . V$. cf. Colisa vulgaris $C . V$.

Colisa lalius $C . V$. cf. Colisa vulgaris $C . V$.

Colisa ponticeriana $C . V$. cf. Colisa vulgaris $C . V$.

Colisa sota $C . V .91,42$.

Colisa unicolor $C . V .91,42$.

Colisa vulgaris C. V. 91, 94 .

Collia nasus Schl. cf. Coilia nasus Gïnth., Coilia (Chaetomus) nasus Blkr.

Collichthys Gthr. 468, 330.

Collichthys biaurita Gthr. 425, I42.

Collichthys biauritus Gthr. 452, I 5 .

Collichthys lucida Gthr. 307, I42. 411, 252. 425, I 42. 468, 33 O. 
Collichthys microdon Blkr. 425, I42. 452, r6.

Conger $K p$. Klein 175, 28. 351, I 6 .

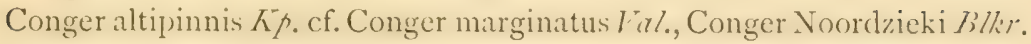

Conger anago T. Schl. 90, 52. 253, 235. cf. Ophisoma anago Blk.

Conger anagoïdes Blkr. 88, 76. 95, I I 2. 275, 50. 287, 62. 296, I I 2. cf. Ophisoma anagoïdes $B l k r$.

- Conger bagio Cant. 51, 59. 71, 777. 88, 22. 90, 54. 91, 78. 191, 2. 270, 59. 272, 28. cf. Muraenesox bagio Pct.

- Conger balearicus Cuv. 90, 53 .

Conger brasiliensis Ranz. cf. Brachyconger savanna Blkr.

Conger communis Costa cf. Conger vulgaris Cuv.

-Conger fasciatus Rich. 425, I23.

Conger flavipinnatus Benn. cf. Pisoodonophis cancrivorus $K p$.

- Conger habenatus Rich. 119, I5.

Conger hamo T. Schl. 90, 54. cf. Conger bagio Cant., Muraenesox bagio Pet.

Conger japonicus Blkr. 487. 497, 32 .

Conger leptognathus Blkr. 175, 27.

Conger lepturus Rich. 174, 49. 270, 59. cf. Uroconger lepturus $K p$. Conger limbatus Cast. cf. Brachyconger savanna Blkr.

Conger longirostris Bcnu. cf. Conger talabon Cuv., Muraenesox bagio Pet.

Conger marginatus $\mathrm{Val}$. 459, 72. 495, 22.

Conger microstoma Eyd. Soul. cf. Pisoodonophis boro $K p$.

- Conger moniliger Wus. L. B. cf. Muraenesox bagio Peters.

Conger neoguinaicus Blkr. 232, 22. 491, 56. cf. Ophisoma neoguinaicum Blkr.

Conger Noordzieki Blkr. 163, 86. 170, 480. 236, 332. 270, 59. 275, 50. $315,237.320,272.352,125.364,295.391,277$.

Conger oxyrhynchus Ey'd. Soul. cf. Muraenesox bagio Petcrs.

Conger oxyurus $K . v . H$. Uroconger lepturus $K p$.

-Conger Salviani IVill. cf. Conger vulgaris Cu».

Conger singaporensis Blkr. 51, 59. 88, 2 I.

Conger singapurensis Blkr. cf. Muraenesox singapurensis Blkr.

Conger talabon Cuv. 16, 6. 25, I2. 51, 77. 55, 4I4. 85, 456. 88, I 8 . $91,78.156,218.270,59.272,28$.

Conger talabon Cant. cf. Muraenesox talabon Blkr.

Conger talabonoïdes Blkr. 88, 20. cf. Muraenesox talabonoides Blkr.

- Conger tricuspidatus $88,2 \mathrm{I}$.

-Conger urolophus T. Schl. 90, 53. cf. Ophichthys urolophus Gthr., Ophisurus urolophus.

Conger uropterus T. Schl. 88, 26. 90, 54. cf. Myrus uropterus Günth., Ophisurus uropterus.

Conger vulgaris $C u v .90,53.157,235.236,332,334.253,235.275$, 5 I. 352, I $25.394,288.425$, I $23.497,25$.

Congermuraena $K p .175,28.351$, iा 6 . 
Congerodon $K p .175,28.351$, II7.

Congrogadus reticulatus Gthr. 395, 293.

Congromuraena megastoma Giunth. cf. Ophisoma megastoma Blkr.

Congrus angustidens Rich. cf. Muraenesox bagio Peters.

Congrus brevicuspis Rich. cf. Muraenesox bagio Peters.

Congrus curvidens Rich. cf. Muraenesox singapurensis Blkr.

Congrus habenatus Rich. 90, 53 .

Congrus hamo Rich. cf. Muraenesox bagio Peters.

Congrus lepturus Rich.cf. Conger lepturus Rich., Uruconger lepturus $K \not$.

Congrus leucophaeus Rich. cf. Conger vulgaris Cuv.

Congrus protervus Rich. cf. Muraenesox bagio Peters.

Congrus tricuspidatus Rich. cf. Congrus bagio Cant., Muraenesox bagio Peters.

Congrus vulgaris Rich. cf. Conger vulgaris Cuv.

Conodon C. V. 468, 272 .

Conodon pacifici Gthr. 468, 272.

Conodon Plumieri C. V. 468, 272.

Conorhynchus Blkr. 306, Iо2.

Conorhynchus conirostris Blkr. 306, 102.

Conorhynchus glossodon Blkr.396, 300.459, 102.491, 55.495, 22.497, 25 .

Conostomus C. Dum. 306, 102.

Coracinus Gron. 468, 294.

Coracinus insignitus Gron. cf. Prochilus ephippium Blkr.

Coracinus vittatus Gron. cf. Prochilus bifasciatus Blkr.

Coradion $K p .468,304$.

Coradion chrysozonus $K p .468,304.474,3$ I6. 481, 37. 491, '44.

Coradion melanopus Blkr. 474, 3г6. 481, 39 .

Coradion merlangus Blkr. 495, I4.

Coricus Cuv. 292, ro8.

Coris Lac. 292, 98.

Coris aygula Lac. 459, 83. 495, 10.

Coris caudimacula Gthr. cf. Hemicoris candimacula Blkr.

Coris Cuvieri Gthr. 459, 83. 495, го.

Coris erythropterus Blkr. 459, 83. 495, го.

Coris formosa Blkr., Gthr. 293, 281. 364, 276. 459, 83. 491, 40.

Coris Gaimardi Gthr. 293, 282. 320, 266.

Coris Greenoughi Blkr., Gthr. 293, 282. 320, 266. 354, I44. 358, I85. $364,276$.

Coris guineënsis Blkr. 302, 30.

Coris semipartita Gthr. cf. Hologymnosus semipartitus Blkr.

Corvina Cuv. 1, 553. 468, 327, 329.

Corvina adusta Jen. cf. Johnius adustus Blkr.

Corvina albida $C . V .91,36.425$, I42. cf. Pseudosciaena albida $B l k r$.

Corvina albiflora Rich. 425, 142.

Corvina amblyceps Blkr.411, 25 ז. 425, I42. 
Corvina amoyensis Blkr. 425, 142.

Corvina anei $C . V .91,36$.

Corvina axillaris $C . V .91,36$.

Corvina Belengeri $C . V .51,56.91,36$. cf. Corvina Kuhlii $C . V$., Johnius Belengeri Cant.

Corvina carouna C. V. 91, 36. cf. Johnius carutta $B l$.

Corvina carutta $C . V .91,36$. cf. Johnius carutta $B l$.

Corvina catalea $C . V .16,4.18$, I 8.25, S. 51, 56. 56, 444. 91, 36. 425, 142. cf. Corvina diacanthus Blkr., Johnius diacanthus Cant., Pseudosciaena diacanthus $B / k r$.

Corvina celebica Blkr. 109, 244. cf. Psendosciaena miles Blkr. Corvina chaptis $C . V .91,36$. cf. Pseudosciaena diacanthus Blkr. Corvina coitor $C . V .91,36.425, \mathrm{I} 42$.

Corvina crocea Blkr. 425, I42.

Corvina cuja C. V. 91, 36. cf. Pseudosciaena cuja Blkr.

Corvina dentex $C . V .468,330$.

Corvina diacanthus Blkr. 425, 142.

Corvina dorsalis Peters cf. Pseudosciaena miles Blkr.

Corvina Dussumierii $C . V .51,56.91,36.425$, I42. cf. Johnius Dussumieri Cant.

Corvina Goldmanni Blkr. 112, 37r. cf. Pseudosciaena Goldmani Blkr. Corvina grypota Rich. 425, I42.

Corvina hypostoma Blkr. 86, 499. 127, 345. cf. Johnius hypostoma Blkr. Corvina jubata Blkr. 121, I60. cf. Johnius jubatus Blkr.

Corvina Kuhlii $C . V$. 18, I 8. 25, 7. 55, 4II. 156, 213. 178, 2. cf. Johnius Belengeri Cant., J. Kuhlii.

Corvina lobata C. V. 91, 36 .

Corvina macrophthalmus Blkr. 395, 292. 425, r42. cf. Pseudosciaena macrophthalmus Blkr.

Corvina maculata $C . V .91,36$.

Corvina miles C.V. 18, I 7. 25, 7. 91, 36. cf. Pseudosciaena miles $B / k r$. Corvina nalla-katchelee Rich. cf. Corvina coitor C. $V$.

Corvina oscula C.V. 468,324 .

Corvina plagiostoma Blkr. 16, Iо. 18, I7. 25, 7. 31, Iоo. cf. Pseudosciaena plagiostoma Blkr.

Corvina platycephala $K$. v. $H_{\text {., }}$ C. $V$. cf. Johnius diacanthus Cant., Pseudosciaena diacanthus Blkr.

Corvina polycladiscus Gthr. cf. Pseudosciaena polycladiscus Blkr.

Corvina polykladiskos Blkr.55, 420. cf. Pseudosciaena polycladiscus Blkr.

Corvina sampitensis Blkr. 55, 42 I. cf. Pseudosciaena miles Blkr.

Corvina semiluctuosa $C . V .2,522.91,36.425$, 142. cf. Johnius semiluctuosus $K n$.

Corvina sina C. $Y .91,36.118,82.408,238.425$, 142. cf. Corvina Kuhlii $C . V$.

Corvina sina Schl. cf. Pseudosciaena Schlegeli Blkr. 
Corvina soldado Cant. cf. Pseudosciaena miles Blkr.

Corvina trachycephalus Blkr. 35, 269. 42, 58.45, I95, 200. 49, 4I8. 55, 4II. cf. Johnius trachycephalus Blkr.

Corvina Wolffii Blkr. 42, 66. 45, I95. 49, 4r8. 55, 4I J. cf. Pseudosciaena miles $B l k r$.

Corydoras Lac. 306, 83 .

Corydoras edentatus Spix cf. Oxydoras niger Blkr.

Corydoras Geoffroyi Lac. cf. Corydoras punctatus Blkr.

Corydoras punctatus Blkr. 306, 83. 336, 4.

Coryphaena argyrurus $C . V .91,46$.

Coryphaena chrysurus Lac., C. $V$. 10, 67, 68. 46, 213. 91, 46. 270, 28. 275, 40. cf. Coryphaena hippurus $L$.

Coryphaena fasciolata Pall. cf. Lampugus fasciolatus $C . V$.

Coryphaena hippurus $L .459,97.495$, I8. 497, I6.

Coryphaena japonica Schl. cf. Coryphaena hippurus $L$.

Coryphaena lineolata Raf. cf. Novacula lineolata Blkr.

Coryphaena pentadactyla $B l$. cf. Hemipteronotus pentadactylus Blkr., Novacula pentadactyla $C . V$.

Coryphaena scomberoides Lac. 176, 7. 275, 40.

Coryphaena sinensis Lac. cf. I atilus argentatus C. $V$.

Coryphaena virens Park 145, 382.

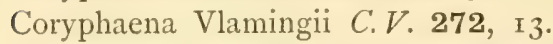

Coryphaenoides altipinnis Günth. 497, 21.

Coryphaenoides asper Gïnth.497, 2 I.

Coryphaenoides leptolepis Günth. cf. Macruroplus leptolepis.

Coryphaenoides longifilis Giunth. 497, 2 I.

Coryphaenoides nasutus Gïnth. 497, 2 I.

Coryphaenoides villosus Gïnth. cf. Lepidorynchus villosus.

Coryphopterus Gill 453, 316, 3 I 7 .

Coryphopterus glaucofrenum Gill 453, 3 I 7 .

Corystion chirophthalmus Blkr. 364, 291.

Corystion orientale Blkr. 315, 236.396, 297. 425, I 26.491, 49.495, r6.

Corystion orientalis Blkr. 354, I49. 358, I9I. 364, 29r. 459, 76. 497, I3.

Corystion volitans Blkr. 459, 76 .

Corythobatus Cant. 473, 298.

Corythobatus echinatus Cant. cf. Cocotropus echinatus $K p$.

Corythobatus monodactylus Blkr. 364, 286. 425, I4I. cf. Minous oxycephalus Blkr.

Corythobatus trachycephalus Blkr. 364, 286.

Corythobatus woora Cant.425, 14 I. cf. Minous monodactylus, woora C. $V$.

Corythoichthys fasciatus $K p .270,72.296,103.315,230.318,250$. $320,265.340,364.354$, I 44.358, I $84.364,273.394,286$. cf. Syngnathus conspicillatus Jen.

Corythoichthys penicillus Blkr. 288, 69 . 
Cossyphodes macrodon Blkr. 270, 37. 275, 19. 285, 244. 287, 43 .

Cossyphus $\mathrm{Val}$., $\mathrm{MC} \mathrm{Cl} .292$, 106. 306, 120.

Cossyphus anthioides Gthr. 293, 296. 364, 277. 459, 82. 495, 9.

Cossyphus atrolumbus $C . V$. 179, 5. 459, 82. 495, 9.

Cossyphus axillaris $C . V .459,82.495,9$.

Cossyphus bilunulatus $C . V .179,4.293,296.459,82.495,9$.

Cossyphus bodianus $\mathrm{Val}$. cf. Cossyphus rufus Gthr.

Cossyphus cyanostolus Rich. cf. Choerops cyanostolus Blkr.

Cossyphus diana $C . V$. 106, 86. 270, 37. 293, 296. 296, 104. 364, $277.459,82.495,9$.

Cossyphus dimidiatus $C$. $V$. cf. Labroides latovittatus, paradiseus $B l k r$.

Cossyphus echis Guich. cf. Pseudocheilinus hexataenia Blkr.

Cossyphus Gouldii Rich. 119, 13.

Cossyphus japonicus Blkr. 118, Ir 4. cf. Choerops japonicus Blkr.

Cossyphus leucosticticus Gthr. 459, 82. 495, 9.

Cossyphus macrodon Blkr. 11, 10. 12, 45. 51, 58. 185, 225. 237, 438. cf. Choerops macrodon Blkr.

Cossyphus macrurus Gthr. 459, 82. 495, 9.

Cossyphus maldat C. $V$. 179, 5. cf. Cossyphus macrurus Gthr.

Cossyphus maxillosus $\mathrm{Val} .459,82$.

Cossyphus mesothorax $C . V$. 11, 13. 12, 47. 270, 37. 293, 296. 315, 231. 358, I85. 364, 277. 391, 276. cf. Bodianus mesothorax Blkr.

Cossyphus ommapterus Rich. cf. Choerops Schoenleini Blkr.

Cossyphus opercularis Pet. cf. Pteragogus opercularis Gthr.

Cossyphus opercularis Guich. cf. Trochocopus opercularis Gthr.

Cossyphus oxycephalus Blkr. 297, I29. cf. Harpe oxycephalus Blkr.

Cossyphus perditio C. $V .179,5$.

Cossyphus quadrilineatrus $C . V .143,53$.

Cossyphus reticulatus C.V. 269, 72. cf. Semicossyphus reticulatus Schl.

Cossyphus rufus Gthr. 459, 82 .

Cossyphus Schoenleinii Agass. 11. 7. 12, 42. 10, 67, 68, 46, 2 I3.

109, 252. 275, 19. 287, 43 .

Cossyphus spilotes Guich. 459, 82 .

Cossyphus vulpinus Rich. 119, I3.

Cossyphus zosterophorus Blkr. 163, 75. cf. Cossyphus anthioides Gthr.

Cottapistus Blkr. 473, 298.

Cottapistus cottoides Blkr. 475, 75 .

Cottus grunniens $B l$. cf. Batrachus grunniens $C . V$.

Cottus hemilepidotus Tiles. cf. Hemilepidotus Tilesii $C$. $V$.

Cottus insidiator $L$. Gm. cf. Platycephalus indicus $B l k r$.

Cottus insidiator Forsk. L. cf. Platycephalus insidiator $\mathrm{Bl}$.

Cottus intermedius Schl. cf. Centridermichthys intermedius Gir.

Cottus japonicus Pall. cf. Hippocephalus japonicus Srons.

Cottus madagascariensis Lac. cf. Platycephalus indicus Blkr.

Cottus pollux Gïnth. 497, Ix. 
Cottus scaber $L_{\text {. }}, B$ l. cf. Platycephalus scaber Bl. Schn., C. V. Cottus uncinatus Schl. cf. Centridermichthys uncinatus Ger.

Cotylephorus Szons. 306, Io8.

Cotylephorus Blochii Stons. cf. Platystacus cotylephorus Bl.

Cotylopus Guich. 453, 3І 3.

Cotylopus acutipinnis Guich.453, 3І3. 459, 78.

Cotylopus parvipinnis Guich. 459, 78 .

Crayracion Klein 367, I8.

Crayracion astrotaenia Blkr. 364, 27 I. 368, 36.

Crayracion bondarus $B l k r .368,37$.

Crayracion carduus Blkr. 368, 36.

Crayracion erythrotaenia Blkr. 364, 271. 368, 36. 388, 27 I.

Crayracion firmamentum Blkr. 497, 27.

Crayracion fluviatilis Blkr. 368, 36. 395, 290.

Crayracion hispidus Blkr. 459, 69. 495, 23.

Crayracion immaculatus Blkr. 364, 27 I. 368, 38. 395, 290. 425, I 2 I. 437, 36. 459, 69. 495, 23.

Crayracion implutus Blkr. 364, 27 I. 368, 37. 394, 285. 425, I 2 I. 459, 103. 491, 57 .

Crayracion leiurus $B l k r .368,36$.

Crayracion lineatus Blkr. 364, 27I. 368, 36. 425, I21. 459, 69 . $495,23.497,27$.

Crayracion manillensis $B l k r .364,27$ I. 368, 36.

Crayracion mappa Blkr. 364, 27 r. 368, 37. 491, 57.

Crayracion margaritatus Blkr. 394, 285.

Crayracion meleagris Blkr. 368, 37. 394, 285 .

Crayracion nigropunctatus Blkr. 364, 27x. 368, 38. 389, 273. 396, $298,459,69.491,57.495,23$.

Crayracion palembangensis Blkr. $368,3^{6}$.

Crayracion stellatus Blkr. 368, 37. 425, I21. 459, 69. 495, 23.

Crayracion testudineus Blkr. 364, 27 I. 368, 37. 395, 290.

Crenidens C. $V .468,284$.

Crenidens Forskalii $C . V .468,284$.

Crenidens melanichthys Rich. cf. Girella punctata Gr., Melanichthys crenidens Blkr.

Crenidens punctatus Rich. cf. Girella punctata $G r$.

Crenidens sarissophorus Cant. cf. Girella sarissophorus Cant.

Crenidens simplex Rich. 119, 9. 468, 296.

Crenidens tephraeops Rich. 119, 9. 468, 296.

Crenidens triglyphus Rich. 119, 9. 269, 78.

Crenidens zebra Rich. 119, 9.

Crenilabrus Cuv. 1, 552, 292, I08.

Crenilabrus anthioides Benn. cf. Cossyphus anthioides Gthr.

Crenilabrus aurigarius Rich. cf. Duymaeria aurigaria Blkr.

Crenilabrus elegans $K$. v. H. cf. Cossyphus nesothorax Cuv. Val. 
Crenilabrus enneacanthus Blkr. 72, I20.

Crenilabrus flagellifer $T$. Schl. cf. Crenilabrus nematopterus $B l k r$.

Crenilabrus flagellifer Schl. cf. Duymaeria japonica Blkr.

Crenilabrus leucozona Blkr. 185, 238.

Crenilabrus nematopterus Blkr. 47, 250.

Crenilabrus oligacanthus Blkr. 50, 489. 51, 68. 235, 369. 275, I9. cf. Choerops oligacanthus Blkr.

Crenilabrus rubellio Rich. cf. Duymaeria aurigaria Blkr.

Crenilabrus spilogaster Blkr. 102, 4I6. 118, I13. cf. Duymaeria spilogaster Blkr.

Crinodus Gill 468, 3 I 7 .

Crinodus lophodon Gill 468, 3 I 7 .

Cristallogobius Gill 453, 3 ro.

Cristiceps australis $C . V .119$, I2.

Cromileptes Swns. 468, 257.

Cromileptes altivelis Stons. 364, 28 r. 395, 29 I.425, 137.451, $26.468,257$.

Cromileptes miniatus Swns, cf. Epinephelus miniatus Blkr.

Cromileptes myriaster Szons. cf. Epinephelus argus Bl. Schn.

Crossocheilichthys 314, I92.

Crossocheilos v. Hass. 261, 423.

Crossocheilos (Crossocheilichthys) cobitis Blkr. 270, 50.

Crossocheilos (Crossocheilichthys) Langei Blkr. 270, 50.

Crossocheilos (Crossocheilos) oblongus v. Hass., Blkr. 86, 525. 170, $476,477.270,5 \circ$.

Crossocheilus v. Hass. 314, 192.

Crossoderma madagascariensis Guich. cf. Caracanthus madagascariensis Blkr.

Crossorhinus barbatus $M . H .119,17.497,3$.

Crossorhinus dasypogon Blkr. 383, 400. 396, 298. 398, 306.437, 36.

Crotalopsis $K p$. 351, II 8 .

Cryptocentrus Ehr. 453, 322.

Cryptocentrus castaneus Blkr.497, I9.

Cryptocentrus diproctotaenia Blkr. 469, r 43.

Cryptocentrus Knutteli Blkr. 497, I9.

Cryptocentrus leptocephalus Blkr.469, I46.

Cryptocentrus liolepis Blkr. 469, I 45.

Cryptocentrus papuensis Blkr. 491, 54.

Cryptopterus $K p .351$, I 19.

Cryptosmilia Cope 468, 301 .

Cryptosmilia luna Cope cf. Harpochirus punctatus Cant.

Ctenogobius Gill 453. 321.

Ctenogobius criniger Blkr. 491, 54 .

Ctenogobius cylindricus Blkr. 461, 129.

Ctenogobius fasciatus Gill 453, 32 r.

Ctenogobius gracilis Blkr. 461, 127. 
Cténogobius grammatogaster Blkr. 461, I 24 .

Ctenogobius gymnauchen Blkr. 497, I9.

Ctenogobius notophthalmus Blkr. 461, I 26.

Ctenolabrus $V$ al. 292, Iо7.

Ctenolabrus flagellifer C. $V .47,250.289,8$ I.

Ctenolates Gthr. 468, 267.

Ctenopharyngodon idellus Gthr. cf. Jeeuciscus idellus $\mathrm{Val}$.

Ctenopharyngodon laticeps Steind. cf. Leuciscus idellus $V a l$.

Ctenops nobilis $M c$ Cl. 91, 42 .

Ctenops vittatus Blkr. 498, 24.

Ctenotrypauchen Steind. 453, 33I.

Ctenotrypauchen chinensis Steind. cf. Trypauchen chinensis Steind.

Culius Blkr. 151, 4II. 453, 303. 460, rо3, го5.

Culius acanthopomus Blkr. 151, 4II. 270, 4I. 354, I5O. 364, 293. cf. Culius melanosoma $B l k r$.

Culius fuscus Blkr. 296, III. 354, i 50.358 , I92. 364, 293. 396, 298. 425, г $28.453,3 \circ 3.459,78.478,40.495$, I 7 .

Culius insulindicus Blkr. 460, I07. 478, 48.

Culius macrocephalus Blkr. 164, 70. 354, I50. 478, 45.

Culius macrolepis Blkr. 460, I09. 478, 50.

Culius melanosoma Blkr. 151, 4I2. 270, 41. 296, I I I. 320, 27 I. 358, I $92.430,233.478,43$.

Culius niger $B l k r .151,4$ I . 156, 215. 231, I48. 236, 331. 237, $43^{8}$. 270, 41. 275, 45. 279, I00. 287, 57. cf. Culius fuscus Blkr.

Culius oxycephalus Blkr. 478, 47. 497, I9.

Culius pseudacanthopomus Blkr. 151, 4I I. 270, 4I. cf. Culius fuscus $B l k r$.

Culter Basil. 261, 438. 314, 2 I4.

Culter alburnus Basil. cf. Culter recurviceps Blkr.

Culter brevicauda Gthr. 411, 252. 415, 69. 420, 82. 425, 145. 496, 3 .

Culter Dabryi Blkr. 415, 70. 420, 84. 425, I45.

Culter erythropterus Bas. 345, 27. 415, I3. 425, 145.

Culter erythropterus $B l k r$. cf. Culter ilishaeformis Blkr.

Culter erythropterus Kner cf. Culter Kneri Blkr.

Culter exiguus Basil. cf. Pseudoculter exiguus Blkr.

Culter hypselonotus Blkr. 411, 252. 415, 72. 420, 85. 425, x45.

Culter ilishaeformis Blkr. 411, 252. 415, 67. 420, 80.425, I 45 .

Culter Kneri Blkr. 411, 252.415, I4. 425, I45.

Culter leucisculus Bas., Kner cf. Hemiculter leucisculus Blkr.

Culter mongolicus Basil. cf. Culter hypselonotus Blkr.

Culter oxycephalus Blkr. 411, 252. 415, 74. 420, 87. 425, I 45 .

Culter pekinensis Kner cf. Parabramis pekinensis Blkr.

Culter pekinensis Bas. cf. Pseudoculter pekinensis Blkr.

Culter recurviceps Blkr. 415, I3. 425, I 25.

Curtus macrolepidotus Bl. Schn. cf. Pempheris moluca $C$. $V$.

Cyanichthys coeruleus $K p$. cf. Diodon coeruleus $Q$. $G$. 
Cybium 1, 553 .

Cybium chinense $C . V .425$, г 3 г. 497, т5.

Cybium clupeoideum $C . V .119$, го.

Cybium Commersonii C. $V .2,5$ I6. 10, 67. 46, 212. 51, 57. 91, 42. 275, 38. 288, 74. 425, I3 1. 459, 100. 495, I8. cf. Cybium konam Blkr.

Cybium Croockewitii Blkr. 32, I6 I. 56, 445. 57, 37. 275, 38 .

Cybium flavo-brunneum Sintth 266, 53.

Cybium guttatum C. V. 2, 516. 10, 66. 16, 4. 25, 8. 46, 2 I2. 51, 57. $56,445.57,38.91,42.191,2.270,29.272,13.275,38.288$, 74. 356, І $73.364,289.425$, І 3 I.

Cybium interruptum $C . V .2,5$ I6. 25, S. cf. Cybium lineolatum $C . V$.

Cybium konam Blkr. 37, 357. 56, 445. 57, 39. 91, 42. 127, 345 . $270,29.275,38.287,52.296$, 109. 356, I 73.

Cybium Kuhlii C. $V$. 2, 5 I 6 .

Cybium lineolatum $C . V .2,5$ r6. 16, 4. 25, 8. 51, 57. 57, 40. 91, 42. 227, 407. 288, 74 .

Cybium Mertensii C. $V .425$, г3 г.

Cybium niphonium $C . V .497$, I5.

Cybium sara Benn. cf. Acanthocybium sara Gill.

Cybium tritor C. V. 302, 72 .

Cychla cuning Bl. Schn. cf. Caesio erythrogaster $K . v . H$.

Cychla erythrura $B l$. Schn. cf. Gerres oyena $C . V$.

Cychla fares Rüpp. cf. Aphareus rutilans $C . V$.

Cycleptus Raf. 261, 428. 314, I90.

Cyclichthys orbicularis $K p .275$, I2. 288, 68 .

Cyclocheilichthys Blkr. 261, 431. 314, I99.

Cyclocheilichthys (Anematichthys) apogon Blkr. 235, 37r. 270, 52. $271,2.272,22$.

Cyclocheilichthys (Anematichthys) apogonides Blkr. 356, I75.

Cyclocheilichthys (Cyclocheilichthys) armatus Blkr. 270, 52 .

Cyclocheilichthys Dumerili 347, 37 .

Cyclocheilichthys (Cyclocheilichthys) Dumerili Blkr. 356, 175.

Cyclocheilichthys (Siaja) heteronema Blkr. 272, 22.

Cyclocheilichthys (Anematichthys) janthochir Blkr. 272, 22.

Cyclocheilichthys (Cyclocheilichthys) macracanthus Blkr. 270, 52. 356, I 75 .

Cyclocheilichthys (Siaja) macropus Blikr. 272, 22.

Cyclocheilichthys (Siaja) microlepis Blkr. 270, 52. 272, 22.

Cyclocheilichthys (Cyclocheilichthys) repasson Blk). 270, 52.

Cyclocheilichthys (Siaja) siaja Blkr. 270, 52. 272, 22.

Cyclocheilichthys (Cyclocheilichthys) sinensis Blkr. 487. 496, 10.

Cyclogobius Stcind. 453, 319.

Cyclopoma $A g .468,263$.

Cyclopterus dentex Pall. cf. Gobiesox dentex Cuv. 
Cymatogaster Gill 468, 290.

Cymatogaster aggregatus Gill 468, 290.

Cymolutes Gthr. 292, ro2.

Cymolutes praetextatus Gthr. 293, 294. 354, I44. 364, 277. $459,83.495$, го.

Cynaedus Gron. 468, $28 \mathrm{I}$.

Cynichthys Szons. 468, 256.

Cynichthys flavo-purpuratus Steains. cf. Epinephelus flavocoeruleus Blkr.

Cynocephalus (Scoliodon) acutus Blkr. 495, 8.

Cynocephalus (Prionace) japonicus Blkr. 497, 3.

Cynocephalus (Hypoprion) Mackloti Blkr. 491, 39.

Cynocephalus (Scoliodon) macrorhynchus Blkr. 496, 2.

Cynocephalus (Prionace) melanopterus Blkr. 491, 39. 497, 3 .

Cynocephalus (Prionace) pleurotaenia Blkr. 491, 39.

Cynoglossus abbreviatus Gthr. 425, I3 $\mathrm{T}$.

Cynoglossus aurolimbatus Blkr. 425, г 30.

Cynoglossus favośquamis Blkr. 425, I3I.

Cynoglossus grammicus Blkr. 425, I3 I.

Cynoglossus javanicus Gthr. 364, 274.

Cynoglossus Kopsii Gthr. 364, 274. 395, 290.

Cynoglossus lineolatus Steind. 430, 234.

Cynoglossus lingua Büch.91, I53.

Cynoglossus macrolepidotus Gthr. 425, I3 I.

Cynoglossus melampetalus Gthr. 425, г3 I.

Cynoglossus monopus Blkr. 425, I3 I.

Cynoglossus nigrolabeculatus Blkr. 425, I3I.

Cynoglossus oligolepis Blkr. 430, 234.

Cynoglossus oxyrhynchus Gthr. 364, 274 .

Cynoglossus polytaenia Blkr. 364, 274.

Cynoglossus potous Gthr. 356, I72.

Cynoglossus puncticeps Gthr. 425, I3 I.

Cynoglossus quadrilineatus Gthr. 364, 274. 395, 290.

Cynoglossus trigrammus Gthr. 425, I31.

Cynoglossus xiphoideus Gthr. 356, I 73 .

Cynoscion Gill 468, 33 .

Cynoscion regalis Gill 468, $33 \circ$.

Cyprinella Gir. 261, 438. 314, 212. 329, 264.

Cyprinion Heck. 261, 427.

Cyprinodon Lac. 261, 439.

Cyprinodon cundinga Ham. Buch. cf. Megalops kundinga Blkr.

Cyprinodon orthonotus Petrs. cf. Fundulus orthonotus Gthr.

Cyprinopsis Fitz. 314, I9I.

Cyprinus Art. 1, 552. 261, 429. 314, 212.

Cyprinus abbreviatus Rich. cf. Carassius auratus Blkr.

Cyprinus acuminatus Rich. cf. Carpio vulgaris Rüpp. 
Cyprinus atrovirens Rich. cf. Carpio vulgaris Rüpt.

Cyprinus auratus $B l .75$, II.

Cyprinus auratus L., Rich. cf. Carassius auratus Blkr.

Cyprinus bacaila Buch. Ham. cf. Leuciscus bacaila C. $V$.

Cyprinus bendilisis Buch.91, I35.

Cyprinus bimaculatus Buch. Ham. cf. Systomus ticto $\mathrm{MCC}$.

Cyprinus boga Buch. Ham. cf. Gobio boga $\mathrm{MC} C$.

Cyprinus calbasu Buch. Ham. cf. Rohita calbosu C. $V$.

Cyprinus canius Buch. Ham. cf. Systomus gelius $\mathrm{MCCl}$.

Cyprinus cantonensis Osb. cf. Leuciscus idellus $\mathrm{Val}$.

Cyprinus carassioides (Gray) Rich. cf. Carassius auratus Blkr.

Cyprinus catle Buch. Ham. cf. Catla Buchanani C. $V$.

Cyprinus chinensis Basil. cf. Carpio vulgaris Rüpp.

Cyprinus conirostris Schl. cf. Carpio flavipinnus Blkr., Carpio vulgaris $R a p p$.

Cyprinus cosuatis Buch. Ham. cf. Leuciscus cosuatis C. $V$.

Cyprinus Daniconius H. Buch. 25, 22.

Cyprinus danrica Buch. Ham. cf. Nuria danrica C. $V$.

Cyprinus devario Buch. 91, I38.

Cyprinus flammans Rich. cf. Carpio vulgaris Rïpp.

Cyprinus flavipinnis $K . v . H .166,345.170,477$. cf. Carpio vulgaris $R a p p$.

Cyprinus floripenna $K . v . H .2,5$ I 2. cf. Cyprinus flavipinnis $K . v . H$.

Cyprinus fossicola (Gr.) Rich., Gthr. cf. Carpio fossicola Blkr.

Cyprinus gelius Buch. Ham. cf. Systomus gelius $\mathrm{MC} C l$.

Cyprinus gibelioides Cant. cf. Carassius auratus Blkr., Carassius gibelioides Blkr.

Cyprinus gohama Buch.91, I36.

Cyprinus haematopterus Schl. cf. Carpio vulgaris Rapp.

Cyprinus hybiscoides Rich. cf. Carpio vulgaris Rüpp.

Cyprinus jogia Ham. Buch. cf. Nuria danrica $C$. $V$.

Cyprinus Langsdorfii $\mathrm{Val}$. cf. Carassius auratus $B l k r$.

Cyprinus latius Buch. 91, r36.

Cyprinus laubuka Buch. Ham. cf. Leuciscus laubuka C. $V$.

Cyprinus lineatus $\mathrm{Val}$. cf. Carassius auratus $\mathrm{Blkr}$.

Cyprinus macrophthalmus Bl., Basil. cf. Carassius auratus Blkr.

Cyprinus Maillardi Guich. cf. Carassius auratus Nils., Blkr.

Cyprinus mauritianus Benn. cf. Carassius auratus Nils.

Cyprinus melanotus T. Schl. 269, 94. cf. Carpio melanotus Blkr., Carpio vulgaris $R a p p$.

Cyprinus mola Ham. Buch. 25, 21. cf. Leuciscus mola $\mathrm{ICC}_{\mathrm{C}} \mathrm{Cl}$.

Cyprinus morar Buch. Ham. cf. Leuciscus morar $\mathrm{MC} C$.

Cyprinus nancar Buch. 91, 60.

Cyprinus nigro-auratus Lac. cf. Carpio vulgaris Rüpp.

Cyprinus obesus Basil. cf. Carpio vulgaris Rüpp. 
Cyprinus pangusia Buch.91, 135 .

Cyprinus phutunio Buch. Ham. cf. Systomus phutunio $C$. $V$.

Cyprinus (Puntius) phutunio Ham. Buch. cf. Puntius (Puntius) phutunio Ham. Buch.

Cyprinus phutunipungto Ham. Buch. cf. Puntius (P.) phutunio Ham. Buch.

Cyprinus quadrilobatus Basil. cf. Carassius auratus Blkr.

Cyprinus ranipungti Buch. Ham. cf. Systomus gelius $\mathrm{MC} C l$.

Cyprinus rasbora $\mathrm{Ham}$. Buch. 25, 2 I. cf. Leuciscus rasbora $\mathrm{MC} \mathrm{Cl}$.

Cyprinus rohita Buch. Ham. cf. Rohita Buchanani C. $V$.

Cyprinus rubro-fuscus $L a c$. cf. Carpio vulgaris Rüpp.

Cyprinus sarana 91, I27.

Cyprinus sculponeatus Rich. cf. Carpio vulgaris Rüpp.

Cyprinus semiplotus $M c C l .91,60$.

Cyprinus sophore Buch. Ham. cf. Systomus sophore $\mathrm{Mc} \mathrm{Cl}$.

Cyprinus sutiha Buch. Ham. cf. Nuria danrica C. $V$.

Cyprinus ticto Buch. Ham. cf. Systomus ticto $\mathrm{Mc} C l$.

Cyprinus tor Ham. Buch. cf. Labeobarbus tor Blkr.

Cyprinus viridi-violaceus $L a c$. cf. Carpio vulgaris Rüpp.

Cyprinus vittatus $C . V$. cf. Cyprinus flavipinnis $K . v . H$.

Cypsilurus micropterus Blkr. 372, I28.

Cyrene Heck. 314, I02.

Cyrene festiva Heck. cf. Dangila festiva Blkr.

Dactylanthias Blkr. 468, $25 \mathrm{I}$.

Dactylanthias aplodactylus Blkr. 426, I68.468, 25 I.

Dactylopagrus Gill 468, 3I5.

Dactyloptera orientalis T. Schl. cf. Dactylopterus japonicus Blkr.

Dactylopterus bispinosus Swns. 91, 34.

Dactylopterus cheirophthalmus Blkr. 115, 494. 275, 42.

Dactylopterus japonicus Blkr. 102, 396. 118, 72. cf. Corystion orientalis Blkr.

Dactylopterus macracanthus Blkr. 114, 449. 275, 42.

Dactylopterus orientalis C. $V$., Schl. 14, 4. 47, 226. 54, 264. 115, 495. 270, 25. 275, 42. 296, I Iо. cf. Corystion orientale Blkr.

Dactylopus Bennetti Gill cf. Vulsus dactylopus Gthr.

Dactylosargus Gill 468, 3 r7.

Dactylosparus Gill 468, 3I5.

Dajaus diemensis Rich. 119, II. cf. Agonostoma Forsteri Gthr.

Dajaus Forsteri Rich. 119, II.

Dalophis Raf. 351, I20.

Dalophis anceps Cant. 83, 247. 88, 5. cf. Sphagebranchus anceps $K p$.

Dalophis marmorata Blkr. 83, 247. 88, 37. 106, Ioo. cf. Callechelys marmoratus Blkr.

Dalophis moluccensis Blkr. 83, 246. 88, 70. cf. Sphagebranchus moluccensis $K p$. 
Dalophis orientalis $M C C$ Cl. 83, 248. 88, 37. 91, 78 .

Dalophis polyophthalmus Blkr. 76, 299. 83, 247.88, 69. 207, 2 II. cf. Sphagebranchus polyophthalmus $K p$.

Damalichthys Gir. 468, 290.

Dangila Val. 261, 424. 314, I92.

Dangila Cuvieri C. V. 25, 19. 67, 596. 170, 476. 211, 242. 225, $35^{8}, 270,5 \mathrm{I}$.

Dangila fasciata Blkr. 76, 297. 270, 51. 272, 22.

Dangila festiva $B l k r .162,16.272,22$.

Dangila leptocheilos $V a l .2,5$ I3. 272, 2 I.

Dangila Leschenaultii $C . V .91,64$.

Dangila microlepis Blkr. 67, 595.

Dangila ocellata Blkr. 270, 51. 272, 22.

Dangila siamensis Blkr. 347, 37. 356, I75.

Dangila spilurus $B / k r .35,272.42,60.45$, x96. 49, 420. 55, 413. $272,22$.

Dangila sumatrana Blkr. 67, 596. 270, 51. 271, 2.

Dangila urostigma $V a l$ 2, 513 .

Danio Ham. Buch. 314, 203 329, 264.

Danio lineolatus Blkr. 326, 247. 335, 19.

Danio micronema Blkr. 326, 250. 335, 21 .

Dascyllus 1, 553 .

Dascyllus amanus C. $V . \mathbf{2 3 7}, 438$.

Dascyllus aruanus $C . V .6,2.9,633.47,246.54,237.91,52.95$, го8. 156, 2т6. 157, 234. 167, 373. 184, 202. 188, 460. 234, 354. 270, 36. 275, 20. 288, 70. cf. Tetradrachmum arcuatum Blkr., Cant., Tetradrachmum aruanum Cant.

Dascyllus aruanus $C$. $V$. var. Blkr. cf. Dascyllus melanurus Blkr.

Dascyllus aruanus var. Schl., Mïll. cf. Tetradrachmum melanurus Blkr.

Dascyllus marginatus Steind. cf. Tetradrachmum reticulatum Blkr.

Dascyllus melanurus Blkr. 95, 109. 184, 202. 275, 20. 285, 244. cf. Tetradrachmum melanurus Blkr.

Dascyllus niger Blkr., Rüpp. 6, 10. 47, 227. 54, 237. cf. Tetradrachmum trimaculatum $B l k r$.

Dascyllus polyacanthus Blkr. 141, 503. 163, 71. 167, 373. cf. Acanthochromis polyacanthus Gill.

Dascyllus reticulatus Osc. Cart. cf. Tetradrachmum reticulatum Blkr.

Dascyllus trimaculatus Rüpp., C. $V .47,230.184,202.231$, I 48.296 , ı04. Dascyllus niger Blkr., Tetradrachmum trimaculatum Blkr.

Dascyllus unicolor Benn. cf. Tetradrachmum trimaculatum Blkr.

Dascyllus xanthosoma Blkr. 47, 247. 156, 216. 184, 202. cf. Tetradrachmum reticulatum, xanthosoma Blkr.

Dascyllus xanthosoma Blkr. 47, 247. 156, 2 16. 184, 202. cf. Tetradrachmum reticulatum, xanthosoma Blkr. 
Dascyllus xanthurus Blkr. 72, II7. cf. Pomacentrus (Pomacentrus) violascens Blkr.

Dasyatis micrura var. J. E. Gray cf. Pteroplatea japonica T. Schl.

Dasybatis asterias Blkr. 459, 68. 495, S.

Dasybatis batis Blkr. 425, I 20.

Dasybatis chinensis Blkr. 425, I20.

Dasybatis isotrachys Blkr. 497, 4 .

Dasybatis kenojei Blkr. 425, I20. 497, 4 .

Dasybatis Meerdervoorti Blkr. 497, 4.

Dasybatis undulata Blkr. 425, I20.

Datnia C. $V .468,267$.

Datnia ambigua Rich. 119, 6. 468, 267.

Datnia argentea $C . V .13,52.25,7.91,32.129,439.270$, I 8.364 , 28o. 369, 42.425, I39. 468, 267. cf. Therapon (Datnia) argenteus Blkr.

Datnia Bennetti Blkr. 91, 32 .

Datnia cancellata $C . V .13,4.129,438.275,24.364,280$. cf. Therapon (Datnia) cancellatus Blkr.

Datnia cancellatoïdes Blkr. 76, 247. 129, 439. cf. 'Therapon (Datnia) argenteus Blkr.

Datnia caudivittata Rich. 119, 6.

Datnia elliptica Rich. 119, 6.

Datnia micracanthus Blkr. 275, 55. cf. Therapon (Datnia) micracanthus Blkr.

Datnia plumbea Kner cf. Therapon (Datnia) plumbeus Blkr.

Datnia polota Cant. cf. Datnioïdes polota Blkr.

Datnia Rosenbergii Blkr. 283, 237. 358, 186. cf. Therapon (Datnia) Rosenbergii Blkr.

Datnia virgata $C . V .91,32$. cf. Therapon (Datnia) theraps Blkr.

Datniaeformes 468,266 .

Datnioides Blkr. 468, 272.

Datnioïdes microlepis Blkr. 85, 442. 239, тог. 272, I I. 356, I73.

Datnioides polota Blkr. 85, 441. 91, 32. 191, I. 239, I01. 270, I9. 272, I I. 356, х73. 468, 272.

Darnioides quadrifasciatus Blkr. 468, 272.

Davalla 306, ro8.

Decapterus Blkr. 128, 4I 7. 297, I36.

Decapterus fasciatus Blkr. 425, I3I.

Decapterus kiliche Blkr. 91, 44 .

Decapterus kurra Blkr. 37, 358. 46, 213. 57, 50. 91, 44. 124, 303 . 275, 39 .

Decapterus kurroïdes Blkr. 128, 420.

Decapterus lajang Blkr. 124, 302. 315, 235. 364, 289.

Decapterus macrosoma Blkr. 37, 358. 57, 87, 167, 372. 275, 39.

Decapterus maruadsi Blkr. 118, 100. 124, 303. 411, 252. 425, I31. 497,14 . 
Decapterus muroadsi Blkr. 118, Ior. 425, I31. 497, I4.

Decodon Gthr. 292, I05.

Deltentosteus Gill 453, 316, 3r7.

Dendrochirus Srons. 473, 296.

Dentex C. $V .$, Blkr. 1, 553. 438, 40.468, 336.

Dentex argyrozona $C . V .266,59$.

Dentex balinensis Blkr. 231, I 55. 441, 38 .

Dentex Blochii Blkr. 44, 176. 91, 38. 127, 345. 227, 406. 270, 2 I. 441, 27. cf. Dentex japonicus blkr.

Dentex celebicus Blkr. 109, 245. 275, 27. 441, I9.

Dentex cynodon $C: V .46,215$.

Dentex fasciatus Sol. 90, 26.

Dentex filamentosus C. $V .468,279.495,12$.

Dentex (Heterognathodon) filamentosus Stcind. cf. Dentex filamentosus $C . V$.

Dentex furcosus $C . V .91,38.364,283.441$, I 2 .

Dentex gracilis Blkr. 438, 42. 441, 34 .

Dentex griseus T. Schl., Blkr. 106, 80. 118, 88. 227, 406. 231, 145. 270, 2 r. 438, 42. cf. Gymnocranius griseus, lethrinoïdes Blkr., Synagris griseus Blkr.

Dentex guliminda Blkr. 91, 38. 288, $7 x$.

Dentex hasta C. $V .91,38$. cf. Sparus Cuvieri, Schlegeli, Blkr.

Dentex hexodon $Q . G .320,268.441$, II.

Dentex hypselognathus Blkr. 438, 4I. 441, 9.

Dentex hypselosoma Blkr. 102, 402. 118, 89. 253, 234. cf. Synagris hypselosoma Blkr.

Dentex isacanthus Blkr. 438, 4r. 441, I3.

Dentex jahngarah Blkr. 91, 38 .

Dentex japonicus Blkr. 356, 173. 411, 25 1. 425, I38.

Dentex lethrinoides Blkr. 19, I I. 31, 102. cf Dentex griseus T. Schl. Gymnocranius lethrinoides Blkr., Lobotes microprion Blkr.

Dentex luteus C. $V .91,38$.

Dentex lycogenis Benı. cf. Gnathodentex aurolineatus Blkr.

Dentex macrocephalus $C . V .266,52$.

Dentex marginatus $C . V .70,725.441$, II.

Dentex marmoreus Sol. cf. Saurus synodus $C$. $V$.

Dentex mesoprion Blkr. 76, 255. 270, $21.287,46.441,30$.

Dentex metopias Blkr. 163, 51.441, I6.

Dentex microdon Blkr. 46, 2 I9. 275, 27. 438, 42. cf. Gymnocranius microdon Blkr.

Dentex mulloides Blkr. 67, 576. 270, 21. 364, 283.441, 22.

Dentex nebulosus Sol. cf. Saurida nebulosa $C$. $V$.

Dentex nematophorus Blkr. 86, 500. 156, 21 4. 270, 21. 441, 23.

Dentex nematopus Blkr. 46, $219.275,27.441,29$.

Dentex nemurus Blkr. 163, 49. 316, 242. 396, 299. 441, I8. 
Dentex obtusis $v$. Hass. cf. Dentex tolu $C . V$.

Dentex obtusus $S$. Mill. 10, 66, 68, 46, 2 1 2. 275, 27 .

Dentex Ovenii Blkr. 109, 246. 275, 27. 441, I4.

Dentex Peronii $C . V .70,725.441$, II.

Dentex pristipoma Blkr. cf. Aprion (Aprion) pristipoma Blkr., Chaetopterus pristipoma $B l k r$.

Dentex rivulatus Rüpp., Klunz. 46, 215. cf. Gymnocranius rivulatus Kluns.

Dentex ruber C. $V .19$, I2. 25, 8. 46, 216. 156, 214. 275, 27. 396, 297. 441, 9.

Dentex ruber Blkr. (nec C. $V$.) cf. Dentex gracilis Blkr.

Dentex rupestris $C . V$. 266, 59 .

Dentex setigerus $C . V$. T.Schl.90, 32. 253, 235. cf. Dentex sinensis Blkr.

Dentex sinensis Blkr. 425, I38.441, 25.497, 7 .

Dentex striatus $C . V .91,38$.

Dentex sumbawensis Blkr. 237, 439. 441, 35 .

Dentex sundanensis Blkr. 438, 42. 441, I5.

Dentex taeniopterus $C . V$. 19, Iт. 25, 8. 46, 216. 270, 21. 275, 27. 287, 46. 356, 173. 364, 283. 411, 25 I. 441, 7.

Dentex tambuloïdes Blkr. 78, 465. 275, 27. 441, 31.

Dentex tambulus C. V. 19, 12. 40, 478. 52, 89. 185, 222. 228, 424 . $235,364.275,27$.

Dentex tambulus Blkr. (nec $C$. $V$.) cf. Dentex sundanensis Blkr.

Dentex Thunbergii $C$. $V$. cf. Synagris Thunbergii Blkr.

Dentex tolu C. $V$. 19, I3. 51, 56. 56, 444. 90, 33. 91, 38. 237, 436. 270, 2 I. 441, 20. 491, 43 .

Dentex upeneö̈des Blkr. 70. 725. 441, 33 .

Dentex vulgaris Cuข. 438, 40.468, 278 .

Dentex waigiensis Q. G. cf. Lutjanus Quoyi Blkr.

Dentex xanthopterus Blkr. 2, 522. cf. Dentex lethrinoïdes Blkr., Gymnocranius lethrinoides Blkr.

Dentex zysron Blkr. 156, 2 I9. 237, 436. 441, 37 .

Denticini 468, 278 .

Dermatolepis Gill 468, $25^{8}$.

Dermogenys pusilla $K$.. . H. cf. Hemiramphus fluviatilis Blkr.

Dermogenys pusillus $K$. v. H. 375, I65.

Dermogenys sumatranus Blkr. 375, 167 .

Deuteropterus Gill 468, 275.

Devario Heck. 261, 433. 314, 2 1 4. 328, 258. 329, 264.

Diabasis Desm. 468, 27 I, 336.

Diacope $C . V .1,553.468,276$.

Diacope alboguttata $C$. $V$. cf. Lutjanus alboguttatus Blkr., Lutjanus rivulatus $B l k r$.

Diacope analis $C$. $V$. cf. Lutjanus analis Blkr., Lutjanus flavipes Blkr. Diacope angulus Benn. cf. Lutjanus coeruleovittatus Blkr. 
Diacope annularis $K u h l(R \ddot{p} p p$.$) cf. Lutjanus chirtah B l k r$., Mesoprion annularis $C \cdot V$.

Diacope argentimaculata $C$. $V$. cf. Lutjanus argentimaculatus $B l k r$.

Diacope aurantiaca $C$. $V$. cf. Lutjanus flavipes $B l k r$.

Diacope axillaris $C . V$. cf. Lutjanus butonensis Blkr., Lutjanus marginatus Blkr.

Diacope bitaeniata $C . V .10,66,68.438,44$. cf. Lutjanus lunulatus Bl. Schn., Mesoprion bitaeniatus Blkr.

Diacope bohar $C$. $V$. cf. Lutjanus bohar Bl. Schn. $\left(B l k r_{0}\right)$.

Diacope bottonensis $C$. $V$. cf. Lutjanus butonensis Blkr., Mesoprion bottonensis Blkr.

Diacope Calveti $C . V ., Q . G$. cf. Lutjanus malabaricus Blkr., Mesoprion Calveti Blkr.

Diacope civis C. $V$. cf. Lutjanus civis Blkr.

Diacope coeruleopunctata $C$. $V$. cf. Lutjanus rivulatus $B l k r$.

Diacope coeruleopunctatus $C$. $V$. cf. Mesoprion coeruleopunctatus Blkr.

Diacope coeruleovittata C. $V$. cf. Lutjanus coeruleovittatus Blkr.

Diacope decemlineata $C$. $V$. cf. Lutjanus quinquelineatus Blkr., Mesoprion octolineatus Blkr.

Diacope dondiawah $C . V$. cf. Mesoprion Russellii Blkr.

Diacope duodecimlineata $C$. $V$. cf. Lutjanus duodecimlineatus Blkr., Lutjanus octovittatus Blkr.

Diacope Ehrenbergii Klunz. cf. Lutjanus oligolepis Blkr.

Diacope flavipes $C . V$. cf. Lutjanus flavipes Blkr.

Diacope fulviflamma Rüpp., $C$. $V$. cf. Lutjanus fulviflamma $B l k r$., Mesoprion fulviflamma Blkr., monostigma $C . V$.

Diacope immaculata $C$. $V$. cf. Lutjanus marginatus Blkr., Mesoprion immaculatus Blkr.

Diacope kasmira Klunz. cf. Lutjanus bengalensis Blkr.

Diacope lineata $Q$. G. cf. Lutjanus lineatus Blkr.

Diacope lineolata Rupp. 438, 43. cf. Lutjanus erythropterus Bl., Mesoprion lineolatus Blkr.

Diacope macolor $C$. $V$. cf. Lutjanus macolor Blkr., Mesoprion macolor Blkr.

Diacope marginata $C . V$. 13, 44. cf. Lutjanus marginatus Blkr., Mesoprion marginatus $B l k v$.

Diacope marginatus C. V. 2, 526 .

Diacope melanura Rüpp. cf. Lutjanus butonensis Blkr.

Diacope metallicus $K . \% . H ., C . V .2,524,525$. cf. Mesoprion sanguineus Blkr.

Diacope miniata C. $V$. cf. Epinephelus miniatus Blkr.

Diacope monostigma C. $V$, Kluns. cf. Lutjanus fulviflamma Blkr., Lutjanus lioglossus Blkr.

Diacope nigra $C . V$. cf. Lutjanus macolor Blkr. 
Diacope notata $C$. $V$. 10, 66. cf. Mesoprion notatus, Russellii, Blkr. Diacope notatus $C . V .2,526$.

Diacope olivaceus $C . V$. 2, 526 .

Diacope octolineata $C$. $V$. cf. Lutjanus bengalensis Blkr., Lutjanus quinquelineatus $B l k r$. , Mesoprion octolineatus $B l k r$.

Diacope octolineatus $C . V .2,526$.

Diacope octovittata $C$. $V$. cf. Lutjanus bengalensis Blkr.

Diacope quadriguttata $C$. $V$. cf. Lutjanus bohar Bl. Schn. (Blkr.), Mesoprion quadriguttatus Blkr.

Diacope rivulata $C . V$. cf. Lutjanus alboguttatus Blkr., Lutjanus rivulatus $B l k r$., Mesoprion rivulatus $B l k r$.

Diacope rivulatus $C . V .2,526$.

Diacope rufolineata $C$. $V$. cf. Lutjanus amboinensis $B l k r .438,44$.

Diacope rufolineatus $C$. $V$. cf. Mesoprion rufolineatus $B l k r$.

Diacope sanguinea (Ehr.) C. $V$. cf. Mesoprion sanguineus Blkr.

Diacope Sebae C. $V$. 2, 526. 10, 66. cf. Lutjanus Sebae Blkr., Mesoprion Sebae Blkr.

Diacope siamensis $C . V$. cf. Lutjanus Sebae Blkr.

Diacope sparus Schl. cf. Lutjanus sparus Blkr.

Diacope striata $C . V ., Q . G$. cf. Lutjanus lineatus Blkr., Mesoprion striatus Blkr.

Diacope timoriensis Q.G. cf. Lutjanus malabaricus Blkr.

Diacope vaigiensis $Q . G$. cf. Diacope waigiensis $Q . G$., Lutjanus waigiensis Blkr.

Diacope vitianus Hombr. Jacq. cf. Lutjanus amboinensis Blkr.

Diacope vitta Schl. cf. Lutjanus vitta Blkr., Mesoprion vitta Blkr.

Diacope waigiensis Q.G. cf. Lutjanus marginatus $B l k r$.

Diacope xanthopus $C . V .13,44$. cf. Lutjanus marginatus Blkr., Mesoprion marginatus $B l k r$.

Diacope xanthozona $K . v . H .2,526$. cf. Mesoprion unimaculatus $Q . G$.

Diagramma Cuv. 1, 553. 468, 27 I.

Diagramma affine Gthr. cf. Plectorhynchus crassispina Blkr.

Diagramma albovittatum Rïpp. 143, 46. 275, 25. cf. Plectorhynchus albovittatus Blkr.

Diagramma balteatum $K . v$. $H$. cf. Diagramma punctatum Ehr., Plectorhynchus pictus Blkr.

Diagramma Blochii C. V.91, 38. 288, 7 I.

Diagramma centurio $C$. $V$. cf. Plectorhynchus pictus Blkr.

Diagramma chaetodonoides Blkr. 275, 25. 287, 45. cf. Plectorhynchus chaetodontoides Lac.

Diagramma chrysotaenia Blkr. 137, 303. 275, 25. 287, 45. cf. Plectorhynchus chrysotaenia Blkr.

Diagramma cinctum T.Schl. 90, 30. cf. Plectorhynchus cinctus Blkr.

Diagramma cinerascens $C$. $V$. cf. Diagramma punctatum Ehr., Plectorhynchus pictus $B l k r$. 
Diagramma crassispinum Rüpp. 2, 523. 18, 26. 25, 8. 55, 4Ir. 56, 444. 157, 232. 184, 299. 270, 2 1. 272, Ix. $275,25.287,45.288$, 7x. cf. Plectorhynchus crassispina Blkr.

Diagramma Cuvieri Playf. cf. Plectorhynchus Cuvieri Blkr.

Diagramma foetela $C$. $V$. cf. Plectorhynchus foetela Blkr.

Diagramma gaterina $C . V$. cf. Plectorhynchus gaterina Blkr.

Diagramma Goldmani Blkr. 275, 25.

Diagramma Goldmanni Blkr. 79, 602. cf. Plectorhynchus Goldmani Blkr.

Diagramma griseum $C . V$. cf. Plectorhynchus griseus $B l k r$.

Diagramma haematochir Blkr. 96, I75. cf. Plectorhynchus Goldmani Blkr.

Diagramma japonicum Blkr.90, 3 I. cf. Parapristipoma trilineatum Blkr.

Diagramma Lessonii C. $V .78,463$. cf. Plectorhynchus Lessoni Blkr.

Diagramma lineatum $C . V .72$, I12.91, 38. 167, 37 1. 184, 299. 275, 25. 309, I55. cf. Plectorhynchus lineatus Blkr.

Diagramma lineatum Rüpp. (nec $C$. $V$.) cf. Diagramma punctatum $E h r .$, Plectorhynchus pictus Blkr.

Diagramma nigrum Day cf. Plectorhynchus crassispina Blkr.

Diagramma ocellatum $K$. $v$. H. cf. Plectorhynchus pictus Blkr.

Diagramma orientale $C . V .17,3.18,23.91,38.231,145.236,330$. 270, $21_{\text {\& }}$ cf. Plectorhynchus orientalis Suns.

Diagramma pardalis $(K . v . H) \quad. C . V .2,523$. cf. Diagramma plectorhynchos $C . V$., Plectorhynchus chaetodontoides Lac.

Diagramma pica $C$. $V$. cf. Plectorhynchus orientalis Szons.

Diagramma pictum C. $V .2,523.287,45$. cf. Diagramma punctatum Elor., Plectorhynchus pictus Blkr.

Diagramma plectorhynchos $C . V .18,24.51,56$.

Diagramma plectorhynchus $C . V .2,523$. cf. Plectorhynchus chaetodontoides $L a c$.

Diagramma poecilopterum C. V. 91, 38 . cf. Plectorhynchus pictus, poecilopterus, Blkr.

Diagramma poecilopterus $C$. cf. Plectorhynchus pictus Blkr.

Diagramma polytaenia Blkr. 71, 755. 275, 25. 330, 266. cf. Diagramma polytaenioïdes Blkr., Plectorhynchus polytaenia Blkr.

Diagramma polytaenioides Blkr. 101, 377. of. P'lectorhynchus polytaenia, polytaenioïdes, Blkr.

Diagramma porosa Rich. 119, S.

Diagramma punctatissimum Playf. cf. Plectorhynchus punctatissimus Blkr.

Diagramma punctatum (E/hr) C. V. 18, 25. 46, 21 2. 50, 47 I. 51, 56 . $54,235.56,444.91,38.126,344.156,214.227,407.234,352$. 270, 22. 275, 25. 288, 7I. 296, 106. cf. Plectorhynchus pictus (Ellr.) Blkr.

Diagramma punctulatum $C . V .2,522$. 
Diagramma radja Blkr. 84, 336. 184, 299. 275, 25. 309, I 55. cf. Plectorhynchus radja Blkr.

Diagramma reticulatum Gthr. of Plectorhynchus reticulatus $B l k r$.

Diagramma Sebae Blkr. 18, 24. 47, 226. 157, 232. 170, 479. 270, 22. cf. Plectorhynchus Cuvieri Blkr.

Diagramma Sibbaldi Benn. cf. Plectorhynchus orientalis Swns.

Diagramma Thunbergii Cuv. cf. Plectorhynchus pertusus Blkr.

Diapterus Ranz. 468, 285, 336.

Diapterus abbreviatus Blkr. 358, I86. 364, 279. 395, 291. 396, 299. $440,248$.

Diapterus acinaces Blkr. 440, 242.

Diapterus argyreus Blkr. 396, 297. 440, 245. 459, 92.

Diapterus decacanthus Blkr. 349, 58. 425, x40.

Diapterus equula Blkr. 425, I40. 497, 8 .

Diapterus filamentosus Blkr. 315, 23r. 316, 241. 320, 269. 364, 279. 396, 299. 425, I $40.440,237.491,43.495$, I 2 .

Diapterus japonicus Blkr. 349, 59. 425, I40. 497, 8 .

Diapterus kapas Blkr. 320, 269. 364, 279. 395, 291. 440, 246.

Diapterus limbatus Blkr. 440, 249.

Diapterus lineolatus Blkr. 459, 93.

Diapterus macracanthus Blkr. 440, 239.

Diapterus macrosoma Blkr. 315, 231. 316, 241. 320, 269. 338, 360. 364, $279.396,299.440,244$.

Diapterus oyena Blkr. 315, 232. 320, 269. 354, I46. 364, 279. 440, 252. 459, 93. 495, I 2 .

Diapterus philippinus Blkr. 440, 242.

Diapterus poetie Blkr. 338, 360. 364, 279. 395, 291. 440, 250.459, 93.

Diapterus punctatus Blkr. 425, I 40. 440, 24 I.

Diapterus Richii Blkr. 459, 93.

Dicentrarchus Gill 468, 263.

Dicerobatis eregoodo Cant. 91, 82 .

Dicerobatis japonica Blkr. 497, 4

Dicerobatis Kuhlii Blkr., Gthr. 91, 82. 459, 69.495, 8.

Dicotylichthys punctulatus $K p .266,57.459,69.495,23$.

Dictyosoma Bürgeri v. d. Hoeven 93, 9. 497, 20.

Dictyosoma Temminckii Blkr. 90, 42.

Dillonia Heck. 261, 427. 314, I96.

Dinematichthys iluocoeteoïdes Blkr. 125, 319. 156, 215. 157, 233. 182, I 59. 184, 20r. 270, 44.

Diodon L. 1, 552. 2, 506. 367, I7.

Diodon atinga $L$. $(B l$.$) 266, 57. 270, 66. cf. Diodon punctatus Cur.$

Diodon atringa $L .315,229.318,249.320,264$.

Diodon coeruleus Q. G. 368, 33. 491, 56 .

Diodon hystrix $L$. $(B l$.$) 275, I2. cf. Diodon punctatus Cuv., Para-$ diodon hystrix Blkr. 
Diodon maculifer $K p$. 266. cf. Paradiodon maculifer $B l k r$.

Diodon mola Pall. cf. Molacanthus mola Blkr.

Diodon nicthemerus Cu\%. 119, 25. cf. Diodon orbicularis $B 1$.

Diodon novemmaculatus Cur\%, Schl. 66, 567. 156, 21 S. 157, 236. cf. Paradiodon maculatus, novemmaculatus Blkr.

Diodon orbicularis $B l .80,92.266,57.364,27$ I. 368, 33. 394, $2 S_{5}$. $459,70$.

Diodon punctatus $L_{.}, C$ Cur. 47, 228. 54, 238. 62, 19. 157, 236. 358, 183. cf. Diodon atringa $L$.

Diodon quadrimaculatus Cuv. 163, 94. 288, 68.

Diodon reticulatus Blkr. 459, I03.

Diodon sexmaculatus Cuv. 266, 57. 270, 66. 275, I2. 287, 39. 320, $265.338,360.339,362.354,143$.

Diodon tigrinus Cuv. 459, 70. 497, 26.

Diodon triedricus Cant. cf. Diodon orbicularis $B /$.

Dionda Gir. 261, 426. 314, 206.

Dioplites Raf. 468, 26 I.

Diphreutes Cant. 468, 304 .

-Diphreutes chrysostoma Blkr. 358, rS9. 364, 287 .

Diphreutes chrysostomus Blkr. 315, 234. cf. 'Taurichthys chrysostomus Blkr.

Diphreutes macrolepidotus Cant. 234, 315. 358, IS9. 364, 2S7. 394, 288. cf. Heniochus macrolepidotus C. $V$., Taurichthys macrolepidotus Blkr.

Diphreutes varius Bl., Blkr. 358, I90. 364, 287. cf. Taurichthy's varius $C . V$.

Diphreutes viridis Blkr. 364, 287. cf. Taurichthys varius C. $V$.

Diplectrum Holbr. 468, 255.

Diplocheilichthys Blkr. 261, 423. 314, I94.

Diplocheilichthys pleurotaenia Blkr. 270, 5 r.

Diplocheilos erythropterus $v$. Hass. cf. Labeo erythropterus $\mathrm{Val}$.

Diplocheilus v. Hass. 314, I94.

Diplodus Raf. 468,283 .

Diplogrammus goramensis Gill cf. Synchiropus goramensis Blkr.

Diplolepis Stcind. 468, 329.

Diplomystes C. Dum. 306, 92 .

Diplomystes papillosus Dum, 306, 92.

Diploprion K. v. H. 1, 553. 468, 259.

Diploprion bifasciatum $K . v . H .10,66.13,3.46,2$ Ir. 98, 20 S. 118, 59. 157, 232. 275, 30. 287, 47. 320, 269. 354, I 47. 364, 284. 395, 292. 425, 139. 451, 124. 468, 259. 497, 6.

Diplopriontini 468, 259.

Diplopterus pulcher Gray'. cf. Luciocephalus pulcher Blkr.

Diprotacanthus Blkr. 292, 104.

Diproctacanthus xanthurus Blkr. 293, 295. 358, IS5. 364, 277. 
Dipterodon Lac., Cuv. 468, 276, 294.

Dipterodon capensis $C . V .266,63.468,294$.

Dipterodon hexacanthus Lac. cf. Amia aurea Blkr.

Dipterygonotini 468, 274. 470, r 53 .

Dipterygonotus Blkr. 10, 70. 468, 275.470, I54.

Dipterygonotus leucogrammicus Blkr. 10, 7 I. 445, I58.468, 275. 470, I54. cf. Emmelichthys leucogrammicus Blkr.

Dischistodus annulatus Blkr. 483, 40. 485, 89.

Dischistodus bifasciatus Blkr. 485, 88 .

Dischistodus Cartieri Blkr. 485, 82 .

Dischistodus chrysopoëcilus Blkr. 483, 40. 485, 84 .

Dischistodus fasciatus Gill 485, 86.

Dischistodus notophthalmus Blkr. 483, 40. 485, $\mathrm{S}_{2}$.

Dischistodus prosopotaenia Blkr. 483, 40. 485, So.

Dischistodus trimaculatus Blkr. 483, 40. 485, 79 .

Discognathichthys Blkr. 261, 423. 314, I91. 326, 242. 329, 262.

Discognathus Heck. 261, 423. 314, 191. 329, 262.

Ditrema Schl. 297, 141. 468, 29 .

Ditrema brevipinna Gthr. 468, 290.

Ditrema Temminckii Blkr. 90, 33. 468, 291. 497, S.

Doidyxodon $\mathrm{Val}$. 468, 294.

Doidyxodon Freminvillei $\mathrm{Val} .468,294$.

Doras Lac. 306, 84 .

Doras affinis Kner cf. Amblydoras affinis Blkr.

Doras asterifrons $K n e r^{\circ} \mathrm{cf}$. Astrodoras asterifrons Blkr.

Doras Blochii $\mathrm{Val}$. cf. Acanthodoras cataphractus Blkr.

Doras brevis Kner 303, I3.

Doras carinatus Lac. 303, 13. 306, 84. 336, 3 I.

Doras (Oxydoras) carinatus Kner cf. Doras carinatus Lac.

Doras cataphractus $\mathrm{Val}$. cf. Acanthodoras cataphractus Blkr.

Doras costatus Lac. cf. Platydoras costatus Blkr.

Doras dentatus Kner cf. Platydoras costatus Blkr.

Doras fimbriatus Kner 303, I3.

Doras granulosus $\mathrm{Val}$. cf. Pterodoras granulosus Blkr.

Doras Humboldti $A g$. Oxydoras niger Blkr.

Doras humeralis Kner 303, I3.

Doras lipophthalmus Kner 303, I3.

Doras lithogaster Heck. cf. Lithodoras lithogaster Blkr.

Doras maculatus $V a l$. cf. Pterodoras granulosus Blkr.

Doras niger $V a l$. cf. Oxydoras niger Blkr.

Doras (Oxydoras) niger Kner cf. Oxydoras Kneri Blkr.

Doras (Oxydoras) Orbignyi Kröy. cf. Rhinodoras Orbignyi Blkr.

Doras oxyrhynchus $V a l$. cf. Doras carinatus Lac.

Doras polygramma Heck. cf. Acanthodoras cataphractus Blkr.

Doras punctatus Kner 303, I3. 
Doras stenopeltis Kner 303, 13.

Doratonotus Gthr. 292, 102.

Dormitator Gill 453, 305.460, 103, 106.

Dormitator lineatus Gill 453, 305.

Dorosoma chacunda Blkr. 395, 294.

Dorosoma chrysopterus Blkr. 425, r4S.

Dorosoma maculatum Blkr. 425, 148 .

Dorosoma nasus Blkr.497, 25.

Dorosoma Osbecki Blkr. 425, I48.

Dorosoma punctatum Blkr. 425, I48. 497, 25 .

Dorosoma triza Blkr. 425, I48.

Dorsuarius Comm. 468, 293.

Doryichthys auronitens $K p .275, I 4$.

Doryichthys Bernsteini Blkr. 382, 39 S.

Doryichthys boaja Gthr. 425, г26.

Doryichthys brachysoma Blkr. 459, 75. cf. Doryichthys Valenciennii Gthr.

Doryichthys brachyurus Gthr. 459, 75 .

Doryichthys deokhatoides Blkr. 270, 72. 272, 8 .

Doryichthys excisus $K p .495$, I 6 .

Doryichthys manadensis Blkr. 270, 72. 275, I3.

Doryichthys millepunctatus $K p .459,75$.

Doryichthys spinosus $K p . \mathbf{2 7 2}, 8 . \mathbf{2 7 5}$, I4. cf. Doryichthys boaja Gthr.

Doryichthys Valenciennii Gthr. 495, I6. cf. Doryichthys brachysoma Blkr.

Doryramphus melanopleura Blkr. 270, 72.

Drepane Cuv. 1, 553. 468, 3०1.

Drepane longimana $C . V .2,520.22,23.25,8.32$, I60. 45, 195. 46, 212. 49, 419. 51, 56. 55, 412. 56, 445. 468, 3ог. cf. Harpochirus punctatus Cant.

Drepane punctata C. V. 2, 520. 16, 4. 25, 8. 91, 40. 119, 9. 156, 2 I 4. 158, 274. 227, 407. 231, I 46. 270, 27. 272, II. 275, 29. 287, 47. 288, 72. 296, 107. 309, г 55. 468, 301. cf. Drepane longimana C. $V$., Harpochirus punctatus Cant.

Drepanis C. $V .468,30 \mathrm{I}$.

Dules C. V. 468, 254 .

Dules argenteus Klunz. cf. Moronopsis taeniurus Gill.

Dules auriga $C \cdot V .468,254$.

Dules Bennetti Blkr. 91, 32. cf. Moronopsis taeniurus Gill.

Dules caudavittatus $C$. $V$. cf. Moronopsis caudavittatus Blkr.

Dules fuscus $C$. $V$. cf. Moronopsis fuscus $B l k r$.

Dules guamensis $C . V$. cf. Moronopsis rupestris Gill.

Dules leuciscus Jen. cf. Moronopsis ciliatus Blkr.

Dules maculatus C. $V .10,66,68.13,4.46,2$ I . 84, 333. 270, I6. 275, 24. 296, Iс6. 354, I46. cf. Moronopsis ciliatus Blkr. 
Dules malo $C . V$. cf. Moronopsis ciliatus Blkr.

Dules marginatus $C . V .13,4.67,373.156,213.231$, I44. 270, I6. 271, 2. 275, 24. 276, 65. 354, I46. cf. Moronopsis ciliatus Blkr.

Dules rupestris C. $V .98,209.296$, го6. 354, I46. cf. Moronopsis rupestris Blkr., Gill.

Dules taeniurus $C . V .13,49.25,7.236,333.270$, I6. 354, I46. cf. Moronopsis taeniurus Gill, Paradules taeniurus Blkr.

Dules vanicolensis $C$. $V$. cf. Moronopsis rupestris Gill.

Dussumieria acuta $C . V .51,59.90,49.91,72.270,56.288,79$. $390,275.425,147.496,3$.

Dussumieria elopoides Blkr. 296, i I2. 364, 294. 395, 294. 396, 300.

Dussumieria elopsoides Blkr. 16, I2. 25, I1. 39, 42 I. 59, I2. 127, 345. 235, 372. 270, 56. 425, I 47 .

Dussumieria Hasseltii Blkr. 39, 422. 59, I3. 127, 345. 227, 408. 270, 56. 275, 48. 287, 60. 296, II2. 316, 243. 364, 294. $382,398$.

Ditymaeria Blkr. 142, 52, 289, 81. 292, 95.

Duymaeria amboinensis Blkr. 142, 54. 293, 277.

Duymaeria aurigaria Blkr. 142, 53. 425, I35.

Duymaeria enneacanthus Blkr. 142, 54. 293, 277.

Duymaeria flagellifer Blkr. 142, 53 .

Duymaeria japonica Blkr. 142, 53. 497, 5 .

Duymaeria nematopterus Blkr. 142, 54. 293, 277.

Duymaeria rubellio Blkr. 142, 53.

Duymaeria spilogaster Blkr. 142, 54. 497, 5 .

Echelus microchir Blkr. 348, 40. 352, I25.

Echeneis 1, 552. 2, 508 .

Echeneis albescens T. Schl. 232, 20. 491, 50. 497, I5. cf. Remora albescens $B l k r$.

Echeneis borboniensis Guich.459, 98.

Echeneis brachyptera Lowe 497, I5. cf. Remora brachyptera Blkr.

Echeneis lophioides Guich. 459, 98 .

Echeneis lunata Bancroft cf. Echeneis neucrates $L$.

Echeneis naucrates $L$. (Lac.) 459, 98. cf. Echeneis neucrates $L$., Leptecheneis naucrates Gill.

Echeneis neucrates $L .51,58.55,414.59,22.91,52.119$, I2. 127, 345. 156, 2 I5. 157, 234. 222, 317. 231, I48. 235, 370. 236, 33 I. $270,44.272$, I 7. 275, 46. 288, 77. 302, го6. 308, І 52. 309, г 56 . 315, 236. 316, 242. 354, I 50. 358, 192. 364, 294. 395, 293. 398, 306. 425, г32. 437, 37. cf. Leptecheneis neucrates Gill.

Echeneis Nieuhofii Blkr. 76, 279. 270, 44.

Echeneis pallida Schl. cf. Echeneis brachyptera Lowe.

Echeneis remiligo $A$. Dum. 459, 99.

Echeneis remora L., Bl. 131, 71. 266, 55. 459, 99. 495, is. cf. 
Echeneis borboniensis Guich., Echeneis remiligo A. Dum., Remora remora Blkr.

Echeneis remoroïdes Blkr. 131, 70. 157, 234.

Echeneis vittata Lotve cf. Echeneis neltcrates $L$.

Echidna $J$. R. Forst. 351, $12 \mathrm{I}$.

Echidna amblyodon Blkr. 308, I 52. 317, 247. 320, 272. 352, r 30. 354, I 5 ०. $364,296$.

Echidna catenata Blkr. 362, 242.

Echidna delicatula Blkr. 317, 247. 352, I30. 354, I 50. 364, 297 :

Echidna dizona Blkr. 317, 247. 320, 272.

Echidna polyzona Blkr. 317, 247. 352, r 30.354, I50. 358, I93. $364,297$.

Echidna rhodochilus Blkr. 317, 247. 352, I30. 354, I50.

Echidna variegata Forst. 317, 247. 318, 252. 320, 272. 352, 130. 354, I5I. 358, I93. 364, 297. 394, 288. 425, 124. 459, 73. 491, $56.495,23$. cf. Muraena variegata Forst., Poecilophis variegata.

Echidna xanthospilos 317, 247.

Echidna xanthospilus Blkr. 352, г 30.

Echidna zebra Blkr. 352, I30. 354, I 5 I. 364, 297. 396, 301. 459, 73. 495,23 .

Echiichthys vipera Blkr. 291, II 7 .

Echinorhinus obesus Smith. 266, 58.

Echinorhinus spinosus $B p$. 266, $5^{8}$.

Echiopsis $K p .351$, II 9 .

Echiopsis intertinctus $K \phi$. cf. Ophichthys intertinctus Blkr.

Edelia Cast. 468, 334.

Edelia vittata Cast. 468,334 .

Elacate 1, 553 .

Elacate atlantica $C . V$. cf. Elacate nigra Gthr.

Elacate bivittata $C . V .2,5$ 16. 25, 8. 51, 57. cf. Elacate motta C.V., Elacate nigra $G t h r$.

Elacate canada Holbr. cf. Elacate nigra Gthr.

Elacate hexacantha 2, $5^{\text {r6. }}$

Elacate malabarica $C . V$. cf. Elacate motta $C . V$, nigra Gthr.

Elacate mottah C. $V .56,443.57,42.90$, ^. 91, 42 171, 245. 191, 2. 222, 3 17. 270, 28. 272, I3. 287, 52.288, 74. cf. Elacate nigra Gthr.

Elacate nigra Gthr. 296, I09. 302, 14. 364, 289. 395, 292. 425, I32. 459, 98. 497, I5.

Elacate pondiceriana $C . V$. cf. Elacate motta $C . V$.

Elapsopis $K p .351$, I 19 .

Elapsopis versicolor $K p$. cf. Ophichthys versicolor Blkr.

Elastoma Sions. 438, 45. 468, 276.

Elastoma oculatum Szons. 425, I37. cf. Etclis oculatus blkr.

Eleginus bursinus $C$ \% $119, \mathrm{~S}$.

Eleotriformes 453, 30x. 
Eleotrii $453,302.460,103$.

Eleotrini 453, 301. 460, 103 .

Eleotriodes Blkr. 184, 2 r2.

Eleotriodes cyanostigma Blkr. 188, 460. 354, I50.

Eleotriodes Hasseltii Blkr. 354, I50. cf. Valenciennesia Hasselti Blkr.

Eleotriodes Helsdingenii Blkr. 184, 2 I2. cf. Valenciennesia Helsdingenii Blk).

Eleotriodes heteropterus Blkr. 272, r 7. cf. Ptereleotris heteropterus Blkr.

Eleotriodes microlepis Blkr. cf. Ptereleotris microlepis Gill.

Eleotriodes muralis Blkr. 184, 20I. 267, I 40. 270, 4I. 275, 44. 285, 245. 287, 57. 296, II . 320, 271,358 , r92. 364, 293. cf. Valenciennesia muralis Blkr.

Eleotriodes periophthalmus Blkr. cf. Amblyeleotris periophthalmus Blkr. Eleotriodes sexguttata Blkr. 167, 372. 354, I50. 364, 293.

Eleotriodes sexguttatus Blkr. 184, 201, 234, 355. 270, 4r. 271, 2. 276, 65. 316, 242. cf. Valenciennesia sexguttata Blkr.

Eleotriodes strigata Blkr. 354, I50. 364, 293.

Eleotriodes strigatus Blkr. 275, 44. 287, 57. 318, 252. 320, 27x. cf. Valenciennesia strigata Blkr.

Eleotriodes taenionotopterus Blkr. 231, I48. cf. Asterropteryx taenionotopterus Blkr.

Eleotrioides Blkr. 450, 372. 453, 307 .

Eleotrioides cyanostigma Blkr. cf. Brachyeleotris cyanostigma Blkr.

Eleotris Gron. 1, 553. 453, 302.460, 103, 104.

Eleotris acanthopomus Blkr. 76, 275. 91, 106: cf. Culius melanosoma Blkr.

Eleotris amboinensis Blkr. 84. 343. cf. Butis amboinensis Blkr.

Eleotris aporos Blkr: 94, '59. 296, I I I. 309, г 56. 310, г6o. cf. Ophiocara aporus Blkr.

Eleotris aporus Blkr. 364, 293.

Eleotris belobrancha $C . V .10,67,68,15,3.46,213.81$, I67. cf. Belobranchus Quoyi Blkr.

Eleotris brachysoma Blkr. 425, I28, 152.

Eleotris brachyurus Blkr. 15, 20. 25, 9. 91, ro6. cf. Culius fuscus Blkr. Eleotris butis Cant., Gthr. 91, 50. cf. Butis butis, prismaticus, Blkr.

Eleotris canina Blkr. 15, 20. 16, 5. 25, 9. cf. Odonteleotris canina Blkr.

Eleotris cantherius Rich. cf. Eleotris oxycephalus Schl., Culius oxycephalus Blkr.

Eleotris Cantoris Gthr. 358, x92. 364, 293. cf. Ophiocara porocephalus Blkr.

Eleotris caperatus Cant. cf. Butis caperatus Blkr. Prionobutis koilomatodon $B l k r$.

Eleotris cyanostigma Blkr. 130, 452. 450, 375. 453, 306. 459, 103. cf. Brachyeleotris cyanostigma Blkr.

Eleotris cyprinoides $C . V ., B l k r .76,277.270,40.287,57$. cf. Asterropteryx cyprinoides, modestus, Blkr. 
Eleotris danius Blkr. 91, 50.

Eleotris dasyrhynchus Gthr. 453, 305. cf. Prionobutis dasyrhynchus Blkr.

Eleotris flammans Cant. 425, I28.

Eleotris fusca Gthr. cf. Culius fuscus Blkr.

Eleotris gobioïdes C. V. 119, 12. 453, 304.

Eleotris guavina $\mathrm{Val} .453,302$.

Eleotris gymnocephalus Steind. 453, 305.

Eleotris gymnopomus Blkr. 76, 274. cf. Butis gymnopomus Blkr.

Eleotris gyrinoïdes Blkr. 76, 272. 91, 105. 270, 40. 275, 44. 478, 20.

Eleotris gyrinus $C . V .91,105.453,302$.

Eleotris Hasseltii Blkr. 34, 253. 151, 4I2. 453, 3 IS.

Eleotris Helsdingenii Gthr. cf. Valenciennesia Helsdingenii Blkr.

Eleotris heterolepis Gthr. cf. Pogoneleotris heterolepis Blkr.

Fleotris heteropterus Blkr. 139, 422. 149, 103. cf. Ptereleotris heteropterus Blkr.

Eleotris Hoedti Blkr. 103, 496. 156, 21 5. 275, 44. 320, 27 г. 354, I50. 364, 293. 396, 300. cf. Ophiocara Hoedti Blkr.

Eleotris humeralis $C . V .15,22.16,5.25,9$. cf. Butis butis Blkr.

Eleotris ikeineur Montrouz. cf. Valenciennesia longipinnis Blkr.

Eleotris incerta $B l y t / 2$ cf. Culius fuscus Blkr.

Eleotris koilomatodon Blkr. 15, 21. 16, 5. 25, 9. cf. Prionobutis koilomatodon $B l k r$.

Eleotris kuak Thioll. cf. Ophiocara ophiocephalus Gill.

Eleotris lanceolata $B l$. Schn. cf. Apocryptes changua $C . V$.

Eleotris leuciscus Blkr. 76, 278.270, 40. cf. Asterropteryx leuciscus Blkr.

Eleotris lineato-oculatus Kner cf. Valenciennesia muralis Blkr.

Eleotris longipinnis Benn. cf. Valenciennesia longipinnis Blkr.

Eleotris macrodon Blkr. 91, I04. 453, 302.

Eleotris madagascariensis $\mathrm{Val}$. 4.22, Iо2. 459, 46, 78 .

Eleotris margaritacea $C . V .130,453$. cf. Ophiocara ophiocephalus Gill.

Eleotris marmorata Blkr. 55, 424. 191, 3. 270, 40. 272, I7. 356 , I74. 453, 303. cf. Oxyeleotris marmorata Blkr.

Eleotris mauritiana $B c m$. cf. Eleotris nigra $Q . G$.

Eleotris mauritianus Benn. cf. Culius fuscus Blkr.

Eleotris melanopterus Blkr.69, 706. cf. Butis butis, melanopterus, Blkr.

Eleotris melanosoma Blkr. 69, 705. 91, го6. cf. Culius melanosoma Blkr.

Eleotris melanostigma Blkr. 25, 9. 26, 3. 35, 261. 42, 59. 45, I95. 49, 419. 55, 412. cf. Butis melanostigma Blkr.

Eleotris melanurus Blkr. 15, 21. 25, 9. cf. Culius fuscus Blkr.

Eleotris microlepis Blkr. 149, ro2. 156, 2I 5. 453, 307. cf. P'tereleotris microlepis Gill.

Eleotris mogurnda Rich. 119, 12. 331, 448. 453, 303.

Eleotris muralis Q. G. 34, 25 . 54, 276. 91, 52. cf. Valenciennesia muralis Blkr. 
Eleotris niger $Q$. G. cf. Culius fuscus Blkr.

Eleotris nigra $Q . G ., C . V .15,3.91,105.453,303$. cf. Culius fuscus Blkr. Eleotris nudiceps Cast. 453, 3or.

Eleotris obscura Schl. 453, 305. cf. Odontobutis obscura Blkr.

Eleotris ophicephalus $K . v . H .2,5^{1} 3.15,22.25,9.185,224.250$, 206. 270, 40. 275, 44. 287, 57. 288, 77. 296, III. 354, I50. 358, I02. 364, 293 .

Eleotris ophiocephalus $K . v . H .425$, I $28.453,303.459,78$. cf. Ophiocara ophiocephalus Gill.

Eleotris oxycephala Schl. cf. Culius oxycephalus Blkr.

Eleotris oxycephalus Schl. 425, I28. cf. Culius oxycephalus Blkr.

Eleotris periophthalmus Blkr. 78, 477. 450, 374. 453, 307. cf. Amblyeleotris periophthalmus Blkr.

Eleotris porocephala $V a l$. cf. Ophiocara porocephalus $B l k r$.

Eleotris porocephaloides Blkr. 86, 5 II. 270, 40. 275, 44. cf. Ophiocara porocephalus Blkr.

Eleotris porocephalus C. $V .84,344.156,2$ I 5. 270, 40. 275, 44. 287, 57. 288, 77. cf. Eleotris ophiocephalus $K . v . H$.

Eleotris potamophilus Gthr. 425, I28.

Eleotris prismatica Blkr. 15, 23. 16, 5. 25, 9. cf. Butis prismaticus Blkr. Eleotris pseudacanthopomus $B l k r .76,276.91$, I06. cf. Culius fuscus Blkr.

Eleotris radiata $Q . G .119$, I 2 .

Eleotris seminudus Gthr. 453, 304.

Eleotris sexguttata C. $V .34,253.54,277.174,42$. cf. Eleotriodes sexguttata Blkr., Valenciennesia sexguttata Blkr.

Eleotris siamensis Gthr. 356, I 74 .

Eleotris sinensis Gthr. cf. Bostrychus sinensis Lac.

Eleotris soaresi Playf. 459, ro3. cf. Culius fuscus Blkr.

Eleotris strigata $C . V .15,3.142,48.170,478.450,372.453,307$.

Eleotris strigata Thioll. cf. Valenciennesia longipinnis Blkr.

Eleotris strigata Bl. Schn. cf. Valenciennesia strigata Blkr.

Eleotris taenioptera Gthr. cf. Belobranchus Quoyi Blkr.

Eleotris taenionotopterus Blkr. 159, 298. cf. Asterropteryx taenionotopterus Blkr.

Eleotris Tolsoni Blkr. 104, 542. 270, 4I. cf. Ophiocara Hoedti Blkr.

Eleotris urophthalmoïdes Blkr. 76, 273. 91, I05. 270, 41. 272, I7. cf. Oxyeleotris urophthalmoïdes Blkr.

Eleotris urophthalmus $B l k r .45, .202 .49,419.55,4$ I2. 91, 105. 272, I7. 356, I74. cf. Oxyeleotris urophthalmus Blkr.

Eleotris viridis Blkr. 15, 22. 16, 5. 25, 9. cf. Ophiocara ophiocephalus Gill.

Eleotris Wolffii Blkr. 34, 253. 35, 26I, 270. 42, 59. 45, 195. 49, 419. 55, 4r2. cf. Butis melanostigma Blkr.

Eleutheractis Cope 468, $25^{8}$.

Eleutherochir opercularioides Blkr.494, I03.

Eleutheronema tetradactylum Blkr. 297, r24. 
Eleutheronema tetradactylus Blkr. 296, I Iо. 356, х74. 395, 293. $425,143$.

Eleutheronema tridactylum Blkr. 297, I 24.

Elopichthys Blkr.261, 436. 314, 2 I2.

Elopichthys bambusa Blkr. 345, 27.415, x. 425, r44.

Elopichthys dahuricus 466,535 .

Elopichthys dauricus Blkr. cf. Elopichthys bambusa Ijlkr., Elopichthys dahuricus.

Elops 1, 552.

Elops cundinga Cant. cf. Megalops cyprinoides Blkr.

Elops cyprinoides Gm. cf. Megalops cyprinoides Blkr.

Elops inermis Mitch. cf. Elops saurus $L$.

Elops javanicus $K$. $v$. H. cf. Dussumieria elopsoides Blkr.

Elops machnata Forsk., Rich., Rüpp. cf. Elops saurus $L$.

Elops purpurascens Rich. cf. Elops saurus $L$.

Elops saurus $L .(C . V$.$) 2, 510. 10, 67. 16, 6. 25, I . 46, 214. 59,$ I 4. $91,72.266,56.275,48.287,60.425$, I 47.459 , Iо2. 495 , 22. 497,25 .

Embiotoca $A g .468,290$.

Embiotoca Jacksoni $A g .468,290$.

Embiotocoidei 468, 289 .

Emmelichthys Rich. 468, 275. 470, I 53 .

Emmelichthys leucogrammicus Blkr. 20, го. 31, 1о3. 46, 2 I2. 54, 235. 275, 28. cf. Dipterygonotus leucogrammicus Blkr.

Emmelichthys nitidus Rich. 119, 9.

Emmelichthys Schlegelii Rich.497, 8.

Enchelycore $K p .351,121$.

Enchelynassa $K p .351$, I 21 .

Enchelynassa Bleekeri $K p$. cf. Gymnothorax Bleckeri Blkr.

Enchelyopus $A$ g. 351, I I3.

Enchelyopus haumela Blkr. 296, xo9. 309, I56. 316, 242. 320, 270. 358 , I $90.364,289.395,292.396,300.411,252$.

Enchelyopus lepturus Blkr. 302, 73.

Enchelyopus muticus $B l \mathrm{kr} .425, \mathrm{r}_{3} \mathrm{r}$.

Enchelyopus savala Blkr. 395, 292. 425, I31. cf. Lepturus savala Blkr. Engraulis 1, $55^{2}$.

Engraulis balinensis Blkr. 17, I1. cf. Engraulis Russellii Blkr.

Engraulis boelama $C . V .459$, 102. 495, 22.

Engraulis breviceps Cant. 288, 79.

Engraulis brevifilis $C . V .91,74$.

Engraulis Brownii $C . V .51,59.55,414.56,446.60,39.91,74$. 119, I 5. 127, 345. 200, 48. 231, 150. 270, 58. 272, 26. 275, 49. 288, 79. 296, I I 2. cf. Stolephorus Browni Blkr., Commersonianus Lac.

Engraulis Commersonianus Rich. cf. Engraulis Brownii C. $V$., Stolephorus japonicus Blkr. 
Engraulis Commersonii Lac., Cuv. 2, 510. 16, 6. 25, Ix. cf. Engraulis Brownii $C . V$.

Engraulis crocodilus Blkr. 26, I 5. 35, 262. 42, 60. 45, 197. 49, 42 I. $55,414.60,35 \cdot 272,26$.

Engraulis Dussumierii $C . V .51,59.60,43.425,148$.

Engraulis encrasicholoïdes Blkr. 46, 2 I4. 53, 173. 54, $238.60,37$. 147, 469. 171, 245. 231, I 50. 270, 58. 275, 49. 296, I I 2 .

Engraulis encrasicholus $C . V .118, \mathbf{1} 20.266,56$.

Engraulis fasciata Lesueur cf. Engraulis Brownii C. $V$.

Engraulis Grayi Blkr. 50, 492. 56, 445. 60, 41. 91, 74. 178, 2. 227, 408. cf. Stolephorus (Thryssa) Hamiltoni Blkr.

Engraulis Hamiltoni Blkr. 91, 74. 270, 58. 272, 26. 275, 49. 287, 6I. 288, 79. 296, I I 2 .

Engraulis heteroloba Rüpp. 10, 73. 118, I20. cf. Stolephorus (Stolephorus) heterolobus Blkr.

Engraulis japonica Gthr. cf. Stolephorus japonicus Blkr.

Engraulis japonicus T. Schl. 118, r19. cf. Engraulis ringens Jen., Stolephorus (Stolephorus) japonicus Blkr.

Engraulis kammalensis Blkr. 16, I3. 25, I2. cf. Engraulis rhinorhynchos Blkr.

Engraulis malabaricus $C . V .91,74$.

Engraulis melanochir Blkr. 16, 13. 25, 12. 42, 6о. 45, 197. 49, 42 1. 55, 4I4. 60, 36. 239, I02. 270, 58. 272, 26.

Engraulis mystacoides Blkr. 60, 42. 91, 74. 127, 345. 178, 2. 267, 1 40. $270,58.272,26.287,6$ I.

Engraulis mystax $C . V$. 51, 59. 60, 43. 91, 74. 270, 58. 288, 79. 425, I48. cf. Stolephorus (Thryssa) Valenciennesi Blkr.

Engraulis nasus $C$ v. 119, I5.

Engraulis nesogallicus Benn. cf. Engraulis boelama $C$. $V$., Engraulis brelama Cuv.

Engraulis Pfeifferi Blkr. 55, 433. 178, 2. 272, 26.

Engraulis phasa $C . V .91,74$.

Engraulis polynemoides Gthr. 459, гоз.

Engraulis poorawah Blkr. 425, 148 .

Engraulis purava $C . V .91,74$.

Engraulis purawa $\mathrm{Val} .288,79$.

Engraulis rhinorhynchos Blkr. 55, 434. 56, 445. 60, 40. 158, 274 . $191,2.270,58.272,26.275,49.287,6$ I.

Engraulis rhinorhynchus Blkr. 231, I 50.

Engraulis ringens Jen. 497, 25 .

Engraulis Russellii Blkr. 50, 472. 60, 38. 91, 74. 194, 26. 227, 408. 231 , I 50. 234, 356. 235, 373. 270, 58. 275, 49. 287, 6 г. 296, II2. cf. Stolephorus (Stolephorus) indicus Blkr.

Engraulis setirostris C. $V$. , Blkr. 32, I60. 56, 446. 60, 44. 91, 74 . 127, 345. 156, 2 I 7. 231, I 50. 270, 58. 275, 49. 425, I48. 
Lingraulis stolephorus $C .2,5$ Io.

Engraulis taty $C . V .60,36.91,74.191,2.267,140.270,58.272$, 26. 287, 61. 288, 79 .

Engraulis telara $C . V .91,147$.

Engraulis telaroides Blkr. 16, r3. 25, I2. cf. Engraulis taty $C . V$.

Engraulis tenuifilis $C$. $V$. cf. Engraulis taty $C . V$.

Engraulis tri Blkr. 55, 435. 56, 446. 60, 40. 272, 27. 425, I 48.

Engraulis Zollingeri Blkr. 10, 73. 46, 214. 60, 38. 231, 172. 237, 438. 275, 49. cf. Stolephorus (Stolephorus) Zollingeri Blkr.

Enneacanthus Gill 468, 250.

Enneacanthus obesus Gill 468, 250.

Enneacentrus Gill 468, 256.

Ennichthys Gill 468, 29 I.

Enoplosus Lac. 468, 268 .

Enoplosus armatus Lac., Cv. 119, 5. 468, 268.

Epalzeorhynchos Blkr.261, 423.

Epalzeorhynchos kalfopterus Blkr. 136, 270. 270, 50. 272, 2 I.

Epalzeorhynchus Blkr. 314, 192.

Eperlanus sinensis Basil. cf. Hypomesus sinensis Glhr.

Ephippion Bibr. 367, 19.

Ephippus $C . V ., C u v .468,301,302$.

Ephippus argus Cuथ. 468, 302.481, 26.

Ephippus faber $C . V .468,301$.

Ephippus falcatus Cuv. cf. Harpochirus punctatus Cint.

Ephippus longimanus Cuv. cf. Harpochirus punctatus Cant.

Ephippus melanopus Blkr. 2, 520. cf. Ephippus orbis $C . V$., llarches orbis Cant.

Ephippus punctatus Cuv. cf. Harpochirus punctatus Cant.

Ephippus terla Cuv. cf. Harpochirus punctatus Cant.

Ephippus orbis C. V. 2, 520. 22, 22. 51, 56. 91, 40. 235, 366. 270, 27. 288, 72. 468, 301. cf. Ilarches orbis Cant.

Epibulus Cuv. 282, 95 .

Epibulus insidiator $C . V .11,40.12,97.126,344.130,458.156$, $217.270,39.275$, I6. 287, 42. 293, 276. 296, 104. 364, 275. 425, I $35.459,84.495$, Iо.

Epinephelini 468, 254.

Epinephelus Bl. 468, 256.

Epinephelus afer $B l .302, I_{3} .468,256$.

Epinephelus aka-ava Blkr.497, 5.

Epinephelus albofuscus Blkr. 354, r46. 425, I37. cf. Epinephelus maculatus Blkr.

Epinephelus alboguttatus Blkr. 309, I55. 315, 232. 318, 25I. 354, I 46. 358, I $87.364,28$ r. 369, 42. 394, 287. 396, 299. cf. Epinephelus coeruleopunctatus Blkr.

Epinephelus altivelioides Blkr.364,28 I cf. Epinephelus corallicola Blkr. 
Epinephelus amblycephalus Blkr. 364, 281. 451, 66.

Epinephelus amboinensis Blkr. 364, 2S I. cf. Epinephelus undulosus Blkr. Epinephelus analis Blkr. 438, 45. 451, 45. 459, 88 .

Epinephelus angularis Blkr. 459, 88. 495, r r.

Epinephelus areolatus Blkr. 459, 88. 497, 5 .

Epinephelus argus Bl. Schn., (Blkr.) 396, 296. 451, 57. 459, 8 . 491, 42. 495, II. cf. Epinephelus miniatus Blkr.

Epinephelus aurantius Blkr. 315, 232. 391, 276.451, 42.459, S8.491, 4r. Epinephelus awoara Blkr. 425, I37. 451, 98. 497, 5 .

Epinephelus bataviensis Blkr. 364, 28r. cf. Epinephelus ongus Blkr. Epinephelus biguttatus Blkr. 364, 28r.

Epinephelus boelang Blkr. 395, 29I. 451, 49. 459, 88. 491, 4I.

Epinephelus boenack Blkr. 354, I46. 364, 28 г. 425, I37. cf. Epinephelus boelang, formosus, Blkr.

Epinephelus bontoides Blkr. 364, 28I. 451, 85. 491, 4I.

Epinephelus brunneus Blkr. 425, I37.

Epinephelus celebicus Blkr. 364, 28I. 451, 69. 459, 88 .

Epinephelus chlorostigma Blkr. 459, 88.

Epinephelus coeruleopunctatus Blkr. 451, I08. 491, 4I.

Epinephelus corallicola Blkr. 438, 46. 451, 83 .

Epinephelus crapao Blkr. 320, 268. 346, 3r. 364, 28 r. 395, 29I. cf. Epinephelus pantherinus Blkr.

Epinephelus cyanopodus Blkr. 425, I37.

Epinephelus cyanostigma Blkr. 318, 251. 364, 28I. 451, 55.

Epinephelus cyanostigmatoides Blkr. 315, 232. 316, 241. 318, 25 I. 354, I 46. 358, I87. 364, 28 r. 394, 287. cf. Epinephelus miniatus Blkr. Epinephelus cylindricus Blkr. 459, 88. 495, I r.

Epinephelus dermochirus Blkr. 459, 88.

Epinephelus dermopterus Blkr. 497, 6.

Epinephelus diacanthus Blkr. 425, I37. 497, 6.

Epinephelus dictiophorus Blkr. 451, II 5 .

Epinephelus dispar Blkr. 459, 88.

Epinephelus epistictus Blkr. 497, 6.

Epinephelus erythraeus $B l k r .459,88,495$, I I.

Epinephelus fasciato-maculatus Pet. 497, 6.

Epinephelus fasciatus Blkr.451, r r9. 459, 88. 495, I r. 496, 2.497, 6 . Epinephelus flavocoeruleus Blkr. 459, I 7, 8S. 495, гі.

Epinephelus flavocoeruleus $B l k r$. (var, melanometopon) 422, 96.

Epinephelus formosus Blkr. 451, 59. 459, 89. 495, I I.

Epinephelus fuscoguttatus Blkr. 438, 46. 451, 93 .

Epinephelus Gilberti Blkr. 396, 299. 425, I37. 451, 9I.

Epinephelus Goldmani Blkr. 316, 24r. 451, 99.

Epinephelus guttatus $B l k r .315,232.320,268.354$, I46. 358, I 87 . 364, 281. 391, 276. 425, 137. cf. Epinephelus argus Bl. Schn.

Epinephelus hexagonatus Blkr. 315, 232. 316, 241. 318, 251. 320, 
268. 354, г46. 358, т87. 364, 281. 396, 296. 425, ז37. cf. Epinephelus merra $B l$.

Epinephelus Hoedti Blkr. 364, $281.451,63$.

Epinephelus Hoevenii Blkr. 315, 232. 320, 268. 354, I46. 358, i 87 . 364,28 I. 451 , I Iо.

Epinephelus horridus Blkr. 315, 232. 320, 268. 396, 299. 425, 137. cf. Epinephelus fuscoguttatus Blkr.

Epinephelus Janseni Blkr. 451, 8o.

Epinephelus janthinopterus Blkr. 438, 45. 451, 40.

Epinephelus japonicus Krusenst. cf. Epinephelus fasciato-maculatus Pet., Epinephelus merra Bl., Serranus trimaculatus $C . V$.

Epinephelus kawamebari Blkr.425, I37. 497, 6.

Epinephelus lanceolatus $B l k r .451,73$.

Epinephelus latifasciatus Blkr. 497, 6.

Epinephelus leopardus Blkr. 438, 45. 451, 6r. 459, S9. 495, I I.

Epinephelus leucogrammicus Blkr. 315, 232. 318, 25 I. 320, 268. 364, 28r. cf. Anyperodon leucogrammicus Gthr.

Epinephelus lineatus Blkr. 425, I38.

Epinephelus lutra Blkr.459, 89. 495, rI.

Epinephelus macrospilus Blkr. 364, 281.394, 287.451, 8r. 30/, 52 Epinephelus maculatus Blkr. 438, 46. 451, 75. 459, 88. 491, 4I.

Epinephelus marginalis Bl. (Blkr.) 315, 232. 318, $25 \mathrm{r} .320,268$. 364, 281. 394, 287. 425, 138. cf. Epinephelus fasciatus $B l k r$. Serranus marginalis $C . V$.

Epinephelus merra Bl. (Blkr.) 320, 268. 438, 46. 451, S8. 459, S9. 491, 4I. 495, I I. 496, 2.

Epinephelus microdon Blkr. 451, 95.

Epinephelus microprion Blkr. 309, I 55. 315, 232. 354, I46. 358, г87. 364, 28г. 425, r38. 451, 47.

Epinephelus micropsion Blkr. 491, 42.

Epinephelus miltostigma Blkr. 438, 45. 451, 43.

Epinephelus miniatus Blkr. 451, 53. 459, 88. 491, 42. 495, I I.

Epinephelus morrhua Blkr. 459, 89.495, I r.

Epinephelus multinotatus Blkr. 495, Ir.

Epinephelus mystacinus Blkr. 459, 88. 495, II.

Epinephelus nebulosus Blkr. 438, 45. 451, II7. 497, 6.

Epinephelus Nigri Blkr.302, 45 .

Epinephelus nigripinnis Blkr. 451, 39.

Epinephelus octocinctus Blkr. 408, 238.

Epinephelus ongus Blkr. 451, i I 3 .

Epinephelus pantherinus Blkr. 451, 78.459, 89.

Epinephelus pardalis Blkr. 315, 232. 318, 251.320, 268. 354, 146.

358, I87. 364, 28 $\mathrm{x}$. cf. Epinephelus Gilberti $B / k \%$.

Lpinephelus Playfayri Blkr. 495, 2.

Epinephelus poecilonotus Blkr. 497, 6. 
Epinephelus Polleni Blkr. 401, 336. 459, I9, 89.

Epinephelus polyphekadion Blkr. 451, 97.

Epinephelus polypodophilus Blkr. 364, 28I. 451, гог.

Epinephelus polystigma Blkr. 354, I46. 364, 28r. cf. Epinephelus summana Blkr.

Epinephelus punctatissimus Blkr.425, r 38.

Epinephelus Quoyanus Blk7.364, 28I.

Epinephelus Reevesii Blkr. 425, I38.

Epinephelus Retouti Blkr. 401, 339. 459, 21, S9. 495, II.

Epinephelus rhyncholepis Blkr. 320, 268. 451, 107.

Epinephelus rivulatus $B l k r .459,89.495$, I I.

Epinephelus ruber $B l$. cf. Epinephelus fasciatus $B l k r$.

Epinephelus salmonoides Blkr. 459, 89. 495, I I.

Epinephelus Sebae Blkr. 315, 232. 320, 268. 364, 28I. cf. Epinephelus maculatus Blkr.

Epinephelus semipunctatus Blkr. 477, 6 .

Epinephelus sexfasciatus Blkr. 425, I38. 451, Iо3.

Epinephelus Sonneratii Blkr. 459, 89.

Epinephelus spilurus Blkr. 315, 232.318, 25 I. 364, 2S r. cf. Epinephelus leopardus Blkr.

Epinephelus stellans Blkr. 364, 28 r. 425, г $38.438,46.451,86.459,89$.

Epinephelus stigmapomus Blkr. 425, I38.

Epinephelus summana Blkr. 438, 46. 451, I05.

Epinephelus susuki Blkr. 425, I38. 497, 6.

Epinephelus trimaculatus Blkr.425, г 38 .

Epinephelus tsirimenara Blkr.459, 89.497, 6.

Epinephelus tumilabris Blkr. 459, 89.

Epinephelus undulosus Blkr. 396, 296. 451, 65.

Epinephelus unicolor Blkr. 459, 89.495, I I.

Epinephelus ura Blkr. 497, 5.

Epinephelus urodelus Blkr.315, 232. 316, 24I. 364, 28r, 451, 5I. $459,89.491,42.497,6$.

Epinephelus urophthalmus Blkr. cf. Anyperodon urophthalmus Blkr. Epinephelus variegatus Blkr.425, $\mathrm{r}_{3} 8$.

Epinephelus variolosus $B l k \%$. 354, г 46. 356, г $73.364,28$ г. 425, г 38 . 451, 7 I. 459, 89. 495, II.

Epinephelus Waandersii Blkr. 451, 68.

Epinephelus zanana Blkr. cf. Epinephelus leopardus Blkr.

Eques Bl. 468, 324.

Eques americanus Bl. 468, 324 .

Eques lanceolatus Cast. 468, 324.

Equiti 468, 324.

Equula 1, 553 .

Equula bindoides $B ! k r .37,372.46,213.57,83.235,367.236$, 33 I. 237, 437. 275, 40. 287, 54 . 
Equula bindus $C . V .91,46.288,75$.

Equula Blochii C. $V, 91,46$.

Equula brevirostris $C . V .2,5$ I8. 16, 5. 25, 9. 91, 46. cf. Equula dacer $C . V$.

Equula caballa $C . V$. (Blkr.) 2, 5 IS. 9, 633. 16, 5, 25, 9. 37, 372 . 51, 57. 57, 81. 91, 46. 288, 75. cf. Equula gomorah C. $V$.

Equula cara $C$. $V$. cf. Equula filigera $C$. $V$.

Equula coma C. $V .37,372.57,8$ г. 91, 46.

Equula dacer C. $V .51,57.57,8$ r. 91, 46. 235, 367.

Equula dentex $C$. $V$. cf. Gazza minuta $B l k r$., Leiognathus minuta Blkr. Equula Dussumierii C. V. 91, 46. cf. Leiognathus Dussumieri Blkr.

Equula ensifera C. $V .2,5$ I $8.35,26$ I. 42, 59. 45, I95.46, 213. 49, 419. 54, 237. 55, 412. 57, 80. 91, 46. 156, 215. 178, 2. 194, 26. 200, 48. 227, 407. 235, 367. 270, 31. 272, I4. 275, 40. 284, 239. 287, 54. 296, іто. 309, I56. cf. Leiognathus edentulus Blkr.

Equula fasciata $C . V$. cf. Equula filigera $C . V$., Leiognathus fasciatus $B l k r$. Equula filigera $C . V .2,5$ I8. 25, 9. 50, 47 I. 51, 57. 53, I65. 55, 412. 57, 79. 91, 46. 156, 215. 184, 200. 231, 147. 235, 367. 270, 3т. 272, 14. 275, 40. 288, 75. 296, гто. 309, г 56. cf. Leiognathus fasciatus $B l k r$.

Equila gerreoides. Blkr. 37, 37 I. 55, 4I2. 57, SI. 90, 39. 227, 407. $235,367.270,3$ ․ 272, I $4.287,54$.

Equula gomorah $C . V$. 2, 5I8. 32, I6о. 46, 213. 56, 445. 57, 82. 91, 46. 156, 2I5. 227, 407. 231, I47. 234, 353. 237, 437. 270, 31. 275,4 ․ $287,54.288,75.296$, r 1 . cf. Leiognathus splendens Blkr.

Equula gracilis Blkr. 109, 249. 231, 147. 275, 40.

Equula insidiatrix $C . V .2,5$ I $8.16,5.25,9.46,2$ I $3.51,58.57$, 84. 91, 46. 231, 147. 270, 31. 275, 40. 288, 75. cf. Leiognathus insidiator Blkr.

Equula interrupta $C . V .57,85.91,46.127,345.237,437.270,3$ I. 287, 54. cf. Leiognathus interruptus $B l k r$.

Equula lineolata C. V. 56, 445. 57, I $83.90,38.156,215.200,48$. 270,3 I. $275,40.287,54$.

Equula longimana Cant. 51, 57. 288, 75 .

Equula longispinis $C . V$. cf. Equula filigera $C . V$.

Equula minuta $C$. $V$. cf. Gazza minuta Blkr.

Equula nain $C$. $V$. cf. Gazza minuta Blkr.

Equula nuchalis Temm. Schl. 90, 38. cf. Leiognathus nuchalis Blk\%. Equula oblonga $C . V .46,2$ 13. 57, 84. 90, 38. 91, 46. 231 , 1 47. $275,40.287,54.288,75$.

Equula parviceps $C$. $V$. cf. Leiognathus parviceps $B l k r$.

Equula rivulata $T$. Schl., C. $V$., 90, 38. cf. Leiognathus rivulatus Blkr. Equila ruconius $C . V .91,9^{6 .}$

Equula serrulifera Rich. 119, I I. 
Equula splendens $C$. $V$. cf. Leiognathus splendens Blkr.

Eremophilus Humb. 306, II 2.

Eremophilus Mutisii Humb. 306, I I2.

Erethistes Mïll. Trosch. 306, го6.

Erethisthes pusillus $M$. Trosch. 91, 58. 306, Iо6.

Erotelis Poey 453, 302.

Erythrichthys Schl. 468, 275. 470, I53.

Erythrichthys leucogrammicus Gthr. 354, I47. 364, 283. cf. Dipterygonotus leucogrammicus Blkr.

Erythrichthys Schlegeli Gthr. 468, 275. 470, I 54 .

Erythrodon Rüpp. 367, ro.

Erythrodon niger Rüpp. 364, 272. 368, 21. 425, I22. 459, 71.491, 52. $495,20$.

Esomus Stons. 261, 434. 314, 2 I 3.

Esomus daurica Heck. 356, I76.

Esomus thermoicos Heck. 335, 3.

Esox australis Cv. 119, I4.

Esox belone Forsk. cf. Mastacembelus choram Blkr.

Esox brasiliensis Brozen cf. Hemiramphus vittatus $\mathrm{Val}$.

Esox cancila Buch. Ham. cf. Belone cancila C. $V$.

Esox chirocentrus Lac. cf. Chirocentrus dorab Cuv.

Esox ectuntio 91, I46.

Esox far Forsk. cf. Hemiramphus Commersonii $C . V$.

Esox indica $M c$ Cl. 91, 72.

Esox panchax Buch. cf. Panchax Buchanani $C$. $V$.

Esox sphyraena $L$. cf. Sphyraena vulgaris $C . V$.

Esox synodus $L$. cf. Saurus synodus $C$. $V$.

Eteira $K p$. 468, 306.

Eteira Taunay Kaup cf. Megaprotodon strigangulus Blkr.

Eteira triangularis $K a u p$ cf. Megaprotodon strigangulus $B l k r$.

Etelis C. V. 438, 44. 468, 276, 336 .

Etelis carbunculus $C . V .459,90.468,276.495$, I 2 .

Etelis coruscans $\mathrm{Val} .459,90$.

Etelis oculatus Blkr. 497, 7 .

Etroplus coruchi C. V. 91, 52. cf. Pseudetroplus coruchi Blkr.

Etroplus maculatus $C . V$. 91, 52. 297, 126.

Etroplus meleagris C. $V .91,52$.

Etrumeus micropus Blkr. 90, 48. 497, 25.

Etsgilus fumosus Brev. cf. Rhombotides fumosus Blkr.

Euanemus Mïll. Trosch. 306, rog.

Euanemus colymbetes Blkr. cf. Auchenipterus colymbetes Blkr.

Euanemus dentatus Blkr. cf. Auchenipterus dentatus $\mathrm{Val}$.

Euchistodus declivifrons Gill cf. Glyphidodon (Glyphidodon) septemfasciatus $C . V$.

Euchoristopus Gill 453, 326. 
Euchoristopus argentilineatus Blkr. 491, 54.

Euchoristopus Koelreuteri Gill 491, 54. 495, I 7.

Eucinostomus Baird, Gir. 468, $28_{5}$.

Euctenogobius Gill 453, 323 .

Euctenogobius badiis Gill 453, 324 .

Eucyclogobius Gill 453, 3 I9.

Eugobii 453, 3 r6.

Eumesogrammus hexagrammus Gill 497, 20.

Eupomacentrus (Brachypomacentrus) albifasciatus Blk\%. 483, 40. 485,

75. $491,+7$.

Eupomacentrus ater Lién. 487.

Eupomacentrus (Brachypomacentrus) ater Blkr. 495, 5 .

Eupomacentrus (Eupomacentrus) lividus Blkr.483, to. 485, 73. 495, I 5 .

Euprotomicrus Labordii Gthr. 459, 68. 495, 8.

Euryglossa ovalis $K p .288,69$.

Eurymyctera $K p$. 351, I 2 I.

Eurymyctera crudelis $K p$. cf. Gymnothorax crudelis $B l k r$., Gymnothorax schismatorhynchus Blkr.

Eurypegasus draconis Blkr. 318, 250. 358, I $8_{3} .364,274$.

Eutropiichthys Blkr. 299, 398. 306, Iо7.

Eutropiichthys vacha Blkr. 306, I07.

Eutropius Miull. Trosch. 299, 398. 306, го6.

Eutropius brachypopterus Blkr. 270, 46. cf. Pseudeutropius brachypopterus Blkr.

Eutropius niloticus Blkr. 306, I06.

Evoplites Gill 468, 276.

Evoplites pomacanthus Gill cf. Lutjanus bengalensis Blkr.

Evorthodus Gill 453, 3II.

Evorthodus breviceps Gill 453, $31 \mathrm{r}$.

Exocoetus 1, 552.

Exocoetus affinis Gthr. 459, IоI.

Exocoetus agoo T. Schl. 497, 24. cf. Exocoetus unicolor $C$. $V$.

Exocoetus alatus Soland. cf. Exocoetus poëcilopterus $V a l$.

Exocoetus altipennis C. $V .266,56$.

Exocoetus apus $V a l .425$, I 49.

Exocoetus arcticeps Gthr. 425, I49.

Exocoetus bahiensis Rang 496, 3 .

Exocoetus bicolor $\mathrm{Val}$. 373, I32.

Exocoetus brachycephalus Gt/ur. 425, I 49 .

Exocoetus brachypterus Sol., Rich. 425, I 49.

Exocoetus brachysoma Blkr. 372, III. 459, IoI.

Exocoetus chloropterus $C . V$. 266, 56 .

Exocoetus Commersonii Lac., C. $V$. 372, I25. cf. Exocoetus brachysoma Blkr.

Exocoetus evolans L. 266, 76. 425, I 49. 459, гог. 495, 2 г. 
Exocoetus exsiliens L. (Gm., Lac.) 425, r49. cf. Exocoetus Commersonii Lac.

Exocoetus fasciatus Les., Rich. cf. Exocoetus exsiliens L. (Gm.), Exocoetus nigripennis $\mathrm{Val}$.

Exocoetus hexazona Blkr. 75, 206. 372, I I8.

Exocoetus javanicus $K . v . H .2,5 \mathrm{I} 2$.

Exocoetus katoptron Blkr. 372, I15.

Exocoetus mento C. V. 58, 21. 91, 72. 235, 372. 275, 47. 315, 236. cf. Parexocoetus mento Blkr.

Exocoetus micropterus $C . V .91,72.119$, I4. 142, 63. 354, I50. cf. Cypsilurus micropterus Blkr.

Exocoetus monocirrhus Rich. 425, r49.

Exocoetus neglectus Blkr. 372, II2.

Exocoetus nigripennis $C . V .75,207.163,86.287,60.288,78.372$, I 20. $388,272.491,55$.

Exocoetus obtusirostris Gïnth. 496, 3 .

Exocoetus oligolepis Blkr. 372, 109. cf. Exocoetus agoo Sihl.

Exocoetus opisthopus Blkr. 372, I2I.

Exocoetus oxycephalus Blkr. 71, 77I. 275, 47. 372, I24. 437, 39.

Exocoetus poëcilopterus $\mathrm{Val}$. 372, I 18 .

Exocoetus Polleni Blkr. 373, r3o.

Exocoetus speculiger $C . V .71,772.119$, I4. 136, 273. 221, 314. $270,55.364,294.372$, I22. 388, 272.459 , гог. 495, 2 I. cf. Exocoetus katoptron Blkr.

Exocoetus spilonotopterus Blkr. 372, Ir3.

Exocoetus spilopterus $V a l .372$, I 6 .

Exocoetus spilopus C. $V$. 119, 14. cf. Exocoetus bicolor $V a l$.

Exocoetus splendens Clarck Abel cf. Exocoetus evolans $L$.

Exocoetus Solandri $C . V .459$, Iог.

Exocoetus unicolor C. $V .58,21.71,772.175,22.227,408.231$, I 49. 270, 55. 275, 47. 287, 60. 296, III. 364, 294. cf. Exocoetus oligolepis Blkr.

Exocoetus volitans Don., Forst., Penn. cf. Exocoetus evolans $L$.

Exoglossum Raf. 261, 425. 314, 205.

Exostoma Blyth 306, т05.

Exostoma Andersonii Day 425, 149. 430, 233.

Exostoma Berdmorei Blyth 306, 105.

Felichthys filamentosus Swns. cf. Pimelodus filamentosus Blkr.

Felichthys nodosus Stens. cf. Pseudauchenipterus nodosus Blkr.

Fierasfer acus Brinn. 495, 2 I.

Fierasfer Brandesii Blkr. 184, 204. 188, 461.

Fierasfer gracilis Blkr. 247, 202. 358, I92. 364, 294. 396, 300. 491, 54 .

Fierasfer Homei $K p .315,236.320,272.358$, I 92. 364, 294. 459, 74.

Fierasfer lumbricoides Blkr. 358, I92. 
Fierasfer parvipinnis $K p, 495,2 \mathrm{I}$.

Fistularia chinensis Schl. cf. Aulostoma chinense Cu\%.

Fistularia Commersonii Rüpp. cf. Fistularia immaculata Comm.

Fistularia immaculata Comm. 54, 28 r. 90, 4. 91, 54, г65. 93, I I. 119, I2. $157,234.167,372.196,37.207,2$ I . 231, I48. 235, 370. $270,44.275,46.287,58.288 .77 .296$, III. 309, I 56.

Fistularia immaculata Cut\%, Schl. cf. Cannorhynchus serratus Blkr., Solenostomus serratus Blkr., Gill.

Fistularia paradoxa Pall. cf. Solenostoma paradoxum Lac.

Fistularia serrata Cuv., Gthr. cf. Cannorhynchus serratus Blkr., Solenostomus serratus Gill.

Fistularia tabaccaria L. (White) 302, 107. cf. Cannorhynchus tabaccaria Blkr., Fistularia immaculata Comm.

Fundulichthys Blkr. 261, 439 .

Fundulichthys virescens Blkr. 497, 24.

Fundulus Lac. 261, 44I.

Fundulus orthonotus Gthr. 459, rox.

Fundulus virescens Schl. cf. Fundulichthys virescens Blkr.

Gadopsis marmoratus Rich. 119, 8.

Gadus (Boreogadus) Bottemannei Blkr.447, 462

Gadus pseudomorrhua Blkr. 425, I30, I5I.

Gagata Blkr. 306, 90.

Gagata typus Blkr. 306, 90 .

Galaxias alepidotus Rich. 119, I4.

Galaxias attenuatus $C v$. 119, I 4 .

Galaxias brocchus Rich.119, I4.

Galaxias fasciatus $C v$. 119, I4.

Galaxias reticulatus Rich. 119, I4.

Galaxias scriba $C$. 119, I4.

Galaxias truttaceus $C$. 119, I4.

Galeichthys $V a l .306,90$.

Galeichthys Blochii Val. cf. Pimelodus filamentosus Blkr.

Galeichthys feliceps C. $V .266,76.306,90$.

Galeichthys Gronovii $V a l$. cf. Pimelodus bagre Lac.

Galeichthys stanneus Rich. cf. Batrachocephalus mino Gthr.

Galeocerdo tigrinus $M . H .91,80.459$, го3.

Galeoides microps Steind. 425, 149. 430, 234.

Galeoides polydactylus Gthr. 302, I5.

Galeus canis Rond. 266, 58.

Galeus japonicus $M . H .497,3$.

Gallichthys aegyptiacus Ehr. cf. Carangoïdes gallichthys Blkr.

Gallichthys chevola C. $V$. cf. Carangoïdes gallichthys Blkr.

Gallichthys major C. V. 2, 517. 17, 3. 25, S. cf. Carangoïdes gallichthys Blkr., Citula gallus Blkr. 
Gallichthys minor 2, 5I7.

Gallus virescens Lac. cf. Carangoïdes gallichthys Blkr.

Gambusia Poey 261, 440.

Garra Ham.Buch. 314, I91. 326, 242. 329, 262.

Garra (Garra) ceylonensis Blkr. 326, 239. 335, 8 .

Gasteroschisma melampus Rich. 119, II.

Gasterosteus glaucus (Forst.) Bl. Schn. cf. Glaucus Rondeletii Will.

Gasterosteus japonicus Houtt. cf. Monocentris cataphracta Bl. Schn., Monocentris japonus Bl. Schn.

Gasterosteus obolarius $C$. 497, I4.

Gasterosteus ovatus $L$. cf. Trachinotus mookalee $C$. $V$.

Gasterosteus rhombeus Forst. cf. Psettus Sebae $C$. $V$.

Gasterosteus saltatrix $L$. cf. Temnodon saltator $C$. $V$.

Gasterosteus volitans $L$. cf. Pseudomonopterus (Pterois) volitans Blkr., Pterois volitans $C . V$.

Gastrophysus alboplumbeus Blkr. 106, 104. 270, 65.

Gastrophysus argenteus Lac., J. Miül. 119, I6. 231, I5I. 235, 374. 272, 7. 275, 12. 287, 40.

Gastrophysus glaber Blkr. 119, I6.

Gastrophysus Hamiltonii Blkr. 119, I6.

Gastrophysus Honckenii Blkr., J. Mïll. 109, 258. 266, 57. 275, I 2.

Gastrophysus implutus Blkr. 188, 462.

Gastrophysus laevigatus Blkr. 302, 22.

Gastrophysus lunaris J. Mïll. 156, 2 18. 157, 235. 191, 2. 194, 26. 198, 45. 227, 408. 235, 374. 270, 65. 272, 7. 275, I2. 287, 4 0. 288, 68. cf. Tetraödon lunaris Cu\%.

Gastrophysus oblongus $J$. Müll. 235, 374. 272, 7. 287, 40. 288, 68. cf. Tetraödon oblongus $B l$.

Gastrophysus patoca J. Mïll. cf. Tetraödon patoca Ham. Buch.

Gastrophysus Richei Blkr. 119, 24.

Gastrophysus rubripes Blkr. 269, 68.

Gastrophysus stictonotus Blkr. 175, 30.

Gastrophysus vermicularis Blkr. 118, I25.

Gastrophysus xanthopterus Blkr. 269, 69 .

Gastrotokeus biaculeatus Heck. 184, 204. 234, 357. 235, 374. 270. 73. 275, I3. 287, 40. 288, 69. 296, I03. 309, I 54. 315, 230. 318, 250. 338, 360. 340, 364. 354, I 43. 358, I83. 364, 273. 394, 286. 395, 290. 396, 298. 425, I26. 459, 75. 491, 49. 497, I3.

Gazza 1, 553 .

Gazza argentaria Gthr. 316, 242. 320, 270. 354, I49. 358, I91. 364, 290. 395, 293.

Gazza equulaeformis Rupp. 2, 518. 76, 261. 127, 345. 184, 200. 231, I $47.235,3^{6} 7.270,3$ I. $275,40.287,53.288,75.296$, I 10.309, I56. 316, 242. 364, 290. 396, 300. 491, 50. cf. Gazza minuta Blkr. Gazza minuta Blkr. 46, 213. 50, 471. 51, 58. 57, 85. 76, 259. 91, 
46. $231,147.237,437.270,31.271,2.275,40.276,65.316$, 242. $364,290.459,98$.

Gazza tapeinosoma Blkr. 76, 260. 156, 2r5. 200, 48. 227, 407. 231, I 47. 234, 353. 235, 367. 237, 437. 270, 31. 275, 40. 287, 54 . 296, I го. cf. Gazza argentaria Gthr.

Gempylus coluber $C$. $V$. 425, I 3 I. 496, 2.

Gempylus prometheus $C . V .142,43.459$, 100.

Gempylus Solandri C. V. 119, I0. 142, 43.

Genicanthus Swons. 168, 307.

Genicanthus caudovittatus Blkr. 459, 96. cf. Holacanthus caudovittatus Gthr.

Genicanthus Lamarcki Swons. 354, I48. 358; 190. 364, 287. 365, 340. cf. Holacanthus Lamarcki Sions.

Genidens Cast. 306, 91.

Genidens Cuvieri Cast. 306, $9 \mathrm{r}$.

Genyatremus Gill 468, 27 I.

Genyonemus Gill 468, 325 .

Genyonemus lineatus Gill 468, 325.

Genyoroge Cant. 468, 276.

Genyoroge alboguttata Day cf. Lutjanus rivulatus Blkr.

Genyoroge amboinensis Gthr. cf. Lutjanus amboinensis Blkr.

Genyoroge bengalensis Gthr. cf. Lutjanus bengalensis Blkr., Lutjanus quinquelineatus Blkr.

Genyoroge bottonensis Gthr. cf. Lutjanus butonensis Blkr.

Genyoroge coeruleopunctata $G t h r$. cf. Lutjanus rivulatus $B l k r$.

Genyoroge duodecimlineata Gthr. cf. Lutjanus octovittatus Blkr.

Genyoroge macolor Gthr. cf. Lutjanus macolor Blkr.

Genyoroge marginata Gthr. cf. Lutjanus marginatus Blkr.

Genyoroge melanospilos Gthr. cf. Lutjanus amboinensis Blkr.

Genyoroge melanura Gthr. cf. Lutjanus butonensis Blkr.

Genyoroge nigra Gthr. cf. Lutjanus macolor Blkr.

Genyoroge notata Cant. cf. Lutjanus Russelli Blkr.

Genyoroge octovittata Gthr. cf. Lutjanus bengalensis Blkr.

Genyoroge rivulata Gthr. cf. Lutjanus rivulatus Blkr.

Genyoroge sebae Gthr. cf. Lutjanus sebae Blkr.

Genytremus Gill 468, 27 I.

Geotria australis Gray 119, I9.

Gerranus Tankervillae Benn. cf. Cirrhites Forsteri Blkr., Cirrhites pantherinus $C \cdot V$.

Gerreiformes 468, 285 .

Gerres Cu\%. 1, 553. 297, 141. 468, $2 S_{5}$.

Gerres abbreviatus Blkr. 20, Ir. 31, I03. 54, 237. 56, 445. 185, 223. 270, 24. 275, 29. 287, 47. cf. Diapterus abbreviatus Blkr.

Gerres acinaces Blkr. 97, I94. 188, 459. 275, 29. cf. Diapterus acinaces Blkr. 
Gerres argyreus Cuv. 20, x3. cf. Diapterus argyreus Blkr.

Gerres equula T. Schl. 118, 92. cf. Diapterus equula, oyena Blkr.

Gerres filamentosus $C . V$. 2, $521.10,66.16,4.20$, то. 25, 8. 46, 2 I2. $91,40.119,9.167,37$ I. 170, 479. 227, 407. 231, 145. 234, 353. 237, 436. 270, 24. 275, 29. 281, 108. 287, 47. 288, 71. 296, I05. 309, I55. cf. Diapterus filamentosus $C . V$., Blkr.

Gerres japonicus Blkr. 102, 404. 118, 93. cf. Diapterus japonicus Blkr.

Gerres kapas Blkr. 50, 482. 53, т6r. 156, 21 4. 157, 232. 235, 365 . 270, 24. 275, 29. 287, 47. cf. Diapterus kapas Blkr.

Gerres limbatus C. V. 91, 40. 288, 7 I. cf. Diapterus limbatus Blkr.

Gerres lineolatus Gthr. cf. Diapterus lineolatus Blkr.

Gerres lucidus C. $V .91,40$.

Gerres macracanthus Blkr. 97, I95. 156, 2 I4. 235, 365. cf. Diapterus macracanthus Blkr.

Gerres macrosoma Blkr. 94, 56. 147, 469. 156, 2 I4. 231, I45. 287, 47. 309, I 55. cf. Diapterus macrosoma Blkr.

Gerres melanopterus Blkr. 302. 44.

Gerres oblongus C. $V$., Blkr. 2, 52 I. 91, 40. cf. Diapterus oyena Blkr. Gerres oyena $C$. $V$.

Gerres octactis Blkr. 302, 43.

Gerres oyena C. $V .2,52$ I. 16, 4. 20, I2. 25, 8. 156, 214. 188, 459. 231, I45. 270, 24. 275, 29. 287, 47. cf. Diapterus oyena Blkr.

Gerres philippinus Gthr. cf. Diapterus philippinus Blkr.

Gerres Plumieri C. $V .468,285$.

Gerres poetie C. V., Blkr. 2, 521. 16, 4. 20, I1. 25, 8. 50, 471. 56, 444. 91, 40. 147, 469. cf. Diapterus poetie Blkr.

Gerres punctatus C. $V .2,52$ r. 91, 40. cf. Diapterus punctatus Blkr.

Gerres punctatus Blkr. cf. Diapterus filamentosus Blkr. Gerres filamentosus $C . V$.

Gerres punctatus Kner cf. Diapterus macracanthus Blkr.

Gerres Richii C. $V$. cf. Diapterus Richii Blkr.

Gerres singapurensis Steind. cf. Diapterus kapas Blkr.

Gerres subfasciatus $C . V$. 119, 9 .

Gerres waigiensis $Q$. G. cf. Diapterus argyreus Blkr.

Gibelion Heck. 261, 433. 314, 201.

Gila Baird, Gir. 261, 436. 314, 213. 329, 264.

Gillia Gthr. 453, 3I5.

Gillichthys Coop. 453, 3 I 5 .

Gillichthys mirabilis Coop. 453, 3I5.

Ginglymostoma (Ginglymostoma) brevicaudatum Gthr. 459, 68 .

Ginglymostoma cirratum $M$. H. 396, 296.

Ginglymostoma concolor M. H., Cant. 91, 8o. cf. Ginglymostoma Rüppellii Blkr.

Ginglymostoma (Nebrius) concolor Gthr. 459, 68. 
Ginglymostoma Rüppellii Blkr. 51, 83. 64, 91. 287, 36. 288, 66. cf. Ginglymostoma (Nebritis) concolor Gthr.

Girardinus Poey. 261, 439.

Girella Gr. 468, 295.

Girella leonina Gthr. 425, I39.

Girella melanichthys Blkr. 497, 8 .

Girella punctata Gr. 425, 139. 468, 295 .

Girella sarissophorus Cant., Blkr. 51, 64. 288, 7r. cf. Proteracanthus sarissophorus Gthr.

Girella zebra Rich., Steind. (Klunz.) 468, 296.

Girellichthys Klunz. 468, 296.

Glaucosoma Schl. 468, 253.

Glaucosoma Bürgeri Schl., Rich. 428, г3S. 468, 253. 497, 5.

Glaucosoma hebraicum Rich. 119, 6.

Glaucus calcar Blkr. 302, I4.

Glaucus Rondeletii IVill. 302, 75.

Glossamia Gill 468, 259.

Glossogobius Gill 453, 315, 320.

Glossogobius brunneus Gill 497, 19.

Glossogobius filosus $B l k r$. 495, I7.

Glossogobius giuris Blkr. 495, 17. 496, 3 .

Glossogobius olivaceus Blkr. 497, I9.

Glyphidodon ').

Glyphidodon affinis Gthr. 425, I36. cf. Glyphidodon affinis, bengalensis $C . V$.

Glyphidodon albocinctus Kner cf. Glyphidodontops zonatus Blkr.

Glyphidodon albofasciatus Homlr. Jacq. 354, I 45. 358, i S6. 364, 27 .

Glyphidodon albofasciatus $G t h r$. cf. Glyphidodontops albofasciatus bikr.

Glyphidodon amboinensis Gthr. cf. Pomacentrus taeniurus Blkr.

Glyphidodon anabatoides Day cf. Chromis lepisurus Blkr.

Glyphidodon anabatoides Gthr. cf. Pomacentrus (Pomacentrus) anabatoides Blkr.

Glyphidodon antjerius K.v.H., Gthr. 320, 267. 354, I45. 358, I86. 364, 278. 394, 286. 396, 299. 425, I36. 437, 38. 459, 84. cf. Glyphidodontops antjerius $B l k r$.

Glyphidodon antjerius Day cf. Glyphidodontops unimaculatus $B l k r$.

Glyphidodon antjerius Kner cf. Glyphidodontops zonatus Blkr.

Glyphidodon assimilis Gthr. 320, 267. 358, IS6. 364, 278. 369, 42. cf. Glyphidodontops uniocellatus Blkr.

Glyphidodon aureus K.v.H., Gthr. 364, 278. cf. Glyphidodon (Amblyglyphidodon) aureus Blkr.

Glyphidodon (Amblyglyphidodon) aureus Blkr. 485, ro9.

Glyphidodon azureus Gthr. cf. Glyphidodontops cyaneus Blkr.

I) Some species of Glyphidodon appear also under Glyphisodon. 
Glyphidodon Bankieri Gthr., Rich. 358, I86. 425, I36.

Glyphidodon batjanensis Blkr., Gthr. 354, I45. cf. Glyphisodon (Stegastes) batjanensis Blkr.

Glyphidodon Behnii Gthr. cf. Paraglyphidodon Behnii Blkr.

Glyphidodon bengalensis $C . V .346,3$ 1. 364, 278. 395, 291. 425, г 36.

Glyphidodon (Glyphidodon) bengalensis C. $V$. 483, 40. 485, 99. $491,47$.

Glyphidodon bonang Gthr. cf. Paraglyphidodon bonang Blkr.

Glyphidodon breviceps Gthr. cf. Amblypomacentrus breviceps Blkr.

Glyphidodon cingulatus Kner cf. Glyphidodontops zonatus Blkr.

Glyphidodon cingulum Klunz. cf. Glyphis (Stegastes) leucozona Blkr.

Glyphidodon coelestinus $C . V$. 315, 231. 320, 267. 358, I 86.364 , 278.425, г $36.459,85.496,2$.

Glyphidodon (Glyphidodon) coelestinus (Sol.) C. $V .483,40.485$, Iог. $491,47.495$, I 5. 497, Iо.

Glyphidodon cyaneus Q. G. 320, 273 .

Glyphidodon Dickii Lién. 320, 274. 358, г86. 364, 278. 459, S4.

Glyphidodon (Stegastes) Dickii Blkr. 483, 40. 485, I I5. 495, I 5.

Glyphidodon dispar Gthr. cf. Glyphidodontops unimaculatus Blkr.

Glyphidodon florulentus Gthr. cf. Glyphis (Stegastes) leucozona Blkr.

Glyphidodon gigas Lién. cf. Glyphidodon sordidus Rüpp.

Glyphidodon lacrymatus $Q . G$. 315, 231. 320, 267. 358, I86.

Glyphidodon lacrymatus Gthr. cf. Glyphidodon (Stegastes) lacrymatus $B l k r$.

Glyphidodon (Stegastes) lacrymatus Blkr. 483, 40. 485, I I I. 491, 47 .

Glyphidodon leucogaster Blkr. 320, 267 . 354, 145. 358, I86. 364, 278.

Glyphidodon leucogaster Gthr. cf. Glyphidodon (Amblyglyphidodon) leucogaster Blkr.

Glyphidodon (Amblyglyphidodon) leucogaster Blkr. 485, ro8.

Glyphidodon leucozona Blkr. 394, 286.

Glyphidodon leucozona Gthr. cf. Glyphisodon (Stegastes) leucozona Blkr.

Glyphidodon limbatus $C . V .459,85$.

Glyphidodon margariteus $C . V .459,85$.

Glyphidodon melanopus Blkr. 320, 267.

Glyphidodon melanopus Gthr. cf. Paraglyphidodon melanopus Blkr.

Glyphidodon melas K. \%.H. 358, I 86. 364, 278. 394, 286.

Glyphidodon melas Gthr. cf. Paraglyphidodon melas Blkr.

Glyphidodon modestus Schl., Mïll., 354, I 45. 364, 278. 425, г 36.

Glyphidodon modestus Gthr. cf. Glyphidodontops modestus Blkr.

Glyphidodon mutabilis Osc. Cart. cf. Glyphidodontops mutabilis Blkr.

Glyphidodon nemurus Blkr. 354, I45. 364, 278. cf. Glyphidodon Bankieri Rich.

Glyphidodon nivosus Gthr. cf. Glyphidodon (Stegastes) lacrymatus Blkr. Glyphidodon notatus Day' cf. Glyphidodon (Glyphidodon) sordiclus Rüpp. Glyphidodon oxyodon Gthr. cf. Paraglyphidodon oxyodon Blkr. 
Glyphidocton plagiometopon (sthr. cf. Glyphidodon (Hemiglyphiclocton) plagiometopon Blkr.

Glyphidodon (Hemiglyphidodon) plagiometopon Blkr. 485, ro3.

Glyphidodon rahti $C . V .309,155.315,23$ I. 318, 250. 320, 267. 354, 145. 358, 186. 364, 278.425, 1 36. 437, 38. cf. Glyphidodon (Glyphidodon) coelestinus $C . V$.

Glyphidodon rhyncholepis Blkr. 406, 231. 459, 8, 85 .

Glyphidodon Schlegeli Blkr. 315, $23 \mathrm{I}$.

Glyphidodon Schlegelii Gthr. ef. Glyphidodon (.Imblyglyphidodon) ternatensis Blkr.

Glyphidodon septemfasciatus $C . V .320,267.354$, I 45. 364, 278. 425, I36. $459,85.485,97.495$, I5.

Glyphidodon sinensis Blkr. 425, I36, I 49.

Glyphidodon smaragdinus Brov. cf. Glyphidodon (Amblyglyphidodon) trifasciatus Blkr.

Glyphidodon sordidus $C$. $V$., Rüpp. 316, 241. 364, 279. 425, 136.459, S $_{5}$. $485,95.495$, I 5 .

Glyphidodon sparoides $C . V .459,85$.

Glyphidodon (Glyphidodon) sparoides $C . V$. 495, I5.

Glyphidodon taenioruptus Osc. Cart. cf. Glyphidodontops albofasciatus Blkr.

Glyphidodon termatensis Gthr. cf. Glyphidodon (Amblyglyphidodon) ternatensis Blkr.

Glyphidodon (Amblyglyphidodon) ternatensis B/kr. 315, 23I. 483, 40. 485 , I06.

Glyphidodon trifasciatus Blkr. 315, 231. 318, 250. 358, I86. 364, 279.

Glyphidodon trifasciatus Gthr. cf. Glyphidodon (Amblyglyphidodon) trifasciatus $B l k r$.

Glyphidodon (Amblyglyphidodon) trifasciatus Blkr. 483, +o. 485, 105. 497, го.

Glyphidodon unifasciatus Kner \& Stind. cf. Glyphidodon (Stegastes) Dickii Blkr.

Glyphidodon unimaculatus $C . V .315,231.320,267.354,145.358$, I86. $364,279.382,397$.

Glyphidodon unimaculatus Gthr. cf. Glyphidodontops unimaculatus Blkr.

Glyphidodon uniocellatus $Q . G .320,267$.

Glyphidodon uniocellatus Gthr. cf. Glyphidodontops uniocellatus IBkr.

Glyphidodon waigiensis $Q . G .$, C. V. 320, 267. 364, 279. 396, 296. 437,38 .

Glyphidodon Westermani Blkr. 322, 30. cf. Dischistodus annulatus Ptrs. Glyphidodon xanthonotus Gthr. cf. Paraglyphidodon xanthonotus lilkr. Glyphidodon xanthozona Blkr. 358, I86. 364, 279.

Glyphidodon xanthozona Gthr. cf. Gilyphiclodontoges allofasciatus Blkr. Glyphidodon xanthurus Blkr., Gthr. 316, 241. 318, 250. 358, I86. 364, 279. cf. Paraglyphidodon xanthurus Blkr. 
Glyphidodon zonatus $C . V .364,279$.

Glyphidodontops albofasciatus $B l k r .483,4$ I. 485, I36.

Glyphidodontops antjerius Blkr. 483, 4I. 485, I34. 491, 47. 495, I5.

Glyphidodontops cyaneus Blkr. 485, I30.

Glyphidodontops modestus Blkr. 483, 4I. 485, т3 г. 491, 47.

Glyphidodontops mutabilis Blkr. 485, I42.

Glyphidodontops unimaculatus Blkr. 483, 4I. 485, I40. 491, 47.

Glyphidodontops uniocellatus $B l k r .483,4$ I. 485, I33.

Glyphidodontops zonatus Blkr. 483, 4I. 485, I38. 491, 47.

Glyphisodon 1, 553 .

Glyphisodon albofasciatus Hombr. Jacq. cf. Glyphidodontops albofasciatus Blkr.

Glyphisodon amboinensis Blkr. 163, 72. cf. Pomacentrus (Pomacentrus) taeniurus Blkr.

Glyphisodon anabatoides Blkr. 2, 523. 6, 28, 8, I57. 234, 354. cf. Pomacentrus (Pomacentrus) anabatoides Blkr.

Glyphisodon andjerinus K. v. H. 6, 2 .

Glyphisodon andjerinus Schl. cf. Glyphisodon antjerius $K . v . H$.

Glyphisodon andjerinus Schl. Mïll. cf. Glyphidodontops antjerius Blkr.

Glyphisodon angulatus Blkr. 2, 523. cf. Chromis cinerascens Blkr.,

Glyphisodon antjerius $C . V .6,4.130,454.157,234.167,373.171$. 245. 184, 202. 236, 33I, 334. 270, 36. 275, 20. cf. Glyphidodontops antjerius $\mathrm{Blkr}$.

Glyphisodon ater Ehr. cf. Glyphisodon melas K. v. H., Paraglyphidodon melas Blkr.

Glyphisodon aureus $K$. v. H. 2, 523. 6, 21. 275, 20. cf. Glyphidodon (Amblyglyphidodon) aureus $B l k r$.

Glyphisodon azur Q. G. cf. Glyphidodon cyaneus $Q$. $G$.

Glyphisodon azureus $C . V .6,5$. cf. Glyphidodon cyaneus $Q . G$., Glyphidodontops cyaneus $B l k r$.

Glyphisodon balinensis Blkr. 17, 9. cf. Glyphidodontops unimaculatus Blkr.

Glyphisodon bandanensis Blkr. 47, 248. cf. Chromis lepisurus Blkr., Heliases frenatus $C . V$.

Glyphisodon Bankieri Gthr., Rich. cf. Parapomacentrus Bankieri Blkr.

Glyphisodon batjanensis Blkr. 112, 373. cf. Glyphisodon (Stegastes) batjanensis Blkr.

Glyphisodon (Stegastes) batjanensis Blkr. 485, II 4.

Glyphisodon Behnii Blkr. 6, 25. 8, I56. 17, 9. cf. Paraglyphidodon Behnii Blkr.

Glyphisodon bengalensis $C . V .2,523.6$, Ir. 9, 633. 10, 67. 46, 213 . $51,58.56,445.91,52.158,274.167,373.185,225.222,317$. 236, 334. 275, 20.

Glyphisodon biniar Montrouz. cf. Glyphidodon (Glyphidodon) coelestinus $($ Sol.) C. $V$. 
Glyphisodon biocellatus $C . V .6,2 S .76,286$. cf. Glyphidodon antjerius K. . H., Glyphidodontops antjerius Blkr.

Glyphisodon bonang Blkr. 67, 582. 170, 478. 270, 36. cf. Paraglyphidodon bonang Blkr.

Glyphisodon breviceps $M$. Schl. 6, 2. 270, 36. cf. Amblypomacentrus breviceps Blkr.

Glyphisodon Brownriggii C. I. 91, 52. cf. Glyphidodontops antjerius Blkr.

Glyphisodon coelestinus (Sol.) $C$. $V$. 6, I5, I6. 10, 67. 46, 2 I3. 51, 58. 91, 52. 126, 344. 156, 216. 157, 234. 158, 274. 170, 480. $184,202.185,225.222,3$ I $7.234,355.270,36.275,20.296$, 105. Glyphisodon curassao C. $V$. cf. Glyphidodon (Amblyglyphidodon) trifasciatus Blkr.

Glyphisodon cyaneus Q.G. cf. Glyphidodontops cyaneus Blkr.

Glyphisodon Dickii Lién. cf. Glyphidodon (Stegastes) Dickii Blkr.

Glyphisodon gigas Lićn. cf. Glyphidodon (Glyphidodon) sordidus Rüpp.

Glyphisodon glaucus C. $V .17,9$.

Glyphisodon javanicus $\vartheta$. Hass. cf. Chromis cinerascens Blkr.

Glyphisodon lacrymatus $Q . G .124,303.167,373.170,478.271,2$. 276, 65. cf. Glyphidodon (Stegastes) lacrymatus Blkr.

Glyphisodon leucogaster Blkr. 2, 523. 6, 26. 8, I56. 126, 344. cf. Glyphidodon (Amblyglyphidodon) leucogaster Blkr.

Glyphisodon leucozona Blkr. 236, 338. cf. Glyphisodon (Stegastes) leucozona Blkr.

Glyphisodon (Stegastes) leucozona Blkr. 483, 40. 485, II 2.

Glyphisodon melanopus Blkr. 148, 82. cf. Paraglyphidodon melanopus Blkr.

Glyphisodon melas $K . v . H .2,523.6,23.10,67.46,2$ г $3.48,348$. 156, 2 16. 275, 20. 287, 44. cf. Paraglyphidodon melas Blkr.

Glyphisodon modestus M. Schl. 6, 2. 236, 331, 334. 270, 36. 272, 9. cf. Glyphidodontops modestus Blkr.

Glyphisodon modestus T. Schl. 42, 59. 45, 196. 49, 4r9. 55, 4 I2. 76, 285. 156, 2I6. 171, 245. 208, 239 .

Glyphisodon nemurus Blkr. 164, 73. 234, 355. cf. Parapomacentrus Bankieri Blkr.

Glyphisodon nivosus Hombr. Jacq. cf. Glyphidodon (Stegastes) lacrymatus Blkr.

Glyphisodon oxyodon Blkr. 176, I4. 275, 20. 285, 244. cf. Paraglyphidodon oxyodon Blkr.

Glyphisodon phaiosoma Blkr. 17, 9. 231, 148. cf. Glyphidodontops modestus Blkr.

Glyphisodon plagiometopon Blkr. 51, 67. cf. Glyphidodon (Hemiglyphidodon) plagiometopon Blkr.

Glyphisodon punctulatus $C$. $V$. cf. Glyphisodon antjerius $K . v$. $I$.

Glyphisodon quadrifasciatus Blkr. 6, I7. 8, r55. cf. Glyphidodon (Glyphidodon) coelestinus (Sol.) C. $V$. 
Glyphisodon rahti $C . V .2,523.6,2.10,67.46,2$ 13. 51, 58. 54, $287.91,52$. 156, 216. 171, 245. 167, 373. 185, 225. 234, 355 . 235, 369. 236, 33I, 334. 250, 206. 270, 36. 275, 20. 288, 70. 296, го5. cf. Glyphidodon (Glyphidodon) coelestinus (Sol.) C. $V$.

Glyphisodon Rossii Blkr. 105, 48. cf. Glyphisodon antjerius $K$. v. H., Glyphidodontops zonatus Blkr.

Glyphisodon saxatilis Rüpp. cf. Glyphidodon (Glyphidodon) coelestinus (Sol.) C. $V$., Glyphidodon rahti $C . V$.

Glyphisodon Schlegelii Blkr. 73, I38. 184, 202. 275, 20. cf. Glyphidodon (Amblyglyphidodon) ternatensis Blkr.

Glyphisodon septemfasciatus $C . V .67,582$. 156, 2 I6. 167, 373. 171, 245. 185, 225. 236, 334. 270, 36. 275. 20.

Glyphisodon smaragdinus Brev. cf. Glyphidodon (Amblyglyphidodon) trifasciatus $B l k r$.

Glyphisodon sordidus $C . V .6$, 16. 25, ro. 91, 52. 185, 225. 188, 460. $236,334.270,36$.

Glyphisodon ternatensis Blkr. 73, I37. cf. Glyphidodon (Amblyglyphidodon) ternatensis $B l k r$.

Glyphisodon trifasciatus $B l$., Blkr. 2, 523. 6, I9. 8, I56. 156, 2 I6. 157, 234. 275, 2I. cf. Glyphidodon (Amblyglyphidodon) trifasciatus Blkr.

Glyphisodon unimaculatus $\left(K . v . H_{0}\right) C . V .6,2.42,59.45$, I96. 49, 419. 55, 4I2. 76, 284. 127, 345. 167, 373. 182, I59. 184, 202. 188, 460. 231, 148. 236, 33r, 334. 270, 37. 272, 9. 275, 2 1. 285, 244. cf. Glyphidodontops unimaculatus Blkr.

Glyphisodon uniocellatus $Q . G .6,2.72$, I I9. 184, 202. 296, I05. cf. Glyphidodontops uniocellatus Blkr.

Glyphisodon Vidal Q.G. cf. Glyphidodon (Stegastes) lacrymates Blkr.

Glyphisodon violaceus Brev. cf. Paraglyphidodon melas Blkr.

Glyphisodon waigiensis $Q . G ., C . V .6, \mathrm{I}_{3} .46,2 \mathrm{I}_{3} .78,484.156$, 2 I6. 184, 203. 275, 2 I. cf. Glyphidodon (Glyphidodon) coelestinus (Sol.) $C . V$.

Glyphisodon xanthonotus Blkr. 234, 357. cf. Paraglyphidodon xantho. notus $B l k r$.

Glyphisodon xanthozona $B l k r .76,283.270,37.275,2$. cf. Glyphidodontops albofasciatus $B l k r$.

Glyphisodon xanthurus Blkr. 84, 345. 164, 74. 167, 373. cf. Paraglyphidodon xanthurus Blkr.

Glyphisodon zonatus C. $V .6,2$. cf. Glyphisodon antjerius $K . v . H$., Glyphidodontops zonatus Blkr.

Glyphodes Guich. 468, 282.

Glyphodes aprionoides Guich. 459, 90. 468, 282 .

Glyptauchen Gthr. 473, 297.

Glyptocephalus Gottsch.294, 428.

Glyptocephalus saxicola Gottsch. 294, 428. 
Glyptosternon $M C C l .306$, I04.

Glyptosternon labiatus $M c C l .91,5^{8}$.

Glyptosternon pectinopterus $M c C l .91,58$.

Glyptosternon platypogon Blkr. 185, 225. 190, I. 200, 48. 270, 47. 271, I.

Glyptosternon platypogonides $B l k r .270,47$.

Glyptosternon reticulatus $\mathrm{MC} \mathrm{Cl.} \mathrm{91,58.306,} \mathrm{ro4.}$

Glyptosternon striatus $M C C \% 91,58$. cf. Glyptothorax striatus Blyth.

Glyptosternon sulcatus $M c C l .91,58$. cf. Pseudecheneis sulcatus Blyth.

Glyptothorax Blyth 306, I04.

Glyptothorax striatus Blyth 306, I05.

Gnathanacanthus Blkr. 119, 20. 473, 299.

Gnathanacanthus cutaneus 479, I34.

Gnathanacanthus Goetzeei Blkr. 119, 21. 473, 299. 479, I34.

Gnathanodon Blkr. 128, 4I8. 297, I38,

Gnathanodon margarita Blkr. 425, I3 $\mathrm{r}$.

Gnathanodon petaurista Blkr. 459, 99.

Gnathanodon speciosus Blkr. 32, I60. 50, 47 I. 51, 57. 54, 236. 56, 445. 57, 72. 91, 46. 119, 10. 126, 344. 184, 200. 185, 223. 222, 3т7. 270, 30. 271, 2. 275, 40. 276, 65. 288, 74. 315, 235. 358, I91. $364,290.395,293.396,300.459,99.495, \mathrm{r} 8$.

Gnathocentrum Guich. 468, 309.

Gnathocentrum centrognathum Guich. cf. Zanclus canescens Gthr.

Gnathodentex Blkr. 438, 41. 468, 279.

Gnathodentex aurolineatus Bttr. 438, 41. 441, 49. 468, 279.495 , 2.

Gnatholepis Blkr. $453,318$.

Gnathophis $K p .351$, II 7 .

Gnathopogon Blkr. 261, 435. 314, 202.

Gnathopogon bimaculatus Blkr. 326, 245. 335, r7.

Gnathopogon elongatus Blkr. 497, 23.

Gnathopogon gracilis Blkr. 497, 23.

Gnathopogon javanicus Blkr. 353, I37.

Gnathypops papuensis Blkr. 455, 473 .

Gnathypops Rosenbergii Blkr. 455, 474.

Gobiesox cephalus Lai. 425, I29.

Gobiesox dentex Cuv. 266, 74 .

Gobiesox tudes Rich. cf. Gobiesox cephalus Lac.

Gobiichthys Klunz. 453, 324.

Gobiiformes 453, 308 .

Gobiini 453, 314 .

Gobileptes Szons. 453, 327 .

Gobio Cuv. 261, 435. 314, 208.

Gobio angra $M C C l .91,64$.

Gobio anisurus $M C C l .91,64$.

Gobio barbus Schl. cf. Hemibarbus barbus Blkr. 
Gobio bendilisis C. $V .91,64$.

Gobio bicolor $M c C l .91,64$.

Gobio boga $M c$ Cl. 91, r34.

Gobio chapalio C. $V .91,64$.

Gobio curmuca $M C \mathrm{Cl}$. 91, 64, I35.

Gobio esocinus Schl. cf. Pseudogobio esocinus Blkr.

Gobio heterodon Blkr. 345, 26. cf. Saurogobio heterodon Blkr.

Gobio javanicus Blkr. 166, 358 .

Gobio limnophilus $M_{c} C l .91,64$.

Gobio microcephalus Blkr. 166, 357.

Gobio rivularis Basil. cf. Pseudogobio rivularis Blkr.

Gobio sinensis Blkr. 345, 26. cf. Pseudogobio rivularis Blkr.

Gobiodon (K. v. H.), Blkr. 151, 407. 453, 309:

Gobiodon ceramensis Gthr. 364, 292. cf. Gobiodon quinquestrigatus Blkr.

Gobiodon ceramensis Blkr. 354, I50. 358, I92. 425, I 29.

Gobiodon citrinus Day cf. Gobiodon hypselopterus Blkr.

Gobiodon coryphaenula Blkr. 459, 78 .

Gobiodon erythrophaios Blkr. 151, 409. 184, 201. 237, 438. 315, 236. cf. Gobiodon quinquestrigatus $B l k r$.

Gobiodon erythrophaius Blkr. 354, I50. 358, 192.

Gobiodon erythrospilus Blkr. 461, 122. 491, 53 .

Gobiodon heterospilos Blkr. 151, 409.

Gobiodon heterospilus Blkr. 354, I50. 453, 309.

Gobiodon histrio $K . v . H .453,309$.

Gobiodon hypselopterus Blkr. 461, I20.

Gobiodon melanosoma Blkr. 184, 201. 320, 27 1. 354, 150. 358, 192. 364, 292. 394, 288. cf. Paragobiodon melanosoma Blkr.

Gobiodon micropus Gthr. 425, I29.

Gobiodon quinquestrigatus $B l k r$. 157, 233. 184, 201. 237, 438. 242, I 42. $275,44.320,27$ I. 354, I 50. 394, 288.461 , I I 791,53 . cf. Gobiodon erythrospilus Blkr.

Gobiodon rivulatus Gthr. 495, I 7 .

Gobiodon xanthosoma Blkr. 184, 2 о I. 275, 44. 320, 27 I. 354, I 5 0.

358, I92. 364, 292. cf. Paragobiodon xanthosoma Blkr.

Gobiodontini 453, 308 .

Gobioidei 453, $30 \mathrm{I}$.

Gobioides Lac. 453, 329.

Gobioides anguilliformis Lac. cf. Gobioides coeculus Blkr.

Grobioides brachysoma Blkr. 430, 233.

Gobioides Broussoneti Lac. 453, 329.

Gobioides coeculus Blkr. 425, I29.

Gobioides Hermannianus Blkr. 425, I29.

Gobioides melanurus Blkr. 425, I29.

Gobioides ruber Buch.cf. Trypauchen vagina $C$. $V$. 
Gobioides rubicundus 73uch.453, 330. cf. Amblyopus Hermannianus C. $V$.

Gobioides rugosus Blkr. 425, I 29.

Gobiomoroides Lac. 453, 302.

Gobiomorphus Gill 453, 303. 460, I03, I05.

Gobiomorus taiboa Lac. cf. Valenciennesia strigata Blkr.

Gobion erythrophaios Blkr. cf. Gobiodon quinquestrigatus Blkr.

Gobionelli 453, 325.

Gobionellus Gir. 453, 325 .

Gobionellus microlepis Blkr. 425, 128.

Gobionichthys Blkr. 314, I94.

Gobiopsis Steind. 453, 3I5.

Gobiopsis macrostomus Steind. 453, 315.

Gobiopsis oligactis Blkr. 461, I 3 .

Gobiopterus Blkr. 453, 3 Ir.

Gobiopterus brachypterus Blkr. 472, 290.

Gobiopterus variegatus lilkr. 472, 29I.

Gobiosoma Gir. 453, 3 ro.

Gobiosoma fasciatum Plfr. 453, 3 Iо.

Gobiosoma molestum Gir. 453, 3 10.

Gobius Art. 1, 553. 453, 316, 317.

Gobius acutipennis $C . V .91,50.288,76$.

Gobius albopunctatus $C . V .459,77$.

Gobius anjerensis Blkr. 34, 251.453, 3 IS.

Gobius apogonius Cant. 288, 76.

Gobius auchenotaenia Blkr. 384, 4I5. 459, 56, 77 .

Gobius baliuroides Blkr. 15, 26. 16, 5 .

Gobius baliurus (K. ข. H.) C. V. 15, 31. 275, 43. 287, 56. 364, 292.

Gobius bato Buch. cf. Apocryptes bato C. $V$.

Gobius batrachocephalus Pall. 453, 317.

Gobius belosso Blkr. 110, 316. 156, 2 I5.

Gobius biocellatus $C . V .91,50$.

Gobius Blokzeyli Blkr. 284, 240.

Gobius Bontii Blkr. 15, 27. 16, 5. 25, 9.

Gobius boea $C$. $V$. cf. Gobius kokius $C . V$.

Gobius borneënsis Blkr. 26, Iо. 35, 26r. 42, 59. 45, I95. 49, 4 19 . 55, 4I2. 270, 4I. 272, I6.

Gobius brevifilis $C . V .91,50$. cf. Gobius auchenotaenia Blkr.

Gobius brevirostris Gthr. 425, I28.

Gobius brunneus Sihl. cf. Glossogobius brunneus Gill.

Gobius bynoënsis Rich. 119, 12. 453, 323.

Gobius caninoïdes Blkr. 54, 274. 69, 702. 143, 50. 184, 2 I I. 275, 43 . 281 , Іо8. 354, I $49.364,292.437,37$.

Gobius caninus $C . V .2,514.15,27.16,5.25,9.50,472.54,275$. $56,445.156,215.227,408.275,43.287,56.395,293.425,128$. 459, 103. 
Gobius castaneus O'Shaugness. cf. Cryptocentrus castaneus Blkr.

Gobius catebus $C$. $V$. cf. Gobius kokius $C . V$.

Gobius cauerensis Blkr. 76, 269. 270, 41.

Gobius celebius $C . V$. 10, 67, 68. 15, 3. 46, 2 ×3. 110, 318. 129, 443 . 170, 476. 270, 41. 275, 43. 354, I 49 .

Gobius ceramensis Blkr. 69, 704. cf. Gobiodon quinquestrigatus Blkr.

Gobius changua Buch. cf. Apocryptes changua $C$. $V$.

Gobius chinensis Osb. 425, I 28.

Gobius chlorostigma Blkr. 15, 27. 16, 5. 25, 9. 51, 58. 54, 275. 55, 4I 2. 90, 43. 235, 369. 272, I6. 275, 43. 364, 292. 453, 32 I.

Gobius chlorostigmatoïdes Blkr. 15, 26. 16, 5. 25, 9. 143, 51. 178, 2. 272 , 16.

Gobius chuno Buch. 91, 50.

Gobius coalitus Benn. 459, 77. cf. Bathygobius coalitus Blkr.

Gobius cocosensis Blkr. 105, 47.

Gobius coeruleus Lac. 459, 77 .

Gobius coeruleus Lién. cf. Awaous Lienardi Blkr., Gobius Lienardi Blkr.

Gobius Commersonii C. $V$. 459, 77. cf. Awaous Commersoni Blkr.

Gobius coryphaenula $C$. $V$. cf. Gobiodon coryphaenula Blkr.

Gobius criniger $C . V .15,3.56,453.91,50.119$, 12. 275, 43. 287, 56. 364, 292. 437, 37. cf. Ctenogobius criniger Blkr.

Gobius crista galli $V a l .453,3$ I5.

Gobius cryptocentrus $V a l .453,322$.

Gobius cyanoclavis Cant. 288, 76 .

Gobius cyanomos Blkr. 15, 25. 16, 5. 25, 9. 235, 369. 287, $5^{6}$.

Gobius cyanotaenia Blkr. 78, 475 .

Gobius decussatus Blkr. 129, 442. 156, 2 I 5. 275, 43. 354, I 49.

Gobius diadematus Riupp. 453, 306.

Gobius Doriae Gt/ur. 453, $3 \dot{1} \dot{5}$.

Gobius echinocephalus Rüpp. cf. Paragobiodon echinocephalus Blkr.

Gobius elapoides Gïnth. cf. Pterogobius elapoides Blkr.

Gobius elegans (K. v. H.) C. $V .15,3.91,50$. cf. Acentrogobius ornatus Blkr.

Gobius eleotrioides Blkr. 15, 25. 270, 41. 275, 43. 287, 56 .

Gobius erythrinus $K . v . H$. cf. Gobius tentacularis $C$. $V$.

Gobius erythrophaios Blkr. 9, 637. 15, 29. cf. Gobiodon quinquestrigatus Blkr.

Gobius fasciato-punctatus Rich. cf. Gobius giuris $H . B$.

Gobius filifer $C . V .459,77$.

Gobius filosus C. $V$. 459, 77. cf. Glossogobius filosus Blkr.

Gobius flavimanus $T$. Schl. 90, 42. 453, 320. cf. Acanthogobius flavimanus Gill.

Gobius Fontanesii Blkr. 71, 764. 275, 43. 364, 292.

Gobius fuscus Rüpf. cf. Bathygobius punctillatus Blkr., Gobius nebulopunctatus $C, V$. 
Gobius fusiformis Blkr. 15, 30. 25, 9. 129, 443. cf. Gobius celebius $C . V$. Gobius gastrospilos Blkr. 78, 477. cf. Stigmatogobius gastrospilus Blkr.

Gobius giuris Buch. 15, 24. 16, 5. 25, 9. 91, 50. 270, 41. 358, 1 92. 364, 292. 425, 12S. 459, 77. cf. Glossogobius giuris $B l k r$.

Gobius gobiodon Day 453, 309 .

Gobius Goldmanni Blkr. 53, г67. 320, $27 \mathrm{r}$.

Gobius gracilis Girard 453, 319 .

Gobius grammepomus Blkr. 15, 34. 25, 10. 129, 443. 135, 200. 211, 24I. 231, I 47. 270, 4I. 275, 43. 296, III. 354, I 50. 364, 292. cf. Awaous personatus Blkr.

Gobius Grandidieri Playf. 459, Iо3.

Gobius grandinosus Eyd. Soul. cf. Gobius caninus $\mathrm{Val}$.

Gobius gutam Buch. 91, 50.

Gobius gymnauchen Blkr. 269, 84. cf. Ctenogobius gymnauchen Blkr.

Gobius gymnocephalus Blkr. 78, 473. 425, I2S.

Gobius gymnopomus Blkr. 76. 270. 270, 4r. 284, 240.453, 3 I 8.

Gobius Hasseltii Blkr. 34, 250. cf. Sicydium cyanocephalus $C$. $V$., Sicyopterus cynocephalus Blkr.

Gobius hasta $T$. Schl. 71, 765. 453, 325. cf. Synechogobius hasta Gill.

Gobius hemigymnopomus Blkr. 143, 50. 185, 224. 275, 43 .

Gobius histrio $K . v . H .15,4$.

Gobius Hoevenii Blkr. 49, 426. 55, 412. 272, I6.

Gobius hypselosoma Blkr. 384, 407. 459, 5I, 77.

Gobius interstinctus Rich. 34, 250. 54, 275. 119, I2. 285, 245. 296, I I I. 317, 246. 318, 252.320, 27 I. 358, 192.364, 292. cf. Gobius ornatus Rüpp.

Gobius isognathus Blkr. 384, 4II. 459, 53, 77 .

Gobius janthinopterus Blkr. 69, 702. 185, 224. 235, 370. 296, III. 358, 192 .

Gobius javanicus Blkr. 148, 88. 354, I 50.

Gobius Knutteli Blk). 175, r6. cf. Cryptocentrus Knutteli Blkr., Paragobius Knutteli Blkr.

Gobius Koelreuteri Pall. cf. Periophthalmus Koelrenteri C. $V$.

Gobius kokius $C . V .2,5$ I $4.15,24.16,5.25,9.35,26$ I. 42, 59. 45, I95. 49, 4I9. 55, 4I2. 56, 445. 91, 50. 227, 408. 270, 4I. 272. I6. $275,44.284,239.287,56.288,76.354$, I 49.356 , I 74. cf. Gobius giurus Buch.

Gobius Kuhlii Blkr. 34, $25 \mathrm{I}$.

Gobius Lagerstroemianus L. cf. Acanthogobius eleotris Blk\%.

Gobius lagocephalus Pall. cf. Sycidium lagocephalum C. $V$.

Gobius lanceolatus $B l .453,326$.

Gobius lentiginosus Rich. 119, I2.

Gobius Lienardi Blkr. 459, 77. cf. Awaous Lienardi Blkr.

Gobius macrognathos Blkr. 269, 83.

Gobius macrognathus Blkr.453, 309. cf. Gymnogobius macrognathus Gill. 
Gobius macrorhynchus Blkr. 384, 403. 453, 316. 459, 48, 77 .

Gobius macrurus Blkr. 15, 35. 16, 5. 25, 10. 129, 443. cf. Gobius tentacularis $C . V$.

Gobius madagascariensis Blkr. 384, 405. 459, 49, 77 .

Gobius margariturus Rich. 425, I28.

Gobius melanocephalus Blkr. 15, 33. 25, ıо. cf. Gobius gramnepomus Blkr.

Gobius melanopterus Blkr. 384, 409. 459, 52, 77.

Gobius melanopus Cast. 263, 455 .

Gobius melanosoma Blkr. 69, 703. 453, 309. cf. Paragobiodon melanosoma Blkr.

Gobius melanostigma Blkr. 15, 32. 287, 56. 453, 3 I 8 .

Gobius melanurus Blkr. 15, 31. 25, I0. 185, 224. 453, 319.

Gobius microlepis Blkr. 15, 35. 16, 5. 25, 1о. 113, 436 .

Gobius modestus Blkr. 15, 28. 16, 5. 25, 9 .

Gobius mystacinus $C . V$. 15, 4 .

Gobius nebulopunctatus $C . V .174,40.270,42.459,77$. cf. Bathygobius nebulopunctatus, punctillatus Blkr.

Gobius nebulopunctatus Rüpp. cf. Gobius albopunctatus $C . V$.

Gobius Newberri Gir. 453, 319.

Gobius niger Lac. cf. Awaous Commersoni Blkr., Gobius Commersonii $C . V$.

Gobius niger $L .453,3$ r6, 3r 7 . cf. Gobius nox Blkr.

Gobius nigripinnis $C . V .459,77$.

Gobius Nilssoni Düb. Kor. 453, 310.

Gobius niveatus $C . V .15,4$.

Gobius notacanthus Blkr. 184, 2 I0. 437, 37. 453, 32 I.

Gobius nox Blkr. 34, 248. 156, $215.270,42.394,288$.

Gobius nudiceps $C V$. 266, 73 .

Gobius nunus Buch. 91, 50.

Gobius occellaris Brouss. 453, 320. 459, 77. cf. Awaous ocellaris Blkr.

Gobius oligolepis Blkr. 86, 508. 270, 42.

Gobius olivaceus Schl. 408, 244. cf. Glossogobius olivaceus Blkr.

Gobius ommaturus Rich. 453, 319. cf. Acanthogobius ommaturus Blkr.

Gobius ophicephalus Jen. 453, 310.

Gobius ophthalmonema Blkr. 155, 208.

Gobius ophthalmoporus Blkr. 84, 340.

Gobius ophthalmotaenia Blkr. 105, 46. 275, 44. 425, I28.

Gobius ornatus Rïpp. 394, 288. 396, 300. 459, го3.

Gobius oxypterus Blkr. 138, 400. 453, 3I I. cf. Leptogobius oxypterus Blkr.

Gobius padangensis Blkr. 34, 249. 236, 331, 270, 42.

Gobius pallidus C. $V .459,77$. cf. Awaous pallidus $B / k r$.

Gobius papilio $C . V .459,77$. cf. Amblygobius semicinctus Blkr.

Gobius papuanus Peters of. Cryptocentrus papuensis Blkr. 
Gobius papuensis C. $V .15,4$. cf. Oxyurichthys papuensis Blkr.

Gobius pasuruensis Blkr. 15, 32. 25, ro.

Gobius pavoninoides Blkr. 15, 33. 16, 5. 287, $5^{6 .}$

Gobius peelmotta Blkr. 91, 50.

Gobius periophthalmoïles Blkr. 34, 249. 167, 372. 184, 201. 185, 224. $234,356.270,42.275,44.287,56.320,27$ I. 354, I 5 0. 358, I92. 364, 292.

Gobius personatus Blkr. 15, 34. 25, го. 129, 443. cf. Awaous personatus Blkr. Gobius grammepomus Blkr.

Gobius petrophilus Blkr. 78, 476. 184, 201. 231, I 47. 236, 331, 333. 270, 42. 320, 27 I. 354, I49. 358, 192. 364, 292. cf. Bathygobius petrophilus Blkr.

Gobius Pflaumii Blkr. 90, 42. cf. Acentrogobius Pflaumi Blkr.

Gobius phaiomelas Blkr. 15, 28. 16, 5. 25, 9. 287, 56.

Gobius phaiosoma Blkr. 15, 30. 129, 443. cf. Gobius celebius $C . V$.

Gobius phaiospilosoma Blkr. 15, 30. 16, 5. 25, 9.

Gobius phalaena C. V. 47, 244. 53, I6 I. 54, 237. 156, 2 I 5. 157, 233. 167, 372. 184, 20I. 188, 460. 234, 356. 275, 44. 315, 236. 318, 252. 320, 27 r. 358, I92. 364, 292. cf. Amblygobius semicinctus Blkr.

Gobius platycephalus Rich. 425, I28. 453, 3 I6.

Gobius pleurostigma Blkr. 15, 28. 25, 9. 91, I03. 272, I6. 453, 323 . cf. Stigmatogobius sadanundio $B l k r$.

Gobius Plinianus Buch. 15, 40. cf. Boleophthalmus Boddaerti $C$. $V$.

Gobius poicilosoma Blkr. 15, 31. 25, 10.

Gobius polycynodon Blkr. 15, 25. 16, 5. 25, 9.

Gobius polyophtpalmus Blkr. 78, 474.

Gobius polyzona Blkr. 384, 4I3. 459, 55, 77 .

Gobius punctillatus Rüpp. cf. Bathygobius punctillatus $B l k r$.

Gobius puntang Blkr. 50, 486. 287, 56. 315, 236. 320, 27 I. 354, I49. 358, I92. 364, 292. 395, 293. cf. Gobius puntangoïdes Blkr.

Gobius puntangoides Blkr. 83, 242. 167, 372. 185, 224. 275, 44 . $287,56.395,293$.

Gobius quadrimaculatus $\mathrm{Val} .453,3$ I 7 .

Gobius quinquestrigatus $C . V .9,633.15,29.80, S_{2}$. cf. Gobiodon quinquestrigatus Blkr.

Gobius quinquestrigatus $B l k r$. cf. Gobiodon erythrospilus Blkr.

Gobius Reichei Blkr. 86, 509. 270, 42.

Gobius Richardsonii Blkr. 86, 508. 270, 42. 275, 44.

Gobius ripilepis Rich. 425, I 28 .

Gobius rivulatus Rüpp. cf. Gobiodon rivulatus Gïnth.

Gobius rubrotaeniatus Lién. 459, 78. 495, 17.

Gobius Russelii C. V. 2, 514. 91, 50.

Gobius russus Cant. 288, 76 .

Gobius sadanundio Buch.91, I02. cf. Stigmatogobius sadanundio Blkr. 
Gobius samberanoensis Blkr. 384, 4I7. 459, 57,78 .

Gobius Schlegelii Gthr. 302, xо3. 453, 32 I.

Gobius semicinctus Benn. 459, 78. cf. Amblygobius semicinctus Blkr.

Gobius semidoliatus $C . V .453,323$.

Gobius semifasciatus Kner 453, 323.

Gobius setosus C. $V .91,50$.

Gobius soporator C. $V$. 459, 78. cf. Bathygobius soporator $B l k r$.

Gobius sphynx $C . V .15,4.95$, 103. 167, 372. 184, 201. 296, I1 r. 358, 192. 364, 292. 453, 322. cf. Amblygobius sphynx Blkr.

Gobius spilurus Blkr. 15, 32. 16, 5. 25, I0.

Gobius stethophthalmus Blkr. 34, 248. 185, 236. 234, 356. 287, 56.

Gobius stigmothonus Rich. cf. Acanthogobius stigmothonus Blkr.

Gobius striatus Bl. Schn. cf. Boleophthalmus Boddaerti C. $V$.

Gobius strigatus Brouss. cf. Valenciennesia strigata Blkr.

Gobius Stuvitzii Düb. Kor. 453, 3 то.

Gobius sublitus Cant. 453, 320.

Gobius sumatranus Blkr. 106, 83. 270, 42.

Gobius tambujon Blkr. 110, 319. 231, 147.

Gobius tannao Osb. cf. Acanthogobius tannao Blkr.

Gobius Temminckii Blkr. 15, 33. 16, 5. 25, 10.

Gobius tentacularis C. $V$. 15, 4. 113, 434. 129, 443.

Gobius tjilankahanensis Blkr. 34, $25 \mathrm{r}$.

Gobius unicolor $K . v, H .15,27$.

Gobius vagina $B l$. Schn. cf. Trypauchen vagina $C . V$.

Gobius venenatus $C . V .2,5$ I $4.91,50$.

Gobius Vergeri Blkr. 384, 4I8. 459, 58, 78 .

Gobius virgo Schl. 453, 322. cf. Pterogobius virgo Gill.

Gobius viridipunctatus $C . V .2,5$ 14. 91, 50.

Gobius Voigtii Blkr. 106, 83. 156, 2 I5. 175, I7. 234, 356. 270, 42. $275,44.287,56$.

Gobius xanthosonia.Blkr. 69, 703. cf. Paragobiodon xanthosoma Blkr. Gobius xanthotaenia Blkr. 137, 308.

Gobius xanthozona Blkr. 15, 34. 16, 5. 25, 1о. 191, 2. 270, 42. 272, I7. 453,319 .

Gobius Yokohamae Gthr. cf. Acentrogobius Yokohamae Blkr.

Gompherus fuscus $\mathrm{Val} .497,5$.

Gomphosus Lac. 292, 97.

Gomphosus Cepedianus Q. G. 11, 7. 12, 42 .

Gomphosus coeruleus Lac. 76, 292. 91, 54. 270, 38. 293. 278. 459, 82. 495, 9 .

Gomphosus fuscus C. V. 91, 54. cf. Gomphosus varius Lac.

Gomphosus melanotus Blkr. 130. 457. 293, 278.

Gomphosus notostigma Blkr. 293, 304.

Gomphosus tricolor $Q . G$. 143, 54. 270, 38. 275, 16. 293, 278. 364, 276.425 , I 35 . 
Gomphosus varius Lac. 459, 82. 495, 9.

Gomphosus viridis Benn. cf. Gomphosus coeruleus Lac.

Gonenion serra $R a f$. cf. 'Temnodon saltator $C . V$.

Goniistius Gill 468, 315.

Goniistius zonatus Gill 468, 315.

Gonioperca Gill 468, 257.

Gonioperca albomaculata Gill 468, 257.

Gonioplectrus Gill 468, 255.

Gonochaetodon Blkr. 468, 306.

Gonoproctopterus Blkr. 261, 430. 314, I99.

Gonopterus Gron. 468, 309.

Gonopterus moerens Gron. cf. Zanclus cornutus C. $V$.

Gonorhynchus abbreviatus Schl. 497, 22.

Gonorhynchus brevis 91, I36.

Gonorhynchus Greyi $C . V$. 459, IоI.

Gonorhynchus Gronovii $C . V .266,56$. cf. Gonorhynchus Greyi $C . V$.

Gonorhynchus macrosomus $91, \mathbf{1} 36$.

Gonostoma 1, $55^{2}$.

Gonostoma javanicum $K$. $. H .2,509$. cf. Anodontostoma Hasseltii Blkr.

Gramma Poey 468, 321 .

Gramma loreto Pocy 468, $32 \mathrm{I}$.

Grammateus Poey 468, $28 \mathrm{I}$.

Grammatopleurus lagocephalus Gill 497, I I.

Grammisteiformes 468, 258.

Grammistes Art. 468, 258.

Grammistes compressus Lićn., Lich. cf. Grammistes ocellatus Blkr.

Grammistes Forsteri Bl. Schn. cf. Paracirrhites Forsteri Blkr.

Grammistes furcatus $B l$. Schn. cf. Pristipoma furcatum Blkr.

Grammistes lineatus $B l$. Schn. cf. Diagramma lineatum $C$. $V$., Plectorhynchus lineatus $B l k r$.

Grammistes ocellatus Blkr. 423, I09. 459, 23, 89. 495, II.

Grammistes orientalis $B l$. Schn. 72, 105. 157, 232. 167, 370. 184, 199. 236, 330, 333. 270, 16. 275, 29. 277, 89. 315, 233. 318, 25 I. 354, I 47.358 , I $88.364,284.382,398.437,38.451$, г 29.459 , 89. $468,258.491 .42 .495$, II.

Grammistes pictus $B l$. Schn. cf. Diagramma punctatum Elrr., Plectorhynchus pictus $B l k r$.

Grammistes punctatus C. V. 163, 31. 389, 273. 423, 108. 451, 127. $459,24,89$.

Grammistes quinquelineatus $B l$. Scln. cf. Lutjanus quinquelineatus Blkr., Mesoprion octolineatus Blkr.

Grammistes servus $B l$. Schn. cf. Therapon (Datnia) jarbua Blkr.

Grysteini 468, 26 I.

Grystes C. V. 468, 26I. 
Grystes lunulatus Guich. 459, 89. 468, 262.

Grystes macquariensis C. V. 119, 6.

Grystes Peelii Mitch. 119, I9.

Grystes salmonoides C. V. 468, 272.

Grundulus Val. 261, 440.

Guathagnus elongatus Gill 497, 18.

Guavina Blkr. 453, 302. 460, 103.

Guiritinga Blkr. 306, 90.

Guiritinga Commersonii Blkr. 306, 90.

Gunellus roseus $V a l .449,369$.

Gunnellichthys Blkr. 176, 9.

Gunnellichthys pleurotaenia Blkr. 176, I0. 184, 201. 275, 46. 364, 293.

Gunnellini 449, 368.

Gunnellops Blkr. 449, 368 .

Gunnellus crassispina Schl. cf. Centronotus crassispinis Gill.

Gunnellus nebulosus Schl. cf. Centronotus nebulosus Gill.

Güntheria Blkr. 292, IоI.

Güntheria coeruleovittata Blkr. 293, 291. 318, 250. 320, 266. 354, I 44. 358, I85. 364, 277. cf. Güntheria scapularis Blkr., Platyglossus (Güntheria) scapularis Blkr.

Güntheria scapularis Blkr. 394, 286. 437, 38. 459, $8_{3}$.

Güntheria trimaculata Blkr. 293, 291. 318, $250.320,266.338,360$. 354, I $44.358,185.364,277.369,42.425$, I 35 .

Gymnapistus Stons. 473, 299.

Gymnapistus barbatus Siens. 475, 85 .

Gymnapistus hypselopterus Gthr. 338, 360.

Gymnapistus hypselopterus Blkr. cf. Paracentropogon longispinis Blkr.

Gymnapistus leucogaster Blkr. 475, 88 .

Gymnapistus marmoratus Blkr., Szons. 320, 269. 475, $\mathrm{S}_{7}$.

Gymnapistus niger Blkr. 475, 86.

Gymnapistus rubripinnis Gill 497, I2.

Gymneleotris Blkr. 453, 304. 460, $103,105$.

Gymnetrus capensis C. $V$. 266, 54 .

Gymnetrus Russelii Shaw, C. V. 91, 48.

Gymnobutis Blkr. 453, 304. 460, 103, 105.

Gymnocaesio Blkr. 468, 273. 470, r 52.

Gymnacaesio gymnopterus Blkr. 468, 274. 470, 152.

Gymnocephalus Bl. 468, 266.

Gymnocephalus schraetser Bl.468, 266.

Gymnocranius Blkr., Klunz. 438, 41. 468. 278.

Gymnocranius frenatus Blkr. 438, 43. 441, 46 .

Gymnocranius griseus Blkr. 437, 38. 438, 42. 441, 43. 495, 12. 497, 7 .

Gymnocranius lethrinoides Blkr. 438, 42. 441, 45 .

Gymnocranius microdon Blkr. 441, 48. 459, 9I.

Gymnocranius rivulatus Klunz. 459, 91. 468, 278.495 , I 2. 
Gymnocrotaphus Gthr. 468, 295.

Gymnocrotaphus curvidens Gthr. 468, 295.

Gymnogobiini 453, 309 .

Gymnogobius Gill 453, 309.

Gymnogobius macrognathus Gill 497, I9.

Gymnomuraena Lac. 88, 38. 351, 122.

Gymnomuraena Bennetti Gthr. 459, 73.

Gymnomuraena concolor Blkr. 459, 73. 495, 23.

Gymnomuraena doliata Lac. cf. Muraena zebra Cıv., Echidna zebra Blkr.

Gymnomuraena fasciata $K p$. cf. Echidna zebra Blkr.

Gymnomuraena macrocephalus Blkr. 348, 54. 352, I35. 364, 297.

Gymnomuraena marmorata Lac. cf. Gymnomuraena pantherina Blkr.

Gymnomuraena micropterus Blkr. 352, I35. 354, I5I. 358, I93.

364, 297. 382, 398. 394, 288. 396, 301. 437, 37 .

Gymnomuraena pantherina Blkr. 352, I35. 364, 297.

Gymnomuraena tigrina Blkr. 352, I36. 364, 297. 459, 73. 495, 23.

Gymnomuraena xanthopterus Blkr. 352, I36. 364, 297.

Gymnomuraena zebra Lac. 320, 272.

Gymnomuraena zebra Shaw, Kp. cf. Muraena zebra Cut', Echidna zebra Blkr.

Gymnostomus Heck. 261, 427. 314, I97.

Gymnostomus (Gymnostomus) macrolepis Blkr. 411, 252. 415, 32. 425, I 43 .

Gymnostomus molitorella Blkr. 415, 8. 416, г20. 425, I43.

Gymnothorax Bl. 351, 121 .

Gymnothorax afer Bl. 302, I 7.

Gymnothorax Agassizi Blkr. 352, I31. 364, 296.

Gymmothorax albimarginatus Blkr. 352, I3 I. 354, I5 I. 364, 296. $497,26$.

Gymnothorax annulatus $B l$. Schn. cf. Leiuranus colubrinus $K p$., Ophisurus colubrinus Rich.

Gymnothorax aterrimus Blkr. 362, 244.

Gymnothorax Augusti Blkr. 459, 72 .

'Gymnothorax aulopterus Blkr. 459, 72. 495, 22.

Gymnothorax Bleekeri Blkr. 352, 136.

Gymnothorax Blochi Blkr. 352, I31. 364, 296.

Gymnothorax borneënsis Blkr. 312, r69. 348, 52. 352, I 31 .

Gymnothorax Boschi Blkr. 352, 131. 397, 302.

Gymnothorax bullatus Blkr. 315, 237. 352, I3r. 354, I5 I. 425, I24.

Gymnothorax buroensis Blkr. 352, I3I. 354, I 5 I. 358, 193. 364, 296.

Gymnothorax cancellatus Blkr. 352, r 3 r. 364, 296. 459, 73.

Gymnothorax caninus Blkr. 352, r36. 396, 298.

Gymnothorax catenatus Bl. cf. Echidna catenata Blkr., Muraena catenata Blkr. 
Gymnothorax ceramensis Blkr. 320, 272. 352, I31. 354, I5I. 358, I 93. 364, 296. 394, 288. 396, 300. 397, 302 .

Gymnothorax cerino-niger Blkr.425, I 24.

Gymnothorax chilospilus Blkr. 348, 52. 352, I32. 354, I 51. 364, 296.

Gymnothorax chlorostigma Blkr. 352, 132.

Gymnothorax cinerascens Blkr. 459, 72 .

Gymnothorax colubrinus Blkr. 315, 237. 318, 252. 320, 272.

Gymnothorax crudelis Blkr. 312, r68. 320, 272.

Gymnothorax Duivenbodei Blkr. 315, 237. 352, I32.

Gymnothorax echidna Bl. Schn. cf. Echidna variegata J. R. Forst., Muraena variegata Forst.

Gymnothorax fasciatus $B l$. Schn. 88, 36. cf. Ophisurus colubrinus Rich., Ophisurus fasciatus Rich.

Gymnothorax fimbriatus Blkr. 437, 37. 459, 72. 495, 22.

Gymnothorax flavimarginatus Blkr. 352, 132. 358, 193. 364, 296. $459,72.491,56.495,22$.

Gymnothorax floresianus Blkr. 315, 237. 318, 252.

Gymnothorax formosus Blkr. 348, 5 1. 352, I32.

Gymnothorax funebris Ranz. 362, 245.

Gymnothorax griseobadia Blkr. 354, I5 r. 358, 193. 364, 296.

Gymnothorax griseobadius Blkr. 352, I32. 491, 56.

Gymnothorax helena Blkr. 459, 73.

Gymnothorax isingleena Blkr. 495, 22.

Gymnothorax isingleenoides Blkr. 312, I 70. 315, 237. 320, 272. 352, I32. 354, г 5 т. 358, г 93. 364, 296. 397, 302. 491, 56. cf. Gymnothorax fimbriatus Blkr.

Gymnothorax isingteena Blkr. 352, I 32. 459, 73 .

Gymnothorax jacksoniensis Blkr. 331, $45^{\circ}$.

Gymnothorax javanicus Blkr. 348, 51, 352, I32.

Gymnothorax Kidako Blkr. 497, 26.

Gymnothorax macassariensis Blkr.425, I24. cf. Gymnothorax makassariensis Blkr.

Gymnothorax macrosiphon Blkr. 425, I24, I54.

Gymnothorax maculaepinnis Blkr. 302, I29.

Gymnothorax makassariensis Blkr. 312, I68. 352, I32. cf. Gymnothorax macassariensis Blkr.

Gymnothorax margaritophorus Blkr. 348, 53. 352, 132. 364, 296. Gymnothorax marmoratus Bl., Blkr. 352, г36. 396, 298.

Gymnothorax mauritianus Blkr. 459, 73. 495, 22.

Gymnothorax melanospilus Blkr. 352, I33. 354, I5I.

Gymnothorax meleagris Blkr. 459, 73. 495, 22.

Gymnothorax micropoecilus Blkr. 317, 246. 320, 272. 352, I33. 364, 296. 396, 300.

Gymnothorax monochrous Blkr. 315, 237. 352, I33. 364, 296.

Gymnothorax moringua Blkr. 459, 73 . 
Gymnothorax Mulleri Blkr. 352, I33.

Gymnothorax nebulosus Bl. Schu. cf. Echichna variegata J. R. Forst., Muraena variegata Forst.

Gymnothorax nubilus Blkr. 459, I03.

Gymnothorax ocellatus $A g .348,49$.

Gymnothorax pantherinus Blkr. 308, I52. 315, 237. 320, 272. cf. Gymnothorax pictus Blkr.

Gymnothorax pardalis Blkr. 352, I33.459, 73. 495, 22.497, 26.

Gymnothorax Peli Blkr. 302, I30.

Gymnothorax Petelli Blkr. 352, I33. 364, 296. 459, 73. 495, 22.

Gymnothorax pictus Blkr. 352, I33. 358, I93. 364, 296. 459, 73. 491,56 .

Gymnothorax polyophthalmus Blkr. 352, 133 .

Gymnothorax polyuranodon Blkr. 352, I33. 354, I5 I. 358, I93. 364, 296.396, 30I.

Gymnothorax prosopeion Blkr. 309, I 56. 320, 272. 352, I33. 358, I93. 364, 296.

Gymnothorax pseudothyrsoidea Blkr. 495, 23.

Gymnothorax pseudothyrsoideus Blkr. 352, 134. 364, 296.

Gymnothorax punctato-fasciatus Blkr. 312, I67. 352, I34. 354, I5I. $364,296$.

Gymnothorax Reevesii Blkr. 425, r24. 495. 23.

Gymnothorax reticularis Bl., Blkr. 352, I34. 354, I 5 I. 358, 193. 364, 296. 397, 302 .

Gymnothorax reticulatus $B l$. Schn. cf. Gymnothorax reticularis $B l$.

Gymnothorax rhodocephalus Blkr. 348, 50. 352, 134. 364, 296.

Gymnothorax Richardsoni Blkr. 352, т 34. 358, т93. 364. 296. 382, 398. 394, 288.

Gymnothorax sagenodeta Blkr. 364, 296. 459, 73. 495, 23.

Gymnothorax sagenodetus Blkr. 352, I34.

Gymnothorax schismatorhynchus Blkr. 352, I34.

Gymnothorax scoliodon Blkr. 952, 134.

Gymnothorax similis Blkr. 497, 26.

Gymnothorax stellifer Blkr. 459, 73 .

Gymnothorax tessellatus Blkr. 352, I 24. 382, 398. 425, I 24. 437, 37 . $459,73.491,56.495,23$.

Gymnothorax thyrsoides Blkr. 352, I 35 .

Gymnothorax thyrsoideus Blkr. 425, I 24.

Gymnothorax tigrinus Blkr. 459, 73 .

Gymnothorax tile Blkr. 352, I35. 459, 73.

Gymnothorax Troscheli Blkr. 315, 237.

Gymnothorax unicolor Blkr. 459, 73.

Gymnothorax venosus Blkr. 320, 272.

Gymnothorax zebra Bl. Schn. cf. Muraena zebra Cuv.

Gymnura micrura $K u h l$ cf. Pteroplatea micrurus $M$. $H$. 
Haemulon $C . V .468,27$ I.

Haemulon elegans Cuv. 468, 27 I.

Haemulopsis Steind. 468, $27 \mathrm{I}$.

Halicampus koilomatodon Blkr. 270, 71. 408, 238. 497, I3.

Halichoeres Rüpp. 1, 553. 292, 99.

Halichoeres amboinensis Blkr. 293, 285. 364, 276.

Halichoeres bicolor Blkr. 293, 285. 364, 276 .

Halichoeres binotopsis Blkr. 293, 285. 315, 230. 320, 266. 358, 184 . 364, 276. 382, 397. 394, 286.

Halichoeres chloropterus Blkr. 293, 285. 364, 276. 425, 135. cf. Platyglossus (Halichoeres) chloropterus Blkr.

Halichoeres coeruleovittatus Rüpp. 292, Iог.

Halichoeres eximius Rïpp. cf. Julis (Halichoeres) hortulanus $C$. $V$.

Halichoeres guttatus Blkr. 293, 286. 354, I44. 358, I 84 364, 276. 425, I35.

Halichoeres Hartzfeldi Blkr. 393, 286. 315, 230. 354, I 44. 364, 276.

Halichoeres Horsfieldi Blkr. 356, I 73

Halichoeres Hyrtli Blkr. 293, 286.

Halichoeres javanicus Blkr. 293, 286.

Halichoeres kawarin Blkr. 293, 286. 320, 266.

Halichoeres Kneri Blkr. 293, 307.

Halichoeres leparensis Blkr. 293, 287. 315, 230. 320, 266. 338, 360, 354, I44. 364, 276.

Halichoeres miniatus Blkr. 293, 287. 315, 230. 320, 266. 364, 276. $394,286.425$, I 35 .

Halichoeres modestus Blkr. 293, 287. 320, 266. 354, I44. 364, 276. Halichoeres nigrescens Blkr. 293, 287. 320, 266. 354, I 44. 425, I 35 . Halichoeres pardaleocephalus Blkr. 293, 287.

Halichoeres podostigma $B l k r .293,288.318,250.354$, 144. 364, 277. Halichoeres poecila Blkr. 293, 288. 315, 230. 318, 250. 320, 266. 354, I 44. 364, 276. 382, 397. 437, 38.

Hailchoeres poecilopterus Blkr. 363, 251. 425, I35. cf. Platyglossus (Parajulis) poecilopterus $B l k r$.

Halichoeres prosopeïon Blkr. 293, 288. 364, 277.

Halichoeres pseudominiatus Blkr. 293, 2SS. 318, 250. 320, 266. 364, 277. Halichoeres pyrrhogramma Blkr. 363, 253. cf. Platyglossus (Parajulis) pyrrhogramma Blkr.

Halichoeres Reichei Blkr. 293, 288. 354, I44. 358, I85. 364, 277.

Halichoeres Schwarzi Blkr. 293, 288, 296, 104. 309, r54. 315, 230. $320,266.338,360.340,364.354$, I 44.358, I $84.364,277.394,286$. Halichoeres solorensis Blkr. 293, 289. 320, 266. 358, I85. 364, 277 . $394,286$.

Halichoeres tenuispinis Blkr. 349, 57, 425, I35.

Halichoeres timorensis Blkr. 293, 289. 320, 266.

Halichoeres variegatus Rüpp. 292, 99. 
Halientaea stellata $C . V .54,279.93$, 10. 358, г $\delta_{4} .425$, r $22.497,20$.

Haligenes Gt/hr. 302, 40.

Haligenes guineënsis Blkr. 302, $4 \mathrm{I}$.

Haliperca Gill 468, 255.

Halosaurus aftinis Gïnth. 497, 2 I.

Hamiltonia Swons. 468, 292.

Hamiltonia ovata Szons. 468, 292.

Hampala v. Hass 1, 552. 261, 430. 314, 200.

Hampala ampalong Blkr. 270, 53. 272, 23.

Hampala macrolepidota $K$. v. H. 225, 357. 270, 53. 272, 23. 288, 78. 356, г76. cf. Capoeta macrolepidota $K . v$. $H$.

Hapalogenys Rich. 468, $27 \mathrm{I}$.

Hapalogenys analis Rich. cf. Hapalogenys mucronatus Gthr.

Hapalogenys maculatus Rich. 118, 65. 497, 7. cf. Hapalogenys nigripinnis Gthr.

Hapalogenys mucronatus Gthr. 307, 139. 425, I39.

Hapalogenys nigripinnis Gthr., Rich. 425, I39. 497, 7.

Hapalogenys nitens Rich. 468, 271.

Haploactis cottoides $v$. d. Hoez. cf. Aploactis aspera Blkr.

Haplochilus homalonotus Gthr. 459, гог.

Haplochilus nuchimaculatus Guich. 459, ror.

Haplochilus Playfairii Gthr. 459, гог.

Haplochilus (Panchax) rubropunctatus Steind. 430, 234.

Haplodactylus Gthr. 468, 3 I 7 .

Haplodactylus lophodon Gthr. 468, 3 I 7 .

Haploidonotus Gill 468, 324 .

Hara Blyth 306, 95 .

Hara aspera Gthr. 425, 125.

Hara Buchanani Blyth 306, 95.

Harengula abbreviata $C$. 119, I 5 .

Harengula arabica $C$. $V$. cf. Clupea (Harengula) arabica Blkr.

Harengula (Sardinella) clupeoides Blkr. 395, 294.

Harengula dispilonotus Blkr. 56, 456. 60, 49. 234, 356. 287, 6I.

Harengula hypselosoma Blkr. 128, 427.

Harengula (Spratella) kowala Blkr. 395, 294. 396, 300.

Harengula Kunzei Blkr. 155, 209. 185, 226. 315, 236. 364, 295. $394,288$.

Harengula melanurus Blkr. 83, 245. 147, 469. 156, 217. 231, I 50. $275,48.296$, II $2.315,236.316,243.338,361.358$, I92. 364, 295. 382, 398. cf. Harengula (Paralosa) Valenciennesi Blkr.

Harengula moluccensis Blkr. 79, 609. 118, IIS. 127, 345. 147, 469. 156, 2 I7. 231, I50. 237, 438. 270, 56. 275, 49. 287, 6 г. 296, г 1 2. $315,236.320,272.338,36$ I. 354, I 50. 358, I92. $364,295$. Harengula punctata $C . V .90,49$.

Harengula spilura Guich. cf. Clupea (Harengula) spilurus Blkr. 
Harengula (Paralosa) Valenciennesi Blkr. 396, 300.

Harengula zunasi Blkr. 102, 4I7. 118, II7.

Harpe oxycephalus Blkr. 497, 5 .

Harpochirus Cant. 468, 30I.

Harpochirus longimanus Cant. cf. Harpochirus punctatus Cant.

Harpochirus punctatus Cant. 354, I48. 356, I 73. 364, 283. 395, 292. 396, 299. 425, 140. 459, 94. 468, 3० I. 481, 2 I. 491, 44. cf.

Drepane longimana $C . V$.

Harpodon microps Les. 270, $5^{6}$.

Harpodon nehereus Gthr. 425, 147.

Harpodon ophiodon Les. 235, 372. 272, 27. 287, 62. 288, 79.

Harpurus Desjardini Blkr. 459, 97. 495, I9.

Harpurus fasciatus Forst. cf. Acanthurus triostegus Bl. Schn.

Harpurus gemmatus Blkr. 459, 97. 495, I9.

Harpurus glaucopareius Forst. cf. Acanthurus glaucopareius $C$. $V$.

Harpurus hypselopterus Blk\%. 318, 252.

Harpurus lunulatus 459, 97. 495, 19.

Harpurus monoceros Forst. cf. Naseus fronticornis Comin.

Harpurus rhombens Blkr. 315, 235. 318, 252. 320, 27 1. 340, 365 . 354, I49. 358, г 90. 364, 288. 388, 272. 394, 288. 459, 97. 491, 51. 495, I9.

Harpurus Rüppelli Blkr. 315, 235. 338, 360. 358, I90. 364, 288. $391,276.459,97.491,5$ I. 495, I9.

Harpurus suillis Blkr.459, 97. 495, I9.

Hectoria Cast. 468, 336.

Heliases amboinensis Blkr. 424, III. cf. Chromis amboinensis Blkr.

Heliases analis $C . V .6,2.364,279.425$, I36. cf. Chromis analis Blkr.

Heliases axillaris $C . V$. cf. Chromis axillaris Blkr.

Heliases cinctus Playf. cf. Chromis cinctus Blkr.

Heliases cinerascens $C . V .6,3$ r. 126, 344. 275, 20. 320, 267. 358, เ86. 425, I36. cf. Chromis cinerascens Blkr.

Heliases coeruleus C. V. 6, 2. 130, 455. 320, 267. 354, I 45. 358, г 86 . 364, 279. 437, 38. cf. Chromis lepisurus Blkr.

Heliases frenatus C. $V .69,7$ I0. 234, 355. 237, 438. 275, 20. 315, 231. 318, 250. 354, I 45. 358, I 86. 364, 279.

Heliases frenatus, Blkr. cf. Chromis lepisurus Blkr.

Heliases lepisurus $C . V ., B l k r .6,2.9,633$. cf. Chromis lepisurus Blkr. Heliases macrochir Blkr. 84. 346. 354, I45. cf. Chromis analis Blkr. Heliases notatus Schl. 425, I36. cf. Chromis notatus Blkr.

Heliases reticulatus Rich. cf. Tetradrachmum reticulatum $B l k r$.

Heliases ternatensis Blkr. 145, 377. 167, 373. 184, 203. 315, $23 \mathrm{I}$. 316, 24r. 318, 250. 354, I 45. 437, 38. cf. Chromis ternatensis Blkr. Heliases xanthochir Blkr. 315, 231. 318, 25I. 354, I45. 358, I 86. 364,279 .

Heliases xanthochirus Blkr. 47, 248. cf. Chromis xanthochir Blkr. 
Heliases xanthurus Blkr. 95, 107. 287, 44. cf. Chromis xanthurus Blkr. Heliastes analis Gthr. cf. Chromis analis Blkr.

Heliastes cinerascens Gthr. cf. Chromis cinerascens Blkr.

Heliastes coeruleus Gthr. cf. Chromis lepisurus Blkr.

Heliastes frenatus Gthr.cf. Chromis lepisurus Blkr.

Heliastes lepidurus Gthr. cf. Chromis lepisurus Blkr.

Heliastes ternatensis Gthr. cf. Chromis ternatensis Blkr.

Heliastes xanthochir Gthr. cf. Chromis xanthochir Blkr.

Heliastes xanthurus Gthr. cf. Chromis xanthurus Blkr.

Helicophagus Blkr. 174, 45. 299, 399. 306, 107.

Helicophagys typus Blkr. 174, 46. 270, 46. 306, Iо8.

Helicophagus Waandersi Blkr. 270, 46.

Helmichthys oculus Petcrs cf. Leptocephalus oculus Blkr.

Helostoma 1, 553 .

Helostoma oligacanthum Blkr. 2, 520. cf. Helostoma Temminckii (K. $\because . H.) C: V$.

Helostoma striolatum $K . v . H$. cf. Helostoma Temminckii $(K . v . H) C .$.$V .$

Helostoma tambakkan Blkr. 2, 520. cf. Helostoma Temminckii ( $K$. ข. H.) C. $V$.

Helostoma 'Temminckii $\left(K . v . H_{*}\right) \quad C . V .2,520.21,9.35,26$ r. 42,

59. 45, I95. 49, 4I9. 55, 4I I. 239, гог. 270, 34. 272, го. 356,

I 73. 498 , I 5 .

Helotes $C . V .468,267$.

Helotes octolineatus Jen. 119, 6.

Helotes polytaenia Blkr. 94, 53. 309, I55. cf. Therapon (Pelates) quadrilineatus Blkr.

Helotes sexlineatus C. $V .40,478.44$, I 71. 50, 47 1. 51, 55 52, 89. $119,6.185,222.346,3$ г. 358, т 86 395, 29 г. 468, 267. cf. Therapon (Helotes) sexlineatus $B l k r$.

Helotosoma $K p .468,298$.

Helotosoma servus $K p .468,298$. cf. Atypichthys strigatus Gthr.

Hemanthias Steind. 468, 252.

Hemerocoetes acanthorhynchus $C .119$, I 2 .

Hemiancistrus Blkr. 306, 78 .

Hemiancistrus medians Blkr. 306, 78 .

Hemiarius Blkr. 306, 90.

Hemiarius Stormi Blkr. 306, 90.

Hemibagrus Blkr. 306, 96.

Hemibagrus Hoevenii Blkr. 356, I75.

Hemibagrus limbatus Blkr. 425, I25.

Hemibagrus macropterus Blkr. 413, 257. 425, I25.

Hemibagrus nemurus Blkr.306, 96. 356, 175.

Hemibarbus Blkr. 261, 431. 314, I98.

Hemibarbus barbus Blkr. 497, 23.

Hemibarbus dissimilis Blkr.411, 252. 415, $21.425,144$. 
Hemibarbus maculatus Blkr. 511, 252. 415, I9. 425, I44.

Hemicaranx Blkr. 297, r37. 302, 8I.

Hemicaranx marginatus Blkr. 297, 138. 302, 8r.

Hemicetopsis Blkr.300, 403. 306, III.

Hemicetopsis candira Blkr. 306, II I.

Hemichaetodon Blkr. 468, 305 .

Hemichromis Pet. 302, 38 .

Hemichromis fasciatus Pet. 302, 38 .

Hemicoris Blkr. 292, 99.

Hemicoris batuensis Blkr. 293, 283 .

Hemicoris caudimacula Blkr. 293, 283. 422, 98. 459, 6, 83. 495, ro.

Hemicoris cingulum $B l k r .459,83.495$, то.

Hemicoris variegata Blkr. 293, 283. 320, 266. 364, 276.

Hemiculter Blkr. 261, 432. 314, 210

Hemiculter leucisculus Blkr. 411, 252. 415, 76. 416, I 2 I. 425, I45.

Hemiculter machaeroides Blkr. 415, I 5. 425, I45.

Hemigaleus macrostoma Blkr. 64, 46. 287, 37 .

Hemigaleus microstoma Blkr. 64, 46. 287, 37. 364, 270.

Hemiglyphidodon plagiometopon Blkr. 496, 8 .

Hemigobius Blkr. 453, 3 I9.

Hemigymnus Gthr. 292, гог.

Hemigymnus fasciatus Gthr. 293, 292. 364, 277. 459, 82.495, 9. 497, 5 .

Hemigymnus leucomos Gthr. 293. 293.

Hemigymnus melanopterus Gthr. 358, I85. 395, 291. 491, 41.

Hemigymnus melapterus Gthr. 293, 293. 315, 23r. 320, 266. 364, $277.495,9$.

Hemilepidotus Tilesii C. 497, I I.

Hemiloricaria Blkr. 306, 8r.

Hemiloricaria caracasensis Blkr. 306, 8r.

Hemilutjanus Blkr. 468, 277.

Hemiodon Kner 306, 82.

Hemiodon acipenserinus $K$ Kner cf. Hemiodontichthys acipenserinus Blkr.

Hemiodon depressus Kner 306, 82.

Hemiodon platycephalus Kner cf. Pseudohemiodon platycephalus Blkr.

Hemiodontichthys Blkr. 306, 82

Hemiodontichthys acipenserinus Blkr. 306, 82 .

Hemioplites Cope 468, 249.

Hemioplites simulans Cope 468, 249.

Hemipimelodus Blkr. 306, 9т.

Hemipimelodus borneënsis Blkr. 270, 47. 272, 19. 306, 92. 356, 175.

Hemipimelodus macrocephalus Blkr. 226, 384 . 270, 47. 272, I9.

Hemiplatystoma Blkr. 306, 97.

Hemiplatystoma tigrinum Blkr. 306, 98.

Hemipteronotus Lac. 292, Iо3.

Hemipteronotus celebicus Blkr. 293, 294. 364, 277. 
Hemipteronotus immaculatus Blkr. 459, 83. 495, 10.

Hemipteronotus melanopus Blkr. 293, 294. 354, 144. 364, 277.

Hemipteronotus pentadactylus Blkr. 293, 294. 308, I52. 309, I 54. 315, 231. 354, 144. 358, I $84.364,277.425$, r $35.459,83.491,4$ r. Hemipteronotus quinquemaculatus Lac. 292, 103.

Hemipteronotus spilonotus Blkr. 293, 295. 364, 277.

Hemipteronotus tessellatus Blkr. 459, 83. 495, Iо.

Hemipteronotus 'Twistii Blkr. 293, 295. 296, I04. 315, 23I. 491, 4I.

Hemirhamphodon phaiosoma Blkr. 375, г68.

Hemirhamphodon pogonognathus Blkr. 375, I69.

Hemirhamphus 1, $55^{2}$.

Hemirhamphus amblyurus Blkr. 16, II. 25, II. 58, I6. cf. Zenarchopterus amblyurus $B / k r$.

Hemirhamphus balinensis Blkr. 231, I70. cf. Hemirhamphus intermedius Cant.

Hemirhamphus Bleekeri Kner cf. Zenarchopterus amblyurus $B l k r$.

Hemirhamphus borneënsis Blkr. 35, 273. 42, 68.45, I96. 49, $42 \mathrm{r}$. 55, 414. 58, 23. 69, 7r2. 270, 55. 272, 25. 356, г76. cf. Zenarchopterus amblyurus Blkr.

Hemirhamphus brachynotopterus Blkr. 91, I46.

Hemirhamphus brevirostris Cuv. 2, 512. cf. Hemirhamphus marginatus Blkr.

Hemirhamphus Brownii $\mathrm{Val}$. cf. Hemirhamphus vittatus $\mathrm{Val}$.

Hemirhamphus Buffonis Val. 69, 7 I I. 184, 203. 235, 37 I. 270, 55. 275, 47. 287, 6о. 288, 78. 296, III. 358, i92. cf. Zenarchopterus Buffonis Blkr.

Hemirhamphus Cantori Blkr. 375, I45. 425, 149.

Hemirhamphus Commersonii Lac. 2, 512.

Hemirhamphus Commersonii $C . V$. 25, r I. 58, I7. 235, 37 ז. cf. Hemirhamphus far Rüpp.

Hemirhamphus dispar $C . V .103,498.157,235.167,374.184,203$. 185, 226. 270, 55. 275, 47. cf. Zenarchopterus dispar Gill.

Hemirhamphus Dussumierii $C . V .32$, I6o. 46, 2 14. 51, 59. 56, 445 . 58, I8. 156, 2 I 7. 157, 235. 170, 480. 188, 46 I. 227, 408. 231, I $49.234,356.270,55.275,47.288,78.315,236.316,243$. 320, 272. 354, I50. 356, I76. 364, 294. 375, г50. 459, гог.

Hemirhamphus erythrorhynchos Les., $C . V$. 10, 67, 68. 46, 2 I 4. 231, I 7 I. $275,47$.

Hemirhamphus erythrorhynchus Les., C. $V .459$, гог. 495, 2 I.

Hemirhamphus far Rüpp. 270, 55. 275, 47. 287, 60. 296, I I I. 309, I $56.315,236.316,243.320,272.364,294.375,146.425$, I 49 . 459, rог. 495, 2r. cf. Hemirhamphus Commersonii C. $V$.

Hemirhamphus fasciatus $B l k r .80,89.375$, I $52.394,288$.

Hemirhamphus fluviatilis Blkr. 29, 95. 58, I6. 170, 476, 477. 270, 55. cf. Dermogenys pusillus $K$. $\%$. 
Hemirhamphus Gaimardi $C . V$. 50, 472. 55, 4I4. 56, 445. 58, 20. 119, I4. 156, 21 7. 270, 55. 272, 25. 275, 47. 287, 60. 315, 236. $364,294.375$, I $58.390,275.395,294.491,55$.

Hemirhamphus Georgii $C . V$. 58, 19. 91, 72, 165. 178, 2. 231, I 49. $272,25.287,60.288,78.459$, гог. 495, 2 г.

Hemirhamphus Georgii Cant. cf. Hemirhamphus Cantori Blkr.

Hemirhamphus Gernaerti $V a l .425$, I 49. 497, 24.

Hemirhamphus guineënsis Blkr. 302, II9.

Hemirhamphus intermedius Cant. 375, I54. 425, I49.

Hemirhamphus japonicus Brev. 497, 24.

Hemirhamphus leucopterus $C . V .91,72$.

Hemirhamphus limbatus $C . V .91,72$.

Hemirhamphus longirostris $C . V .2,5$ 12. 91, 72.

Hemirhamphus longirostris Blkr. cf. Hemirhamphus Cantori Blkr., Hemirhamphus Georgii $C . V$.

Hemirhamphus lucens $\mathrm{Val}$. 375, I60.

Hemirhamphus Lütke $V a l .354$, I 50.375, I 5 o.

Hemirhamphus marginatus Blkr. 375, I48. 390, 275.

Hemirhamphus marginatus Les. cf. Hemirhamphus vittatus $\mathrm{Val}$.

Hemirhamphus melanochir $C . V .119$, I4 cf. Hemirhamphus intermedius Cant.

Hemirhamphus melanurus $C . V .10,67,68.46,2$ I4. 50, 472. 58, r9. 235, 372. 275, 47. 296, III. 375, I 56. 395, 294. 425, I 49 .

Hemirhamphus neglectus Blkr. 375, 157.

Hemirhamphus occipitalis Gill.497, 24.

Hemirhamphus phaiosoma Blkr. 52, 99. 58, 26. 185, 226. cf. Hemirhamphodon phaiosoma Blkr.

Hemirhamphus pogonognathus Blkr. 82, 193. 185, 226. cf. Hemirhamphodon pogonognathus Blkr.

Hemirhamphus Quoyi C. V. 50, 491. 51, 59. 58, 26. 156, 217. 235, $372.270,-55.271,2.272,25.275,47.276,65.364,294.375$, I 53. 395, 294. 437, 39. 491, 55 .

Hemirhamphus Reynaldi $C . V .91,72.459$, IоI.

Hemirhamphus Russelli $C . V .58$, I $7.91,72.126,344.167,374$. $275,47.287,60.315,236.318,252.364,294$. cf. Hemirhamphus marginatus Blkr.

Hemirhamphus Russelli Blkr. 227, 408.

Hemirhamphus sajori T. Schl. 118, I I6. 497, 24.

Hemirhamphus Schlegeli Blkr. 302, r2o.

Hemirhamphus sinensis Gthr. 425, I 49.

Hemirhamphus sumatranus Blkr. 86, 526. 270, 55. cf. Dermogenys sumatranus Blkr.

Hemirhamphus tridentifer Cant. 288, 78 .

Hemirhamphus unifasciatus Ram., Rang. 459, I01. 495, 2 I.

Hemirhamphus vittatus $V a l .302$, I 18 . 
Hemirhombus Blkr. 294, 425. 332, 455 .

Hemirhombus guineënsis Blkr. 294, 425. 302, 25.

Hemisciaena Blkr. 307, r40. 468, 328 .

Hemisciaena lucida Blkr. 307, r4r. 425, r42.468, 328. cf. Sciaena lucida Rich.

Hemisciaenini 468, 327 .

Hemiscyllium malayanum M.H. 112, 376.

Hemiscyllium trispeculare Rich. 339, 362 .

Hemiscyllium variolatum A. Dum. cf. Parascyllium variolatum Gill.

Hemisilurus Blkr. 299, 395. 306, I 6.

Hemisilurus heterorhynchus Blkr. 270, 48. 306, I 6.

Hemisilurus schilbeides Blkr. 270, 48. 272, 20.

Hemisilurus scleronema Blkr. 324, 74.

Hemisorubim Blkr. 306, 97.

Hemisorubim platyrhynchos Blkr. 306, 97.

Hemisynodontis Blkr. 306, 87.

Hemisynodontis mèmbranaceus Blkr. 304, 55. 306, 87 .

Hemisynodontis nigrita Blkr. 304, 55 .

Hemisynodontis schall Blkr. 304, 55. 363, 266.

Hemisynodontis zambezensis Blkr. 304, 55 .

Hemitaurichthys Blkr. 468, 304.

Hemitaurichthys polylepis Blkr. 468, 304. 474, 316. 481, 50. 491, 44. cf. Hemitaurichthys zoster Blkr.

Hemitaurichthys sexfasciatus Blkr. 474, 3 I6.

Hemitaurichthys zoster Blkr. 495, I4.

Hemitautoga Blkr. 292, IоI.

Hemitautoga centiquadra Blkr. 293, 292. 309, I54. 318, 250.320, 266. 354, ז 44.358, I $84.364,277.425$, I $35.459,83$.

Hemitautoga notophthalmus Blkr. 293, 292. 320, 266. cf. Platyglossus (Hemitautoga) notophthalmus Blkr.

Hemithylacus leiaspis $K p$. cf. Coelonotus liaspis $G t h r$.

Hemixiphophorus Blkr. 261, 440.

Heniochus C. $V ., K p .1,553.468,304$.

Heniochus acuminatus $C$. $V$. cf. Heniochus macrolepidotus $C$. $V_{\text {。, }}$ Taurichthys macrolepidotus Blkr.

Heniochus chrystomus Lay \& Benn., C. V. 184, 200. 318, 252. cf. Taurichthys chrysostomus Blkr.

Heniochus drepanoides Thioll. cf. Taurichthys chrysostomus Blkr.

Heniochus macrolepidotus $C . V .2,520.9,633.10,66.22,2$ I. 46, 212. $51,56.54,235.91,40.126,344.196,37.231$, I 46.270 , 26. $275,35.288,73.296$, 108. 316, 242. 320, 270.330, 266. 346, 31. 354, I48. cf. Diphreutes macrolepidotus Cant., Taurichthys macrolepidotus Blkr.

Heniochus macrolepidotus Brev. cf. Taurichthys monoceros Blkr.

Heniochus melanistion $B / k r .95,9 S$. cf. Taurichthys chrysostomus Blkr. 
Heniochus monoceros $C . V .2,520.22,2 \mathrm{I}$. cf. Taurichthys monoceros Blkr.

Heniochus permutatus Ed. Benn. cf. Taurichthys chrysostomus Blkr. Heniochus varius Gthr. 320, 270. cf. Taurichthys varius C. $V$.

Heptanchus indicus Cuv., M. H. 119, I8. 266, 58. 425, I 19. 497, 3 . Heptapterus Blkr. 306, I Io.

Heptapterus mustelinus Blkr. 306, Iro. 336, 4.

Heptapterus surinamensis Blkr. 298, 387. 336, 9г.

Heptatrema cirrhatum Schl. cf. Bdellostoma Bürgeri Gir.

Herpetoichthys $K p$. 351, II9.

Hesperanthias Loze 438, 45. 468, 276.

Heterandria Baird, Gir. 261, 439.

Hetereleotris Blkr. 453, 306.460, 103, 106.

Heterobagrus Bocourti Blkr. 337, 355. 356, I75.

Heterobranchus Geoffr. 1, 552, 306, I 20.

Heterobranchus bidorsalis Geoffr. 306, I20.

Heterobranchus isopterus Blkr. 302, Io8.

Heterobranchus macronema Blkr. 302, rog.

Heterobranchus sextentaculatus Spix cf. Rhamdia Queleni Blkr.

Heterobranchus tapeinopterus Blkr. 70, 732. 272, 20.

Heteroconger polyzona Blkr. 400, 332.

Heterodon zonatus Blkr. 2, 523. 9, 634. cf. Heterognathodon xanthopleura Blkr., Pentapus caninus Blkr.

Heterodontus Philippi Blainv. 119, I8. 425, I I9.

Heterodontus zebra Gray 118, 127. 142, 71. 497, 3.

Heterognathodon Blkr. 438, 4I. 468, 279.

Heterognathodon bifasciatus Blkr. 9, 636. 18, 30. 51, 56. 53, г6о. 158, 273. 185, 222. 275, 27. 285, 244. 296, 106. 309, 155. 315, 233. 316, 242. 320, 269. 358, r88. 364, 283. 438, 43. cf. Pentapus bifasciatus, caninus, Blkr.

Heterognathodon Hellmuthii Blkr. 80, 75. 267, 139. cf. Pentapus Hellmuthi Blkr.

Heterognathodon macrurus Blkr. 18, 31. 31, Iог. cf. Pentapus macrurus Blkr.

Heterognathodon microdon Blkr. 78, 464. 364, 283. cf. Pentapus microdon Blkr.

Heterognathodon nemurus Blkr. 71, 754. 275, 27. 320, 269. cf. Pentapus nemurus Blkr.

Heterognathodon xanthopleura Blkr. 9, 634. 18, 31. 31, Iо I. 234, 352. 270, 21. 275, 27. 287, 46. 296, 106. 315, 233. 320, 269. 364, $283.438,43$. cf. Pentapus caninus Blkr.

Heterophthalmus Blkr. 143, 42. 273, 7.

Heterophthalmus katoptron Blkr. 143, 43. 214, 253. 273, 9. 275, 22. cf. Anomalops katoptron Blkr.

Heterophthalmus palpebratus 214, 254 . 
Heteropneustes J. Mïll 306, т20.

Heteroprosopon Blkr. 294, 429.

Heteroprosopon cornutus Blkr. 497, 2 I. cf. Parophrys cornuta Gthr. Hexacanthus Nordm. 453, 309.

Hexagrammus asper Stcll. cf. Chirus hexagrammus Stell.

Hexagrammus Stelleri Tiles. cf. Chirus hexagrammus Stcll.

Hexanchus griseus Raf. 459, 68.

Hexanematichthys Blkr. 306, 90.

Hexanematichthys hymenorrhinus Blkr. 298, 377. 336, 57.

Hexanematichthys leptaspis Blkr. 324, 7o. cf. Arius (Hexanematichthys) leptaspis Blkr.

Hexanematichthys leptocassis Blkr. 288, 65. 324, 72.

Hexanematichthys sundaicus Blkr. 191, 2. 227, 408. 235, 370. 270, 45. 272, I8. 287, 58. 288, 78. 306, 90.

Hexanematichthys surinamensis Blkr. 298, $380.336,55$.

Hippichthys heptagonus Blkr. 16, I5. 25, I2. cf. Syngnathus heptagonus Blkr.

Hippocampus abdominalis Less, 119, 28.

Hippocampus antiquorum Leach 497, I3.

Hippocampus brevirostris Cuv. 93, г6.

Hippocampus brevirostris Schl. cf. Hippocampus antiquorum Leach.

Hippocampus borboniensis $A$. Dum. 459, 75 .

Hippocampus Camelopardalus Bianc. 495, I6.

Hippocampus chinensis Basil. cf. Hippocampus trimaculatus Leach.

Hippocampus comes Cant. 143, 80. 275, I3. 287, 40. 288, 69. 320, 265. 459, 75. 495, г6.

Hippocampus coronatus Schl. 408, 238. 497, I3.

Hippocampus filamentosus H. Clocq. cf. Hippocampus ramulosus Leach.

Hippocampus foliatus Shato. 119, I 7 .

Hippocampus fuscus Rüpp. 93, 16.

Hippocampus gracilissimus Schl. cf. Acentronura gracillima $K p$.

Hippocampus guttulatus Cuv. 425, г $26.459,75.495$, I6. cf. Hippocampus kuda Blkr.

Hippocampus hystrix $K p .459,75.497$, I3.

Hippocampus japonicus $K p$. cf. Hippocampus antiquorum Leach.

Hippocampus kampylotrachelos Blkr. 106, ro7. 270, 64. 287, 40.

Hippoєampus kuda Blkr. 51, 82. 89, 26. 143, 79. 196, 37. 235, 374. 238, 88. 270, 64. 275, г 3. 296, 103. 315, 230.316, 24 I. 320, 265. 354, I $43.358, I 83.364,273.382,397.395,290.491,49$.

Hippocampus longirostris Schl. 425, I26. 497, г3.

Hippocampus manadensis Blkr. 143, 79. 275, 13.

Hippocampus mannulus Cant. 143, 79. 288, 69.

'Hippocampus melanospilus Blkr. 103, 505. 275, I3. 287, 40. $308,152$.

Hippocampus Nohnikei Blkr.54, 306. 90, 55. 93, I6. 497, I3. 
Hippocampus moluccensis Blkr. 54, 305, 307. cf. Hippocampus guttulatus Cuv., Hippocampus kuda Blkr.

Hippocampus polytaenia Blkr. 99, 338. 275, I3. 287, 40. 318, 250. 358, I83. 364, 273 .

Hippocampus ramulosus Leach 459, 75. 495, I6.

Hippocampus taeniopterus Blkr. 54, 306. cf. Hippocampus kuda Blkr.

Hippocampus trimaculatus Leach 425, I 26.

Hippocampus Whitei Blkr. 119, I7.

Hippocephalus japonicus Sins. 497, I3.

Hippoglossoides Gottsch. 294, 427.

Hippoglossus Cuv. 118, т23 294, 425.

Hippoglossus dentex Rich. cf. Hippoglossus erumei Cuv., Psettodes erumei Blkr.

Hippoglossus erumei Cu\%. 2, 509. 25, т2. 38, 401. 50, 472. 51, 59. 56, 446. 61, I3. 91, 76. 194, 26. 227, 408. 270, 61. 287, 40.288, 69. 294, 424. cf. Psettodes erumei Blkr.

Hippoglossus goniographicus Rich. cf. Psettodes goniographicus Blkr.

Hippoglossus nalaka Cuv. cf. Hippoglossus erumei Cu\%.

Hippoglossus olivaceus Schl. cf. Chaenopsetta olivacea Blkr.

Hippoglossus orthorhynchus Rich. cf. Psettodes erumei Blkr.

Hippoglossus pinguis Mus. L. B. 294, 426.

Hippoglossus vulgaris Cuv. 294, 425.

Histiophorus dubius Blkr. 425, I33, I5 $\mathrm{I}$.

Histiophorus gladius Lac. 459, Iо0. 495, 18 .

Histiophorus gracilirostris $C$. $V$. cf. Xiphias velifer Gthr.

Histiophorus indicus C. $V .270,29$. cf. Histiophorus gladius Lac.

Histiophorus orientalis Schl. 497, I5.

Histiopteriformes 468, 266.

Histiopterus Schl. 468, 269.

Histiopterus acutirostris Schl. 497, 7 .

Histiopterus labiosus Gthr. 468, 269.

Histiopterus recurvirostris Rich. 119, I0.

Histiopterus typus Schl. 468, 269. 497, 7.

Holacanthiformes 468, 307 .

Holacanthus Lac. 1, 553. 468, 307.

Holacanthus alternans $C . V .459,96$. cf. Acanthochaetodon alternans Blkr.

Holacanthus annularis $C . V .2,520.51,57.91,40.288,73$.

Holacanthus annularis Lac. 22, 26. 91, 40. 196, 37. 270, 26. 275, 35 . 468, 308. cf. Acanthochaetodon annularis Blkr.

Holacanthus arcuatus Gr. 482, 22.

Holacanthus aruset Lac. cf. Acanthochaetodon maculosus Blkr.

Holacanthus asfur Cuv. cf. Acanthochaetodon asfur Blkr.

Holacanthus asfur b. var, c. Khmz. cf. Acanthochaetodon maculosus Blkr. 
Holacanthus biaculeatus Lac. cf. Premnas biaculeatus Blkr.

Holacanthus bicolor Bl., Lac , C. V. 80, 77. 270, 26. 315, 234. 318, 252. 338, 360. 358, 190. 364, $287.437,39.481$, 1 $27.482,22$. 491,45 .

Holacanthus bispinosus Gthr. 364, 287. 481, 125. 482, 22.

Holacanthus caudovittatus Gthr.482, 22.495, I4. cf. Genicanthus caudovittatus Blkr.

Holacanthus chrysocephalus Blkr. 113, 42S. cf. Chactodontoplus chrysocephalus Blkr.

Holacanthus chrysurus $C$. $V$. cf. Acanthochaetodon chrysurus Blkr.

Holacanthus ciliaris Lac. 482, 22 .

Holacanthus coerulescens Rüpp. cf. Acanthochaetodon maculosus Blkr.

Holacanthus coeruleus Elhr., C. $V$. cf. Acanthochactodon striatus Blkr.

Holacanthus cyanotis Gthr. 482, 22.

Holacanthus diacanthus Blkr. 163, 57. cf. Holacanthus bispinosus Gthr. Holacanthus diacanthus Gthr. 425, I40. 459, 96. 481, г38. 482, 22. $491,45.495,14$.

Holacanthus dimidiatus Blkr. 273, 1 I. cf. Chaetodontoplus dimidiatus Blkr.

Holacanthus Duboulayi Gthr. cf. Chaetodontoplus Duboulayi Blkr.

Holacanthus dux Lac. 71, 757. 275, 35. 318, 252. 320. 270. 358, I90. cf. Holacanthus diacanthus Gthr.

Holacanthus flavissimus C. $V .482,22$.

Holacanthus flavo-niger Lac. cf. Chaetodon Meyeri Bl. Sc/n., Tetragonoptrus (Citharoedus) Meyeri Blkr.

Holacanthus formosus Cirst. 482, 22.

Holacanthus Forsteni Mus. L. B. cf. Holacanthus diacanthus Gthr.

Holacanthus geometricus Lac. cf. Acanthochaetodon nicobariensis $B$. Sclun., Blkr., Holacanthus nicobariensis Blkr.

Holacanthus haddaja $C$. $V$. cf. Acanthochaetodon maculosus Blkr.

Holacanthus iburu Montrouz. cf. Acanthochaetodon semicirculatus Blkr.

Holacanthus ignatius Playf. cf. Acanthochaetodon lepidolepis Blkr., Holacanthus lepidolepis Blkr.

Holacanthus imperator $C . V$., Lac. 71, 758. 143, 48. 275, 35. 364, 287. 425, I 40. 459, 96. cf. Acanthochaetodon imperator Blkr.

Holacanthus Lamarckii Lac. 95, 100. 481, 122. 482, 22. 491, 45.

Holacanthus lepidolepis Blkr. 78, 468.459, 96. cf. Acanthochaetodon lepidolepis Blkr.

Holacanthus leucopleura Blkr. 80, 79. 275, 35. 315, 234. 318, $25^{2}$. 358, 190. 364, 287. 394, 288. cf. Holacanthus tibicen C. $V$.

Holacanthus lineatus Rüpp. cf. Acanthochaetodon maculosus Blkr.

Holacanthus loriculus Gthr. 482, 22.

Holacanthus luteolus $C V .482,22$.

Holacanthus maculosus C. $V$. cf. Acanthochaetodon maculosus Blkr. 
Holacanthus melanosoma Blkr. 80, 78. 273, I2. 394, 288. cf. Chaetodontoplus melanosoma Blkr.

Holacanthus melanospilus Blkr. 163, 56. 481, I 24. 482, 22.

Holacanthus mesoleucos Lac., C. V. 22, 26. 113, 429. 126, 344. 157, 233. 275, 35. 287, 50. 296, Iо8. 309, I56. 316, 242.

Holacanthus mesoleucus $C . V$. 358, 190.

Holacanthus mesoleucus Lac. cf. Chaetodontoplus mesoleucus Blkr.

Holacanthus mokhella $C$. $V$. cf. Acanthochaetodon mokhella Blikr.

Holacanthus monophthalmus Kner. cf. Holacanthus cyanotis Gthr.

Holacanthus multispinis Plfr. 482, 22.

Holacanthus navarchus $C . V .95,99.318,252.364,287.481,{ }_{3} 6$. $482,22$.

Holacanthus nicobariensis Bl., Blkr. 128, 413. 320, 270. 358, I90. cf. Acanthochaetodon nicobariensis Blkr.

Holacanthus nicobariensis $C . V .396,300$.

Holacanthus nox Blkr. 84, 338. 184, 200. 481, I31. 482, 22.

Holacanthus ocularis Pet. 482, 22.

Holacanthus passer $V a l .482,22$.

Holacanthus poecilus Pet. cf. Acanthochaetodon lepidolepis Blkr.

Holacanthus pseudannularis Blkr. 183, I70. cf. Acanthochaetodon annularis Blkr.

Holacanthus semicirculatus C. $V$. 56, 452. 128, 4I4. 184, 200. 236, $33 \mathrm{I}, 333.255,24 \mathrm{I} .270,26.275,35.320,270.354$, I 48.358 , 190. 364, 287. 396, 297. cf. Acanthochaetodon semicirculatus Blkr.

Holacanthus semicirculatus Blkr. cf. Acanthochaetodon striatus Blkr., Holacanthus striatus Rüpp.

Holacanthus septentrionalis T. Schl. 269, 78. 425, r40. 468, 307. cf. Chaetodontoplus septentrionalis Blkr.

Holacanthus septentrionalis Rich. cf. Acanthochaetodon annularis Blkr.

Holacanthus sexstriatus $K$. \%. H. 2, 520. 22, 25. 51, 57. 275, 35 . $364,287.481,140.482,22$.

Holacanthus striatus Rüpp. 128, 4I4. 287, 50. cf. Acanthochaetodon striatus Blkr.

Holacanthus strigatus Gill 482, 22.

Holacanthus tibicen $C . V .481$, I29. 482, 22. 491. 45 .

Holacanthus tricolor Lac. 425, I 40. 468, 308. 482, 22.

Holacanthus trimaculatus Lac. 47, 242. 364, 287. 459, 96. 481, I33. 482, 22. 491, 45. 495, I 4 .

Holacanthus uniocellatus Kner cf. Holacanthus cyanotis Gthr.

Holacanthus Vroliki Blkr. 84, 339. 315, 234. 316, 24\%. 318, 252. 320, 270. 354, I 48.358 , I90. 481, I32. 482, 22. 491, 45.

Holacanthus xanthometopon Blkr. 76, 258. 184, 200. 270, 27. 396, 300. 481, I35. 482, 22.

Holacanthus xanthurus Benn. 91, 40. 482, 22.

Holanthias Gthr. 468, 252. 
Holconotus $A g .468,29 \mathrm{I}$.

Holconotus rhodopterus $A g$. 468, 29 I.

Holocentrum 1, 553 .

Holocentrum albo-rubrum Lac. 270, I5. 275, 21. 287, 44. cf. Holocentrum rubrum Rüpp.

Holocentrum albo-rubrum Rich. cf. Holocentrum rubrum Gthr.

Holocentrum argenteum C. V. 13, 4. 180, I. 429, 208. 491, 48.

Holocentrum aurolineatum Lac. 459, 86. 495, I5.

Holocentrum binotatum Q. G. 184, 207. 429, 229.491, 48.

Holocentrum caudimaculatum Rüpp. 429, 2 I9. 459, S6. 491, 48.

Holocentrum christianum Ehr., $C$. $V$. cf. Holocentrum sammara $C$. $V$.

Holocentrum cornutum Blkr. 83, 240. 275, 21, 429, 222.

Holocentrum diadema $C$. $V$., Lac. 13, 4. 54, 259. 157, 232. 182, I 59. 242, I 42. 270, I 5. 275, 2 I. 309, I 55. 315, 232. 318, 25 I. 320, 267. 425, I36. 429, 2 10. 459, 86. 491, 48. 495, I 5 .

Holocentrum diploxiphus Gthr. 495, I5.

Holocentrum hastatum $C . V .302, \mathrm{I}_{3}$.

Holocentrum lacteo-guttatum $C$. $V$. cf. Holocentrum punctatissimum $C$. $V$.

Holocentrum laeve Gthr. 459, 86. cf. Holocentrum sammara C. $V$.

Holocentrum laticeps $C . V .13,4$. cf. Holocentrum rubrum Rüpp.

Holocentrum leo C. V. 13, 4. 111, 355. 157, 232. 275, 21. cf. Holocentrum spiniferum Rüpp., Gthr.

Holocentrum leonoïdes Blkr. 10, 7I. 13, 54. 46, 2 I 1. 157, 232. 167, 37 I. 275, 22. 296, 105. 309, I55. 315, 23r. 318, 25 r. 320, 267. cf. Holocentrum caudimaculatum Rüpp.

Holocentrum macropus Gthr. 459, 86. 495, r 5 .

Holocentrum maculatum $M C C$. cf. Labrax japonicus $C . V$.

Holocentrum marginatum $C$. $V$. cf. Holocentrum rubrum Rüpp.

Holocentrum melanospilus Blkr. 179, 2. 429, 226.

Holocentrum melanotopterus Blkr. 137, 302. 167, 370. 236, 330. $275,22.429,2$ I 7 .

Holocentrum microstoma Gthr. 429, 203.

Holocentrum operculare $C . V .47,233.270$, I 5. 429, 2 II.

Holocentrum orientale C. V. 2, 527. 10, 66. 13, 53.46, 2 II. 51, 55 . 91, 34, I65. 126, 344. 127, 345. 156, .213. 157, 232. 158, 273. 167, 371. 171, 245. 185, 222. 208, 239. 227, 406. 234, 352.236, 330, 333. 250, 205. cf. Holocentrum rubrum Rüpp., Holocentrus ruber Gthr.

Holocentrum poecilopterus Blkr. 111, 356. 429, 207.

Holocentrum punctatissimum C. V. 76, 248. 167, 37 1. 184, 198. 234, $352.236,33 \circ, 333.270$, I $5.275,22.315,232.429,2 \times 5.491,48$. Holocentrum rubrum Gthr., R̈̈pp. 296, ro5. 309, г55. 425, г36. $429,224.437,3$ S. 459, S6. 491, 48. 495, I 5. 497, то.

Holocentrum samara Rüpp. cf. Holocentrum sammara $C . V$.

Holocentrum sammara $C . V_{\text {. }}$ E/hr. 9, 636. 13, 53. 54, 235. 66, 555. 
167, 37I. 184, I98. 188, 458. 270, I5. 275, 22. 296, I05. 315, 232. 429,2 I 3. $459,86.491,48.495$, г5.

Holocentrum spiniferum $C$. $V$. cf. Holocentrum caudimaculatum Rüpp. Holocentrum spiniferum Rüpp., Gthr. 91, 34. 296, го5. 396, 296. 429, 205. 459, 86. 495, I6.

Holocentrum spinosissimum Schl. 497, ro.

Holocentrum spinosissimum Rich. cf. Holocentrum rubrum Gthr., Rüpp.

Holocentrum tahiticum Kner cf. Holocentrum sammara $C . V$.

Holocentrum tiereoïdes Blkr. 84, 334. 275, 22. 315, 231. 429, 228.

Holocentrum violaceum $B l k r .84,335.429,22 \mathrm{I}$.

Holocentrus albofasciatus $L a c$. cf. Sebastes albofasciatus $C$. $V$., Sebastes marmoratus $C$. $V$., Sebastichthys marmoratus Blkr.

Holocentrus albofuscus Lac. cf. Epinephelus maculatus Blkr., Serranus maculatus Blkr.

Holocentrus bengalensis $B l$. cf. Lutjanus bengalensis Blkr., Mesoprion octolineatus Blkr.

Holocentrus bifasciatus $B l$. Schn. cf. Amphiprion bifasciatus Bl. Schn., Prochilus bifasciatus Blkr.

Holocentrus binotatus C. $V .364,279$.

Holocentrus boutton Lac. cf. Lutjanus butonensis Blkr.

Holocentrus calcarifer $B l$. cf. Lates nobilis $C$. $V$.

Holocentrus caudimaculatus Rüpp. cf. Holocentrum caudimaculatum Rupp.

Holocentrus ciliatus Lac. cf. Scolopsides lycogenis C. $V$., Scolopsis ciliatus Gthr.

Holocentrus coeruleopunctatus $B$ l. cf. Epiniphelus coeruleopunctatus $B l k r$.

Holocentrus cornutus Blkr. 318, 25 I. 354, I46. 358, I86. 364, 279.

Holocentrus diacanthus Lac. cf. Pomacentrus pavo Lac., Pomacentrus (Pomacentrus) pavo Lac.

Holocentrus diadema $C . V$. 354, I46. 358, I86. 364, 279.

Holocentrus diadema Lac. cf. Holocentrum diadema $C . V$.

Holocentrus erythraeus $B l$. Schn. cf. Epinephelus fasciatus $B l k r$.

Holocentrus flavocoeruleus $L a c$. cf. Epinephelus flavocoeruleus Blkr.

Holocentrus Forskalii Lac. cf. Epinephelus fasciatus Blkr.

Holocentrus gymnosus Lac. cf. Epinephelus flavocoeruleus Blkr.

Holocentrus hexagonatus Bl. Schn. cf. Epinephelus merra Bl., Serranus hexagonatus $C$. $V$.

Holocentrus jarbua Lac. cf. Therapon (Datnia) jarbua Blkr.

Holocentrus laevis Gthr. 364, 279.

Holocentrus lanceolatus $B l$. cf. Epinephelus lanceolatus Blkr., Serranus lanceolatus $C$. $V$.

Holocentrus leonoides Blkr. 354, I46. 364, 279. cf. Holocentrum caudimaculatum Rüpp.

Holocentrus leopardus Lac. cf. Paracanthistius leopardinus Blkr., Plectropoma leopardinus $C . V$. 
Holocentrus maculatus $B l .438,46$. cf. Epinephelus maculatus $B l k r$., Serranus maculatus Blkr.

Holocentrus malabaricus Bl. Schn. cf. Epinephelus polypodophilus Blkr.

Holocentrus marginatus Lac. cf. Epinephelus fasciatus Blkr., Serranus marginalis $C . V$.

Holocentrus melanotopterus $B l k \% .318,25$ 1. 354, I 46. 358, I 86. 364, 280.

Holocentrus merra Lac. cf. Epinephelus merra $B /$.

Holocentrus microstoma Gthr. 364, 280.

Holocentrus miles Lac, cf. Corvina miles $C$. $V$., Pseudosciaena miles Blkr.

Holocentrus occanicus $L a c$. cf. Epinephelus fasciatus blkr.

Holocentrus ongus $B l$. cf. Epinephelus ongus Blkr.

Holocentrus opercularis C. V. 318, $25 \mathrm{r}$.

Holocentrus pantherinus Lac. cf. Epinephelus pantherinus Blkr.

Holocentrus punctatissimus C.V. 358, x $86.364,280$.

Holocentrus quinquelinearis $B l$. cf. Lutjanus quinquelineatus $B$ ? kr. Mesoprion octolineatus Blkr.

Holocentrus quinquelineatus $B l$., Blkr. cf. Lutjanus quinquelineatus $B l k r$.

Holocentrus quadrilineatus $B l$. cf. Therapon quadrilineatus $C . V$., Therapon (Pelates) quadrilineatus Blkr.

Holocentrus radjabon Lac. cf. Plectorhynchus pictus Blkr.

Holocentrus rosmarus Lac. cf. Epinephelus fasciatus Blkr.

Holocentrus ruber Rüpp. 320, 267. 354, I 46. 358, r86. cf. Holocentrum rubrum Rüpp.

Holocentrus ruber Gthr. 364, 280. 394, 287. 395, 29 I.

Holocentrus ruber Benn. cf. Holocentrum caudimaculatum Rïpp.

Holocentrus salmoides Lac. cf. Epinephelus polypodophilus Blkr.

Holocentrus samara Rüpp. cf. Holocentrum sammara $C V$.

Holocentrus sammara Rïpp., C. V., Ehr. 13, 54. 316, 24r. 318, 25 r. $320,268.354$, I 46.358, I $86.364,280$.

Holocentrus servus $B l$. cf. Therapon (Datnia) jarbua Blkr., Therapon servus $C$. $V$.

Holocentrus Sonnerat Lac. cf. Premnas biaculeatus Blkr.

Holocentrus spinifer Gthr. 364, 280.

Holocentrus spinifer Rüpp. cf. Holocentrum caudimaculatum Rüpp., Holocentrum spiniferum Gthr.

Holocentrus tauvinus Bl. Schn. cf. Epinephelus Gilberti Blkr.

Holocentrus violaceus Blkr. 320, 268.

Hologymnosus Lac. 292, 97.

Hologymnosus fasciatus Lac. 292, 97. 293, 2Sז. 318, 250. 364, 276. $459,83.495$, Iо.

Hologymnosus oxyrhynchus Blkr. 293, 28r. 354, I44.

Hologymnosus semipartitus Blkr. 459, 83. 495, Io.

Holoxenus cutaneus Gthr. cf. Gnathanacanthus cutaneus.

Homaloptera v. Hass. 1, 552. 261, 422.

Homaloptera anisurus Blkr. 91, 70. 
Homaloptera Brucei Blkr. 74, I58. 91, 70.

Homaloptera erythrorhina v. Hass. 74, I 57.

Homaloptera fasciata $v$. Hass. 270, 50.

Homaloptera gymnogaster Blkr. 74, I63. 270, 50.

Homaloptera javanica v. Hass. 270, 50.

Homaloptera lineolata $74, x_{5}^{8}$.

Homaloptera lissorhynchus Blkr. 91, 70.

Homaloptera maculata Blkr. 74, 158. 91, 70.

Homaloptera nasuta Blkr. 74, I58.91, 70.

Homaloptera ocellata v. Hass. 74, I 57. 270, 5 o.

Homaloptera ophiolepis Blkr. 74, r6o. 270, 50.

Homaloptera pavonina 74, i $5^{8}$.

Homaloptera polylepis Blkr. 74, I62. 170, 476, 477 .

Homaloptera (Octonema) rotundicauda Mart. 425, I 49. 430, 234.

Homaloptera salusur Blkr. 74, I6 . 270, 50.

Homaloptera Wassinkii Blkr. 74, 163. 170, 476.

Homaloptera Zollingeri Blkr. 74, I59. 170, 477.

Homodon Bris. 468, 275.

Homoprion Hollor. 468, 327 .

Hopladelus Raf., Gill 306, го2.

Hopladelus olivaris Gill 306, 103.

Hoplegnathiformes 468, 284 .

Hoplegnathoidei Blkr. 93, 6.

Hoplegnathus Rich., Gthr. 468, 284.

Hoplegnathus Conwayi Rich. 119, Iо. 468, 284 .

Hoplegnathus fasciatus Rich. 90, 4, 34.93, 6. 425, I34. 497. 8.

Hoplegnathus maculosus Rich. cf. Hoplegnathus punctatus Rich.

Hoplegnathus punctatus Rich. 425, г34. 497, 8.

Hoplichthys Langsdorffi $C . V$. 175, I I. 253, 235. 425, I27. cf. Hoplichthys pusillus Blkr.

Hoplichthys pusillus Blkr. 497, I3.

Hoplisoma Stons. 306, 83.

Hoplopagrus Gill 468, 280.

Hoplopagrus Güntheri Gill 468, 280.

Hoplosoma Gill 306, 83 .

Hoplosternum Gill 306, 82.

Hoplosternum laevigatum Gill 306, 83. 336, 24 .

Hoplosternum longifile Gill 336, 27.

Hoplosternum Stevardii Gill cf. Hoplosternum laevigatum Gill.

Hoplosternum thoracatum Gill 336, 26.

Hoplunnis Kp. 351, II5.

Hudsonius Gir. 261, 437. 314, 2 II.

Huro C. $V .468,26 \mathrm{I}$.

Huro nigricans $C . V .468,26$ r.

Hybognathus $A g$. 261, 426. 314, 206. 
Hybopsis $A g .261,437.314,2$ I .

Hyborhynchus $A g$. 261, 426. 314, 206.

Hydrargyra Lac. 261, 440.

Hydrocyon lineatus Schl. 302, I25.

Hylomyzon $A g .261,428.314,190$.

Hymenophysa $M C$ Cl. 220, 303. 261, 421.

Hymenophysa curta Blkr. cf. Botia curta Gthr.

Hymenophysa Mac Clellandi Blkr. 225, 358. 270, 49. 272, 20.

Hymenophysa macracanthus Blkr. 270, 49. 272, 21.

Hynnis insanus $V$ arl. 459, 99.

Hypeneus Cant. 468, 333 .

Hypeneus flavolineatus $\mathrm{Val}$. cf. Mulloides flavolineatus Blkr.

Hyperistius Gill 468, 248.

Hyperoglyphe Gthr. 468, 268.

Hyperoglyphe porosa Gthr. 468, 268.

Hyperprosopon Gill 468, 291.

Hypnos subnigrum $A$. Dum. 119, I9.

Hypocritichthys Gill 468, 291.

Hypogymnogobius Blkr. 453, 3 I 8.

Hypolophus sephen W. H. 16, 6. 25, I3. 51, 60. 64, 77. 91, 82. $288,67.459,68$.

Hypomerus olidus Gthr. 497, 24.

Hypomesus sinensis Gthr. 425, 147.

Hypophthalmichthys Blkr. 261, 433. 314, 201.

Hypophthalmichthys Dabryi Guich.490, 2 Io. cf. Hypophthalmichthys molitrix Blkr.

Hypophthalmichthys mantschuricus Blkr. of. Hypophthalmichthys molitrix Blkr.

Hypophthalmichthys mantschuricus Kner of. Hypophthalmichthys nobilis Blkr.

Hypophthalmichthys microlepis Blkr. 415, I6. 425, I46.

Hypophthalmichthys molitrix Blkr. 415, 83. 425, I 46.490, 2 I I. 496, 3 .

Hypophthalmichthys nobilis Blkr.415, 85.425, I 46. 490, I I 5.496, 3 . cf. Hypophthalmichthys Simoni Guich.

Hypophthalmichthys Simoni Guich.490, 2 I I. cf. Hypophthalmichthys nobilis Blkr.

Hypophthalmus Spix 306, 109.

Hypophthalmus davalla Schomb. 306, Io8.

Hypophthalmus edentatus Spix 306, Iog.

Hypophthalmus fimbriatus Kner cf. Pseudohypophtalmus fimbriatus Blkr.

Hypophthalmus longifilis $\mathrm{Val} .336,8 S$.

Hypophthalmus marginatus $V a l .336,4$.

Hypophthalmus niloticus Rüpp. cf. Eutropius niloticus Blkr.

Hypoplectrodes Gill 468, 255.

Hypoplectrus Gill 468, 255. 
Hypoplectrus puella Gill 468, 256.

Hypoplites Gill 468, 276.

Hypoprion notatus Blkr. cf. Hemigaleus macrostoma Blkr.

Hypopterus Gill 468, 264.

Hypopterus macropterus Gill 468, 264 .

Hyporthodus Gill 468, 256.

Hypostomus Blkr. 306, 77.

Hypostomus aurantiacus Cast. cf. Parancistrus aurantiacus Blk\%.

Hypostomus barbatus $V a l$. cf. Pseudancistrus barbatus Blkr.

Hypostoma flava Szons. cf. Plecostomus brasiliensis Blkr.

Hypostomus guacari Lac. cf. Plecostomus brasiliensis Blkr.

Hypostomus guttatus $V a l$. cf. Pseudancistrus guttatus Blkr.

Hypostomus plecostomus $\mathrm{Val}$. cf. Plecostomus brasiliensis Blkr.

Hypostonus serratus $\mathrm{Val}$. cf. Pseudacanthicus serratus $B l k r$.

Hypostomus Temminckii $\mathrm{Val}$. cf. Ancistrus Temmincki Blkr.

Hypostomus verres $\mathrm{Val}$. cf. Plecostomus brasiliensis Blkr.

Hypseleotrini 453, 305. 461, I03.

Hypseleotris Gill 453, 305 .

Hypseleotris cyprinoides Gill cf. Asterropteryx cyprinoides Blkr.

Hypselobagrus Blkr. 306, 96.

Hypselobagrus macronema Blkr. 306, 96. 356, I75.

Hypselobagrus tengara Blkr. 356, I 75 .

Hypselobagrus Wolffi Blkr. 356, I75.

Hypselobarbus Blkr. 261, 430. 314, r99.

Hypsigenys Gthr. 292, I06.

Hypsinotoidei 468, 3 I 0.

Hypsinotus Schl. 468, 310.

Hypsinotus benhatatate Blkr. 468, 3ro. 497, 9.

Hypsipops microlepis $B / k r .331,445$.

Hypsolepis Baird 261, 433. 314, 208.

Hypsurus $A g .468,290$.

Hysterocarpus Gill 468, 290.

Hysterocarpus Traskii Gill 468, 290.

Iaus Heck. 261, 438 .

Icania cynoglossa $K p .288,69$.

Ichthyapus Bris. 351, I20.

Ichthyobus Raf. 261, 429. 314, г9т.

Ichthyocampus Belcheri $K p$. 272, 8. 425, I26.

Ichthyocampus carce $K p . \mathbf{2 7 0}, 7$ I.

Ichthyophis Less., Rich. 351, I 22.

Ichthyophis guttatus Rich. cf. Channomuraena vittata Rich.

Ichthyophis micropterus Blkr. 318, 252. 320, 272.

Ichthyophis pantherinus Less. 334, 464. cf. Gymnomuraena pantherina $B l k \%$. 
Ichthyophis tigrinus Less. 320, 272. 334, 463. cf. Gymnomuraena tigrina Blkr.

Ichthyorhamphus Cast. 468,284 .

Ichthyscopus elongatus Blkr. cf. Guathagnus elongatus Gill.

Ichthyscopus inermis Szuns. 497, I8.

Ictalurus Raf., Gill 306, го3.

Ictalurus coerulescens Gill 306, 103.

Ictalurus furcifer Gill 336, 4.

Icthelis Raf. 458, 249 .

Icthelis macrochir Raf. 468, 249.

Ictyopogon Raf. 453, 30I.

Idus Heck. 314, 212. 329, 264.

Ilarches Cant. 468, 30 r.

Ilarches orbis Cant 425, r40. 468, 30r. 481, 24. cf. Ephippus orbis $C . V$.

Ilisha abnormis $G r$. cf. Ilisha elongata Blkr.

Ilisha elongata $B l k r .425$, I $48.497,25$.

Ilisha macrogaster Blkr. 381, 300.

Ilisha megalopterus Blkr. 395, 294.

Ilisha novacula Blkr. 381, 302. 425, 148.

Ilisha Schlegeli Blkr. 411, 252.

Iluocoetes 125, 3 I9.

Incisidens Gill 468, 296.

Irex indicus $V a l .459,99$.

Isistius brasiliensis Gill $\mathbf{4 5 9}, 68$.

Isocephalus Heck. 314, 194. 329, 262.

Isopisthus Gill 468, 335.

Isopisthus parvipinnis Gill 363, 255. 468, 335 .

Isurus cornubicus $G r .497,3$.

Johnii 468, 325 .

Johnius $B l .468,327$.

Johnius adustus Blkr. 363, 260.

Johnins amazonicus Cast. cf. Otolithus amazonicus Blkr.

Johnius Belangeri Blkr. 287, 49. 288, 72. 425, I42.

Johnius Belengeri C. V., Cant. 2, 523. 452, 46.

Johnius Cantori Blkr. 452, 51.

Johnius carutta $B l .452,48.468,327$.

Johnius catalea $C . V .2,523$.

Johnius cataleus Cuv. cf. Johnius diacanthus Cant., Pseudosciaena diacanthus Blkr.

Johnius celebicus Blkr. 272, I2. 275, 33. cf. Pseudosciaena miles Blkr. Johnius coitor C. V. 2, 523 .

Johnius coitor Blk\%.cf. Corvina miles $C . V$, Johnius Belengeri Cant.

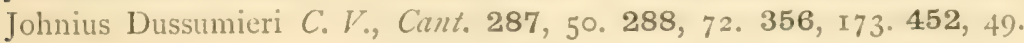


Johnius diacanthus Cant. 166, 326. 235, 364. 287, 50. 288, 72. cf. Pseudosciaena diacanthus Blkr.

Johnius Goldmanni Blkr. 231, I45. 296, ro8. cf. Pseudosciaena Goldmanni Blkr.

Johnius heterolepis Blkr. 446, $45^{6}$.

Johnius hypostoma Blkr. 270, 23. 452, 42 .

Johnius jubatus Blkr. 270, 23. 272, I2. 452, 52 .

Johnius Kuhlii Blkr. 191, I. 235, 364. 270, 23. 272, I2. 287, 5 ०.

288, 73. 425, I42. cf. Johnius Belengeri Cant.

Johnius maculatus Bl. Schn. 288, 73. cf. Johnius Cantori Blkr.

Johnius melanotis Cast. 287, 50."

Johnius microlepis Blkr. 190, I I. 226, 384. 270, 23. 287, 50. cf. Pseudosciaena microlepis Blkr.

Johnius miles Blkr. 235, 364. 272, т 2. 275, 33. 288, 73. cf. Pseudosciaena miles Blkr.

Johnius Novae-hollandiae Blkr. 452, 44.

Johnius plagiostoma Blkr. 287, 50. 356, I73. cf. Pseudosciaena plagiostoma Blkr.

Johnius polykladiskos Blkr. 272, I2. 275, 33. cf. Pseudosciaena Vogleri Blkr.

Johnius resplendens Hombr. Jacq. cf. Pseudosciaena macrophthalmus Blkr.

Johnius ruber $B l$. Schn. 468, 330. cf. Otolithus ruber $C$. $V$.

Johnius sampitensis Blkr. 272, I2.

Johnius semiluctuosus Kner 452, 54 .

Johnius soldado Cant. 231, I45.

Johnius tenlo Blkr. 425. I 42.

Johnius trachycephalus Blkr. 270, 23. 272, I 2. 452, 4I.

Johnius Valenciennei Eyd. Soul. cf. Corvina diacanthus Blkr., Pseudosciaena diacanthus Blkr.

Johnius Wolffii Blkr. 191, I. 272, I2.

Julis Cuv. 1, 552. 292, 97.

Julis Abhortani C. V. 459, 82.495, 9.

Julis aeruginosus $C . V$. cf. Julis trilobata $C . V$.

Julis amblycephalus Blkr. 293, 278. 358, I $84.364,276$.

Julis (Julis) amblycephalus $B l k r .148,83.167,373.275$, I 7 .

Julis (Halichoeres) amboinensis Blkr. 142, 55 .

Julis (Halichoeres) annularis K. v. H. 11, 6. 12, 42. 86, 513. 157, 234. 170, 479. 171, 245. 236, 331. 270, 37. cf. Platyglossus marginatus Blkr.

Julis (Julis) annulatus C. $V$. 137, 3 II. 275, I7. cf. Hologymnosus fasciatus Lac.

Julis (Halichoeres) argus Benn. 11, 7. 12. 42. 270, 37. 275, I 7.

Julis auricularis $C v$. 119, 13 .

Julis axillaris Q. G. cf. Stethojulis axillaris Gthr. 
Julis (Halichoeres) balteatus Q. G. 47, 253. 71, 768. 156, 217. 157, 234. 167, 373. 184, 203. 231, I48. 270, 37. 275, I 7. cf. Stethojulis albovittata Gthr.

Julis (Halichoeres) bandanensis Blkr. 47, 254. 184, 203. 236, 334 . 275, I 7 .

Julis (Halichoeres) batuensis Blkr. 157, 240.

Julis bicatenatus Benn. cf. Julis trilobata $C$. $V$.

Julis bicolor $C$. $V$. cf. Julis Commersoni $C$. $V$.

Julis (Halichoeres) binotopsis Blkr. 17, 7. 53, r 7u. 184, 203. 236, 331. 285, 243.

Julis (Halichoeres) Cantori Blkr. 288, 70.

Julis (Halichoeres) casturi Blkr. 71, 768. 156, 217. 167, 373. 170, $478.171,245.184,203.234,355.236,33$ 1. 250, 206.270, 38. 275, 1 7. Julis caudimacula Q.G. cf. Hemicoris caudimacula Blkr.

Julis (Julis) celebicus Blkr. 137, 3I3. 182, I60. 184, 203. 234, 355 . 236, 334. 275, I 7.

Julis (Halichoeres) chrysotaenia Blkr. 78, 488.

Julis (Julis) cingulum C. V. 91, 52. cf. Hemicoris cingulum Blkr.

Julis Commersonii C. $V .459,82.495,9$.

Julis coris $C$. $V$. cf. Coris aygula Lac.

Julis cupido T.Schl., 11, 30. 12, 58. 25, 10. 293, 278, 497, 5 .

Julis (Julis) cupido T. Schl. 170, 479. 236, 331. 277, 88.

Julis Cuvieri Benn. cf. Coris Cuvieri Gthr.

Julis (Halichoeres) Cuvieri Blkr. 8, 160. 11, 24. 12, 54. 126, 344. $158,274.275,17.287,43$.

Julis cyanogaster $C . V .143,56$.

Julis cyanogrammus Rich. 119, I3.

Julis (Halichoeres) cyanopleura Blkr. 78, 489. 292, Iоo.

Julis decussatus C. $V$. cf. Julis (Halichoeres) hortulanus $C$. $V$., Hemitautoga centiquadra Blkr., Platyglossus (Hemitautoga) centiquadra Blkr.

Julis (Halichoeres) diechismenacanthoïdes Blkr. 72, I2 I. 125, 324. $234,355.275$, I 7 .

Julis (Halichoeres) diechismenacanthus Blkr. 68, 645. 134, I55.

Julis doliatus $C$. $V$. cf. Julis (Julis) annulatus $C$. $V$., Hologymnosus fasciatus $L a c$.

Julis dorsalis Q.G., $\mathrm{Val} .11,6.12,42.66,564.91,52.156,216$. $157,234.167,373.171,245.184,203.228,424.236,331.270$, 37. 275 I 7. $285,243.293,279.308$, I 52. 315, 230. 318, 250. $320,266.358, \mathrm{r} S 4.364,276.396,299.425, \mathrm{I} 35.437,38.459$, S2. $491,40: 495,9$.

Julis (Halichoeres) Dussumieri C. V. 91, 54. 288, 70.

Julis elegans $K . v . H .11,6.12,42$. cf. Platyglossus (Güntheria) scapularis Blkr.

Julis (Halichoeres) elegans $K \cdot v . I I .54,2 S_{9} .156,2$ I $7.157,234.167$, 
373. 184, 203. 234, 355. 270, 38. 275, x8. 277, 88. 287, 43. cf. Güntheria scapularis Blkr.

Julis erythrogaster $C . V .143,56$.

Julis erythropterus $C$. $V$. cf. Coris erythropterus Blkr.

Julis exornatus Rich. cf. Halichoeres nigrescens Blkr.

Julis (Halichoeres) Finlaysonii C. $V$. 91, 54.

Julis formosus $C$. $V$. cf. Julis trilobata $C . V$.

Julis (Halichoeres) formosus Stens. 81, I69. 91, 54. 275, x8.

Julis (Halichoeres) formosus Blkr. cf. Coris formosa Blkr.

Julis Gaimardi Q. 11, 4. 12, 42.

Julis genivittata $C . V$. cf. Julis hebraica $C$. $V$.

Julis (Halichoeres) Geoffroyii Q.G. 143, 58. 275, I8. 292, 100.

Julis (Julis) Girardi Blkr. 231, I68. 292, 99.

Julis Güntheri Blkr. 293, 279.

Julis Hardwickii Gray cf. Julis lunaris Cuv. $\mathrm{Val}$.

Julis (Halichoeres) Harloffii Blkr. 8, I 59. 11, 22. 12, 53. 25, то.

157, 234. 184, 203 208, 239. 231, I 48. 234, 355. 236, 33 r, 334.

270, 38. 275, 18. 277, 88.

Julis (Halichoeres) Hartzfeldii Blkr. 66, 563 228, 424. 250, 206275 , IS. Julis hebraica $C . V .459,82$.

Julis (Halichoeres) heteropterus Blkr. 163, 78. 292, 98.

Julis (Halichoeres) Hoevenii Blkr. 47, 250. 156, 2 I 7. 184, 203. 234, 355. 267, I39. cf. Platyglossus Hoevenii Blkr.

Julis hortulanus $C$. $V$. cf. Hemitautoga centiquadra Blkr., Platyglossus (Hemitautoga) centiquadra Blkr.

Julis (Halichoeres) hortulanus C. $V .78,486.91,54.157,234.167$, 373. 182, I6о. 184, 203. 228, 424. 270, 38. 275, I 8.

Julis (Halichoeres) hortulanus Blkr. cf. Platyglossus (Hemitautoga) centiquadra Blkr.

Julis (Halichoeres) Hyrtlii Blkr. 143, 60. 156, 2 I 7. 167, 373. 235, 369236,33 I, 334. 275, I8.

Julis (Halichoeres) interruptus Blkr. 47, 252. 53, т6r. 54, 237. 231, I 48. 275, I8. cf. Stethojulis interrupta Blkr.

Julis Janseni Blkr. 293, 279. 354, I44. 364, 276.

Julis (Julis) Janseni Blkr. 143, 56. 167, 373. 170, 479. 236, 331 . $270,37.275$, I 7 .

Julis (Halichoeres) javanensis Blkr. 170, 479.

Julis (Julis) javanicus Blkr. 236, $33 \mathrm{I}$.

Julis (Halichoeres) javanicus Blkr. 166, 34I.

Julis (Halichoeres) kallochroma Blkr. 76, 289. 156, 217. 270, 38.

Julis (Halichoeres) kallopisos Blkr. 84, 348. 275, I8.

Julis (Halichoeres) kallosoma Blkr. 54, 289. 156, 217. 167, 373.

184, 203. 231, I $48.236,33^{2}, 334.270,38.275$, I $8.277,88$.

285,244 .

Julis (Halichoeres) kawarin Blkr. 53, I72. 275, I8. 
Julis Lamarrii $C . V .459,83.495,9$.

Julis (Halichoeres) leparensis Blkr. 70, 730. 147, 469. 156, 2 I 7. 170, 48o. 184, 203. 185, 225. 234, 355. 236, 33r, 334. 270, 38. 275, I8. 287,43 .

Julis Leschenaulti $C . V$. cf. Güntheria scapularis Blkr., Platyglossus (Güntheria) scapularis Blkr.

Julis (Julis) leucorhynchos Blkr.143, 57. 275, I7.

Julis lineolatus $C v$. 119, 13 .

Julis lunaris $C . V .11,28.12,56.46,213.91,52.126,344.156$, 216. 167, 373. 171, 245. 185, 225. 234, 355. 236, 331. 270, 37. 275, I7. 287, 43. 293, 279. 296, 104. 309, 154. 315, 230. 318, 250. $320,266.340,364.354$, r 44.358, I $84.364,276.369,42$. $394,286.425$, г $35.437,38.459,83.491,40.497,5$.

Julis lutescens Brev. cf. Julis lunaris $C$. $V$.

Julis (Halichoeres) margaritophorus Blkr. 78, 487.

Julis (Halichoeres) marginatus v. H. 292, 99.

Julis Matthaei C. $V .459,83.495,9$.

Julis (Julis) melanochir Blkr. 163, 77. 293, 280. 491, 40. 496, 2.

Julis (Halichoeres) melanurus Blkr. 47, 25 I. 80, 87. 167, 373, 184, 203. 234, 355. 275, r8. cf. Platyglossus melanurus Blkr., Platyglossus (Platyglossus) melanurus Blkr.

Julis meleagris $C . V$. cf. Julis (Halichoeres) Geoffroyii Q.G.

Julis meniscus $C . V .11,4.12,42$. cf. Julis lunaris $C . V$.

Julis miniatus $K . v . H .11,6.12,42$. cf. Halichoeres miniatus Blkr. Julis (Halichoeres) miniatus $(K . v . H). C . V .17,8.53$, I 7 1. 80, 87.

142, 6o. 157, 234. 170, 48o. 234, 355. 236, 332. 248, 203. 270, 38. 275, 18. cf. Halichoeres miniatus Blkr.

Julis (Halichoeres) modestus Blkr. 8, I60. 9, 633, 638. 11, 26. 12, 55. 126, 344. 158, 274. 185, 225. 285, 244. 287, 43.

Julis (Halichoeres) mola Cuv. 91, 54. 126, 344. 127, 345. 185, 225.

227, 408. 231, I 49. 235, 369. 270, 38. 275, IS. 287, 43.

Julis (Halichoeres) nebulosus C. $V .91,54$.

Julis notatus Rich. 119, I3. cf. Pseudolabrus celidotus Blkr.

Julis (Halichoeres) notophthalmus Blkr. 8, 158. 11, 20. 12, 52. 17, 4. cf. Julis (Halichoeres) mola Cuv.

Julis (Halichoeres) notopsis $K . v . H .11,6.12,42.76,290.157$, 234. 185, 225. 236, 332, 334. 270, 38. 275, IS. 285, 244.

Julis (Julis) oxyrhynchos Blkr. 164, 74 .

Julis (Halichoeres) pardaleocephalus Blkr. 17, S. 236, 332. 270, 3 S. Julis pavo Val. 302, 3r.

Julis (Halichoeres) phaiopus Blkr. 76, 291. 157, 235 .

Julis (Halichoeres) phaiotaenia Blkr. 125, 322. 157, 235.

Julis (Halichoeres) phekadopleura Blkr. 17, 8. 156, 217. 167, 373. 182, I60. 236, 332, 334. 270, 38. 275, I8. 285, 244. cf. Stethojulis phekadopleura Gthr.

INDEX BL̨EEKER. 
Julis (Halichoeres) podostigma Blkr. 99, 332.

Julis poecila Lay \& Benn. cf. Halichoeres poecila Blkr.

Julis poëcilopterus Schl., Richds. cf. Halichoeres poëcilepterus Blkr. Platyglossus (Parajulis) poecilopterus Blkr.

Julis (Julis) polyophthalmus Blkr. 156, 2 I 7. 185, 225.

Julis (Halichoeres) polyophthalmus Blkr. 70, 731. 127, 345. 157, 235 .

167, 374. 170, 480. 234, 355. 236, 334 .

Julis porphyrocephalus Benn. cf. Julis lunaris $C . V$.

Julis praetextatus, Q. G., Gthr. 11, 6. 12, 42. cf. Cymolutes praetextatus Gthr.

Julis (Halichoeres) prosopeion Blkr. 84, 347. cf. Platyglossus (Paraplatyglossus) prosopeion $B l k r$.

Julis (Halichoeres) pseudominiatus Blkr. 142, 62. 170, 479. 236, 332.

270, 38. 275, I8. 277, 88. 285, 244.

Julis (Halichoeres) purpureolineatus C. $V .91,54$.

Julis purpureus Rüpp. 143, 56 .

Julis pyrrhogramma T. Schl. 143, 6r. cf. Halichoeres pyrrhogramma Blkr., Platyglossus (Parajulis) pyrrhogramma Blkr.

Julis (Halichoeres) pyrrhogrammatoïdes Blkr. 78, 490. 143, 6r.

Julis (Julis) quadricolor Less. 143, 55. 167, 373. 236, 339. 275, I 7 .

293, 280. 364, 276. 497, 5 .

Julis (Halichoeres) quadricolor Less. 91, 54.

Julis (Halichoeres) Reichei Blkr. 170, 479. 174, 43. 236, 332. 250, 207. 270, 38. 285, 244.

Julis (Halichoeres) Renardi Blkr. 47, 253. 167, 374. 275, 18. 285, 244. Julis rosea $Q . G$. cf. Julis (Julis) annulatus $C . V$.

Julis rubecula Rich. 119, 13 .

Julis rubiginosus Rich. 119, I3.

Julis scapularis Benn. cf. Güntheria scapularis Blkr., Platyglossus

(Güntheria) scapularis $B l k r$.

Julis (Halichoeres) Schröderii Blkr. 180, 2.

Julis (Julis) Schwanefeldi Blkr. 76, 288. 270, 37. 293, 28 r.

Julis (Halichoeres) Schwarzii Blkr. 8, r59. 16, 5. 17, 7. 53, 61.

Julis semidecorata Less. cf. Julis (Halichoeres) hortulanus $C$. $V$.

Julis semifasciatus $C$. $V$. cf. Julis dorsalis $Q$. G., Julis (Julis) dorsalis $Q . G$. Julis (Halichoeres) solorensis Blkr. 80, 86. cf. Halichoeres solorensis Blkr. Julis (Julis) Souleyeti $C . V$. 122, I76. 236, 33 r, 334. cf. Julis umbrostigma Rüpp.

Julis (Halichoeres) spilurus Blkr. 47, 252. 167, 374. 184, 203. 188, 460. 275, I8. cf. Platyglossus (Güntheria) trimaculatus Blkr.

Julis stellatus $C . V$. cf. Coris Cuvieri Gthr., Julis (Halichoeres) formosus Szons.

Julis (Julis) strigiventer Bcnn. 185, 225. cf. Stethojulis strigiventer Gthr. Julis (Halichoeres) strigiventer Binn. 47, 25 I. 54, 238. 235, 369. 275, I8. $285,244.287,43$. 
Julis taenianotus $Q$. G. cf. Novaculichthys macrolepidotus Blkr.

Julis (Halichoeres) Temminckii Blkr. 78, 49r. 158, 274. 227, 408.

234,355 .

Julis thersites Richds. cf. Halichoeres poecilopterus Blkr.

Julis (Halichoeres) timorensis Blkr. 53, I 7 I.

Julis trilobata $C . V .425$, I $35.459,83.495,9$.

Julis trimaculata $Q$. G. cf. Güntheria trimaculata Blkr.

Julis turcica Riss. cf. Julis pavo $\mathrm{Val}$.

Julis umbrostigma Rïff. 293, 2S I. 364, 276.425, I 35.459, 3.495 , г 0.

Julis unimaculata Lowe. cf. Juiis pavo $\mathrm{Val}$.

Julis (Julis) urostigma Blkr. 76, 287. 157, 234. 236, 331. 270, 37 .

275, I 7 .

Julis viridis $\mathrm{Val}$. cf. Julis lunaris C. $V$.

Julis (Julis) Vrolikii Blkr. 156, 217.

Julis (Halichoeres) Vrolikii Blkr. 125, 323. 157, 235. 158, 274. 285, 244.

Julis (Halichoeres) zeylonicus Bemn. 91, 54.

Karodon punctatus Schl. cf. Hoplegnathus punctatus Rich.

Katethostoma malacopterus 493, 59 .

Kentrocapros Kp. 367, 16 .

Keris 137, 305. 163, 62 .

Keris amboinensis Blkr. 54, 272. cf. Naseus amboinensis Blkr.

Keris anginosus $C$. $V$. cf. Acronurus anginosus Blkr., Priodon anginosus Blkr.

Ketengus Blkr. 306, 92.

Ketengus typus Blkr. 7, 9. 8, 167. 16, 5. 25, 10. 35, 271. 42, 59.

45, I96. 49, 420. 55, 4I3. 267, I40. 270, 47. 272, I9. 287, 59.

$288,78.306,92.356$, I 75 .

Kowala albella C. $V .91,74$.

Kowala lauta Cant. 288, 79. cf. Harengula zunasi Blki.

Kowala thoracata C. $V .91,74.288,79$.

Kryptopterichthys Blkr. 299, 395. 306, i I6.

Kryptopterichthys bicirrhis Blkr. 270, 48. 272, I9.

Kryptopterichthys lais Blkr. 272, 20.

Kryptopterichthys macrocephalus Blkr. 270, 48.

Kryptopterichthys palembangensis Blkr. 270, 48. 306, II6.

Kryptopterus Blkr. 299, 395. 306, I 6 .

Kryptopterus limpok Blk\%. 270, 48, 272, I9.

Kryptopterus micropus Blkr. 270, 48. 272, 19. 306, I 6.

Kuhlia Gill 468, 268.

Kuhlia ciliata Gill cf. Moronopsis ciliatus Blkr.

Kurtus 1, 553 .

Kurtus argenteus $B l$. Schn. 468, 3 I2.

Kurtus Blochii C. V. 2, 5 18. 16, 4. 25, 9.

Kurtus Blochii Lac. cf. Kurtus indicus $\mathrm{Bl}$. 
Kurtus cornutus $C . V$. 2, 518. cf. Kurtus indicus $B l$.

Kurtus indicus $B l .51,57.57,78.91,46.178,2.235,367.272,14$. 288, 75. 425, I33.

Kurtus macrolepidotus $B l$. Schn. cf. Pempheris mangula $C$. $V$. Kyphosus Lac. 468, 293.

Labeo Cuv. 261, 423. 314, I94. 329, 262.

Labeo cetopsis Kner cf. Saurogobio cetopsis Blkr.

Labeo curchius $M C C l .91,64$.

Labeo cursa $C . V .91,64$.

Labeo diplostomus C. $V .91,64$.

Labeo Dussumierii $C . V .91,64$.

Labeo dyocheilus $C . V .91,64$.

Labeo erythropterus $C . V$. 166, 360. 170, 476.

Labeo falcifer $C$. $V$. cf. Lobocheilos falcifer $v$. Hass.

Labeo fimbriatus $C . V .91,64$.

Labeo malacostomus $C . V .91,64$.

Labeo molitorella Gthr. cf. Gymnostomus molitorella Blkr.

Labeo morar C. $V .91,66$.

Labeo oblongus $C . V$. cf. Crossocheilos oblongus $v$. Hass.

Labeo velatus Cuv. 91, I32.

Labeobarbus Rüpp. 1, 552. 261, 431. 314, I98.

Labeobarbus douronensis Blkr. 270, 52 .

Labeobarbus macrolepis Heck. 91, 60:

Labeobarbus progeneius $C . V .91,60$.

Labeobarbus soro Blkr. 270, 52 .

Labeobarbus tambra Blkr. 166, 355. 170, 476. 270, 52 .

Labeobarbus tambroides Blkr. 106, 92. 170, 477. 200, 48. 270, 52 . $271,2$.

Labeobarbus tor Blkr. 91, 60, I65. 335, го.

Labracini 468, 262 .

Labracinus Mus. L. B. 468, 320. $1465: 5$

Labracoglossa argenteiventris Peters 497, I I.

Labrastum Guich. 289, 81.

Labrax Klein 468, 263.

Labrax agrammus Schl. cf. Agrammus Schlegelii Gïnth.

Labrax hexagrammus Pall. cf. Chirus hexagrammus Stell., C. $V$.

Labrax japonicus $C . V .90,23.463,263$. cf. Lateolabrax japonicus Blkr.

Labrax lupus Cuv. 468, 263.

Labrax octogrammus Schl. 449, 371 .

Labrax waigiensis Q. G., C. $V .13,3.50,479.51,55$. cf. Paammoperca waigiensis Blkr.

Labrichthys Blkr. 292, I04. 331, 444.

Labrichthys Arago Blkr. cf. Novaculichthys macrolepidotus $B l k r$.

Labrichthys celidota Gthr. cf. Pseudolabrus celidotus Blkr. 
Labrichthys cyanotaenia Blkr. 99, 331. 292, 104. 293, 295. 318, 250. $320,267$.

Labrichthys rubiginosus Gthr. cf. Pseudolabrus rubiginosus Blkr.

Labristoma Szons. 468, 320.

Labroïdes Blkr. 292, 104.

Iabroïdes dimidiatus Blkr. 495, 10.

Labroïdes latovittatus Blkr. 163, 73. 184, 203. 293, 295. 320, 267. 358,185 .

Labroïdes paradiseus Blkr. 47, 249. 167, 373. 184, 203. 188, 460. 236, 331. 270, 37. 275, I 7. 292, 104. 293, 296. 296, 104. 315, 23r. 320, 267. 354, I 44. 358, r85. 364, 277. 369, 42. 396, 296.

Labroïdes xanthurus Blkr. 143, 52. 275, I7. 292, I04.

Labroperca Gill 468, 256.

Labrus L. 292, 107.

Labrus albovittatus Bonnat. cf. Julis (Halichoeres) balteatus Q. G.

Labrus angulosus Lac. cf. Holocentrum sammara $C$. $V$.

Labrus annulatus $L a c$. cf. Julis (Julis) annulatus $C . V$.

Labrus Arago Q. G. cf. Novaculichthys macrolepidotus Blkr.

Labrus argentatus Lac. cf. Lutjanus argentimaculatus Blkr.

Labrus auratus Raf. 468, 249.

Labrus axillaris Benn. cf. Cossyphus axillaris $C . V$.

Labrus badis Buch. cf. Badis Buchanani Blkr.

Labrus bilunulatus Lac. cf. Cossyphus bilunulatus $C$. $V$.

Labrus botryocosmus Rich. 119, I3.

Labrus celidotus Forst. 119, I3. cf. Pseudolabrus celidotus Blkr.

Labrus centiquadrus Comm. 292, I0I. cf. Julis (Halichoeres) hortulanus $C . V$.

Labrus centiquadrus Lac. cf. Hemitautoga centiquadra Blkr.

Labrus chloropterus $B l$. cf. Halichoeres chloropterus Blkr.

Labrus Commersoni Lac. cf. Pristipoma hasta $C$. $V$.

Labrus cothinus Rich. cf. Pseudolabrus rubiginosus Blkr.

Labrus cyanodus Rich. 119, I3.

Labrus dario Buch. 91, 107.

Labrus diana Lac. cf. Cossyphus diana $C$. $V$.

Labrus doliatus Lac. cf. Julis (Julis) annulatus $C$. $V$.

Labrus fasciatus $B l$. cf. Tautoga fasciata $C . V$.

Labrus formosus Bcnn. cf. Coris formosa Gthr., Julis (Halichoeres) formosus Sions.

Labrus fuliginosus Lac. cf. Tautoga fasciata C. $V$.

Labrus furcatus Lac. cf. Aphareus furcatus Gthr.

Labrus fuscus Lac. cf. Novacula taeniurus Blkr.

Labrus gallus Gmel. cf. Julis lunaris $C$. $V$.

Labrus guttatus $B l$. cf. Halichoeres guttatus Blkr.

Labrus hebraicus Riss. cf. Julis pavo $\mathrm{Val}$.

Labrus hololepidotus Lac. cf. Sciaena hololepidota $C$. $V$. 
Labrus hortulanus Lac. cf. Julis (Halichoeres) hortulanus C. $V$.

I abrus iris Rich. 119, r3.

Labrus jaculator Shaw cf. Toxotes jaculator $C$. $V$.

Labrus jaculatrix Lac. cf. Toxotes jaculator $C$.

Labrus japonicus $C$. $V$. cf. Choerops japonicus Gthr., Blkr.

Labrus latovittatus Rïpp. cf. Labroïdes latovittatus Blkr., Labrus paradiseus Blkr.

Labrus latovittatus Lac. cf. Malacanthus taeniatus C. $V$.

Labrus leopardus Lac. 438, 45. cf. Epinephelus leopardus Blkr.

Labrus leucosticticus Benn. cf. Cossyphus leucosticticus Gthr.

Labrus lineatus $B l$. Schn. cf. Julis pavo $\mathrm{Kal}$.

Labrus longirostris Lac. cf. Diapterus oyena Blkr., Gerres oyena $C . V$.

Labrus lorius Bibl. Banks cf. Julis lunaris Cuv. $\mathrm{Val}$.

Labrus Iunaris L. cf. Julis lunaris Cuv. $\mathrm{Val}$.

Labrus macrodontus Lac. 292, 106. cf. Cossyphus macrodon Blkr.

I abrus macrogaster Lac. cf. Glyphidodon (Glyphidodon) bengalensis C.V.

Labrus maculosus Gron. cf. Julis pavo $\mathrm{Val}$.

Labrus marmoratus Lac. cf. Cirrhites marmoratus Gill, Cirrhitichthys marmoratus $B l k r$.

Labrus melapteronotus Lac. cf. Tautoga fasciata $C$. $V$.

Labrus melapterus $B l$. cf. Tautoga melapterus $C$. $V$.

Labrus mesothorax $B l$. Schn. cf. Cossyphus mesothorax $C$. $V$.

Labrus octogrammus Pall. cf. Octogrammus Pallasi Blkr.

Labrus octovittatus Lac. cf. Lutjanus octovittatus Blkr.

Labrus ornatus Sol. cf. Julis lunaris $C$. $V$.

Labrus oyena Forsk. cf. Diapterus oyena Blkr.

Labrus oyena Lac. cf. Gerres oyena $C . V$.

Labrus poecilopleura $C . V .119$, r3. cf. Pseudolabrus celidotus Blkr.

Labrus pulcherrimus Forst. cf. Julis (Julis) dorsalis $Q . G$.

Labrus punctulatus Lac. cf. Serranus punctulatus $C$. $V$., Variola louti Blkr.

Labrus quadrilineatus Rüpp. 143, 53.

Labrus reticulatus $V a l .292$, 105.

Labrus reticulatus $T$. Schl. cf. Cossyphus reticulatus $C . V$.

Labrus rubiginosus Schl. 292, I02, cf. Pseudolabrus rubiginosus Blkr.

Labrus semidiscus Lac. cf. Julis (Julis) annulatus C. $V$.

Labrus sexfasciatus Lac. cf. Glyphidodon (Glyphidodon) coelestinus (Sol.) C. $V$.

Labrus spilonotus Benn. cf. Cossyphus macrurus Gthr.

Labrus syriacus Bl. Schn. cf. Julis pavo Val.

Labrus taeniurus Lac. 292, I02. cf. Novacula taeniurus Blkr.

Labrus tetracanthus Lac. cf. Percis cancellata $C$. $V$.

Labrus trichopterus $L$., Pall. cf. Trichopodus trichopterus Lac.

Labrus zeylonicus Forst. cf. Julis lunaris $C$. $V$.

Lacertus peregrinus Rond. cf. Saurida tombil C. $V$.

Lachnolaimus Cuv. 292, I07. 
Lactarius delicatulus $C . V .2,518.16,4.25,8.51,57.57,74.91$, 46. $127,345.156,215.178,2.270,28.272,13.275,38.288$, 74. 364, 289. 396, 300. 425, I32.

Lactophrys Stons. 367, I5.

Lactophrys cornutus Szons. 316, 24x. 320, 265. 354, I43. cf. Ostracion cornutus $L$.

Lactophrys diaphanus $K p$. cf. Ostracion diaphanus $B l$. Schn.

Laemargus Labordii $M$. $H$. cf. Euprotomicrus Labordii Gthr.

Lagocephalus laevigatus Gill cf. Gastrophysus laevigaetus Blkr.

Lagodon Holbr. 468, 283.

Lais Blkr. 299, 398. 306, 107.

Lais hexanema Blkr. 270, 46. 272, I9. 306, 107.

Lama guliminda Russ. cf. Dentex tolu $C$. $V$.

Lamna cornubica Flem. cf. Isurus cornubicus $G r$.

Lamnostoma $K$. 351, I20.

Lamnostoma bicolor $K p \cdot \mathbf{2 7 2}, 28$. cf. Sphagebranchus bicolor $K p$.

Lampris luna Risso 425, I33.

Lampugus fasciolatus $C . V .266,66.364,290$.

Larimodon $K p .468,329$.

Larimus C. $V .468,328$.

Larimus breviceps C. $V .468,328$.

Larimus Peli Blkr. 302, 63.

Latebrus Poey 468. 335.

Lateolabrax Blkr. 118, 53. 468, 263.

Lateolabrax japonicus $B l k r .118,53.425$, I $37.468,263.496,2.497,6$.

Lates C. V. 1, 553. 468, 263.

Lates calcarifer $C . V .288,7$ I. 356, I73. cf. Lates nobilis $C . V$.

Lates calcarifer Gthr. 425, I37.

Lates heptadactylus Cant. 272, I0. 275, 24. 287, 45.

Lates nobilis $C . V .2,524.9,632.13,27.16,4.25,7.26,4.35$, 26 I. $42,58.49, .418 .51,55.55,4$ I . 91, 30. 227, 406. 235, 362.

Lates nobilis Rich., 91, 30. cf. Lates calcarifer Gthr.

Lates punctulatus Cant. cf. Labrax japonicus $C . V$.

Latilus $C . V .1,553.468,279$.

Latilus argentatus $C . V$. 118, 85. 425, I39. 459, 78. 468, 280. 495, I 2. 497,7 .

Latilus doliatus $C . V .459,78$. cf. Caulolatilus doliatus Blkr.

Latilus upeneiformis Blkr. cf. Dentex ruber $C . V$.

Latilus upeneoides Blkr. 2, 523. cf. Dentex gracilis Blkr.

Latridopsis Gill 468, 3 г6.

Latris Rich.468, 3 I 6.

Latris hecateia Rich.468, 3 I6.

Latrunculini $453,310$.

Latrunculus Gthr. 453, 312.

Latrunculus albus Gthr. 453, 312. 
Laubuca Blkr. 261, 438. 314, 2 I5.

Lavinia Gir. 261, 425. 314, 206.

Lebias Cuv. 261, 439.

Leiarius Blkr. 306, 99.

Leiarius longibarbis Blkr. 306, 99.

Leiobatis akajei Blkr. 497, 4 .

Leiobatis (Trygon) Bennetti Blkr. 425, I20.

Leiobatis dadong Blkr. 395, 290.

Leiobatis Gerrardi Blkr. 491, 39.

Leiobatis Kuhli Blkr. 364, 271. 395, 290. 491, 39. 497, 4 .

Leiobatis (Trygon) nuda Blkr. 487. 497, 33 .

Leiobatis (Trygon) pastinaca Blkr. 425, r $20.459,68$.

Leiobatis polylepis Blkr. 495, 8 .

Leiobatis (Himantura) uarnak Blkr. 320, 264. 364, 27 I. 425, I 20. $459,68.491,40$.

Leiobatis uarnakoides Blkr. 364, $27 \mathrm{r}$.

Leiobatis (Trygon) zugei Blkr. 395, 290. 425, I20. 497, 4.

Leiobatis walga Blkr. 364, 27 I.

Leiocassis Blkr. 269, 87. 306, 94.

Leiocassis Ellenriederii Blkr. 271, ro.

Leiocassis longirostris 497, 22 .

Leiocassis micropogon Blkr. 185, 225. 235, 370. 270, 46. 272, I8.

Leiocassis poecilopterus Blkr. 272, I8. 306, 95.

Leiodon Srens. 367, I8.

Leiodon patoca Blkr. 364, 271. 368, 38. 395, 290. 396, 298. 425, I 2 г. $459,69$.

Leiodon Waandersi Blkr. 368, 38.

Leioglossus Blkr. 128, 418. 297, I37.

Leioglossus carangoides $B l k r .37,367.57,70.267$, I40. 270, 30. 287, 53. 396, 300.

Leiognathus argenteus Lac. cf. Equula ensifera $C . V$.

Leiognathus (Aulostoma) chinense Lac. 495, 19.

Leiognathus Dussumieri Blkr. 459, 98.

Leiognathus edentulus Blkr. 315, 235. 346, 3 I. 354, I48. 358, I9 I. $364,290.437,37.459,98.495$, I 8 .

Leiognathus fasciatus Blkr. 316, 242. 320, 270. 364, 290. 395, 293. $396,297.437,37.459,98.495,18$.

Leiognathus gerreoides Blkr. 356, I74.

Leiognathus gomorah Blkr. 315, 235.

Leiognathus insidiator $B l k r .364,290.425$, I32. 459, 98.

Leiognathus interruptus Blkr. 364, 290. 425, I32.

Leiognathus leuciscus Blkr. 364, 290.

Leiognathus lineolatus Blkr. 364, 290.

Leiognathus (Gazza) minuta Blkr. 495, I 9.

Leiognathus nuchalis Blkr. 425, I32, 497, I6. 
Leiognathus oblongus Blkr. 320, 270. 364, 290.

Leiognathus parviceps Blkr. 459, 98. 495, I9.

Leiognathus rivulatus $B l k r .425$, I32. 497, I6.

Leiognathus splendens Blkr. 320, 270. 346, 3r. 356, I 74. 354, I 48. 358, I91. 364, 290. 425, г32. 437, 37. 459, 98. 495, I9.

Leiopsis Benn. 468, 279 .

Leiostomus Lac. 468, 325 .

Leiostomus lineatus Ayr. 468, 325.

Leiostomus xanthurus Lac.468, 325 .

Leiosynodontis Blkr. 306, 88 .

Leiosynodontis maculosus Blkr. 304, 55. 306, 88.

Leiosynodontis nebulosus Blkr. 304, 55 .

Leiuranus Blkr. 88, 24. 351, II9.

Leiuranus colubrinus $K p$., Blkr. 352, I26. 425, I23.459, 72.495, 22.

Leiuranus fasciatus $K p$. 358, I93. 364, 296.

Leiuranus Lacepedii Blkr. 88, 36. 157, 235. 188, 46r. 236, $33^{2}$. 270, 6o. cf. Leiuranus colubrinus $K p$.

Leiurus Swns. 367, Io.

Leiurus stellatus Blkr. 364, 272. 368, 2 I. 425, 122. 459, 71. 495, 20.

Leius ferox Kner cf. Isistius brasiliensis Gill, Tristius brasiliensis Gill.

Lembus Gthr. 453, 302 .

Lemnisoma thyrsitoides Less. cf. Gempylus coluber $C . V$.

Lentipes Gthr. 453, 314.

Lepadogaster dentex Bl. Schn. cf. Gobiesox dentex Cuv.

Lepadogaster puniceus Rich. 119, 12.

Lepadogaster testar Bl. Schn. cf. Gobiesox dentex Cuv.

Lepibema Raf. 468, 263 .

Lepidamia Gill 468, 259.

Lepidamia kalosoma Gill cf. Amia kallosama Blkr.

Lepidocephalichthys Blkr. 323, $3^{8 .}$

Lepidocephalichthys balgara Blkr. 323, 42.

Lepidocephalichthys Hasseltii Blkr. 323, 42.

Lepidocephalichthys thermalis Blkr. 323, 42. 335, 6.

Lepidocephalus Blkr. 220, 303. 261, 422. 323, 38 .

Lepidocephalus Hasseltii Blkr. 279, Iоr.

Lepidocephalus macrochir Blkr. 270, 50.323, 42.

Lepidochaetodon Blkr. 468, 306.

Lepidogobius Gill 453, 319.

Lepidogobius gracilis Gill 453, 3 I9.

Lepidoleprus australis Rich. 119, I4. 449, 370.

Lepidopus argyreus $C . V .266,53$.

Lepidopus tenuis Gïnth. 497, I 5 .

Lepidorhynchini 449,369 .

Lepidorhynchus villosus 497,2 I.

Lepidosteus sinensis Blkr. 425, I48. 
Lepidotrigla Bürgeri Gthr. 425, I26. 496, 2. 497, I3.

Lepidotrigla stonuchii Stnd. 497, I3.

Lepidotrigla vanessa Gthr. 425, I26.

Lepidozygus tapeinosoma Gthr. 485, 7 r.

Lepipterus $C . V .468,327$.

Lepisacanthus japonicus Lac. cf. Monocentris cataphracta Bl. Schn.

Lepodontiformes 468, 299.

Lepodus Raf. 468, 299.

Lepodus saragus Raf. 468, 299.

Lepomis Raf. 468, 249, 336.

Leptaspis leptolepis Blkr. 57, 7 r, 87.

Leptecheneis naucrates, neucrates Gill 491, 50. 497, I5.

Leptobarbus Blkr. 261, 435. 314, 202.

Leptobarbus Hoevenii Blkr. 270, 54. 272, 24. 356, г76.

Leptocephalichthys hypselosoma Blkr. 143, 69. 275, 52 .

Leptocephalus Bas. 314, 2 ro.

Leptocephalus dentex Cant. 288, 80.

Leptocephalus mongolensis Bas. cf. Chanodichthys mongolicus Blkr. Leptocephalus mongolicus Bas. cf. Chanodichthys mongolicus Blkr.

Leptocephalus Morrisii Penn., Gm. 128, 428. 266, 56 .

Leptocephalus oculus Blkr. 459, 73.

Leptocephalus taenia Less., Cuv. 128, 428. 266, 56. 491, 56.

Leptocephalus taenioides Blkr. 364, 297.

Leptochromis Blkr.468, $32 \mathrm{I}$.

Leptognathus Swons. 88, 23. 351, I I8.

Leptogobius Blkr. 453, 3I I.

Leptogobius oxypterus Blkr. 472, 292.

Leptojulis Blkr. 292, Ioo.

Leptojulis cyanopleura Blkr. 293, 289.

Leptojulis pyrrhogrammatoides Blkr. 293, 289 .

Leptonurus chrysostigma Blkr. 16, 14. 25, 1 2. cf. Coilia Dussumieri C. $V$.

Leptopegasus natans Blkr. 425, I25.

Leptorhinophis $K p$. 351, II9.

Leptorhynchus Smith, Lowe 351, I I5, I I8.

Leptorhynchus capensis Smith 266, 56 .

Leptorhynchus serpens Blkr. 497, 26.

Leptosynanceia asteroblepa Blkr. 458, I8. 491, 49.

Lepturus argenteus Gill cf. Enchelyopus lepturus Blkr.

Lepturus savala Blkr. 459, roo.

Lethrinus $C . V .1,553.468,28 \mathrm{I}$.

Lethrinus amboinensis Blkr. 103, 490. 140, 436. 318, 251. 358, i 87 . $433,33 \mathrm{r}$.

Lethrinus amboinensis $K n e r$ cf. Lethrinus harak Rüpp.

Lethrinus anatarius Rich. 425, 138.

Lethrinus argenteus $C . V .459,91$. 
Lethrinus atlanticus $C . V .468,28 \mathrm{r}$.

Lethrinus borbonicus $C . V .459,9 \mathrm{I}$.

Lethrinus centurio $C . V$. cf. Lethrinus nebulosus $C . V$.

Lethrinus choerorhynchus $C . V .497,8$.

Lethrinus chrysostomus Rich. 119, 9.

Lethrinus cinereus C. $V .91,3^{8}$.

Lethrinus cocosensis Blkr. 105, 40. 275, 27. 309, I55. 433, 34I. cf. Lethrinus sordidus $C . V$.

Lethrinus coeruleus $C . V .459,9 \mathrm{I}$.

Lethrinus croceopterus $C . V .459,9$ I.

Lethrinus elongatus $C$. $V$. cf. Lethrinus variegatus Elor.

Lethrinus erythrurus $C . V .91,38$. cf. Lethrinus harak Rüpp.

Lethrinus esculentus $C . V$. cf. Lethrinus nebulosus $C . V$.

Lethrinus fasciatus $C . V .91,3^{8}$.

Lethrinus fasciatus Kner cf. Lethrinus cocosensis Blkr.

Lethrinus fraenatus $C . V .91,3^{8 .}$

Lethrinus genivittatus $C . V$. 459, 9 I.

Lethrinus Güntheri Blkr. 444, I 53.497, 8.

Lethrinus haematopterus $T$. Schl., Rich. 118, 91. 425, I38. 433, 323. 497,8 .

Lethrinus haematopterus Gthr. cf. Lethrinus Güntheri Blkr.

Lethrinus harak Rüpp., C. V. 19, r5. 227, 406. 228, 424. 234, $35^{2}$. $270,20.287,46.316,242.358,187.394,287.396,299.433,338$. Lethrinus hypselopterus Blkr. 433, 326.

Lethrinus insulindicus Blkr. 433, 334.

Lethrinus Jagorii Pet. 433, 332.

Lethrinus kallopterus Blkr. 143, 47. 275, 27. 296, I06. 433, 324. 491,43 .

Lethrinus karwa $C . V .91,38$.

Lethrinus korely $C . V .91,38$.

Lethrinus latidens $C . V$. cf. Monotaxis grandoculis Blkr., Sphaerodon grandoculis Rüpp.

Lethrinus latifrons Rüpp. 46, 220. 54, 237. 184, 198. 275, 27. 309, I 55. 315, 233. 318, $251.320,268.358,187.364,282$. cf. Lethrinus variegatus $E h r$.

Lethrinus latifrons $C . V$. 354, 147 .

Lethrinus leutjanus $C . V .19,14.270,20.364,282.433,340$.

Lethrinus longirostris Playf. 459, 9I. cf. Lethrinus rostratus $K$. v. $H$. Lethrinus maculatus $C . V .91,38$.

Lethrinus mahsena $C . V .495$, I2.

Lethrinus mahsenoides $C$. $V .19$, I 5.275, 27. 320, 268. 364, 2S2.459, 9I.

Lethrinus mahsenoides Ehr. cf. Lethrinus insulindicus Blkr.

Lethrinus microdon $C$. $V .354$, r47. cf. Lethrinus variegatus Ehr.

Lethrinus miniatus $C . V .491,43$.

Lethrinus Moensii Blkr. 140, 435. 296, Io6. 316, 242. 433, 328. 
Lethrinus nebulosus $C . V .459,9$ 1. 495, I2.

Lethrinus nematacanthus Blkr. 102, 403. 118, 90.433, 327. 497, 8.

Lethrinus olivaceus $C$. $V$. 354, r 47. cf. Lethrinus rostratus $K . v . H$.

Lethrinus opercularis $C . V$. 2, 522. 19, 14. 25, 8. 50, 47 I. 53, 160. 56, 444. 91, 38. 126, 344. 156, 2 I 4. 167, 37 I. 170, 479. 185, 222. 208, 239. 227, 406. 231, I 45. 234, 352. 270, 20. 275, 27. 287, 46. 308, I 52. 315, 233. 316, 242. 318, 25 1. 320, 268. 358, I $87.364,282.395,29$ I. 425, I38. 433, 337.

Lethrinus ornatus $C . V$. 184, I98. 228, 424. 231, 145. 270, 20. 275, 27. 315, 233. 318, 251. 320, 268. 358, 187. 364, 282. 433, 335 . $437,38.459,9$ I. $491,43$.

Lethrinus ramak $C . V .459,9 \mathrm{I}$.

Lethrinus reticulatus $C . V$. 95, 96. 275, 27. 296, 106. 315, 233. $364,282,433,329.491,43$.

Lethrinus rhodopterus $B l k r .51,65.315,233$. cf. Lethrinus harak Rüpp.

Lethrinus Richardsonii Gthr. cf. Lethrinus haematopterus Rich.

Lethrinus rostratus $(K$. v. $H$.) C. $V .19$, I3. 25, 8. 231, I45. 275, 27. 308, I 52.358, I $87.364,282.433,343$.

Lethrinus semicinctus $C . V$. cf. Lethrinus latifrons Rüpp., Lethrinus variegatus Ehr.

Lethrinus sordidus $C$. $V . \mathbf{4 3 3}, 342.491,43$. cf. Lethrinus cocosensis Blkr. Lethrinus striatus Steind. 459, 92.

Lethrinus variegatus Elr. 433, 332. cf. Lethrinus latifrons Rüpp.

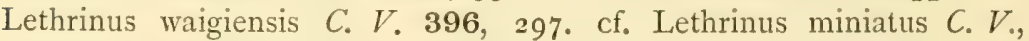
Lethrinus rostratus $K . v . H$.

Lethrinus xanthotaenia $B l k r .44,176.54,237$. cf. Lethrinus ornatus $C . V$.

Leucaspius Heck. Kner. 261, 437. 314, 2 I I. 329, 263.

Leuciscus Rond. 1, 552. 261. 437. 314, 2 I2. 329, 264.

Leuciscus abramioïdes Blkr. 91, 66.

Leuciscus acanthopterus Blkr. 91, 66.

Leuciscus acinaces $C . V$. 91, 66 .

Leuciscus acra $C . V .91,66$.

Leuciscus acutirostris $G r$. cf. Hemiculter leucisculus Blkr.

Leuciscus acutus Brouss. cf. Hemiculter leucisculus Blkr.

Leuciscus aeneus $V a l .415$, Iо. 416, I2 I. 425, I 44.

Leuciscus aequipinnatus Blkr. 91, 66.

Leuciscus aethiops Bas. 415, 45. 416, I2 I. 425, I44. cf. Leuciscus piceus Rich.

Leuciscus alkootee Blkr. 91, 66.

Leuciscus anjona $C . V .91,66$.

Leuciscus anjono $\mathrm{McCl} . \mathbf{9 1}, \mathrm{I} 40$.

Leuciscus apiatus $M C C l .91,66$.

Leuciscus argenteus Bas. cf. Xenocypris macrolepis Blkr.

Leuciscus argyrotaenia Blkr. 25, 2 I.

Leuciscus ariza $C . V .91,66$. 
Leuciscus atpar $C . V .91,66$.

Leuciscus bacaila $C . V .91,66,137$.

I.enciscus balitora $C . V .91,66$.

Leuciscus balookee $B$ lkr. 91, 66.

Leuciscus bambusa Rich. cf. Elopichthys bambusa Blkr.

Leuciscus bankanensis Blkr. 82, I92.

Leuciscus barila $C . V .91,66$.

Leuciscus barna $C . V .91,66$.

Leuciscus bata $C . V .91,66$.

Leuciscus bicirratus Blkr. 91, 66.

Leuciscus binotatus Blyt/ cf. Puntius (Puntius) binotatus Blkr.

Leuciscus bobree Blkr. 91, 66.

I.euciscus boga $C$. $V$. cf. Gobio boga $M C C l$.

Leuciscus bola C. $V .91,66$.

Leuciscus borelio $C . V .91,66$.

Leuciscus brachialis Blkr. 91, 66.

Leuciscus bramula Val. cf. Parabramis bramula Blkr.

Leuciscus cachius $C . V .91,66$.

Leuciscus catla Val. cf. Catla Buchanani C. $V$.

Leuciscus cephalotaenia Blkr. 52, 97. 56, 445. 185, 226.

Leuciscus chedra $M C C l .81,66$.

Leuciscus chevanella $V a l$. cf. Pseudobrama Dumerili Blkr.

Leuciscus chitul Sykes 91, 66.

Leuciscus chrysops C. $V .91,66$.

Lenciscus clupeoïdes C. $V .91,66$, г 38 .

Leuciscus corëensis $V a l$. cf. Barilius corëensis Blkr.

Leuciscus cosuatis C. V. 91, 139 .

Leuciscus cotio $C . V$. $91,66$.

Leuciscus cultellus $C . V .91,66$.

Leuciscus cupreus $\mathrm{Val} .415$, I0. 425, I44.

Leuciscus curriculus Rich. cf. Squaliobarbus curriculus Gthr.

Leuciscus cyanotaenia Blkr. 25, 21. 170, 477. 211, 242. 231, I49.

Leuciscus dancena Blkr. 91, 66.

Leuciscus dandia $C . V .91,66$, I40. cf. Rasbora dandia Blkr.

Leuciscus daniconius $M C C l .91,66$, I40.

Leuciscus devario C. $V .91,66$.

Leuciscus dubius Blkr. 345, I9. cf. Leuciscus aethiops Basil.

Leuciscus dusonensis Blkr. 26, I4. 35, 262. 42, 60. 45, 196. 49, 420.

55,4 I 3 .

Leuciscus Dussumierii $C . V .91,138$.

Leuciscus Duvaucelii $C . V .91,66$.

Leuciscus Einthovenii Blkr. 49, 434. 55, 4I3. 185, 226.

Leuciscus elanga Blkr. 91, 66.

Leuciscus elingulatus $M C$ Cl. $91,68$.

Leuciscus filamentosus $C . V .91,68$. 
Leuciscus fintella $V a l .415$, то. 416, I 2 I. 425, I 44.

Leuciscus gatensis $C . V .91,68$.

Leuciscus gobioïdes $B l k r .91,68$.

Leuciscus goha $C . V .91,68$.

Leuciscus gora $C . V .91,68$.

Leuciscus guganio $C . V$. 91, 68 .

Leuciscus hakuensis Gill 497, 23.

Leuciscus Helfrichii Blkr. 162, I5.

Leuciscus hemistictus Rich. 415, I I. 416, I 2 I. 425, I 44.

Leuciscus hoalius $C . V .91,68$.

Leuciscus homospilotus Rich. cf. Xenocypris homospilotus Blkr.

Leuciscus hypophthalmichthys $G r$. cf. Hypophthalmichthys molitrix $B l k r$.

Leuciscus hypophthalmus $G r$. cf. Hypophthalmichthys molitrix Blkr.

Leuciscus idellus $V$ al. 415, 47. 425, I44. 496, 3 .

Leuciscus isurus $C . V .91,68$.

Leuciscus jaya $C . V .91,68$.

Leuciscus jesella $\mathrm{Val}$. cf. Xenocypris tapeinosoma Blkr.

Leuciscus jorah Blkr. 91, 68.

Leuciscus kalochroma Blkr. 35, 272. 42, 60. 45, I96. 49, 420. 55, 4 I 3 .

Leuciscus lateristriatus $(K . v . H$.$) Blkr. 106, 94. 170, 477. 200, 48$. cf. Leuciscus cyanotaenia Blkr.

Leuciscus laubuka $C . V .91$, I38.

Leuciscus leptosoma Blkr. 136, 269.

Leuciscus leucerus Blkr. $\boldsymbol{9 1 ,} 68$.

Letıciscus lineolatus Blyth cf. Danio lineolatus Blkr.

Leuciscus machaeroides Rich. cf. Hemiculter machaeroides Blkr.

Leuciscus macropus Schl. cf. Barilius macropus Blkr.

Leuciscus mahecola C. $V . \mathbf{9 1}, 68, \mathbf{1 4 0 .}$

Leuciscus melettina $C . V$. 91, 68, I4I.

Leuciscus Mertensii Guich. cf. Leuciscus idellus $V a l$.

Leuciscus microlepis Blkr. 91, I41.

Leuciscus minor Schl. cf. Barilius (Barilius) minor Blkr.

Leuciscus mola $M c C l .91$, I 40.

Leuciscus molitorella Val. 416, I20. cf. Gymnostomus molitorella Blkr.

Leuciscus molitrix $\mathrm{Val}$. cf. Hypophthalmichthys molitrix Blkr.

Leuciscus morar $M C C l .91,136$.

Leuciscus mosario $C . V .91,68$.

Leuciscus musiha $C . V .91,68$.

Leuciscus nesogallicus $C . V .459$, I02. 495, 2 I.

Leuciscus nobilis $G r$. cf. Hypophthalmichthys nobilis $B l k r$.

Leuciscus novacula $\mathrm{Val} . \mathbf{9 1}, 68$.

Leuciscus osteographus Blkr. 91, 68.

Leuciscus Owenii Blkr. 91, 68.

Leuciscus oxygaster C. $V .85,453.170,476.191,3$.

Leuciscus oxygastroïdes Blkr. 55, 431 . 
Leuciscus pangusia $C v .91,68$.

Leuciscus parvus Schl. cf. Pseudorasbora parva Blkr.

Leuciscus perseus Blkr. 91, 68.

Leuciscus petrophilus Blkr. 91, 68.

Leuciscus phula C. $V$. 91, 68.

Leuciscus piceus Rich. 415, II. 425, I 44.

Leuciscus piscatorius Blkr. 91, 68.

Leuciscus platypus Schl. cf. Barilius platypus Blkr.

Leuciscus plenus Brouss. cf. Xenocypris plena Blkr.

Leuciscus potail Blkr. 91, 68.

Leuciscus presbyter $C . V .91,68, \mathbf{1} 40$.

Leuciscus pusillus Schl. cf. Pseudorasbora parva Blkr.

Leuciscus pusillus $T$. Schl. cf. Pseudorasbora pusilla Blkr.

Leuciscus rasbora $\mathrm{MC} \mathrm{Cl} .91, \mathrm{I} 40$.

Leuciscus recurviceps Rich. 26, I4. cf. Culter recurviceps Blkr.

Leuciscus rhomboidalis Val. cf. Parabramis bramula Blkr.

Leuciscus rosetta $V a l .415$, Iо. 416, I2 I. cf. Hypophthalmichthys nobilis Blkr.

Leuciscus rupeculus Blkr. 91, 68.

Leuciscus sandkhol Sykes 91, 68.

Leuciscus scalpellus C. $V .91,68$. cf. Chela scalpella Blkr.

Leuciscus Schwenkii Blkr. 174, 47.

Leuciscus Sieboldi Schl. cf. Barilius Sieboldi Blkr.

Leuciscus solio $C . V . \mathbf{9 1}, 68$.

Leuciscus stigma $C . V .91,68$.

Leuciscus sucatio $C . V .91,68$.

Leuciscus sulphureus C. V. 91, 68.

Leuciscus sumatranus Blkr. 67, 60I.

Leuciscus teekaree Blkr. 91, 68.

Leuciscus Temmincki Si hl. cf. Barilius Temmincki Blkr.

Leuciscus teretiusculus Bas. cf. Rasbora teretiuscula Blkr., Squaliobarbus curriculus Gthr.

Leuciscus thermalis $C . V .91,68$. cf. Puntius (Puntius) thermalis Blkr.

Leuciscus thynnoïdes Blkr. 67, 599.

Leuciscus tila C. $V .91,68$.

Leuciscus tileo $C . V .91,68$.

Leuciscus trinema Blkr. 67, 600 .

Leuciscus tschiliensis Basil. cf. Leuciscus idellus $\mathrm{Val}$.

Leuciscus uncirostris Schl. cf. Opsariichthys uncirostris $B l k r$.

Leuciscus uranoscopus Blkr. 26, I4. 35, 262.42, 60.45, I96.49, 420. 55, 4I3.

Leuciscus vagra $C . V .81,68$.

Leuciscus vandella $\mathrm{Val} .415, \mathrm{I}$. $425, \mathrm{I} 44$.

Leuciscus variegatus Schl. cf. Sarcocheilichthys variegatus Blkr.

Leuciscus xanthurus Rich. 425, 145. cf. Pseudobrama Dumerili Blkr. 
Leuciscus zeylonicus Benn. 91, 68. 335, 3.

Leucos Heck. 261, 437. 314, 2 12. 329, 264.

Leucosoma chinensis Gr. cf. Salanx chinensis Gthr.

Leucosoma Reevesii Gr. of Salanx chinensis Gthr.

Leucosomus Heck. 261, 437. 314, 2 I I. 329, 264.

Liachirus nitidus Gthr. 430, 233.

Lichia 1, 553.

Lichia amia C. $V .266,53$.

Lichia glauca Gthr. cf. Glaucus Rondeletii Will.

Lichia glaucus C. V. 266, 53. cf. Glaucus Rondeletii Will.

Lichia glaycos Risso cf. Glaucus Rondeletii Will.

Lichia lysan Rüpp. cf. Chorinemus Commersonianus $C$. $V$.

Lichia moadetta Ehrenb. cf. Chorinemus Sancti Petri C. $V$.

Lichia tetracantha Bowd. cf. Glaucus Rondeletti Will.

Limamuraena $K p$. 351, I2 .

Limanda Gottsch. 294, 427.

Limanda vulgaris Gottsch. 294, 427.

Limia Poey 261, 440.

Linophora $K p .468,306$.

Linophora auriga $K p$. 468, 306. cf. Tetragonoptrus (Linophora) auriga Blkr.

Linophora ephippium $K p$. cf. Tetragonoptrus (Rabdophorus) ephippium Blkr.

Liocaesio Blkr. 468, 274 . 470, I 53.

Liocaesio cilindricus Blkr. 470, $\mathrm{r} 53$.

Liocassis crassilabris Gthr. 425, 125.

Liocassis longirostris Gïnth. cf. Leiocassis longirostris.

Liomonacanthus Blkr. 367, r3.

Liomonacanthus pardalis Blkr. 368, 27. 425, I22. 491, 53. 495, 20.

Lioperca Gill 468, 257.

Liopropoma Gill 468, 262.

Liopropoma aberrans Gill 468, 262.

Liostomus Gill 468, 325.

Lissorhynchus Blkr. 261, 422. 314, I91. 326, 242. 329, 262.

Lithodoras Blkr. 306, 85 .

Lithodoras lithogaster Blkr. 303, I 5. 306, 85 .

Lithognathus Szons. 468, 28r.

Lithognathus capensis Srons. 266, 52. 468, 28 I.

Liuranus semicinctus Gthr. cf. Leiuranus colubrinus Blkr.

Lobocheilos v. Hass. 261, 424. 314, I94.

Lobocheilos caudatus Blkr. 91, 66.

Lobocheilos cobitis Blkr. 86, 523. 136, 268.

Lobocheilos falcatus Blkr. 91, 66.

Lobocheilos (Lobocheilos) falcifer v. Hass., Blkr. 86, 522. 170, 476, $477.270,5 \mathrm{I}$. 
Lobocheilos gotyla Blkr. 91, 66.

Lobocheilos heterorhynchos Blkr. 86, 524 .

Lobocheilos lamta Blkr. 91, 66.

Lobocheilos lucas Blkr. 166, 362 .

Lobocheilos pleurotaenia Blkr. 136, 267.

Lobocheilos ricnorhynchus Blkr. 91, 66.

Lobocheilos rohitoïdes Blkr. 166, 363 .

Lobocheilos Schwanefeldii Blkr. 86, 523. 170, 476.

Lobocheilos (Lobocheilos) Schwanefeldi Blkr. 270, 5 I.

Lobotes Cuv. 1, 553. 468, 270.

Lobotes auctorum Gthr. 395, 29r. 396, 299.

Lobotes citrinus Rich. 425, I39.

Lobotes erate $C . V .2,522.16,4.18,26.25,8.50,47$ I. 51, 56. 91, 38. 178, 2. 267, I39. 270, I9. 272, II. 275, 25. 288, 71.

358, I86. 364, 280. cf. Lobotes auctorum Gthr.

Lobotes Farkharii $C . V$. 2, 523. cf. Lobotes erate $C . V$.

Lobotes hexazona Blkr. 26, 9. 35, 26I. 42, 59. 45, I95. 49, 4 I 8 . 55, 4II. cf. Datnioïdes polota Blkr.

Lobotes incurvus Rich. cf. Lobotes surinamensis Blkr.

Lobotes microprion Blkr. 44, I74. 438, 42. cf. Dentex griseus T. Schl., Gymnocranius lethrinoides Blkr.

Lobotes surinamensis Blkr., Cuv. 425, I39. 468, 270.

Lonchurus $B l .468,325$.

Lonchurus ancylodon $B l$. Sc/ $n$. cf. Ancylodon jaculidens $C$. $V$.

Lonchurus barbatus $B l .468,326$.

Lonchurus lanceolatus Gthr. 488, 326.

Lophiogobius Gthr. 453, 33I.

Lophiogobius ocellicauda Gthr. 453, 331.

Lophius chironectes Lac. cf. Antennarius chironectes Comm.

Lophius cynocephalus Smith 266, 55.

Lophius geographicus $Q . G$. cf. Antennarius raninus Cant.

Lophius gibbus Mitch. cf. Antennarius laevigatus Blkr.

Lophius hirsutus Lac. cf. Brachionichthys hirsutus Blkr.

Lophius hispidus $B l$. cf. Antennarius hispidus Cant.

Lophius histrio L. cf. Antennarius histrio Gthr.

Lophius histrio Lac. cf. Antennarius pinniceps Comm.

Lophius histrio var. marmoratus $B l$. Schn. cf. Antennarius raninus $C a n t$.

Lophius muricatus Shaw cf. Halieutaea stellata $C$. $V$.

Lophius pictus Shaw cf. Antennarius chironectes Comm.

Lophius piscatorius 90, 47 .

Lophius raninus Tiles. cf. Antennarius raninus Cant.

Lophius setigerus Wahl 90, 46. 425, 122. 497, 20.

Lophius stellatus $I V a h l, B l$. Schn. cf. Halieutrea stellata C. $V$.

Lophius striatus Shaw cf. Antennarius hispidus Cant.

Lophius viviparus $B l$. Schn. cf. Lophius setigerus $\| \mathrm{V} a h l$.

INDEX BLEEKER. 
Lophius vomerinus $C . V .90,47.266,55$.

Lophogobius Gill 453, 3 r4.

Lophogobius chrysosoma Blkr. 461, II4.

Lophotes Capellei Schl. 497, I8.

Loricaria L. 306, 80.

Loricaria amazonica Cast. cf. Loricariichthys maculatus Blkr.

Loricaria barbata Kner cf. Oxyloricaria barbata Blkr.

Loricaria carinata Cast. cf. Loricaria dura $L$.

Loricaria cataphracta Bl. Schn. cf. Ancistrus Temmincki Blkr.

Loricaria cataphracta $L$. cf. Loricaria dura $L$.

Loricaria cataphracta Bonn. cf. Loricariichthys maculatus Blkr.

Loricaria cirrhosa $B l$. Schn. cf. Loricaria dura $L$.

Loricaria cirrhosa, var. maculata $B l$. Schn. cf. Loricariichthys maculatus Blkr.

Loricaria dura L. 306, 80. 336, i 8.

Loricaria laeviuscula $V a l$. cf. Loricariichthys laeviusculus $B l k r$.

Loricaria lima Kner cf. Rineloricaria lima Blkr.

Loricaria maculata $B l$. cf. Loricariichthys maculatus $B l k \%$.

Loricaria plecostomus L. cf. Plecostomus brasiliensis Blkr.

Loricaria setifera $L a c$. cf. Loricaria dura $L$.

Loricaria setigera $\mathrm{Val}$. cf. Loricaria dura $L$.

Loricariichthys Blkr. 306, 8o.

Loricariichthys laeviusculus Blkr. 306, 80 .

Loricariichthys maculatus Blkr. 306, 8o. 336, I6.

Lota breviuscula Rich. 119, I4.

Lota phycis Schl. cf. Lotella phycis Blkr.

Lotella phycis Blkr. 497, 2 I.

Lotella Schlegelii $K p$. cf. Loteila phycis Blkr.

Loxodon macrorhinus $M . H .459,67$.

Luciobarbus Heck. 314, I98.

Luciobrama 411, 253.

Luciobrama macrocephalus Blkr. 421, 90. 425, I44.

Luciobrama typus Blkr. 411, 252. 415, 51. cf. Luciobrama macrocephalus Blkr.

Luciocephalus 259, 395 .

Luciocephalus pulcher Blkr. 35, 274. 42, 60. 45, I96. 49, 421. 52, 99. 55, 4I 4. 58, 24. 185, 226. 235, 37 I. 272, 10. 498, 29.

Luciogobïiformes 453, $33 \mathrm{I}$.

Luciogobius Gill 453, 33I.

Luciogobius guttatus Gill 453, 331. 497, 20.

Lucioperca Cü. 468, 265.

Lucioperca sandra Cuv. 468, 265.

Luciosoma Blkr. 261, 433. 314, 204.

Luciosoma (Luciosoma) setigerum Blkr. 136, 264. 170, 476. 270, 54.

Luciosoma (Luciosoma) spilopleura Blkr. 136, 265. 270, 54. 356, 76. 
Luciosoma (Trinematichthys) trinema Blkr. 270, 54. 272, 24.

Lutjanini 468, 275 .

Lutjanus $B l .468,276,294,336$.

Lutjanus agennes Blkr. 302, 49.

Lutjanus alboguttatus Blkr. 459, 90.

Lutjanus amboinensis Blkr. 315, 232. 316, 24r. 354, г 46. 358, I \&7. 364,28 I. 396, 299. $437,38.438,44.442,40.491,43$.

Lutjanus analis Blkr. 459, 90. 495, I 2.

Lutjanus annularis Blkr. 296, Iо6. 316, 24I. 346, 31. 364, 28 I. 395, 29r. cf. Lutjanus chirtah Blkr.

Lutjanus argentimaculatus Blkr. 438, 44. 442, 84. 459, 90. 491, 43.

Lutjanus aruanus $B l$. cf. Dascyllus aruanus $C$. $V$.

Lutjanus aruanus Lac. cf. Tetradrachmum arcuatum Cant.

Lutjanus aurantius Lac. cf. Plectorhynchus orientalis Szons.

Lutjanus aureovittatus Lac. cf. Scolopsides Vosmeri C. $V$., Scolopsis Vosmaeri $C . V$.

Lutjanus bengalensis Blkr. 296, I06. 309, I55. 315, 232. 318, 25 I. 320, 268. 354, I 46. 358, г87. 364, 28 I. 396, 296, 299. 403, 74. 425 , г38. $442,34.459 .35,90.491,43.495$, I $2.497,7$.

Lutjanus biguttatus Blkr. 438, 44. 442, 32. 491, 43.

Lutjanus Bleekeri Gthr. 308, I5I. 309, I55. 315, 233. 364, 28 I. cf. Lutjanus biguttatus Blkr.

Iutjanus Blochii Lac. cf. Lutjanus lutjanus $B l$.

Lutjanus bohar Blkr., Bl. Schn. 364, 281. 442, 57. 459, 90. 491, 43.

Lutjanus bottonensis Blkr. 296, I06. 309, I55. 315, 232. 316, 24 I. $320,268.354$, I 46.358, I $87.364,282.437,38$.

Lutjanus butonensis Blkr. 442, 46. 491, 43.

Lutjanus Calveti Blkr. 320, 268.

Lutjanus cayennensis Lac. cf. Otolithus cayennensis Blkr.

Lutjanus chaetodonoides Lac. cf. Plectorhynchus chaetodontoides Lac.

Lutjanus chirtah Blkr.425, I38. 442, 42. 491, 43. 496, 2.

Lutjanus christat Blkr. 497, 7 .

Lutjanus chrysotaenia Blkr. 296, I06. 315, 233. 364, 282. 395, 29 I. $442,22.496,2$.

Lutjanus civis Blkr. 459, 90 .

Lutjanus coeruleopunctatus Blkr. 296, го6. 320, 268. 354, г46. 364, 282. 396, 299. cf. Lutjanus rivulatus Blkr.

Lutjanus coeruleovittatus Blkr. 459, 90.495, I2.

Lutjanus decussatus Blkr. 315, 233. 318, 25I. 320, 268. 358, г 87. $364,282.437,38.442,79$.

Lutjanus dentex Blkr. 364, 282. cf. Aprion (Aprion) pristipoma Blkr.

Lutjanus diacanthus Lac. cf. Corvina catalea C. $V$., Johnius diacanthus Cant., Pseudosciaena diacanthus Blkr.

Lutjanus dodecacanthoides Blkr. 364, 282. 442, 48. 
Lutjanus dodecacanthus Blkr. 354, I46. 364, 282. 438, 44. cf. 'Lutjanus malabaricus Blkr.

Lutjanus dubius Blkr. 425, I38, I50.

Lutjanus duodecimlineatus $B l k r .459,90.495$, I 2.

Lutjanus ellipticus Lac. cf. Scolopsides bilineatus C. $V$., Scolopsis bilineatus $C . V$.

Lutjanus endecacanthus Blkr. 302, 48.

Lutjanus ephippium Bl. cf. Amphiprion ephippium Bl.Schl., Prochilus ephippium Blkr.

Lutjanus erythrinus Blkr. 459, 90.

Lutjanus erythrognathus Blkr. 459, 90.

Lutjanus erythropterus $B l .364,282.438,43.442,29$. cf. Mesoprion erythropterus $C$. $V$.

Lutjanus eutactus Blkr. 302, $5 \mathrm{r}$.

Lutjanus flavipes Blkr. 442, 68.

Lutjanus fulviflamma Blkr. 296, ro6. 309, 155. 315, 233. 320, 268. 354, г 46. 358, г87. 364, 282. 395, 29 г. 425, т $38.442,6$ r. 459, 90. $491,43.495,12$.

Lutjanus fuscescens Blkr. 296, го6. 358, г 87.425, I 38.442, I5.

Lutjanus gembra Blkr. 296, Iо6. 320, 268, 358, I87. 364, 282. 425, I38.

Lutjanus griseoides Guich. 459, 90.

Lutjanus griseus Blkr. 302, I3.

Lutjanus guinëensis Blkr. 302, 46.

Lutjanus gymnocephalus Lac. cf. Ambassis gymnocephalus Blkr., Ambassis nalua $C . V$.

Lutjanus hamrur Lac. cf. Priacanthus hamrur $C . V$.

Lutjanus hasta $B l$. cf. Pristipoma hasta $C$. $V$.

Lutjanus hexagonus $L a c$. cf. Myripristis hexagonus $C$. $V$.

Lutjanus Johnii Blkr., Lac. 364, 282. 395, 291. 396, 296. 425, I38. $442,20.459,90$.

Lutjanus jourdin Lac. cf. Amphiprion bifasciatus Bl. Schn., Prochilus bifasciatus $B l k r$.

Lutjanus lineatus $B l$., Blkr. 296, Iо6. 316, 241. 358, I87. 364, 282. 396, 296, 299. 442, 7०.

Lutjanus lioglossus Blkr. 438, 44. 442, 74. 491, 43.

Lutjanus lunulatus $B l$. Schn. 438, 44. 442, 64 .

Lutjanus lunulatus $L a c$. cf. Mesoprion lunulatus $C$. $V$.

Lutjanus lutjanus Bl., Blkr. 358, 187. 364, 282. 442, 27. 459, 9r. 468,277 .

Lutjanus macolor Blkr. 442, 86. 491, 43.

Lutjanus maculatus Lac. cf. Pristipoma caripa $C$. $V$., Pristipoma maculatum Gthr.

Lutjanus malabaricus Blkr. 438, 44. 442, 50. 497, 7.

Lutjanus marginatus Blkr. 296, I06. 309, I55. 315, 233. 316, 242. 
320, 268. 354, I 46.358, I $87.364,282.369,42.442,72.459,90$. $491,43.495$, I2.

Lutjanus melanotaenia Blkr. 316, 244. 438, 44. 442, 66.

Lutjanus microchir Blkr. 315, 233. 364, 282. cf. Aprion (Aprion) virescens $C . V$.

Lutjanus microstoma Lac. cf. Pristipoma hasta $C$. $V$.

Lutjanus modestus Blkr. 302, 5 o.

Lutjanus monostigma Blkr. 309, I55. 315, 233. 358, I87. 364, 282. 395, 29r. cf. Lutjanus lioglossus Blkr.

Lutjanus nematophorus Blkr. 442, I 2.

Lutjanus niger $B l$. Schn. cf. Lutjanus macolor $B l k r$.

Lutjanus notatus Blkr. 315, 233. cf. Lutjanus Russelli Blkr.

Lutjanus octovittatus Blkr. 403, 70. 459, 27, 91. 495, I2.

Lutjanus oligolepis Blkr. 438, 43. 442, I7.

Lutjanus olivaceus Blkr. 396, 296. cf. Lutjanus lutjanus Bl.

Lutjanus paraemulon $B l k r .425$, I38, I 50.

Lutjanus percula Lac. cf. Amphipion percula $C$. $V$., Prochilus percula Blkr.

Lutjanus pictus Lac. cf. Diagramma punctatum Ehr., Plectorhynchus pictus Blkr.

Lutjanus polymna var. Lac. cf. Prochilus percula Blkr.

Lutjanus polymnus Lac. cf. Amphiprion percula $C$. $V$., Prochilus polymnus Blkr.

Lutjanus pomacanthus Blkr. 364, 282.

Lutjanus quadriguttatus $B l k r .296$, I06. 320, 268.

Lutjanus quinquelineatus $B l k r .403,72.425$, I38.442, 37.459, 33, 9 I.

Lutjanus Quoyi Blkr. 396, 296.

Lutjanus rangus $C . V$., Blkr. 425, I38. 442, 59.

Lutjanus rivulatus Blkr. 425, r38.442, 8r, 459, 91.

Lutjanus Russelli Blkr. 358, I87. 364, 282. 442, 76. 497, 7.

Lutjanus sambra Blkr. cf. Lutjanus argentimaculatus Blkr.

Lutjanus scandens Lac. cf. Anabas scandens $C . V$., Anabas testudineus Cur.

Lutjanus Sebae Blkr. 296, го6. 356, г73. 364, 282. 396, 297.442, 53. 459, 9I. 491, 43 .

Lutjanus semicinctus Blkr. 296, ro6. 316, 24r. 491, 43.

Lutjanus semicinctus Q.G. 364, 282. 396, 297. 442, 55. cf. Mesoprion semicinctus $C$. $V$.

Lutjanus sparus Blkr. 425, I38. 497, 7 .

Lutjanus striatus Blkr. 354, I46.

Lutjanus testudo Lac. cf. Anabas scandens Cur., Anabas testudineus Cuv.

Lutjanus trifasciatus $B l$. Schn. cf. Premnas biaculeatus Blkr., Premnas trifasciatus $C . V$.

Lutjanus unimaculatus Blkr. 318, $25 \mathrm{I}$.

Lutjanus unimaculatus Q.G. cf. Lutjanus Johnii Blkr. 
Lutjanus vitta Blkr. 315, 233. 358, I87. 364, 282. 396, 297. 425, г $38.437,38.438,43.442,25.459,9$ I. $497,7$.

Lutjanus Vosmaeri Lac. cf. Scolopsides Vosmeri C. $V$., Scolopsis Vosmaeri $C$. $V$.

Lutjanus waigiensis Blkr. 396, 297.

Lutodeira 1, $55^{2}$.

Lutodeira chanos Rüpp. cf. Chanos salmoneus $C$. $V$.

Lutodeira indica Rüpp. 354, I50.

Lutodeira indica $v$. Hass. 2, 5 I0. cf. Chanos orientalis $C . V$.

Lutodeira lubina Blkr. 354, I 5 o.

Luxilus $R a f .261,436.314,2$ I . 329, 263.

Lycodalepis Blkr. 449, 369 .

Lycodeoidei. 449, 369 .

Lycodes mucosus Rich. 449, 369 .

Lycodes variegatus Gthr. 449, 369 .

Lycodontis $\mathrm{MCCl}$. 351, I 2 I.

Lycodontis literata $M C C l$. cf. Gymnothorax tile Blkr., Muraena tile Cant.

Lycodontis longicaudata Blkr. cf. Strophidon sathete Blkr.

Lycodontis punctata $M c C l$. cf. Gymnothorax tile Blkr. Muraena tile Cant. Lycogenis $K . v . H .468,336$.

Lycogenis argyrosoma $K . v . H$. cf. Scolopsides lycogenis $C$. $V$.

Machaerium nebulatum Blkr.51, 76. 59, 31. 287, 57.

Machaerium reticulatum Blkr. 70, 734. 158, 274. 185, 224. 199, 45. cf. Congrogadus reticulatus Gthr.

Machaerium reticulatum Rich. 287, 57 .

Machaerium subducens Rich. 119, I5.

Macolor Blkr. 468, 276.

Macolor typus Blkr. 275, 25. 315, 232. 354, 146. 364, 281. 369, 42. 396, 299. cf. Lutjanus macolor Blkr.

Macquaria $C . V .468,268$.

Macquaria australasica $C . V .119,9.468,268$.

Macrocephalus Browne 468, 336.

Macrochirichthys Blkr. 261, 439. 314, 2 I 5.

Macrochirichthys uranoscopus Blkr. 239, Iо2. 270, 54. 272, 25.356, I 76.

Macrochyrus Szons. 473, 296.

Macrochyrus miles Szons. cf. Pseudomonopterus (Pterois) volitans Blkr.

Macrognathus aculeatus Lac. cf. Rhynchobdella ocellata $C . V$.

Macrognathus argus Blkr. 356, I74.

Macrognathus armatus Lac. 356, I74. cf. Mastacembelus armatus $C$. $V$.

Macrognathus erythrotaenia Blkr. 356, I 74.

Macrognathus maculatus Blkr. 356, I74.

Macrognathus ocellatus Buch. cf. Rhynchobdella ocellata C. $V$. Macrognathus pancalus Buch. cf. Mastacembelus pancalus $C$. $V$. Macrognathus undulatus $M c C l$. cf. Mastacembelus armatus $C$. $V$. 
Macrones C. Dum. 306, 96.

Macrones Lamarrii C. Dum. 306, 96.

Macrones sinensis Blkr. 425, I25.

Macropharyngodon Blkr. 292, I00. 331, 445.

Macropharyngodon Geoffroyi 293, 289. 364, 277.

Macropodis pugnax Cant. cf. Betta picta Blkr.

Macropodus viridi-auratus Lac. 425, 136.

Macrops C. Dum.438, 45. 468, 276.

Macropteronotus Lac. 306, г2о.

Macropteronotus fuscus Lac. cf. Clarias fuscus Gthr.

Macropteronotus hexacicinnus Lac. cf. Clarias hexacicinnus Rich.

Macropteronotus magur Buch. cf. Clarias magur C. $V$.

Macrourus denticulatus Rich. 119, I5.

Macrourus japonicus Schl. 449, 370. cf. Oxymacrurus japonicus Blkr.

Macrurini. 449, 370.

Macruroidei 449,369 .

Macruroplus Blkr. 449, 369 .

Macruroplus leptolepis 497, 2 r.

Macrurus macrochir Gïnth. cf. Oxymacrurus macrochir.

Macrurus parallelus Gïnth. cf. Oxymacrurus parallelus.

Macrurus serratus Lowe 449, 369 .

Maena Cuv. 468, 273. 470, r51.

Maena vulgaris Cuv.468, 273.470, I5I.

Maenas Klein 468, 273.

Maenichthys $K p .468,291$.

Maenini 468, 273. 470, I5I.

Malacanthus brevirostris Guich. 459, 8o.

Malacanthus Hoedtii Blkr. 232, 18. 459, So. 491, 53. 495, I7.

Malacanthus latovittatus Q. G., Blkr., Val. 315, 236. 318, 252. 364, 292. $391,276.459$, 80. $491,53.495$, I 7 .

Malacanthus taeniatus $C . V .11,7.12,42.46,218.275,43$. cf. Malacanthus latovittatus Blkr., Q.G.

Malacobagrus Blkr. 306, Ioo.

Malacobagrus filamentosus Blkr. 306, Ioo.

Malapterurus Lac. 306, II 7 .

Malapterurus beninensis Murr. 302, I6.

Malapterurus coila Buch. cf. Ailia coila Blkr.

Malapterus (Ailia) bengalensis $G r$. cf. Ailia coila Blkr.

Mastacembelus anastomella Blkr. 379, 224. 425, I 49.

Mastacembelus annulatus Blkr. 379, 229. 394, 288. 395, 293. 396, 300. 425, I $48.437,39.459,100.497,24$.

Mastacembelus armatus $C . V .91,97$.

Mastacembelus brachyrhynchus Blkr. 379, 232.

Mastacembelus cancila Blkr. 356, I 76 .

Mastacembelus canciloides Blkr. 379, 223. 
Mastacembelus caudatus Blkr., $M c$ Cl. 91, 48, 98.

Mastacembelus caudimacula Blkr. 356, г 76. 364, 294.

Mastacembelus choram Blkr. 379, 227. 459, 100. 495, 21. cf. Mastacembelus annulatus $B l k r$.

Mastacembelus crocodilus Blkr. 379, 226.

Mastacembelus erythrotaenia Blkr. 24, 6. 26, то. 35, 26r. 42, 59. 45, I95. 49, 4I9. 55, 412. 91, 98. 239, 102. 270, 39. 272, I4.

Mastacembelus esocinus Blkr. 425, I49.

Mastacembelus fasciatus Blkr. 425, I49.

Mastacembelus giganteus Blkr. 315, 236. 364, 294. cf. Mastacembelus annulatus Blkr.

Mastacembelus gracilis Blkr. 379, 230.

Mastacembelus haleppensis $C . V .91,98$.

Mastacembelus leiuroides Blkr. 320, 272. 379, 233.

Mastacembelus leiurus $B l k \%$. 364, 294. 395, 294. 437, 39.

Mastacembelus macrolepis Blkr. 379, $22 \mathrm{r}$.

Mastacembelus maculatus C. $V .$, Rvolt. 52, 93. 91, 98. 170, 476, 477 . 185, 223. 239, 102. 270, 39. 271, I. 272, 14.

Mastacembelus marginatus Blkr. 2, 517. cf. Mastacembelus unicolor K. v. H.

Mastacembelus marmoratus $C$. $V$. cf. Mastacembelus armatus $C$. $V$.

Mastacembelus melanostigma Blkr. 459, roo.

Mastacembelus ornatus Blkr. 91, 48 .

Mastacembelus pancalus $C . V .91,98$.

Mastacembelus pictus Mus. L. B. cf. Mastacembelus erythrotaenia Blkr.

Mastacembelus platurus $B l k r .364,294.379,234.459$, го0. 495, 2 I.

Mastacembelus ponticerianus $C$. $V$. cf. Mastacembelus armatus $C$. $V$.

Mastacembelus punctatus $C$. $V$. cf. Mastacembelus pancalus $C$. $V$.

Mastacembelus schismatorhynchus Blkr. 315, 236. 497, 24.

Mastacembelus strongylurus Blkr. 379, 220. 395, 293.425, 149.491, 55 .

Mastacembelus unicolor $(K$. v. H.) C. $V .24,5.25,9.91,98.170$, $476,477.235,370.270,39.272,14$.

Meda Gir. 261, 432. 314, 207.

Megalaspis Blkr. 128, 417. 297, I36.

Megalaspis Rottleri Blkr. 46, 213. 51, 57. 56, 445. 57, 49. 91, 44 . 119, 10. $178,2.227,407.231$, I46. 237, 437. 270, 29. 272, I4. $275,39.288,75.296$, го9. 356, г74. 358, г91. 364, 289. 395, 293. 425, I3r.

Megalepis Bianconi 468, 333 .

Megalops atlanticus $\mathrm{Val}$. cf. Megalops giganteus Blkr.

Megalops curtifilis Rich. cf. Megalops indicus C. $V$., Megalops kundinga Blkr.

Megalops cyprinoides Blkr. 380, 290. 459, 102. 495, 22.

Megalops cyprinoides Cuv. cf. Megalops giganteus Blkr.

Megalops filamentosus L., Lac. 2, 510. 380, 286. 
Megalops giganteus Blkr. 380, 282 .

Megalops indicus $C . V .10,67.16,6.25$, II. 46, 214. 59, I5. 91, 72. 119, I4. cf. Megalops cyprinoides Blkr.

Megalops indicus Blkr. cf. Megalops filamentosus Lac.

Megalops indicus $V a l$., Blkr. cf. Megalops macropterus Blkr.

Megalops kundinga Blkr. 380, 288. 425, 147.

Megalops macropthalmus Blkr. 39, 421. 59, I5. cf. Megalops cyprinoides Blkr.

Megalops macropterus Blkr. 380, 284. 395, 294.

Megalops setipinnis Rich.270, 56. 275, 48. 284, 240. 287, 60. 288, 79. 320, 272. 354, 150. 358, r92. 364, 294. cf. Megalops indicus C. $V$, Megalops kundinga Blkr.

Megaprotodon Guich.468, 306.

Megaprotodon bifascialis Guich.4468, 307. cf. Megaprotodon strigangulus Blkr.

Megaprotodon strigangulus Blkr. 468, 307. 474, 320. 481, 109. 491, 45. 495, I 4 .

Melambaphes Gthr. 468, 295.

Melanichthys T. Schl. 269, 76. 468, 295.

Melanichthys crenidens Blkr. 269, 76. cf. Girella melanichthys Blkr.

Melanichthys tricuspidatus Blkr. 269, 78 .

Melanodactylus Blkr. 306, 95 .

Melanodactylus nigrodigitatus Blkr. 306, 95.

Melanogenes macrocephalus Blkr. 302, 36 .

Melanogenes microcephalus Blkr. 302, 37.

Melapterurus electricus Lac. 306, Ir7.

Melapterus $\mathrm{Val}$. 292, 108.

Meletta lile C. $V .91,74$.

Meletta Novae-Hollandiae $C \%, 119$, I5.

Meletta obtusirostris $C$. $V$. cf. Clupea (Harengula) obtusirostris Blkr.

Meletta Schrammii Blkr. 60, 30. 231, 150.

Meletta venenosa $C$. $V$. cf. Clupea (Harengula) venenosa Blkr.

Melichthys Stons. 367, Io.

Melichthys niger $B l k r$. cf. Melichthys ringens Blkr.

Melichthys ringens Sions., Blkr. 368, 2 I. 425, I22, 459, 7 I.

Melichthys ringens $K p$. cf. Balistes ringens $L$.

Melichthys vidua $K p$, Blkr. 364, 272. 368, 22. 388, 27 I. 425, I22. 491,52 .

Mendosoma Gay (Guich.) 468, 3 I6.

Mendosoma lineatum Gay 468, 3I6.

Mene Anna-carolina Lac. 497, i6.

Mene maculata C. V. 2, 518. 10, 67. 25, 9. 46, 213. 57, 86. 90, 4 . $91,46.227,408.270,3$ 1. 275, 40. 287, 54. 288, 75. 364, 290. 391, 276. 425, 133. cf. Mene Anna-carolina Lac.

Menephorus Gill 468, $25^{6}$. 
Menticirrhus Gill 468, 326.

Merlangus productus $A y r .425$, I 30 .

Merluccius vulgaris Cuv. 266, 54 .

Mesogobius Blkr. 453, 317 .

Mesogonistius Gill 468, 250.

Mesogonistius chaetodon Gill 468, 250.

Mesoprion C. V. 1, 553. 468, 276 .

Mesoprion alboguttatus Blkr. 91, 32 .

Mesoprion amboinensis Blkr. 54, 259. 231, I44. 275, 25. cf. Lutjanus amboinensis Blkr.

Mesoprion annularis C. V. 2, 526. 10, 66. 13, 47. 16, 4. 25, 7. 40, 478. 46, 211. 51, 55. 52, 89. 56, 444. 91, 32. 118, 63. 127, 345 . 156, 212. 158, 273. 227, 406. 231, I44. 270, 19. 275, 25. 185, 222. 288, 71. cf. Lutjanus annularis, chirtah, christat Blkr.

Mesoprion argentimaculatus Gthr. cf. Lutjanus argentimaculatus Blkr. Mesoprion aurolineatus C. $V .91,32$.

Mesoprion aurolineatus Day cf. Lutjanus fulviflamma Blkr.

Mesoprion bengalensis Kner cf. Lutjanus quinquelineatus Blkr.

Mesoprion bitaeniatus Blkr. 13, I1. 46, 2 II. 275, 25 .

Mesoprion bitaeniatus Gthr. cf. Lutjanus lunulatus Bl. Schn.

Mesoprion Bleekeri Gthr. 438, 43. cf. Lutjanus biguttatus Blkr.

Mesoprion bohar Gthr. cf. Lutjanus bohar Bl. Schn.

Mesoprion bottonensis $C$. $V$. cf. Lutjanus bottonensis Blkr.

Mesoprion bottonensis Blkr. 13, 12. 44, I70. 54, 237. 157, 232. 167, 370. 184, 198. 231, r44. 236, 330. 275, 26. cf. Lutjanus butonensis Blkr.

Mesoprion boutonensis Blkr. 270, 19. cf. Lutjanus butonensis Blkr.

Mesoprion Calveti Blkr. 13, I I.

Mesoprion caroui C. $V .91,32.288,7$ I. 438, 43. cf. Lutjanus erythropterus $B$.

Mesoprion caudalis $C$. $V$. cf. Lutjanus Johni Lac.

Mesoprion chirtah C. V. 91, 32. cf. Lutjanus chirtah Blkr.

Mesoprion chrysotaenia Blkr. 44, I 70. 50, 47 I. 51, 55. 54, 236. 143, 40. 156, 212. 158, 273. 185, 222. 275, 26. cf. Lutjanus chrysotaenia Blkr.

Mesoprion coeruleopunctatus Blkr. 44, I69. 91, 32, 127, 345. 171, 245. 231, I44. 234, 352. 270, x9. 275, 26. cf. Lutjanus rivulatus Blkr.

Mesoprion decussatus $(K . v$. H. $)$ C. $V .13,43.54,236.126,344$. 156, 212. 171, 245. 185, 222. 231, I44. 236, 330. 270, 19. 275, 26. cf. Lutjanus decussatus Blkr.

Mesoprion dentex Blkr. 270, I9. cf. Aprion (Aprion) pristipoma Blkr., Chaetopterus pristipoma $B l k r$.

Mesoprion dodecacanthoides Blkr. 103, 489. 237, 436. cf. Lutjanus dodecacanthoides Blkr.

Mesoprion dodecacanthus Blkr. 72, ro4. cf. Lutjanus malabaricus Blkr. 
Mesoprion dondiava Q. G. cf. Lutjanus fulviflamma Blkr.

Mesoprion dondiava $C$. $V$. cf. Mesoprion unimaculatus $Q . G$.

Mesoprion Ehrenbergii Pet. cf. Lutjanus oligolepis Blkr.

Mesoprion enneacanthus Blkr. 13, 40. cf. Lutjanus vitta Blkr.

Mesoprion erythrinus Gthr. cf. Lutjanus erythrinus Blkr.

Mesoprion erythrognathus $C$. $V$. cf. Lutjanus erythrognathus $B l k r$.

Mesoprion erythropterus C. $V$., Gthr. 9, 635. 13, 47. 156, 2 I 2. 235, 363. 236, 330. 270, 19. 275, 26. cf. Lutjanus erythropterus $B l$.

Mesoprion erythrozona 2, 526 .

Mesoprion etapee Less, cf. Lutjanus quinquelineatus Blkr.

Mesoprion flavipinnis $C . V .91,32$.

Mesoprion fulviflamma $Q . G .208,239.222,3$ I6.

Mesoprion fulviflamma Blkr. 66, 553. 71, 750. 127, 345. 156, 2 I2. 167, 370. 170, 479. 185, 222. 200, 48. 234, 352. 235, 363. 248, 202. 270, I9. 275, 26. 287, 46. cf. Lutjanus fulviflamma Blkr.

Mesoprion fuscescens $C . V$., Blkr. 10, 66, 68. 13, 4. 46, 2 II. 135, 197. 275, 26. cf. Lutjanus fuscescens Blkr.

Mesoprion Gaimardi Blkr. cf. Lutjanus marginatus Blkr.

Mesoprion gembra C. V. 76, 246. 91, 32. 156, 212. 231, I 44. 270, I9. 272, II. 275, 26. 287, 46. 288, 7 I. 438, 44. cf. Lutjantis argentimaculatus, gembra Blkr.

Mesoprion gorëensis C. $V$. cf. Lutjanus guinëensis Blkr.

Mesoprion griseoides Guich. cf. Lutjanus griseoides Guich.

Mesoprion gudgutia $C . V .91,32$.

Mesoprion hoteen Rich. cf. Lutjanus fuscescens Blkr.

Mesoprion immaculatus $C . V .13,4,45$. cf. Lutjanus argentimaculatus Blkr.

Mesoprion janthinuropterus Blkr. 71, 75I. 127, 345. cf. Lutjanus lineatus Blkr.

Mesoprion janthinurus Blkr. 94, 52. cf. Lutjanus butonensis Blkr., Mesoprion bottonensis Blkr.

Mesoprion Johnii C. $V$., Blkr. 91, 32. 126, 344. 156, 212. 227, 406. $235,3^{6} 3.270,20.272$, I I. 275, 26. 287, 46. 288, 7 I. cf. Lutjanus Johnii Lac., Blkr.

Mesoprion lineatus Gthr. cf. Lutjanus lineatus Blkr.

Mesoprion lineolatus Blkr., C. V. 13, 46. 156, 212. 231, I45. 237, 436. 270, 20. 275, 26. 438, 44. cf. Lutjanus biguttatus Blkr.

Mesoprion lineolatus Gthr. cf. Lutjanus erythropterus $B l$.

Mesoprion lunulatus $C . V .13,4.270,75 \cdot 271,2.276,65$. cf. Lutjanus lunulatus Bl. Schn.

Mesoprion lutjanus C. V. 13, 4. 227, 406. 231, 145. 234, 352. 235, 363. 236, 330. 270, 20. 275, 26. 287, 46. cf. Lutjanus lutjanus Bl. Mesoprion macolor Blkr. 13, I2. 71, 752. cf. Lutjanus macolor Blkr. Mesoprion madras C. $V .13,44.54,237.126,344$. cf. Lutjanus lutjanus $B l$. 
Mesoprion malabaricus $C . V .13,4.86,497.91,32.270,20.438,44$. cf. Lutjanus malabaricus Blkr.

Mesoprion marginatus Blkr., C. V. 66, 554. 91, 32. 167, 370. 188, 459. 231, I 45. 236, 330. 270, I9. 275, 26. cf. Lutjanus marginatus Blkr.

Mesoprion melanospilos Blkr. 71, 750. cf. Lutjanus amboinensis Blkr. Mesoprion microchir Blkr. 84, 332. cf. Aprion (Aprion) virescens C. $V$. Mesoprion Mitchelli Gthr. 470, I 52.

Mesoprion monostigma C. $V ., 13,42.50,47$ r. 156, 2r3. 167, 370. 184, 198. 185, 222. 188, 459. 235, 363. 236, 330, 333. 250, 206. 270, 20. 287, 46. 438, 44. cf. Lutjanus fulviflamma, lioglossus Blkr.

Mesoprion monostigma Blkr. (nec $C . V$.) cf. Lutjanus monostigma Blkr.

Mesopriou nematophorus Blkr. 275, 56. 287, 46. cf. Lutjanus nematophorus Blkr.

Mesoprion notatus Blkr. 46, 2 II.

Mesoprion octolineatus Blkr. 13, 40. 46, 2 I I. 54, 234. 91, 32. 119, 5 . 126, 344. 156, 213. 170, 478, 479. 200, 48. 227, 406. 231, 145. 234, 352. 270, 20. 275, 26. cf. Lutjants bengalensis, quinquelineatus Blkr.

Mesoprion olivaceus $C . V . \mathbf{1 3}, 4$. cf. Lutjanus lutjanus $B l$., Lutjanus olivaceus Blkr.

Mesoprion Ophuysenii Blkr. 270, 74. 438, 43. cf. Lutjanus vitta Blkr.

Mesoprion phaiotaenia Blkr. 56, 444 .

Mesoprion phaiotaeniatus Blkr. 13, 43. cf. Lutjanus vitta Blkr.

Mesoprion pomacanthus Blkr. 128, 407. cf. Lutjanus bengalensis Blkr., Lutjanus quinquelineatus Blkr.

Mesoprion quadriguttatus Blkr. 47, 233. 157, 232. 236, 330. 270, 20. 275, 26. cf. Lutjanus bohar Bl. Schn., Blkr.

Mesoprion quinquelineatus $C . V .13,4.91,32$.

Mesoprion rangus Day cf. Lutjanus argentimaculatus Blkr.

Mesoprion rangus $C . V .13,4.51,55.91,32.231$, I $54.237,436$. 288, 7 I. cf. Lutjanus rangus C. V., Blkr.

Mesoprion rivulata Blkr. 91, 32 .

Mesoprion rivulatus $B l k r .13,9$.

Mesoprion rufolineatus $B l k r .13$, I2. cf. Lutjanus amboinensis Blkr.

Mesoprion Russelli Blkr. 13, 41. 40, 478. 52, 89. 56, 444. 91, 32. 127, 345. 156, 2 І 3. 157, 232. 185, 222. 228, 424. 270, 20. 275, 26. 284, 239. 288, 7 I. cf. Lutjanus Russelli Blkr.

Mesoprion sanguineus Blkr. 13, 48. 16, 4. 25, 7. cf. Lutjanus chirtah Blkr., Mesoprion annularis C. $V$.

Mesoprion Sebae Blkr. 13, 45. 46, 2 I I. 91, 32. 185, 222. 250, 206. 270, 20. 275, 26. cf. Lutjanus Sebae Blkr.

Mesoprion semicinctus $C . V$. 13, 4. 84, 331. 184, I98. cf. Lutjanus semicinctus Q. G., Blkr.

Mesoprion sillao $C . V .91,32$. 
Mesoprion sparus Schl. cf. Lutjanus sparus Blkr.

Mesoprion spilurus Blkr. 91, 32.

Mesoprion striatus Blkr. 13, 44. 54, 237. 170, 478. 171, 245. 228, 424. 270, 20. 275, 26. 287, 46. cf. Lutjanus lineatus Blkr.

Mesoprion taeniops $C . V$. 10, 66, 68. 13, 4. 46, 2 Ir. 275, 26. 438, 44. cf. Lutjanus argentimaculatus Blkr.

Mesoprion timoriensis Gthr. cf. Lutjanus malabaricus Blkr.

Mesoprion unimaculatus $Q . G .10,66.13,42.16,4.25,7.46,2$ I I. 55, 4II. cf. Lutjanus Johni Lai.

Mesoprion unimaculatus $C . V$., Rich. 13, 42. 50, 47 I. cf. Lutjanus Johnii Blkr.

Mesoprion vitta Blkr., Gthr. 13, 44. 119, 6. 127, 345. 185, 222. 231, I45. 235, 363. 270, 20. 275, 26. 287, 46. cf. Lutjanus vitta Blkr.

Mesoprion xanthopterygius Blkr. 13, 46. 46, 2 ז I. cf. Lutjanus erythropterus $B l$.

Mesoprion xanthopus Blkr. 91, 32.

Mesoprion yapilli Rich. cf. Lutjanus argentimaculatus Blkr.

Mesoprion yapilli $C . V .91,32$. cf. Lutjanus Johni Lac.

Mesopristes Blkr. 468, 267 .

Mesopristes macracanthus Blkr. 2, 523. cf. Datnia argentea C. $V$, Therapon (Datnia) argenteus $B l k r$.

Metrogaster $A g .468,290$.

Microcanthini 468, 298.

Microcanthus Szens. 468, $298,336$.

Microcanthus strigatus Srens. 468, 298. 497, 8 .

Microdonophis $K p .351$, I 9 .

Microdonophis altipinnis Kp. cf. Muraenopsis altipinnis Blkr., Ophichthys altipinnis $B / \mathrm{kr}$.

Microichthys Rüpp. 468, 260.

Microichthys Coccoi Rüpp. 468, 260.

Microlepidotus Gill 468, 27 I.

Micrometrus Gill 468, 290.

Micronema Blkr. 299, 395. 306, I 6.

Micronema Bleekeri Boc. 347, 37. 356, I75.

Micronema hexapterus Blkr. 239, I02. 270, 48. 272, 20. 356, I75.

Micronema micruropterus 226, 386.

Micronema phalacronotus Blkr. 226, 386. 270, 48. 272, 20.

Micronema typus Blkr. 226, 386. 270, 48. 306, I I6. 356, I75.

Microperca Cast. 468, 260.

Microperca Yarrae Cast. 468, 260.

Microphis boaja Blkr. 270, 72. 272, 8. 356, I 72.

Microphis brachyurus Blkr. 270, 72. 275, I4. 354, I44.

Microphis cuncalus $K p .270,72$.

Microphis dactyliophorus Blkr. 270, 72. 364, 273.

Microphis heterosoma Blk\%.270, 72. 272, 8. 
Microphis polyacanthus Blkr. 270, 72. 275, I4.

Micropogon C. $V .468,326$.

Micropogon costatus De Kay 468, 326.

Micropogon lineatus $C . V .468,326$.

Micropterus Lac. 468, $26 \mathrm{I}$.

Micropteryx chrysurus Gthr. 302, 84. 425, I32.

Micropteryx cosmopolita $A g$. cf. Micropteryx chrysurus Gthr.

Micropus Gr. 297, I40.

Micropus apistus Blkr. 354, I48. 358, I 89. cf. Caracanthus apistus Blkr.

Micropus maculatus $G r$. cf. Caracanthus madagascariensis Blkr.

Micropus unipinna Gr. cf. Caracanthus unipinna Blkr.

Microsicydium Blkr. 453, 314.

Microsicydium gymnatichen Blkr. 472, 284 .

Microstomus Gottsch. 294, 428.

Minomus Gir. 261, 428. 314, I 89 .

Minous Cuv. 473, 298.

Minous Adamsii Rich. cf. Corythobatus monodactylus Blkr., Minous monodactylus $C . V$.

Minous monodactylus $C . V .14,9.25,7.56,444.227,407.270,25$. $275,32.287,49.459,87.473,298.475,63.495$, х 6.

Minous oxycephalus Blkr. 475, 65 .

Minous pusillus T. Schl. 497, I2. cf. Aploactis pusillus Blkr.

Minous trachycephalus Gthr. 475, 62 .

Minous woora C. V. 76, 251. 91, 36. 270, 25. 288, 72. cf. Corythobatus woora Cant., Minous monodactylus $C . V$.

Minous woora Blkr. cf. Minous oxycephalus Blkr.

Mionorus Krefft 468, 259.

Misgurnus Lac: 323, 39.

Misgurnus anguillicaudatus Blkr., Gthr. 307, I48. 323, 42. 425, 146. $496,3.497,22$.

Misgurnus barbatuloides Blkr. 323, 43 .

Misgurnus bifurcatus Blkr. 323, 42.

Misgurnus decemcirrosus Blkr. 323, 42.

Misgurnus dichachrous Blkr. 323, 43. 411, 253. 425, I46. 497, 22.

Misgurnus enalios Blkr. 323, 43. 408, 238. 497, 22.

Misgurnus fossilis Lac. 323, 42 .

Misgurnus maculatus Blkr. 323, 43.

Misgurnus micropus Blkr. 323, 42.

Misgurnus pectoralis Blkr. 323, 42 .

Misgurnus polynema Blkr., Gthr. 323, 43. 425, I46. 497, 22.

Misgurnus psammismus Blkr. 323, 42.

Misgurnus rubripinnis $B l k r .323,43$.

Mogurnda Gill 453, 303. 460, I03, I05.

Moharra Poey 468, 336.

Mola Heck. 261, 433. 314, 202. 
Molacanthus mola Blkr. 302, I2.

Molacanthus Pallasii Szons. cf. Molacanthus mola Blkr.

Mollienisia Les. 261, 440.

Monacanthus Cuv. 1, 552. 2, 506. 367, II.

Monacanthus aspersus Holl. 275, II.

Monacanthus brachyderma Blkr. 269, 70.

Monacanthus Broekii Blkr. 175, 35. cf. Paramonacanthus Brockii Blkr.

Monacanthus Cantori Blkr. 235, 374. 315, 230.

Monacanthus Cantoris Blkr. 51, 80. 63, i7.

Monacanthus chinensis Cuv. 364, 272. 368, 25. 395, 290. 425, I 22. $437,36$.

Monacanthus choirocephalus Blkr. 63, I9. 156, 218. 275, I1. 281, IoS. 287, 39. cf. Monacanthus komuki Blkr.

Monacanthus chrysospilos Blkr. 72, I26. 157, 236. 234, 357. 275, I I.

Monacanthus cirrhifer T. Schl. 175, 31. 497, I7. cf. Paramonacanthus setifer Blkr.

Monacanthus cryptodon Blkr. 128, $43 \mathrm{I}$.

Monacanthus curtorhynchos Blkr. 128, 430.

Monacanthus Dumerili Holl. 459, 7 I. 495, 20.

Monacanthus Freycineti Holl. 459, 7I. 495, 20.

Monacanthus geographicus Cuv. 50, 472. 51, 59. 63, I 7. 275, I I. 288, 68. cf. Monacanthus chinensis Cuv.

Monacanthus granulatus Rich. 119, I6.

Monacanthus hajam Blkr. 63, I8. 185, 226. 227, 408. 285, 243. 287, 39. 354, I 43. 364, 272. 368, 26. 437, 36. cf. Monaccnthus Helle ri Stcind.

Monacanthus Helleri Steind. 430, 233.

Monacinthus hippocrepis Holl.cf. Pseudomonacanthus hipjocrepis Blkr.

Monacanthus Houttuyni Blkr. 86, 533.

Monacanthus hystrix Cuv., Gr. 119, I6. 320, 265.

Monacanthus (Amanses) hystrix Burt. 84, $35 \mathrm{r}$.

Monacanthus isogramma Blkr. 166, 367. 235, 374. 368, 26.425, I22.

Monacanthus janthinosoma Blkr. 103, 504. 157, 236. 184, 204.

Monacanthus japonicus Rich., Cuv. 119, 16. 497, I7.

Monacanthus Komuki Blkr. 90, 55. 93, I3. 497, I7.

Monacanthus kryptodon Blkr. 364, 272.

Monacanthus kurtorhynchus Blkr. 364, 272.

Monacanthus lineolatus Rich. cf. Paramonacanthus lineolatus $B l k r$.

Monacanthus longirostris Cur. cf. Oxymonacanthus longirostris Blkr.

Monacanthus macrurus Blkr. 156, 226. 287, 39. 364, 272.

Monacanthus megalurus Rich. 425, I22.

Monacanthus melanocephalus Blkr. 80, 95. 157, 236. 296, 103. 315, 230. $320,265.364,272.368,26.394,285$.

Monacanthus melanuropterus Blkr. 71, 781. 275, Ir.

Monacanthus modestus Gïnth. cf. Paramonacanthus modestus Blkr. 
Monacanthus nematophorus Gthr. cf. Paramonacanthus nematophorus Blkr.

Monacanthus nemurus Blkr. 63, 20. 287, 39.

Monacanthus oblongus $T$. Schl. 175, 34. 253, 235. cf. Paramonacanthus oblongus Blkr.

Monacanthus pardalis Rüpp. 234, 357. 236, 332. 270, 64. 275, II. $315,230.318,249.320,265.354,143.358,183.364,272 . \mathrm{cf}$. Liomonacanthus pardalis Blkr.

Monacanthus penicilligerus Cuv. 51, 60. 231, I73. 288, 68.

Monacanthus rudis Rich. 119, I6.

Monacanthus scopas Cuv.. cf. Amanses scopas Blkr.

Monacanthus setifer Benn. 175, 32.

Monacanthus sinensis Cuv. 119. I6. 270, 65 .

Monacanthus sulcatus Holl. cf. Monacanthus isogramma Blkr.

Monacanthus tomentosus Cuv. 54, 235. 63, I9. 126, 344. 275, II. 287, 39. 288, 68. 309, I 54. 315, 230. 318, 249. 358, x83. 364, 272. 368, 26. 396, 298. 425, I 22. 437, 37 .

Monacanthus tomentosus Blkr. cf. Monacanthus hajam Blkr.

Monacanthus trachyderma Blkr. cf. Paramonacanthus trachyderma $B l k r$.

Monacanthus trichurus Blkr. 72, I25. 184, 204. 275, 12. 296, 103. 315, 230. 320, 265. 354, I43, 358, I83. cf. Monacanthus tomentosus Cuv.

Monacanthus variabilis Rich. cf. Aleuterius variabilis Rich.

Monacanthus vittatus Rich. 119, I6.

Moniana Gir. 314, 212. 329, 264.

Monocentris carinata Cuv. cf. Monocentris cataphracta Bl. Schn., Monocentris japonicus $B l$. Schn.

Monocentris cataphracta $B l$. Schn. 90, 26. 93, 5 .

Monocentris japonicus $B l$. Schn. 296, I05. 497, Iо.

Monocentris japonicus $C . V .425$, I36. cf. Monocentris cataphracta Bl. Schn.

Monoceros biaculeatus Bl. Schn. cf. Naseus fronticornis Comm.

Monoceros ecornis Ehr. cf. Naseus lituratus C. $V$.

Monoceros Raii Bl. Schn. cf. Naseus fronticornis Comm.

Monocirrhus Heck. 468, 319.

Monocirrhus polyacanthus Heck. 468, 3 I9.

Monodactylus argenteus Blkr. 316, 242. 354, I48. 358, I90. 364, 287. 395, 292. 425, I33. 459, 94. 495, г 8.

Monodactylus falciformis Lac. 425, т 33. 459, 94 .

Monodactylus orbicularis Blkr. 459, 94 .

Monodactylus rhombeus Cant. cf. Psettus rhombeus $C . V$.

Monoprion Poey 468, 259.

Monopterus cinereus Rich. cf. Monopterus javanensis Lac.

Monopterus helvolus Rich. cf. Monopterus javanensis Lac.

Monopterus javanensis Lac. 88, 59. 108, I64. 158, 274. 170, 476, 
$47 \% .191,2.200,48.219,302.225,358.238,88.255,24$ 1. 270, 61. $271,2.272,27.275,5$ 0. 279, гог. 284, 240. 287, 63. 356, I 76. 358 , I93. 395, 294. 425, I $24,496,4$.

Monopterus javanicus Cuv. 25, 22.

Monopterus laevis Ricl. cf. Monopterus javanensis Lac.

Monopterus marmoratus Rich. cf. Monopterus javanensis Lac.

Monopterus xanthognathus Rich. cf. Monopterus javanensis Lac.

Monotaxis Benn. 468, 28r.

Monotaxis grandoculis Blkr. 468, 28I. 491, 43.

Monotaxis indica Benn. 468, $28 \mathrm{I}$.

Morara Blkr. 261, 424. 314, I93.

Morara siamensis Blkr. 347, 37. 356, 175 .

Mordacia mordax Gray 119 , I9.

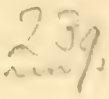

Moringua Gr. 351, I I 4.

Moringua bicolor $K p .320,272.352$, I 24.

Moringua linearis $G r$. cf. Moringua lumbricoidea Rich., Moringua raitaborua Cant.

Moringua lumbricoidea Rich. 425, I23.

Moringua macrochir Blkr. 131, 7 1. 157, 235. 352, I 24.

Moringua microchir Blkr. 72, I24. 88, 66. 91, I55. 157, 235. 182, I60. 236, 332. 270, 59. 275, 51. 315, 237. 352, I 24. 364, 295.

Moringua raitaborua Cant. 91, I54.

Mormyrini 449, 367 .

Mormyrodini 449,367 .

Mormyroidei 449,367 .

Mormyrus tamandua Gthr. 449, 367 .

Mormyrus zanclirostris Gthr. 449, $3^{67}$.

Morone Mitch. 468, 263.

Morone americana Gill 468, 263.

Morone rufa Mitch. 468, 263.

Moronopsis Gill 468, 268, 336 .

Moronopsis caudavittatus Blkr. 459, 90. 495, I I.

Moronopsis ciliatus Blkr. 417, 376. 459, 90. 491, 42.

Moronopsis fuscus Blkr. 459, 90. 495, I I.

Moronopsis rupestris Gill, Blkr. 417, 379. 459, 90. 495, I I.

Moronopsis taeniurus Gill 417, 374. 459, 90. 468, 268.

Morulius Buch. 261, 424. 314, I95.

Morulius chrysophekadion Blkr. 356, I75.

Morulius dinema 347,37 .

Motella pacifica Schl. 425, I30. 497, 2 I.

Moxostoma Raf. 261, 429. 314, I9o.

Mrigala Blkr. 261, 427. 314, 197. 329, 262.

Mrigala sinensis Blkr. 415, 7. 416, I20. 425, I 43 .

Mugil 1, 553 .

Mugil acutus $C$. 119, I I. 
Mugil adustus Blkr. 86, 503. cf. Mugil borneënsis Blkr.

Mugil affinis Gthr. 425, r 43 .

Mugil albula Forst. cf. Agonostoma Forsteri Gthr.

Mugil albula Buch. cf. Mugil Buchanani Blkr.

Mugil amarulus C. V. 91, 48. 138, 400. 270, So. 272, 47.

Mugil ashanteënsis Blkr. 302, 91.

Mugil axillaris C. V. 218, 28o. 231, 147. 235, 367. 236, 333. 270, 32. $271,3.275,4$ I. 287, 54. 296, І го. 315, 235. 320, 27 1. 354, I 49. 364, 29I. 425, I 43. 459, 79. 491, 53. 495, I 8 .

Mugil belanak Blkr. 166, 337. cf. Mugil bontah Blkr.

Mugil bontah Blkr., Russ. 91, 48. 166, 336. 218, $278.227,407$. 231, I 47. $235,367.270,33.272,49.287$, 54. 356, I 74. 425, I 43 .

Mugil borbonicus Cant., C. $V .218,279.235,375.270,84.288,75$. $459,79$.

Mugil bornëensis Blkr. 45, 201. 49, 419. 50, 472. 55, 412. 218, 278. 270, 33. 272, 55. 275, 4I. 320, 27I. 364, 29I. 395, 293.

Mugil brachysoma Blkr. 138, 399. cf. Mugil sundanensis Blkr.

Mugil Broussonetii C. $V .270,80$.

Mugil Buchanani Blkr. 91, 99. 270, 8o.

Mugil Cantoris Blkr. 91, 100. 270, 80.

Mugil capensis C. $V . \mathbf{2 6 6}, 69$.

Mugil carinatus Ehr. 91, 48. 459, 79.

Mugil cascasia Buch. 91, 48, 100. 270, 82 .

Mugil cephalotus $C$. $V$., Cant. 2, 514. 16, 5. 25, 9. 51, 58. 218, 277 . 272,5 г. 288, 76. 422, го०. 425, г 43. 459, 45, 79. 496, 2. cf. Mugil bontah Russ.

Mugil cephalus Buch.272, 52 .

Mugil ceramensis Blkr. 69, 699. 158, 274. 167, 372. 218, 277. 270, 33. 272, 48. 275, 41. 358, 191. 364, 291. 496, 2.

Mugil cirrhostomus Forst. 272, 47.

Mugil coeruleomaculatus Lac. 50, 484. 156, 2 I 5. 157, 233. 170, 479. 188, 460. 218, 279. 270, 33, 84. 271, 5. 275, 4I. 276, 65. 315, 235. 316, 242. 320, 27 I. 354, I49. 358, г9І. 364, 29 I. 395, 293. $396,300.459,79.491,53.495$, I 8.

Mugil Constanciae C. $V .266,54$.

Mugil corsula Buch. 91, гог. 270, 82.

Mugil cunnesius $C . V$., Blkr. 2, 514. 16, 5. 25, 9. 32, I60. 51, $5^{8}$. $56,454.69,701.91,48,99.218,278.270,33.271,8.275,41$. 288, 75. 354, I 49. 364, 29 г.

Mugil cunnesius Cant. cf. Mugil axillaris $C . V$.

Mugil curema C. $V .270,80.272,55$.

Mugil cylindricus $C . V$. 25, 9. 76, 266. 156, 2x5. 270, 80. 272, 47. 309, I 56. cf. Mugil axillaris C. $V$.

Mugil dobula Gthr. 425, I43. 
Mugil Dussumierii C. V. 9, 637. 16, 5. 25, 9. 91, 48. 166, 339. 270, 8o. cf. Mugil bontah Blkr.

Mugil Dussumieri Blkr. cf. Mugil oligolepis Blkr.

Mugil Engeli Blkr. 218, 277. 231, 147. 270, 78.

Mugil euronotus Smith. 266, 54.

Mugil Ferrandi $Q . G$. 119, I1.

Mugil Forsteri Bl. Schn. cf. Agonostoma Forsteri Gthr.

Mugil grandisquamis $C . V .272,42$.

Mugil haematocheilus $T$. Schl. 118, 107. 272, 59. 497, I7.

Mugil haematochilus Schl. 425, I43. 496, 2.

Mugil haematochilus Rich. cf. Mugil sinensis Blkr.

Mugil heterocheilos Blkr. 135, I 98. 170, 478. 218, 280. 275, 57. 296, 1 I 0.

Mugil heterochilus Blkr. 358, I91. 364, 29 I.

Mugil japonicus T. Schl. 90, 4I. 272, 59. 425, I43. 497, I7.

Mugil javanicus Blkr. 69, 701. cf. Mugil sundanensis Blkr.

Mugil labiosus C. V.98, 2 I3. 218, $278.270,33.271,6.272,55.320,27$ I.

Mugil Lauvergnii Eyd. Soul. 271, 4. 425, 143.

Mugil liza $C . V .270,80.272,55$.

Mugil macrocheilos Blkr. 105, 43. 218, 280.

Mugil macrolepidotus $C . V$. 2, $5 \times 4$.

Mugil macrolepidotus Rich. cf. Mugil cephalotus $C$. $V$.

Mugil macrolepidotus Rüpp. cf. Mugil melanochir K.v. H., Mugil vaigiensis $Q . G$.

Mugil macrolepis Smith 266, 54. 270, 8o.

Mugil macrolepis Blkr. 55, 422. 69, 701. cf. Mugil oligolepis Blkr.

Mugil melancranus Rich. 425, I 43 .

Mugil melanochir K.v.H. 16, 5. 25, 9. 55, 423. 56, 445. 91, 48. 170, 479. 184, 201. cf. Mugil vaigiensis $Q . G$.

Mugil melanopterus Ehr. cf. Mugil melanochir K.v. H., Mugil vaigiensis $Q$. $G$.

Mugil melinopterus $C . V$. 270, So.

Mugil multilineatus Smith 266, 54.

Mugil oligolepis Blkr. 218, 275. 237, 437. 272, 40.

Mugil Ophuysenii Blkr. 218, 279. 270, 82 .

Mugil öur Forsk. 272, 52. cf. Mugil cephalotus $C . V$.

Mugil parmatus Cant. 138, 400. 218, 276. 272, 42. 288, 76.

Mugil parsia Buch. 53, r66. 56, 445. 69, 701. 91, 48, 99. 270, 82. cf. Mugil axillaris $C . V$.

Mugil pederaki C. $V .91,48$.

Mugil Peronii $C . V$. 119, II. 270, 80.

Mugil Perusii C. V. 270, so.

Mugil planiceps C. V. 91, Ior. 270, So. 425, I 43 .

Mugil Richardsonii Smith 266, 54 .

Mugil robustus Gt/ur. 459, 79 .

Mugil Rossii Blikr. 105, 45. 218, 276. 272, 42. 
Mugil Schlegeli Blkr. 302, 92.

Mugil sinensis Blkr. 425, I 43 .

Mugil Smithii Gthr. 459, I04.

Mugil Speigleri Blkr. 191, 2. 218, 279. 272, $5^{8 .}$

Mugil squamopennis Srens. cf. Mugil corsula Buch.

Mugil strongylocephalus Rich. 270, 81. 425, I 43.

Mugil subviridis $C . V .91,48.270,80$.

Mugil sundanensis $B l k r .76,265.178,2.218,276.231,147.235$, 368. 270, 33. 272, 45. 287, 55. 320, 27 I. 395, 293.

Mugil Troscheli Blkr. 218, 277. 270, 80. 272, 15. 275, 41. 459, ro4. Mugil vaigiensis $Q . G$. 218, 276. 248, 203. 270, 33. 272, 42, 43 . 275, 4I. 296, I Iо. 315, 236. 320, 27 . Mugil waigiensis $Q . G$.

Mugil Valenciennesii Blkr. 218, 277. 270, 8r.

Mugil ventricosus Rich. 425, I43.

Mugil waigiensis Q.G. 235, 368. 236, 33r. 287, 55. 288, 76. 364, 291. 395, 293. 396, 297, 300. 425, 143.

Mugil xanthurus Rich. cf. Mugil haematochilus Schl.

Mullhypeneus Poey 468, 333.

Mulloidei 468, 332.

Mulloides Blkr. 468, 333.

Mulloides auriflamma Klunz, cf. Mulloides flavolineatus Blkr.

Mulloides flavolineatus Blkr. 13, I2. 69, 697. 188, 459. 236, 330, 333. $275,33.296$, то8. $315,234.320,269$. 354, I 47. 358, I 89. $364,285.425$, I $42.459,8$ o. 464 , I 5. 468, 333. 495, I 4.

Mulloides japonicus Blkr. 497, ıо.

Mulloides Lathamii Blkr. 119, 7.

Mulloides mauritianus Blkr. 459, 8o. 495, I4.

Mulloides pinnivittatus Steind. 497, Iо.

Mulloides ruber Klunz. cf. Mulloides flavolineatus Blkr.

Mulloides vanicolensis Blkr. 79, 6ог. 167, 37 I. 315, 234. 464, I4. Mulloides zeylonicus Blkr. 13, I2. 91, 34. 464, I6. 491, 46.

Mullus Kl. 468, 334 .

Mullus aureo-vittatus Shaw cf. Mulloides flavolineatus Blkr.

Mullus auriflamma Forsk. cf. Mulloides flavolineatus Blkr.

Mullus auriflamma Lac. cf. Parupeneus macronema Blkr., Upeneus lateristriga $C . V$.

Mullus bandi Shaw cf. Upeneoïdes vittatus Blkr., Upeneus vittatus C. $V$.

Mullus barbatus $L$. 468, 334 .

Mullus barberinus Lac. cf. Parupeneus barberinus Blkr., Upeneus barberinus $C . V$.

Mullus bensasi $T$. Schl. cf. Upeneus bensasi Rich., Upeneoïdes bensasi Blkr.

Mullus bifasciatus Lac. 402, 342. cf. Parupeneus bifasciatus Blkr.

Mullus cherserydros Lac. cf. Parupeneus cherserydros Blkr., Upeneichthys chrysopleuron Blkr. 
Mullus chrysopleuron $T$. Schl. cf. Upeneus (Upeneichthys) chrysopleuron Blkr.

Mullus dispilurus Playf. cf. Parupeneus dispilurus Blkr.

Mullus dubius Schl. cf. Upeneus dubius Gthr., Rich.

Mulius erythrinus $v$. Hass. cf. Upeneus sulphureus $C$. $V$.

Mullus fasciatus White of. Amia fasciata Gill., Blkr., Apogon quadrifasciatus $C . V$.

Mullus fasciatus Thunb. cf. 'Tautoga fasciata $C$. $V$.

Mullus flavolineatus Lac. cf. Mulloïdes flavolineatus Blkr.

Mullus indicus Shaze cf. Parupeneus indicus Blkr., Upeneus Russellii Blkr.

Mullus lateristriga $C$. $V$. cf. Upeneus lateristriga $C . V$.

Mullus luteus Playf. cf. Parupeneus luteus Blkr.

Mullus macronema Lac. cf. Upeneus lateristriga $C$. $V$., Upeneus barberinus $C . V$., Parupeneus macronema Blkr.

Mullus malabaricus Playf. cf. Parupeneus indicus Blkr.

Mullus micronemus Playf. cf. Parupeneus macronema Blkr.

Mullus multifasciatus $Q$. G. cf. Parupeneus multifasciatus Blkr.

Mullus oxycephalus Playf. cf. Parupeneus cherserydros Blkr.

Mullus pleurostigma Playf. cf. Parupeneus pleurostigma Blkr.

Mullus radiatus Shaw. cf. Parupeneus cherserydros Blkr.

Mullus subvittatus Schl. cf. Upeneus vittatus $C$. $V$., Upeneus subvittatus Blkr.

Mullus tragula Playf. cf. Upeneus tragula Rich.

Mullus trifasciatus Lac. 402, 342. cf. Upeneus trifasciatus $C . V$.

Mullus vittatus Forsk. cf. Upeneoides vittatus Blkr., Upeneus vittatus C. $V$.

Muraena Art., Cuv. 1, 552. 351, I I3, I 2 I.

Muraena Agassizi Blkr. 130, 458. 236, 332. cf. Gymnothorax Agassizi Blkr.

Muraena albimarginata $T$. Schl. 164, 77. cf. Gymnothorax albimarginatus $B l k r$.

Muraena alusis Blkr. cf. Echidna catenata Blkr.

Muraena amblodon Blkr. 459, 72 .

Muraena amblyodon Blkr. 143, 72. 234, 356. 275, 51. cf. Echidua amblyodon Blkr.

Muraena annulata $A h l$ cf. Leiuranus colubrinus $K p$.

Muraena annulata Thunb., Schl. cf. Ophisurus colubrinus Rich.

Muraena arenata Cuv. cf. Gymnothorax thyrsoideus Blkr.

Muraena atomaria Sol. cf. Gymnothorax pictus Blkr.

Muraena Augusti Gtlır. cf. Gymnothorax Augusti Blkr.

Muraena auloptera De Fil. cf. Gymnothorax aulopterus Blkr.

Muraena australis Blkr. 320, 272. 352, I23.

Muraena avisotis Blkr. 425, I23.

Muraena bagio Buch. cf. Conger bagio Cant., Muraenesox bagio Peters.

Muraena balearica De la Roche 90, 53. cf. Conger balearicus Cuv. 
Muraena batuensis Blkr. 157, 24I. 171, 245. cf. Gymnothorax flavimarginatus Blkr.

Muraena bengalensis $G r$. cf. Anguilla Elphinstonei Sykes, Muraena maculata Buch.

Muraena bicolor Rndt. cf. Gymnothorax reticularis $B l$.

Muraena bilineata Rüpp. cf. Gymnothorax flavimarginatus Blkr.

Muraena Blochii Blkr. 88, 49. 106, 102. 130, 460. 170, 479. 188, 46r. 270, 60. 272, 28. cf. Gymnothorax Blochi Blkr.

Muraena Boschii Blkr. 88, 52. 106, 103. 270, 60. cf. Gymnothorax Boschi Blkr.

Muraena bostoniensis Les., Blkr. 425, r23. 497, 25.

Muraena Brummeri Blkr. 230, I37. cf. Strophidon Brummeri Blkr.

Muraena bullata Rich. 33, I62. 35, 262. 42, 6о. 45, I97. 49, 42 I. 55, 4I 4. 88, 5. 136, $276.171,245.236,332,334.255,241.270$, 6o. 272, 28. cf. Gymnothorax bullatus Blkr.

Muraena buroënsis Blkr. 164, 79. 182, I60. 188, 461. 236, 332. cf. Gymnothorax buroensis Blkr.

Muraena cancellata Rich. 86, 53x. 88, 5, 74. 119, I6. 125, 326. 157, 235. 188, 46I. 208, 239. 236, 332. 270, 60. 287, 63. cf. Gymnothorax cancellatus Blkr.

Muraena canina $Q . G .88,5$. cf. Gymnothorax caninus Blkr.

Muraena catenata Rich. 88, 42. cf. Echidna catenata Blkr.

Muraena catenata Blkr. (nec Rich.) 142, 66. cf. Gymnothorax punctofasciatus Blkr.

Muraena ceramensis Blkr. 54, 297. 88, 51. 275, 51.cf. Gymnothorax ceramensis Blkr.

Muraena cerino-nigra Rich. cf. Gymnothorax cerino-niger Blkr.

Muraena chlorostigma Blkr. 182, I60. cf. Gymnothorax chlorostigma Blkr.

Muraena cinerascens Rüpp. cf. Gymnothorax cinerascens Blkr.

Muraena clathrata $B / k r .425$, I 23.

Muraena colubrina Rich. 88, 5. 99, 335. 157, 235. 171, 245. 188, 46r. 236, 332. 248, 203. 270, 60. 275, 51. cf. Gymnothorax reticularis $B l$.

Muraena colubrina Bodd. cf. Leiuranus colubrinus $K p$., Ophisurus colubrinus Rich.

Muraena conger $L$. cf. Conger vulgaris $C u$.

Muraena congeroides Blkr. 270, 87. cf. Gymnothorax crudelis Blkr., Gymnothorax schismatorhynchus Blkr.

Muraena crudelis Blkr. cf. Gymnothorax schismatorhynchus Blkr.

Muraena dizona Blkr. 286, 260. cf. Echidna polyzona Blkr.

Muraena Duivenbodei Blkr. 145, 385. cf. Gymnothorax Duivenbodei Blkr.

Muraena Dussumieri Blkr. 459, 72.

Muraena fasciata Sol. cf. Gymnothorax reticularis $B l$. 
Muraena fasciata Banks, Sol. cf. Muraena colubrina Rich.

Muraena fasciata Thunb. 88, 36. cf. Ophisurns colubrinus Rich.

Muraena fasciata Ahl cf. Ophisurus fasciatus Rich.

Muraena favaginea 91, $\mathbf{r} 66$.

Muraena fimbriata Benn. cf. Gymnothorax fimbriatus Blkr.

Muraena flavimarginata Rüpp. (nec $K p$.) 88, 41. cf. Gymnothorax flavimarginatus Blkr.

Muraena flavimarginata $K p$. cf. Gymnothorax mauritianus $B l k r$.

Muraena floresiana Blkr. 99, 334. cf. Gymnothorax ceramensis Blkr.

Muraena fusca Zuiezo. cf. Sphagebranchus fuscus Blkr.

Muraena geographica Banks, Sol. cf. Echidna variegata $J_{0}$ R. Forst.

Muraena geometrica Rüpp. 88, 37 .

Muraena gracilis Rich. 88, 42.91, 78. cf. Gymnothorax tile Blkr.

Muraena grisea Cuv. 17, II. 270, 60. cf. Gymnothorax Richardsoni Blkr., Muraena Troschelii Blkr.

Muraena griseobadia Rich. 88, 42. 125, 325. 130, 460. 156, 2 I8. 157, 235. 167, 374. 228, 424. 231, x 50. 270, 60. cf. Gymnothorax griseobadius Blkr., Gymnothorax thyrsoideus Blkr.

Muraena guttata Soland. 88, 42 .

Muraena guttata $K p$. cf. Gymnothorax Augusti Blkr.

Muraena gymnopterus Blkr. 88, 52. cf. Muraenichthys gymnopterus Blkr.

Muraena halmaherensis Blkr. 309, I58. 352, I23.

Muraena helena L. 88, 4r. 119, I6. cf. Gymnothorax helena Blkr.

Muraena hepatica Rüpp. cf. Gymnothorax albimarginatus Blkr.

Muraena isingleena Rich. 88, 4I. cf. Gymnothorax isingleena, isingleenoides, isingteena $B l k r$.

Muraena isingleenoides Blkr. 88, 48. cf. Gymnothorax isingleenoides Blkr., Muraena isingteena Rich.

Muraena isingteena Rich. 136, 277. 156, 218. 157, 235. 171, 245. 236, 332. 248, 203. 270, 60. cf. Gymnothorax isingteena, tessellatus Blkr.

Muraena isingteena Blkr. cf. Gymnothorax isingleenoides Blkr.

Muraena japonica Blkr. 408, 238. 496, 4 .

Muraena javanica Blkr. 236, 347. cf. Gymnothorax javanicus Blkr.

Muraena Johannae Blkr. 459, I03.

Muraena Kidako Schl. cf. Gymnothorax Kidako Blkr.

Muraena labiata Blkr. 495, 22.

Muraena latirostris Blkr. 425, I23.

Muraena leopardina (Mus. L. B.) cf. Gymnothorax bullatus Blkr.

Muraena lita Rich. 54, 294. 88, 47. 145, 383. cf. Gymnothorax pictus Blkr.

Muraena literata Blkr. 91, 78. cf. Gymnothorax tile Blkr., Muraena tile Cant.

Muraena (Strophidon) literata $M C$ Cl. 88, 42. 
Muraena Mac Clellandi Blkr. 88, 4I.

Muraena macrurus Blkr. 110, 324. cf. Thyrsoidea macrurus Blkr.

Muraena maculata Buch. 88, I5, 66. 315, 237. 346, 31. 352, I23. 354, I50. 358, I93. 364, 295. 396, 298. cf. Anguilla Elphinstonei Sykes.

Muraena maculosa Cuv. cf. Ophisurus Ophis Lac.

Muraena malgumora Blkr. 352, I23.

Muraena manillensis Blkr. 346, 31. 352, I 23.

Muraena marmorata $Q . G .88,5$. cf. Gymnothorax marmoratus Blkr.

Muraena marmorata Kner cf. Muraena mauritiana Blkr.

Muraena mauritiana Blkr. 425, I23. 459, 72. 491, 56. 495, 22.

Muraena mauritiana $K p$. cf. Gymnothorax mauritianus Blkr.

Muraena melanospilos Blkr. 136, 279. 270, 61. cf. Gymnothorax melanospilus Blkr.

Muraena melanostigma (Mus. L. B.) cf. Gymnotherax bullatus Blkr.

Muraena meleagris Shaw 88, 42. cf. Gymnothorax meleagris Blkr.

Muraena micropoecilus Blkr., Rich. 130, 459. 236, 332. 287, 63. cf. Gymnothorax micropoecilus Blkr.

Muraena micropterus Blkr. 54, 298. 88, 50. 130, 460. Gymnomuraena micropterus Blkr.

Muraena minor T. Schl. 54, 296. 88, 4I. 118, 123. 348, 49. cf. Priodonophis reticularis $B l k r$.

Muraena moa Blkr. 352, I24.

Muraena molendinaris Benn. cf. Echidna zebra Blkr.

Muraena monaca Cocco cf. Gymnothorax unicolor Blkr.

Muraena monochrous Blkr. 145, 384. 287, 63. cf. Gymnothorax monochrous Blkr.

Muraena moringua Cuv. 88, 41. cf. Gymnothorax moringua Blkr.

Muraena nebulosa $A h l$ cf. Echidna variegata Forst.

Muraena nebulosa Thunb. cf. Muraena variegata Forst.

Muraena nubila Gthr. cf. Gymnothorax nubilus Blkr.

Muraena nubila Rich. 88, 4I. 119, I6. cf. Gymnothorax Petelli Blkr.

Muraena ocellata Agass. 88, 4I.

Muraena ophis Rüpp. 54, 296. 88, 41. cf. Echidna variegata J. R. Forst.

Muraena pantherina. $M_{C}$ Cl., Cuv. 88, 5. 171, 245. 236, 332. 270, 6r. 275, 5 I.

Muraena pantherina Blkr. cf. Gymnothorax pictus Blkr.

Muraena pardalis T. Schl. 175, 30. 188, 461. cf. Gymnothorax pardalis Blkr.

Muraena pavonina Rich. 88, 42.

Muraena pekinensis Basil. cf. Muraena latirostris Blkr.

Muraena Petelli Blkr. 148, 84. 163, 92. 171, 245. cf. Gymnothorax Petelli Blkr.

Muraena Pfeifferi Blkr. 81, I73. 88, 72. 275, 52. cf. Gymnothorax pictus Blkr. 
Muraena picta $A h l$. cf. Gymnothorax pictus Blkr.

Muraena polyopthalmus Blkr. 176, I5. 275, 52. cf. Gymnothorax polyophthalmus Blkr.

Muraena polyuranodon Blkr. 83, 248. 88, 75. 270, 61. 275, 52. cf. Gymnothorax polyuranodon $B l k r$.

Muraena polyzona Rich., Blkr. 88, 42. 143, 73. 188, 461. 236, 332. 275, 52. cf. Echidna polyzona Blkr.

Muraena prasina Rich. 119, I6.

Muraena pratbernon Q.G.88, 4I.

Muraena Pratbernoniana 125, 325 .

Muraena prosopeion Blkr. 76, 300. 88, 73. 188, 462. 208, 239. 236, 332, 334. 270, 61. cf. Gymnothorax prosopeion Blkr.

Muraena pseudothyrsoïdea Blkr. 71, 778. 88, 44. 275, 52. cf. Gymnothorax pseudothyrsoidea $B l k r$.

Muraena punctata Bl. Schn. 88, 4I.

Muraena punctata Blkr. 91, 78. cf. Gymnothorax tile Blkr., Muraena tile Cant.

Muraena (Strophidon) punctata $M C C l .88,42$.

Muraena python $K p$. cf. Gymnothorax tessellatus $B l k r$.

Muraena raitaborua Buch. cf. Moringua raitaborua Cant.

Muraena Reevesii Rich. cf. Gymnothorax bullatus Blkr., Gymnothorax Reevesii Blkr.

Muraena reticularis $B l k r .91,78$.

Muraena reticularis $C u v$. cf. Gymnothorax reticularis $B$.

Muraena reticulata $\operatorname{Rüpp} .88,37$.

Muraena reticulata Rich. 55, 4I4. 88, 5, 37. 148, 85. 272, 28. cf. Gymnothorax reticularis $B l$., Priodonophis reticularis Blkr.

Muraena Richardsonii Blkr. 54, 296. 88, 45. 184, 204. 208, 239. 270, 6r. cf. Gymnothorax Richardsoni Blkr.

Muraena sagenodeta Rich. 88, 4I. cf. Gymnothorax sagenodeta Blkr., Gymnothorax sagenodetus Blkr.

Muraena sathete Buch., Cant. 54, 298. 88, 52. 288, So.

-Muraena sathete Rich. 88, 5. 91, 78. cf. Strophidon sathete Blkr.

Muraena schismatorhynchus Blkr. 76, 301. 88, 7I. 270, 6I. cf. Gymnothorax schismatorhynchus Blkr.

Muraena scoliodon Blkr. 54, 297. 88, 43. 106, 100. 270, 61. cf. Gymnothorax scoliodon Blkr.

Muraena serpens $L$. cf. Ophisurus serpens Lac.

Muraena sidat Blkr. 352, I24.

Muraena sidera Rich. 119, I6.

Muraena siderea Rich. 88, 5. cf. Gymnothorax pictus Blkr.

Muraena similis Rich. 88, 41. cf. Gymnothorax similis Blkr.

Muraena sordicla Cui'. cf. Echidna catenata Blkr., Nuraena catenatia filkr.

Muraena stellifer Rich. 88, 41. 348, 53.

Muraena stellifera Rich. cf. Gymnothorax stellifer Blkr. 
Muraena talabon 2, 508 .

Muraena (Conger) talabon Cuv. cf. Muraenesox talabon Blkr.

Muraena tenebrosa Soland. 88, 4I.

Muraena tessellata Rich. 86, 530. 88, 4I, 74. 126, 344. 157, 235. 171, 245. 188, 462. 270, 61. 287, 63. cf. Gymnothorax tessellatus Blkr. Muraena thyrsoïdea Buch. 54, 298. 88, 52.

Muraena thyrsoidea Rich. 71, 779. 88, 5. 288, So. cf. Gymnothorax macassariensis, thyrsoideus Blkr.

Muraena tigrina Cuv. cf. Gymnothorax bullatus Blkr.

Muraena tigrina Rüpp. 88, 37. cf. Gymnothorax tigrinus Blkr.

Muraena tile Buch., Cant. 91, I59. 139, 427. 158, 274. 191, 2. 270, 6r. 272, 28. 287, 63. cf. Gymnothorax tile Blkr.

Muraena Troscheli Blkr. 88, 45. 106, гог. 231, I50. 270, 6r. 271, 2. 276, 65. cf. Gymnothorax Richardsoni Blkr.

Muraena Valenciennei Eyd. Soul. cf. Gymnothorax cancellatus Blkr.

Muraena variegata Forst., Blkr. 54, 295. 88, 47. 171, 245. 188, 462. 236, 332. 275, 52 .

Muraena variegata Rich. 119, I6. 170, 479. 184, 204. 270, 6r. cf. Echidna variegata Forst.

Muraena variegata $Q$. G. cf. Gymnothorax pictus Blkr., Muraena Pratbernoniana.

Muraena variegata Mus. Brit. cf. Muraena lita Rich.

Muraena venosa $K p$. cf. Gymnothorax Richardsoni Blkr., Gymnothorax scoliodon Blkr.

Muraena vermiculata $R i c h .88,42.91,78$. cf. Gymnothorax tile Blkr.

Muraena virescens Blkr. 459, 72.

Muraena viridis (prasina) Rich. 88, 42 .

Muraena xanthospilos Blkr. 236, 348. cf. Echidna xanthospilus Blkr.

Muraena zebra Blkr., Shaw 88, 5. 270, 61.

Muraena zebra Cuv. 163, 93. 164, 80.236, 332. cf. Echidna zebra Blkr.

Muraenesox $M C C l .175,28.351$, Ir6.

Muraenesox bagio $K p$., Cant., Petrs. 275, 50. 287, 62. 288, So. 352, I $24.395,294.396,298.425$, I $23.497,26$.

Muraenesox bengalensis $\mathrm{Mc} \mathrm{Cl}$. cf. Conger bagio Cant., Muraenesox bagio Petrs.

Muraenesox exodentata $M c$ Cl. 88, I9. cf. Muraenesox talabon Blkr.

Muraenesox exodon $\mathrm{McCl}$. cf. Muraenesox talabon Blkr.

Muraenesox Hamiltonii $\mathrm{Mc} C$. cf. Conger bagio Cant., Muraenesox bagio Petrs.

Muraenesox lanceolata $M C C l$. 88, ig. cf. Muraenesox talabon Blkr. Muraenesox pristis $K p$. cf. Muraenesox talabon Blkr.

Muraenesox savanna $K p$. cf. Brachyconger savanna $B l k r$.

Muraenesox serradentata $M c C l$. cf. Conger talabon Cuv., Muraenesox talabon Blkr.

Muraenesox singapurensis Blkr. 287, 62. 352, I24. 425, I23. 
Muraenesox talabon Blkr. 275, 50. 287, 62. 352, I24.

Muraenesox talabonoides Blkr. 352, r25.

Muraenesox tricuspidata $M C C l$. cf. Conger bagio Cant., Muraenesox bagio Petrs.

Muraeni 351, Ir3.

Muraenichthys Blkr. 351, II7.

Muraenichthys gymnopterus Blkr. 78, 506. 88, 71. 157, 235. 275, 5I. 352, I 25 .

Muraenichthys gymnotus Blkr. 163, 90. 352, 125.

Muraenichthys macropterus Blkr. 163, 91. 352, I25.

Muraenichthys macrostomus Blkr. 348, 38. 352, 126. 364, 295.

Muraenichthys microstomus Blkr. 348, 39.

Muraenichthys Schultzei Blkr. 166, 366. 170, 479. 352, 126.

Muraenoblenna Lac. 54, 298. 88, 38.

Muraenoblenna tigrina Blkr. cf. Ichthyophis tigrinus Less., Gymnomuraena tigrina $B l k r$.

Muraenoblenna tigrina $K p$. 163, 93. 170, 480. 188, 46r. 275, 5 I. cf. Ichthyophis pantherinus Less., Gymnomuraena pantherina Blkr. Gymnothorax polyuranodon Blkr.

Muraenophis Lac. 351, I2 I.

Muraenophis catenula Lac. cf. Echidna catenata Blkr., Muraena catenata Bikr.

Muraenophis colubrina Lac. cf. Gymnothorax reticularis Bl., Muraena colubrina Rich.

Muraenophis flaveola Less. 54, 297. 88, 45. cf. Gymnothorax Richardsoni Blkr.

Muraenophis grisea Lac. 88, 46. cf. Muraena grisea Cuv.

Muraenophis pantherina Lac. cf. Gymnothorax pictus Blkr.

Muraenophis reticularis Lac. cf. Gymnothorax reticularis $B l$.

Muraenophis sathete Bucl. cf. Strophidon sathete Blkr.

Muraenophis tile Buch. cf. Gymnothorax tile Blkr., Muraena tile Cant.

Muraenophis undulata Lac. cf. Echidna catenata Blkr., Muraena catenata Blkr.

Muraenophis unicolor De la Roch. cf. Gymnothorax unicolor Blkr.

Muraenopsis Les. 351, II9.

Muraenopsis altipinnis Blkr. 313, ISo.

Muraenopsis Bernsteini Blkr. 309, I 57. cf. Ophichthys Bernsteini Blkr.

Muraenopsis marginatus Blkr. 313, I79. cf. Ophichthys cephalozona Blkr.

Murraya Cast. 468, 267.

Mustelus manazo Blkr. 102, 422. 118, I26. 425, II9. 497, 3.

Mustelus megalopterus Smith 266, 57.

Mustelus vulgaris $M . H$. 119, I8. 266, 57.

Mustelus vulgaris $T$. Schl. cf. Mustelus manazo Blkr.

Mycteroperca Gill 468, 256. 
Myctophum asperum Rich. 430, 234.

Myctophum boops Rich. 425, I 47 .

Myctophum Coccoi Blkr. 430, 234.

Myctophum coruscans Blkr. 459, Iо2.

Myctophum hians Rich.459, I02. 495, 2 I.

Myctophum spinosum Blkr. 430, 234.

Myctophum tenuicauda Blkr. 430, 234.

Myliobatis 1, $55^{2}$.

Myliobatis aquila Risso, C. Dum. 266, 59. 459, 69.

Myliobatis aquila Bonap. cf. Myliobatis Nieuhofii $M$. $H$.

Myliobatis aquila T. Schl. cf. Myliobatis tobijei Blkr.

Myliobatis cyclurus $V$. Hass. cf. Myliobatis maculatus $G r$.

Myliobatis macroptera $\int . M$. cf. Aëtobatis narinari $M$. $H$.

Myliobatis maculatus Gr., M.H. 25, 13. 64, 84. 91, 82. 191, 2. 272, 6. $287,38.288,67.395,290.425$, т 20.

Myliobatis milous $V a l ., M . H .25$, I3. 64, 87. 425, I20.

Myliobatis milous Cant. cf. Myliobatis vespertilio Blkr.

Myliobatis narinari Cuv. 2, $507 \mathrm{cf}$. Aëtobatis narinari $M . H$.

Myliobatis Nieuhofi M.H. 64, 85. 91, 82. 119, I9. 191, 2. 270, 68.

$272,7.287,38.288,67.395,290.425$, г20.

Myliobatis ocellatus $K u \hbar l$ cf. Aëtobatis narinari $M . H$.

Myliobatis oculeus Rich. cf. Myliobatis milous $M . H$.

Myliobatis tobijei Blkr. 102, 425. 118, I30. 425, I20. 497, 4 .

Myliobatis vespertilio $B l k r .64,85.288,67$.

Mylocheilus $A g .261,425.314,205$.

Mylopharodon Ayr., Ag. 261, 425. 314, 205.

Myriodon Bris. 468, 257.

Myriodon scorpaenoides Bris. 50, 480. 51, 55. 119, 6. 185. 222. 234, 352. 267, r39. 275, 24. cf. Myriodon waigiensis Gthr.

Myriodon waigiensis Gthr. 320, 268. 364, 281. 395, 291. 396, 296. 451, I 22.

Myriodontini 468, 257 .

Myripristis 1, 553 .

Myripristis adustus Blkr. 72, I08. 157, 232. 167, 371. 315, 232.354, I46. $364,280.428$, r93.

Myripristis archiepiscopus $V a l .459,86$.

Myripristis axillaris C. $V .459,86.495$, r6.

Myripristis Bleekeri Gthr. 315, 232. 358, r86. 364, 280. 428, r8 I. $491,48$.

Myripristis borbonicus C. V. 422, 94. 459, I5, 86 .

Myripristis botche C. V. 2, 526. 13, 52. 47, 229. 91, 34. 364, 280. cf. Myripristis adustus, macrolepis Blkr.

Myripristis hexagonus C. V. 13, 4. 47, 229. 54, 262. 156, 213. 236, 330. $275,22.354$, I 46.428, I $85.459,86.495$, I 6.

Myripristis hexagonus Blkr. cf. Myripristis macrolepis Blkr. 
Myripristis indicus Blkr. 428, I83. 459, 86.

Myripristis japonicus $C . V .175$, I 0.425, I $36.459,86.495,16.497$, го.

Myripristis kuntee $C . V .91,34.459,86.495$, I6.

Myripristis lima $C . V .459,86.495$, I 6.

Myripristis macrolepis Blkr. 428, I95.

Myripristis melanophrys Szens. cf. Myripristis murdjan Rüpp.

Myripristis melanostictus Blkr. 428, 187, 237.

Myripristis microphthalmus Blkr. 54, 26r. 364, 280. 428, г9r.

Myripristis murdjan Rüpp. 72, го9. 157, 232. 184, I98. 188, 458. 275, 22. 358, I $86.364,280.428$, 188.

Myripristis Pahudi Blkr. 321, 22. 428, I83. 491, 48.

Myripristis parvidens $C . V$. 54, 260. 167, 371. 184, I98. 275, 22. 296, Iо5. 309, I $55.315,232.320,268.354$, I $46.364,280.388$, 272. 428, 190.

Myrispristis pralinius $C . V .47,234.54,262.167,37$ 1. 275, 22. cf. Myrispristis Bleekeri Gthr.

Myripristis pralinius Gthr. cf. Myripristis indicus Blkr.

Myripristis pralinius Rich. cf. Myripristis japonicus $C . V$.

Myripristis refulgens $\mathrm{Val} .459,86$.

Myripristis seychellensis $C . V .459,86.495, \mathrm{I} 6$.

Myripristis trachyacron Blkr. 321, 24. 428, I 85 .

Myripristis violaceus C. $V ., B l k r .47,234.54,262.309$, I 55. 364, 280. 428, 192.

Myripristis vittatus $C . V .459,86.495$, г6.

Myrophis Liütk. 351, II7.

Myrophis heterognathos Blkr. 193, 9. cf. Ophisoma heterognathus Blkr.

Myrophis uropterus Blk\%. 175, 2S. 253, 235.

Myrus $K p .351$, I I 7 .

Myrus uropterus Gïnth. 497, 26.

Mystriophis $K p .351$, I 18 .

Mystriophis rostellatus $K p .497,26$.

Mystus ascita Gron. cf. Pseudorhamdia ascita Blkr., Pseudorhamdia macronema Blkr.

Mystus Badgee Sykes cf. Notopterus Bontianus $C . V$.

Mystus carinatus Szens. cf. Doras carinatus Lac.

Mystus carolinensis Gron. cf. Pimelodus bagre Lac.

Mystus chitala Buch. cf. Notopterus Buchanani C. $V$.

Mystus costatus Srons. cf. Platydoras costatus Blkr.

Mystus Gronovii Szons. cf. Platydoras costatus Blkr.

Mystus kapirat $G r$. cf. Notopterus Bontianus $C$. $V$.

Myxus analis Kner 425, I43.

Nandoidei 468,3 r 7 .

Nandus Cuv. 468, 3 I8.

Nandus marmoratus C. $V .91,107$. 
Nandus nebulosus Blkr. 52, 92. 56, 445. 185, 224. 271, I. 272, 9. 287, 44. 454, $45^{8}$.

Nannoperca Gthr. 468, 260.

Nannoperca australis Gthr. 468, 260.

Narcacion fuscomaculatus Blkr. 495, 8.

Narcacion marmoratus Blkr. 459, 68. 495, 8 .

Narcacion Polleni Blkr. 376, r71. 459, I, 68.

Narcine brasiliensis Henle 266, 58.

Narcine indica Henle 91, 82. 288, 67. cf. Narcine timlei $M$. $H$.

Narcine lingula Rich. 425, 120.

Narcine tasmaniensis Rich. 119, I9.

Narcine timlei $M . H .10,68.46,2$ r $4.78,5$ r $.91,82.275$, 1 . 287, 37. 425 , т20. 497,4 .

Naseus Blkr. 163, 62 .

Naseus amboinensis Blkr. 167, 372. 170, 478. 184, 201. 231, I 47. 234, 354. 275, 37. 287, 5 I. 315, 235. 354, I49. 358, г9o. 364,288 .

Naseus anginosus Blkr. 275, 37. 320, 27 I. 358, I9०. 364, 288.

Naseus annulatus Blkr. 137, 304. 157, 233. 184, 201. 236, 331. 250, 206. $270,32.275,37.315,235.320,27$ I. 354, I 49. 358, I90. $364,288$.

Naseus brachycentron $C . V .396,297$.

Naseus brevirostris $C . V .81,165.137,306.275,57.364,288.459$, 97. 491, 51. 495, I9.

Naseus dahuricus Bas. cf. Elopichthys bambusa Blkr., Elopichthys dahuricus.

Naseus dauricus Bas. cf. Elopichthys dahuricus.

Naseus fronticornis Comm., C. V. 157, 238. 318, 252. cf. Naseus unicornis Gthr.

Naseus hexacanthus Blkr. 157, 233. 318, 252. 320, 271. 358, I90. 364, 288 .

Naseus Hoedtii Blkr. 84, 339. cf. Naseus brevirostris C. $V$.

Naseus leptopeltis Blkr. 459, 97.

Nàseus lituratus $C . V .71,763.119$, г $.157,233.167,372.275,37$.

318, 252. 320, 27 1. 364, 288. 459, 97.491, 52. 495, I 9.

Naseus longicornis $C$. $V$. cf. Naseus fronticornis Comm.

Naseus marginatus $C$. $V$. cf. Naseus annulatus Blkr.

Naseus punctulatus $C . V .495$, r9.

Naseus scalprum Blkr. 269, 79. 425, г 34. 497, г6.

Naseus tapeinosoma Blkr. 137, 306. 320, 27 I. 364, 288.

Naseus thynnoides Blkr. 163, 61. 491, 52 .

Naseus tuber Comm. 459, 97. 495, 19.

Naseus tuberosus Gthr. 318, 252. 358, I9o.

Naseus unicolor Lién. 459, 97. 495, r9.

Naseus unicornis Gthr. 396, 297. 425, I34. 459, 97. 495, I 9. 497, г6. 
Naseus Vlamingii $C . V .78,472.234,354.275,37$. cf. Naseus tuber Comm.

Naucrates ductor C. V. 425, 132. 459,.98.497, I5.

Naucrates indicus $C . V .91,42.270,28.364,289$. cf. Naucrates ductor $C . V$.

Nebris $C . V .468,327$.

Nebris microps C. V. 363, 26r. 468, 328 .

Nebrius concolor Rüpp. cf. Ginglymostoma Rüppellii Blkr.

Nemacheilus v. Hass. 323, 37 .

Nemacheilus arenatus $B k \% .323,40$.

Nemacheilus argyrogramma $B l k r .323,40$.

Nemacheilus barbatulus Blkr. 323, 40.

Nemacheilus boutanensis Blkr. 323, 4I.

Nemacheilus chlorosoma Blkr. 323, $4 \mathrm{I}$.

Nemacheilus cincticauda Blkr. 323, 4r.

Nemacheilus corica Blkr. 323, $4 \mathrm{I}$.

Nemacheilus fasciatus $v$. Hass. 323, 40. cf. Cobitis fasciata C. $V$.

Nemacheilus frenatus Blkr. 323, 40.

Nemacheilus guttatus Blkr. 323, 4I.

Nemacheilus insignis $B l k r .323,40$.

Nemacheilus Jaklesi Blkr. 323, 40.

Nemacheilus leopardus Blkr. 323, 40.

Nemacheilus marmoratus Blkr. 323, 40.

Nemacheilus montanus Blkr. 323, 4r.

Nemacheilus notostigma Blkr. 323, 4I. 327, 254. 335, 5 .

Nemacheilus nudus Blk\%. 343, т2. 425, I46.

Nemacheilus nurga Blkr. 323, 40.

Nemacheilus panthera Blkr. 323, 40.

Nemacheilus persus Blkr. 323, 40.

Nemacheilus phoxocheilus Blkr. 323, 4I.

Nemacheilus rupecula Blkr. 323, 4r.

Nemacheilus savona Blkr. 323, 4I.

Nemacheilus scaturiginus $B l k r .323,40$.

Nemacheilus spilopterus Blkr. 323, 4I.

Nemacheilus subfuscus Blkr. 323, 4I.

Nemacheilus tigris Blkr. 323, 40.

Nemacheilus turio Blkr. 323, 40.

Nemacheilus vittatus Blkr. 323, 40.

Nemacheilus zonalterans $B l k r .323,4$ I.

Nemacheilus zonatus Blkr.323, $4 \mathrm{~T}$.

Nemadactylus Rich.468, 3 I 6.

Nemadactylus concinnus Rich. 119, 8. 468, 3 I6.

Nemaphaerus maculosus $K . \%$. H. cf. Trichopodus trichopterus Lac.

Nematodactylus Gill 468, 3 r6.

Nematogenys Gir. 306, II 2. 
Nematogenys inermis Gir. 306, I I 2.

Nemichthys Rich. 351, I I5.

Nemipterus Srens. 468, 279.

Nemipterus filamentosus Szons. 468, 279.

Nemobrama Webbii $\mathrm{Val}$. cf. Polymixia nobilis Loze.

Nemophis Lessonii $K p$. 459, 73.

Neochaetodon Cast. 468, 336.

Neocirrhites Cast. 468, $33^{6 .}$

Neoconger Gir. 351, II 7 .

Neomaenis Gir. 468, 276.

Neosebastes Guich. 473, 295.

Neotephraeops Cast. 468, 296.

Neotephraeops zebra Cast. 468, 296.

Nestis cyprinoides $C . V$. cf. Agonostoma Telfairi Benn.

Nestis dobuloides $C . V$. cf. Agonostoma dobuloides Gthr.

Nettastoma Kp., Raf., Rich. 88, 39. 175, 28. 351, II5, I22.

Nettastoma parviceps Gïnth. 497, 26.

Netuma Blkr. 306, 90.

Netuma dubia Blkr. 298, 382. 336, 63 .

Netuma Herzbergi Blkr. 336, 60.

Netuma nasuta Blkr. 227, 408. 270, 45. 275, 46. 287, 58. 306, 90.

Netuma proops Blkr. 336, 62.

Netuma quadriscutis Blkr. 336, 59.

Niphon C. $V .468,264$.

Niphon spinosus $C . V .468,264.497,6$.

Nocomis Gir. 261, 437. 314. 2 I . 329, 264.

Nomeus 1, 553 .

Nomeus Gronovii Gthr. 425, I32. 459, I00. 495, I8.

Nomeus maculatus $V a l$. cf. Nomeus Gronovii Gthr.

Nomeus Mauritii C. $V$. 302, r5. cf. Nomeus Gronovii Gthr.

Nomeus nigrofasciatus Rüpp. 2, 5r8. cf. Seriola Rüppelii $C . V$.

Notacanthoidei 449, 368.

Notacanthus Risosanus Fil. Veran. 449, 368.

Notacanthus sexipinnis Rich. 119, Iо.

Notidanus griseus Cuv. cf. Hexanchus griseus Raf.

Notidanus indicus Cuv. cf. Heptanchus indicus $M . H$.

Notobranchus orthonotus Pet. cf. Fundulus orthonotus Gthr.

Notopterus 1, $55^{2}$.

Notopterus Bontianus C. V. 39, 423. 46, 214. 59, 17.91, I 51. 275, 49. cf. Notopterus kapirat $B l k r$.

Notopterus bornëensis Blkr. 49, 437. 55, 414. 59, 26. 91, I50. 92, 57. 272,27 .

Notopterus Buchanani C. V. 67, 605. 91, I 49.

Notopterus chitala $G r$. cf. Notopterus Buchanani C. $V$.

Notopterus hypselonotus Blkr. 59, 27. 67, 604. 92, 59. 270, 59. 272, 27 . 
Notopterus hypselopterus Blkr. 91, I50.

Notopterus kapirat Blkr. 2, 510. 59, 27. 91, I51. 92, 55. 225, $35^{8}$. 270, 59. 275, 49. 356, I76. cf. Notopterus Bontianus $C . V$.

Notopterus kapirat Lac. 10, 67. 425, 148.

Notopterus lopis Blkr. 2, 510. 25, х2. 39, 422. 59, 17. 91, I50. 92, 6o. $170,476.225,35^{8}, 272,27$.

Notopterus maculatus $\mathrm{V}$ al. cf. Notopterus Buchanani $C$. $V$.

Notopterus maculosus Blkr. 49, 438. 55, 414. 59, 26. 91, I50. 92, $58,270,59.272,27$.

Notopterus ocellifer Cast.; Alb. 356, г76.

Notopterus ornatus Gr. 91, I5I.

Notopterus Pallasii C. V. 91, 76, 152.

Notothaenia coriiceps Rich. 119, I2.

Noturus Raf. 306, 1о3.

Noturus flavus Raf. 306, 103.

Novacula Risso 292, I03.

Novacula celebica Blkr. 143, 6ז. 275, т6.

Novacula cephalotaenia Blkr. 99, 333.

Novacula dea Blkr. 175, 20. 425, 135. 497, 5.

Novacula Hoedtii Blkr. 142, 59. 167, 374. 275, r7.

Novacula immaculata $\mathrm{Val}$. cf. Hemipteronotus immaculatus Blkr.

Novacula javanica Blkr. 293, 305 .

Novacula julioïdes Blkr. 47, 254. 54, 237. 167, 374. 184, 203. 250, 207.

Novacula kallosoma Blkr. 250, 207. 273, 5 .

Novacula lineolata Blkr. 302,"33.

Novacula macrolepidota $B l k r$. cf. Novaculichthys macrolepidotus $B l k r$.

Novacula melanopus Blkr. 163, 82 .

Novacula novacula Cuv. cf. Novacula lineolata Blkr.

Novacula pavo Blkr. 145, 378. cf. Xyrichthys pavo $\mathrm{Val}$.

Novacula pentadactyla $C . V .10,67,68.11,7.12,42.46,222.54$, 237. 91, 54. 231, I49.275, I7. cf. Hemipteronotus pentadactylus Blkr.

Novacula Plinii Riss. cf. Novacula lineolata Blkr.

Novacula praetextata Blkr.250, 207. 275, I 7 .

Novacula punctulata $C . V .81$, I70. 231, 149. 275, I7. cf. Hemipteronotus pentadactylus $B l k r$.

Novacula sexmaculata $C$. $V$. cf. Novacula pentadactyla $C$. $V$.

Novacula spilonotus Blkr. 163, 83 .

Novacula taeniurus Blkr. 143, 62. 250, 207. 275, I7.

Novacula tessllata $C$. $V$. cf. Hemipteronotus tessellatus $B l k r$.

Novacula tetrazona Blkr. 231, I69.

Novacula Twistii Blkr. 145, 38x. cf. Hemipteronotus 'Twistii Blkr.

Novaculichthys Blkr. 292, ro2.

Novaculichthys kallosoma Blkr. 293, 293. 364, 277.

Novaculichthys macrolepidotus Blkr. 293, 293. 358, I84. 364, 277. $396,296.491,4 I$. 
Novaculichthys taeniurus Blkr. 293, 293. 318, 250.320, 266. 364, 277. 459, 83. 495, го.

Nuria Val. 261, 434. 314, 2 ×3.

Nuria dangila $C . V .91,62$.

Nuria danrica $C . V$. 91, I30.

Nuria rerio $C . V .91,62$.

Nuria thermoicos C. $V . \mathbf{9 1}, 62$, I31. cf. Esomus thermoicos Heck.

Nuria thermophilos C. $V .91,62$.

Oblada Cuv. 468, 283 .

Oblada melanura Cuv. 468, $2 \delta_{3}$.

Oblada tricuspidata $C$. 119, 9.

Oblata $C . V .468,283$.

Oblata tricuspidata $C . V .269,77$.

Ochetobius elongatus Gthr. 415, II. 425, I 44 .

Octogrammus Blkr. 449, 370.

Octogrammus Pallasi Blkr. 449, 37 r. 497, Ir.

Octonematichthys Blkr. 306, 95 .

Octonematichthys nigrita Blkr. 306, 95.

Ocyurus Gill 468, 276.

Odax Cuv. 290, 229. 292, Io8.

Odax algensis Rich. 119, I3.

Odax balteatus Cv. 119, I3.

Odax borbonicus $C$. $V$. cf. Odax radiatus $G t h r$.

Odax lineatus Rich. 119, i3.

Odax moluccanus C. V. 11, 7. 12, 42. 143, 64. 275, I5. 290, 229. 292, 96. cf. Pseudodax moluccanus Blkr.

Odax pullus $C$ v. 119, 13 .

Odax radiatus Gthr. 459, $8 \mathrm{r}$.

Odax vittatus Rich. 119, I3.

Odontamblyopus Blkr. 453, 330.

Odontamblyopus Lacepedii Blkr. 497, 20.

Odontanthias Blkr. 468, 252.

Odontanthias borbonicus Blkr. 459, г6, 88. 495, го.

Odontanthias chrysostictus Blkr. 431, 237.

Odontanthias rhodopeplus Blkr. 431, 236.

Odontaspis taurus $M . H . \mathbf{2 6 6}, 58$.

Odonteleotris Gill 453, 302. 460, 103, 104.

Odonteleotris canina Blkr. 478, I8.

Odonteus $A g .468,282$.

Odonteus sparoides $A g$. 468, 282 .

Odontobutis Blkr. 453, 305. 460, 103, I05.

Odontobutis obscura $B l k r .478,56.496,3.497$, I8.

Odontogobius Blkr. 453, 323 .

Odontolabrax Blkr. 425, I49. 468, 336. 
Odontolabrax typus Blkr. 425, I37, I50.

Odontonectes erythrogaster Gthr. cf. Caesio erythrogaster $K$. v. H.

Odontoscion Gill 468, 329.

Odontoscion dentex Gill 468, 330.

Odontostomus 273, I3.

Ognichodes Swns. 453, 329 .

Oligolepis Blkr. 453, 318 .

Oligorus Gthr.468, 262, 336 .

Oligorus macquariensis Gthr. 468, 262.

Olisthops Rich. 290, 229. 292, 109.

Olisthops cyanomelas Rich. 119, I3.

Olistus atropus $C$. $V$. cf. Carangoïdes atropus Blkr.

Olistus malabaricus $C$. $V$. cf. Carangoïdes citula Blkr., Citula malabarica Blkr.

Olistus Rüppelli $C$. $V$. cf. Carangoïdes citula Blkr.

Olyra $\mathrm{Mc} \mathrm{Cl}$. 306, 103.

Olyra laticeps $M c C l .91,58$. cf. Branchiosteus laticeps Gill.

Olyra longicaudata $M C C .91,58.306$, I03.

Ompok Lac. 299, 395. 306, II5.

Ophicardia $M C C l .88,57$.

Ophicardia Phayriana $M C C$. cf. Monopterus javanensis Lac.

Ophicardia xanthognatha Rich. cf. Monopterus javanensis Lac.

Ophichthys Ahl 351, I I9.

Ophichthys adspersus Gthr. 425, I 23.

Ophichthys altipinnis Blkr. 352, I 26.

Ophichthys amboinensis Blkr. 348, 45. 352, I26. 364, 296.

Ophichthys amoyensis Blkr. 349, 6r. 425, I 23.

Ophichthys apicalis Blkr. 425, I23.

Ophichthys bangko Blkr. 352, I27.

Ophichthys Bernsteini Blkr. 352, I27.

Ophichthys Bonapartei Blkr. 352, 127. 364, 296.

Ophichthys breviceps Blkr. 352, I27.

Ophichthys Broekmeyeri Blkr. 352, I27.

Ophichthys celebicus Blkr. 352, 127.

Ophichthys cephalozona Blkr. 349, 62. 352, I27. 358, I93. 364, 296. $425,123.497,26$.

Ophichthys compar Blkr. 352, I27.

Ophichthys conger $A h l \mathrm{cf}$. Conger vulgaris $C u v$.

Ophichthys dicellurus Gthr. 425, I 24.

Ophichthys Diepenhorsti Blkr. 352, 127.

Ophichthys grandoculis Blkr. 352, I 27.

Ophichthys intertinctus Blkr. 362, 234.

Ophichthys lumbricoides Blkr. 352, I27.

Ophichthys Mac Clellandi Blkr. 352, I2S.

Ophichthys macrochir Blkr. 352, I28. 
Ophichthys magnioculis Blkr. 362, 237.

Ophichthys melanochir Blkr. 348, 44. 352, I28. 364, 296.

Ophichthys pallens Gthr. 425, r24.

Ophichthys parilis Blkr. 362, 238.

Ophichthys polyophthalmus Blkr. 348, 43. 352, I28. 364, 296."

Ophichthys punctatus Swns. cf. Amphipnous cuchia J. Mïll.

Ophichthys rutidodermatoides Blkr. 352, I28.

Ophichthys rutidoderma Blkr. 352, т28.

Ophichthys spadiceus Blkr. 459, 72 .

Ophichthys stenopterus Cope 497, 26.

Ophichthys urolophus Gïnth.497, 26.

Ophichthys versicolor Blkr. 352, I28.

Ophidium aculeatum $B$. cf. Rhynchobdella ocellata $C$. $V$.

Ophidium aculeatum Basil. cf. Rhynchobdella sinensis Blkr.

Ophidium mastacembelus Shave cf. Mastacembelus haleppensis $C . V$.

Ophidium rostratum Shane cf. Rhynchobdella ocellata C. $V$.

Ophidium simak $W a l b$. cf. Mastacembelus haleppensis $C$. $V$.

Ophiocara Gill 453, 303. 460, 103, 105.

Ophiocara aporus Blkr. 478, 33.

Ophiocara Hoedti Blkr. 478, 35. 491, 53.

Ophiocara ophiocephalus Gill 478, 28.

Ophiocara porocephalus Blkr. 478, 30.

Ophiocephalus 1, 553.

Ophiocephalus affinis Gthr. cf. Ophiocephalus punctatus $B l$.

Ophiocephalus amphibeus $\mathrm{MCCl}$.91, 42 .

Ophiocephalus argus Cant. 411, 252. 425, I27.

Ophiocephalus aurantiacus $B u c h$. cf. Ophiocephalus marginatus $C$. $V$., Ophiocephalus gachua Buch.

Ophiocephalus bankanensis Blkr. 70, 726. 82, 187. 272, I 5. 498, 5 I. Ophiocephalus barca Buch. 91, 42.

Ophiocephalus bivittatus Blkr. 2, 5r9. cf. Ophiocephalus micropeltes K. v. H.

Ophiocephalus chena Buch. cf. Ophiocephalus striatus $B \%$

Ophiocephalus cora-mota $C$. $V$. cf. Ophiocephalus marginatus $C . V$, Ophiocephalus gachua Buch.

Ophiocephalus cyanospilos Blkr. 76, 256. 270, 33 .

Ophiocephalus cyanospilus Blkr. 498, 45 .

Ophiocephalus diplogramma Day cf. Ophiocephalus micropeltes $K . v . H$.

Ophiocephalus fuscus $C$. $V$. cf. Ophiocephalus marginatus $C . V$., Ophiocephalus gachua Buch.

Ophiocephalus gachua Buch. 498, 38 . cf. Ophiocephalus marginatus $C . V$. Ophiocephalus grandinosus $C . V .91,42.425$, х 27.

Ophiocephalus guachua Pet. cf. Ophiocephalus gachua Buch.

Ophiocephalus indicus $M C C l .91,42$. cf. Ophiocephalus punctatus $B l$. Ophiocephalus iris $C . V$. 425, 127. cf. Ophiocephalus marulioïdes Blkr. 
Ophiocephalus jovis Rich. 425, т27.

Ophiocephalus karrouvei Lac. cf. Ophiocephalus punctatus Bl.

Ophiocephalus kelaertii Gthr. cf. Ophiocephalus gachua Buch.

Ophiocephalus lata Buch. cf. Ophiocephalus punctatus $B l$.

Ophiocephalus latus $H$. $B$. cf. Ophiocephalus punctatus $B l$.

Ophiocephalus leucopunctatus Sykes 91, 42.

Ophiocephalus limbatus $C$. $V$. cf. Ophiocephalus marginatus $C . V$, Ophiocephalus gachua Buch.

Ophiocephalus lucius $K . v$. H. 2, 519. 21, I3. 25, S. 26, 3. 35, 26 I. 42, 59.45, I95.49, 4I9. 55, 4II. 56, 445. 191, 3. 185, 223. 239, IOI. $270,33.272$, I 5. 356 , I 74. $496,2.498,53$.

Ophiocephalus maculatus $C . V$. 425, I27. 498, 50.

Ophiocephalus marginatus $C . V$. 16, 4. 21, I4. 25, 8. 52, 89. 56, $445.91,42.170,476,477.185,223.211,24$ I. 270, 33. 272, 15. 279, ıоo. cf. Ophiocephalus gachua Buct.

Ophiocephalus marulioïdes Blkr. 49, 424. 52, S9. 55, 4II. 185, 223. 235, 368. 272, I 5. 498, 40.

Ophiocephalus marulius Buct. 91, 42.

Ophiocephalus melanopterus Blkr. 139, 420. 270, 34.272, r 5.498, 4 I . Ophiocephalus melanosoma Blkr. 156, 214. 235, 368. 271, I. 272, I 5. 356, I 74. 498, 46 .

Ophiocephalus melasoma Blkr. 49, 424. 55, $4 \mathrm{r}$.

Ophiocephalus micropeltes (K. v. H.) C. V. 21, r2. 35, 26r. 42, 59. 45, 195. 49, 419. 55, 4I1. 235, 368. 270, 33. 272, I6. 356, 174. 498,55 .

Ophiocephalus miliaris $C . V .425$, I 27.

Ophiocephalus montanus $M C C l .91,42$. cf. Ophiocephalus gachua Buch.

Ophiocephalus mystax Blkr. 82, I88. cf. Ophiocephalus melanosoma Blkr.

Ophiocephalus ocellatus $C . V .425$, I27.

Ophiocephalus pekinensis Basil. cf. Ophiocephalus argus Cant.

Ophiocephalus planiceps $(K . v . H) C .. V ., B l k r .2,5$ r9. cf. Ophiocephalus striatus $B l$.

Ophiocephalus pleurophthalmus Blkr. 35, 270.42, 59. 45, 195. 49, 4 I $.55,4$ II. 174, 37. 190, I. 270, 34. 272, I6. 498, 48.

Ophiocephalus polylepis Blkr. 67, 57 S. 270, 34. 271, I. 498, 50.

Ophiocephalus punctatus $B l .91,95.498,36$.

Ophiocephalus puticola Rich.cf. Ophiocephalus grandinosus $C . V$.

Ophiocephalus rhodotaenia Blkr. 49, 425. 55, 4II. 70, 726. 272, I6. 498,47 .

Ophiocephalus serpentinus C. $V .70,726$. cf. Ophiocephalus micropeltes $(K . v, H). C \cdot V$.

Ophiocephalus siamensis Gthr. 356, I74.

Ophiocephalus sowarah Blkr. 2, 519.91, 42. cf. Ophiocephalus striatus Bl. 
Ophiocephalus Stevensi Blkr. 85, 444. 239, ror. 270, 34. 272, I6. 356, I 74. cf. Ophiocephalus micropeltes $\left(K . v . H_{0}\right) C . V$.

Ophiocephalus striatus Bl., C. V., Blkr. 2, 519. 10, 66. 21, I3. 25, 8 . 26, 4. 35, 26 I. 42, 59. 45, I95. 46, 2 I $2.49,4 \mathrm{I} 9.55,4 \mathrm{II} .56$, 445. 91, 42. 170, 476, 477. 191, 2. 225, 357. 231, I46.239, 10I. 255,24 I. 271, I. 272, I6. $275,42.279$, гоо. $287,55.288,76$. 356, I 74. 364, 29I. 411, 252. 425, I27. 496, 2. 498, 42 .

Ophiocephalus Theophrasti $\mathrm{Val} .91,42$.

Ophiocephalus urophthalmus Blkr: 67, 578. 190, I. cf. Ophiocephalus pleurophthalmus Blkr.

Ophiocephalus vagus $P$ et. cf. Ophiocephalus striatus $B l$.

Ophiocephalus whrahl Lac., Buch. cf. Ophiocephalus striatus Bl.

Ophiogobius Gill 453, 3 Io.

Ophioscion Gill 468, 327.

Ophisoma Swns. 351, I 6.

Ophisoma anago Blkr. 497, 25.

Ophisoma anagoides Blkr. 352, I25. 364, 295.

Ophisoma heterognathus Blkr. 497, 26.

Ophisoma megastoma Blkr. 497, 26.

Ophisoma myriaster Blkr. 497, 26.

Ophisoma neoquinaicum Blkr. 352, I25.

Ophisternon $M C C l .88,57$.

Ophisternon bengalensis $J . M ., M c C l .272,27.275,50$. cf. Symbranchus immaculatus $B l$.

Ophisternon caligans Blkr. 288, I80.

Ophististhius Gill 468, 293.

Ophististius tahmel Gill cf. Pimelepterus cinerascens Day.

Ophisurophis $K p .351$, I20.

Ophisurus Lac., Kp. 90, 54. 351, I I 8, I 20.

Ophisurus acuminata Szons. cf. Pisoodonophis boro Kp., Ophisurus boro Buch.

Ophisurus alternans Q.G. 88, 36. 270, 59. cf. Ophisurus colubrinus Rich., Ophisurus fasciatus Rich.

Ophisurus (Microdonophis) altipinnis $K p$. 275. $5 \mathrm{I}$.

Ophisurus altipinnis Blkr. cf. Muraenopsis altipinnis Blkr.

Ophisurus apicalis Benn. 88, 5. 270, 59. cf. Ophichthys Diepenhorsti Blkr.

Ophisurus baccidens Cant. 70, 736. 88, 5.

Ophisurus bangko Blkr. 88, 67. 275, 51. cf. Ophichthys bangko Blkr.

Ophisurus bengalensis $C u v$. cf. Pisoodonophis boro $K p$.

Ophisurus Bonapartei Blkr. 163, 87. cf. Ophichthys Bonapartei Blkr.

Ophisurus boro Buch., Cant. 70, 736. 88, 5. 91, r56. 170, 48o. 255, 24I. 287, 62. 288, so. cf. Pisoodonophis boro $K p$.

Ophisurus brachysoma Blkr. 71, 776. 88, 55. 275, 51. cf. Pisoodonophis brachysoma $K p$. 
Ophisurus breviceps Rich. 88, 25 .

Ophisurus breviceps Cant. cf. Ophisurus lumbricoïdes Blkr.

Ophisurus Broekmeyeri Blkr. 143, 7 r. 275, 51. cf. Ophichthys Broekmeyeri Blkr.

Ophisurus cancrivorus Rich. 70, 736. 71, 777. 88, 5. 119, х5. 287, 62. 288, 8o. cf. Pisoodonophis cancrivorus $K p$.

Ophisurus caudatus $M C C$. 88, 27. cf. Ophisurus boro Buch., Pisoodonophis boro $K p$.

Ophisurus celebicus Blkr. 143, 70. 275, 51. cf. Ophichthys celebicus Blkr.

Ophisurus cheilopogon Blkr. 275, 59. cf. Cirrhimuraena chilopogon Jlkr.

Ophisurus chrysospilos Blkr. cf. Ophichthys Bonapartei Blkr., Ophisurus Bonapartei $B l k r$.

Ophisurus cirrhocheilos Blkr. 163, 89. 270, 87. cf. Brachysomophis cirrhocheilus Blkr.

Ophisurus colubrinus Rich. 88, 5. 91, 78. 149, I06. 188, 461. 236,

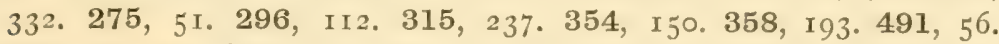
497, 26. cf. Leiuranus colubrinus $K p$., Ophisurus fasciatus $L a c ., R i c h$.

Ophisurus colubrinus Lac. 320, 272.

Ophisurus compar Rich. 88, 5. 270, 60. cf. Ophichthys apicalis Blkr., Ophichthys compar Blkr.

Ophisurus crocodilinus Benn. cf. Brachysomophis crocodilinus Blkr.

Ophisurus dicellurus Rich. 88, 25. cf. Ophichthys dicellurus Gthr.

Ophisurus Diepenhorsti Blkr. 270, 85. cf. Ophichthys Diepenhorsti Blkr.

Ophisurus fasciatus Rich. 88, 5. 352, I29. 364, 296. 369, 42. 394, 288. 397, 302. cf. Ophisurus colubrinus Rich.

Ophisurus fasciatus, $L a c .459,72$. cf. Leiuranus colubrinus $K p$, Leiuranus Lacepedii 'Blkr., Ophisurus colubrinus Rich.

Ophisurs grandoculis Cant. 88, 5. 288, 8o. cf. Ophichthys grandoculis Bilkr.

Ophisurus guttulatus Blkr. 362, 239.

Ophisurus harancha Buch. 88, 27. cf. Ophisurus boro Buch., Pisoodonophis boro $K p$.

Ophisurus haranche Rich. cf. Ophichthys pallens Gthr.

Ophisurus Hoevenii Blkr. 81, 172. 88, 67. 275, 5I. cf. Pisoodonophis Hoevenii $K p$.

Ophisurus hyala Buch. 88, 25. 91, 158.

Ophisurus hypselopterus Blkr. 49, 42r. 55, 414. 70, 737. 88, 34 . $272,28$.

Ophisurus hypselopterus $K p$. cf. Pisoodonophis hypselopterus $K p$.

Ophisurus immaculata Szons. cf. Ophisisurus boro Buch., Pisoodonophis boro $K p$.

Ophisurus intertinctus Rich. 88, 25. cf. Ophichthys intertinctus Blkr.

Ophisurus lumbricoides Blkr. 88, 32. 288, 80. cf. Ophichthys lumbricoides Blkr. 
Ophisurus Mac Clellandi Blkr. 88, 33. 143, 72. cf. Ophichthys Mac Clellandi Blkr.

Ophisurus macrochir Blkr. 88, 26. 113, 446. cf. Ophichthys macrochir Blkr.

Ophisurus macrorhynchus Blkr. 88, 28. 90, 54. cf. Leptorhynchus serpens Blkr.

Ophisurus maculatus Schl. cf. Ophichthys intertinctus Blkr.

Ophisurus maculosus Lac., Cuv. 88, 29. 236, 332.

Ophisurus maculosus Rich. cf. Ophisurus ophis Lac.

Ophisurus marginatus $V a l$. cf. Muraenopsis marginatus Blkr., Ophichthys cephalozona Blkr.

Ophisurus minimus $M C C l .88,25$. cf. Ophisurus hyala Buch.

Ophisurus multizonus $C u v$. cf. Leiuranus colubrinus $K p$.

Ophisurus ocellatus Rich. 88, 25.

Ophisurus oculatus Schl. 362, 240.

Ophisurus ophis Lac. 352, I29. 354, I 50. 358, I93. 459, 72. cf. Ophiurus maculosus Cav.

Ophisurus pallens Rich. 88, 25. cf. Ophichthys pallens Gthr.

Ophisurus pardalis $V$ al. $88,26$.

Ophisurus parilis Rich. 88, 25. cf. Ophichthys parilis Blkr.

Ophisurus polyodon Blkr. 270, 86. cf. Cirrhimuraena polyodon Blkr. Ophisurus porphyreus $T$. Schl. 88, 25. cf. Mystriophis rostellatus $K p$. Ophisurus potamophilus! Blkr. 85, 458. 88, 68. 272, 28. Pisoodonophis potamophilus $K p$.

Ophisurus puncticulata Stons. cf. Ophisurus hyala Buch.

Ophisurus regius Shaw. 88, 25.

Ophisurus rostellatus Rich. 88, 25 .

Ophisurus rostratus Q. G. 88, 29. cf. Muraenesox bagio Pct., Ophisurus serpens Lac.

Ophisurus rostratus $M_{C} C l .88,27$. cf. Ophisurus hyala Buch.

Ophisurus rutidermatoides Blkr. cf. Ophichthys rutidermatoides Blkr.

Ophisurus rutidoderma Blkr. 88, 30. cf. Ophichthys rutidoderma Blkr.

Ophisurus rutidodermatoides Blkr. 88, $3 \mathrm{I}$.

Ophisurus Schaapi Blkr. 70, 735. 88, 53. cf. Pisoodonophis Schaapi Kp.

Ophisurus semicinctus Rich.70, 736. 88, 26.

Ophisurus semicinctus Lay \& Benn. cf. Leiranus colubrinus $K p$.

Ophisurus serpens Lac. 93, r2. cf. Leptorhynchus serpens Blkr.

Ophisurus serpens Rich. 88, 25.

Ophisurus serpens T. Schl. cf. Ophisurus macrorhynchos Blkr.

Ophisurus sinensis Rich. 70, 736. 88, 26. cf. Pisoodonophis cancrivorus $K p$.

Ophisurus spadiceus Rich. 88, 25. 349, 62. cf. Ophichthys apicalis Blkr., Ophichthys spadiceus Blkr.

Ophisurus sugillatus Rich. 88, 25.

Ophisurus urolophis 88,25 . 
Ophisurus uropterus $88,26$.

Ophisurus vermiformis $M C C l .88,27.91,78$.

Ophisurus versicolor Rich. 88, 5. cf. Ophichthys versicolor $\mathrm{Blkr}$.

Ophisurus vimineus Rich. cf. Leiuranus colubrinus $K p$.

Ophithorax $M C C l .88,23$.

Ophithorax colubrina $M C C$. cf. Gymnothorax reticularis Bl., Muraena colubrina Rich.

Ophiurus baccidens Cant. 51, 59. cf. Pisoodonophis baccidens Blkr. Ophiurus boro Cant. cf. Ophisurus boro Ham.

Ophiurus breviceps Cant. (nec Rich.) cf. Ophichthys breviceps Blkr. Ophiurus hyala Cant. cf. Ophisurus hyala Buch.

Ophiurus hypselopterus Blkr. 42, 69. 45, I97.

Ophiurus maculosus Cuv. 47, 258.

Opisthognathus Castelnaui Blkr. 275, 45. 287. 57. 296, I I I. 455, 469. Opisthognathus nigromarginatus R'üf . cf. Opisthognathus Sonneratii Cut'.

Opisthognathus papuensis Blkr.400, 333. cf. Gnathypops papuensis Blkr.

Opisthognathus Rosenbergii Blkr. 156, 220. cf. Gnathypops Rosenbergii Blkr.

Opisthognathus solorensis Blkr. 80, 81. 184, 201. 364, 293. 394; $287.455,47 \mathrm{I}$.

Opisthognathus Sonneratii Cuv. 46, 22 r. 91, 50. 246, 202. cf. Opisthognathus Castelnaui Blkr.

Opisthopterus macrognathus Blkr. 381, 299.

Opisthopterus tartoor Gill 381, 296.

Opistocheilus Blkr. 261, 425. 314, I96. 329, 262.

Oplectognathus Rich. 468, 284.

Oplegnathus Rich. 468, 284 .

Oplichthys Langsdorfii Eyd. Soul. cf. Hoplichthys Langsdorfii $C . V$.

Oplopomus Steind. 453, $32 \mathrm{I}$.

Opsariichthys Blkr. 314, 203. 329, 263.

Opsariichthys acutipinnis Guich. cf. Barilius (Barilius) acutipinnis Blkr.

Opsariichthys uncirostris Blkr. 497, 24.

Opsarius $M C$ Cl. 261, 436. 314, 203, 215. 329, $263,264$.

Opsarius argyrotaenia Blkr. 225, 358 .

Opsarius bacaila $M c C l$. cf. Leuciscus bacaila $C$. $V$.

Opsarius coreënsis Blkr. cf. Barilius coreënsis Blkr.

Opsarius elongatus Kner of Ochetogobius elongatus Gthr.

Opsarius macropus Blkr. cf. Barilius macropus Blkr.

Opsarius (Opsarius) minor Blkr. cf. Barilius (Barilius) minor Blkr.

Opsarius parous Kner cf. Pseudorasbora parva Blkr.

Opsarius platypus Blkr. cf. Barilius platypus Blkr.

Opsarius Sieboldi Blkr. cf. Barilius Sieboldi Blkr.

Opsarius Temmincki Blkr. cf. Barilius Temmincki Blkr.

Opsarius uncirostris Blkr. cf. Opsariichthys uncirostris Blkr.

Orectolobus ornatum $B p$. cf. Chiloscyllium ornatum Blkr. 
Oreinus $M C C l .261,427.314$, I96.

Oreonectes platycephalus Gthr. 425, I46.

Orestias $\mathrm{Val}$. 261, 44I.

Orthagoriscus Bl. Schn. 367, I6.

Orthagoriscus lanceolatus Lién. 459, 69. 495, 23.

Orthagoriscus mola Bl. Schn. 425, I21. 459, 69. 497, 26.

Orthagoriscus oblongus $B l .266,57$.

Orthagoriscus oblongus Bl. Schn. cf. Orthagoriscus truncatus Flcm.

Orthagoriscus oxyuropterus Blkr. 439, ${ }_{5} \mathrm{r}$.

Orthagoriscus spinosus Cuv. cf. Orthagoriscus mola Bl. Schn.

Orthagoriscus truncatus Flem. 425, I21. 497, 26.

Orthichthys velitaris Gill 364, 274.

Orthodon Gir. 261, 426. 314, 206.

Orthopristis Gill 468, $27 \mathrm{I}$.

Orthostoechus Gill 468, 27 I.

Orthostomus Kner 453, 308. 460, 104, 106.

Orthostomus amblyopinus Kner 453, 308. 478, 107.

Osmerus japonicus Brev. cf. Hypomerus olidus Günth.

Osmerus mehereus Buch. cf. Saurus ophiodon Cuv.

Osphromenus 1, 553 .

Osphromenus Deissneri Blkr. 235, 376. cf. Parosphromenus Deissneri Blkr.

Osphromenus gorami Lac. cf. Osphromenus olfax Comm.

Osphromenus gourami Lac. cf. Osphromenus olfax Comm.

Osphromenus Leerii Blkr. 356, 173.

Osphromenus notatus $K . v . K$. cf. Osphromenus olfax Comm.

Osphromenus olfax $C . V . \mathbf{5 1 9}, \mathbf{2}$. cf. Osphromenus olfax Comm.

Osphromenus olfax Comm. 16, 4. 21, 10. 25, 8. 26, 4. 35, 26r. 42, 59. 45 , I95. 49, 419. 55, 4II. 170, 476, 477. 239, 101. 270, 34 . $272,9.288,70.356$, I 73.425 , I 36.459, S5. 495, I5. 498, I 7 . Osphromenus satyrus Blkr. 2. 5r9. cf. Osphromenus olfax Comm.

Osphromenus siamensis Gthr. 356, 173 .

Osphromenus striatus Gthr. 356, 173. cf. Ctenops vittatus Blkr.

Osphromenus trichopterus Gthr. 356, I73.

Osphromenus trichopterus Kner cf. 'Trichopodus trichopterus Lac.

Osphromenus trichopterus var. Cantoris Gthr. cf. 'Trichopodus trichopterus Lac.

Osphromenus trichopterus var. Koelreuterei Gthr. cf. Trichopodus trichopterus Lac.

Osphromenus trichopterus var. Leerii Gthr. cf. Trichopodus trichopterus Lac.

Osphromenus vittatus $K$. v. $H$. cf. Ctenops vittatus $B l k r$., Trichopus striatus Blkr.

Osteobrama Hcck. 261, 432. 314, 201.

Osteogeneiosus Blk\% 306, 93 . 
Osteogeneiosus Blochii Blkr. 3, I75. 4, 288. 5, 41. 16, 5. 25, Iо.

Osteogeneiosus Cantoris Blkr. 91, I20.

Osteogeneiosus gracilis Blkr. 3, I75. 4, 288. 5, 41. 7, 7. 25, Iо.

Osteogeneiosus ingluvies Blkr. 3, I 74. 4, 287. 5, 40.

Osteogeneiosus longiceps Blkr. 3, I 74. 4, 287. 5, 40.

Osteogeneiosus macrocephalus Blkr. 3, r73. 4, 287. 5, 39. 7, 6. 16, 5,25, I0. 272, IS. 287, 59 .

Osteogeneiosus militaris Blkr. 51, 58. 91, 58. 288, 78. 306, 93.

Osteogeneiosus Valenciennei Blkr. 235, 370.

Osteogeneiosus Valenciennesi Blkr. 3, I75. 4, 288. 5, 4I. 227, 408. 287, 59 .

Osteoglossum formosum M. Schl. 35, 262. 42, 60. 45, 197. 49, 436. 55, 414. 235, 372. 270, 58. 272, 27.

Osteoglossum prionostoma Basil. cf. Coilia (Chaetomus) Playfairi Blkr.

Osteorhynchus Lac. 468, 259.

Ostichthys aureus Langsd. cf. Myripristes japonicus $C . V$.

Ostorhinchus Fleurieu Lac. cf. Amia aurea Blkr., Apogon roseipinnis C. $V$.

Ostracion Art. 1, 552. 2, 506. 367, I5.

Ostracion aculeatus Houtt. cf. Aracana aculeata Gïnth.

Ostracion arcus Bl. Schn. 358, 183. cf. Ostracion (Acanthostracion) arcus Blkr.

Ostracion (Acanthostracion) arcus Blkr. 364, 273. 368, 30. 395, 290. 425, I21. 459, 70. 491, 56. 495, 23. 497, 27.

Ostracion (Ostracion) arcus $B l$. Schn. 394, 285 .

Ostracion (Aracana) auritus Shaw 119, 26.

Ostracion bicuspis Blum. cf. Ostracion concatenatus Bl., Ostracion (Acanthostracion) concatenatus Blkr.

Ostracion brevicornis T. Schl. cf. Ostracion diaphanus Bl. Schn.

Ostracion brevicornis Schl. cf. Ostracion (Acanthostracion) cornutus Blkr.

Ostracion concatenatus Bl. 266, 57. 331, 443. cf. Ostracion (Acanthostracion) concatenatus Blkr.

Ostracion (Acanthostracion) concatenatus Blkr. 425, I 2 1. 459, 70. $497,27$.

Ostracion cornutus $L .17,4.47,228.51,60.54,235.63,32.65$, 490. 156, $218.157,236.184,204.188,462.196,37.199,45$. $235,374.237,439.270,65.275$, 10. 288, 68. 296, 102. 315, 230. cf. Ostracion (Acanthostracion) arcus Blkr., Ostracion (Ostracion) arcus Bl. Sclun., Ostracion (Acanthostracion) cornutus Blkr.

Ostracion cornutus Bl., Blkr.90, 54. cf. Ostracion (Acanthostracion) arcus Blkr.

Ostracion (Acanthostracion) cornutus Blkr. 364, 273. 368, 30. 425, I 2 I. $497,27$.

Ostracion cubicus Blkr. 188, 462. 
Ostracion cubicus L., Bl. 9. 639. 47, 231. 54, 235. 63, 35. 157, 236. $270,65.275$, го. $287,38.315,230.318,250.338,360.354$, 143. cf. Ostracion (Ostracion) tetragonus $L$.

Ostracion cubicus $B$ l. var. immaculatus Blkr. 90, 55 .

Ostracion cyanurus Rïpp. 47, $23 \mathrm{I}$.

Ostracion diaphanus Bl. Sihn. 175, 38. 266, 57. cf. Ostracion (Acanthostracion) cornutus Blkr.

Ostracion (Aracana) flavigaster Rich. 119, I 7 .

Ostracion Fornasini Bianc. cf. Ostracion (Acanthostracion) Fornasini Blkr.

Ostracion (Acanthostracion) Fornasini Blkr. 364, 273. 368, 30. 459, 7०. 495,23 .

Ostracion (Acanthostracion) gibbosus Blkr. 497, 27.

Ostracion (Acanthostracion) Gronovii Blkr. 365, 302.

Ostracion (Acanthostracion) guineënsis Blkr. 365, $3 \circ 2$.

Ostracion immaculatus $T$. Sclul. cf. Ostracion cubicus $B l$. var. immaculatus Blkr.

Ostracion lenticularis Rich. 119, I7.

Ostracion lentiginosus $\mathrm{Bl}$. Schn. cf. Ostracion punctatus Lac.

Ostracion maculatus $Q . G .266,57$.

Ostracion maculatus Holl. cf. Ostracion (Acanthostracion) tricornis $L$.

Ostracion meleagris Shaze cf. Ostracion punctatus Lac.

Ostracion nasus Bl. 63, 33. 267, 139. 287, 38. 288, 68.

Ostracion (Ostracion) nasus Bl. 358, I83. 364, 273. 368, 3 I.

Ostracion notacanthus Blkr. 365, $3 \circ 3$.

Ostracion (Aracana) ornatus Rich. 119, I 7.

Ostracion (Ostracion) ornatus Guich. apud Holl. 368, 3 I.

Ostracion pentacanthus Blkr. 163, $9^{8 .}$

Ostracion punctatus Lac. 149, 108. 315, 230.

Ostracion punctatus Ehr. cf. Ostracion cubicus Bl.

Ostracion (Ostracion) punctatus Lac. 358, I83. 364, 273. 368, 3 I. 388,27 1. 394, 285. 459, 70. 491, 56. 495, 23.

Ostracion quadricornis Bl. cf. Ostracion (Acanthostracion) Gronovii Blkr.

Ostracion quadricornis Blkr. cf. Ostracion (Acanthostracion) guineensis Blkr.

Ostracion quadricornis L. 270, 65. 302, 20. cf. Ostracion (Acanthostracion) quadricornis $B l k r$.

Ostracion (Acanthostracion) quadricornis L., Blkr. 365, 302. 368, 30. $459,70$.

Ostracion (Ostracion) Renardi Blkr. 142, 68. 358, I83. 364, 273. $368,32$.

Ostracion rhinorhynchos Blkr. 63, 34. 65, 490. 126, 344. 215, 26 I. 227, 409. 275, I0.

Ostracion (Ostracion) rhinorhynchus Blkr. 368, 31. 394, 285. 
Ostracion (Ostracion) Sebae Blkr. 47, 259. 63, 34. 364, 273. 368, 32 . $491,56$.

Ostracion (Ostracion) solorensis Blkr. 80, 96. 318, 250. 354, I 43 . 364, 273. 368, 33. 394, 285 .

Ostracion (Aracana) spilogaster Rich. 119, 27.

Ostracion stellifer $\mathrm{Bl}$. Schn. cf. Ostracion concatenatus $B l$.

Ostracion stictonotus T.Schl. 90, 54.93, I 5. cf. Aracana aculeata Gïnth.

Ostracion striatus Shaw cf. Ostracion (Aracana) auritus Shaze.

Ostracion tesserula Cant. 54, 305. 288, 68. cf. Ostracion (Ostracion) tetragonus $L$.

Ostracion tetragonus L. 358, I 83 .

Ostracion (Ostracion) tetragonus L. 364, 273. 368, 32. 394, 285. 425, โ 2 I. $459,70.491,56.495,23.497,27$.

Ostracion (Acanthostracion) tricornis L. 365, 302 .

Ostracion (Ostracion) triqueter L. 459, 70.

Ostracion turritus Forsk. 63, 31. 90, 54. 93, I5.

Ostracion turritus Forst. cf. Ostracion (Acanthostracion) gibbosus Blkr.

Ostracion (Tetrosomus) turritus Forsk., Swns. 358, I83. 364, 273. $368,29$.

Ostracion undecimaculeatus Smith cf. Ostracion diaphanus Bl. Schn.

Ostracion Valentini Blkr. 9, 639. cf. Ostracion cornutus $L$.

Ostracium cornutum Cant. cf. Ostracion cornutus $L$.

Otolithi 468, 329 .

Otolithus Cuv. 1, 553. 468, 330.

Otolithus aequidens $C . V .266,64.468,329$.

Otolithus amazonicus $B l k r .363,257$.

Otolithus argenteus $\left(K . v . H_{.}\right)$C. $V .2,522.10,66.16,4.18,15$. 25, 7. 32, I60. 46, 212. 51, 56. 56, 444. 91, 36. 156, 213. 270, 23. 272 , I 2. $275,33.288,72.356$, I 73. 425, I 4 r. 452, 9. 459, I04. Otolithus aureus Rich. 425, 141 .

Otolithus biauritus Cant. 51, 56. 178, 3. 272, 1 2. 288, 72. cf. Collichthys biauritus Gthr.

Otolithus biauritus $C . V .468,330$.

Otolithus bornëensis Blkr. 35, 268. 42, 58. 45, I95. 49, 4I S. 55, 411. 272, 12. cf. Pseudosciaena borneënsis Blkr.

Otolithus cayennensis Blkr. 363, $25^{8}$.

Otolithus Goldmanni B?kr. 364, $2 \$ 5$. cf I'seudosciaena Goldmanni Blkr.

Otolithus lateoides Blkr. 2, 523. 18, I6. 31, 98. 452, 7 .

Otolithus macrophthalmus Blkr. 18, 16. 25, 7. 31, 99. 235, $3^{64}$. 275, 33. 287, 49. cf. Corvina macrophthalmus Blkr., Pseudosciaena macrophthalmus Blkr.

Otolithus maculatus $(K . v . H$.$) C. V .18$, I $5.25,7.51,56.272$, I 2. 288, 72, 356, I73. 452, I2.

Otolithus microdon Blkr. 16, 10. 18, r6. 25, 7. 31, 99. 178, 2. 270, 23. 272, 12. 287, 49. 288, 72. cf. Collichthys microdon Blkr. 
Otolithus mi-uy Blkr. 425, I4I.

Otolithus Reevesii Rich. 425, I4I.

Otolithus regalis $C . V .468,330$.

Otolithus ruber C. $V .51,56.91,36.288,72.452$, II. 468, 330.

Otolithus submaculatus Blyth cf. Otolithus ruber $C . V$.

Otolithus toeroe $C$. $V$. cf. Otolithus cayennensis Blkr.

Otolithus tridentifer Rich. 425, I4I.

Otolithus versicolor C. V. 91, 36. 288, 72 .

Otolithus versicolor Cant. cf. Otolithus lateoides Blkr.

Otolithus Vogleri Blkr. 76, 253.270, 23. cf. Pseudosciaena Vogleri Blkr.

Oxuderces dentatus $V a l .425$, 129.

Oxybeles Brandesii Blkr. 36, 278. 47, 228. 54, 238. 59, 24. 107, I62. 116, 495. 173, 255 .

Oxybeles gracilis Blkr. 149, 105 .

Oxybeles Honei Rich. 119, I5.

Oxybeles lumbricoïdes Blkr. 107, I63.

Oxycephas japonicus $K p$. cf. Oxymacrurus japonicus $B l k r$.

Oxychaetodon Blkr. 468, 306.

Oxycirrhitei 468, 3 I 4 .

Oxycirrhites Blkr. 163, 39. 468, 3 r 4 .

Oxycirrhites typus Blkr. 163, 40. 459, 93. 463, I 7. 468, 314. 495, I3.

Oxyconger Blkr. 351, in6.

Oxydoras Kner 306, 85.

Oxydoras Kneri Blkr. 303, I4. 306, 85 .

Oxydoras niger Blkr. 303, I 4. 336, 34 .

Oxyeleotris Blkr. 453, 302. 460, 103, 104.

Oxyeleotris marmorata Blkr. 478, 22.

Oxyeleotris urophthalmoides Blkr. 478, 25 .

Oxyeleotris urophthalmus Blkr. 478, 23.

Oxygaster v. Hass. 1, 552. 314, 2 I 5. 329, 264.

Oxylabrax Blkr. 468, 264, 336 .

Oxyloricaria Blkr. 306, $8 \mathrm{r}$.

Oxyloricaria barbata Blkr. 306, 8I.

Oxymacrurus Blkr. 449, 370.

Oxymacrurus japonicus Blkr. 497, $2 \mathrm{I}$.

Oxymacrurus macrochir 497, 2 I.

Oxymacrurus parallelus 497, 2 I.

Oxymetopon Blkr.286, 258.453, 308. 460, то4, го6.

Oxymetopon typus Blkr. 286, 259. 320, 27I. 453, 308. 478, 109.

Oxymonacanthus Blkr. 367, I3.

Oxymonacanthus chrysospilus Blkr. 354, I 43. 358, I $\delta_{3} .364,272$.

Oxymonacanthus longirostris Blkr. 368, 27. 459, 7 r. 495, 20.

Oxymormyrus Blkr. 449, 367 .

Oxyrhina glauca $M$. H. 497, 3 .

Oxyurichthys Blkr. 453, 324 . 
Oxyurichthys auchenolepis Blkr. 469, I38.

Oxyurichthys belosso Blkr. 275, 44. 287, 56. 453, 324.

Oxyurichthys microlepis Blkr. 227, 408. 287, 56. cf. Gobionellus microlepis Blkr.

Oxyurichthys opthalmonema Blkr. 315, 236.

Oxyurichthys papuensis Blkr. 491, 54.

Oxyurichthys tentacularis Blkr. 270, 42 .

Pachylabrus Gill 468, 290.

Pachymetopon Gthr. 468, 295.

Pachymetopon grande Gthr. 468, 295.

Pachypops Gill 468, 326.

Pachypops trifilis Gill 468, 326.

Pachystomus Heck. 261, 433. 314, 203. 329, 263.

Pachyurus Sans., Agass. 351, х14. 468, 327.

Pachyurus linearis Szons. cf. Moringua raitaborua Cant.

Pachyurus squamipinnis $A g .468,327$.

Pagellus Cuv. 468, 28r.

Pagellus afer $P a p p$. 266, 52 .

Pagellus lithognathus $C . V .468,28 \mathrm{I}$.

Pagrichthys Blkr. 266, 60. 468, 28 r.

Pagrichthys Castelnaui Blkr. 266, 6r.

Pagrus Cuv. 1, 553. 468, 28r.

Pagrus cardinalis Gthr. 425, I 38 .

Pagrus chinensis Steind. 430, 234.

Pagrus filamentosus $C . V .270,22$. cf. Sparus filamentosus $B l k r$.

Pagrus guttulatus C. $V$. 119, 9. cf. Pagrus unicolor $C . V$.

Pagrus heterodon Blkr. 94, 54. 157, 232. 270, 22. 275, 28. 438, 46. cf. Sphaerodon grandoculis Rüpp.

Pagrus laniarius $C . V$. 266, 52 .

Pagrus laticeps Steind. cf. Sparus laticeps Blkr.

Pagrus latus Rich. 119, 9.

Pagrus longifilis $C . V .71,756.91,3^{8.275,28 . c f . ~ S p a r u s ~ s p i n i f e r ~ F o r s k . ~}$ Pagrus macrocephalus Basil. 425, I39.

Pagrus major Gthr. 425, I38.

Pagrus micropterus Cuv. 119, 9.

Pagrus spinifer C. $V . \mathbf{9 1}, 3^{8}$. cf. Sparus spinifer Forsk.

Pagrus unicolor C. V. 119, 9. 331, 445. 425, 138. 430, 234. cf. Pagrus chinensis Steind.

Pallasia Nardo 367, I7.

Panchax Val. 261, 44I.

Panchax Buchanani C. $V$. 25, 22. 91, I 44. 170, 476. 270, 54. 272, 25. 279, IоI. 356, I76.

Panchax Kuhlii C. $V$. cf. Panchax Buchanani $C$. $V$., Panchax melanotopterus Blkr. 
Panchax lineatum C. $V .91,72, \mathrm{I} 45$.

Panchax melanotopterus Blkr. 25, 22. 26, 3. 35, 262. 42, 6o. 45, I96. 49, 421. 55, 4I4. 58, 22. cf. Panchax Buchanani $C$. $V$.

Panchax panchax Cant. cf. Panchax Buchanani $C$. $V$.

Panchax pictum C. V. 91, I45. cf. Betta picta Blkr.

Pangasius $V a l .299,398.306$, Iо7.

Pangasius Buchanani C. $V .91$, I 8. 306, го7.

Pangasius djambal Blkr. 4, 290. 5, 57. 91, ir 8 .

Pangasius hexanema Blkr. 67, 588.

Pangasius juaro Blkr. 67, 589.

Pangasius Larnaudi Boc. 347, 37. 356, I75.

Pangasius macronema Blkr. 26, II. 35, 26г. 42, 59. 45, г96. 49, 420. 55, 413. 239, го2. 272, I 9. 356, I75.

Pangasius micronema Blkr. 225, 357.

Pangasius micronemus Blkr. 7, 8. 8, I66. 25, Iо.

Pangasius polyuranodon Blkr. 55, 425. 270, 47. 272, I9. cf. Pseudopangasius polyuranodon $B l k r$.

Pangasius rios Blkr. 45, 205. 49, 420. 55, 4r3. 272, I9.

Parabagrus Blkr. 306, I00.

Parabagrus pusillus Blkr. 306, гоo.

Parabalistes Blkr. 367, ro.

Parabembras Blkr. 449, 370.

Parabembras curtus Blkr. 497, I 2.

Parabramis Blkr. 345, 21.

Parabramis bramula Blkr. 411, 253. 415, 78. 425, 146. 496, 3 .

Parabramis pekinensis Blkr. 345, 22. 411, 252. 415, 80. 425, 146.

Paracaesio Blkr. 468, 273. 470, I52.

Paracaesio xanthurus Blkr. 459, 92. 468, 273. 470, I 52.

Paracanthistius Blkr. 468, $256,336$.

Paracanthistius leopardinus Blkr. 451, г4. 468, 256. 497, 6.

Paracanthistius maculatus Blkr. 451, x6. 459, 89. 495, Ir.

Paracanthistius melanoleucus Blkr. 459, 89. 495, II.

Paracanthistius oligacanthus Blkr. 451, I9.

Paracanthobrama Blkr. 345, 23.

Paracanthobrama Guichenoti Blkr. 345, 24. 415, I2. 425, I 45.

Paracanthurus hepatus Blkr. 318, 252. 358, I90. 364, 288. 459, 97.

491, 5.

Paracara typus Blkr. 488, I93.

Paracentropogon Blkr. 473, 297.

Paracentropogon leucoprosopon Blkr. 475, 69.

Paracentropogon longispinis Blkr. 475, 67.

Parachaetodon Blkr. 449, 371. 468, 303.

Parachaetodon ocellatus Blkr. 468, 304. 474, 3I5. 481, 35 .

Parachaetodon oligacanthus Blkr. cf. Parachaetodon ocellatus Blkr.

Parachaeturichthys Blkr. 453, 325 .

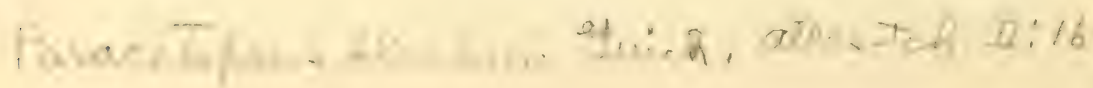


Parachaeturichthys polynema Blkr. 497, r9.

Paracheilognathus Blkr. 314, 213. 329, 264.

Paracheilognathus rhombeus Blkr. 497, 23.

Parachilognathus imberbis Blkr. 411, 252. 415, 37. 425, I44.

Parachromis Polleni Blkr. cf. Paratilapia Polleni Blkr.

Paracirrhites Blkr. 468, 3r4.

Paracirrhites amblycephalus Blkr. 463, 9.

Paracirrhites arcatus Blkr. 459, 93. 463, 8. 495, т3.

Paracirrhites cinctus Blkr. 459, 93. 495, г3.

Paracirrhites Forsteri Blkr. 459, 93. 463, 6. 468, 314. 491, 44. 495, I3.

Paracobitis Blkr. 323, 37.

Paracobitis malapterurus Blkr. 323, 40.

Paraconodon Blkr. 468, 272.

Paradentex Blkr. 468, 278 .

Paradiodon Blkr. 367, I 7 .

Paradiodon hystrix Blkr. 364, 27 1. 368, 33. 425, I 2 I. 459, 69, 495, 23 .

Paradiodon maculatus Blkr. 425, I2 1. 459, 69.

Paradiodon maculifer Blkr. 495, 23.

Paradiodon novemmaculatus Blkr. 364, 27 I. 368, 34. 491, 57.497, 26.

Paradiodon quadrimaculatus Blkr. 364, 27 I. 368, 34.

Paradiplomystes Blkr. 306, 92.

Paradiplomystes coruscans Blkr. 306, 92.

Paradules Khunz., Blkr. 468, 267, 268.

Paradules maculatus Blkr. 364, 280.

Paradules marginatus Blkr. 358, I86. 364, 280. cf. Moronopsis ciliatus Blkr.

Paradules obscurus Klunz. 468, 267.

Paradules rupestris Blkr. 320, 267. 364, 280. cf. Moronopsis rupestris Gill.

Paradules taeniurus Blkr. 425, r39. cf. Moronopsis taeniurus Gill.

Paraglyphidodon Behnii Blkr. 485, I 2 I.

Paraglyphidodon bonang Blkr. 485, II 8.

Paraglyphidodon melanopus Blkr. 485, I I9.

Paraglyphidodon melas Blkr. 483, 40. 485, I23. 497, Iо.

Paraglyphidodon oxycephalus Blkr. 476, 399. 485, I25.

Paraglyphidodon oxyodon Blkr. 485, I 27.

Paraglyphidodon xanthonotus Blkr. 485, I 26.

Paraglyphidodon xanthurus Blkr. 485, I22. 491, 47.

Paragobiodon Blkr. 453, 309 .

Paragobiodon echinocephalus $B l k r .425$, I 29. 495, 17 .

Paragobiodon melanosoma Blkr. 459, 78. 491, 54 .

Paragobiodon xanthosoma Blkr. 491, 54.

Paragobius Blkr. 453, 322.

Paragobius Knutteli $B$ lkr. 425, I28.

Paragobius sinensis Blkr. 425, I28, 152 .

INDEX BLEEKER. 
Parahemiodon Blkr. 306, 8o.

Parahemiodon typus Blkr. 298, 373. 306, 8०. 336, 20.

Paralabrax Gir. 468, 255.

Paralabrax fasciatus $B l k r .425$, I 37, I 50.

Paralabrax guttatus Blkr. 425, I37, I50.

Paralaubuca typus Blkr. 344, I6. 356, I 76.

Paralonchurus Boc. 468, 325.

Paralonchurus Petersii Boc. 468, 325.

Paraluteres Blkr. 367, I4.

Paraluteres prionurus Blkr. 368, 28. 491, 53 .

Paralycodes Blkr. 449, 369 .

Paramacrurus Blkr. 449, 370.

Parambassis Blkr. 468, 292.

Parambassis apogónides Blkr. 457, I9. 468, 292.

Parambassis microlepis Blkr. 457, 20.

Paramia Blkr. 468, $26 \mathrm{r}$.

Paramia amblyuropterus Blkr. 354, ז47. cf. Pseudamia amblyuropterus Blkr.

Paramia lineata Blkr. 364, 284. 459, 93. cf. Paramia macrodon Blkr.

Paramia macrodon Blkr. 456, 75. 459, 94. 468, 26r. 495, Ir.

Paramia octolineata Blkr. cf. Paramia macrodon Blkr.

Paramia quinquelineata $B l k r .315,233.354$, I 47. 364, 284. 394, $287.456,76$.

Paramia singapurensis Blkr. 456, 78 .

Paramonacanthus Blkr. 367, I2.

Paramonacanthus Broekii Blkr. 497, I 7 .

Paramonacanthus choirocephalus Blkr. 368, 27.

Paramonacanthus cryptodon Blkr. 368, 27 .

Paramonacanthus curtorhynchus Blkr. 368, 27.

Paramonacanthus Knerii Steind. 425, I 22.

Paramonacanthus lineolatus Blkr.425, I22.

Paramonacanthus modestus Blkr. 497, I 7 .

Paramonacanthus nematophorus Blkr. 425, r22.

Paramonacanthus nemurus Blkr. 368, 27.

Paramonacanthus oblongus Blkr. 497, I7.

Paramonacanthus setifer Blkr. 425, I22.

Paramonacanthus trachyderma Blkr. 497, I7.

Paranandus Day 468, 3I8.

Parancistrus Blkr. 306, 79.

Parancistrus aurantiacus Blkr. 306, 79 .

Paranguilla Blkr. 351, II3.

Paranthias Guich. 468, 253.

Parapegasus natans $L .491,49$.

Parapercis cancellata Blkr. 318, 252.320, 27 I. 354, I 49. 364, 292.437, 37.

Parapercis cylindrica Blkr. 315, 236. 318, 252. 320, 271. 338, 36r. 
339, 362. 354, I49.358, г 9г. 364, 292. 394, 288. 425, г27.437, 37. 491, 53 .

Parapercis hexophthalmus Blkr. 388, 272. 459, 78. 491, 53.

-Parapercis nebulosa Blkr. 459, 78 .

Parapercis pulchella Blkr. 425, I27. 497, I 8.

Parapercis punctulata Blkr. 459, 78. 495, I 7 .

Parapercis sexfasciata $B l k r .408,238.425,127.497$, r 8.

Parapercis tetracanthus Blkr. 354, I49. 364, 292.

Paraphoxinus Blkr. 314, 209. 329, 263.

Paraplagusia bilineata Blkr. 364, 274. 459, 77.

Paraplagusia japonica Blkr. 497, 22.

Paraplagusia marmorata Blkr. 364, 274. 459, 77. 497, 22.

Paraplesichthys $K p$. 468, 335 .

Paraplesiops Blkr. 468, 322.

Paraploactis Blkr. 473, 300.

Paraploactis trachyderma Blkr. 355, I69. 473, 300.

Parapocryptes Blkr. 453, 327 .

Parapomacentrus Bankieri Blkr. 483, 39. 485, 67.

Parapomacentrus polynema Blkr. 485, 65 .

Parapriacanthus Steind. 468, 3 I 2.

Parapriacanthus Ransonneti Steind. 468, 312. 497, 9.

Parapristipoma Blkr. 468, 27 r.

Parapristipoma trilineatum Blkr. 443, 22. 468, $271.497,7$.

Parapterois Blkr. 473, 296.

Parapterois heterurus Blkr. 475, 55.

Pararhynchobdella Blkr. 449, 368.

Paraschizothorax Blkr. 329, 262.

Parascorpaena Blkr. 473, 295.

Parascorpaena bandanensis Blkr. 475, 26. 491, 48 .

Parascorpaena mauritiana Blkr. 459, 87. 495, г6.

Parascorpaena picta Blkr. 459, 87. 475, 25.

Parascorpis Blkr. 462, 380. 468, 277.

Parascorpis typus Blkr.462, 38I. 468, 277.

Parascyllium variolatum Gill 425, I 19.

Paraserranus Blkr. 468, 255.

Paraserranus Hasseltii Blkr. 451, 7. 468, 255.

Parasilurus Blkr. 299, 394. 306, II4.

Parasilurus asotus Blkr. 425, I25.

Parasilurus japonicus Schl., Blkr. 306, i I 4. 363, 268.408, 23S, 497, 22.

Parastromateus niger Blkr. 356, r 74 .

Paratilapia Polleni Blkr. 399, 308. 459, Io, S4.

Parauchenipterus Blkr. 306, 88.

Parauchenipterus galeatus Blkr. 306, 88, 336, 45.

Pardachirus marmoratus Gthr. cf. Achirus marmoratus Lac.

Pareiodon Kner 306, II 3 . 
Pareiodon microps Kner 306, II3.

Pareleotrini 453, 306. 460, I03.

Parephippus Gill 468, 300.

Parepinephelus Blkr. 468, 257.

Paretroplus Damii Blkr. 399, 3 г3. 459, I3, 84.

Paretroplus polyactis Blkr. 488, 195 .

Parexocoetus mento Blkr. 372, I26.496, 3 .

Paristiopterus Blkr.468, 268.

Parma microlepis Gthr. cf. Hypsipops microlepis Blkr.

Parophrys bicoloratus Blkr. 425, I30.

Parophrys cornuta Gthr. 425, I30.

Parosphromenus Deissneri Blkr. 498, 20.

Paroxyurichthys typus Blkr. 469, I4I.

Parupeneus Blkr. 468, 334 .

Parupeneus barbarinoides Blkr. 315, 234. 358, I89. 364, 285. 464, 22. cf. Parupeneus Kneri Blkr.

Parupeneus barberinus Blkr. 315, 234. 320, 269. 354, I48. 358, г89. $364,285.437,38.459$, 104. 464, 25. 468, 334. 491, 46.

Parupeneus bifasciatus Blkr. 402, 345. 459, 40, 80. 495, I4.

Parupeneus bilineatus Blkr. 364, 285. 464, 38 .

Parupeneus Brandesi Blkr. 364, 285. cf. Parupeneus pieurostigma Blkr.

Parupeneus cherserydros Blkr. 459, 80. 464, 35. 491, 46. 495, I 4 .

Parupeneus chrysopleuron Blkr. 425, 142.

Parupeneus crassilabris Blkr. 464, 33. 491, 46.

Parupeneus cyclostomus Blkr. 364, 285. 459, 8o. 495, I4.

Partipeneus cyprinoides Blkr. 459, 80. 495, I4.

Parupeneus dispilurus Blkr. 459, I04.

Parupeneus fraterculus $B l k r .459,80$.

Parupeneus indičus Blkr. 354, I48. 364, 285. 396, 297, 299. 425, I 42. 459,8 o. $464,27.497$, Iо.

Parupeneus Janseni Blkr. 354, I47. 364, 285. 464, 34 .

Parupeneus Kneri Blkr. 425, I42.

Parupeneus luteus Blkr. 364, 285. 459, 80. 464, 32. 491, 46. 495, I 4.

Parupeneus macronema Blkr. 437, 38.459, 80. 464, 24. 495, I 5.

Parupeneus macronemus Blkr. 364, 285.

Parupeneus multifasciatus Blkr. 402, 346. 425, I42. 437, 38. 459, 42. 464, 20. 491, 46. 495, 15. 496, 2.

Parupeneus oxycephalus Blkr. 364, 285. cf. Parupeneus cherserydros Blkr.

Parupeneus pleurospilus Blkr. 338, 360. 364, 285. 464, 31. 497, Iо.

Parupeneus pleurostigma Blkr. 459, So. 464, 29. 495, I 5 .

Parupeneus Russelli Blkr. 315, 234. 320, 269. 358, I89. cf. Parupeneus indicus Blkr.

Parupeneus spilurus Blkr. 491, 63. 497, Io. 
Parupeneus trifasciatus Blkr. 315, 234. 316, 242. 318, 251. 320, 269. 358, I89. 364, 285. cf. Parupeneus multifasciatus Blkr.

Parupeneus xanthospilurus Blkr. 464, 37.

Pastinaca akajei Bürg. cf. Trygon akajei $M$. $H$.

Pastinachus uarnak Rüpp. 2, 507. cf. Trygon uarnak M. H.

Pataecus fronto Rich. 119, 8.

Pegasus draco L. 459, 76 .

Pegasus draconis $L .89,27.296$, го3. 425, I 25.

Pegasus latirostris Rich. cf. Pegasus draconis $L$.

Pegasus natans L. 89, 28. 156, 2 I 8. 270, 64. 287, 40. cf. Parapegasus natans $L$.

Pegasus natans Rich. 89, 28. cf. Leptopegasus natans Blkr.

Pegasus natans $\mathrm{Val}$. cf. Pegasus draconis $L$.

Pegasus pristis Blkr. 67, 606. cf. Parapegasus natans $L$., Pegasus natans $L$.

Pegasus volans Rich. cf, Leptopegasus natans Blkr., Pegasus natans $L$.

Pegasus volans L. 54, 307. 425, I 25. cf. Pegasus draconis $L$.

Pegasus volitans $L$. 358, r83. 364, 274.

Pegusa impar Gthr. 333, $45^{8 .}$

Pelamis coerulea Aldrovand. cf. Thynnus thunnina $C . V$.

Pelamys japonicus Blkr. 497, I5.

Pelamys macropterus Blkr. 364, 289. 497, r5.

Peiamys orientalis Schl. cf. Sarda orientalis Blkr.

Pelamys pelamys Blkr. 364, 289. 497, I5.

Pelamys sarda $C . V .459$, I00.

Pelamys sibi Blkr. 497, I5.

Pelamys thunnina Blkr. 364, 289 .

- Pelamys thunninus Blkr. 497, I5.

Pelates C. $V .468,267$.

Pelates quadrilineatus $C . V .468,267$. cf. Therapon Cuvieri Blkr., Therapon (Pelates) quadrilineatus $B l k r$.

Pelates quinquelineatus $C$. $V$. cf. Therapon Cuvieri Blkr., 'Therapon (Pelates) quadrilineatus Blkr.

Pelates sexlineatus $C . V$. cf. Therapon Cuvieri Blkr., Therapon (Pelates) quadrilineatus Blkr.

Pelecus $A g .261,439.314 .215 .329,264$.

Pellona affinis Cant. 270, 57. 272, 26. 287, 6r. 288, 79. cf. Pellona Grayana C. $V$.

Pellona africana Blkr. 302, 122.

Pellona amblyuropterus Blkr. 60, 21. 270, 57. 272, 26.

Pellona brachysoma Blkr. 60, 22. 270, 57.387, 6r.

Pellona ditchela $C . V .91,72$.

Pellona ditchoa C. $V .60,24.91,72.127,345.267$, I $40.270,57$. 287, 6r.

Pellona Dussumierii $C . V .91,72$. 
Pellona filigera $C . V .91,72$.

Pellona Grayana $C . V .32$, I60. 35, 262. 42, 60. 45, г96. 49, 42 I. $51,59.55,414.56,445.60,25.191,2.227,408$. cf. Ilisha elongata $B$ lkr.

Pellona Hoevenii Blkr. 60, 21. 69, 7 I2. 127, 345. 270, 57. 275, 49. 287, 61. 309, I56. 316, 243. 356, г76. 358, I92. 364, 295.

Pellona Iserti $\mathrm{Val}$. cf. Pellona africana Blkr.

Pellona Leschenaultii $C . V$. 16, 6. 25, I I. 91, 72.

Pellona Leschenaultii Blkr. cf. Pellona Russellii Blkr.

Pellona melastoma $C . V .91,72$.

Pellona micropus C. $V .91,72$.

Pellona motius C. $V . \mathbf{9 1}, 72$.

Pellona novacula $V a l$. cf. Ilisha novacula $B l k r$.

Pellona pristigastroides Blkr. 60, 20. 178, 2. 272, 26.

Pellona Russellii Blkr. 51, 72. 55, 4I4. 60, 23. 91, 74. 235, 372. 272, 26. 364, 295. cf. Ilisha megalopterus Blkr.

Pellona Schlegelii Blkr. 102, 418. 118, Ir8. cf. Ilisha elongata Blkr.

Pellona soborni C. V. 91, 74 .

Pellona xanthopterus Blkr. 49, 439. 55, 414. 60, 49. 272, 26.

Pelor aurantiacum Schl. 425, I4I. 497, 12.

Pelor brachyrhynchus Blkr. 458, 5 .

Pelor Cuvieri Gray 76, 252. 270, 25. 287, 49. 425, I4 I. 458, 8, 9. Pelor didactylus Blkr., Gthr. 287, 49. 296, ro7. 340, 365. 358, r89. 364, 286. 425, I 4 I. 458, 7 .

Pelor filamentosum $C . V .459,87.495$, I6.

Pelor japonicum C. $V$., Schl. 193, 3. 425, I4I. 497, I2.

Pelor maculatum $C . V .14,4.396,297.425$, I4I. cf. Pelor didactylus Gthr.

Pelor obscurum C. V. 83, 24r. 275, 32. cf. Pelor didactylus Gthr.

Pelor sinense $C . V .425$, I4r. cf. Pelor Cuvieri $G r$.

Pelor tigrinum Rich. cf. Pelor japonicum $C . V$.

Pelteobagrus Blkr. 342, 9.

Pelteobagrus calvarius Blkr.425, 125. 496, 3 .

Pempherichthys Klunz. 468, 312.

Pempheridoidei. 468, 3 I 2.

Pempheris Cuv. 1, 553. 468, 3 I2.

Pempheris adustus Blkr. 484, 50.

Pempheris malabarica C. $V$. cf. Pempheris mangula $C \cdot V$., Pempheris moluca $C \cdot V$.

Pempheris mangula C.V. 2, 52r. 22, 30.54, 235. 91, 40. 222, 3 I6. 275, 36. 309, 156. 364, 287. 459, 94. 484, 45. 491, 44. 495, г4. Pempheris mangula Gthr. cf. Pempheris adustus Blkr.

Pempheris mangula Kner cf. Pempheris vanicolensis C. $V$.

Pempheris moluca, molucca C. $V$. 2, 521. 22, 30. 91, 42. 167, 37 I. 
185, 223. 275, 36. 287, 51. 288, 73. 315, 235. 320, 270. 364, 287. 425, 133. 497, 9. cf. Pempheris mangula C. $V$.

Pempheris moluca Blkr. cf. Pempheris vanicolensis C. $V$.

Pempheris nesogallica $C . V .125,314$. cf. Pempheris mangula $C . V$, Pempheris vanicolensis $C . V$.

Pempheris otaitensis C. V., Kner 22, 30. 425, I33. 484, 5I. cf.

Pempheris mangula $C$. $V$., Pempheris oualensis $C . V$.

Pempheris oualensis $C . V .47,242.275,36.309$, I $56.315,235$. $320,270.364,287.484,47$.

Pempheris Schwenkii Blkr. 125, 314. 157, 233. 270, 27. 484, 52.

Pempheris taitensis $C$. $V$. cf. Pempheris mangula $C$. $V$., Pempheris otaitensis $C . V$.

Pempheris touea Cuv. 468, 312.

Pempheris vanicolensis $C . V . \mathbf{2 2}, 30.484,48$. cf. Pempheris mangula $C$. $V$.

Pentaceros C. $V .468,269$.

Pentaceros capensis C. $V .266,52.468,269$.

Pentaceros Richardsoni Smith 266, 52. 468, 270.

Pentacerotiformes 468, 269.

Pentanemus Art. 302, 86.

Pentanemus quinquarius Gthr. 302, 86.

Pentapodus nubilus Cant. cf. Pentapus nubilus Gthr.

Pentaprion Blkr. 468, 285.

Pentaprion gerreoides Blkr. 20, 13. 31, 104. 270, 24. 275, 29. 440, 254. 468, 285 .

Pentaprion longimanus Blkr. 468, 285.

Pentapus C. V. I, 553. 438, 41. 468, 279.

Pentapus aureolineatus $C . Y .94,55.188,459.309$, I $55.364,2 S_{3}$.

438, 41. 468, 279. cf. Gnathodentex aurolineatus BlkT.

Pentapus bifasciatus Blkr. 395, 29x. cf. Pentapus caninus Blkr.

Pentapus caninus Blkr. 438, 43. 441, 6r. 491, 43.

Pentapus curtus Guich. 459, 9I.

Pentapus dux Cuv. 459, $9 \mathrm{I}$.

Pentapus Hellmuthi Blkr. 394, 287. 441, 57. 491, 43.

Pentapus iris $C$. 119, 9.

Pentapus macrurus Blkr. 441, 60.

Pentapus microdon Blkr. 441, 56.

Pentapus nemurus Blkr. 398, 306. 437, 38. 441, 58.

Pentapus nubilus Cant., Gthr. 288, 7 1. 441, 53.

Pentapus paradiseus Gthr. cf. Pentapus setosus C. $V$.

Pentapus setosus $C . V .40,478.44,175.51,56.52,89.126,344$.

185, 222. 228, 424. 235, 364. 275, 27. 296, I06. 395, 291. 441, 54.

Pentapus vitta $C$ v. 119, 9 .

Pentapus vittatus C. V. 459, 91. 468, 279.

Pentapus xanthopleura Kner cf. Pentapus caninus Blkr.

Pentaroge Gthr. 473, 299. 
Pentaroge marmorata Gthr. cf. Gymnapistus marmoratus Swns.

Perca Art. 468, 265.

Perca aberrans Poey 468, 262.

Perca amboinensis Will. cf. Pseudomonopterus (Pterois) volitans Blkr.

Perca arcata Parkins cf. Paracirrhites arcatus Blkr.

Perca argentata Bl. Schn. cf. Lutjanus argentimaculatus Blkr.

Perca argentea Benn. cf. Moronopsis taeniurus Gill.

Perca aurata Mungo Park cf. Scolopsides Vosmeri C. V., Scolopsis Vosmaeri $C . V$.

Perca bilineata Thunb. cf. Grammistes orientalis Bl. Schn.

Perca chuantsi Basil. cf. Siniperca chuatsi Gill.

Perca chuatsi Basil. cf. Siniperca chuatsi Gill.

Perca ciliata K. v. H. 13, 3. cf. Moronopsis ciliatus Blkr.

Perca cirrhosa Thunb. cf. Scorpaena cirrhosa $C V$., Scorpaenopsis cirrhosus Blkr.

Perca cottoïdes $L$. cf. Apistus cottoïdes $C$. $V$., Cottapistus cottoides $B l k r$.

Perca diagramma $L$. cf. Diagramma lineatum $C$. $V$.

Perca fasciata Forsk. cf. Epinephelus fasciatus Blkr.

Perca flavo-purpurca Benn. cf. Epinephelus flavocoeruleus Blkr.

Perca fluviatilis $L .468,265$.

Perca fulviflamma $B l$. Schn. cf. Lutjanus fulviflamma Blkr., Mesoprion fulviflamma $B$ lkr.

Perca fusca Thunb. cf. Epinephelus boelang Blkr.

Perca grunniens Forst. cf. Pristipoma guoraca $C$. $V$.

Perca hexagonata Forst. cf. Epinephelus merra Bl., Serranus hexagonatus $C . V$.

Perca lineata $L$. cf. Diagramma lineatum $C$. $V$., Plectorhynchus lineatus $B l k r$.

Perca louti Forsk. cf. Variola louti Blkr.

Perca lunulata $M$. Park 438, 44. cf. Lutjanus lunulatus Bl. Schn., Mesoprion lunulatus $C$. $V$.

Perca maculata Forst. cf. Serranus variolosus $C . V$.

Perca miniata Forsk. 113, 42 r. cf. Epinephelus miniatus Blkr., Plectropoma leopardinus $C$. $V$.

Perca murdjan Lac., Bl. Schn. cf. Myripristis murdjan Rüpp.

Perca pentacantha Lac. cf. Grammistes orientalis Bl. Schn.

Perca pertusa Thunb. cf. Plectorhynchus pertusus Blkr.

Perca picta Thunb. cf. Diagramma punctatum Ehr., Plectorhynchus pictus Blkr.

Perca polymna $L$. cf. Prochilus polymnus Blkr.

Perca polymna Bonn. cf. Prochilus Sebae Blkr.

Perca polyzonias J. R. Forst. cf. Lutjanus bengalensis Blkr., Mesoprion octolineatus Blkr.

Pérca pulchella Benn. cf. Holocentrum diadema $C . V$.

Perca rubra $B l$. Schn. cf. Holocentrum rubrum Rüpp. 
Perca saltatrix $L$, cf. Temnodon saltator $C$. $V$.

Perca samara, sammara $B l$. Schn. cf. Holocentrum sammara $C$. $V$.

Perca scandens Dald. cf. Anabas scandens $C$. $V$., Anabas testudineus $C u$.

Perca septemfasciata Thunb. cf. Epinephelus semipunctatus Blkr.

Perca spinifera Bl. Schn. cf. Holocentrum spiniferum Gthr.

Perca summana Forsk. cf. Epinephelus summana Blkr.

Perca taeniata J. R. Forst. cf. Paracirrhites Forsteri Blkr.

Perca tauvina Forsk. cf. Epinephelus Gilberti Blkr.

Perca triacantha Lac. cf. Grammistes orientalis $B l$. Schn.

Perca trilineata Thunb. cf. Parapristipoma trilineatum Blkr.

Perca urodela Forst. cf. Epinephelus urodelus Blkr., Serranus urodelus C. $V$.

Perca variolosa Forst. cf. Epinephelus variolosus Blkr., Serranus variolosus $C . V$.

Perca vittata Park. cf. Lutjanus bengalensis Blkr., Mesoprion octolineatus Blkr.

Percae 468, 247.

Percaeformes 468, 258, 334.

Perca-Labrax Schl. 468, 263.

Perca-Labrax japonicus 'Temm. Schl. cf. Labrax japonicus $C . V$.

Percamia Blkr. 468, 260.

Percarina Nordm. 468, 266.

Percarina Demidoffii Nordm. 468, 266.

Percichthys Gir. 468, 265.

Percichthys chilensis Gir. 468, 265.

Percichthys ciliata Gthr. cf. Moronopsis ciliatus Blkr.

Percilia Gir. 468, 265 .

Percini 468, 265 .

Percis Klein 1, 553. 468, 266.

Percis cancellata C. $V .141$, 501, 236, 331. 309, I56. cf. Parapercis cancellata Blkr.

Percis caudimaculata Rüpp. 13, 54. 81, I63. 188, 459. 275, 43.

Percis colias C. V. 119, 6 .

Percis cylindrica C.V. 13, 55. 47, 235. 167, 37 I. 184, I99. 275, 43. 287, 56. cf. Parapercis cylindrica Blkr.

Percis cylindrica Rüpp. cf. Percis caudimaculata Rupp.

Percis Emeryana Rich. 90, 26. 119, 6.

Percis hexophthalma Ehr., C. $V$. 2, 527. cf. Parapercis hexophthalmus Blkr., Percis caudimaculata Rüpp.

Percis maculata Bl. Sclın. 91, 34 .

Percis nebulosa $C . V .90,26.119,6$.

Percis nebulosa Q.G. cf. Parapercis nebulosa Blkr.

Percis nycthemera $C . V .119,6$.

Percis ocellata C. V. 13, 4 .

Percis polyophthalma Ehr. cf. Percis caudimaculata Rüpp. 
Percis pulchella T. Schl. 90, 26. 253, 235. cf. Parapercis pulchella Blkr.

Percis punctulata $C$. $V$. cf. Parapercis punctulata Blkr.

Percis sexfasciata $T$. Schl. 118, 66. 253, 235. cf. Parapercis sexfasciata Blkr.

Percis tetracanthus Blkr. 78, 458. 167, 37r. 236, 331. 270, 40.

Percis xanthozona Blkr. 13, 55. 287, 56 .

Percoidei 468, 248.

Percosoma Gill 468, 265.

Perilampus $M c$ Cl., Blkr. 261, 434. 314, 203, 2 r 4. 328, 258. 329, 264.

Perilampus devario $M c C l .328,258$.

Perilampus guttatus $M_{c} C l$. cf. Leuciscus laubuka $C . V .91$, I38.

Perilampus macropterus $M c C l$. cf. Nuria danrica $C$. $V$.

Perilampus macrourus $M c C l$. cf. Nuria danrica $C$. $V$.

Perilampus recurvirostris $\mathrm{Mc} C l$. cf. Nuria danrica $C . V$.

Periophthalmini 453, 326.

Periophthalmodon Blkr. 453, 326.

Periophthalmodon modestus Blkr. 497, I9.

Periophthalmus Bl. Schn. 453, 326.

Periophthalmus argentilineatus $C . V$. 15, 4. 54, 276. 156, 215. 147, 469. 235, 370. 270, 43. 315, 236. 320, 27 1. 338, 361. 354, I 5 0. 358, 192. 364, 292. 382, 398. 388, 272. 396, 297, 300. cf. Euchoristopus argentilineatus $B \mathrm{lkr}$.

Periophthalmus bornëensis Blkr. 26, II. 35, 261. 42, 59. 45, 195. 49, 4I9. 55, 4I2. 270, 43. 272, I7. 356, I74.

Periophthalmus chrysospilos Blkr. 70, 728, 281, I08.

Periophthalmus dipus Blkr. 110, 320. 270, 43. 318, 252.

Periophthalmus Freycineti C. V. 15, 4. 320, 271. 491, 54.

Periophthalmus kallopterus Blkr. 84, 342.

Periophthalmus kalolo Less. cf. Periophthalmus Koelreuteri $V$ al.

Periophthalmus Koelreuteri Bl. Schn. 15, 4. 91, 50.

Periophthalmus Koelreuteri C. $V .34,252.91,50.354$, I50. 382 , 398. 396, 298. 453, 326. 459, 78. cf. Euchoristopus Koelreuteri Gill.

Periophthalmus Koelreuteri Blkr. cf. Periophthalmus dipus Blkr.

Periophthalmus modestus $T$. Schl. 253, 235. 269, 82. 425, I29. cf. Periophthalmodon modestus Blkr.

Periophthalmus novemradiatus $C . V .91,50$.

Periophthalmus papilio $B l$. Schn. 91, I66. 302, 104. 453, 326.

Periophthalmus Schlosseri Bl. Schn., C. $V$. 15, 39. 25, 10. 51, 58 .

191, 2. 272, I7. 288, 77. 356, I75. 364, 293. 396, 300. 453, 327.

Periophthalmus septemradiatus $C . V .91,50$.

Periophthalmus tredecimradiatus $C . V .91,50$.

Peristedion moluccense Blkr. 27, 24, 47, 226. 142, 30.

Peristedion orientale Sihl. 238, 408. 497, I3.

Petrometopon Gill 468, 256. 
Petromyzon branchialis Gthr. 425, I23.

Petromyzon fluviatilis $L .425, \mathrm{I} 23$.

Petromyzon japonicus $v$. Mart. 497, 28.

Petromyzon marinus $L .425$, I23.

Petronason psittacus Szens. cf. Scarus psittacus Forsk.

Petroskirtes amblyrhynchos Blkr. 163, 64 .

Petroskirtes amboinensis Blkr. 72, II4.

Petroskirtes anema Blkr. 54. 273. 296, Irr. 354, I49. 358, I9I. 364, 293. 425, I 29.

Petroskirtes anolius Blkr. 119, I I.

Petroskirtes bankanensis Blkr. 70, 727. 156, 215. 287, 57. 358, r 9r. 364, 293.

Petroskirtes Bankieri Rich.425, I29.

Petroskirtes barbatus Pet. 459, 74. 495, 20.

Petroskirtes breviceps Blkr. 91, 48.

Petroskirtes dispar Gthr. 425, I 29.

Petroskirtes Dussumieri Blkr. 459, 74 .

Petroskirtes elegans Stnd. 497, 20.

Petroskirtes filamentosus Gïnth. 491, 54 .

Petroskirtes Heyligeri Blkr. 224, 340. 270, 43.

Petroskirtes hypselopterus Blkr. 128, 423. 316, 242.

Petroskirtes japonicus Blkr. 408, 246. 497, 20.

Petroskirtes kallosoma Blkr. 185, 227.

Petroskirtes marmoratus Blkr. 459, 74. 495, 20.

Petroskirtes mitratus Rüpp. 47, 244.

Petroskirtes mitratus Blkr. cf. Petroskirtes hypselopterus Blkr.

Petroskirtes paradiseus Blkr. 103, 495.

Petroskirtes polyodon Blkr. 275, 45. 364, 293.

Petroskirtes punctatus Blkr. 91, 48 .

Petroskirtes rhinorhynchos Blkr. 54, 273. 184, 201. 275, 45.

Petroskirtes rhinorhynchus Blkr. 354, I 49. 358, I9r.364, 293. 369, 42.

Petroskirtes rostratus Blkr. 163, 62. cf. Petroskirtes filamentosus Gthr.

Petroskirtes solorensis Blkr. 80, 8I, 394, 288.

Petroskirtes taeniatus Blkr. 184, 201. 364, 293.

Petroskirtes tapeinosoma Blkr. 163, 64. 275, 45.

Petroskirtes Temmincki Blkr. 47, 243. 184, 201. 315, 236. 317, 246.

$318,252.354$, I 49.358 , I9 I. 364, 293. 369, 42 .

Petroskirtes thepassii Blkr. 73, I36. 315, 236. cf. Petroskirtes marmoratus Blkr.

Petroskirtes variabilis Cant. 51, 58. 288, 77.

Petroskirtes zebra Blkr. 392, 279.

Phaetonichthys Blkr. 468, 256.

Phagorus $\mathrm{MCCl}$. 306, I 20.

Phalacronotus Blkr. 299, 395. 306, I 6 .

Phalacronotus leptonema Blkr. 270, 48, 306, Ir6. 
Phalacronotus micropogon Blkr. 226, 384. 270, 49. 272, 20.

Phalacronotus micruropterus Blkr. 239, I02.

Phanerodon Gir. 468, 290.

Pharopteryx Rüpp. 468, 322 .

Pharopteryx nigricans Rü $p p$. 468, 322. cf. Plesiops nigricans, Rüpp.

Philypni 453, 30r. 460, 103.

Philypnodon Blkr. 453, 301. 460, 103, 104.

Philypnoides surakartensis Blkr. 15, I9. 25, 9. 35, 26r. 42, 59. 45, I95. 49, 4I9. 55, 4I2. cf. Ophiocephalus gachua Buch.

Philypnus $V a l .453,302.460,103$, 104.

Philypnus dormitator $\mathrm{Val} .453,302$.

Philypnus ocellicauda Rich. 15, 20. 287, 57. cf. Bostrichthys sinensis Lac., Bostrychus sinensis Lac.

Philypnus ophicephalus Blkr. 15, 20. 16, 5. 25, 9. cf. Bostrychus sinensis Lac.

Philypnus sinensis Rich. cf. Bostrichthys sinensis Lac., Bostrychus sinensis Lac.

Pholidichthys Blkr. 151, 406.

Pholidichthys leucotaenia Blkr. 151, 406. 354, I 49.

Phoxinellus Heck., Blkr. 261, 434. 314, 209. 329, 263.

Phoxinus Rond. 261, 434. 314, 209.

Phractocephalus $A g$. 306, Iо०.

Phucocoetes 125, 3I9.

Pikea Steind. 468, 262.

Pikea lunulata Steind. 468, 262.

Pimelepteriformes 468, 293.

Pimelepterus Lac. 468, 293.

Pimelepterus altipinnis $C . V .70,727.188,459.231$, I46. cf. Pimelepterus cinerascens $D a y$, Pimelepterus tahmel Rüpp.

Pimelepterus altipinnoides Guich. 459, 94.

Pimelepterus Boscii Lac. 468, 294.

Pimelepterus cinerascens Day 481, I2. 491, 44. 495, I3. 497, 8.

Pimelepterus Dussumierii C. V.91, 40. cf. Pimelepterus cinerascens Day.

Pimelepterus fuscus $C . V$. 266, 53. 459, 94.

Pimelepterus indicus $K . v . H .10,66.46,212.275,29$. cf. Pimelepterus cinerascens Day.

Pimelepterus lembus C. V. 78, 469. 157, 233. 275, 29. 296, 107. 364, 283. 481, I 4 .

Pimelepterus marciac Q.G. 10, 66. 46, I2. 275, 29.

Pimelepterus marciac C. $V$. cf. Pimelepterus waigiensis $Q . G$.

Pimelepterus oblongior $C . V .288,72.481$, I5.

Pimelepterus oblongior Cant. cf. Pimelepterus lembus $C . V$.

Pimelepterus Reynaldi $C$. $V$. cf. Pimelepterus cinerascens Day.

Pimelepterus tahmel Rïpp. 275, 29. 316, 242. 318, 251. 320, 269. 338, 360. 364, 283. 394, 287.459, 94. cf. Pimelepterus cinerascens Day. 
Pimelepterus ternatensis Blkr. 79, 604. 315, 233. 364, 283. cf. Pimelepterus lembus $C$. $V$.

Pimelepterus waigiensis Q.G. 364, 283. 396, 297. 481, I 7. 491, 44.

Pimelodus Lac., Gill 1, 552. 306, 92, гог.

Pimelodus abbreviatus $K . v . H$. cf. Bagrus gulio C. $V$.

Pimelodus anisurus $\mathrm{MCCl} .91,5^{8}$.

Pimelodus aor Buch. cf. Bagrus aor C. $V$.

Pimelodus argenteus Lac. cf. Netuma Herzbergi Blkr.

Pimelodus asperus $M c C l$. cf. Hara aspera Gthr.

Pimelodus auratus Geoffr. cf. Chrysichthys auratus Blkr.

Pimelodus bagarius Bui ., C.V.7, 10. 25, 10. cf. Bagarius Buchanani Blkr.

Pimelodus bagre Lac. 306, 92. 336, 66.

Pimelodus batasio Buch. cf. Batasio Buchanani Blyth.

Pimelodus batasius Buch. cf. Bagrus tengara $C$. $V$.

Pimelodus biscutatus Gcoffr. cf. Auchenaspis biscutatus Blkr.

Pimelodus Blochii Val. cf. Pseudariodes clarias Blkr.

Pimelodus borneënsis Blkr. 49, 430. 55, 4r 3 .

Pimelodus botius Buch. 91, 58 .

Pimelodus cantonensis $\mathrm{Val}$. cf. Amiurus cantonensis Gthr.

Pimelodus catus $L$. cf. Amiurus catus Gill.

Pimelodus cavasius Buch. cf. Bagrus cavasius C. $V$.

Pimelodus cenia Buch. 91, 58.

Pimelodus clarias Geoffr. cf. Hemisynodontis schall Blkr.

Pimelodus coerulescens Raf. cf. Ictalurus coerulescens Gill.

Pimelodus conirostris $V a l$. cf. Conorhynchus conirostris Blkr.

Pimelodus conta Buch. 91, $5^{8}$.

Pimelodus coruscans Licht. cf. Paradiplomystes coruscans Blkr.

Pimelodus ctenodus $A g$. cf. Pseudocallophysus ctenodus Blkr.

Pimelodus cyanochloros Blkr. 7, Iт. 8, I68. 25, Iо. 52, 89. 170, 477. 190, I.

Pimelodus fasciatus Lac. of Pseudoplatystoma fasciatum Blkr.

Pimelodus filamentosus Blkr. 336, 67 .

Pimelodus filamentosus Licht. cf. Malacobagrus filamentosus Blkr.

Pimelodus fulvidraco Rich. cf. Pelteobagrus calvarius $B l k r$.

Pimelodus furcifer $\mathrm{Val}$. cf. Ictalurus furcifer Gill.

Pimelodus gagata Buch. 91, 58. cf. Gagata typus Blkr.

Pimelodus gagora Buch. cf. Arius gagora C. $V$.

Pimelodus galeatus Lac. cf. Parauchenipterus galeatus Blkr.

Pimelodus gogra Blkr. 91, $5^{8}$.

Pimelodus gulio Buch. cf. Bagrus gulio C. $V$.

Pimelodus guttatus Lac. cf. Amiurus guttatus Gthr.

Pimelodus hara Buch. 91, 58.

Pimelodus indicus $\mathrm{MCC} C \mathrm{Cl} . \mathbf{8 1}, 5^{8}$.

Pimelodus jatius Buch. 91, $5^{8}$.

Pimelodus javus K. v. H., Val. 3, I36. 5, 2. 
Pimelodus kuturnee Blkr. 91, $5^{8 .}$

Pimelodus maculatus Lac. cf. Pseudorhamdia maculata Blkr.

Pimelodus mangois Buch. 91, 58.

Pimelodus melanogaster Blkr. 106, 89.

Pimelodus mong Rich. cf. Arius (Pseudarius) falcarius $B l k r$.

Pimelodus mustelinus $V a l$. cf. Heptapterus mustelinus Blkr.

Pimelodus nangra Buch. 91, 58.

Pimelodus nella $C . V$. 91, $5^{8}$.

Pimelodus nemurus $K$. v. H. cf. Bagrus nemurus $V a l$.

Pimelodus nigrodigitatus Lac. cf. Melanodactylus nigrodigitatus Blkr.

Pimelodus nodosus Lac. cf. Pseudauchenipterus nodosus Blkr.

Pimelodus ornatus Kner cf. Pseudorhamdia ornata Blkr.

Pimelodus pangasius Buch. cf. Pangasius Buchanani $C$. $V$.

Pimelodus pirinampu $A g$. cf. Pinirampus typus Blkr.

Pimelodus planiceps $K . v$. H. cf. Bagrus planiceps $V a l$.

Pimelodus platypogon $K$. v. H., Val. 3, т36. 5, 2. 67, 591. 91, 58 . $170,476,477$.

Pimelodus platypogonides Blkr. 136, 272.

Pimelodus pleurostigma Blkr. 113, $44^{2}$.

Pimelodus pusillus Ranz. cf. Parabagrus pusillus $B / k r$.

Pimelodus Quelini Q. G. cf. Rhamdia Quelini Blkr.

Pimelodus rama Buch. cf. Rama Buchanani Blkr.

Pimelodus raninus $\mathrm{Val}$. cf. Pseudopimelodus raninus $B l k r$.

Pimelodus rita Buch. cf. Rita Buchanani Blkr.

Pimelodus rugosus Blkr. 7, I I. 8, I68. 25, Iо. 113, 443. 170, 477.

Pimelodus Sebae $V a l$. cf. Rhamdia Quelini Blkr.

Pimelodus silondia Buch. cf. Silundia gangetica $C$. $V$.

Pimelodus Stegelichii Mïll. cf. Rhamdia Stegelichi Blkr.

Pimelodus telchitta Buch. 91, $5^{8 .}$

Pimelodus tengara $B u c h$. cf. Bagrus tengara $C$. $V$.

Pimelodus vacha Buch. cf. Eutropiichthys vacha Blkr.

Pimelodus variegatus Blkr. 3, I77. 4, 288. 5, 43. 7, Iо.

Pimelodus viridescens Buch. 91, $5^{8}$.

Pimelodus zonatus Blkr. 113, 444.

Pimelodus zungaro Humb. cf. Zungaro Humboldtii Blkr.

Pimelonotus Gill 306, ror.

Pimelotropis Gill 306, ro2.

Pimelotropis lateralis Gill 306, 102.

Pimephales Raf. 261, 425. 314, 204.

Pinirampus Blkr. 306, I00.

Pinirampus typus Blkr. 306, Iо0.

Pinjalo Blkr.468, 273. 470. I 52.

Pinjalo typus Blkr. 2, 251. 445, I 79. 468, 273. 470, I52.cf. Caesio pinjalo $B l k r$.

Piramutana Blkr. 306, 99. 
Piramutana piramuta Blkr. 306, Iо0.

Pirarara Spix 306, xoo.

Pirarara hemiliopterus Blkr. 306, Iоo.

Piratinga Blkr. 306, 99.

Piratinga reticulata Blkr. 306, 99.

Pisoodonophis $K p$. 351, I 19 .

Pisoodonophis baccidens Blkr. 352, I 28.

Pisoodonophis boro $K p$. 352, 1 28. 364, 296. 382, 398.425, 124. 491, 56.

Pisoodonophis brachysoma $K p .352$, I 28.

Pisoodonophis cancrivorus $K p$. 352, I28. 364, 296. 425, I 24. 459, 72. 495, 22. 496, 4 .

Pisoodonophis fasciatus $K p$. cf. Ophisurus fasciatus Rich.

Pisoodonophis guttulatus $K p$. cf. Ophisurus guttulatus $B l k r$.

Pisoodonophis Hoevenii $K p$. 352, I28.

Pisoodonophis hypselopterus $K p$. 352, I29.

Pisoodonophis lumbricoides $K p$. cf. Ophichthys lumbricoides Blkr.

Pisoodonophis Mac Clellandi $K p$. cf. Ophichthys Mac Clellandi Blkr.

Pisoodonophis maculosus $K p$. cf. Ophisurus ophis $L a c$.

Pisoodonophis micropterus Blkr. 313, 182. 352, r29.

Pisoodonophis moluccensis Blkr. 361, 2 I4.

Pisoodonophis oculatus $K p$. cf. Ophisurus oculatus Schl.

Pisoodonophis oligodon Blkr. 349, 59. 425, x 24.

Pisoodonophis potamophilus $K p$. 352, I29.

Pisoodonophis rutidermatoides $K p$. cf. Ophichthys rutidermatoides $B l k r$.

Pisoodonophis rutidoderma $K p$. cf. Ophichthys rutidoderma $B l k r$.

Pisoodonophis Schaapi $K p$. 352, 129. 364, 296.

Plagioscion Gill 468, 329.

Plagiusa aurolimbata Rich. cf. Cynoglossus aurolimbatus Blkr.

Plagiusa favosquamis Rich. cf. Cynoglossus favosquamis Blkr.

Plagiusa grammica Rich. cf. Cynoglossus grammicus Blkr.

Plagiusa melampetala Rich. cf. Cynoglossus melampetalus Gthr.

Plagiusa nigrolabeculata Rich. cf. Cynoglossus nigrolabeculatus Blkr.

Plagiusa puncticeps Rich. cf. Cynoglossus puncticeps Gthr.

Plagusia 1, $55^{2}$.

Plagusia abbreviata $G$ r. cf. Cynoglossus abbreviatus Gthr.

Plagusia bengalensis Blkr. 91, I52.

Plagusia bilineata $B l ., K . v . H ., C . V .2,509.16,6.25,12.32$, 160. 56, 446. 270, 62. 287, 41. 288, 69.

Plagusia bilineata Blkr. cf. Paraplagusia bilineata Blkr.

Plagusia bilineata Cant. cf. Plagusia quadrilineata $K . v . H$.

Plagusia Blochii Blkr. 38, 41 1. 61, 21. 91, 76. 170, 480.

Plagusia borneënsis Blkr. 191, 6.

Plagusia brachyrhynchos Blkr. 38, 4I4. 51, 59. 61, 24. 91, I52.

Plagusia Cantoris Blkr. 91, 76, I53.

Plagusia cynoglossa Cant. 91, 76. 
Plagusia favosquamis Rich. 113, 446.

Plagusia Feldmanni Blkr. 85, 455. 166, 99.

Plagusia grandisquamis Cant. 113, 445 .

Plagusia japonica T. Schl. 175, 26.

Plagusia japonica Schl. cf. Paraplagusia japonica Blkr.

Plagusia javanica $K$. v. H. 38, 4I4. 56, 446.61, 24. 91, I52. cf. Arelia javanica $K p$., Plagusia monopus Blkr.

Plagusia Kopsii Blkr. 50, 494. 54, 235. 61, 31. 91, I52. 106, 99. cf. Cynoglossus Kopsii Gthr.

Plagusia lida Blkr. 2, 509. 38, 413. 46, 2 I 4. 61, 23. 91, I52.

Plagusia macrolepidota Blkr. 38, 4I5. 61, 25. 113, 445. 227, 408.

Plagusia macrorhynchos Blkr. 38, 4I3. 61, 22.

Plagusia marmorata Blkr. 38, 4II. 61, 20. 157, 235. 231, I50. 270, 62. 275, I4. 287, 4I. 296, то3. cf. Paraplagusia marmorata Blkr.

Plagusia melanopterus Blkr. 38, 4I5. 61, 25. 91, I52. 191, 2.231, I50. cf. Cynoglossus monopus Blkr.

Plagusia melanorhynchus Blkr. 26, I5. cf. Achiroüdes melanorhynchus Blkr.

Plagusia microlepis Blkr. 38, 4r3. 42, 6о. 45, 197. 49, 42r. 55, 4r4. 61, 31. 106, 99.

Plagusia monopus Blkr. 16, 6. 17, I r. 25, I2. cf. Plagusia melanopterus Blkr.

Plagusia oligolepis Blkr. 113, 445.

Plagusia oxyrhynchos Blkr. 38, 4r6. 61, 26. 91, I52.

Plagusia polytaenia Blkr. 86, 529. 127, 345 .

Plagusia potous Cuv. 2, 509. 38, 402. 51, 59. 55, 414. 61, 23. 91, 76, I53. 191, 2.

Plagusia quadrilineata $K . \% . H .38,4$ I2. 50, 472. 51, 59. 61, 21.91, I 53. 178, 2. cf. Cynoglossus quadrilineatus Gthr.

Plagusia sumatrana Blkr. 86, 529. 91, I52.

Plagusia Waandersii Blkr. 106, 98.

Plagyrus Raf. 261, 433. 314, 208.

Plataciformes 468, 308.

Platax Cuv. 1, 553. 468, 309.

Platax albipunctatus Riupp. cf. Platax teira Cuv.

Platax anagon Montrouz. cf. Platax pinnatus Blkr., Platax teira Cuv. Platax arthriticus Gthr. cf. Platax batavianus C. $V$.

Platax arthriticus $C . V .51,57.270,27.288,73.394,287.395,292$. 396, 299. 459, 94. cf. Platax teira Cuv.

Platax batavianus $C . V .2,52$ I. 22, 28. 156, 2 I $4.185,223.235,366$. 255,24 I. $270,27.275,35.287,5$ I. $315,234.364,287.481$, r65. cf. Platax arthriticus $C$. $V$.

Platax bataviensis C. $V .40,478.52,89.126,344$.

Platax Blochii C. V. 2, 521. 16, 4. 22, 27. 51, 57. 54, 235. 56, 445 .

71, 759. cf. Platax vespertilio Cuv. 
Platax Boersi Blkr. 71, 758. 156, 2 14. 184, 200. 188, 459. 275, 35. 315, 234. 358, I9o. 364, 287. cf. Platax teira Cuv.

Platax Ehrenbergii $C$. $V$. cf. Platax Blochii $C$. $V$., Platax vespertilio $C u v$. Platax expansus Thioll. cf. Platax pinnatus $B / k r$.

Platax Gaimardi C. V. 71, 759. cf. Platax teira Cuv.

Platax gampret Blkr. 22, 29. 31, I05. 51, 57. 275, 35. 296, ro8.cf. Platax pinnatus Blkr.

Platax guttulatus $C$. $V$. cf. Platax vespertilio $C u v$.

Platax japonicus Temm. Schleg. cf. Platax teira $C$. $V$.

Platax Leschenaldi C. V. 2, 52x. 9, 633. 16, 4. 25, 8. cf. Platax teira $C . V$.

Platax melanosoma Blkr. 273, го. 481, I62.

Platax ocellatus C. V. 51, 57. 91, 165. 288, 73. 468, 304. cf. Parachaetodon ocellatus Blkr.

Platax orbicularis C. $V$. 106, 81. 119, 9. 157, 233. 270, 27. 287, 5 I. 459, 94. cf. Platax teira Cuv.

Platax orbicularis Rüpp. cf. Platax vespertilio Cuv.

Platax pentacanthus $C . V$. cf. Platax orbicularis $C . V$, Platax vespertilio Cuv.

Platax pinnatus Blkr. 481, I63.

Platax punctulatus $C$. $V$. 320, 270. cf. Platax teira Cur'.

Platax Raynaldi C. V. 22, 28. 31, 105. 91, 40. cf. Platax verpertilio Cur.

Platax teira C. $V .22,28.50,471.51,57.91,40.119,9.156,214$. 157, 233. 158, 274. 188, 459. 237, 437. 270, 27. 275, 35. 288, 73. 296, го8. 315, 235. 346, 3т. 358, г 90. 364, 287. 395, 292. 425, г 33. 459, 94. 468, 309. 481, г57.491, 45. 495, г3. 497, 9.

Platax teira Riupp. cf. Platax vespertilio Cuv.

Platax vespertilio Rüpp. 171, 245. 270, 27.

Platax vespertilio Cuv. 91, 40. 156, 2r4. 157, 233. 167, 371. 184, 200. 194, 26. 231, I 46. 234, 353. 235, 366. 242, I 42. 275, 35 . 287, 5 I. 288, 73. 296, го8. 315, 235. 316, 242. 354, г 48.358 , I 90. $364,287.394,287.395,292.396,299.425$, I33. 459, 94 . 481, I60. 491, 45. 495, 13. 497, 9. cf. Platax Blochii C. $V$.

Platax vespertilio Cant., T. Schl. cf. Platax teira Cuv.

Platax vespertilio japonicus Schl. cf. Platax teira Cuv.

Platax xanthopus Blkr. 22, 28. 31, 105. cf. Platax teira Cu\%.

Platessa Cuv. 118, I23. 294, 427.

Platessa asperrima Schl. cf. Clidoderma asperrimum Blkr.

Platessa bicolorata Basil. cf. Parophrys bicoloratus Blkr.

Platessa chinensis Rich. cf. Tephrinectes sinensis Gthr.

Platessa cornuta T. Schl. 118, I2 1. 253, 235. 294, 429. cf. Heteroprosopon cornutus $B l k r$.

Platessa Jenynsii Blkr. 119, I5.

Platessa microcephalus Flem. 294, 428.

INDEX BLEEKER. 
Platessa plana Mus. L. B. 294, 428.

Platessa Russellii Gray 51, 59. 287, 41. 288, 69.

Platessa variegatus Schl.cf. Pleuronectes variegatus Günth.

Platessa velafracta Rich. cf. Tephrinectes sinensis Gthr.

Platophrys Szons. 294, 426.

Platophrys (Platophrys) assimilis Blkr. 425, I30.

Platophrys (Platophrys) grandisquama Blkr. 425, I30. 497, 2 I.

Platophrys (Arnoglossus) intermedius Blkr. 370, 47.

Platophrys (Platophrys) lunatus Blkr. 459, 76.

Platophrys (Platophrys) myriaster Blkr. 425, I30. 497, 2 I.

Platophrys (Platophrys) pantherinus Blkr. 310, I60. 318, 250. 338, 360. 354, I 44.358, I $84.364,274.394,286.396,296.437,37$. 459, 76. 495, $2 \mathrm{I}$.

Platophrys (Platophrys) pavo Blkr.425, I30. 495, 2 I.

Platophrys (Platophrys) polyophthalmus Blkr. 370, 46.

Platophrys (Arnoglossus) tapeinosoma Blkr. 370, 49. 425, I30.

Platycara $M C$ Cl. 261, 423. 329, 262.

Platycara nasuta $M C C l$. cf. Homaloptera nasuta.

Platycephaloidei 449, 370.

Platycephalus 1, 553 .

Platycephalus asper $C . V .425$, I27. 497. I 2.

Platycephalus bassensis $C$. $V$. 119, 7 .

Platycephalus bataviensis Blkr. 78, 460. 156, 2 І3. 275, 42. 287, 55 . 296, I I0. 364, 29I. 499, 2 I.

Platycephalus bobossok Blkr. 78, 46r. 235, 369. 287, 55. 395, 293. $499,23$.

Platycephalus borbonicus $C . V .459,76$.

Platycephalus Bosschei Blkr. 267, I40. 499, I6.

Platycephalus Cantori Blkr. 499, 26.

Platycephalus carbunculus $C . V .91,34.119,7.288,76$.

Platycephalus carbunculus Cant. 91, 34. cf. Platycephalus Cantori Blkr.

Platycephalus celebicus Blkr. 114, 450. 275, 42. 364, 291. 499, 3 o.

Platycephalus chacca $G r$. cf. Platycephalus indicus Blkr.

Platycephalus cirronosus Rich. 119, 7.

Platycephalus clavulatus Cant. 288, 76. cf. Platycephalus punctatus $C . V$. Platycephalus crocodilus Voy. Krusens\%. cf. Platycephalus guttatus $C$. Platycephalus cultellatus Rich. 425, I27.

Platycephalus endrachtensis $Q . G$. 119, 7. cf. Platycephalus indicus Blkr. Platycephalus fasciatus $G t h r$. cf. Platycephalus punctatus $C$. $V$.

Platycephalus fuscus $C . V .119,7.497$, I2.

Platycephalus guttatus $C . V .425$, I27. 497, I 2.

Platycephalus indicus Blkr. 491, 49. 497, I2. 499, 8.

Platycephalus inops $C 2.119,7$.

Platycephalus insidiator Bl. Schn., C. V. 2, 524. 14, 6. 16, 4. 25, 7 . 35, 26 1. 40, $478.42,58.45$, 195. 46, 2 12. 49, 418. 52, 89. 55, 
4II. 91, 34. 170, 479. 184, 200. 185, 223. 270, 39. 272, 16. 275, 42. 287, 55. 288, 76. 364, 29r. 391, 277. 395, 293. 425, 127. 459, 76. cf. Platycephalus indicus Blkr.

Platycephalus isacanthus $C . V .14,4.50,481.51,63.157,232.275$, 42. 310, I60. 315, 236. 354, I49. 395, 293. 396, 297. 499, 20.

Platycephalus isacanthus Blkr. cf. Platycephalus nematophthalmus Gthr. Platycephalus japonicus Tiles., $C . V .90,28.118,78.425$, I27.497, I2. Platycephalus laevigatus $C . V .119$, I9.

Platycephalus macracanthus Blkr. 409, 253. 499, 22.

Platycephalus macrolepis Blkr. 102, 399. 118, 76. 253, 235.497, I 2. Platycephalus maculosus Pet. cf. Platycephalus bobossok Blkr.

Platycephalus malabaricus Gthr. cf. Platycephalus Cantori Blkr.

Platycephalus malabaricus $C . V .91,34.425$, I27. cf. Platycephalus punctatus $C \cdot V$.

Platycephalus malayanus Blkr. 86, 498. 236, 333. 270, 39. 364, 29 I. $491,49.499,27$.

Platycephalus Meerdervoortii Blkr. 269, 80. 408, 238. 497, I2.

Platycephalus neglectus Trosch.425, r 27.cf. Platycephalus scaber Bl.Schn.

Platycephalus nematophthalmus Gthr. 499, го.

Platycephalus polyodon Alkr. 78, $462.275,42.358$, r9 r. 364, 29 r. 499,'25.

Platycephalus pristiger $C \cdot V .10,66,68.14,4.46,212.102,400$ $118,77.155,205.231$, I $45.275,42.287,55.296$, т1о. 315, 236. 354, I 49. 364, 291. 491, 49. 499, 29.

Platycephalus pristis Pet. cf. Platycephalus celebicus $B l k r$.

Platycephalus punctatus $C . V .27,25.56,444.91,34.155,207$. 156, 213. 270, 40. 287, 55. 358, т91. 364, 291. 425, т27. 497, I 2. 499, I3.

Platycephalus Quoyi Blkr. 155, 206. 308, I52. 315, 236. 320, 27 I. 338, 36г: 364, 29 г. 499, г г.

Platycephalus Ransonneti Steind. 499, I9.

Platycephalus rodericensis $C$. $V$. cf. Platycephalus scaber $B l$.

Platycephalus rudis Gïnth.497, I2.

Platycephalus scaber Bl. Schn., C. V. 2, 524. 10, 66. 14, 6. 16, 4. 25, 7. 32, I6о. 46, 21 2. 55, 4 II. 56, 444. 91, 34, I65. 156, 2 I3. 191, 2. 222,3 16. 227, 407. 270, 40. 272, I6. 275, 42. 287, 55 . 356, I $74.459,76.499$, I 4 .

Platycephalus serratus $C . V .91,34.102,400.118,77$.

Platycephalus sihamus $B l$. Schn. cf. Sillago sihama Rüpt.

Platycephalus spathula $B l$. cf. Platycephalus insidiator $B l$., Platycephalus indicus $B / k r$.

Platycephalus spinosus $T$. Schl。 118, 77. 253, 235.425, г27.497, I2.

Platycephalus sumatranus $B c n n .270,40$.

Platycephalus sumatrensis Benn. 14, 4.

Platycephalus sundaicus Blkr. 499, 18.

Platycephalus suppositus Trosch. cf. Platycephalus scaber Bl. Schn. 
Platycephalus tasmanius Rich. 119, 7.

Platycephalus tentaculatus Kner cf. Platycephalus nematophthalmus Gthr.

Platycephalus timoriensis $C . V$. 14, 4. 320, 27I. cf. Platycephalus scaber $B l$. Schn.

Platycephalus tuberculatus $C . V .91,36$.

Platycephalus vittatus $C . V .91,36$. cf. Platycephalus scaber Bl. Schn.

Platydoras Blkr. 306, 86.

Platydoras armatulus Blkr. 303, I6.

Platydoras costatus Lac., Blkr. 303, I6. 306, 86. 336, 38.

Platydoras dentatus Blkr. 303, I6.

Platyglossus Klein 292, 99.

Platyglossus (Hemitautoga) centiquadra Blkr. 491, 41. 495, 10.

Platyglossus (Halichoeres) chloropterus Blkr. 495, Iо.

Platyglossus Hoevenii Blkr. 293, 284. 315, 230. 316, 24r. 318, 250. $320,266.340,364.354$, I 44.358, I $85.364,276.369,42.394$, 286. 437, 37 .

Platyglossus kallochroma Blkr. 293, 284.

Platyglossus marginatus Blkr. 293, 283. 320, 266. 364, $276.459,83$. Platyglossus (Platyglossus) marginatus Blkr. 495, го.

Platyglossus melanurus Blkr. 293, 284. 318, 250. 320, 266. 354, 144 . 358, I85. 364, $276.394,286.491,40$.

Platyglossus (Hemitautoga) notophthalmus Blkr. 491, $4 \mathrm{r}$.

Platyglossus notopsis Blkr. 293, 284. 320, 266. 358, r85. 364, 276.

Platyglossus (Parajulis) poecilopterus Blkr. 497, 5 .

Platyglossus (Paraplatyglossus) prosopeion Blkr. 491, 40.

Platyglossus purpurescens Blkr. 293, 284. 320, 266. 364, 276.

Platyglossus (Parajulis) pyrrhogramma Blkr. 497, 5.

Platyglossus scapularis Gthr. cf. Güntheria scapularis Blkr.

Platyglossus (Güntheria) scapularis Blkr. 491, 4I. 495, Iо.

Platyglossus tenuispinis Gthr. cf. Halichoeres tenuispinis $B l k r$.

Platyglossus (Güntheria) trimaculatus $B l k r .491,41$.

Platyglossus Vroliki Blkr. 293, 285.

Platygobii 453, 3I5.

Platygobius Blkr. 453, 316.

Platyinius Gill 438, 44. 468, 276.

Platynematichthys Blkr. 306, 99.

Platynematichthys punctulatus Blkr. 306, 99.

Platyptera aspro $K . v . H .10,67.15,3.46,2$ 13. 137, 310. 270, 40. $275,45.284,240$.

Platyptera melanocephala $v$. Hass. cf. Platyptera aspro $v$. Hass.

Platyptera sinensis Blkr. 425, I27, I 52.

Platypterus Swns. 473, 297.

Platypterus flavescens $\mathrm{Val}$. cf. Platypterus aspro $v$. Hass.

Platypterus fusco-virens Szens. cf. Paracentropogon longispinis Blkr. 
Platypterus longispinis Szons. cf. Paracentropogon longispinis Blkr.

Platypterus taenianotus Sions. cf. Amblyapistus taenianotus Blkr.

Platyrhina Schoenleinii M. H. 91, 82 .

Platyrhina sinensis $M . H .51,60.288,67.425$, I20. 497, 4.

Platysomatichthys Blkr. 294, 425 .

Platysome Lién. 468, 336.

Platysomos Spixii Szons. cf. Vomer setipinnis Ayr.

Platysomos Brownii Szuns. cf. Vomer setipinnis Ayr.

Platysomus micropteryx Szuns, cf. Vomer setipinnis $A y r^{\circ}$

Platystacus $B l$. 306, $x \mathrm{x} 8$.

Platystacus anguillaris $B l$. cf. Plotosus lineatus $\mathrm{Val}$.

Platystacus cotylephorus Bl. 306, i i $8.336,95$.

Platystacus laevis $B l$. cf. Aspredo batrachus $L$.

Platystacus nematophorus Blkr. 298, 371. 336, 96.

Platystacus verrucosus $B$. cf. Bunocephalus verrucosus Kner.

Platystoma $A g$. 306, 98.

Platystoma affine $V a l$. cf. Brachyplatystoma Vaillanti Blkr.

Platystoma fasciatum $\mathrm{Val}$. cf. Pseudoplatystoma fasciatum Blkr.

Platystoma platyrhynchos $\mathrm{Val}$. cf. Hemisorubim platyrhynchos Blkr.

Platystoma punctifer Cast. cf. Pseudoplatystoma fasciatum $B l k r$.

Platystoma sturio $K n e r$ cf. Platystomatichthys sturio Blkr.

Platystoma tigrinum $\mathrm{Val}$. cf. Hemiplatystoma tigrinum Blkr.

Platystoma Vaillantii $V a l$. cf. Brachyplatystoma Vaillanti Blkr.

Platystomatichthys Blkr. 306, 98.

Platystomatichthys sturio Blkr. 306, 98.

Plecoglossus altivelis 497, 24.

Plecostomus Art. 306, 77.

Plecostomus aculeatus Gron. cf. Ancistrus Temmincki Blkr.

Plecostomus bicirrosus Gron. cf. Plecostomus brasiliensis Blkr.

Plecostomus brasiliensis Blkr. 306, 78. 336, 7 .

Plecostomus cataphractus Gron. cf. Loricarichthys maculatus Blkr.

Plecostomus flagellaris Gron. cf. Loricaria dura $L$.

Plecostomus maculatus Stons, cf. Loricariichthys maculatus Blkr.

Plectorhynchus Lac. 468, 27 I.

Plectorhynchus albovittatus Blkr.432, 280.

Plectorhynchus balteatus Cant. cf. Diagramma punctatum Ehr., Plectorhynchus pictus $B l k r$.

Plectorhynchus celebicus Blkr. 432,285 .

Plectorhynchus chaetodonoides Lac. 315, 232. 369, 42. 468, 27I. cf.

Diagramma plectorhynchos $C . V$.

Plectorhynchus chaetodontoides Lac. 432, $2 S_{2}$.

Plectorhynchus chaetonoides Lac. 364, 282.

Plectorhynchus chrysotaenia Blkr. 432, 279.

Plectorhynchus cinctus Blkr. 425, I39. 497, 7.

Plectorhynchus crassispina Blkr. 315, 232. 316, 24I. 354, I 47. 358, 
I $87.364,282.396,299.425$, I39. $432,277.459,92.495$, I2.

Plectorhynchus Cuvieri Blkr. 432, 291. 491, 42.

Plectorhynchus foetela Blkr. 425, I39.

Plectorhynchus gaterina Blkr.425, 139. 459, 92. 495, 12.

Plectorhynchus Goldmani Blkr. 315, 232. 316, 241. 320, 268. 432, 292.

Plectorhynchus griseus $B l k r .459,92.495$, I 2.

Plectorhynchus haematochir Blkr. 315, 232. 320, 268. cf. Plectorhynchus Goldmani $B l k r$.

Plectorhynchus Lessonii Blkr. 315, 232. 364, 282. 396, 297.432, 288.

Plectorhynchus lineatum Blkr. 315, 232.

Plectorhynchus lineatus Blkr. 320, 268. 338, 360. 354, 147. 358, I $87.364,282.369,42.432,286$.

Plectorhynchus orientale Blkr. 315, 232.

Plectorhynchus orientalis Stens., Blkr. 320, 268. 354, I47. 358, I87. $432,295.495$, I 2.

Plectorhynchus pertusus Blkr. 497, 7 .

Plectorhynchus pictus Blkr. 364, 282. 389, 273. 408, 238. 425, I 39. 432, 298. 459, 92. 497, 7.

Plectorhynchus poecilopterus Blkr. 425, I39.

Plectorhynchus polytaenia Blkr. 315, 232. 391, 276. 394, 287.432, 296.

Plectorhynchus polytaenioides Blkr. 364, 282. 394, 287. cf. Plectorhynchus polytaenia Blkr.

Plectorhynchus punctatissimus Blkr. 459, 92 .

Plectorhynchus punctatus Blkr. 358, I87. 364, 282. cf. Plectorhynchus pictus Blkr.

Plectorhynchus radja $B l k r .315,232.354,147.364,282.432,289$.

Plectorhynchus reticulatus Blkr. 425, I39.

Plectorhynchus Sebae Blkr. 364, 283. cf. Plectorhynchus Cuvieri Blkr.

Plectranthias Blkr. 468, $25^{2}$.

Plectroperca Peters 468, 255.

Plectroperca Berendtii Pet. cf. Siniperca chuatsi Gill.

Plectroplites Gill 468, 267.

Plectroplites ambiguus Gill 468, 267.

Plectropoma C. V., Gill 1, 553. 468, 256, 263, 336.

Plectropoma aculeatum $C . V$. 266, 5 I.

Plectropoma anthioides Gthr. 431, 237. 468, 252.

Plectropoma areolatum Rüpp. cf. Paracanthistius leopardinus Blkr.

Plectropoma calcarifer Cuv. 468, 263 .

Plectropoma cyanostigma Blkr. 2, 525. cf. Paracanthistius leopardinus Blkr., Plectropoma leopardinus $C . V$.

Plectropoma dentex C. $V .119,5$.

Plectropoma ephippium $C . V .13,4$.

Plectropoma leopardinum $C . V .119,5$. cf. Paracanthistius leopardinus Blkr.

Plectropoma leopardinum T. Schl. cf. Plectropoma leopardinus C. $V$. 
Plectropoma leopardinus $C . V .113,420$.

Plectropoma leopardus Rich. cf. Plectropoma leopardinus $C . V$., Acanthistius leopardinus Blkr., Paracanthistius leopardinus Blkr.

Plectropoma macrophthalmus Tschudi, Kner 468, 277.

Plectropoma maculatum Rüpp. cf. Paracanthistius leopardinus Blkr.

Plectropoma maculatum C. $V$. 2, 525. 13, 39. 51, 55. 113, 418. 275, 24. cf. Paracanthisthius maculatus Blkr., Plectropoma leopardinus C. $V$.

Plectropoma melanoleucum $C$. $V$. cf. Paracanthistius melanoleucus Blkr.

Plectropoma oligacanthus Blkr. 113, 422. 275, 24. cf. Paracanthistius oligacanthus Blkr.

Plectropoma punctatum Q. G. cf. Plectropoma maculatum C. $V$., Paracanthistius maculatus Blkr.

Plectropoma serratum C. $V$. 119, I9. cf. Acanthistius serratus Gill.

Plectropoma susuki $C$. $V$. cf. Epinephelus susuki Blkr.

Plesiops Cuv. 468, 322.

Plesiops Bleekeri Gthr. 468, 322.

Plesiops coeruleolineatus Rüpp. 72, пा6. 127, 345. 156, 2 I6. 157, 234. 167, 372. 170, 479.184, 202.185, 224. 188, 460. 231, 148. 234, 354. 236, 33I. 270, 35. 275, 2 I. 315, 23 I. 318, 25 I. 320, 267. 354, I 45. 358, I86. 364, 279. cf. Plesiops nigricans Rïpp., Gthr. Plesiops corallicola Müll., Gthr. 76, 280. 170, 479.270, 35.364, 279. Plesiops corallicola $K$. \%. H. 425, 136. cf. Plesiops nigricans Rüpp.

Plesiops melas Blkr. 17, 9. 53, r6r. cf. Plesiops coeruleo-lineatus Rüpp., Plesiops nigricans Rüpp.

Plesiops nigricans Gthr. 382, 397. 394, 286. 396, 299.

Plesiops nigricans Rüpp. 465, 27. 468, 322. 491, 46. 495, I4. cf. Plesiops corallicola $K, v$. $H$.

Plesiops oxycephalus Blkr. 125, 320. 157, 234. 364, 279. 465, 29.

Plesiopsini 468, 322.

Pleuronectes Art. 294, 427.

Pleuronectes arnoglossus 294, 427.

Pleuronectes bicoloratus Blkr. 425, I30.

Pleuronectes bilineatus Bl. cf. Plagusia Blochii Blkr.

Pleuronectes chrysopterus Bl. Sclnn. cf. Pseudorhombus Russelli Gthr.

Pleuronectes erumei Bl. Schn. cf. Hippoglossus erumei Cuv.

Pleuronectes limandoides Bl. 294, 427.

Pleuronectes macrolepidotus $L$. cf. Citharus macrolepidotus.

Pleuronectes nasutus Pall. cf. Pegusa impar. Gthr.

Pleuronectes pan Buch. cf. Solea pan Cuv., Synaptura pan Cant.

Pleuronectes platessa L. 294, 428 .

Pleuronectes sinensis Lac. cf. Tephrinectes sinensis Gthr.

Pleuronectes triocellatus Bl. Schn. cf. Rhombus triocellatus Cuv.

Pleuronectes variegatus Gïnth. 497, 22.

Pleuronectes Yokohamae Giunth. 497, 22.

Pleuronectes zebra Bl. cf. Solea zebra C. $V$. 
Plotosus Lac. 1, 552. 306, II9.

Plotosus albilabris $C . V$. 3, I36. 5, 2. 51, 70. 185, 226. 267, 140. $270,49.275,46.287,59.288,78.395,293$.

Plotosus anguillaris Cuv. 119, I4. 127, 345. 156, 2 I 7. 157, 235. 167, 374. Plotosus anguillaris Cant. cf. Plotosus lineatus C. $V$.

Plotosus anguillaris Lac. 184, 203. 234, 356. 235, 371. 250, 207. $270,49.275,46.287,59.354$, I 50. 358, 192. 364, 294. cf. Plotosus arab Blkr.

Plotosus arab Blkr. 296, III. 306, IIg. 395, 293. 396, 298, 300. 425, I $25.437,37.459,75.491,55.495,2$ I. 496, 3. 497, 22.

Plotosus canius Buch. 91, I25. 191, 2. 227, 408. 235, 371. 239, I02. $270,49.272,20.275,46.287,59.288,78.356,175$. $425, \mathrm{r} 25$.

Plotosus castaneoides Blkr. 50, 490. cf. Plotosus arab.Blkr.

Plotosus castaneus $C . V .91, .58$.

Plotosus horridus Blkr. 3, I83. 5, 49. 25, II.

Plotosus ikapor Less. cf. Plotosus arab Blkr.

Plotosus limbatus $C . V . \mathbf{9 1}, \mathbf{5 8 .}$

Plotosus lineatus C. V. 3, I80. 4, 288. 5, 46. 10, 67. 25, I I. 46, 2I3. 51, 58. 54, 238. 56, 445. 90, 5. 91, 60. cf. Plotosus anguillaris Cuv.

Plotosus lineatus ( $C . V$. .) Schl. cf. Plotosus arab Blkr.

Plotosus macrocephalus $V a l .3,136.5,2.320,272$.

Plotosus macrophthalmus Blkr. 3, I 79. 4, 288, 5, 45. 46, 2 r4.

Plotosus malignus Ehrenb. cf. Plotosus lineatus $\mathrm{Val}$.

Plotosus marginatus Raffl. 3, r42, r8 1. 5, 47. cf. Plotosus lineatus $V a l$.

Plotosus megastomus Rich. 119, I4.

Plotosus microceps Rich. 119, 14.

Plotosus multiradiatus Blkr. 3, I83. 4, 288. 5, 49.

Plotosus plumbeus Raffl. 3, I36. 5, 2.

Plotosus tandanus Mitch. 119, I9.

Plotosus unicolor $K$. v. H., C. V. 3, х $S_{2} .4,288.5,48.7,7.16 .5$. 25, II. 32, I60. 51, 58. 56, 445. 178, 2.

Plotosus viviparus Blkr. 3, r82. 4, 288. 5, 48 .

Pneumabranchus $\mathrm{MC} \mathrm{Cl}$. 88, 57 .

Pneumabranchus albinus $M_{C} C l$. cf. Amphipnous cuchia $J$. Müll.

Pneumabranchus leprosus $M c C l$. cf. Amphipnous cuchia $J$. Müll.

Pneumabranchus striatus $\mathrm{Mc} C \mathrm{Cl}$. cf. Amphipnous cuchia $J$. Mïll.

Podabrus centropomus Rich. 425, I4I.

Podabrus cottoides Rich. 425, I4I.

Poecilia Bl. Simn. 261, 44r.

Poecilia fusca Bl. Schn. 91, 105. cf. Eleotris nigra Q. G., Culius fuscus Blkr.

Poecilia latipes T. Schl. cf. Aplocheilos latipes T. Schl., Aplocheilus, Aplochilus latipes Blkr. 
Poecilia omalonota A. Dum. cf. Haplochilus homalonotus Gthr.

Poecilocephalus $K p$. 351, II9.

Poecilocephalus Bonaparti $K p$. cf. Ophichthys Bonaparti Blkr., Ophisurus Bonapartei Blkr.

Poecilocephalus Markwarti $K p$. cf. Ophichthys Bonapartei Blkr.

Poecilophis $K p$. 351, I2I.

Poecilophis catenatus $K p$. cf. Echidna catenata Blkr.

Poecilophis delicatulus $K p$. cf. Echidna delicatula $B l k r$.

Poecilophis ornata $K p$. cf. Echidna xanthospilus $B l k r$.

Poecilophis polyzonus $K p$. cf. Echidna polyzona Blkr.

Poecilophis variegata $K p$. 348, 49. cf. Echidna variegata $\int$. R. Forst.

Pogoneleotris Blkr. 460, 103, 105.

Pogoneleotris heterolepis $B l k r .478,37$.

Pogonias Lac. 468, 324 .

Pogonias fasciatus Lac. 468, 324 .

Pogonias nigripinnis Schl. cf. Hapalogenys nigripinnis Rich.

Pogonichthys Gir. 261, 437. 314, 2rr. 329, 264 .

Pogonognathus 10, 70 .

Pogonognathus barbatus Blkr. 10, 73. 46, 214. 50, 472. 51, 60. 63, 24. 235, 374. 275, I2. 288, 68. cf. Psilocephalus barbatus Sions.

Pogonoperca Gthr. 468, 258.

Pogonoperca ocellata Gthr. cf. Grammistes ocellatus Blkr.

Pogonoperca punctata Gthr. cf. Grammistes ocellatus Blkr.

Polemius $K p .473,296$.

Polemius alatus $K p$. cf. Apistus alatus $C . V$.

Polotus Blyth 468, $27 \mathrm{I}$.

Polyacanthonotus Blkr. 449, 368 .

Polyacanthus 1, 553 .

Polyacanthus chinensis C. $V$. cf. Polyacanthus opercularis Rich.

Polyacanthus cupanus $C . V .91,42$.

Polyacanthus Deissneri Gthr. cf. Parosphromenus Deissneri Blkr.

Polyacanthus Einthovenii Blkr. 49, 423. 55, 4ri. 270, 34. 272, 10. cf. Polyacanthus Hasseltii C. $V$.

Polyacanthus fasciatus $C$. $V$. cf. Colisa vulgaris $C . V$.

Polyacanthus Hasseltii C. $V .2,520.21,9.191,3.270,34.272$, 10. $498, \mathrm{r} 2$.

Polyacanthus Helfrichii Blkr. 121, I62. 178, 2. 272, 10. cf. Polyacanthus Hasseltii $C . V$.

Polyacanthus Kuhlii Blkr. 2, 520. cf. Polyacanthus Hasselti C. $V$.

Polyacanthus olivaceus $v$. Hass. cf. Polyacanthus Hasseltii $C$. $V$.

Polyacanthus opercularis Rich. 425, I 36.

Polyacanthus paludosus Rich. cf. Polyacanthus opercularis Rich.

Polycaulus elongatus Gthr. 364, 286. 425, I4I. 458, 20. 496, 2.

Polycentroidei 468, 3I9.

Polycentrus Miull. Trosch. 468, 3 I9. 
Polycentrus Schomburgkii Mïll. Trosch. 468, 3 I9.

Polycirrhus Boc. 468, 325.

Polymixia nobilis Lozve 459, 86.

Polynemus 1, 553.

Polynemus Artedii Benn. cf. Pentanemus quinquarius Gthr.

Polynemus aureus Buch. cf. Polynemus longifilis $C$. $V$.

Polynemus bornëensis Blkr. 178, 2. 272, I4.

Polynemus decadactylus $B l .46,2$ I 5 .

Polynemus diagrammicus Blkr. 2, 527. 13, 60. 25, 7 .

Polynemus dubius Blkr. 91, 92. 270, 25. 272, 14.

Polynemus heptadactylus $C . V .2,527.13,60.25,7.156,213,270$, 25. 288, 75 .

Polynemus hexanemus $C . V .2,527.13,59.25,7.227,407.270$, 25. 288, 75 .

Polynemus indicus Shaw 91, 34. 113, 427. 235, 365. 287, 54. 288, 75. cf. Polynemus uronemus $C$. V., Trichidion indicum Blkr.

Polynemus kuru Blkr. 79, 600. 147, 469.

Polynemus lineatus $M C C$. cf. Polynemus indicus Share.

Polynemus lineatus Lac. 13, 58. cf. Polynemus plebejus Brouss., Trichidion plebejus Blkr.

Polynemus longifilis C. $V .35,268.42,58.45$, I95. 49, 418. 55, 418. 91, 9I.

Polynemus macronema Blkr. 55, 419. 91, 92.

Polynemus macronemus $\mathrm{Pel}$. cf. Pentanemus quinquarius Gthr.

Polynemus macrophthalmus Blkr. 190, I0. 270, 25.

Polynemus melanochi $C . V$. 13, 60. 127, 345. 156, 2 13. 270, 25.

Polynemus microstoma Blkr. 46, 217. 231, 146. 237, 437. 275, 40. cf. Trichidion microstoma Blkr.

Polynemus multifilis Schl. 272, I5.

Polynemus multiradiatus Gthr. cf. Trichidion multiradiatum Blkr.

Polynemus paradiseus L. cf. Polynemus longifilis C.V., Trichidion paradiseus Blkr.

Polynemus Pfeifferi Blkr. 76, 249. 270, 25.

Polynemus plebejus $C . V .13,58.16,4.25,7.90,4.91,34$.

Polynemus plebejus $M C C l .91,34$. cf. Polynemus indicus Shaze.

Polynemus plebejus Gthr. cf. Trichidion microstoma Blkr.

Polynemus plebejus Brouss. 2, 524, 527. 13, 58. 53, 160. 91, 34 . $119,7.127,345.170,479.231,146.270,25.275,4$ г. 288, 75 . cf. Trichidion plebejus Blkr.

Polynemus ploteus $M C C l$. cf. Polynemus indicus Shaze.

Polynemus polydactylus Blkr. 55, 4r7.

Polynemus quadrifilis $C$. $V$. cf. Trichidion quadrifilis Blkr.

Polynemus quaternarius Park. 13, 57. cf. Polynemus tetradactylus Shazo.

Polynemus quinquarius $L$. cf. Pentanemus quinquarius Gthr., Polynemus longifilis $C, V$. 
Polynemus risua Buch. cf. Polynemus longifilis C. $V$.

Polynemus sele Buch. cf. Polynemus indicus Share, Polynemus plebejus Brouss.

Polynemus sexfilis C. $V$. cf. Trichidion sexfilis Blkr.

Polynemus sextarius $B l .2$, 527. 13, 59. 91, 34. 267, I40. 270, 26. 288, 75. cf. Trichidion sextarium Blkr.

Polynemus teria Buch. 13, 57. cf. Polynemus tetradactylus Shazo.

Polynemus tetradactylus $C . V .2,524,527.10,66.13,57.16,4.25$, 7. 32, г6о. 46, 2 г2. 50, 47 г. 55, 4х . 56, 444. 91, 34. 158, 273. 191, x. 194, 26. 270, 26. 272, I 5. 275, 41. cf. Eleutheroma tetradactylus Blkr.

Polynemus tetradactylus Shaw 13, 57. 51, 55. 91, 34. 119, 7 . 288, 75.

Polynemus toposui Buch.. cf. Polynemus longifilis C. $V$.

Polynemus tridactylus Blkr. 2, 524. 13, 57. 25, 7. 287, 54. cf. Elenttheronema tridactylum $B l k r$.

Polynemus uronemus $C . V .13,58.16,4.25,7.51,55$. cf. Polynemus indicus Shaze.

Polynemus xanthonemus $C . V .13,60.91,34$. cf. Trichidion xantho nema Blkr.

Polyodon (Spatularia) angustifolium Kp. cf. Polyodon gladius Mart.

Polyodon gladius Mart. 425, I 2 I.

Polyprion Cuv. 468, 253.

Polyprion cernium Cuv. 468, 253.

Polyprion japonicus Langsd. 44, I72. cf. Priacanthus japonicus $C$. $V$. Polypriontini 468, 253.

Polypterichthys Valentini Blkr. 79, 608. 157, 234. 247, 202. cf. Aulostoma chinense Lac.

Polysteganus Klunz. 468, 278.

Polyuranodon $K p .351$, I2I.

Polyuranodon Kuhli $K p$. cf. Gymnothorax polyuranodon Blkr.

Pomacanthodes Gill 468, 308.

Pomacanthodes zonipectus Gill cf. Chaetodon zonipectus Blkr.

Pomacanthus Cuv., Lac. 468, 308, 309.

Pomacanthus arcuatus Lac. 482, 2 I. cf. Chaetodon arcuatus L.

Pomacanthus asfur Lac. cf. Acanthochaetodon asfur Blkr.

Pomacanthus aureus $C, V$. cf. Chaetodon aureus $B l$.

Pomacanthus balteatus $C . V$. cf. Chaetodon aureus $B l$.

Pomacanthus canescens Lac. cf. Zanclus canescens Gthr.

Pomacanthus cingulatus $C$. $V$. cf. Chaetodon cingulatus Blkr.

Pomacanthus paru Lac. cf. Chactodon pura Bl.

Pomacanthus quinquecinctus $C . V$. cf. Chaetodon arcuatus $L$.

Pomacentrus 1, 533. 2, 524 .

Pomacentrus albifasciatus M. Schl. 6, 2. 10, 67, 68. 46, 213. 106, S5. 156, 2 I6. 157, 234. 270, 35. 275, I9. 296, I04. 320, 267. 338, 
36o. 354, r45. cf. Eupomacentrus (Brachypomacentrus) albifasciatus Blkr., Pomacentrus leucopleura Blkr.

Pomacentrus albofasciatus Day cf. Dischistodus prosopotaenia Blkr.

Pomacentrus (Pseudopomacentrus) amboinensis Blkr. 400, 334. 485, $5^{8}$.

Pomacentrus (Pomacentrus) anabatoides Blkr. 485, $5 \mathrm{I}$.

Pomacentrus annulatus Peters cf. Dischistodus annulatus Blkr.

Pomacentrus aruanus Rupp. cf. Dascyllus aruanus $C . V$., Tetradrachmum arcuatum Cant.

Pomacentrus ater Lién. 459, 85. 487. cf. Eupomacentrus (Brachypomacentrus) ater Blkr.

Pomacentrus (Pomacentrus) azysron Blkr. 485, 50.

Pomacentrus bankanensis Blkr. 156, 2 I6. 157, 234. 182, I59. 184, 202. 185, 224. 234, 354. 235, 369. 275, 19. 287, 43. 315, 23 I. $320,267.354$, I 45.358, I $85.364,278.388,27$ I. 425, г 36.437 , 38. 459, 85. cf. Pomacentrus (Pseudopomacentrus) trilineatus Blkr.

Pomacentrus bifasciatus Blkr. 99, 330. 285, 244. 315, 23 I. 318, 25 . 320, 267. 354, I45. cf. Dischistodus bifasciatus Blkr.

Pomacentrus bilineatus Casteln. cf. Pomacentrus (Pseudopomacentrus) trilineatus Blkr.

Pomacentrus biocellatus Rüpp. cf. Pomacentrus (Pseudopomacentrus) trilineatus Blkr.

Pomacentrus brachialis $C . V .6,2$. cf. Pomacentrus (Pseudopomacentrus) melanopterus Blkr.

Pomacentrus chrysopoecilus $K$. v. H. 6, 2. 54, 284. 184, 202. 185, 224. 234, 354. 270, 35. 275, I9. 287, 43. 354, 145. 358, I85. 364, 278. 388, 27x. cf. Dischistodus chrysopoecilus Blkr.

Pomacentrus chrysurus $C . V$. cf. Pomacentrus (Pseudopomacentrus) trilineatus Blkr.

Pomacentrus (Pomacentrus) coeruleus Q. G. 459, 85. 495, I 5 .

Pomacentrus (Pomacentrus) cyanomos Blkr. 148, 89. 156, 216. 270, $35.485,45$.

Pomacentrus cyanospilos Blkr. 69, 709. cf. Eupomacentrus (Eupomacentrus) lividus Blkr.

Pomacentrus dimidiatus Blkr. 476, 385 .

Pomacentrus (Pseudopomacentrus) dimidiatus Blkr. 485, 63.

Pomacentrus dorsalis Gill 425, I36. cf. Pomacentrus (Pseudopomacentrus trilineatus $B l k r$.

Pomacentrus emarginatus C. $V .6,2.54,284.287,43.318,250$. 354, I 45. 364, $278.396,296$. cf. Pomacentrus (Pseudopomacentrus) trilineatus Blkr.

Pomacentrus enneadactylus Lac. cf. Scolopsides Vosmeri $C . V$., Scolopsis Vosmaeri $C$. $V$.

Pomacentrus falcula Lac. cf. Tetragonoptrus (Oxychaetodon) falcula Blkr.

Pomacentrus fasciatus $(K . v . H$.$) C. V. 6, 2. 9, 633. 78, 482. 185,$ 
224. 275, I 9. 285, 244. 287, 43. 309, I 54. 320, 267. 354, I 45 . 358, I85. 364, 278. cf. Dischistodus fasciatus Gill.

Pomacentrus furcatus Thioll. cf. Pomacentrus (Pomacentrus) pavo Lac. Pomacentrus hogoleuensis Hombr. Jacq. cf. Pomacentrus (Pseudopomacentrus) littoralis Blkr.

Pomacentrus interorbitalis Gthr. cf. Dischistodus prosopotaenia Blkr. Pomacentrus katunko Blkr. 53, I69. 54, 2S4. 156, 2 x6. 170, 479. 208, 239. 234, 354. 235, 369. 270, 35. 275, I9. 287, 43. 315, 231. 320, 267. 354, I 45. 358, I $85.364,278.382,397$. cf. Ponacentrus (Pseudopomacentrus) trilineatus Blkr.

Pomacentrus kumkum Montrouz. cf. Dischistodus notophthalmus Blkr., Eupomacentrus (Eupomacentrus) lividus Blkr.

Pomacentrus leucopleura Blkr. 106, 85. cf. Eupomacentrus (Brachypomacentrus) albifasciatus $B l k r$.

Pomacentrus littoralis $(K . v . H$.$) C. V. 6, 2. 78, 483. 167, 372. 185,$ 224. 231, I48. 270, 36. 275, I9. 318, 250. 320, 267. 354, I 45. 364, 278. 394, 286. 459, 85. cf. Pomacentrus (Pseudopomacentrus) littoralis Blkr.

Pomacentrus (Pseudopomacentrus) littoralis Blkr. 483, 39. 485, 53.

Pomacentrus littoratus Day cf. Pomacentrus (Pseudopomacentrus) trilineatus Blkr.

Pomacentrus lunula Lac. cf. Chaetodon lunula $C$. $V$., 'Tetragonoptrus (Chaetodontops) fasciatus Blkr.

Pomacentrus marginatus $K$. v. $H$. cf. Dischistodus trimaculatus Blkr.

Pomacentrus marginatus Schl. Mïll. cf. Pomacentrus (Pseudopomacentrus) trilineatus $B l k r$.

Pomacentrus melanochir Blkr. 476, 396.

Pomacentrus (Pomacentrus) melanochir Blkr. 485, 49.

Pomacentrus melanopterus Blkr. 66, 562. 231, I48. 234, 354. 318, 250. 354, I 45. 358, I $85.364,278$. cf. Pomacentrus (Pseudopomacentrus) melanopterus Blkr.

Pomacentrus (Pseudopomacentrus) melanopterus Blkr. 485, 55.491, 47 .

Pomacentrus melanotus Blkr. 176, I3. 275, 20. cf. Dischistodus notophthalmus Blkr.

Pomacentrus moluccensis Blkr. 72, I18. 157, 234. 184, 202. 234, 354. 275, 20. 287, 43. 315, 231. 318, 250.320, 267. 354, I 45. 358, r85. 364, 278. 394, 286. cf. Pomacentrus (Pseudopomacentrus) moluccensis Blkr.

Pomacentrus (Pseudopomacentrus) moluccensis Blkr. 485, 56. 491, 47. Pomacentrus Montrouzieri Thioll. cf. Dischistolus notophthalmus Blkr. Pomacentrus nematopterus Blkr. 54, 285. 275, 20. 285, 244. 309, I 54. 315, 23I. 320, 267. 354, I45. 358, x85. 364, 278. cf. Amblypomacentrus breviceps Blkr.

Pomacentrus nigricans $C . V .6,2.10,67,68.46,2$ I $3.275,20.425$, I36. Pomacentrus notophthalmus Blkr. 73, I37. 143, 51. 167, 372. 184, 
202. 275, 20. 315, 23r. 354, I 45. cf. Dischistodus notophthalmus Blkr.

Pomacentrus notostigmus Rich. 54, 286. cf. Dischistodus chrysopoecilus Blkr.

Pomacentrus nuchalis Benn. cf. Tetradrachmum trimaculatum Blkr.

Pomacentrus obscurus Thioll. cf. Eupomacentrus (Eupomacentrus) lividus Blkr.

Pomacentrus ovoides Osc. Cart. cf. Amblypomacentrus ovoides Blkr.

Pomacentrus pavo Lac. 6, 2. 47, 247. 54, 237. 156, 2 16. 167, 373. 184, 202. 185, 224. 234, 354. 270, 36. 275, 20. 315, 231. 316, 24I. 320, $267.340,3^{6} 5.354$, I $45.364,278.369,42.459,85.495$, I 5 .

Pomacentrus (Pomacentrus) pavo Lac.483, 39. 485, 42. 491, 47. 495, I 5 .

Pomacentrus pavoninus Blkr. 80, 85. 394, 286. cf. Pomacentrus (Pomacentrus) pavo Lac.

Pomacentrus perspicillatus Kner cf. Dischistodus prosopotaenia Blkr. Pomacentrus perspicillatus $C$. $V$. cf. Dischistodus trimaculatus Blkr. Pomacentrus polynema Blkr. 76, 283. 270, $3^{6}$ cf. Parapomacentrus polynema Blkr.

Pomacentrus pristiger $C . V$. cf. Eupomacentrus (Eupomacentrus) lividus Blkr., Pomacentrus littoralis $C . V$.

Pomacentrus prosopotaenia Blkr. 51, 67. 54, 284. 156, 2I6. 185, 225. 234, 354. 275, 20. 296, 104. 309, I 55. 320, 267. 354, I 45. cf. Dischistodus prosopotaenia Blkr.

Pomacentrus prosopotaenioïdes Blkr. 54, 286. cf. Eupomacentrus (Eupomacentrus) lividus Blkr.

Pomacentrus punctato-lineatus Cart. cf. Pomacentrus (Pseudopomacentrus) trilineatus Blkr.

Pomacentrus punctatus $C . V$. 184, 202. 270, 36. 354, I45. 358, I 85 .

Pomacentrus punctatus Q.G. 188, 460. 364, 278. 459, 85. cf. Eupomacentrus (Eupomacentrus) lividus Blkr.

Pomacentrus quadrifasciatus $K$. v. $H$. cf. Dischistodus fasciatus Gill.

Pomacentrus rhodonotus Blkr. 76, 282. 156, 2 г6. 270, 36. 364, 278. cf. Pomacentrus (Pseudopomacentrus) rhodonotus Blkr.

Pomacentrus (Pseudopomacentrus) rhodonotus Blkr. 485, 59.

Pomacentrus scolopsis Q. G., Gthr. 459, 85. cf. Eupomacentrus (Eupomacentrus) lividus Blkr.

Pomacentrus setifer Lac. cf. Tetragonoptrus (Linophora) auriga Blkr. Pomacentrus simsiang Blkr. 148, 90. 287, 43. cf. Pomacentrus (Pseudopomacentrus) trilineatus Blkr.

Pomacentrus sordidus Lac. cf. Glyphidodon (Glyphidodon) sordidus Rütp. Pomacentrus summana Lac. cf. Epinephelus summana Blkr.

Pomacentrus taeniometopon Blkr. 54, 283. 156, 216. 234, 354. 270, 36. $275,20.315,23$ I. 354, I 45. 358, I $85.364,278.369,42$. 394, 286. cf. Pomacentrus (Pseudopomacentrus) trilineatus Blkr.

Pomacentrus taeniops C. $V .70,729.86,5$ I2. 167, 373. 185, 225. 
188, 460. 270, 36. 354, I 45. cf. Eupomacentrus (Eupomacentrus) lividus Blkr., Pomacentrus scolopsis $Q . G$.

Pomacentrus taeniops Less. cf. Pomacentrus (Pseudopomacentrus) trilineatus $B l k r$.

Pomacentrus (Pomacentrus) taeniurus Blkr. 142, 51. 185, 225. 354, I 45. 364, $278.483,39.485,47$.

Pomacentrus tapeinosoma Blkr. 145, 376. 315, 231. 364, 278. cf. Lepidozygus tapeinosoma Gthr.

Pomacentrus trifasciatus Blkr. 237, $43^{8}$.

Pomacentrus trilineatus 6,5 .

Pomacentrus trilineatus E $E \%$. cf. Pomacentrus (Pseudopomacentrus) trilineatus Blkr.

Pomacentrus (Pseudopomacentrus) trilineatus Blkr. 483, 39. 485, 60. 491, 47. 497, го.

Pomacentrus trimaculatus Rüpp. cf. Dascyllus niger Blkr., Tetradrachmum trimaculatum Blkr.

Pomacentrus trimaculatus C. $V .6,2.10,67.46,2$ I3. 78, 48 I. 126, 344. 156, 2 I6. 157, 234. 270, 36. 275, 20. 287, 43. 354, I 45 . 358, I85. 364, 278. 425, I36. cf. Dischistodus trimaculatus Blkr.

Pomacentrus tripunctatus $C . V$. cf. Pomacentrus (Pseudopomacentrus) trilineatus $B l k r$.

Pomacentrus vanicolensis $C . V .9,633.17,9.231,148.270,3^{6 .}$ cf. Pomacentrus (Pseudopomacentrus) trilineatus Blkr., Pristotis fuscus Blkr.

Pomacentrus (Pomacentrus) violascens Blkr. 156, 222. 237, 438. 485, 46.

Pomadasys Lac. 468, 27 r.

Pomadasys argenteus Lac. 468, 272.

Pomadesys argyreus Blkr. 495, I2.

Pomadesys hasta Blkr. 495, I 2 .

Pomadesys punctulatus Blkr. 495, I2.

Pomatomus C. V. 468, 26 r.

Pomatomus telescopium Risso 468, $26 \mathrm{I}$.

Pomatoschistus Gill 453, 316, 3 I 7 .

Pomotis Raf. 468, 249.

Pomoxys Raf. 468, 248.

Pomoxys hexacanthus 468, 248.

Porogobius Blkr. 453, $32 \mathrm{I}$.

Porthmeus C. V. 297, r34.

Porthmeus argenteus $C . V .266,53.297$, I34. 302, 79.

Premnas 1, 553. 2, 524 .

Premnas biaculeatus Blkr. 95, 105. 126, 344. 156, 216. 157, 234. 184, 202. 234, 354. 270, 35. 275, I 9. 296, 104. 309, I 54. 320 , 267.358 , I $85.364,277.394,286.396,299.459,85.485$, I $7.491,46$.

Premnas leucodesmus $K . \%$. $H$. cf. Premnas trifasciatus $C$. $V$., Premnas biaculeatus Blkr. 
Premnas semicinctus C. $V .6,7$. cf. Premnas biaculeatus Blkr.

Premnas trifasciatus $C . V .6,9.10,67.46,2$ I3. cf. Premnas biaculeatus Blkr.

Premnas unicolor $C$. $V$. cf. Premnas biaculeatus Blkr.

Priacanthichthys Day 468, 256.

Priacanthiformes 468, 250 .

Priacanthus Cur. 1, 553. 468, 250, 336.

Priacanthus alticlarens $V$ al. 459, 87.

Priacanthus argenteus $C . V .13$, I 2.

Priacanthus benmebari T. Schl. 90, 25. 497, 5 .

Priacanthus Blochii Blkr. 78, 456. 91, I66. 127, 345. 157, 232. 167, 370. 270 , I 5. 275, 22. 296, I05. 315, 232. 354, 146. 364, 280 . cf. Priacanthus hamrur $C . V$.

Priacanthus carolinus $C . V .47,235.358$, I $86.364,280.427$, І 74 . 459, 87. 495, Iо.

Priacanthus dubius Schl. 497, 5. cf. Priacanthus hamrur $C$. $V$.

Priacanthus fanal $C . V$. cf. Priacanthus hamrur $C \cdot V$.

Priacanthus fax $C . V$., Blkr. 2, 526. cf. Priacanthus hamrur $C . V$.

Priacanthus fax $B l k r$. cf. Priacanthus macracanthus $C . V$.

Priacanthus hamruhr $C, V$. cf. Priacanthus hamrur $C . V$.

Priacanthus hamrur $C . V .427$, I76. 437, 38. 459, 87.491, 41.495, го.

Priacanthus holocentrum Blkr. 13, 48. 235, 362. 270, I6. 275, 2. 287, 44. 364, 280. cf. Priacanthus tayenus Rich.

Priacanthus japonicus Schl. 497, 5.

Priacanthus japonicus $C . V .44$, I7 1. 54, 234. 425, 136. 459, 87. cf. Priacanthus Blochii Blkr., Priacanthus hamrur $C$. $V$.

Priacanthus macracanthus $C . V .13,48.90,26$. cf. Priacanthus hamrur C. $V$.

Priacanthus macracanthus Blkr. 364, 280.

Priacanthus macrophthalmus Cuv. 468, 250.

Priacanthus Meyeri Gthr. cf. Pseudopriacanthus niphonius Blkr.

Priacanthus niphonius $C . V .269,73$. cf. Pseudopriacanthus niphonius Blkr.

Priacanthus Schmittii Blkr. 67, 572. cf. Priacanthus tayenus Rich.

Priacanthus speculum $C . V$. 459, 87 .

Priacanthus tayenus Rich. 425, I36. 427, I 72.

Priodon 163, 62.

Priodon anginosus Blkr. 103, 492.

Priodon annularis $C$. $V$. cf. Naseus annulatus $B l k r$.

Priodon annulatus Q.G. cf. Naseus annulatus Blkr.

Priodon hexacanthus Blkr. 128, 42 I.

Priodon tapeinosoma Blkr. 103, 494. cf. Naseus tapeinosoma Blkr. Priodonophis $K p$. 351, I21.

Priodonophis minor Blkr. cf. Priodonophis reticularis Blkr.

Priodonophis moluccensis Blkr. 348, 48. 352, I31. 364, 296. 
Priodonophis reticularis Blkr. 425, т $24.497,26$.

Priolepis Ěrr. 453, 305.

Prionobutis Blkr. 453, 305. 460, 103, 105.

Prionobutis dasyrhynchus Blkr. 478, 7.5.

Prionobutis koilomatodon Blkr. 478, 73 .

Prionodes Jen. 468, 255.

Prionodon annularis $C . V .66,558$.

Prionotus japonicus Blkr. 102, 398. 118, 75. 425, I27. 497, I3.

Prionurus coume Less. cf. Naseus lituratus $C$. $V$.

Prionurus scalprum $C$. $V$. cf. Naseus scalprum Blkr.

Priopis argyrozona $(K . v . H) \quad C . V$. 13, 3. cf. Ambassis gymnocephalus Blkr.

Pristiapogon Klunz. 468, 259.

Pristigaster chinensis Basil. 425, I48.

Pristigaster indicus Szons. 272, 26. 287, 6r. cf. Opisthopterus tartoor Gill.

Pristigaster tardoore Cuv. cf. Opisthopterus tartoor Gill.

Pristigaster tartoor Blkr. cf. Opisthopterus macrognathus Blkr.

Pristigaster tartoor $C . V$. 55, 414. 60, 25. 91, 74. 127, 345. 178, 2 . 227, 408. 270, 57. cf. Opisthopterus tartoor Gill.

Pristiophorus cirratus $M . H .118$, I28. 119, I8. cf. Pristiophorus serratus $M . H$.

Pristiophorus serratus $M . H .497,3$.

Pristipoma Cuv. 1, 553. 468, 27 I.

Pristopoma anas $\mathrm{Val} .459,92$.

Pristipoma argenteum _C. V. 468, 272.

Pristipoma argenteum var. nageb Klunz. cf. Pristipoma nageb Rüpp.

Pristipoma argyreum $C . V .18,22.55,4$ I . 91, 36. 156, 214. 227, 407. 270, I9. 272, II. 287, 45. 432, 313. 459, 92. cf. Pomadesys argyreus $B l k r$.

Pristipoma auritum C. $V .288,7$ г. 356, т 73. 432, 3І 6.

Pristipoma caripa C. V. 2, 523. 18, 2 I. 25, 8. 56, 444. 91, 36. 156, 21 4. 231, I 45. 234, 352. 270, I9. 275, 25. 287, 45. 288, 7I. 346, 3r. 356, I73. cf. Pristipoma maculatum Gthr.

Pristipoma chloronotum Rich. 425, 139.

Pristipoma chrysobalion $(K . v . H) C .$.$V . cf. Pristipoma hasta C . V$.

Pristipoma Commersonii C. $V .91,36$. cf. Pomadesys hasta Blkr., Pristipoma hasta $C . V$.

Pristipoma Commersonii Cant. 91, 36. 275, 25. 287, 45.

Pristipoma Dussumieri $C . V .18,20$. cf. Pristipoma paiheeli $C . V$.

Pristipoma furcatum Blkr. 432, 304.

Pristipoma gallinaceum Rich.425, I39.

Pristipoma grammopoecilum Rich.425, I 39.

Pristipoma guoraca $C . V .2,523.18,23.91,38.170,479.270$, I9. $275,25.288,7$ 1. 364, 280. 432, 315.

Pristipoma hasta $C . V .16,4.18,20.25,8.50,47$ I. 91, 38. 231, INDEX BLEEKER. 
I $45.237,436.270$, I9. $275,25.287,45.316,24$ I. 320, 268. 346, 3 I. 354, I 46.358 , г 86. 364, 280. 369, 42. 395, 291. 396, 299. 425, I 39. 432, 308. 459, 92. cf. Pomadesys hasta Blkr.

Pristipoma japonicum $C . V .175$, I 4. 425, r39. cf. Parapristipoma trilineatum Blkr. cf. Pristipoma hasta $C . V$.

Pristipoma Jubelini $C . V$. 302, 54 .

Pristipoma koakan C. $V ., B l k r .2,522.9,633.10,66.17,3.46$, 2т2. $51,56$.

Pristipoma leucurus $C . V$. 459, 92.

Pristipoma macrophthalmus Blkr. 302, 52.

Pristipoma maculatum Gthr. 364, 280. 396, 299. 425, I39. 432, 306. Pristipoma manadense Gthr. 432, 3 го.

Pristipoma mucronatum Eyd. Soul. cf. Hapalogenys mucronatus Gthr. Pristipoma nageb Rïpp. C. $V$. 16, 4. 18, 2 I. 25, 8. 51, 56. 55, 4 Ir. 158, 273. 170, 479. 227, 407. 237, 436. 270, I9. 272, I1. 275, 25. 364, 280. 395, 291. 425, I39. 432, 310.

Pristipoma nigrum $C . V$., Mert. 18, 26. 288, 7 r. cf. Diagramma crassispinum Rüpp., Plectorhynchus crassispina $B l k r$.

Pristipoma paikeeli C. $V$. 18, 20. 25. 8. 91, 38. 236, 330. 270, 19. 288, 7 . cf. Pristipoma furcatum $B l k r$.

Pristipoma pihloo Rich.425, I39.

Pristipoma punctulatum Rüpp. 459, 92. cf. Pomadesys punctulatus Blkr.

Pristipoma sexlineatum Q. G. cf. Therapon (Pelates) quadrilineatus Blkr. Pristipoma therapon Blkr. 18, 22. 31, 100. 46, 212. 267, r39. 275, 25. 432, 3I2.

Pristipoma unicolor $K, v$. $H$. cf. Pristipoma guoraca $C . V$.

Pristipomatini 468, 270.

Pristipomoides Blkr. 438, 44. 468, 276.

Pristipomoides typus Blkr. 67, 575. cf. Aprion (Aprion) pristipoma Blkr., Chaetopterus pristipoma Blkr.

Pristis antiquorum Lath. 459, 68.

Pristis cirratus Lath., T. Schl. cf. Pristiophorus cirratus $M$. $H$.

Pristis cuspidatus Lath. 270, 67. cf. Squalus cuspidatus Blkr.

Pristis dubius Blkr. 64, 56. 85, 459. 272, 6.

Pristis microdon Lath., M. H. 64, 54. 91, 80. 272, 6.

Pristis pectinatus Lath. 266, $5^{8}$.

Pristis semisagittatus Shave, Cuv., Lath. 25, I3. 51, 60. 64, 53. 91, 8o. $270,67.288,66$.

Pristis zysron Blkr. 49, 442. 55, 441. 64, 55. 272, 6. cf. Pristis dubius Blkr.

Pristocantharus Gill 468, 271.

Pristolepis Jerd. 468, 3I8.

Pristolepis marginatus Jerd. 468, 318.

Pristotis Rüpp. 148, 90. 
Pristotis fuscus Blkr. 17, 9. cf. Pomacentrus (Pseudopomacentrus) trilineatus Blkr.

Pristotis trifasciatus Blkr. 9,637. cf. Amblypomacentrus breviceps Blkr.

Pristotis violascens Blkr. 9, 637. cf. Pomacentrus (Pomacentrus) violascens Blkr.

Proamblys Gill 468, 276.

Proamblys niger Gill cf. Lutjanus macolor Blkr.

Prochilus akallopisus Blkr. 354, r45. 358, i $S_{5} .364,277.485,35$.

Prochilus bifasciatus Blkr. 338, 360. 354, I 45.358, I $85.364,277$. 425, I35. 483, 39. 485, 31. 491, 46.

Prochilus chrysogaster Blkr. 459, 85. 495, I 5 .

Prochilus chrysurus Blkr. 364, 278. cf. Prochilus polymnus Blkr.

Prochilus Clarckii Blkr. 315, 23r. 318, 250. 354, I45. 358, I85. 364, 277. 369, 42. 394, 286. 425, I35. cf. Prochilus polymnus Blkr.

Prochilus ephippium Blkr. 364, 278. 483, 39. 485, 23.

Prochilus frenatus Blkr. 497, Io.

Prochilus fusciventer Blkr. 459, $8_{5} .495, x_{5}$

Prochilus macrostoma Blkr. 480, I36. 485, 25. cf. Prochilus frenatus Blkr.

Prochilus melanopus Blkr. 315, 231. 364, 278. 394, 286. 485, 26. 491, 46.

Prochilus percula Blkr. 316, 241. 320, 267. 340, 364. 354, I 45 . 358, I $85.364,278.369,42.396,299.425$, I $35.483,39.485$, 33. 491,46 .

Prochilus perideraion Blkr. 316, 241. 358, I85. 364, 278. 369, 42. $485,37.491,46$.

Prochilus polylepis Blkr. 480, I35. 485, 22. 491, 46.

Prochilus polymnus Blkr. 483, 39.485, 27. 491, 46.495, I 5.497, 10.

Prochilus Rosenbergii Blkr. 485, 38. 491, 47.

Prochilus Sebae Blkr. 485, 30.

Prochilus trifasciatus Blkr. 459, 85. cf. Prochilus bifasciatus Blkr.

Prochilus xanthurus Blkr. 459, 85. cf. Prochilus polymnus Blkr.

Prognathodes Gill 468, 303.

Prognathodes pelta Gill cf. Prognathodus aculeatus Poey.

Prognathodus Gill 468, $3 \circ 3$.

Prognathodus aculeatus Pocy 468, 303. 474, 3 I 5 .

Prognathodus longirostris Blkr. 474, 315. 481, 33. 495, I4.

Promicrops Gill 468, 256.

Promicropterus Gill 468, 258.

Pronotogrammus Gill 468, 255.

Prosopodasys Cant. 473, 298.

Prosopodasys cottoides Gthr. cf. Cottapistus cottoides Blkr.

Prosopodasys (Prosopodasys) cottoides Gthr. 425, I4I.

Prosopodasys (Tetraroge) crista galli Blkr. 425, I4I.

Prosopodasys depressifrons Gthr., Blkr. 354, I48.475, So.497, I 2. 
Prosopodasys leucogaster Gthr. 358, I88. 364, 286. cf. Gymnapistus leucogaster $B l k r$.

Prosopodasys (Prosopodasys) leucogaster Gthr. 425, I4I.

Prosopodasys (Tetraroge) longispinis Blkr. 425, I4r.

Prosopodasys niger Gthr. 364, 286. cf. Gymnapistus niger Blkr.

Prosopodasys (Prosopodasys) sinensis Blkr. 425, I4r, I 5 I.

Prosopodasys trachinoides Gthr. 395, 292.

Prosopodasys trachinoïdes Cant. 475, 78. cf. Apistus trachinoïdes C. $V$. Prosopodasys (Prosopodasys) trachinoides Gthr. 425, I4I.

Prosopodasys (Centropogon) urostigma Blkr. 425, I4I, I 5 I.

Prosopodasys Zollingeri Gthr. 475, 77 .

Prospinus Poey 468, 256.

Proteracanthiformes 468, 296.

Proteracanthus Gthr. 468, 297.

Proteracanthus sarissophorus Gthr. 468, 297. 481, I8.

Psammoperca Rich. 468, 264.

Psammoperca datnioides Rich.468, 264. cf. Psammoperca waigiensis Blkr.

Psammoperca macroptera Gthr. 468, 264.

Psammoperca waigiensis Blkr. 119, 5. 185, 222. 235, 362. 275, 24 . 287, 45. 395, 29г. 396, 296. 425, г 37. 437, 38. 468, 264.

Psenes anomalus Blkr. 102, 409. 118, 104. 425, I33. cf. Psenopsis anomalus Gill.

Psenes auratus C. $V .91,46$.

Psenes cyanophrys $C . V$. $57,74$.

Psenes fuscus Guich. 459, 94.

Psenes javanicus C. $V . \mathbf{5 7}, 74.163,60$.

Psenes leucurus $C . V .119$, I I. 302, I4.

Psenopsis anomalus Gill 497, 15.

Psephurus gladius Gïnth.497, 27.

Psetta Stons. 294, 424.

Psettodes Benn. 294, 423.

Psettodes erumei Blkr. 364, 274. 395, 290. 425, г 30.

Psettodes goniographicus Blkr. 425, г3о.

Psettus 1, 553 .

Psettus argenteus Rich. 119, 10.

Psettus Commersonii C. $V$. cf. Monodactylus falciformis Lac.

Psettus orbicularis Guich. cf. Monodactylus orbicularis Blkr.

Psettus rhombeus C. V. 2, 521. 22, 29. 51, 57. 91, 40. 156, 2 I4. 184, 200. 185, 223. 235, 366. 270, 27. 275, 35. 287, 51. 288, 73 . 296, I08. cf. Monodactylus argenteus Blkr.

Psettus Sebae C.V. 302, 68.

Pseudacanthicus Blkr. 306, 79.

Pseudacanthicus serratus Blkr. 306, 79. 336, I3.

Pseudageneiosus Blkr. 306, Io8.

Pseudageneiosus brevifilis $B l k r .306$, IoS. 336, 83 . 
Pseudageneiosus dentatus Blkr. 336, 4.

Pseudageneiosus inermis Blkr. 336, 4.

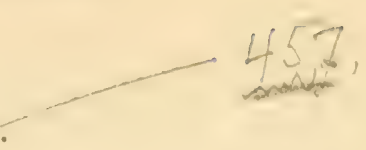

Pseudalutarius nasicornis Blkr. 364, 273 .

Pseudaluteres Blkr. 367, I4.

Pseudaluteres nasicornis Blkr. 437, 37. 459, 71. 495, 20.497, I 7.

Pseudambassis Blkr. 468, 292.

Pseudambassis lala Blkr. 468, 292.

Pseudamia Blkr. 468, $26 \mathrm{I}$.

Pseudamia amblyuropterus Blkr. 456, 8 r.

Pseudamia polystigma Blkr. 364, 284. 456, 8o. 468, 26r.

Pseudancistrus Blkr. 306, 78.

Pseudancistrus barbatus Blkr. 306, 78 .

Pseudancistrus guttatus Blkr. 336, ro.

Pseudanthias Blkr. 468, 252.

Pseudanthias chirospilus Blkr. 426, I6r.

Pseudanthias cichlops Blkr. 426, х66.

Pseudanthias Huchtii Blkr. 426, I60.

Pseudanthias hypselosoma Blkr. 491, 58. 500, 29.

Pseudanthias lepidolepis Blkr. 426, I63.

Pseudanthias manadensis Blkr. 426, 165 .

Pseudanthias pleurotaenia Blkr. 426, r58. 468, 252.

Pseudapocryptes Blkr. 453, 328 .

Pseudariodes Blkr. 306, 99.

Pseudariodes clarias Blkr. 306, 99. 336, 4.

Pseudarius Blkr. 306, 9r.

Pseudarius arius Blkr. 306, 9 I.

Pseudauchenipterus Blkr. 306, 88 .

Pseudauchenipterus nodosus Blkr. 306, 88. 336, 43.

Pseudecheneis Blyth 306, I04.

Pseudecheneis sulcatus Blyth 306, I04.

Pseudechidna Brummeri Blkr. 320, 272.

Pseudetroplus coruchi Blkr. 297, I26.

Pseudeutropius Blkr.299, 398. 306, го6.

Pseudeutropius brachypopterus Blkr. 306, го7.

Pseudobagrichthys Blkr. 306, 94.

Pseudobagrichthys macracanthus Blkr. 356, I75.

Pseudobagrichthys macropterus Blkr. 306, 94. 356, I75.

Pseudobagrus Blkr. 269, 87. 306, 95.

Pseudobagrus aurantiacus Blkr.269, 85. 306, 95. 497, 22.

Pseudobagrus Vachelli Gthr. 425, I25.

Pseudobalistes Blkr. 367, r 1 .

Paeudoblennius anahaze Blkr. 497, Ir.

Pseudoblennius percoides Günth. cf. Pseudoblennius anahaze Blkr. Pseudoblennius pseudoclinus Schl. cf. Pseudoblennius anahaze Blkr. Pseudobrama 411, 253. 
Pseudobrama Dumerili Blkr. 411, 253. 415, I2, 60. 425, I 45.

Pseudobrama hypselosoma Blkr. 416, II9, I2I. 425, I45.

Pseudobrama melanotopterus Blkr. 416, II9, I2 I. 425, I 45.

Pseudocallophysus Blkr. 306, I02.

Pseudocallophysus ctenodus Blkr. 306, Iо2.

Pseudocetopsis Blkr. 300, 403. 306, I I 2.

Pseudocetopsis gobioides Blkr. 306, I 2 .

Pseudocheilinus Blkr. 292, 95.

Pseudocheilinus hexataenia Blkr. 293, 276. 364, 276. 459, 84. 491, 40.

Pseudochromidoidei 468, 320.

Pseudochromis Rüpp.468, 320, $32 \mathrm{x}$.

Pseudochromis (Pseudochromis) adustus Mïll. Trosch. 465, I8.

Pseudochromis cyanotaenia Blkr. 164, 72. 354, I46. 364, 279.

Pseudochromis (Leptochromis) cyanotaenia Blkr. 465, 2 I.

Pseudochromis fuscus Mïll. Trosch. 69, 708. 131, 69. 157, 234.

167, 372. 184, 202. 185, 224. 234, 354. 236, 33 I. 275, 21. 315, 23 I.

318, 25 I. 320, 267. 354, I46. 358, 186. 364, 279. 394, 286. 396, 299. 465, I6.

Pseudochromis melanotaenia $B l k r .320,273.468,32 \mathrm{I}$.

Pseudochromis (Leptochromis) melanotaenia Blkr. 465, 20.

Pseudochromis olivaceus Rüpp.468, $32 \mathrm{I}$.

Pseudochromis polyacanthus Blkr. 145, 375. 315, 23I. 320, 267.

358, I86. cf. Pseudogramma polyacanthus Blkr.

Pseudochromis (Pseudochromis) Ransonneti Steind. 465, r9.

Pseudochromis tapeinosoma Blkr. 72, I I5. 184, 202. 270, 35. 354, I 46.

358, I86. 364, 279 .

Pseudochromis (Leptochromis) tapeinosoma Blkr. 465, 23.

Pseudochromis xanthochir Blkr. 129, 443. 275, 21. 285, 244. 354, I $46.465,17$.

Pseudocoris Blkr. 292, $9^{8 .}$

Pseudocoris heteropterus Blkr. 293, 28 I. 364, 276. 425, I35.

Pseudoculter Blkr. 261, 432. 314, 2 10.

Pseudoculter exiguus Blkr. 415, r4. 425, r 45.

Pseudoculter pekinensis Blkr. 415, I4. 425, I45.

Pseudodax 290, 229. 292, 96.

Pseudodax moluccanus Blkr. 293, 277. 320, 265. 358, r84. 364, 276. 459, 84. 491, 40.

Pseudodoras niger Blkr. cf. Oxydoras niger Blkr.

Pseudogobio Blkr. 261, 425. 314, 205. 411, 253.

Pseudogobio esocinus Blkr. 497, 23.

Pseudogobio rivularis Blkr. 411, 252. 415, 23. 425, I44. 496, I2.

Pseudogobio sinensis Gthr. cf. Pseudogobio rivularis Blkr.

Pseudogobiodon Blkr. 453, 309.

Pseudogobiodon macrochir Blkr. 453, 309. 461, I I6.

Pseudogramma Blkr. 468, $32 \mathrm{I}$. 
Pseudogramma polyacanthus Blkr. 465, 24. 468, 321. 491, 46.

Pseudohemiodon Blkr. 306, 8I.

Pseudohemiodon platycephalus Blkr. 306, 8I.

Pseudohypophthalmus Blkr. 306, ro9.

Pseudohypophthalmus fimbriatus Blkr. 306, ro9.

Pseudojulis Blkr. 292, 99.

Pseudojulis Girardi Blkr. 293, 289.

Pseudolabrus Blkr. 292, ro2.

Pseudolabrus celidotus Blkr. 331, 444.

Pseudolabrus Güntheri Blkr. 297, I3I.

Pseudolabrus rubiginosus Blkr. 363, 250. 425, I35. 497, 5 .

Pseudolaubuca Blkr. 345, 28.

Pseudolaubuca sinensis Blkr. 345, 29. 415, I 5. 425, 146.

Pseudoloricaria Blkr. 306, 80.

Pseudomonacanthus Blkr. 367, I2.

Pseudomonacanthus hippocrepis Blkr. 459, 7I. 495, 20.

Pseudomonacanthus macrurus Blkr. 368, 27.

Pseudomonacanthus tomentosus Blkr. 368, 27.

Pseudomonopterus Kl. 473, 296.

Pseudomonopterus antennatus Blkr. 315, 234. 354, I48. 358, I88. 364, 285. 394, 287. 396, 297. 425, r 4 I. 437, 39. 459, 87. cf. Pseudomonopterus (Pterois) antennatus Blkr.

Pseudomonopterus (Pterois) antennatus Blkr. 475, 48.491, 49. 495, I6. Pseudomonopterus brachypterus Blkr. 354, I48. 358, I88. 364, 285. 394, 287. cf. Pseudomonopterus (Dendrochirus) brachypterus Blkr.

Pseudomonopterus (Dendrochirus) brachypterus Blkr. 475, 53.

Pseudomonopterus heterurus Blkr. 364, 285.

Pseudomonopterus kodipungi Blkr. 346, 31. 459, 87.

Pseudomonopterus (Pterois) kodipungi Blkr. 475, 45. 495, I6.

Pseudomonopterus lunulatus Blkr. 425, I4I.

Pseudomonopterus (Pterois) lunulatus Blkr. 475, 47. 497, r2.

Pseudomonopterus volitans Blkr. 315, 234. 320, 269. 330, 266. 340, 365. 354, I 48.358 , I $88.364,285.369,42.395,292.425$, I 4 I。 459, 87. cf. Pseudomonopterus (Pterois) volitans Blkr.

Pseudomonopterus (Pterois) volitans Blkr. 475, 42. 491, 49. 495, I6. 497, I2.

Pseudomonopterus zebra Blkr. 315, 234. 320, 269. 354, I 4 S. 358, isS. 364, 285. 396, 297. 425, I 4 I. 437, 39. 459, 87. cf. Pseudomonopterus (Dendrochirus) zebra $B l k r$.

Pseudomonopterus (Dendrochirus) zubra Blkr. 475, 50.491, 49. 495, 16.

Pseudomoringua Blkr. 351, II 4 .

Pseudomuraena Johns. 351, $12 \mathrm{I}$.

Pseudopangasius Blkr. 299, 399. 306, 107.

Pseudopangasius nasutus $B l k r .324,72$.

Pseudopangasius polyuranodon Blkr. 306, 107. 
Pseudopentaceros Blkr. 468, 270.

Pseudoperilampus Blkr. 314, 2I4. 328, 258. 329, 264. 411, 253.

Pseudoperilampus ocellatus Kner cf. Rhodeus ocellatus Gthr.

Pseudoperilampus typus Blkr. 328, 258. 497, 24.

Pseudophoxinus Blkr. 261, 431. 314, 209. 329, 263.

Pseudopimelodus Blkr. 306, rо г.

Pseudopimelodus raninus Blkr. 306, 1о .

Pseudoplatystoma Blkr. 306, 97.

Pseudoplatystoma fasciatum Blkr. 306, 97. 336, 72.

Pseudoplesiops Blkr. 184, 2 I 5. 468, 323.

Pseudoplesiops typus Blkr. 184, 2 I 7. 465, 30. 468, 323. 491, 46.

Pseudoplesiopsini 468, 322.

Pseudopleuronectes Blkr. 294, 428 .

Pseudopriacanthus Blkr. 468, $25 \mathrm{I}$.

Pseudopriacanthus niphonius Blkr. 408, 24r. 431, 239. 459, 88

468,25 I. 497, 5 .

Pseudopterus Kl. 473, 296.

Pseudorasbora Blkr. 261, 437. 269, 97. 314, 2 I 2.

Pseudorasbora parva Blkr. 415, Ir. 425, I 44. 497, 23.

Pseudorasbora pusilla Blkr. 269, 98. 497, 23. cf. Pseudorasbora parva Blkr.

Pseudorhamdia Blkr. 306, IoI.

Pseudorhamdia ascita Blkr. 298, 384 .

Pseudorhamdia macronema Blkr. 336, 79.

Pseudorhamdia maculata Blkr. 306, Ior.

Pseudorhamdia ornata Blkr. 336, 77.

Pseudorhombus Blkr. 294, 426.

Pseudorhombus cinnamomeus Blkr. 497, 2 r.

Pseudorhombus lentiginosus Blkr. 358, I84.

Pseudorhombus maculosus Blkr. 425, I 3 o.

Pseudorhombus malayanus Blkr. 370, 43.

Pseudorhombus Mogki Blkr. 315, 230. 354, I44. 358, I84. 364, 273.

Pseudorhombus neglectus Blkr. 370, 44.

Pseudorhombus oligodon Blkr. 497, 2 I.

Pseudorhombus oligolepis Blkr. 497, 21.

Pseudorhombus pantherinus Blkr. 296, Iо3.

Pseudorhombus pentophthalmus Gthr. 425, 130.

Pseudorhombus poëcilurus Blkr. 364, 274.

Pseudorhombus polyspilos Blkr. 296, ro3.

Pseudorhombus polyspilus Blkr. 396, 298. 496, 3 .

Pseudorhombus Russelli Gthr. 364, 274. 395, 290. 425, I30. 491, 54.

Pseudorinelepis Blkr. 306, 79.

Pseudoscarus Blkr. 290, 230.

Pseudoscarus aeruginosus Blkr. 290, 238. 309, I54. 315, 230. 320, 265.358, I $84.437,37.495,8.496,2$. 
Psendoscarus arcuatus Blkr. 356, I 73.

Pseudoscarus balinensis Blkr. 290, 238.

Pseudoscarus bataviensis Blkr. 290, 242.

Pseudoscarus bicolor Blkr.290, 236.

Pseudoscarus Cantori Blkr. 290, 240. 296, I04. 320, 265. 364, 275.

Pseudoscarus capistratoides Blkr. 290, 234.

Pseudoscarus capitaneus Gthr. 459, 8r. 495, 8.

Pseudoscarus caudofasciatus Gthr. 459, 8o. 495, 8.

Pseudoscarus celebicus Blkr. 290, 232. 425, 134.

Pseudoscarus chinensis Steind. 430, 234.

Pseudoscarus chlorodon Blkr. 296, 232.

Pseudoscarus cyanescens Blkr. 459, 81. 495, 8.

Pseudoscarus cyanognathos Blkr. 290, 235. 459, 8 I.

Psendoscarus cyanotaenia Blkr. 290, 233.

Pseudoscarus dimidiatus Blkr. 290, 239. 491, 40.

Pseudoscarus dubius Blkr. 425, r 34 .

Pseudoscarus Dussumieri Blkr. 290, 24I. 459, 8I.

Pseudoscarus falcipinnis Playf. 459, $8 \mathrm{I}$.

Pseudoscarus Forsteni Blkr. 290, 238.

Pseudoscarus frenatus Blkr. 295, 239. 491, 40.

Pseudoscarus ghobban Giüth. 495, 8.

Pseudoscarus gibbus Blkr. 459, 8r.

Pseudoscarus gracilis Steind. 425, I 49. 430, 234.

Pseudoscarus gymnognathus Blkr. 290, 233.

Psendoscarus harid Gthr. 396, 296. 459, 8r. 495, 9.

Pseudoscarus hypselopterus Blkr. 290, 237.

Pseudoscarus janthochir Blkr. 290, 234. 315, 230.

Pseudoscarus javanicus Blkr. 290, 237.

Pseudoscarus Jenynsi Blkr. 290, 242.

Pseudoscarus macrocheilus Blkr. 290, 237. 309, I54.

Pseudoscarus maculiceps Peters 495, 8.

Pseudoscarus maculosus Gthr. 459, 8I. 495, 9.

Pseudoscarus mastax Blkr. 290, 236. cf. Pseudoscarus harid Gthr.

Pseudoscarus microcheilos Blkr. 290, $23 \mathrm{r}$.

Pseudoscarus micorrhinos Blkr. 290, $23 \mathrm{r}$.

Pseudoscarus microrrhinus Blkr. 425, I34.

Pseudoscarus Moensi Blkr.290, $24 \mathrm{I}$.

Pseudoscarus muricatus Blkr. 290, 232.

Pseudoscarus nuchipunctatus Blkr. 290, 235. 320, 265. 364, 275.

425, r34. 459, $8 \mathrm{I}$.

Pseudoscarus oktodon Blkr. 290, 235.

Psendoscarus ovifrons Blkr. 497, 4.

Pseudoscarus pentazona Blkr. 290, 241. 491, 40.

Pseudoscarus psittacus Blkr. 288, 70.

Pseudoscarus pulchellus Blkr. 290, 236. 425, I35. 459, SI. 495, 9. 
Pseudoscarus pyrrhostethus Blkr. 290, 239. 364, 275. 425, I35. $459,8 \mathrm{r} .495,9$.

Pseudoscarus Quoyi Blkr. 290, 234. 315, 230.

Pseudoscarus rhoduropterus Blkr. 290, 233.

Pseudoscarus rivulatus Blkr. 290, 240. 296, 104. 320, 265. 496, 2.

Pseudoscarus rubroviolaceus Blkr. 290, 237.

Pseudoscarus scaber Blkr. 459, 81. 495, 9.

Pseudoscarus Schlegeli Blkr. 290, 242.

Pseudoscarus singapurensis Blkr. 290, 235.

Pseudoscarus spilonotus Kner 495, 4.

Pseudoscarus striatus Gthr. 459, 8I.

Pseudoscarus strongylocephalus Blkr. 290, 23r.

Pseudoscarus sumbawensis Blkr. 290, 242. 320, 265. 459, SI. 491, 40. $495,9$.

Pseudoscarus taeniurus Blkr. 459, 8r. 495, 9.

Pseudoscarus tricolor Blkr. 290, 238. 364, 275.

Pseudoscarus Troscheli Blkr. 290, 232.

Pseudoscarus variegatus Blkr. 459, 8 r. 495, 9.

Pseudoscarus venosus Blkr. 459, 8I. 495, 9.

Pseudoscarus viridis Blkr. 290, 240. 364, 275. 459, 8 I.

Pseudoscarus xanthopleura Blkr. 290, 231.

Pseudosciaena Blkr. 468, 329.

Pseudosciaena (Bairdiella) acanthodes Blkr. 487. 497, 29.

Pseudosciaena albida Blkr. 307, I45. cf. Corvina albida $C . V$.

Pseudosciaena amblyceps Blkr. 307, I42. cf. Corvina amblyceps Blkr.

Pseudosciaena amoyensis Blkr. 307, r 44. cf. Corvina amoyensis Blkr.

Pseudosciaena aquila Blkr. 459, 79.

Pseudosciaena aurata Blkr. 495, I4.

Pseudosciaena bornëensis Blkr. 452, 36 .

Pseudosciaena cuja Blkr. 497, 9.

Pseudosciaena diacanthus Blkr. 452, 27.

Pseudosciaena Goldmani Blkr. 452, 33. 491, 45.

Pseudosciaena japonica Schl. 497, 9.

Pseudosciaena macrophthalmus Blkr. 452, $2 \mathrm{r}$.

Pseudosciaena microlepis Blkr. 452, 23.

Pseudosciaena miles Blkr. 452, 25.

Pseudosciaena plagiostoma Blkr. 452, 3 I.

Pseudosciaena polyactis Blkr. 487. 496, 5 .

Pseudosciaena polycladiscus Blkr. 452, 29.

Pseudosciaena Schlegeli Blkr. 497, 9.

Pseudosciaena surinamensis Blkr. 446, $45^{8}$.

Pseudosciaena Vogleri Blkr. 452, 35 .

Pseudosciaeni 468, 327.

Pseudoserranus Klunz. 468, 255.

Pseudoserranus louti Klunz. cf. Variola louti Blkr. 
Pseudosilurus Blkr. 299, 395. 306, II5.

Pseudosilurus bimaculatus B'lkr. 211, 242. 225, 357. 270, 48. 272, 19. 279, го०.

Pseudosilurus japonicus Blkr. cf. Parasilurus japonicus Blkr.

Pseudosilurus leiacanthus Blkr. 235, 37 r. 270, 48. 272, I9.

Pseudosphromenus opercularis Blkr. 496, 2.

Pseudosynodontis Blkr. 306, 87 .

Pseudosynodontis serratus Blkr. 304, 55. 306, 87.

Pseudotolithus Blkr. 302, 59. 468, 329.

Pseudotolithus brachygnathus Blkr. 302, 62 .

Pseudotolithus macrognathus Blkr. 302, 6r.

Pseudotolithus typus Blkr. 302, 60.

Pseudotrachinus pardalis Blkr. 291, I20. 302, 95.

Pseudotrachinus radiatus Blkr. 291, 1 I 7 .

Pseudoxiphophorus Blkr.261, 440.

Pseudupeneus Blkr. 297, I34. 302, 56. 468, 333.

Pseudupeneus prayensis Blkr. 302, 57. 468, 333.

Psilocephalus Szons. 367, I4.

Psilocephalus barbatus Szens. 368, 29. 395, 290. cf. Pogonognathus barbatus Blkr.

Psilonotus amboinensis $B \mathrm{lkr}$. 357, x $80.364,272$.

Psilonotus Bennetti Blkr. 270, 66. 275, I3. 287, 40. 315, 230. 354, I $43.358, \mathrm{r} 83$.

Psilonotus janthinopterus Blkr. 275, I3. 364, 272.

Psilonotus margaritatus Blkr. 320, 265. 354, I43. 364, 272.

Psilonotus ocellatus Blkr. 364, 272.

Psilonotus striolatus Blkr. 275, I3. 315, 230. 320, 265. 354, 143. $364,272$.

Psilonotus Valentyni Blkr. 358, I83. 364, 272.

Psilorhynchus $M C$ Cl. 261, 422.

Psilosomus Szons. 453, 330.

Pteraclis ocellatus $C . V .459,98$.

Pteraclis trichipterus $C . V .459,98$.

Pteragogus Pet. 292, ro6.

Pteragogus opercularis Gthr. 459, 8 I.

Pteragogus taeniops Pet. 495, 9.

Pterapon Gr. 468, 267.

Pterapon trivittatus Gr. cf. Therapon (Datnia) jarbua Blkr.

Ptereleotrii 460, I04.

Ptereleotris Gill 453, 307.460, 104, 106.

Ptereleotris heteropterus Blkr. 478, I05.

Ptereleotris microlepis Gill 478, I03.

Pterichthys Szons. 473, 296.

Pterichthys alatus Sions, cf. Apistus alatus C. $V$.

Pterocaesio Blkr. 468, 274. 470, I 53. 
Pterocaesio multiradiatus Blkr. 470, I53.

Pterocryptis Pet. 299, 395. 306, гп6.

Pterocryptis gangetica Pet. 306, i 6 .

Pterodoras Blkr. 306, 85 .

Pterodoras dorsalis Blkr. 303, r6.

Pterodoras granulosus Blkr. 303, I6. 306, 86. 336, 36.

Pterodoras murica Blkr. 303, r6.

Pterogobius Gill 453, 322.

Pterogobius elapoides Blkr. 497, I9.

Pterogobius virgo Gill 497, I9.

Pteroidichthys Blkr. 142, 33. 473, 297.

Pteroidichthys amboinensis Blkr. 142, 34. 275, 31. 473, 297. 475, 57.

Pterois C. V. 473, 296.

Pterois antennaria Szens. cf. Pseudomonopterus (Pterois) antennatus Blkr.

Pterois antennata C. $V .14,4.56,450.80,72.91$, I66. 170, 479.

184, I99. 270, 24. 275, 3r. cf. Pseudomonopterus (Pterois) antennatus Blkr.

Pterois brachypterus C. V. 54, 265. 156, 2 13. 275, 3I. cf. Pseudomonopterus (Dendrochirus) brachypterus Blkr.

Pterois cristatus Sions. cf. Pseudomonopterus (Pterois) volitans Blkr.

Pterois geniserra C. $V$. cf. Pseudomonopterus (Pterois) volitans Blkr.

Pterois heterurus Blkr. 142, 33. 473, 296. cf. Parapterois heterurus Blkr.

Pterois kodipungi Blkr. 56, 450. 91, 36. 93, 4. 127, 345. 156, 213. $157,232$.

Pterois kodipungi Benn., Blkr. cf. Pseudomonopterus (Pterois) kodipungi Blkr.

Pterois lunulata T. Schl. 56, 450. 90, 28. 93, 3. cf. Pseudomonopterus (Pterois) lunulatus Blkr.

Pterois miles Benn. 288, 72.

Pterois miles Cant. cf. Pseudomonopterus (Pterois) kodipungi Blkr.

Pterois miles Gthr. cf. Pseudomonopterus (Pterois) volitans Blkr.

Pterois muricata C. V. 91, 36. cf. Pseudomonopterus volitans Blkr., Pseudomonopterus (Pterois) volitans Blkr.

Pterois Russelli $V$. Hass. 270, 24. 275, 31. 287, 49.

Pterois Ruselli Benn. cf. Pseudmonopterus (Pterois) kodipungi Blkr. Pterois volitans C. V. 9, 633. 14, 7. 47, 226. 54, 235. 56, 450. 91, 36. 156, 213. 157, 232. 167, 37I. 182, I59. 196, 37. 199, 45. 234, 353. 236, 330, 333. 270, 24. 275, 31. 473, 296. cf. Pseudomonopterus (Pterois) volitans Blkr.

Pterois zebra C. V. 10, 66, 68. 14, 4. 46, 212. 54, 265. 156, 213. 157, 232. 231, I 45. 270, 24. 275, 31. 295, 96. cf. Pseudomonopterus (Dendrochirus) zebra Blkr.

Pteroleptus Siens. 473, 296.

Pteroleptus longicauda Srons. cf. Psendomonopterus (Pterois) kodipungi Blkr. 
Pteronemus $v$. d. Hocv. 468, 3 I5.

Pteronotus Siens. 306, ror.

Pteroplatea hirundo Lnve 425, 120.

Pteroplatea japonica $T$. Schl. 175, 45. 497, 4. cf. Pteroplatea micrura M. H., Pteroplatea hirundo Lozee.

Pteroplatea micrura M. H. 25, r3. 42, 60. 45, I97. 49, 42I. 51, 60. $55,4 \times 5.64,76.91, S_{2} .267$, I 39. 270, 68. 272, 6. 288, 67. 425, I 20.

Pteroplatea Schlegeli Blkr. 307, I38. 425, I20.

Pteropterus Stons. 473, 296.

Pterurus Stons. 351, I I4.

Pterurus maculatus Stons. cf. Moringua raitaborua Cant.

Pterygophlichthys Gill 306, 78.

Pterygophlichthys duodecimalis Gill 306, 78.

Ptychocheilus $A g .261,436.314,213.329,264$.

Ptychostomus Ag. 261, 428. 314, I90.

Ptyobranchus $M C C l$. 351, I I 4 .

Ptyobranchus arundinaceus $\mathrm{MC} \mathrm{Cl}$. cf. Moringua raitaborua Cant.

Ptyobranchus brevis $M C C l$. cf. Moringua raitaborua Cant.

Ptyobranchus erythraeus $\mathrm{Mc} C$. cf. Moringua raitaborua Cant.

Ptyobranchus gracilis $M c C l$. cf. Moringua raitaborua Cant.

Ptyobranchus Guthrianus $M T C C l$. cf. Moringua raitaborua Cant.

Ptyobranchus multidentatus $M C C l$. cf. Moringua raitaborua Cant.

Ptyobranchus parvidentatus $M C C l$. cf. Moringua raitaborua Cant.

Puntazzo Blkr. 468, 284.

Puntazzo annularis Blkr. 468, 284.

Puntius Buch. 314, 199. 329, 263.

Puntius (Barbodes) ablabes Blkr. 302, Ir 4.

Puntius (Puntius) binotatus Blkr. 335, 3 .

Puntius (Barbobus) Bocourti Blkr. 347, 37. 356, i 76.

Puntius (Puntius) bulu Blkr. 356, г 76 .

Puntius (Barbodes) camptacanthus Blkr. 302, II I.

Puntius (Barbodes) chrysopoma Blkr. 335, I5.

Puntius (Barbodes) deauratus Blkr. 415, 9. 425, I 44.

Puntius (Barbodes) erythropterus Blkr. 356, I 76 .

Puntius (Capoeta) Güntheri Blkr. 415, 9. 425, I 44.

Puntius (Barbodes) javanicus Blkr. 356, з 76.

Puntius (Puntius) phutunio Buch. 335, I2.

Puntius (Barbodes) pleurotaenia Blkr. 326, 242. 335, I3.

Puntius proctozysron Blkr. $345,25$.

Puntius (Puntius) proctozysron Blkr. 356, I 76. 360, 200.

Puntius (Barbodes) rubripinna Blkr.356, r 76.

Puntius (Barbodes) sinensis Blkr. 411, 252.415, 17. 425, 444.

Puntius (Puntius) thermalis Blkr.335, 3 .

Puntius (Barbodes) trispilos Blkr. 302, I I3. 
Pusichthys Szons. 299, 393. 306, I 4 .

Pygaeus $A g$. 468, 272.

Pygaeus gigas $A g .468,272$.

Pygidium Meyen 306, II 2 .

Pyrodon niger Rüpp. 270, 64. 275, I I. 315, 230. 358, I83. cf. Erythrodon niger Riipp.

Rabdophorus Szons. 468, 305.

Rabdophorus ephippium Sains. 468, 305. cf. Tetragonoptrus (Rabdophorus) ephippium Blkr.

Racoma $M c$ Cl. 261, 429. 314, I98.

Raconda Russeliiana Gr. 51, 59. 91, 74. 288, 79.

Raja 1, 552.

Raja africana $B l$. Schn., Blkr. cf. Rhachinotus africanus Cant.

Raja (Rhinobatus) armata Blkr. 364, 27 I.

Raja asterias $L$. cf. Dasybatis asterias Blkr.

Raja batis L. cf. Dasybatis batis Blkr.

Raja capensis Smith 266, 58.

Raja chinensis Basil. cf. Dasybatis chinensis Blkr.

Raja (Syrrhina) Columnae Blkr. 425, I 20.

Raja doiddensis Forsk. cf. Rhynchobatus laevis $M$. $H$.

Raja guttata Shaw cf. Aëtobatis narinari $M$. $H$.

Raja (Rhinobatus) halavi Forst. 425, I 20.

Raja isotrachys Giünth. cf. Dasybatis isotrachys $B l k r$.

Raja kenojei $M$. H., Bürg. 175, 42. 269, 65. cf. Dasybatis kenojei Blkr.

Raja Lemprieri Rich. 119, I9.

Raja ligonifer Blkr. 356, I 72 .

Raja lymma Forsk. cf. Taeniura lymma $M$. $H$.

Raja macrocephala Park cf. Myliobatis Nieuhofii $M$. $H$.

Raja maculata Mont. 266, 58 .

Raja Meerdervoortii Blkr. 269, 66. cf. Dasybatis Meerdervoorti Blkr.

Raja micrura $B l$. Schn. cf. Pteroplatea micrura $M$. $H$.

Raja narinari $B l$. Schn. cf. Aëtobatis narinari $M$. $H$.

Raja Nieuhofii $B l$. Schn. cf. Myliobatis Nieuhofii $M . H$.

Raja poecilura Shaw cf. Pteroplatea micrurus $M$. $H$.

Raja (Syrrhina) polyophthalmus Blkr. 364, 27 I.

Raja quinqueaculeata $Q . G$. cf. Aétobatis narinari $M . H$.

Raja (Rhinobatus) Schlegeli Blkr. 425, I20.

Raja sephen Forsk. cf. Hypolophus sephen $M$. $H$.

Raja Smithii M. H. 266, 58.

Raja Timlei Bl. Schn. cf. Narcine timlei Henle.

Raja narnak Forsk, Bl. Schn. cf. Trygon narnak $M$. $H$.

Raja undulata Lac. cf Dasybatis undulata Blkr.

Rama Blkr. 306, 93.

Rama Buchanani Blkr. 306, 93. 
Rasbora Blkr. 261, 435. 314, 202.

Rasbora argyrotaenia Blkr. 270, 54. 279, гог. 284, 240.

Rasbora bankanensis Blkr. 235, 371.

Rasbora borneënsis. Blkr. 272, 24.

Rasbora Buchanani Blkr. 288, 78 .

Rasbora cephalotaenia Blkr. 235, 371. 272, 24.

Rasbora curricula Blkr. cf. Squaliobarbus curriculus Gthr.

Rasbora dandia Blkr. 335, I8.

Rasbora dusonensis Blkr. 239, 102. 270, 54. 272, 24. 356, I76.

Rasbora Einthovenii Blkr. 235, 37 I. 272, 24. 287, 59.

Rasbora (Bengala) elongata Blkr. cf. Gnathopogon elongatus Blkr.

Rasbora (Bengala) gracilis Blkr. cf. Gnathopogon gracilis Blkr.

Rasbora kallochroma Blkr. 235, 37 r. 272, 24.

Rasbora lateristriata Blkr. 270, 54 .

Rasbora leptosoma Blkr. 270, 54 .

Rasbora macrocephalus Blkr. 353, I39.

Rasbora sumatrensis Blkr. 270, 54 .

Rasbora teretiuscula Blkr. 345, 26. cf. Squaliobarbus curriculus Gthr.

Rasbora tschiliensis Blkr. cf. Leuciscus idellus $V a l$.

Rasborichthys Blkr. 261, 435. 314, 203.

Rasborichthys Helfrichi Blkr. 272, 24.

Rataboura $G r$. 351, I 4 .

Rataboura Hamiltonii Gr. cf. Moringua raitaborua Cant.

Rataboura Hardwickii Gr. cf. Moringua raitaborua Cant.

Regalecus lanceolatus Lac. cf. Synechogobius hasta Gill.

Remora albescens Gill, Blkr. 315, 236. 391, 277. 425, 132.

Remora brachyptera $B l k r .425$, I 32 .

Remora remora Blkr. 425, I32.

Rhachinotus africanus Cant. 78, 514. 288, 67. 302, 12. cf. Urogymnus asperrimus $A$. Dum.

Rhachinotus asperrimus Blkr. 91, 82 .

Rhacochilus $A g$. 468, 290.

Rhacochilus toxotes $A g .468,290$.

Rhamdia Blkr. 306, IоI.

Rhamdia Queleni Blkr. 306, ror. 336, 75.

Rhamdia Sebae Blkr. cf. Rhamdia Queleni Blkr.

Rhambia Stegelichi Blkr. 336, 4 .

Rhamphobatis ancylostomus Gill 425, i I9. 459, 68.

Rhina ancylostomus Bl. Schn. 64, 56. 91, 80. 288, 67. cf. Rhamphobatis ancylostomus Gill.

Rhina japonica Blkr. 497, 3 .

Rhina sinensis $B l$. Schn. cf. Platyrhina sinensis M.H.

Rhina squatina $A$. Dum. 425, I I9.

Rhinacanthus conspicillum Srons. cf. Balistes conspicillum Bl. Schn.

Rhinichthys $A g .261,434.314,209$. 
Rhinobagrus Blkr. 342, 7 .

Rhinobagrus Dumerili Blkr. 342, 7. 425, I25.

Rhinobatus (Syrrhina) annulatus Smith, M.H. 266, 58. 497, 4.

Rhinobatus (Rhinobatus) armatus Gr., M. H. 25, 13. 51, 85. 64, 60.

$91,82.270,67.275$, 10. 396, 298. 491, 39.

Rhinobatus (Syrrhina) Banksii M.H. 119, x9.

Rhinobatus (Syrrhina) Blochii M. H. 266, 58.

Rhinobatus djeddensis Rüpp. cf. Rhynchobatus laevis $M . H$.

Rhinobatus Duhamelii Blainv. cf. Rhynchobatus laevis $M . H$.

Rhinobatus (Rhinobatus) granulatus Cuv. 91, 82 .

Rhinobatus halavi Rüpp. cf. Raja (Rhinobatus) halavi Forst.

Rhinobatus hymnicephalus Rich. cf. Raja (Rhinobatus) Schlegeli Blkr.

Rhinobatus laevis Bl. Schn. cf. Rhynchobatus laevis $M . H$.

Rhinobatus (Rhinobatus) ligonifer Cant. 51, 6o. 55, 4I 5. 64, 59. 272,

6. $288,67.305,73$.

Rhinobatus (Rhinobatus) obtusus $M . H .91,82.266,58$.

Rhinobatus (Syrrhina) polyophthalmus Blkr. 102, 423. 118, r29. cf.

Raja (Syrrhina) Columnae Blkr., Rhinobatus (Syrrhina) annulatus 11. $H$.

Rhinobatus Schlegelii Rich. cf. Raja (Rhinobatus) Schlegeli Blkr.

Rhinobatus (Rhinobatus) Schlegelii $M . H .175,41.497,4$.

Rhinobatus Thouini $v$. Hass, 2, 507. cf. Rhinobatus (Rhinobatus)

ligonifer Cant.

Rhinobatus (Rhinobatus) tuberculatus $C . V .91,82$.

Rhinobatus typus Benn. cf. Rhinobatus (Rlinobatus) armatus $G r$.

Rhinoberyx brachyrhynchus Gill cf. Rhynchichthys brachyrhynchus Blkr.

Rhinodon typicus Smith 266. 58. 459, 68.

Rhinodoras Blkr. 306, 84 .

Rhinodoras Orbignyi Blkr. 303; I4. 306, 84: 336, 33.

Rhinogobio 411, 253.

Rhinogobio typus Blkr. 411, 252. 415, 29. 425, I 44 .

Rhinogobius Gill 453, 320.

Rhinogobius similis Gill 453, 320. 497, I9.

Rhinoptera adspersa M.H., Val. 91, 82. 288, 67 .

Rhinoptera affinis Blkr. 302, x9.

Rhinoptera javanica $M . H .16,6.25$, I3. 64, 89. 179, 6. 425, I20.

Rhinoptera Peli Blkr. 302, I8.

Rhinoscion Gill 468, 329.

Rhinoscion epipercus Blkr. 302, 64 .

Rhodeus Ag. 261, 432. 314, $214.411,253$.

Rhodeus ocellatus Gthr. 411, 252. 415, 34. 425, I44.

Rhodeus sinensis Gthr. 411, 252. 415, 35. 425, 144. 496, 3 .

Rhomboidichthys Blkr. 143, 67. 294, 426 .

Rhomboidichthy's assimilis Gthr.cf. Platophrys (Platophrys) assimilis Blkr. 
Rhomboidichthys lunatus Gthr. cf. Platophrys (Platophrys) lunatus Blkr.

Rhomboidichthys myriaster Blkr. 143, 67. 275, i4. 294, 426. cf. Platophrys (Platophrys) myriaster $B l k r$.

Rhomboidichthys pantherinus Gthr. cf. Platophrys (Platophrys) pantherinus Blkr.

Rhomboplites Gill 468, 276.

Rhombotides achilles Blkr. 425, I34.

Rhombotides bipunctatus Blkr. 425, I34.

Rhombotides Bleekeri 320, 270. 354, 149. 358, 190. 364, 288.

Rhombotides celebicus Blkr. 315, 235. 364, 288. 491, $5 \mathrm{I}$.

Rhombotides chrysosoma Blkr. 354, r 49.

Rhombotides dorëensis Blkr. 491, $5 \mathrm{I}$.

'Rhombotides Dussumieri Blkr. 459, 96. 495, I9.

Rhombotides fumosus Blkr. 497, I6.

Rhombotides gahm Blkr: 364, 288. 459, 97. 495, I9.

Rhombotides gahmoides Blkr. 459, 97.

Rhombotides glaucopareius Blkr. 364, 288.

Rhombotides guttatus Blkr. 459, 96. 495, r9.

Rhombotides Lamarrii Blkr. 459, 96. 495, I9.

Rhombotides leucosternon Blkr. 459, 97. 495, I9.

Rhombotides lineatus Blkr. 315, 235. 320, 270. 364, 288. 391, 276.

396, 297. 437, 37. 491, 51.

Rhombotides lineolatus Blkr. 364, 288.

Rhombotides lunulatus 459, 97. 495, I9.

Rhombotides matoides Blkr. 315, 235. 320, 270. 354, I 49. 358, I 90.

364, 288. 396, 300. 397, 302. 459, 97. 495, I9.

Rhombotides nigro-fuscus $B l k r .364,288.459,97$.

Rhombotides nummifer Blkr. 396, 297.

Rhombotides olivaceus Blkr. 364, 288.491, $5^{\text {I. }}$

Rhombotides pentazona Blkr. 364, 288.

Rhombotides polyzona Blkr. 386, 277. 459, 44, 97.

Rhombotides triostegus Blkr. 315, 235. 320, 27 I. 354, I 49. 358, 190.

364, 288. 391, 276. 394, 288. 459, 97. 491, 51.495, x9.

Rhombotides xanthosoma Blkr. 364, 288.

Rhombus Klein 118, 123. 294, 424.

Rhombus argus $C$. $V .122$, I 78 .

Rhombus arsius Blkr. 91, 76 .

Rhombus aspilos Blkr. 38, 408. 61, I4. 231, I50. 270, 62. cf. Rhombus poecilurus Blkr.

Rhombus balteata Rich. cf. Pseudorhombus Ruselli Gthr.

Rhombus burbonensis $K p$. 459, 76 .

Rhombus cayennensis 294, 427 .

Rhombus chrysoptera Rich. cf. Pseudorhombus Russelli Gthr.

Rhombus cinnamomeus T. Schl. 175, 25. cf. Pseudorhombus cinnamomeus Blkr. 
Rhombus cocosensis Blkr. 122, I79.

Rhombus grandisquama Schl. cf. Platophrys (Platophrys) grandisquama Blkr.

Rhombus grandisquama T. Schl. cf. Rhombus poecilurus $B l k r$.

Rhombus javanicus Blkr. 78, 502. 102, 420. 118, I22.

Rhombus lentiginosus Rich. 38, 401. 55, 4I4. 56, 446. 61, I5. 102, 42 I. 118, I22. 119, 15. 227, 408. 270, 62. 272, 8. 275, 14. 287, 4I. cf. Pseudorhombus Ruselli Gthr., Rhombus poecilurus Blkr.

Rhombus macropterus Q.G. cf. Platophrys (Platophrys) pantherinus $B l k r$.

Rhombus maculosus Cuv. cf. Pseudorhombus maculosus Blkr.

Rhombus mancus 122, I 78 .

Rhombus marginatus $v$. Hass. 91, 76 .

Rhombus maximus Cuv. 294, 424.

Rhombus Mogkii Blkr. 109, 256. 231, I50. 275, I4. 287, 41.

Rhombus myriaster Schl. cf. Platophrys (Platophrys) myriaster Blkr.

Rhombus nauphala Blkr. 91, 76 .

Rhombus oligodon Blkr. 102, 419. 118, 121. cf. Pseudorhombus oligodon Blkr.

Rhombus oligolepis Blkr. 193, 8. cf. Pseudorhombus oligolepis Blkr. Rhombus pantherinus Rüpp. 122, I 78. 147, 469. 157, 235. 170, 480 . 188, 46r. 231, I50. 270, 62. 275, I 4.

Rhombus parvimanus $B c n n$. cf. Platophrys (Platophrys) pantherinus Blkr.

Rhombus pavo Blkr. 122, I77.

Rhombus poecilurus Blkr. 54, 293. 61, 29. 158, 274.

Rhombus polyspilos Blkr. 78, 503. 102, 42 I. 118, I22. 127, 345 . 270, 62. 275, 14. 287, 41. 294, 426.

Rhombus sumatranus Blkr. 38, 409. 47, 228. 54, 235. 61, I4.

Rhombus triocellatus Cuv. 86, 528. 91, 76, 165. 270, 62.

Rhombus Wolffii Blkr. 102, 421. 118, I22. cf. Chaenopsetta Wolffi Blkr.

Rhynchaena Greyi Rich. 119, I4.

Rhynchichthys brachyrhynchos Blkr. 72, ro7.

Rhynchichthys brachyrhynchus Blkr. 364, 280. 429, 231. 459, 86.

Rhynchobatus djeddensis Cant. 270, 67. 275, 10. 287, 37. 288, 67 . 459, 68, 497, 4 .

Rhynchobatus laevis $M . H .10,68.25$, I3. 46, 214. 51, 60. 64, 58 . 91, 82. 364, 27r. cf. Rhynchobatus djeddensis Cant.

Rhynchobdella aculeata Gthr. 358, r9r.

Rhynchobdella aral $B l$. Schn., $C$. $V$. cf. Rhynchobdella ocellata $C$. $V$. Rhynchobdella haleppensis $B l$. Schn. cf. Mastacembelus haleppensis $C$. $V$. Rhynchobdella maculata Roudt. 449, 368. cf. Mastacembelus maculatus C. $V$.

Rhynchobdella ocellata C. V. 2, 5I7. 24, 5. 25, 9. 91, 46. 225, 357 . 239, Iог. 270, 39. 271, I. 272, I4. 279, го०. 356, I 74 .

Rhynchobdella orientalis $B l$. Schn. cf. Rhynchobdella ocellata $C$. $V$. 
Rhynchobdella sinensis Blkr. 410, 249. 411, 252.425, r 34 . Rhynchobdelloidei 449,368 .

Rhynchophorus marginatus $v$. Hass. cf. Mastacembelus unicolor $K . v$. $H$. Rhynchophorus ocellatus $v$. Hass. cf. Mastacembelus maculatus $C . V$. Rhynchotus Gronovii Bibr., Blkr. cf. Chantogaster Valentijni Blkr.

Rhypticus Gthr. 468, 258.

Rhytidostomus Heck. 314, 190.

Richardsonia Cast. 468, 268.

Richardsonia insignis Cast. 468, 269.

Richardsonius Gir. 261, 436. 314, 2 I . 329, 263.

Rinelepis Spix 306, 79.

Rinelepis aspera Spix 306, 79.

Rinelepis genibarbis $\mathrm{Val}$. 306, 79.

Rineloricaria Blkr. 306, 8 ז.

Rineloricaria lima $B l k r .306,8 \mathrm{I}$.

Rita Blkr. 306, 93.

Rita Buchanani Blkr. 91, I23. 306, 93.

Riverina Cast. 468, 267.

Roccus Mitch. 468, 263 .

Rogenia argyrotaenia Blkr. 56, 457.60, 26. 227, 408. 287, 6r. 356, г 76.

Rohita Val. 261, 424. 314, r95.

Rohita Artedii Blkr. 49, 434. 55, 413. 139, 427. 162, 20.

Rohita Belangeri C. V. 91, I32.

Rohita (Rohita) borneënsis Blkr. 162, I 7. 239, I02. 272, 2 I. 356, I 75.

Rohita brachynotopterus Blkr. 136, 266. 139, 427. 270, 5I.

Rohita Buchanani C. V. 91, I33. 139, 427.

Rohita calbosu C. V. 91, I3I.

Rohita chalybeata C. V. 91, I33.

Rohita chrysophekadion Blkr. 25, 20. 67, 598.

Rohita cursis C. V. 67, 599. 91, 64 .

Rohita cyanomelas Blkr. 67, 597.

Rohita Dussumierii C. $V .91,64$.

Rohita Duvaucelii C. $V .91,64$.

Rohita enneaporos Blkr.67, 596. 270, 5 I.

Rohita erythrurus $C . V .67,597.85,45^{2}$.

Rohita fimbriata C. $V .91,64$.

Rohita gonius $C . V .91,64$.

Rohita Hasseltii $C . V .25,20.26,3.35,262.42,60.45$, 196. 49, 420. 55, 413. 85, 450. 139, 427. 162, 20. 211, 242. 225, 358. 270, 5I. 272, 2 I. cf. Rohita leiorhynchos Blkr.

Rohita (Rohita) Hasseltii $\mathrm{Val}$. 356, I75.

Rohita jaolius C. V. 91, 64 .

Rohita (Rohita) kahajanensis Blkr. 162, IS. 271, 2. 272, 2 I.

Rohita Kappenii Blkr. 162, I9. 272, 21.

Rohita koilogeneion Blkr. 166, 359. 
Rohita Kuhli Blkr. 270, 5 I.

Rohita leiorhynchos Blkr. 25, I9. cf. Rohita Hasseltii C. $V$.

Rohita macrochir Blkr.416, II9, I20. 425, I43.

Rohita macronotus Blkr. 91, 64 .

Rohita (Rohita) melanopleura Blkr. 55, 430. 139, 427. 239, 102.270, 51.272, 2 I. 356, I 75.

Rohita (Rohita) microcephalus Blkr. 136, 267. 271, 2.

Rohita moralius C. $V .91,64$.

Rohita nandina $C . V .91,64$.

Rohita nigra 2, 513.

Rohita oligolepis Blkr. 82, 19г.

Rohita polyporos Blkr. 86, 519 .

Rohita Reynauldi C. V. 25, 20. 67, 598 .

Rohita Rouxii C. V.91, 64 .

Rohita (Rohita) Schlegeli Blkr. 49, 432. 55, 4I3. 139, 426. 162, 20.

270, 51. 271, 2. 272, 21. 356, I75.

Rohita triporos Blkr. 67, 598. 270, 51. 272, 2 г.

Rohita vittata $C . V .42,60.45$, I96. 49, 420. 55, 413. 85, 45 1. 170, 476. 225, 358. 270, 5r. 271, 2. 272, 2 I.

Rohita Waandersii Blkr. 70, 733. 139, 427.

Rohitichthys Blkr. 261, 424. 314, 195.

Rohitodes Blkr. 314, 195 .

Rohtee Syk. 261, 432. 314, 200.

Rothee bramula $B l k r$. cf. Parabramis bramula Blkr.

Rohtee microlepis Blkr. 270, 53.

Rohteichthys Blkr. 261, 431. 314, 200.

Rohteichthys microlepis Blkr. 272, 23.

Rüppellia Szons. 453, 309 .

Rypticus Cuv. 468, 258.

Rypticus saponaceus C. V. 468, 258 .

Saccobranchus $V a l .306$, r2o.

Saccobranchus fossilis Blkr. 91, I24. 306, I20. 356, I75.

Saccobranchus singio C. V. 91, 58. cf. Saccobranchus fossilis Blkr.

Salangichthys Blkr. 269, IоI.

Salangichthys microdon Blkr. 497, 24.

Salanx chinensis Gthr. 425, I 47 .

Salanx microdon Blkr. 269, Iо0. cf. Salangichthys microdon Blkr.

Salanx Reevesii Cv. 119, r5.

Salarias 1, 553 .

Salarias aequipinnis Gthr. 364, 293 .

Salarias alticus C. V. 15, 3. 91, 48. 358, I 9r. cf. Alticus saltatorius Comm.

Salarias amboinensis Blkr. 163, 67 .

Salarias arenatus Blkr. 122, I73. 236, 331. 270, 43. 394, 288. 459, 74. 495, 20. 
Salarias bellus Gthr. 425, r 29.

Salarias bilitonensis Blkr. 185, 23 r.

Salarias castaneus $C . V .459,74.495,20$.

Salarias celebicus Blkr. 109, 250. 157, 233. 275, 45. 354, r 49. 364, 293.

Salarias ceramensis Blkr. 69, 701.275, 45.354, 149.358, 1 91. 364, 293.

Salarias chrysospilos Blkr. 163, 66.

Salarias cyanostigma Blkr. 15, I8. 25, 9. 167, 372. 170, 478. 185, 224. 270, 43. 275, 45. 296, III. 315, 236. 320, 272. 364, 293.

Salarias decussatus Blkr. 185, 230.

Salarias diproktopterus Blkr. 164, 69. 354, I 49.

Salarias Dussumieri C. $V . \mathbf{9 1}, 48, \mathbf{4 9 5}, 2 \mathrm{r}$.

Salarias Dussumieri Playf. cf. Salarias striatomaculatus $K u$.

Salarias edentulus Gthr. 364, 293.

Salarias epalzeocheilos Blkr. 236, 344.

Salarias fasciatus $\mathrm{Val.,} \mathrm{Gthr.} \mathrm{315,} \mathrm{236.} \mathrm{318,} \mathrm{252.} \mathrm{320,} 272.354,149$. 358, I9I. 364, 293. 459, 74 .

Salarias Forsteri Val. 34, 255. 320, 272.

Salarias fraenatus $C . V .91,50$.

Salarias frontalis E/ır. cf. Salarias phaiosoma Blkr.

Salarias fuscus Rüpp. 358, I9r. 364, 293. cf. Salarias phaiosoma Blkr.

Salarias gibbifrons Q. G. 34, 256. 270, 43 .

Salarias Goesii Blkr. 232, 19. 491, 54.

Salarias guttatus $C . V .167,379$. cf. Salarias decussatus Blkr.

Salarias Hasseltii Blkr. 34, 257. 122, I 74. 270, 43.

Salarias Hendriksii Blkr. 185, 233. 255, 24 r.

Salarias heteropterus Blkr. 163, 65. 167, 372.

Salarias interruptus Blkr. 164, 68. 354, I49.

Salarias Kingii $C$ \% 119, I r.

Salarias Kuhlii Blkr. 34, 258.

Salarias lineatus $C . V$. 15, 18. 25, 9. 236, 33 I, 333. 285, 245. 394, 288. 491,54 .

Salarias marmoratus $C . V .91,50$.

Salarias melanocephalus Blkr. 15, 18. 25, 9. 185, 224. 188, 460. 236, 33r, 333. 270, 43. 275, 45. 285, 245. 354, I 49. 364, 293. $394,288.437,37.491,54$.

Salarias meleagris C. $V$. 119, I I. 459, 74 .

Salarias nitidus Gthr. 425, I30.

Salarias Oortii Blkr. 34, 257. 174, 39. 236, 333. 270, 43. 285, 245.

Salarias periophthalmus C. $V .$, Blkr. 76, 267. 157, 233. 167, 372. 170, 479. 270. 43. 275, 45. 315, 236. 364, 293. 459, 74.

Salarias phaiosoma Blkr. 125, 317. 156, 215. 157, 233. 167, 372. $270,43.315,236.318,252.354$, 149 .

Salarias (Cirripectes) polyzona Blkr. 392, 278. 437, 37.

Salarias priamensis $B l k r .76,268$. cf. Salarias quadripinnis $C . V$.

Salarias quadricornis $C . V .122$, I 75. 188, 460.425, I30.459, 74. 495, 2 r. 
Salarias quadripinnis C. V. 15, 19. 17, 3. 156, 215. 157, 233. 167, 372. 170, 479.184, 201. 185, 224. 231, I 47.234, 356.270, 43.275, 45 . cf. Salarias fasciatus $G$ thr.

Salarias Raaltenii Blkr. 34, 257.

Salarias Schultzei Blkr. 236, 345 .

Salarias Sebae C. V. 145, 373. 184, 201. 315, 236. 358, I91. 364, 293. 491,54 .

Salarias striatomaculatus Kner 459, 74 488, 197. 495, 2 I.

Salarias striatus $Q . G$., C. V. 122, I74. cf. Salarias arenatus Blkr.

Salarias sumatranus Blkr. 34, $256.156,2$ I $5.170,479.188,460$. 236, 333. 270, 44. 275, 45. 285, 245. 318, 252. 320, 272. 364, 2.93. 394, 288. 491, 54 .

Salarias tetradactylus Blkr. 185, 228 .

Salarias tridactylus Gthr. 318, 252. cf. Alticus saltatorius Comm.

Salarias truncatus Blkr. 270, 44. 318, 252.

Salarias vermiculatoïdes $B l k r .174,40.236,333.270,44$.

Salarias vermiculatus $C . V$. 270, 44. 459, 74 .

Salmo (Plecoglossus) altivelis Schl. cf. Plecoglossus altivelis.

Salmo curilus Pall. 425, r47.

Salmo (Salmo) leptosoma Blkr. 496, I4.

Salmo leucomaenis Pall. 497, 24.

Salmo macrostoma Giüth. 497, 24.

Salmo microps Les. cf. Saurus ophiodon Cuv.

Salmo orientalis $M C$ Cl. 91, 76 .

Salmo orientalis Pall. 497, 24.

Salmo Perryi Brev. 497, 24.

Salmo (Salmo) pomatops Blkr. 496, I5.

Salmo saurus $B l$. cf. Saurus synodus $C . V$.

Salmo tumbil Bl. cf. Saurida tombil $C$. $V$.

Salmophasia Stens. 314, 215. 329, 264.

Samaragdus Pocy 453, 325.

Samaris cristatus $G r .425$, I30.

Sandrus Stark 468, 265.

Sarcocheilichthys Blkr. 261, 435. 314, 208. cf. Sarcochilichthys.

Sarcocheilichthys sinensis Blkr. 496, 3 .

Sarcocheilichthys variegatus Blkr. 497, 23.

Sarcochilichthys 408, 252 .

Sarcochilichthys sinensis Blkr. 411, 252. 415, 3I. 425, I 44 .

Sarcochilichthys teretiusculus Kner cf. Squaliobarbus curriculus Gthr.

Sarcochilichthys variegatus Blkr. 408, 249.

Sarda orientalis Blkr. 497, I 5 .

Sardinella brachysoma Blkr. 60, I9. 267, I40. 270, 56.

Sardinella clupeoides Blkr. 46, 2 14. 60, т9. 71, 773. 270, 57. 275, 48. cf. Harengula (Sardinella) clupeoides Blkr. 
Sardinella leiogaster $\mathrm{Val}$. 54, 238. 60, I8. 71, 774. 90, 50. 91, 72. 270, 57. 275, 48. 287, 61. 296, I I2. 316, 243. 358, I92.

Sardinella leiogastroides Blkr. 109, 255. 275, 48. 315, 236. 364, 295.

Sardinella lemuru Blkr. 78, 500.

Sardinella leucogaster C. V. 46, 2 r4.

Sardinella lineolata $C . V .91,72.354,150$.

Sardinella longiceps $C . V .91,72$.

Sardinella Neohovii $C . V .91,72$.

Sargini 468,282 .

Sargus Gron. 468, 283, 302.

Sargus auriventris Pet. 495, I 2.

Sargus capensis Smith 266, 52.

Sargus ensifer Gron. cf. Premnas biaculeatus Blkr.

Sargus hottentottus Smith 266, 52.

Sargus maculatus Gron. cf. Ephippus argus $C$. $V$.

Sargus vulgaris C. $V .468,283$.

Sarotherodon melanotheron Rïpp. 302, I3.

Sarothrodus Gill 468, 305.

Sarothrodus amplexicollis Poey cf. Tetragonoptrus (Chaetodontops) amplexicollis.

Sarothrodus ataeniatus Poey cf. Tetragonoptrus ataeniatus.

Sarothrodus auriga Blkr. 309, I55. cf. Tetragonoptrus (Linophora) auriga Blkr.

Sarothrodus baronessa Blkr. 309, I55. cf. Tetragonoptrus (Gonochaetodon) triangulum Blkr.

Sarothrodus Bennetti Blkr. 309, I 56. cf. 'Tetragonoptrus (Rabdophorus) Bennetti Blkr.

Sarothrodus bimaculatus Poey cf. Tetragonoptrus (Chaetodontops) ocellatus Blkr.

Sarothrodus capistratus Poey cf. Tetragonoptrus (Hemichaetodon) capistratus Blkr.

Sarothrodus lunula Blkr. 309, I56. cf. Tetragonoptrus (Chaetodontops) fasciatus $B l k r$.

Sarothrodus maculocinctus Gill cf. Tetragonoptrus (T'etragonoptrus) maculocinctus Blkr.

Sarothrodus nigrirostris Gill cf. Tetragonoptrus (Chaetodontops) nigrirostris Blkr.

Sarothrodus oligacanthus Blkr. 309, I56. cf. Parachaetodon ocellatus Blkr.

Sarothrodus princeps Blkr. 309, x 56 . cf. Tetragonoptrus (Linophora) Rafflesi Blkr.

Sarothrodus sedentarius Poey cf. Tetragonoptrus (T'etragonoptrus) sedentarius Blkr.

Sarothrodus striatus Poiy 302, 67. cf. 'Ietragonoptrus ('Tetragonoptrus) striatus Blkr. 
Sarothrodus strigangulus Blkr. 309, I56. cf. Megaprotodon strigangulus Blkr.

Sarothrodus vagabundus Gill 308, ${ }^{2}{ }^{2}$.

Sarothrodus vittatus Blkr. 309, I56. cf. Tetragonoptrus (Rabdophorus) trifasciatus Blkr.

Saurida argyrophanes Gthr. cf. Saurida tumbil $V a l$.

Saurida nebulosa $C . V .54,292.59,30.68,644$. 156, 2 I 7. 167, 374. $170,480.184,203.275$, 50. 287, 62. 309, I56. 315, 237. 318, 252. 320, 272. 354, 150. 358, 193. 364, 295. 394, 288. 398, 306. 437, 39. 459, гог. 491, 55. 495, 21. 496, 3.

Saurida tombil C. V. 46, $214.50,472.51,59.56,446.59,20.91$, 76. 119, I 5. 156, 2 × $7.227,408.275,50.356$, г 76.

Saurida tumbil $\mathrm{Val}$. 234, 356. 270, 56. 288, 79. 391, 277. 395, 294. 396, 298. 425, 147. 459, го1. 495, 2 1. 497, 24.

Sauridichthys ophiodon Blkr. 191, 2.

Saurogobio 411, 253.

Saurogobio cetopsis Blkr. 415, 8. 425, I 43.

Saurogobio Dabryi Blkr. 411, 252. 415, 27. 425, I 43.

Saurogobio Dumerili Blkr. 411, 252. 415, 25. 425, r43. 496, 3 .

Saurogobio heterodon Blkr. 415, 8. 425, I43.

Saurus 1, 552. 273, I3.

Saurus altipinnis Gthr. cf. Synodus altipinnis $B l k r$.

Saurus argyrophanes Rich. cf. Saurida tombil $C . V$.

Saurus badi Cuv. 2, 510. 10, 67. 25, 12. cf. Saurida tombil $C . V$.

Saurus badimottah Rüpp. cf. Saurida tombil $C$. $V$.

Saurus coronatus $v$. Hass. cf. Saurida tombil $C . V$.

Saurus foetens Blkr. 364, 295.

Saurus gracilis $Q . G$. cf. Saurida nebulosa $C$. $V$.

Saurus intermedius Spix cf. Saurus synodus $C$. $V$.

Saurus lucius Schl. cf. Synodus synodus Bl. Schn.

Saurus minutus Lesueur cf. Saurus synodus $C$. $V$.

Saurus myops C. $V .54,292.90,5.91,76.185,226.231$, I 50. 235,

372. $270,56.275,49.288,79.296$, II2. 302, I27. 358, I93.

398, 306. cf. Synodus myops Blkr.

Saurus nehereus Rich. cf. Harpodon nehereus Gthr., Saurus ophiodon Cuv.

Saurus ophiodon Cuv. 16, r6. 25. I2. 32, I60. 51, 59. 56, 446. 59, I8. 91, 76. cf. Sauridichthys ophiodon Blkr.

Saurus synodus $C . V .47,257.59,28.157,235.167,374.182$, г6о. 184, 203. 188, 46 I. 231, I 50. 270, 56. 275, 50. 287, 62. 296, II 2. 309, I 56. 315, 237. 358, I93. 364, 295. cf. Synodus synodus Blkr., Synodus varius Blkr.

Saurus trachinus $T$. Schl. 54, 29I. 56, 446. 59, 29. cf. Saurus myops C. $V$., Synodus myops Blkr.

Saurus variegatus Lac. cf. Saurus synodus $C . V$. 
Saurus varius Gthr. cf. Synodus synodus Blkr.

Saurus varius $\mathrm{Val}$. cf. Synodus varius $B l k r$.

Scabrus scabriusculus Val. 11, 6. 12, 42.

Scaphiodon Heck. 261, 427. 314, 196.

Scardinius $B p .261,438.314,2$ I $2.329,264$.

Scarichthys Blkr. 290, 229.

Scarichthys auritus Blkr. 290, 243. 320, 265. 364, 275. 396, 296. 425, I $34.437,37.459,80.491,40.495,8$.

Scarichthys coeruleopunctatus Blkr. 275, I5. 290, 243. 315, 230. $320,265.354$, I 44.358, I $84.364,275.425$, I $34.459,8$ I. $495,8$.

Scarichthys naevius Blkr. 236, 334. 275, I 5. 358, I84. cf. Scarichthys auritus Blkr.

Scarodon Schl. 468, $2 S_{4}$.

Scarodon fasciatus T. Schl. cf. Hoplegnathus fasciatus Rich.

Scarostoma Kner 468, 284 .

Scartelaos Stuns. 453, 328.

Scarus Forsk., Blkr. 1, 552. 290, 229.

Scarus acroptilus Rich. 119, I3.

Scarus aeruginosus $C . V .11,58.12$, 107. 51, 58. 157, 235. 185, 225. 270, 39. 275, I6. cf. Pseudoscarus aeruginosus Blkr.

Scarus auritus $K . v . H .11,6.12,42$.

Scarus balinensis Blkr. 17, 8. 47, 228 .

Scarus bataviensis Blkr. 166, 342 .

Scarus Blochii C. V. 11, 6. 12, 42. 126, 344. 185, 225. 270, 39 275, 5. 285, 243. 287, 42. cf. Pseudoscarus viridis Blkr.

Scarus capistratoides Blkr. 8, 162. 11, 50. 12, 103.

Scarus capistratus (K. v. H.) Val. 11, 6. 12, 42. 78, 497. 290, 242.

Scarus capistratus Blkr. cf. Pseudoscarus microcheilos Blkr.

Scarus capitaneus $C$. $V$. cf. Pseudoscarus capitaneus Gthr.

Scarus celebicus Blkr. 109, 253. 275, г6.

Scarus chlorodon Jen. 188, $46 \mathrm{I}$.

Scarus chrysopomus Blkr. 8, I63. 11, 53. 12, I05. cf. Pseudoscarus viridis Blkr.

Scarus coeruleopunctatus Rüpp. 95, r 10. 188, 46r.

Scarus (Calliodon) coeruleopunctatus Rüpp. cf. Scarus coeruleopunctatus Rüpp.

Scarus cyanescens $C$. $V$. cf. Pseudoscarus cyanescens $B l k r$.

Scarus cyanognathos Blkr. 8, I65. 11, 63. 12, III.

Scarus cyanotaenia Blkr. 97, 197.

Scarus dimidiatus Blkr. 232, I7. cf. Pseudoscarus dimidiatus Blkr.

Scarus Dussumieri C. V. 78, 494. 236, 332. 287, 42. cf. Pseudoscarus Dussumieri Blkr., Scarus harid Forsk.

Scarus erythrodon $C$. $V$. cf. Pseudoscarus sumbawensis Blkr.

Scarus fasciatus $\mathrm{Val}$. 11, 7. 12, 42. cf. Pseudoscarus rivuiatus Blkr. 
Scarus frenatus Lac. 71, 770. 232, r7. 275, r6. cf. Pseudoscarus frenatus Blkr.

Scarus gallus Forsk. cf. Julis lunaris $C . V$.

Scarus Georgii Benn. cf. Julis (Julis) quadricolor Less.

Scarus ghobban Forsk. cf. Pseudoscarus ghobban Gïnth.

Scarus gibbosus $K . v . H$. cf. Scarus muricatus $C$. $V$.

Scarus gibbus Rüpp. cf. Pseudoscarus gibbus Blkr.

Scarus globiceps $C$. $V .113,440$.

Scarus gymnognathos Blkr. 78, 498.

Scarus harid Forsk. 2, 513. 11, 48. 12, 102. 51, 58. 91, 54. cf. Pseldoscarus harid Gthr.

Scarus harid Blkr. cf. Scarus haridoïdes Blkr.

Scarus haridoïdes Blkr. 126, 344. 270, 39. 287, 42. cf. Pseudoscarus pyrrhostethus $B l k r$.

Scarus hypselopterus Blkr. 78, 496.

Scarus janthochir Blkr. 73, I39. 275, 54 .

Scarus javanicus Blkr. 97, I98.

Scarus Jenynsii Blkr. 188, 46I.

Scarus lacerta $C . V$. 91, 54 .

Scarus limbatus $C . V .11,60.12,109.270,39$.

Scarus limbatus Rich. cf. Pseudoscarus nuchipunctatus Blkr.

Scarus longiceps $C . V .11,7.12,42.290,243$. cf. Pseudoscarus harid Gthr.

Scarus macrocheilos Blkr. 94, 60.

Scarus maculosus Lac. cf. Pseudoscarus cyanescens Blkr., Pseudoscarus maculosus Gthr.

Scarus maschalespilos Blkr. 297, 127.

Scarus mastax Rüpp. 97, I99. 188, 46I.

Scarus melanotis Blkr. 297, I26.

Scarus micrognathos Blkr. 8, 164. 11, 56. 12, 106. 51, 58. 185, 225.

234, 355. 237, 438. 275, I6. cf. Pseudoscarus rivulatus Blkr.

Scarus microrhinos Blkr. 97, $200.113,43^{8}$.

Scarus Moensi Blkr. 275, 54.

Scarus muricatus $C . V$. 11, 46.12, I0I.

Scarus naevius $C . V .71,769$. cf. Scarichthys auritus Blkr.

Scarus nuchipunctatus $C . V .11,62.12$, I10. 47, 228. 275, I6. cf.

Pseudoscarus nuchipunctatus Blkr.

Scarus ovifrons Schl. cf. Pseudoscarus ovifrons Blkr.

Scarus pepo Benn. 91, 54.

Scarus psittacus Forsk. 78, 495. 170, 480. 270, 39. 275, I6. 287, 42.

Scarus psittacus Cant. cf. Pseudoscarus Cantori Blkr.

Scarus pulchellus Rüpp. 113, 440. 275, I6. cf. Pseudoscarus pulchellus Blkr.

Scarus pyrrhostethus Rich. cf. Pseudoscarus pyrrhostethus Blkr.

Scarus Quoyi C. V. 79, 607. 
Scarus quinquefasciatus Benn. cf. Tautoga fasciata $C$. $V$.

Scarus quinquevittatus Lay cf. Julis (Julis) quadricolor Less.

Scarus rivulatoides Blkr. 8, I63. 11, 55. 12, 106. 51, 58. cf. Pseudoscarus rivulatus $B l k r$.

Scarus rivulatus $V a l .11,6.12,42$.

Scarus rubroviolaceus Blkr. 8, 162. 11, 52. 12, 104.

Scarus Russelii C. $V .91,54$.

Scarus scaber $C$. $V$. cf. Pseudoscarus scaber $B l k r$.

Scarus scabriusculus $\mathrm{Val} .290,243$.

Scarus Schlosseri $G m$. cf. Toxotes jaculator $C$. $V$.

Scarus singaporensis Blkr. 51, 69.

Scarus spinidens Q.G. cf. Callyodon spinidens Stons.

Scarus spinidens $C . V .47,257$. cf. Callyodon waigiensis $C . V$.

Scarus striatus $C . V .11,5.12,42$. cf. Pseudoscarus striatus Gthr.

Scarus strongylocephalus Blkr. 113, 439.

Scarus sumbawensis Blkr. 9, 638. 149, 104. 157, 235. 167, 374. 275, I6. cf. Pseudoscarus sumbawensis Blkr.

Scarus taeniurus $C$. $V$. cf. Pseudoscarus taeniurus Blkr.

Scarus tricolor Blkr. 8, I64. 11, 59. 12, I08.

Scarus Troschelii Blkr. 78, 498.

Scarus vaigiensis $C . V .11,7.12,42.71,769$.

Scarus vaigiensis Q.G. 290, 243. cf. Scarichthys auritus Blkr.

Scarus variegatus $C . V$. cf. Pseudoscarus variegatus $B l k r$.

Scarus venosus $C$. $V$. cf. Pseudoscarus venosus $B l k r$.

Scarus viridis $B l$. cf. Pseudoscarus viridis Blkr.

Scarus xanthopleura Blkr. 78, 499.

Scatharus C. $V .468,283$.

Scatharus graecus $C . V .468,28_{3}$.

Scatophagiformes. 468, 302 .

Scatophagus Cuv. 1, 553. 468, 302 .

Scatophagus argus $C . V .2,520.16,4.22,24.25,8.26,3.35,26$ I. 42, 59. 45, 195. 46, $212.49,419.50,47$ 1. 51, 56. 54, 235. 55, 4I . 56, 445. 91, 40. 156, 214. 157, 233. 170, 479.178, 2. 222, 316. 227, 407. 270, 27. 272, х1. 275, 29. 284, 239.288, 72. 296, 107. 395, 292. 468, 302. cf. Cacodoxus argus Cant., Ephippus argus $C . V$.

Scatophagus Bougainvillei C. $V$. cf. Cacodoxus Bougainvillei Blkr, Ephippus argus $C . V$.

Scatophagus fasciatus $C$. $V$. cf. Cacodoxus tetracanthus $B l k r$.

Scatophagus macronotus Blkr. 2, 520. cf. Ephippus argus C. $V$., Scatophagus argus $C . V$.

Scatophagus multifasciatus Rich. 119, 9.

Scatophagus ornatus $C . V .103,492.270,27.296,107$. cf. Cacodoxus ornatus Blkr., Ephippus argus $C . V$.

Scatophagus purpurescens $C . V$. cf. Ephippus argus $C . V$. 
Scatophagus tetracanthus Gthr. cf. Cacodoxus tetracanthus Blkr.

Schedophilus maculatus Gthr. 425, I33.

Schilbe Cuv. 299, 393. 306, I I4.

Schilbe boalis Syk. cf. Wallago Russellii Blkr.

Schilbe garua $C . V .91$, I 10.

Schilbe mystus Cuv. 306, II 4 .

Schilbeichthys Blkr. 299, 393. 306, I I3.

Schilbeodes Blkr. 306, r०3.

Schilum Krynicki 468, 336.

Schismatorhynchos Blkr. 261, 423 .

Schismatorhynchos heterórhynchos Blkr. 270, 50.

Schismatorhynchus Blkr. 314, I 93.

Schistorus Gill 468, 256.

Schistura dario $M C C$. cf. Cobitis dario Buch.

Schizopyge Heck. 261, 427. 314, I96.

Schizothorax Heck. 261, 429. 314, 196. 329, 262.

Schizothorax (Schizothorax) barbatus $M c$ Cl. 91, 62 .

Schizothorax (Racoma) brevis Blkr. 91, 62.

Schizothorax (Racoma) chrysochlora Blkr. 91, 62.

Schizothorax (Schizothorax) curvifrons Heck. 91, 62.

Schizothorax (Schizothorax) Edeniana MC Cl. 91, 62.

Schizothorax (Schizothorax) esocinus Heck. 91, 62.

Schizothorax (Racoma) gobioides Blkr. 91, 62 .

Schizothorax (Oreinus) Griffithii Blkr. 91, 62 .

Schizothorax (Oreinus) guttatus Blkr. 91, 64 .

Schizothorax (Schizothorax) Hügelii Heck. 91, 62.

Schizothorax (Schizothorax) intermedius $M C$ Cl. 91, 62 .

Schizothorax (Racoma) labiatus Blkr. 91, 62.

Schizothorax (Schizothorax) longipinnis Heck. 91, 62.

Schizothorax (Oreinus) maculatus $M C$ Cl. 91, 64 .

Schizothorax (Schizothorax) micropogon Heck. 91, 62.

Schizothorax (Schizothorax) micropogon Heck. 91,.62.

Schizothorax (Schizothorax) nasus Heck. 91, 62.

Schizothorax (Racoma) nobilis Blkr. 91, 62.

Schizothorax (Oreinus) plagiostomus Blkr. 91, 64 .

Schizothorax (Schizothorax) plagiostomus Heck. 91, 62.

Schizothorax (Schizotharax) planifrons Heck. 91, 62.

Schizothorax (Oreinus) progastus Blkr. 91, 64 .

Schizothorax (Schizothorax) proprius $M C$ Cl. 91, 62.

Schizothorax (Schizothorax) Ritchiena $M c$ Cl. 91, 62.

Schizothorax (Schizothorax) sinuatus Heck. 91, 62.

Schuettea Stcind. 468, 300.

Schuettea scalaripinnis Steind. 468, 300.

Sciadeichthys Blkr. 306, 99.

Sciadeichthys pictus Blkr. 306, 99. 
Sciades Miull. Trosch. 306, 91.

Sciades emphysetus Müll. Trosch. 306, 9I. 336, 4.

Sciades pictus Müll. Trosch. cf. Sciadeichthys pictus Blkr.

Sciaena Art., Cuv. 1, 553. 468, 326, 329.

Sciaena adusta Gthr. cf. Johnius adustus Blkr.

Sciaena amblycephalus Blkr. 364, 285.

Sciaena aquila Risso 468, 329. cf. Pseudosciaena aquila $B l k r$.

Sciaena argentata (L.) Gm. cf. Lutjanus argentimaculatus Blkr.

Sciaena argentea $K . v . H$. cf. Corvina miles $C$. $V$.

Sciaena argentimaculata Forsk. 438, 44. cf. Lutjanus argentimaculatus Blkr.

Sciaena aurata Blkr. 459, 79. cf. Pseudosciaena aurata Blkr.

Sciaena bohar Forsk. cf. Lutjanus bohar Bl. Schn.

Sciaena borneënsis Gthr. cf. Pseudosciaena borneënsis Blkr.

Sciaena cataphracta Thunb. cf. Monocentris japonicus C. $V$.

Sciaena chuan-chua Basil. cf. Hemisciaena lucida Blkr.

Sciaena ciliata Lac. cf. Parupeneus cherserydros Blkr.

Sciaena cinerascens Forsk. cf. Pimelepterus cinerascens Day.

Sciaena cirrhosa L. 468, 326 .

Sciaena crocea Rich. cf. Corvina crocea Blkr.

Sciaena cylindrica $B l$. cf. Percis cylindrica $C$. $V$.

Sciaena diacanthus Gthr. cf. Pseudosciaena diacanthus Blkr.

Sciaena Dussumieri Blkr. 425, I42. 452, 56.

Sciaena Dussumieri Gthr. cf. Corvina Dussumieri $C$. $V$. , Johnius Dussumieri Cant.

Sciaena formosa Shaze cf. Epinephelus formosus Blkr., Serranus formosus $C$. $V$.

Sciaena fulviflamma Forsk. cf. Lutjanus fulviflamma Blkr., Mesoprion fulviflamma Blkr.

Sciaena Goldmani Gthr. cf. Pseudosciaena Goldmani Blkr.

Sciaena hamrur Forsk. cf. Priacanthus hamrur $C . V$.

Sciaena harak Forsk. cf. Lethrinus harak Rüpp.

Sciaena hololepidota $C . V .266,63$. cf. Pseudosciaena aquila Blkr.

Sciaena hypostoma Gthr. cf. Johnius hypostoma Blkr.

Sciaena indica $K . v . H$. cf. Sciaena Russelli Blkr., Umbrina Kuhlii $C . V$.

Sciaena jaculatrix Pall. cf. Toxotes jaculator $C$. $V$.

Sciaena japonica Thunb. cf. Monocentris cataphracta Bl.Schn., Monocentris japonicus $B l$. Schn.

Sciaena japonica Schl. cf. Pseudosciaena japonica Schl.

Sciaena jarbua Forsk. cf. Therapon (Datnia) jarbua Blkr., Therapon servus $C . V$.

Sciaena kasmira Forsk. cf. Lutjanus bengalensis Blkr., Mesoprion octolineatus Blkr.

Sciaena Kuhli Blkr. 395, 292. cf. Sciaena Russelli Blkr.

Sciaena lineata Mus. Lugd. Bat. cf. Diagramma lineatum C. $V$. 
Sciaena lineata $L$. cf. Plectorhynchus lineatus Blkr.

Sciaena lucida Rich. 411, 252. 468, 328. cf. Hemisciaena lucida Blkr. Sciaena macrophthalmus Gthr. cf. Pseudosciaena macrophthalmus Blkr. Sciaena macropterus Blkr. 452, 60.

Sciaena maculata Gr. cf. Johnius diacanthus Cant., Pseudosciaena diacanthus Blkr.

Sciaena malabarica $B l$. Schn. cf. Sillago acuta $C$. $V$., Sillago malabarica Cuv., Sillago sihama Rüpp.

Sciaena microdon Gthr. cf. Collichthys microdon Blkr.

Sciaena mi-uy Basil. cf. Otolithus mi-uy Blkr.

Sciaena murdjan Forsk. cf. Myripristis murdjan Rïpp.

Sciaena nigra Forsk. cf. Lutjanus macolor Blkr.

Sciaena (Corvina) Novae-hollandiae Steind. cf. Johnius Novae-hollandiae Blkr.

Sciaena pama $C . V .91,92$.

Sciaena platycephala $K$. v. $H$. cf. Pseudosciaena diacanthus $B l k r$.

Sciaena rubra Forsk. cf. Holocentrum rubrum Rüpp.

Sciaena Russelli Blkr. 452, 58 .

Sciaena safgha Forsk. cf. Ambassis Commersonii C. $V$., Ambassis nalua $C . V$.

Sciaena sammara Forsk. cf. Holocentium sammara $C . V$.

Sciaena spinifera Forsk. cf. Holocentrum spiniferum Gthr.

Sciaena tahmel Forsk. cf. Pimelepterus cinerascens Day.

Sciaena tenlo Basil. cf. Johnius semiluctuosus Kner, Johnius tenlo Blkr.

Sciaena trachycephalus Gthr. cf. Johnius trachycephalus Blkr.

Sciaena vittata Lac. cf. Grammistes orientalis Bl. Schn.

Sciaena vittata Park cf. Holocentrum diadema $C$. $V$.

Sciaena Vogleri Gthr. cf. Pseudosciaena Vogleri Blkr.

Sciaenini 468, 323 .

Sciaenoidei 468,323 .

Sciaenoides Blyth 468, 330.

Sciaenops Gill 468, 327 .

Sclerognathus Val. 314, rgo.

Sclerognathus asiaticus Gthr. cf. Carpiodes asiaticus Blkr.

Scoliodon Russellii Gr. cf. Carcharias (Scoliodon) acutus M. H.

Scolopsides Telf. 1, 553. 468, 270.

Scolopsides aurata Cant. 288, 7 I.

Scolopsides auratus $C . V$. 425, 139.

Scolopsides bilineatus C. V. 2, 523. 18, 28. 47, 226. 96, I75. 134, I 55. 167, 37 I. 234, 352. 275, 27.309, I 55. 315, 233. 338, 360. 358, I88. 364, 283. 394, 287 .

Scolopsides bimaculatus Rüpp. 425, г 39 .

Scolopsides bimaculatus $C$. $V$. cf. Scolopsides monogramma $\left(K . v^{\prime}, H\right.$. $)$

C. $V$., Scolopsis bimaculatus Rüpp.

Scolopsides Bleekeri Gthr. 364, 283. 
Scolopsides cancellatus C. V. 2, 523. 18, 28. 184, I98. 185, 222. $188,459.270,21.275,28.285,244.320,269.354$, I 47.358 , I88. 396, 297. cf. Scolopsides lineatus $Q . G$.

Scolopsides caninus $C . V .9,637.18,34.438,43 . \mathrm{cf}$. Heterognathodon bifasciatus Blkr., Pentapus caninus Blkr.

Scolopsides inermis T. Schl. 118, 83. 253, 235.

Scolopsides inermis Rich. cf. Scolopsides, Scolopsis bimaculatus Rüph.

Scolopsides japonicus Blkr. 425, 139.

Scolopsides kate C. V. 91, 38 .

Scolopsides kurita $C . V .91,38$.

Scolopsides leucotaenia Blkr. 56, 45r. 129, 440. 340, 365. 358, I88. 364, 283. cf. Scolopsis leucotaenia Blkr.

Scolopsides leucotaenioides $B l k r .129,439.185,222.275,2 S .364,2 S_{3}$. cf. Scolopsis leucotaenia Blkr.

Scolopsides lineatus Q. G. 80, 73. 167, 371. 184, I99. 185, 222 270, 21. 275, 28. 296, 107. 315, 233. 354, 1 47. 394, 287. 396, 297.

Scolopsides longulus Rich. 119, 8.

Scolopsides lycogenis $C . V .2,523.9,633.10,66.18,27.46,212$. 54, 235. 156, 214. 157, 232. 227, 406. 234, 352. 270, 21. 275, 28. $287,46.308$, I 52.309, I $55.315,233.316,242.354$, I 47 . 358, I 88, 364, 283. cf. Scolopsis ciliatus Gthr.

Scolopsides margaritifer $C . V .18,30.51,56.126,344.157,232$. 185, 222. 270, 21. 275, 28. 309, I55.354, I47. 364, 283. 396, 297. 425, I39.

Scolopsides monogramma (K. v. H.) C. $V .2,523.18,29.50,47$ r. 51, 56. 54, 236. 56, 444. 91, 38. 231, 145. 275, 28. 296, 107. $315,233.346,3$ I. 364, 283. 395, 29 I.

Scolopsides monogramma Blkr. cf. Scolopsis bimaculatus Rüpp.

Scolopsides pectinatus $K . v . H$. cf. Scolopsis margaritifer $C . V$.

Scolopsides personatus $C . V$. 67, 575. 270, 2 I. 364, 283 .

Scolopsides pomotis Rich. 425, r39.

Scolopsides Rüppelli $C$. $V$. cf. Scolopsides japonicus Blkr.

Scolopsides setifer Blkr. 91, 38 .

Scolopsides taeniatus Ehr., C. $V$. 18, 29. cf. Scolopsides monogramma (K.v. H.) C. V.

Scolopsides taeniopterus $(K . v . H$.$) C. V .18,29.235,364$.

Scolopsides temporalis $C . V$. 396, 297.

Scolopsides tineatus Blkr. cf. Scolopsis bilineatus $C . V$.

Scolopsides torquatus C. $V$. 2, 523. 18, 28. 46, 2 I2. 227, 406. 270, 2 г. $275,28.287,46.346,3$ г. 425, х 39.

Scolopsides Vosmeri $C . V .10,66.18,27.46,212.51,56.91$, I66. 126, 344. 227, 406. 235, 364. 237, 436. 270, 21. 275, 28.

Scolopsis Cur. 468, 270, 336.

Scolopsis argyrosomus $K$. $v$. $H$. cf. Scolopsides Vosmeri $C$. $V$., Scolopsis Vosmeri $C . V$. 
Scolopsis aurata Cant. cf. Scolopsis Vosmeri $C$. $V$.

Scolopsis bilineatus C. V. 434, 359. 491, 42 .

Scolopsis bimaculatus Rüpp. 18, 29. 434, 367. cf. Scolopsides monogramma $(K . v . H) C .$.$V .$

Scolopsis Bleekeri (Gthr.) Blkr. cf. Scolopsis bilineatus C. $V$., Scolopsis cancellatus $C . V$.

Scolopsis cancellatus $C \cdot V: \mathbf{4 3 4}, 355$.

Scolopsis cancellatus Gthr. cf. Scolopsis cancellatus $C . V$.

Scolopsis ciliatus Gthr. 434, 357. 491, 42.

Scolopsis frenatus $C . V .459,9 \mathrm{r} .495, \mathrm{I} 2$.

Scolopsis inermis Schl. 434, 352. 497, 8.

Scolopsis japonicus Day cf. Scolopsis torquatus $C . V$.

Scolopsis kate Cuv. 468, 270.

Scolopsis kurita $C . V .497,8$.

Scolopsis leucotaenia Blkr. 434, $35 \mathrm{I}$.

Scolopsis leucotaenia Gthr. cf. Scolopsis leucotaenia Blkr.

Scolopsis leucotaenioides Gthr. cf. Scolopsis leucotaenia Blkr.

Scolopsis lineatus $Q . G$. cf. Scolopsides cancellatus $C$. $V$., Scolopsis cancellatus $C . V$.

Scolopsis lycogenis C. $V$. cf. Scolopsis ciliatus Gïnth.

Scolopsis margaritifer $C . V$. 434, 349. 496, 2.

Scolopsis monogramma $K$. $\%$. H. 434, 369 .

Scolopsis personatus $C . V .434,364.491,42.495$, I 2.

Scolopsis phaeops Benn. 459, 9 r. 495, I2.

Scolopsis taeniatus Ehr. cf. Scolopsis bimaculatus Rüpp.

Scolopsis taeniopterus C. $V .434,366$.

Scolopsis temporalis $C . V .434,370.491,42$.

Scolopsis torquatus $C . V .434,363$.

Scolopsis trilineatus Kner 434, 353.

Scolopsis Vosmeri C. V. 434, 36r.

Scomber 1, 553 .

Scomber allitteratus Rafinesque cf. Thynnus thunnina $C . V$.

Scomber atun Euphras. cf. Thyrsites atun $C$. $V$.

Scomber auratus Houtt. 497, I4.

Scomber australasicus $C . V .119$, го. 364, 289 .

Scomber brachysoma Blkr. 37, 356. 57, 34 .

Scomber capensis $C$. $V$. cf. Seriola capensis Blkr.

Scomber capensis Forst. cf. Temnodon saltator $C . V$.

Scomber chloris Bl. cf. Micropteryx chrysurus Gthr.

Scomber chrysurus $L$. Gm. cf. Micropteryx chrysurus Gthr.

Scomber ciliaris Bl. cf. Carangoïdes atropus Blkr.

Scomber cordyla L. cf. Megalaspis Rottleri Blkr.

Scomber delphinalis Comm. 57, 35. cf. Scomber kanagurta $C . V$.

Scomber delphinalis $C . V .425$, г 3 г. 459, гоo.

Scomber dentatus Forst. cf. Thyrsites atun $C . V$. 
Scomber edentulus $B l$. cf. Equula ensifera $C . V$.

Scomber filamentosus $M . P$. cf. Carangoïdes blepharis $B l k r$.

Scomber Forsteri Bl. Schn. cf. Chorinemus Commersonianus C. V. 57, 44.

Scomber fulvoguttatus Forsk. cf. Carangoïdes fulvoguttatus Blkr.

Scomber grex Mitch. 266, 67 .

Scomber guttatus Bl. Schn. cf. Cybium guttatum C. $V$.

Scomber janesaba Blk\%. 102, 406. 118, 96. 425, I31. 496, 2.497, I 5 .

Scomber japonicus Houtt. 497, I5.

Scomber kanagurta $C . V .2,515.25,8.56,445.57,34.91,42.270$, 29. $275,38.287,52.356,173.364,289$.

Scomber lactarius $B l$. Schn. cf. Lactarius delicatulus $C$. $V$.

Scomber lanceolatus Bibl. cf. Thyrsites atun $C$. $V$.

Scomber latus Gronov. cf. Micropteryx chrysurus Gthr.

Scomber loo C. V. 46, 2 2. 54, 235. 57, 35. 119, Iо. 170, 479. 231,

I 46. $270,29.275,38.296$, I09. 309, I $56.315,235.320,270$.

$364,289.395,292.396,297,300.491,50$.

Scomber lysan Forsk. cf. Chorinemus Commersonianus C. $T$.

Scomber maculatus Forst. 57, 44. cf. Chorinemus Commersonianus $C . V$.

Scomber maculosus Shaw cf. Cybium konam Blkr.

Scomber madagascariensis Shaze cf. Chorinemus Commersonianus $C$. $V$.

Scomber malabaricus $B l$. Schn。 cf. Carangoüdes talamparah Blkr.

Scomber microlepidotus Rüpp. 288, 74. 425, I31. 459, Iоo.

Scomber minutus $B l$. cf. Gazza minuta Blkr.

Scomber moluccensis Blkr. 142, 40. 296, I09.

Scomber pa-uy Basil. 425, I3I. cf. Scomber microlepidotus Rüpp.

Scomber plumbeus Mitch. cf. Temnodon saltator $C$. $V$.

Scomber pneumatophorus De la Roche 119, I0. 411, 252. 425, I31.

Scomber pneumatophorus major T. Schl. cf. Scomber saba Blkr.

Scomber pneumatophorus minor T. Schl. cf. Scomber janesaba Blkr.

Scomber quadripunctatus Geoffr. cf. 'Thynnus thunnina $C . V$.

Scomber rhombeus Forsk. cf. Psettus rhombeus C. $V$.

Scomber Rottleri $\mathrm{Bl}$. cf. Megalaspis Rottleri Blkr.

Scomber saba Blkr. 102, 405. 118, 95. 497, I5.

Scomber scomber $L .425$, I3 I.

Scomber scombrus $L ., B l .266,53$.

Scomber scombrus (japonicus) Schl. 497, I 5 .

Scomber speciosus Forsk. cf. Gnathanodon speciosus Blkr.

Scomber tapeinocephalus Blkr. 102, 407. 118, 97.497, I5.

Scomberesox saurus Filem. 459, I00. 495, 21 .

Scomberesox scutellatus Les., $C$. $V$. cf. Scomberesox saurus Flem.

Scomberoides Commersonianus Lac., Blkr. 395, 292. 396, 300.459, 98.

Scomberoides delicatulus Blkr. 425, 132.

Scomberoides leucophthalmus $B l k r .425,132$.

Scomberoides lysan Blkr. 425, I32.

Scomberoides orientalis Blkr. 425, 132. 
Scomberoides sancti Petri C. V., Blkr. 315, 235. 338, 360. 346, 3 I. 358, г 9о. 364, 289. 395, 292. 425, 132. 459, 98. 491, 50. 495, I8. 497, I 5 .

Scomberoides tala Blkr. 425, I32.

Scomberoides tol Blkr. 316, 242. 320, 270. 354, I48. 364, 289. 356, I 74. 459, 98. 497, I 5 .

Scomberoides toloo Blkr. 315, 235. 358, r9I. 364, 289. 459, 98.

Scombresox Forsteri C. V. 119, 14.

Scombresox Rondeletii $C . V$. 266, 56 .

Scombresox saira Brez. 497, 24.

Scombrops Schl. 468, 335.

Scombrops cheilodipteroïdes Blkr. 118, 58. 468, 335. 497, ro.

Scombropsiformes 468, 335 .

Scombus glaucus $L$. cf. Glaucus Rondeletii $W$ ill.

Scopelosaurus Blkr. 273, I 2 .

Scopelosaurus Hoedti Blkr. 273, I3.

Scopelus 273, I3.

Scopelus (Dasyscopelus) asper Gthr. cf. Myctophum asperum Rich.

Scopelus boöps Rich. 119, I5.

Scopelus boops $C$. $V$. cf. Myctophum boops Rich.

Scopelus brachygnathos Blkr. 143, 65. 231, 149. 275, 5 o.

Scopelus brachygnathus Blkr. 296, I I 2.

Scopelus Coccoi Cocco cf. Myctophum Coccoi Blkr.

Scopelus coruscans $C$. $V$. 119, I5. cf. Myctophum coruscans Blkr.

Scopelus Dumerilii Blkr. 143, 66. 275, 50.

Scopelus notatus Les. cf. Myctophum hians Rich.

Scopelus spinosus Steind. cf. Myctophum spinosum Blkr.

Scopelus tenuicanda Steind. cf. Myctophum tenuicauda Blkr.

Scorpaena Art. 473, 295.

Scorpaena aculeata Lac. cf. Premnas biaculeatus Blkr.

Scorpaena alepidota $B l$. cf. Synanceia horrida $B l$. Schu.

Scorpaena antennata $B l$. cf. Pseudomonopterus (Pterois) antennatus Blkr., Pterois antennata $C . V$.

Scorpaena aplodactylus Blkr. 69, 698. 320, 269. 340, 365. 354, r48. 358, I 88. 364, 285. cf. Parascorpaena bandanensis Blkr., Scorpaena bandanensis Blkr.

Scorpaena bandanensis Blkr. 47, 237. 69, 699. 167, 37 I. 184, 199. 188, 459. 234, 353. 236, 330. 275, 31. 382, 398. 396, 299. 437, 39. cf. Parascorpaena bandanensis Blkr.

Scorpaena barbata Rüpp. 14, 7. cf. Scorpaena cirrhosa C. $V$. Scorpaena biaculeata $K . v . H$. cf. Minous monodactylus $C . V$. Scorpaena bicapillata Shaw cf. Synanceia verrucosa $B l$. Schn. Scorpaena bicirrata Lac. cf. Synanceia verrucosa Bl. Schn.

Scorpaena brachiata Shaw cf. Synanceia brachio $C$. $V$., Synanceia verrucosa $\mathrm{Bl}$. Schn. 
Scorpaena brachio Lac. cf. Synanceia verrucosa Bl. Schn.

Scorpaena burra Rich. 119, 7 .

Scorpaena Bynoënsis Rich. 119, 7 .

Scorpaena cardinalis Sol., Rich. 119, 7.

Scorpaena carinata $B l$. Schn. cf. Apistus alatus $C$. $V$.

Scorpaena cirrhosa C. $V .14,7.91,36.118$, 79. cf. Scorpaenopsis cirrhosus Blkr.

Scorpaena cottoïdes Forst. 119, 8.

Scorpaena cruenta Sol., Rich. 331, 446.

Scorpaena cyanostigma Blkr. 151, 400. 354, I48. 364, 285. cf. Sebastichthys cyanostigma Blkr.

Scorpaena diabolus $C . V$. 14, 4. 54, 266. 142, 32. cf. Scorpaenopsis diabolus Blkr., Scorpaenopsis gibbosus Blkr.

Scorpaena didactyla Pall. cf. Pelor didactylus Gthr., Pelor obscurum C. $V$.

Scorpaena digitata Gron. cf. Pelor didactylus Gthr.

Scorpaena gibbosa Bl. Schn. 142, 31. 473, 296. cf. Scorpaenopsis gibbosus $B l k r$.

Scorpaena haplodactylus Gthr. cf. Parascorpaena bandanensis Blkr.

Scorpaena horrida L., Bl. cf. Synanceia horrida Bl. Schn., C. $V$.

Scorpaena Koenigii $B$. 54, 265. cf. Pseudomonopterus (Dendrochirus) brachypterus Blkr., Pterois brachypterus $C . V$.

Scorpaena leonina Rich. 425, I4I. cf. Scorpaenopsis cirrhosus Blkr. Scorpaena marmorata Park cf. Scorpaena diabolus C. $V$., Scorpaenopsis diabolus Blkr.

Scorpaena mauritiana $C$. $V$. cf. Parascorpaena mauritiana Blkr.

Scorpaena miles Rich. 119, 8.

Scorpaena miles Benn. cf. Pseudomonopterus volitans Blkr., Pseudomonopterus (Pterois) volitans Blkr.

Scorpaena militaris Rich. 119, 8. cf. Scorpaena cruenta Sol.

Scorpaena miostoma Gïnth. cf. Scorpaena (Parascorpaena) miostoma.

Scorpaena (Parascorpaena) miostoma 497, Ir.

Scorpaena monodactyla $B l$. Schn. cf. Minous monodactylus $C$. $V$.

Scorpaena monstrosa Gron. cf. Synanceia horrida Bl. Schn.

Scorpaena neglecta Schl. 425, I4r. cf. Scorpaenopsis neglectus Blkr.

Scorpaena nesogallica $C$. $V$. cf. Scorpaenopsis gibbosus Blkr.

Scorpaena Novae-Guineae $C . V .14,4$. cf. Sorpaenopsis Novae-Guineae Blkr.

Scorpaena oxycephalus Blkr. 14, 7. cf. Scorpaenopsis oxycephalus Blkr.

Scorpaena papuensis $C . V .14,4.491,48$. cf. Scorpaenopsis NovaeGuineae Blkr.

Scorpaena picta (K. थ. H.) C. V. 10, 66. 14, 4. 46, 212. 275, 32. 287, 49. 288, 72. 473, 296. cf. Parascorpaena picta Blkr.

Scorpaena plebeja Sol. 119, 8.

Scorpaena polylepis Blkr. 44, r73. 473, 295. cf. Scorpaenodes polylepis Blkr., Sebastopsis polylepis Gill. 
Scorpaena polyprion Blkr. 14, 7. 17, 5. 56, 444. 69, 699. 156, 2 I3. 157, 232. 170, 479. 236, 331, 333. 270, 24. 275, 31. 285, 244. 287, 49. 315, 234. 320, 269. 364, 285. 437, 39 .

Scorpaena porcus L. 473, 295.

Scorpaena rawakensis Q.G. 396, 297. cf. Scorpaenopsis Novae-Guinea Blkr.

Scorpaena venosa $C . V .91,3^{6}$.

Scorpaena volitans $B$ l. cf. Pseudomonopterus (Pterois) volitans Blkr., Pterois volitans $C . V$.

Scorpaena waigiensis Q.G. cf. Myriodon waigiensis Gthr.

Scorpaenichthys Blkr. 473, 296.

Scorpaenichthys cirrhosus Blkr. cf. Scorpaenopsis cirrhosus Blkr.

Scorpaenichthys diabolus Blkr. cf. Scorpaenopsis diabolus $B l k r$.

Scorpaenichthys gibbosus Blkr. 156, 21 3. cf. Scorpaenopsis gibbosus Blkr.

Scorpaenichthys oxycephalus Blkr. cf. Scorpaenopsis oxycephalus Blkr. Scorpaenichthys polylepis Blkr. 157, 232. cf. Sebastopsis polylepis Gill. Scorpaenodes Blkr. 473, 296.

Scorpaenodes diabolus Blkr. cf. Scorpaenopsis diabolus Blkr.

Scorpaenodes oxycephalus Blkr. 170, 479. 185, 223.

Scorpaenodes polylepis. Blkr. 167, 37 I. 170, 478. 184, r99. 188, 459. cf. Sebastopsis polylepis Gill.

Scorpaenopsis Heck. 473, 296.

Scorpaenopsis cirrhosus Blkr. 287, 49. 315, 234. 364, 285. 425, I4I. 475, 32. 497, 12.

Scorpaenopsis diabolus Blkr. 236, 33 I. 275, 32. 285, 244. 358, I88. $364,285.396,299.425$, I 4 I. $475,36.491,48$.

Scorpaenopsis gibbosus Blkr. 270, 24. 275, 32. 296, 107. 320, 269. 354, г 48.358, I $88.364,285.425$, г4 г. 437, 39. 459, 87. 475, 38. 495,16 .

Scorpaenopsis neglectus Blkr. 497, I 2 .

Scorpaenopsis nesogallica Heck. cf. Scorpaenopsis gibbosus Blkr.

Scorpaènopsis Novae-Guineae Blkr. 475, 34. 491, 48.

Scorpaenopsis oxycephalus Blkr. 231, I45. 234, 353. 270, 24. 275,

32. 346, 31. 364, 286. 425, I4I. 475, 30.491, 48.

Scorpaenopsis polylepis Blkr. 234, 353. 236, 33r, 333. 275, 32. 315,

234. cf. Sebastes polylepis Gthr., Sebastopsis polylepis Gill.

Scorpidiformes. 468, 297.

Scorpidini 468, 298.

Scorpis C. V. 468, 298.

Scorpis aequipinnis Rich. 119, 9. 331, 447. 468, 299.

Scorpis georgianus C. $V .119,9.468,298$.

Scyllium 1, 55 I.

Scyllium africanum Cuv., M. H. 266, 57. 495, 8.

Scyllium africanum var. pantherina Günth.cf. Scyllium pantherinum Smith. 
Scyllium africanum var. variegata Günth. cf. Scyllium variegatum Smith.

Scyllium barbatum 2, 508 .

Scyllium bivium Smith 266, 57.

Scyllium Bürgeri $M . H .142,69.497,3$.

Scyllium capense Rapp 266, 79.

Scyllium Edwardsii C. $V .266,57$.

Scyllium ferrugineum Less. cf. Ginglymostoma cirratum $M$. $H$.

Scyllium Freycineti Q.G. 64, I7. cf. Chiloscyllium Freycineti Blkr., Chiloscyllium plagiosum $M . H$.

Scyllium heptagonum Rüpp. cf. Stegostoma fasciatum $M$. $H$.

Scyllium maculatum M. H., Gr. 51, 60. 64, I6. 91, 80. 126, 344.

185, 226. 270, 66. 275, 9. 287, 36. 308, I 52. 318, 249. 320, 264.

354, I $43.364,270.358$, I 83.425 , I I9. 491, 39.

Scyllium malaisianum $Q . G$. cf. Chiloscyllium malayanum $M$. $H$.

Scyllium malaisianum Less. cf. Hemiscyllium malayanum $M$. $H$.

Scyllium marmoratum Benn. 425, I I9. cf. Scyllium maculatum Gr.

Scyllium ornatum $G r$. cf. Chiloscyllium ornatum Blkr., Chiloscyllium plagiosum $M$. $H$.

Scyllium pantherinum Smith 266, 57. 495, 8.

Scyllium pardus Temm. cf. Scyllium maculatum $G r$.

Scyllium pictum $M . H$. 266, 57 .

Scyllium plagiosum Bcnn. cf. Chiloscyllium ornatum Blkr., Chiloscyllium plagiosum $M$. $H$.

Scyllium punctatum $v$. Hass. cf. Chiloscyllium punctatum $M$. $H$.

Scyllium quinquecarinatum $\%$. Hass. cf. Stegostoma fasciatum $M . H$.

Scyllium tigrinum 2, 508 .

Scyllium variegatum Smith 266, 57.495, 8.

Scymnus brasiliensis Q. G. cf. Isistius brasiliensis Gill, Tristius brasiliensis Gill.

Scyris 1, 553 .

Scyris alexandrina $C . V .57,69$. cf. Carangoïdes gallichthys Blkr.

Seyris indica $C$. $V$. cf. Carangoïdes gallichthys Blkr.

Scyris indicus Rüpp. cf. Carangoïdes gallichthys Blkr.

Scytalophis Kp. 351, II9.

Scytalophis magnioculis $K p$. cf. Ophichthys magnioculis $B l k \%$.

Scytalophis parilis $K p$. cf. Ophichthys parilis Blkr.

Sebastes Cuv. 473, 294:

Sebastes albofasciatus $C . V .90,30$. cf. Sebastichthys marmoratus Blkr.

Sebastes capensis $C . V .266,52$.

Sebastes caurinus Rich. cf. Sebastes inermis C. $V$.

Sebastes imperialis C. $V .90,30$.

Sebastes inermis C. $V .408,238,243$.

Sebastes inermis Schl. cf. Sebastichthys inermis Blkr.

Sebastes longiceps Rich. 425, I40.

Sebastes macrochir Günth. cf. Sebastichthys macrochir Blkr. 
Sebastes maculatus $C . V$. 266, $5^{2}$.

Sebastes marmoratus C. $V .90,29.425$, I40. cf. Sebastichthys marmoratus Blkr.

Sebastes minutus C. $V$. 14, 4. cf. Sebastichthys minutus Blkr.

Sebastes nematophthalmus Gthr. cf. Sebastichthys nematophthalmus Blkr.

Sebastes nigrocinctus Ayr. 473, 294.

Sebastes norvegicus $C . V .473,294$.

Sebastes oblongus Gïnth. cf. Sebastichthys oblongus Blkr.

Sebastes pachycephalus $T$. Schl. 175, I3. 425, I 40. cf. Sebastichthys pachycephalus Blkr.

Sebastes panda Rich. 473, 295 .

Sebastes pandus Rich. 119, 8.

Sebastes percoïdes Rich. 119, 8 .

Sebastes polylepis Gthr. 354, I48. 358, I88. 364, 286. 437, 39. cf. Sebastopsis polylepis Gill.

Sebastes rhodochrous Gthr. cf. Sebastichthys rhodochrous Blkr.

Sebastes serrulatus Rich. 425, r 40.

Sebastes sinensis $M c C l$. cf. Sebastes marmoratus $C$. $V$., Sebastichthys marmoratus Blkr.

Sebastes strongensis Gthr. cf. Sebastichthys cyanostigma Blkr.

Sebastes strongia Klunz. cf. Sebastichthys cyanostigma Blkr.

Sebastes Vachelli Rich. 425, I40.

Sebastes ventricosus T'Schl. 118, 80. cf. Sebastichthys ventricosus Blkr.

Sebastichthys Gill 473, 294.

Sebastichthys cyanostigma Blkr. 475, I7.

Sebastichthys inermis Blkr. 497, II.

Sebastichthys macrochir Blkr. 497, II.

Sebastichthys marmoratus Blkr. 475, I6. 497, II.

Sebastichthys minutus Blkr. 475, 20.

Sebastichthys nematophthalmus Blkr. 459, 87. 495, I6.

Sebastichthys oblongus Blkr. 497, I I.

Sebastichthys pachycephalus Blkr. 497, I I.

Sebastichthys rhodochrous Blkr. 475, I9.

Sebastichthys ventricosus Blkr.497, II.

Sebastodes Gill 473, 294.

Sebastomus Gill 473, 294.

Sebastoplus Gill 473, 294.

Sebastopsis Gill 473, 295.

Sebastopsis polylepis Gill 475, 21. 491, 48 .

Sebastosomus Gill 473, 294.

Selar Blkr. 128, 417. 297, I37.

Selar boöps Blkr. 57, 51. 127, 345. 147, 469. 156, 2r4. 185, 223. 270, 29. 275, 39. 296, 109.

Selar brevis Blkr. 37, 361. 57, 54 . 
Selar Broekmeyeri Blkr. 138, 398.

Selar declivis Blkr. 119, Iо.

Selar djeddaba Blkr. 288, 75 .

Selar equula Blkr. cf. Carangoïdes equula Blkr.

Selar georgianus Blkr. 119, ro.

Selar Hasseltii Blkr. 37, 359. 46, 2т3. 57, 53. 91, 44. 231, 146. 235, 366. 270, 29. 275, 39. 287, 53. 288, 75. 296, го9. 309, г56. cf. Caranx Hasseltii Blkr.

Selar ire Blkr. $91,44$.

Selar japonicus Blkr. 90, 35. cf. Caranx trachurus Lac., Selar trachurus (japonicus) Blkr.

Selar kalla Blkr. 91, 44.

Selar Kuhli Blkr. 37, 36o, 36r. 46, 2 г 3. 51, 57. 57, 54. 170, 479. 235, 367. 270, 29. 275, 39. 288, 75. cf. Caranx Kuhli Blkr., Caranx xanthurus $C . V$.

Selar macrurus Blkr. 37, 359. 57, 52. 270, 30. 275, 39.

Selar malam Blkr. 37, 362. 50, 47x. 56, 445. 57, 55. 191, 2. 270, 3o. 272, I4. 287, 53 cf. Caranx malam Blkr.

Selar megalaspis Blkr. 86, 502. 156, 2 I 4 .

Selar microchir Blkr. 270, 30.

Selar micropus Blkr. 272, 39.

Selar para Blkr. 57, 56. 91, 44. 156, 214. 227, 407. 235, 367. 270, 3०. $275,39.287,53$.

Selar torvus Blkr. 46, 2I3. 47, 227. 57, 51. 147, 469. 156, 2 r4. 231, I46. 237, 437. 270, 30. 275, 39. 296, 109. 309, I56. cf. Caranx torvus Jen.

Selar trachurus Blkr. 119, xо.

Selar trachurus (japonicus) Blkr. 266, 67.

Selar vari Blkr. 91, 44. 288, 75 .

Sélaroïles Blkr. 57, 87. 128, 418. 297, I38.

Selaroïdes leptolepis Blkr. 46, 213. 51, 57. 270, 30. 275, 39. 364, 290. 496, 2 .

Selenaspis Herzbergii Blkr. cf. Netuma Herzbergi Blkr.

Semicossyphus Gthr. 292, I०5.

Semicossyphus reticulatus Schl. 497, 5 .

Semiplotus Blkr. 261, 424. 314, 195.

Semotilus Raf. 261, 437. 314, 2 II. 329, 264.

Seriola 1, 553 .

Seriola aureovittata T. Schl. 90, 36. 497, I4.

Seriola aurovittata Rich. cf. Seriola Lalandii Risso.

Seriola binotata C. V. 51, 57. 91, 46. 288, 74 .

Seriola bipinnulata $Q . G$. cf. Seriolichthys bipinnulatus $B l k r$.

Seriola capensis Blkr. 266, 65 .

Seriola chloris Holbr. cf. Micropteryx chrysurus Gth\%.

Seriola cosmopolita Cuv. 91, 46. cf. Micropteryx chrysurux Gthr. 
Seriola cultrata Rich. 119, ro.

Seriola Dumerilii Russ. cf. Seriola purpurascens Schl.

Seriola Dussumierii C. $V .91,46$.

Seriola intermedia T. Schl. 90, 37. 497, 14.

Seriola lactaria $C$. $V$. cf. Lactarius delicatulus $C$. $V$.

Seriola Lalandii Risso 425. 132 .

Seriola nigrofasciata Rüpp. 2, 518. 275, 39. 425, I32. cf. Seriola Rüppelii $C$. $V$.

Seriola nigrofasciatus Gthr. 364, 289 .

Seriola purpurascens $T$. Schl. 118, I03. 497, I4.

Seriola purpurascens Rich. cf. Seriola Lalandii Risso.

Seriola quinqueradiata $T$. Schl. 90, 36. 425, I32. 497, I4.

Seriola quinqueradiata Rich. cf. Seriola Lalandii Risso.

Seriola Ruppellii C. $V .57,73.90,37$. cf. Seriola nigrofasciata Rüpp.

Seriola tapeinometopon Blkr. 80, So. 318, 252.364, 2S9.394, $288.491,50$

Seriolichthys bipinnulatus Blkr. 97, I96. 188, 459. 275, 39. 364, 289.

459, roo. $491,50$.

Serranichthys Blkr. 468, 257.

Serranichthys altivelis Blkr. 126, 344.275, 24.287, 45. 288, 70. 296, 106. cf. Cromileptes altivelis Szons.

Serraniformes 468, $25 \mathrm{I}$.

Serranus Cuv. 1, 553. 468, 255 .

Serranus acutirostris $\mathrm{Val} .468,257$.

Serranus aka-ara $T$. Schl. 175, 9. cf. Epinephelus aka-ava Blkr.

Serranus albofuscus Gthr. cf. Epinephelus maculatus Blkr.

Serranus alboguttatus $C . V .72$, 103. 270, 17. 275, 22. 394, 287. cf. Epinephelus alboguttatus Blkr., Epinephelus coeruleopunctatus Blkr. Serranus altivelioides Blkr. 13, 38. 185, 222. 275, 22. 287, 44. 438, 46. cf. Epinephelus corallicola Blkr.

Serranus altivelis $C . V .2,525.13,33.51,55$. cf. Cromileptes altivelis Szons.

Serranus amblycephalus Blkr. 163, 32. cf. Epinephelus amblycephalus Blkr.

Serranus amboinensis Blkr. 54, 258. 275, 22. cf. Epinephelus undulosus Blkr.

Serranus analis $C . V .438,45$. cf. Epinephelus analis Blkr.

Serranus angularis $C . V .91,30$. cf. Epinephelus angularis Blkr.

Serranus ara Schl. cf. Epinephelus ura Blkr.

Serranus areolatus $C$. $V$. cf. Epinephelus areolatus $B l k r$.

Serranus areolatus japonicus Schl. cf. Epinephelus areolatus Blkr.

Serranus argus C. $V$. 13, 3. cf. Epinephelus argus Bl. Schn.

Serranus argyrogrammicus $C$. $V$. cf. Anthias zonatus Gthr.

Serranus aurantius $C . V .67,57$ I. 228, 424. 270, I7. 275, 22. cf. Epinephelus aurantius Blkr.

Serranus awoara Schl. cf. Epinephelus awoara Blkr. 
Serranus bataviensis $B l k r .13,38.126,344.270,17.275,22$. cf. Epinephelus ongus Blkr.

Serranus biguttatus $C . V .13 .4 .91,30.438,44$. cf. Lutjanus biguttatus Blkr.

Serranus boelang $C . V .13,3.91,30.270$, I7. cf. Epinephelus boelang Blkr.

Serranus boenack C. V. 13, 31. 25, 7. 46, 2 I I. 156, 2 I 2. 158, 273. 185, 222. 222, 316. 270, I7. 275, 22. 287, 44. cf. Epinephelus boelang Blkr.

Serranus boenack Pet. cf. Epinephelus formosus Blkr.

Serranus bontoides Blkr. 128, 405. 231, I44. 275, 23. cf. Epinephelus bontoides Blkr.

Serranus bontoo C. V. 2, 525. 46, 215. 91, 30. 128, 406. 288, 70. cf. Epinephelus pantherinus Blkr.

Serranus borbonicus Q.G. cf. Epinephelus flavocoeruleus Blkr.

Serranus borbonius $C$. $V$. cf. Anthias borbonius Gthr., Odontanthias borbonius Blkr.

Serranus borbonius Guich. cf. Epinephelus flavocoeruleus Blkr.

Serranus cabrilla Cuv. 468, 255 .

Serranus celebicus Blkr. 46, 2 I 7. 156, 2 I 2. 231, I 44. 235, 363. 237, 435. 270, r 7. 275, 23. 287, 44. 296, 105. cf. Epinephelus angularis Blkr., Epinephelus celebicus Blkr.

Serranus chlorocephalus $C . V .125,3$ II.

Serranus chlorostigma $C$. $V$. cf. Epinephelus chlorostigma Blkr.

Serranus cichlops Blkr. 76, 245. cf. Pseudanthias cichlops Blkr.

Serranus coeruleopunctatus $C . V$. cf. Epinephelus coeruleopunctatus Blkr.

Serranus coioïdes Cant. 91, 30. 287, 44. 288, 70. cf. Serranus suillus C.V.

Serranus confertus Benn. 13,4.

Serranus confertus Benn. cf. Epinephelus merra $B l$.

Serranus corallicola $K . v . H .10,66.13,3.46,2$ I I. 275, $23.438,46$. cf. Epinephelus corallicola Blkr.

Serranus crapao $C . V .2,525.9,632.13,37.16,4.25,7.46,2$ I 5 . 50,47 r. 51, 55. 54, 234. 55, 4I I. 56, 444. 191, I. 270, 17. 272, 10. $275,23.288,70.296$, 105. cf. Epinephelus crapao Blkr., Epinephelus pantherinus Blkr.

Serranus Cuvieri Smith 266, 5 I.

Serranus cyanopodus Rich. cf. Epinephelus cyanopodus Blkr.

Serranus cyanostigma $K$. v. H. 2, 525. 13, 32. 54, 234. 126, 344. 270, I7. 275, 23. 296, I05. cf. Epinephelus cyanostigma Blkr.

Serranus cyanostigma $V$ al. 46, 2 I r. cf. Epinephelus miniatus Blkr.

Serrants cyanostigmatoides Blkr. 13, 3I. 296, I05. cf. Epinephelus cyanostigmatoides Blkr., Epinephelus miniatus Blkr.

Serranus cylindricus Gthr. cf. Epinephelus cylindricus Blkr.

Serranus decussatus $C . V .228,424$. 
Serranus Delissii Benn. cf. Anthias borbonius Gthr., Odontanthias borbonicus, borbonius Blkr.

Serranus dermochirus $C . V$. 91, 30. cf. Epinephelus dermochirus $B l k r$. Serranus dermopterus Schl. cf. Epinephelus dermopterus Blkr.

Serranus diacanthus $C . V .91,30$. cf. Epinephelus diacanthus Blkr.

Serranus diacopaeformis Benn. 13, 4. cf. Epinephelus pantherinus Blkr.

Serranus diktiophorus $B l k r .143,38.231$, I53. 275, 23. cf. Epine. phelus dictiophorus Blkr.

Serranus dispar Playf. cf. Epinephelus dispar Blkr., Epinephelus fuscoguttatus Blkr.

Serranus epistictus T. Schl. 116, 60. cf. Epinephelus epistictus Blkr.

Serranus erythraeus $C$. $V$. cf. Epinephelus erythraeus Blkr., Epinephelus nigripinnis $B l k r$.

Serranus erythrurus $C . V . \mathbf{9 1}, 30$.

Serranus fasciatus Khunz. cf. Epinephelus fasciatus Blkr.

Serranus faveatus $C . V .9,635.13,37.91,30$. cf. Epinephelus merra Bl., Blkr.

Serranus filamentosus $C . V .459,88.495$, I I.

Serranus flavimarginatus Rüpp. 438, 45. cf. Variola flavimarginata Blkr.

Serranus flavo-coeruleus $C . V .91,30$. cf. Epinephelus flavocoeruleus Blkr.

Serranus formosus C. $V .2,525.13,3$ I. 91, 30. 127, 345. 171, 245 . 185, 222. 222, 3т6. 227, 406. 228, 424. 270, 17. 275, 23. 287, 44. cf. Epinephelus boenack Blkr., Epinephelus formosus Blkr.

Serranus fuscoguttatus Rüpp. 140, 435. 438, 46. cf. Epinephelus fuscoguttatus Blkr.

Serranus Gaimardi $C . V .13,4$. 78, 455. cf. Epinephelus maculatus Blkr.

Serranus geographicus $K . v . H .13,3$. cf. Epinephelus fuscoguttatus Blkr.

Serranus Gilberti Rich.46, 215. 119, 5. cf. Epinephelus Gilberti Blkr. Serranus glaucus Day cf. Epinephelus celebicus Blkr.

Serranus Goldmanni Blkr. 140, 434. cf. Epinephelus Goldmani Blkr. Serranus guttatus $C$. $V$., Peters 13, 32. 91, 30. 270, 17. 275, 23. cf. Epinephelus argus $B l$. Schn., Blkr.

Serranus guttatus $C$. $V$. cf. Epinephelus miniatus Blkr.

Serranus hexagonatus Rich. cf. Epinephelus hexagonatus Blkr.

Serranus hexagonatus $C . V .13,37.46,2$ I5. 97, I9I. 127, 345. 156, 212. 157, 232. 167, 370. 171, 245. 188, 458. 208, 239. 236, 330, 333. 270, I7. 275, 23. 277, 88. 288, 70. 296, 105. 438, 46. cf. Epinephelus merra Bl., Blkr., Epinephelus stellans Blkr., Serranus stellans Rich.

Serranus hirundinaceus Langsdorf ap. C. $V$. cf. Centropristes hirundinaceus $C . V$.

Serranus Hoedtii Blkr. 128, 406. 275, 23. cf. Epinephelus Hoedti Blkr. 
Serranus Hoevenii Blkr. 13, 36. 156, 212. 167, 370. 184, 198. 236, 333. 270, I 7. 285, 244. cf. Epinephelus Hoevenii Blkr.

Serranus Homfrayi Day cf. Epinephelus leopardus Blkr.

Serranus horridus $(K . v . H$.$) C. V .2,525.13,36.51,55.54,236$. 234, 352. 288, 70. 438, 46. cf. Epinephelus fuscoguttatus Blkr., Epinephelus horridus Blkr.

Serranus horridus Cant. cf. Epinephelus lanceolatus Blkr.

Serranus Jansenii Blkr. 167, 376. cf. Epinephelus Janseni Blkr.

Serranus kawamebari Rich. cf. Epinephelus brunneus Blkr.

Serranus kawamebari Schl. cf. Epinephelus kawamebari Blkr.

Serranus Kunhardtii Blkr. 44, I69. 270, I 7. cf. Epinephelus Hoevenii Blkr.

Serranus lanceolatus $C . V .13,35.25,7.91,30.158,273.184$, I98. 275, 23. 288, 70. cf. Epinephelus lanceolatus Blkr.

Serranus latifasciatus Schl. cf. Epinephelus latifasciatus Blkr.

Serranus lemniscatus $C . V .91,30.270$, r $7.438,44$. cf. Lutjanus melanotaenia Blkr.

Serranus leopardus $C$. $V$. cf. Epinephelus leopardus Blkr.

Serranus leucogrammicus (Reinco) C. V. 2, 525. 13, 33. 54, 234. 287, 44. cf. Anyperodon leucogrammicus Gthr.

Serranus leucostigma Ehr. cf. Epinephelus coeruleopunctatus Blkr.

Serranus lineatus $C: V .91,30.125,3$ r. cf. Epinephelus lineatus $B l k r$.

Serranus longispinis Kner cf. Epinephelus maculatus Blkr.

Serranus louti Riupp. 296, ro5. cf. Variola louti Blkr.

Serranus luti $C$. $V$. cf. Epinephelus argus Bl. Schn.

Serranus lutra $C$. $V$. cf. Epinephelus lutra Blkr.

Serranus macrospilos Blkr. 141, 499. 170, 478. 275, 23. cf. Epinephelus macrospilus $B l k r$.

Serranus maculatus Blkr. 151, 398. 275, 23. cf. Epinephelus maculatus Blkr.

Serranus maculosus $C . V$. cf. Epinephelus pantherinus $B / k r$.

Serranus marginalis $C . V .13,34.46,2$ I . 48, 348. 91, 30. 167, 370. 236, 330. 237, 435. 270, I 7. 275, 23. 296, 105. cf. Epinephelus fasciatus Blkr., Epinephelus marginalis Blkr.

Serranus megachir Rich. cf. Epinephelus Gilberti Blkr.

Serranus melanotaenia Blkr. 163, 33. 438, 45. cf. Variola flavinarginata Blkr.

Serranus merra C. $V$. 10, 66, 68. 13, 3. 46, 2 I1. 91, 166. 275, 23. cf. Epinephelus hexagonatus $B l k r$., Epinephelus merra $B l$.

Serranus microdon Blkr. 148, 86. cf. Epinephelus microdon Blkr.

Serranus microprion Blkr. 66, 552, 156, 212. 157, 232. 234, 352. 236, 33०, 333. 270, I 7. 275, 23. 296, Io6. cf. Epinephelus microprion Blkr.

Serranus micropsion Blkr. cf. Epinephelus micropsion Blkr.

Serranus miliaris C. $V .13,4.113,4$ I8. cf. Epinephelus maculatus Blkr. 
Serranus miniatus Rüppp. cf. Epinephelus miniatus Blkr.

Serranus mitis Benn. cf. Serranus filamentosus $C$. $V$.

Serranus moara Temm. Schl. 90, 24. 438, 45. cf. Epinephelus nebulosus Blkr.

Serranus morrhua $C$. $V$. cf. Epinephelus morrhua Blkr.

Serranus multinotatus $P$ et. cf. Epinephelus multinotatus Blkr.

Serranus myriaster $C . V .97$, 192. 119, 5. 156, 2 12. 157, 232. 167, 370. 184, 198. 236, 330. 270, 18. 275, 23. 277, 88. 287, 44. cf. Epinephelus argus Bl. Schn., Blkr., Epinephelus guttatus Blkr.

Serranus mystacinus Poey, Gthr. cf. Epinephelus mystacinus Blkr.

Serranus nebulosus Rich. cf. Epinephelus diacanthus Blkr.

Serranus nebulosus $C . V$. 2, 525. 13, 34. 25, 7. 56, 444. 90, 25 . 227, 406. 236, 330. 270, I8. 287, 44. 438, 45. cf. Epinephelus nebulosus Blkr.

Serranus nigriceps $C . V .13,37$. cf. Epinephelus merra $B l$.

Serranus nigripinnis $C . V, \mathbf{1 4 1}$, 500. cf. Epinephelus nigripinnis Blkr.

Serranus nigrofasciatus Hombr. Jacq. cf. Epinephelus boelang Blkr.

Serranus nouleny $C . V .91,30.438,43$ cf. Lutjanus erythropterus $B l$. Serranus oceanicus $C . V$. cf. Epinephelus fasciatus Blkr.

Serranus octocinctus Temm. Schleg. 90, 24. 118, 62. 253, 234. cf. Epinephelus susuki Blkr.

Serranus octocinctus $C$. $V$. cf. Epinephelus octocinctus Blkr.

Serranus oculatus $C$ v. cf. Etelis oculatus Blkr.

Serranus ongus Pet. cf. Epinephelus ongus Blkr.

Serranus orientalis $C . V$. cf. Diagramma orientale $C$. $V$., Plectorhynchus orientalis Szons.

Serranus pachycentron $C . V .13,32$.

Serranus pantherinus $C . V$. 2, 525. cf. Epinephelus pantherinus Blkr.

Serranus pardalis Blkr. 9, 635. 13, 37. 46, 2I 5. 47, 226. 126, 344. 156, $212.185,222.222,3$ 16. 236, 330. 267, 139. 270, 18. 275, 23. 287, 44. cf. Epinephelus Gilberti Blkr.

Serranus phaenistomus Szons. cf. Variola louti Blkr.

Serranus poëcilonotus T. Schl. 118, 6r. cf. Epinephelus poecilonotus Blkr.

Serranus polyphekadion Blkr. 13, 39. 113, 4I7. cf. Epinephelus polyphekadion $B l k r$.

Serranus polypodophilus Blkr. 13, 37. 185, 222. 235, 363. 275, 23. 287, 44. cf. Epinephelus polypodophilus Blkr.

Serranus polystigma Blkr. 76, 244. 270, I8. 275, 23. 438, 46. cf. Epinephelus summana Blkr.

Serranus punctatissimus Gthr. cf. Epinephelus punctatissimus Blkr.

Serranus punctulatus $C . V .13,4.67,570.91,30.270$, I8. 275, 23. cf. Variola louti Blkr.

Serranus Quoyanus C. $V$. 13, 4. cf. Epinephelus maculatus Blkr.

Serranus rasor Rich. 119, 5 . 
Serranus Reevesii Rich. cf. Epinephelus Gilberti Blkr., Epinephelus Reevesii Blkr.

Serranus reticularis Gthr. cf. Epinephelus ongus Blkr.

Serranus reticulatus $(K . v . H$.$) C. V .2,525.13,3 . \mathrm{cf}$. Epinephelus ongus Blkr.

Serranus rhyncholepis $B l k r .71,749.275,23$. cf. Epinephelus rhyncholepis Blkr.

Serranus rivulatus $C$. $V$. cf. Epinephelus rivulatus $B l k r$.

Serranus roseus $C . V$. 67, 57 r. cf. Epinephelus analis Blkr., Serranus aurantius $C . V$.

Serranus salmoides $C$. $V$. cf. Epinephelus polypodophilus Blkr.

Serranus salmonoïdes Gthr. cf. Epinephelus polypodophilus Blkr.

Serranus salmonoides $C$. $V$. cf. Epinephelus salmonoides $B l k r$.

Serranus scriba Cuv. 468, 255.

Serranus Sebae Blkr. 103, 488. 236, 333. 275, 24. 296, ro6. 438, 46. cf. Epinephelus albofuscus Blkr., Epinephelus maculatus Blkr.

Serranus semipunctatus $C$. $V .91,30$. cf. Epinephelus semipunctatus Blkr.

Serranus sexfasciatus $(K . v . H). C . V .2,525,10,66.13,38.46,2$ I I. 270, I8. 275, 24. 287, 44. cf. Epinephelus sexfasciatus Blkr.

Serranus shihpan Rich. cf. Epinephelus diacanthus Blkr.

Serranus Sonnerati Playf. 485, 2. cf. Epinephelus miltostigma Blkr., Epinephelus Playfayri Blkr.

Serranus Sonneratii C. V. 91, 32. cf. Epinephelus Sonneratii Blkr.

Serranus spilurus $C . V .99,322.167,370.228,424.270$, х8. 296, гоб. cf. Epinephelus leopardus Blkr.

Serranus stellans Rich. 142, 29. 167, 377. 438, 46. cf. Epinephelus stellans Blkr., Serranus hexagonatus $C$. $V$.

Serranus stigmapomus Rich. 119, 5. cf. Epinephelus boelang Blkr., Epinephelus stigmapomus Blkr.

Serranus suillus $C . V$. 2, 525. 46, 2 I 5. 51, 55. cf. Epinephelus pantherinus Blkr.

Serranus summana Playf. 438, 46. cf. Epinephelus summana Blkr.

Serranus tauvina Klunz. cf. Epinephelus Gilberti Blkr.

Serranus Telfairi Benn. cf. Anthias zonatus Gthr., Odontanthias borbonicus Blkr.

Serranus trimaculatus Blkr. cf. Epinephelus diacanthus Blkr.

Serranus trimaculatus $C . V$. 175, 8. 253, 234. cf. Epinephelus fasciatomaculatus Pet., Epinephelus merra Bl., Epinephelus trimaculatus Blkr.

Serranus tsirimenara $T$. Schl. 118, 62. cf. Epinephelus tsirimenara Blkr.

Serranus tumilabrus $C . V .13 ; 39.113,418$. cf. Epinephelus tumilabris Blkr.

Serranus unicolor Lien. cf. Epinephelus unicoior Blkr.

Serranus ura $C$. cf. Epinephelus ura Blkr. 
Serranus urodelus $C . V .105,39.156,2$ 12. 167, 370. 236, 330. 270, I8. 275, 24. cf. Epinephelus urodelus Blkr.

Serranus urophthalmus Blkr. 125, 310. 157, 232. 438, 45. cf. Anyperodon urophthalmus Blkr.

Serranus variegatus Rich. cf. Epinephelus variegatus Blkr.

Serranus variolosus C. $V .2,525.13,35.16,4.25,7.46,2$ I 5. 156, 21 2. 185, 222. 227, 406. 231, I44. 270, I8. 275, 24. 287, 45. cf. Epinephelus variolosus Blkr.

Serranus vitta $Q . G .13$, 3. cf. Lutjanus vitta Blkr.

Serranus Waandersii Blkr. 231, 152. cf. Epinephelus Waandersii Blkr.

Serranus zanana C. V. cf. Epinephelus leopardus Blkr.

Serranus zananella C. V., Blkr. 13, 32. cf. Epinephelus boelang Blkr. Serranus zonatus $C$. $V$. cf. Anthias zonatus $G$ thr.

Seserinus Vachelli Rich. 425, I33. cf. Apolectes niger Cuvv, Stromateus niger $\mathrm{Bl}$.

Setarches Johns. 473, 295 .

Setarches Güntheri Johns. 473, 295.

Shacra Blkr. 261, 436. 329, 263.

Siaja 261, 43I.

Siboma Gir. 261, 425. 314, 205.

Sicydiini 453 , 3I 2 .

Sicydium Val. 453, 3ㄱ.

Sicydium balinense Blkr. 159, 297. cf. Sicyopus balinense Gill.

Sicydium cyanocephalus C. V. 135, 201.

Sicydium cynocephalum C.V.10, 67, 68.15, 4. 46, $213.270,42.275,44$. 296, III. cf. Sicyopterus cynocephalus $B l k r$.

Sicydium cynocephalus Blkr. 231, I48.

Sicydium gymnauchen Blkr. 176, Ir. 275, 44. 453, 3I4. cf. Microsicydium gymnauchen Blkr.

Sicydium lagocephalum $C . V .15,39.25$, го.

Sicydium lagocephalum Blkr. cf. Sicydium cyanocephalus C. $V$., Sicyopterus cynocephalus Blkr.

Sicydium lagocephalus C. V. 459, 78 . cf. Sicyopterus lagocephalus Blkr. Sicydium laticeps C. $V .459,78$.

Sicydium macrostetholepis Blkr. 76, 27 . 270, 43. cf. Sicyopterus macrostetholepis Blkr.

Sicydium microcephalus Blkr. 113, 437. cf. Sicyopterus microcephalus Blkr.

Sicydium micrurus Blkr. 84, 34I. 231, I48. cf. Sicyopterus micrurus Blkr.

Sicydium obscurum Schl. 453, 313. cf. Tridentiger obscurus Gill.

Sicydium Parvei Blkr. 77, 426. cf. Sicyopterus Parvei Blkr.

Sicydium Plumieri Val. 453, 3 13.

Sicydium Stimpsoni Gill 453, 3 r3. 
Sicydium xanthurus Blkr. 76, 27 1. 231, I48. 270, 43. cf. Sicyopterus xanthurus Blkr.

Sicydium zosterophorum Blkr. 159, 296. 453, 3Ir. cf. Sicyopus zosterophorum Gill.

Sicyogaster Gill 453, 314.

Sicyogaster concolor Gill 453, 3 I4.

Sicyopterus Gill 453, 313.

Sicyopterus cynocephalus Blkr. 472, 275.

Sicyopterus lagocephalus Blkr. 495, I7.

Sicyopterus macrostetholepis Blkr. 472, 28 I.

Sicyopterus microcephalus Blkr. 472, 278.

Sicyopterus micrurus Blkr. 472, 280.

Sicyopterus Parvei Blkr. 472, 277.

Sicyopterus xanthurus Blkr. 472, 283.

Sicyopterus (Sicydiops) xanthurus $B l k r$. cf. Sicyopterus xanthurus Blkr.

Sicyopus Gill 453, 3Ir.

Sicyopus balinense Gill 472, 288.

Sicyopus zosterophorum Gill 472, 287.

Sidera $K p .351$, r2 I.

Sidera pantherina $K p$. cf. Gymnothorax pictus Bl., Blkr., Muraena pantherina $J . M c C l$.

Siganus doliatus Cuv. cf. Amphacanthus doliatus $C . V$.

Sillaginichthys Blkr. 468, 332.

Sillaginodes Gill 468, 332.

Sillaginoidei 468, $33 \mathrm{r}$.

Sillaginopsis Gill 468, 332 .

Sillago Cu\%. 1, 553. 2, 512.468, 33 I.

Sillago acuta $C . V .2,524,527.9,633.10,66.13,6$. 16, 4. 25, 7 . $40,478.46,212.50,47$ 1. 51, 55.52, 89.53, г60.55, 411. 56, 444. 90, 28. cf. Sillago malabarica Cuv.

Sillago acuta $C u v .468,332$. cf. Sillago sihama $R \ddot{u} p p$.

Sillago bassensis C. V. 119, 7. cf. Sillago maculata Q.G.

Sillago burrus Rich. 119, 7 .

Sillago chondropus Blkr. 13, 61. 231, 164. 452, 65 .

Sillago ciliata 2,527 .

Sillago domina C. V. 91, 34. 468, 332 .

Sillago erythraea $C$. $V$. cf. Sillago sihama Rüpp.

Sillago japonica T. Schl. 90, 28. 175, I I. 231, I63. 364, 285. 425, 142. 452, 69. 497, 9 .

Sillago macrolepis Blkr. 231, I66. 452, 72.

Sillago maculata $Q . G ., C . V .2,527.13,62.50,47$ I. 119, 7. 231, I6 I. 235, 365. 275, 32. 287, 50. 395, 292. 452, 7 I.

Sillago malabarica Cuv. 91, 34. 156, 2 13. 167, 371. 170, 479. 175, I I. $178,2.185,222.208,239.227,407.231$, I $57.235,365$. 
237, 436. 270, 23. 272, т 2. 275, 32. 284, 239. 287, 50. cf. Sillago sihama Rüpp.

Sillago malabarica Cant. cf. Sillago acuta C. $V$., Sillago sihama Rüpp.

Sillago punctata Cuv. 119, 19. 468, 332.

Sillago sihama Rüpp. 288, 73. 296, 108. 316, 242. 320, 269. 354,

I 47. 358, 189. 364, 285. 395, 292. 425, 142. 452, 67. 459, 8o. 468, 332. 491, 46. 495, I4.

Silundia $V$ al. 299, 399. 306, 108.

Silundia chandramara $C . V .91,58$.

Silundia Childrenii Blkr. 91, 58.

Silundia gangetica $C . V$. 91, I $8.306,108$.

Siluranodon Blkr. 299, 395. 306, I 15.

Siluranodon auritus $B l k r$. 306, I I 5 .

Silurichthys Blkr. 299, 394. 306, I I 5.

Silurichthys phaiosoma Blkr. 185, 225. 235, 37x. 272, I9. 306, I I5.

Silurichthys sinensis Gthr. 425, 125.

Silurodes Blkr. 299, 394. 306, I I5.

Silurodes hypophthalmus Blkr. 270, 47. 306, II 5.

Silurodes macronema Blkr. 270, 48. 272, 19.

Silurodon hexanema Gthr. 425, 125.

Silurus L. 1, 552. 299, 393. 306, Ir 4 .

Silurus anostomus C. $V .91$, I09. cf. Wallago anostomus Blkr.

Silurus apogon Blkr. 42, 67. 45, I96. 49, 420. 55, 4I2. 139, 419. cf.

Silurus micropogon Blkr.

Silurus arab Forsk. cf. Plotosus arab Blkr.

Silurus asotus Basil. cf. Parasilurus asotus Blkr.

Silurus aspredo $L$. cf. Aspredo batrachus $L$.

Silurus attu Bl. Schn. 91, 108. cf. Wallago attu Blkr., Wallago Russellii Blkr.

Silurus auritus Geoffr. cf. Siluranodon auritus Blkr.

Silurus bagre $L$. cf. Pimelodus bagre Lac.

Silurus bagre Bl. cf. Pimelodus filamentosus Blkr.

Silurus bicirrhis $C . V .3$, I 49. 4, 285. 5, I5. 35, 27 I. 55, 4 I 3 .

Silurus bimaculatus $B l .2,5$ I 1. 3, I48. 4, 284. 5, I4. 7, 6.

Silurus boalis Buch. cf. Wallago Russellii Blkr.

Silurus callichthys $L$. cf. Callichthys tamoata $L$.

Silurus calvarius Basil. cf. Pelteobagrus calvarius Blkr.

Silurus canio Buch. 91, 54.

Silurus carinatus $L$. cf. Doras carinatus $L a c$.

Silurus cataphractus $L$. cf. Acanthodoras cataphractus Blkr.

Silurus clarias Bl. cf. Pseudariodes clarias Blkr.

Silurus clarias Hass. cf. Synodontis clarias Cuv.

Silurus costatus $L$. cf. Platydoras costatus Blkr.

Silurus cotylephorus Lac. cf. Platystacus cotylephorus $B l$.

Silurus cous L. cf. Aglyptosternon cous Blkr. 
Silurus cryptopterus Blkr. 35, 270. 42, 59. 45, I96. 49, 420.55, 412. Silurus (Acanthonotus) Cuvieri Gr. cf. Ailia coila Blkr.

Silurus erythropterus $B l$. 91, I I4.

Silurus fasciatus $B l$. cf. Pseudoplatystoma fasciatum $B l k r$.

Silurus fossilis Bl. of Saccobranchus fossilis Blkr.

Silurus galeatus $L$., $B l$. cf. Parauchenipterus galeatus $B l k r$.

Silurus garua Buch. cf. Clupisoma garua Blk\%, Schilbe garua $C$. $V$.

Silurus glanis L. 91, 54. 306, I I 4.

Silurus hemiliopterus Bl. Schn. cf. Pirarara hemiliopterus Blkr.

Silurus Herzbergii Bl. cf. Netuma Herzbergi Blkr.

Silurus hexadactylus Lac. cf. Platystacus cotylephorus $B l$.

Silurus hexapterus Blkr. 45, 203. 49, 420.55, 41 2

Silurus hypophthalmus Blkr. 3, 149. 4, 284. 5, I5. 91, II3.

Silurus japonicus Rich. cf. Parasilurus asotus Blkr.

Silurus japonicus $S c h l$. cf. Parasilurus japonicus Schl., Blkr.

Silurus laïs Blkr. 49, 428. 55, 4I2.

Silurus lamghur Heck. 91, 54.

Silurus leptonema Blkr.67, 584 .

Silurus lima $B l$. Schn. cf. Sorubim lima $B l k r$.

Silurus limpok Blkr. 67, 583 .

Silurus macronema Blkr. 45, 203. 49, 420. 55, 4I 2 .

Silurus maculatus $T h u n b$. cf. Arius ocellatus $B l k r$.

Silurus microcephalus $C$. $V$. cf. Wallago microcephalus $B l k r$.

Silurus micronema Blkr. 91, II3.

Silurus micronemus Blkr. 4, 289. 5, 56. 7, 6. 25, Iо.

Silurus micropogon Blkr. 139, 419.

Silurus militaris $B l$. cf. Ageneiosus militaris $B l k r$.

Silurus mononema $B l k r .7,8.8$, I66. 25, Iо. 91, II 3.

Silurus Mülleri Blkr. 4, 289. 5, 56. 7, 6. 25, Io. cf. Wallago Russellii Blkr.

Silurus mysoricus $\mathrm{Val}$. cf. Callichrous canio Gthr.

Silurus nodosus Bl. cf. Pseudauchenipterus nodosus Blkr.

Silurus olivaris Raf. cf. Hopladelus olivaris Gill.

Silurus (Callichrous) pabda Buch. cf. Callichrous pabda Blkr.

Silurus palembangensis Blkr. 67, $5^{84}$.

Silurus phaiosoma Blkr. 49, 428. 52, $89.55,413$.

Silurus phalacronotus Blkr. 49, 429.55, 412.

Silurus schall Bl. Schn. cf. Hemisynodontis schall Blkr.

Silurus sinensis Rich. cf. Parasilurus asotus Blkr.

Silurus sinensis Lac. cf. Silurichthys sinensis Gthr.

Silurus wallagoo C. $V$. cf. Wallago Russellii Blkr.

Silurus xanthosteus Rich. cf. Parasilurus asotus Blkr.

Siniperca Gill 468, 255 .

Siniperca chuantsi Gill cf. Siniperca chuatsi Gill.

Siniperca chuatsi Gill 425, I37. 468, 255. 496, 4. 497, 5. 
Siniperca matraki Guich. cf. Siniperca chuatsi Gill.

Sirembo Blkr. 175, 22.

Sirembo grandis Gïnth. 497, 20.

Sirembo imberbis Blkr. 175, 24. 497, 20.

Sisor Buch. 306, 83.

Sisor rhabdophorus Buch. 91, 6o. 306, 83 .

Smaris Cuv. 1, 553. 468, 273. 470, I $5 \mathrm{I}$.

Smaris balteatus $C . V .91,38.470$, г 54 .

Smaris gymnopterus Blkr. 445, r81. 470, I 52.

Smaris insidiator $C . V .468,275.470$, I 54 .

Smaris mauritianus Q. G. cf. Caesio coerulaureus Lac.

Smaris oyena Rüpp. cf. Diapterus oyena Blkr., Gerres oyena $C . V$.

Smaris vulgaris Cuv. $470, \mathrm{I}_{52}$.

Smecticus $V a l .468,258$.

Smecticus bicolor Val. 468, $25^{8}$.

Smerdis $A g$. 468, 260.

Smerdis micracanthus $A g .468,260$.

Smiliogaster Blkr. 261, 428. 314, 2 I 4.

Solea $1,55^{2}$.

Solea cornuta C. 2, 509 .

Solea fasciata Basil. 425, I30. cf. Brachirus zebra Swens.

Solea foliacea Rich. cf. Brachirus foliaceus Blkr.

Solea heterorhinos Blkr. 142, 64 .

Solea heterorhinus Günth. 396, 298.

Solea humilis Cant. 288, 69. 395, 290.

Solea impar Benn. cf. Pegusa impar Gthr.

Solea japonica Gïnth. cf. Achirus japonicus Schl.

Solea lascaris $B p$. cf. Pegusa impar Gthr.

Solea liturata Rich. 119, I5.

Solea maculata Cuv. 38, 409. 61, 17. 91, 76. cf. Solea humilis Cant.

Solea nasuta Nordm. cf. Pegusa impar Gthr.

Solea ommatura Rich. 35, 262. 42, 60. 45, I97. cf. Brachirus zebra

Szens., Synaptura ommatura.

Solea ovalis Rich. cf. Brachirus foliaceus Blkr.

Solea ovata Rich. cf. Solea (Microbuglossus) ovata Gthr.

Solea (Microbuglossus) ovata Gthr. 425, rзo.

Solea pan Cuv. 38, 410. 40, 478. cf. Synaptura pan Cant., Blkr.

Solea Russellii Blkr. 38, 401. 61, I 5 .

Solea trichodactyla Cuv. 364, 274.

Solea triophthalmus Blkr. 302, 27.

Solea tubifera Pet. 495, 2 I.

Solea vulgaris Cuv. 266, 56 .

Solea zebra Cuv. 38, 401. 61, I6.

Solegnathus argus Blkr. 119, I 7 .

Solegnathus Blochii Blkr. 89, 24. cf. Gastrotokeus biaculeatus Heck. 
Solegnathus Hardwickii Szons. 91, 80.

Solegnathus polyprion $B l k r .89,25$.

Soleichthys heterorhinos Blkr. 275, I4. 364, 274.

Soleichthys heterorhinus Blkr. 320, 265.

Solenichthys brachyurus Blkr. 364, 273.

Solenichthys cyanopterus Blkr. 358, I83. 364, 273.

Solenichthys paradoxus Blkr. 358, $183.364,274$.

Solenognathus Blochii Blkr. 156, 2 I 8, cf. Gastrotokeus biaculeatus Heck.

Solenognathus Hardwickii $K p$. 425, I26.

Solenognathus lettiënsis Blkr. 274, 3 .

Solenognathus polyprion Blkr. 270, 73. 495, r7. cf. Solenognathus Hardwickii $K p$.

Solenomormyrus Blkr. 449, 368 .

Solenostoma brachyurus Blkr. 128, 433.

Solenostoma cyanopterus Blkr. 128, 434.

Solenostoma paradoxum Lac. 54, 308. 89, 29. 103, 506. cf. Solenostomatichthys paradoxus Blkr.

Solenostomatichthys Bleekeri 459, 76. 495, I 7 .

Solenostomatichthys paradoxus Blkr.459, 76. 495, I 7 .

Solenostomichthys cyanopterus Blkr. 425, I26.

Solenostomus Bleekerii $A$. Dum. cf. Solenostomatichthys Bleekeri.

Solenostomus paradoxus Rich. cf. Solenostomichthys cyanopterus Blkr.

Solenostomus serratus Blkr., Gill 491, 50.496, 2. 497, I4.

Soleotalpa unicolor Gthr. cf. Apionichthys Dumerili $K p$.

Somileptes Sins. 323, 36 .

Somileptes gongota Blkr. 323, 40.

Sorubim Spix 306, 98.

Sorubim jandia Spix cf. Sorubimichthys jandia Blkr.

Sorubim lima Blkr. 306, 98.

Sorubimichthys Blkr. 306, 98 .

Sorubimichthys jandia Blkr. 306, 98 .

Spariformes 468, 270.

Sparini 468, 280.

Sparnodus $A g \cdot 468,282$.

Sparopsis Kner 438, 44. 468, 276.

Sparopsis elongatus $K$ ner $\mathrm{cf}$. Aprion (Aprion) virescens $C . V$.

Sparus Art. 468, 28 r, $33^{6}$.

Sparus aries Blkr. 425, г38.497, 8.

Sparus aurata L. 468, 282.

Sparus aurata Bl. cf. Chrysophrys globiceps C. $V$.

Sparus aurolineatus Lac. cf. Gnathodentex aurolineatus Flkr., l'entapus aurolineatus $C$. $V$.

Sparus bifasciatus Blkr. 364, 282. 459, 92. 495, I2.

Sparus brachio Lac. cf. Novacula taeniurus Blkr.

Sparus brama $B l$. cf. Cantharus Blochii $C$. $V$. 
Sparus brittannus Lac. cf. Diapterus oyena Blkr., Gerres oyena $C . V$. Sparus calamara Blkr. 425, I38.

Sparus cantharus $L .468,283$.

Sparus cardinalis Blkr. 497, 8.

Sparus cardinalis Lac. cf. Chrysophrys cardinalis $C . V$.

Sparus chlorurus $B l .47,230$. cf. Cheilinus trilobatus $C . V$.

Sparus coracinus Blkr. 459, 92. 495, I2.

Sparus cuning $B l$. cf. Caesio erythrogaster $K . v . H$.

Sparus Cuvieri Blkr. 492, 44.

Sparus datnia Blkr. 477, 5. 497, 8.

Sparus decussatus Benn. cf. Julis (Halichoeres) hortulanus $C$. $V$.

Sparus erythrourus $B l$. cf. Diapterus oyena $B l k r$., Gerres oyena $C . V$. Sparus fasciatus $B l$. cf. Cheilinus fasciatus $C$. $V$.

Sparus fasciatus Gron. cf. Glyphidodon (Glyphidodon) coelestinus (Sol.) C. $V$.

Sparus filamentosus Blkr. 459, 92. 495, I2.

Sparus haemisphaerium Lac. cf. Novacula taeniurus Blkr.

Sparus has'a Bl. Schn. 425, I38. 459, 92. 477, 9.

Sparus insidiator Pallas cf. Epibulus insidiator $C$. $V$.

Sparus japonicus Bl. cf. Dentex Blochii Blkr., Dentex japonicus Blkr.,

Dentex tambulus $C . V$.

Sparus laticeps Blkr. 495, I2.

Sparus lepisurus Lac. cf. Lutjanus bohar Bl. Schn., Mesoprion quadriguttatus Blkr.

Sparus madagascariensis Blkr. 459, $9^{2}$.

Sparus maena $L .468,273$.

Sparus major Blkr. 497, 8.

Sparus malabaricus Bl. Schn. 86, 497. cf. Lutjanus malabaricus Blkr., Mesoprion malabaricus $C . V$.

Sparus meaco Lac. cf. Tautoga fasciata $C$. $V$.

Sparus Mylius Bory cf. Prochilus polymnus Blkr.

Sparus nigricans Gron. cf. Tetradrachmum trimaculatum Blkr.

Sparus pantherinus Lac. cf. Cirrtrites pantherinus C. $V$.

Sparus pantherinus C. $V$. cf. Paracirrhites Forsteri Blkr.

Sparus sarba Forsk. 459, 92. 495, I2.

Sparus scandens Shaw cf. Anabas testudineus Cuv.

Sparus Schlegelii Blkr. 477, 2. 497, 8. cf. Sparus hasta Bl. Schn.

Sparus sinensis Lac. cf. Dentex setigerus $C$. $V$., Dentex sinensis Blkr.

Sparus spadiceus 214, 253.

Sparus spinifer Forsk. 459, $9^{2}$.

Sparus testudineus Shaw cf. Anabas scandens Cuv., Anabas testudineus Cuv.

Sparus tranquebaricus Shaw cf. Lutjanus Johni Lac.

Sparus tumifrons Blkr. 497, 8.

Spatularia angustifolium $K p$. cf. Psephurus gladius Gïnth. 
Sphaerodon Rüpp. 468, 28r.

Sphaerodon grandoculis Rüpp. 438, 46. 459, 9 I.

Sphaerodon heterodon Gthr. 309, I 55. 316, 242. 364, 282. cf. Sphaerodon grandoculis Rüpp.

Sphaerodon latidens $K n e r$ cf. Sphaerodon grandoculis Rüpp.

Sphagebranchus $B l .88,38.351,120$.

Sphagebranchus anceps $K p$. 288, 8о. 352, I30.

Sphagebranchus bicolor $K p .313$, I 85.352, I 29.

Sphagebranchus brevirostris $P$ et. cf. Sphagebranchus fuscus Blkr.

Sphagebranchus cephalopeltis Blkr. 302, 128.

Sphagebranchus fuscus Blkr. 459, 72 .

Sphagebranchus Kaupi Blkr. 192, I, 4. 275, 51. 352, I29.

Sphagebranchus lumbricoides Blkr. 348, 46. 352, I29.

Sphagebranchus macrodon Blkr. 313, I 84. 317, 246. 352, I29.

Sphagebranchus marmoratus $K p . \mathbf{2 7 0}, 60$. cf. Callechelys marmoratus Blkr.

Sphagebranchus moluccensis Blkr., Kp. 352, r30. 358, 193. 364, 296.

Sphagebranchus polyophthalmus $K p$. Blkr. 207, 2 I I. 270, 60. 296, I 12. 352, I30.

Sphagebranchus quadratus Rich. cf. Apterichthys quadratus Rich.

Sphagebranchus vimineus $R i c h$. cf. Leiuranus colubrinus $K p$.

Sphyraena 1, 553.

Sphyråena affinis Rüpp. 113, 425. 117, I4.

Sphyraena agam Rüpp. 117, I6.

Sphyraena barracuda C. $V .117$, I6.

Sphyraena becuna Lac. cf. Sphyraena vulgaris $C . V$.

Sphyraena borealis Dek. cf. Sphyraena vulgaris $C . V$.

Sphyraena brachygnathos Blkr. 112, 368. 117, 21. 296, ro9.

Sphyraena chinensis (Lac.) Rich. 13, 56. 117, 18. 425, 133. cf. Sphyraena obtusata $C$. $V$.

Sphyraena Commersonii $C . V .10,66.13,55.16,4.25,7.46,212$. $113,425.117$, 15. 275, 37. 287, 51. 309, 156. 315, 235. 320, 270. $363,265.364,288.459,79.495$, I 8 .

Sphyraena dubia Blkr. 302, 70.

Sphyraena Dussumieri $C . V .117$, 16. 459, 79. 495, 18.

Sphyraena flavicauda Rüpp. 117, I8.

Sphyraena Forsteri $C . V .13,4.113,424.117,13.167,371.231$, I 46. $270,28.275,37.320,270.364,288.491,53$.

Sphyraena guachancho $C . V$. cf. Sphyraena vulgaris $C . V$.

Sphyraena japonica $C . V .$, Schl. 118, 67. 275, 37. 497, I 7 .

Sphyraena jello C. V. 2, 524, 527. 13, 56. 16, 4. 25, 7. 46, 212. 50, 471. 51, 55. 53, I60. 56, 444. 90, 4. 91, 34. 112, 369. 113, 425. 117, I2. $127,345.156,213.227,407.231$, I $46.270,2 S$. 275, 37. 288, 74. 296, 109. 315, 235. 320, 270. 356, I73. 395, 292. 425, г33. 459, 79. 497, I 7 . 
Sphyraena langsar Blkr. 112, 367. 117, r9. 275, 37. 296, го9. 315, 235. 364, 289 .

Sphyraena nigripinnis Schl. 497, I7.

Sphyraena obtusa C. $V .459,79$.

Sphyraena obtusata $C . V$. 13, 56. 16, 4. 25, 7. 51, 55. 54, 235. 91,

34. 112, 364. 117, I 7. 119, 7. 156, 213. 231, I46. 235, 366. 270,

28. 275, 37. 288, 74. 296, I09. 309, I 56. 315, 235. 316, 242. 356,

I 73. 358, І9о. $364,289.396,300.425$, I 33. 495, I8. 497, I7.

Sphyraena spet Lac. cf. Sphyraena vulgaris $C$. $V$.

Sphyraena sphyraena $B l$. Schn. cf. Sphyraena vulgaris $C$. $V$.

Sphyraena viridensis $C$. $V$. cf. Sphyraena vulgaris $C . V$.

Sphyraena vulgaris C. $V . \mathbf{2 6 6}, 53.363,263$.

Sphyrna Blochii $M . H .10,68.16,6.25$, 13. 42, 6o. 45, 197. 46, 2 14. 49, 42 I. 51, 60. 55, 4I5. 64, 4r.

Sphyrna laticephala 2, 508 .

Sphyrna makarran 2, 508.

Sphyrna tudes $V a l$. 16, 6. 25, I3.

Sphyrna tudes M. H. cf. Sphyrna zygaena Rafin.

Sphyrna zygaena Raf. 51, 6o. 64, 42. 91, 8o. 119, I8.

Spicara Raf. 468, 273.

Spinax acanthias Flem. 497, 3. cf. Acanthias vulgaris Risso.

Spinax Blainvillii Bon. cf. Acanthias Blainvillii Risso.

Spirobranchus capensis C. $V .266,54$.

Spondyliosoma Cant. 468, 336.

Spondyliosoma guliminda Cant. cf. Dentex tolu C. $V$.

Spratella fimbriata $C . V .60,27.71,775.91,74.275,49$.

Spratella kowala Blkr. 50, 492. 60, 28. 170, 480. 235, 372. 270, 57 .

287, 6I. 356, I76. 364, 295. cf. Clupea (Harengula) perforata Blkr.,

Harengula (Spratella) kowala Blkr.

Spratella pseudopterus Blkr. 55, 432. 60, 50. 272, 26.

Spratella tembang Blkr. 46, 2I4. 60, 28. 71, 774. 156, 2 г7. 185, 226. 227, 408. 231, I50. 235, 372. 270, 57. 275, 49. 287, 6r.

Spratelloïdes argyrotaenia Blkr. 46, 214. 60, 29. 71, 775. 275, 49.

$315,236$.

Spratelloides delicatulus Gthr. 459, го2. 495, 22.

Spratelloides gracilis Blkr. 497, 25 .

Squaliobarbus curriculus Gthr. 415, 48. 425, 145 .

Squalius $B p .261,437.314,212.329,264$.

Squalus 1, $55 \mathrm{I}$.

Squalus acanthias $L$. cf. Acanthias vulgaris Risso.

Squalus (Carcharinus) amboinensis J.E. Gr. cf. Carcharias (Prionodon) amboinensis $M$. $H$.

Squalus anisodon Lac. cf. Pristiophorus cirratus $M$. $H$.

Squalus carcharias minor Forsk. cf. Carcharias (Prionodon) melanopterus $Q . G$. 
Squalus cuspidatus Blkr. 364, 270.425 , I 19.

Squalus dubius Blkr. 364, 27 I.

Squalus fasciatus $B l$. cf. Stegostoma fasciatum $M$. $H$. .

Squalus lima Banks. 119, I7.

Squalus longicaudus $L$. cf. Stegostoma fasciatum $M$. $H$.

Squalus pantherinus $K . v$. $H$. cf. Stegostoma fasciatum $M . H$.

Squalus Russellii Gr. cf. Carcharinus (Scoliodon) acutus Blkr.

Squalus semisagittatus Shaw cf. Pristis semisagittatus Cuv.

Squalus Spallanzanii Le'su'ur cf. Carcharias (Prionodon)melanopterus ( . G.

Squalus tentaculatus Shaw cf. Pristiophorus cirratus $M$. $H$.

Squalus tiburo $L$. cf. Cestracion tiburo $A$. Dum.

Squalus tigrinus $L$. cf. Stegostoma fasciatum $M$. $H$.

Squalus tuberculatus $B l$. Schn. cf. Chiloscyllum tuberculatum $M . H$.

Squalus ustus Dum. cf. Carcharias (Prionodon) melanopterus Q. G.

Squalus zygaena $L$. cf. Cestracion zygaena Gill.

Squalus zygaena Brinn. cf. Sphyrna zygaena Rafin.

Squalus zysron Blkr. 364, $27 x$.

Squatina japonica Blkr. 175, 40. cf. Rhina japonica Blkr., Rhina squatina $A$. Dum.

Squatina vulgaris Schl. cf. Rhina japonica Blkr.

Stegophilus Reinh. 306, II3.

Stegophilus insidiosus Reinh. 306, Ir 3 .

Stegostoma carinatum Blyth cf. Stegostoma fasciatum $M: H$.

Stegostoma fasciatum $M . H .25$, I2. 64, 23. 91, 8o. 270, 67. 287, 36. 288, 66. 459, 68. cf. Stegostoma tigrinum Gthr.

Stegostoma tigrinum Gthr. 425, II9.

Stellifer Cuv. 468, 327 .

Stenogobius Blkr. 453, 317, 318 .

Stereolepis Ayr. 468, 262.

Stereolepis gigas $A y^{\prime}$. 468, 262.

Sternoptyx diaphana Herm. 425, 147.

Stethochaetus biguttatus Gron. cf. Trichopodus trichopterus Lac.

Stethojulis Gthr. 292, Iоo.

Stethojulis albovittata Gthr. 293, 290. 315, 231. 320, 266. 354, 144. 358, I $85.364,277.459,82$.

Stethojulis axillaris Gtlur., Blkr. 293, 290. 315, 230. 320, 266. 354, I $44.364,277.459,82$.

Stethojulis interrupta Gthr. Blkr. 293, 290. 315, 23r. 317, 246.320, 266. 354, I 44. 358, I $85.364,277.394,286$.

Stethojulis kallosoma Blkr. 293, 290. 315, 23r. 320, 266. 354, I 44. $358,185.364,277.394,286$.

Stethojulis phekadopleura Gthr., Blkr. 293, 290. 315, 231. 320, 266. 354, I 44.358, I $85.364,277.437,38$.

Stethojulis Renardi Blkr. 293, 291. 315, 23I. 320, 266. 354, I44. $358,185.364,277$. 
Stethojulis strigiventer Gthr. 293, 291. 315, 231. 320, 267. 354, I 44. 358, I $85.364,277.459,82.495,9$.

Stethojulis trilineata Gthr. 293, 29r. 296, I04. 320, 266. 364, 277. 369,42 .

Stethopterus Blkr. 88, 24. 351, I I9.

Stethopterus vimineus $B l k r$. cf. Leiuranus colubrinus $K p$.

Sthenopus Rich. 473. 299.

Sthenopus mollis Rich. 473, 299. cf. Trichopleura mollis Gthr.

Stichaeus hexagrammus Schl. cf. Eumesogrammus hexagrammus Gill. Stigmatogobius Blkr. 453, 323 .

Stigmatogobius amblyrhynchus Blkr. 489, 207.

Stigmatogobius gastrospilus Blkr. 489, 206.

Stigmatogobius isognathus Blkr. 489, 203.

Stigmatogobius pleurostigma Blkr. cf. Stigmatogobius sadanundio Blkr.

Stigmatogobius sadanundio Blkr. 489, 2 ог.

Stigmatogobius singapurensis Blkr. 489, 204.

Stigmatophora argus $K p .491,49$.

Stilbe de Kay 261, 436. 314, 2 I I. 329, 263.

Stizostedion Raf. 468, 265 .

Stoasodon narinari Cant. cf. Aëtobatis narinari $M$. $H$.

Stolephorus boelama Blkr. 364, 295.

Stolephorus Browni Blkr. 364, 295. cf. Stolephorus Commersonianus Blkr.

Stolephorus (Stolephorus) Browni Blkr. 395, 294.

Stolephorus Commerrianus Lac. 495, 22.

Stolephorus Commersonianus Blkr. 425, I48.

Stolephorus Commersonianus Lac. 459, ro2. cf. Engraulis Brownii $C$. $V$. Stolephorus encrasicholoides Blkr. 315, 236. 320, 272. 338, $3^{6} \mathrm{r}$. 356, I76. 358, I92. 364, 295.

Stolephorus Hamiltoni Blkr. 356, r76. 358, г92.

Stolephorus (Thryssa) Hamiltoni Blkr. 395, 294.

Stolephorus (Stolephorus) heterolobus Blkr. 381, 305.

Stolephorus (Stolephorus) indicus Blkr. 395, 294. 497, 25.

Stolephorus (Stolephorus) japonicus Blkr. 408, 238. 425, 148. 497, 26. Stolephorus melanochir Blkr. 356, г76.

Stolephorus mystacoides Blkr. cf. Engraulis mystax $B l k r$.

Stolephorus Russelli Blkr. 315, 236. 364, 295.

Stolephorus setirostris Blkr. 364, 295.

Stolephorus surinamensis $B l k r .378$, I 78 .

Stolephorus taty Blkr. 356, I 76.

Stolephorus (Thryssa) Valenciennesi Blkr. 381, 306.

Stolephorus (Stolephorus) Zollingeri Blkr. 381, 303.

Stomianodon chrysophekadion Blkr. 17, ro. cf. Astronesthes chrysophekadion Blkr.

Stromateoïdes aculeatus Blkr. 90, 37. 425, I33. 497, I6. 
Stromateoïdes argenteus Blkr. 91, 46. 425, I33.

Stromateoïdes atokoia Blkr. 37, 369. 57, 76. 91, 46.

Stromateoïdes atoukoia Blkr. 51, 57. 56, 445. 178, 2.

Stromateoïdes atous Blkr. 270, 27. 272, r3. 287, 5r. 288, 73. 411, 252. 425, I33.

Stromateoïdes candidus Blkr. 91, 46 .

Stromateoïdes cinereus Blkr. 37, 368. 51, 57. 57, 75. 90, 37. 91, 46 . 178, 2. 227, 407. 235, 366. 270, 27. 272, 13. 288, 73. 356, I 74. $395,292.411,252.425,133$.

Stromateus 1, 553 .

Stromateus aculeatus Bl. Schn. cf. Stromateoïdes aculeatus Blkr.

Stromateus albus $C . V .37,369.57,76$. cf. Stromateoïdes atokoia Blkr.

Stromateus argenteus $C . V .2,518$.

Stromateus argenteus Euphrasen cf. Stromateoïdes aculeatus Blkr.

Stromateus argenteus Bl. cf. Stromateoïdes argenteus Blkr.

Stromateus atous $C$. $V$. cf. Stromateoïdes atokoia, atoukoia Blkr.

Stromateus candidus $C . V .2,{ }^{\mathrm{I} S} .16,4.25,9$.

Stromateus candidus Blkr. cf. Stromateoïdes atokoia Blkr.

Stromateus capensis Papple. 266, 53.

Stromateus cinereus $B l$. 90, 37. cf. Stromateoïdes cinereus $B l k r$.

Stromateus echinogaster $B$ asil. 425, I33. cf. Stromateoïdes cinereus Blkr.

Stromateus griseus $C$. $V$. cf. Stromateoïdes cinereus Blkr., Stromateus cinereus $B l$.

Stromateus niger $C . V .2,5$ r8. 10, 67. 16, 4. 25, 8.

Stromateus niger $B l .37,370.46,2$ I3. 51, 57. 57, 77. 91, 44. 227, 407. 267, I40. 270, 27. 275, 35. cf. Apolectus niger Cuv.

Stromateus paru $B l$. cf. Stromateus niger $B l$.

Stromateus punctatissimus T. Schl. cf. Stromateoïdes aculeatus Blkr.

Stromateus securifer $C$. $V$. cf. Stromateoïdes cinereus Blkr., Stromateus cinereus $B l$.

Stromateus sinensis Euphr. 37, 369. 57, 76. cf. Stromateoides atokoia Blkr., Stromateoïdes atous Blkr.

Strophidon $M C C l ., B l k r .88,40.351,12 \mathrm{r}$.

Strophidon Brummeri Blkr. 348, 48. 352, I35. 364, 297.

Strophidon flavimarginatus $1 / C \mathrm{Cl}$. cf. Gymnothorax flavimarginatus Blkr.

Strophidon literata $M c C l$. cf. Muraena tile Cant.

Strophidon longicaudata $\mathrm{MCCl}$. cf. Lycodontis longicaudata Blkr., Strophidon sathete Blkr.

Strophidon maculata $M C C l$. cf. Muraena tile Cant.

Strophidon polyodon Blkr. 348, 47. 352, 135. 364, 297. 491, 56.

Strophicion punctata .1C Cl.cf. (iymmothorax tile Blkr., Muratenatile Cirnt.

Strophidon sathete Blkr. 352, I35.

Sturisoma Sions. 306, 8r.

Sturisoma rostratrum Sions. 306, 8r.

Syacium micrurum Ranzani 332, 455. 
Symbranchus caligans Cant. 88, 56 .

Symbranchus immaculatus Bl. 55, 438. 88, 57. 91, 78.

Symphorus Gthr. 468, 278.

Symphorus taeniolatus Gthr. 468, 279.

Symphysanodon typus Blkr. 491, 6r. 500, 28 .

Synagris (Klein) Gthr. 438, 40. 468, $278,279$.

Synagris celebicus Gthr. cf. Dentex celebicus Blkr.

Synagris furcosus $G t / h$. cf. Dentex furcosus $C$. $V$.

Synagris griseus Blkr. 425, I38.

Synagris hexodon Gthr. cf. Dentex hexodon $Q$. $G$.

Synagris hypselosoma Blkr. 441, 40. 496, 2. 497, 7 .

Synagris japonicus Gthr. cf. Dentex Blochi Blkr.

Synagris mesoprion Gthr. cf. Dentex mesoprion Blkr.

Synagris metopias Gthr. cf. Dentex metopias Blkr.

Synagris mulloides Gthr. cf. Dentex mulloides Blkr.

Synagris nematophorus Gthr. cf. Dentex nematophorus Blkr.

Synagris nematopus Gthr. cf. Dentex nematopus Blkr.

Synagris nemurus Gthr. cf. Dentex nemurus Blkr.

Synagris Ovenii Gthr. cf. Dentex Ovenii Blkr.

Synagris Personii Gthr. cf. Dentex Personii C. $V$.

Synagris sinensis Gthr. cf. Dentex sinensis Blkr.

Synagris taeniopterus Gthr. 296, Io6. cf. Dentex taeniopterus $C . V$.

Synagris Thunbergii Blkr. 497, 7 .

Synagris upeneoides Gthr. cf. Dentex upeneoides Blkr.

Synagris zysron Gthr. cf. Dentex zysron Blkr.

Synanceia (Aploactis) aspera Rich. cf. Aploactis aspera Blkr.

Synanceia astroblepa Rich. 14, 4. 55, 419. 191, 2. 272, 12. cf. Leptosynanceia asteroblepa Blkr.

Synanceia bicapillata $C . V .14,4$. cf. Synanceia verrucosa Bl. Schn.

Synanceia brachio $C . V .14,9.47,226.54,237.91,165.157,232$.

188, 459. 238, 87. 275, 32. cf. Synanceia verrucosa Bl. Schn., Synanceichthys verrucosus Blkr.

Synanceia breviceps Rich. cf. Polycaulus elongatus Gthr.

Synanceia didactyla Bl. Schn. cf. Pelor didactylus Gthr.

Synanceia elongata $C . V .14$, Iо. 91, 36. 267, I39. 287, 49. 288, 72. cf. Polycaulus elongatus Gthr.

Synanceia erosa Langsd. 90, 28. 93, 5.

Synanceia erosa Lac. cf. Synanchia erosa Szens.

Synanceia grossa Gr. cf. Synanceia horrida Bl. Schn.

Synanceia horrida Bl. Schn., C. V. 14, 9. 25, 7. 54, 237. 91, 36. 215, 26 r. 235, 365. 270, 25. 275, 32. 287, 49. 354, I48. 358, г 89 . $364,286.395,292.425$, т41. 458 , I $2.459,87$.

Synanceia platyrhynchus Blkr. 458, I4.

Synanceia rubicunda Bl. Schn. cf. Pelor didactylus Gthr., Pelor obscurum $C . V$. 
Synanceia sanguinolenta Ehrenb. cf. Synanceia brachio $C$. $V$., Synanceia verrucosa $B l$. Schn.

Synanceia trachynis Rich. 119, 8. cf. Synanceia horrida Bl. Schn.

Synanceia uranoscopa $B l$. Schn. 91, $3^{6 .}$

Synanceia verrucosa $B l$. Schn. 287, 49. 309, I 55. 458, I5. 459, 87. 495, I6. cf. Synanceia brachio $C$. $V$.

Synanceichthys verrucosus Blkr. 315, 234. 320, 269. 358, I89. 364, 286. 395, 292. 396, 297. cf. Synanceia verrucosa Bl. Schn.

Synanchia erosa Szens. 497, I2.

Synancidium horridum J. Müll., Gthr. cf. Synanceia horrida Bl. Schn.

Synaphobranchus J. Yate Johns. 351, I I4.

Synaphobranchus aftinis Gïnth. 497, 25.

Synaphobranchus bathybius Giunth. 497, 25.

Synaptura aspilos Blkr. 51, 74. 61, 29. 287, 41.

Synaptura Commersoniana Cant. 51. 59. 91, 76.

Synaptura heterolepis Blkr. 142, 65. 320, 265. 358, I84. 364, 274.

Synaptura jerreus 91,76 .

Synaptura macrolepis Blkr. 191, 7. 272, 8.

Synaptura marmorata Blkr. 80, 90. 296, I03. cf. Brachirus heterolepis Blkr.

Synaptura microlepis Blkr. 333, 456 .

Synaptura ommatura 49, 42 I. 55, 4 I 4 .

Synaptura pan Cant. 52, 89. 61, 30.91, 76. 185, 226.287, 41. 356, I 72.

Synaptura pan Blkr. 50, 472. cf. Brachirus pan Szens.

Synaptura panoïdes Blkr. 49, 440. 55, 414. 61, 30. 270, 62. 272, 8 . 287, 41. 356, r72.

Synaptura pectoralis Kaup 333, 457 .

Synaptura potoo 91,76 .

Synaptura quagga Gthr. cf. Brachirus quagga $B l k r$.

Synaptura Russellii Blkr. 91, 76. 272, 8. 287, 41. 288, 69.

Synaptura swinhonis Steind. cf. Brachirus Swinhonis Blkr.

Synaptura zebra Cant. 51, 59. 91, 76. 199, 45. 356, I72. 395, 290. cf. Brachirus zebra Blkr.

Synbranchus cashia Szons. cf. Amphipnous cuchia J. Mïll.

Synbranchus cayennensis $K p$. cf. Synbranchus marmoratus $B$ l.

Synbranchus cuchia Cuv. cf. Amphipnous cuchia J. Mull.

Synbranchus eurychasma $K$. v. $H$. cf. Monopterus javanensis Lac., Monopterus javanicus Cuv.

Synbranchus laevis $M c C l$. cf. Monopterus javanensis Lac., Symbranchus marmoratus $B$ l.

Synbranchus marmoratus $B l .362,247$.

Synbranchus transversalis Bl. Schn. 302, I 7 .

Synbranchus unicolor $\mathrm{Val}$. cf. Synbranchus marmoratus $B l$.

Synbranchus xanthognathus Rich. cf. Monopterus javanensis Lac.

Synchiropus goramense Blkr. cf. Synchiropus goramensis Blkr. 
Synchiropus goramensis Blkr. 494, IоI.

Synchiropus ocellatus Gill cf. Callionymus ocellatus Pall.

Synchiropus opercularioides Gill cf. Eleutherochir opercularioides Blkr.

Synechoglanis Gill 306, 103.

Synechogobius hasta Gill 425, I29. 497, I9.

Syngnathoïdes Blochii Blkr. 47, 259. 51, 60. 54, 235. cf. Solegnathus

Blochii Blkr.

Syngnathus I, $55^{2}$.

Syngnathus acus $L .459,75$.

Syngnathus alternans $G t h r .459,75$.

Syngnathus Andersonii Blkr. 188, 465. 270, 72.

Syngnathus argus Pet. cf. Coelonotus argulus Peters.

Syngnathus biaculeatus $B l$. cf. Gastrotokeus biaculeatus Heck., Solegnathus Blochii Blkr., Syngnathoïdes Blochii Blkr.

Syngnathus bicoarctatus Blkr. 163, 99. 270, 72. 287, 40.

Syngnathus biserialis $K p$. cf. Syngnathus spicifer Rüpp.

Syngnathus boaja Blkr. 26, I6. 35, 262. 42, 60. 45, I97. 49, 421 .

55, 4I5. 89, I4. 178, 2. cf. Microphis boaja Blkr.

Syngnathus brachyrhynchus $K p .459,75$.

Syngnathus brachysoma Blkr. 125, 327. 157, 236. cf. Choeroichthys brachysoma Blkr.

Syngnathus brachyurus Blkr. 89, г6. 106, го5. 170, 476. cf. Doryichthys brachyurus Gthr., Microphis brachyurus Blkr.

Syngnathus budi Blkr. 143, 77. 270, 72. 275, r3.

Syngnathus carce Buch.91, I6r. 139, 430. cf. Ichthyocampus carce $K p$.

Syngnathus conspicillatus Jen. 459, 75. 491, 49. 495, I6.

Syngnathus Coquerelii $A$. Dum. 459, 75 .

Syngnathus cuncalus Buch. 91, I62. cf. Microphis cuncalus $K p$.

Syngnathus cyanospilos Blkr. 95, rr4. 270, 72.

Syngnathus dactyliophorus Blkr. 78, 506.89, I6. cf. Microphis dactyliophorus Blkr.

Syngnathus Delalandei $K p$. 266, 56 .

Syngnathus deocata Buch. 91, So.

Syngnathus deokhatoides Blkr. 89, I7. 106, I06. cf. Doryichthys deokhatoides $B l k r$.

Syngnathus djarong $B l k r .89,22.110,325.139,429.270,72$.

Syngnathus fasciatus $G r$. cf. Corythoichthys fasciatus $K p$., Syngnathus conspicillatus Jen., Syngnathus haematopterus Blkr.

Syngnathus fluviatilis $v$. Hass. 89, 18.

Syngnathus gastrotaenia Blkr. 69, 7 I3. 89, 22. 139, 430. 270, 72. 354, I 43. 358, I83. 364, 273. 382, 397. 459, 75. 491, 49.

Syngnathus haematopterus Blkr. 47, 258. 54, 235. 89, 20. 157, 236.

cf. Corythoichthys fasciatus $K p$., Syngnathus conspicillatus Jen.

Syngnathus Helfrichii Blkr. 139, 428, 270, 72. 272, 8. 287, 40.

Syngnathus heptagonus Blkr. 89, 23. 139, 430. 231, 15 r. 270, 72. 
Syngnathus heterosoma Blkr. 49, 44r. 55, 4r5. 89, r5. 108, r64. cf. Microphis heterosoma Blkr.

Syngnathus Hunnii Blkr. 270, 70, 72.

Syngnathus intermedius Gthr. 425, I26. cf. Trachyrhamphus intermedius $K p$.

Syngnathus Kaupi Blkr. 302, 24.

Syngnathus koilomatodon Blkr. 193, ro. cf. Halicampus koilomatodon Blkr.

Syngnathus leiaspis Blkr. 89, 20. 270, 72.

Syngnathus longirostris Gthr. 425, г 26.

Syngnathus manadensis Blkr. 143, 78. cf. Doryichthys manadensis Blkr.

Syngnathus melanopleura Blkr. 188, 464. cf. Doryramphus melanopleura Blkr.

Syngnathus mento Blkr. 143, 75. 270, 72. 275, 13. $_{3}$

Syngnathus pelagicus L., Osb., Blkr. 266, 56. 270, 72. 430, 233. $459,75.495, \mathrm{r} 7$.

Syngnathus phlegon Risso 266, 56 .

Syngnathus polyacanthus Blkr. 143, 77. cf. Microphis polyacanthus $B / k$ r.

Syngnathus Retzii Blkr. 143, 76. 270, 72. 275, I3.

Syngnathus Schlegeli $K p .425$, I26. 497, х 3 .

Syngnathus serratus $T$. Schl. 90, 55. 425, I26. cf. 'Trachyrhamphus serratus $K p$.

Syngnathus spicifer Rïpp. 16, I 5. 425, I26. cf. Syngnathus gastrotaenia Blkr.

Syngnathus spicifer $K p$. cf. Syngnathus Kaupi Blkr.

Syngnathus subbooko Blkr. 91, 80.

Syngnathus sundaicus Blkr. 89, 2 I.

Syngnathus tapeinosoma Blkr. 100, 376. 139, 429. 270, 72.

Syngnathus Temmincki $K p$. 266, 56 .

Syngnathus tenuirostris Schl. cf. Syngnathus Schlegeli $K p$.

Syngnathus tetragonus $L$. cf. Solegnathus Blochii Blkr.

Syngnathus tetrophthalmus Blkr. 188, 467. 270, 72. 397, 302.

Syngnathus zanzibarensis Gthr. 425, I26.

Synistius Gill 468, 285 .

Synochogobius Gill 453, 325 .

Synodontis Cuv. 306, 87 .

Synodontis arabi $\mathrm{Val}$. cf. Hemisynodontis schall Blkr.

Synodontis batensoda Rüpp. cf. Brachysynodontis batensoda Blkr.

Synodontis clarias Cuv. 304, 55. 306, 87 .

Synodontis maculosus Rüpp. cf. Leiosynodontis maculosus Blkr.

Synodontis membranaceus $I s$. Géoffr. cf. Hemisynodontis membranaceus Blkr.

Synodontis serratus Rüpp. cf. Pseudosynodontis serratus Blkr.

Synodus altipinnis Blkr. 425, 147 .

Synodus chinensis Lai. cf. Elops saurus $L$. 
Synodus fasciatus Lac. cf. Saurus synodus $C . V$.

Synodus macrocephalus Lac. cf. Luciobrama macrocephalus Blkr.

Synodus myops Blkr: 425, I47. 437, 39. 459, гог. 495, 21. 497, 24. Synodus synodus Blkr. 437, 39. 491, 55 .

Synodus synodus $B l$. Schn. 497, 24. cf. Saurus synodus $C$. $V$.

Synodus varius Blkr. 425, I 47. 459, ro2. 495, 2 r.

Synodus vulpes Lac. cf. Albula bananus $C$. $V$.

Sypterus Pallasii Eichze. cf. Temnodon saltator $C$. $V$.

Systomus $M c C l .261,43$ I. 314, I99. 329, 263.

Systomus (Barbodes) ablabes Blkr. 302, I6.

Systomus albuloïdes Blkr. 139, 425 .

Systomus (Barbodes) amblycephalus Blkr. 272, 22.

Systomus apogon $C . V$. 55, 428. 200, 48.

Systomus apogonides Blkr. 225, 358 .

Systomus apogonoïdes Blkr. 138, 410.

Systomus Belangeri Blkr. 91, 60.

Systomus (Barbodes) belinka Blkr. 270, 52.

Systomus bimaculatus $M c C l .91,60$.

Systomus (Systomus) bulu Blkr. 45, 207. 49, 420. 55, 413. 239, 102.

270, 53. 272, 23.

Systomus (Barbodes) Burchelli Blkr. 266, 55 .

Systomus (Barbodes) camptacanthus Blkr. cf. Puntius (Barbodes) camptacanthus Blkr.

Systomus canius $M c C l$. cf. Systomus gelius $M c C l$.

Systomus (Barbodes) carassioides Blkr. 272, 23.

Systomus chrysopterus $M_{C} \mathrm{Cl} .91,60$.

Systomus conchonius $\mathrm{MC} \mathrm{Cl} .91,60$.

Systomus (Barbodes) erythropterus Blkr. 272, 23.

Systomus (Barbodes) fasciatus Blkr. 235, 371. 270, 52. 272, 23.

Systomus gelius $M C C l .91$, I 29.

Systomus (Barbodes) gobionides Blkr. 266, 55 .

Systomus (Barbodes) goniosoma Blkr. 270, 52 .

Systomus (Barbodes) Huguenini Blkr. 270, 53.

Systomus immaculatus $M C C l .91,62$.

Systomus janthochir Blkr. 85, 448.

Systomus (Barbodes) javanicus Blkr. 270, 53.

Systomus (Barbodes) lateristriga Blkr. 235, 37 I. 270, 53. 272, 23. 287, 59 .

Systomus lawak Blkr. 138, 4I I.

Systomus (Capoëta) leiacanthus Blkr. 279, Ior.

Systomus leptosomus $M c C l$. cf. Puntius (Puntius) phutunio Buch., Systomus phutunio $C . V$.

Systomus (Barbodes) maculatus Blkr. 235, 37 I. 270, 53. 272, 23. 284, 240. 287, 59 .

Systomus malacopterus $\mathrm{Mc} C l$. cf. Leuciscus cosuatis $C . V$.

Systomus (Barbodes) marginatus Blkr. 270, 53. 
Systomus melanopterus Blkr. 49, 420. 55, 4r3. 85, 449.

Systomus microlepis Blkr. 42, 6o. 45, I96. 49, 420. 55, 4r3.

Systomus Ogilbii Blkr. 91, 62 .

Systomus (Capoeta) oligolepis Blkr. 270, 53.

Systomus (Capoeta) padangensis Blkr. 270, 53.

Systomus (Barbodes) pallidus Blkr. 266, $5^{6}$.

Systomus pangut Blkr. 91, 62 .

Systomus pausio C. V. 91, 62 .

Systomus pausius 91,62 .

Systomus phutunio $C . V$. 91, I28. cf. Puntius (Puntius) phutunio Buch.

Systomus puntio Blkr. 91, 62 .

Systomus pyrrhopterus $M C C l .91,62$.

Systomus (Barbodes) rubripinnis Blkr. 239, I02.

Systomus (Barbodes) Schwanefeldi Blkr. 270, 53. 272, 23.

Systomus sophore $M_{C} C l$. 91, I27.

Systomus (Capoeta) sumatrensis Blkr. 270, 53.

Systomus terio C. $V .91,62$.

Systomus (Barbodes) tetrazona Blkr. 272, 23.

Systomus tictis $C . V .91,62$.

Systomus ticto $M c C l .91, \mathrm{r} 28$.

Systomus titius C. $V .91,62$.

Systomus (Barbodes) trispilos Blkr. 302, I6.

Systomus truncatus Blkr. 42, 60. 45, I96. 49, 420. 55, 413.

Systomus Vigorsii Blkr. 91, 62.

Systomus Waandersi Blkr. 225, $35^{8}$.

Tachysurus chinensis Lac. cf. Arius (Pseudarius) falcarius Blkr.

Taenianotus Lac. 473, 299.

Taenianotus latovittatus Lac. cf. Amblyapistus taenianotus Blkr., Ma-

lacanthus taeniatus $C . V$.

Taenianotus triacanthus Lac. 128, 4II. 473, 299. 475, 82.

Taenioïdes Lac. 453, 330 .

Taenioïdes anguillaris Blkr. 491, 54.

Taenioïdes coecula cf. Amblyopus Hermannianus $C . V$.

Taenioïdes Hermannianus Lac. 453, 330.

Taeniö̈des Hermannii Lac. cf. Amblyopus Hermannianus C. $V$.

Taeniolabrus filamentosus Stcind. cf. Trichonotus setifer Bl. Schn.

Taeniophis $K p .351$, I 21 .

Taeniotoca $A g .468,290$.

Taeniura lymma $M$. H. 10, 68. 46, 85. 51, 85. 64, 78. 91, 8z. 270,

67.275 , Iо. $288,67.310$, r6o. $315,229.320,264.358,183$.

364, 27 I. 396, 296. 491, 4 ०.

Taeniura melanospilos Blkr. 78, 513.

Taeniura Meyeni M. H. 459, 69. 495, 8.

Taeniurus lymma M. H. 156, 2 I8. 
Tambra Blkr. 261, 430. 314, 199.

Taractes Loner 468, 300.

Taractes asper Lonve 468, 300.

Taurichthyiformes 468, 302 .

Taurichthyini 468, 303 .

Taurichthys C. $V .468,304$.

Taurichthys chrysostomus Blkr. 474, 3 I 5. 481, 45. 491, 44.

Taurichthys macrolepidotus Blkr. 459, 96. 474, 315. 481, 47. 491, 44. 495, 1 $4.497,8$.

Taurichthys monoceros Blkr. 459, 96. 474, 3I5. 481, 44. 495, I4.

Taurichthys varius $C . V .22,25.47,227.157,233.275,35.468$, 304. 474, 3I 5. 481, 42 .

Taurichthys viridus $C . V$. cf. Taurichthys varius $C . V$.

Tautoga Mitch. 292, 108.

Tautoga fasciata $C . V .78,484.91,52,156,216.185,225$. cf. Hemigymnus fasciatus Guich.

Tautoga inscripta Rich. 119, I3.

Tautoga laticlavia Rich. 119, I3.

Tautoga leucomos Blkr. 185, 239.

Tautoga luculenta Rich. 119, I3.

Tautoga melanopterus C. V. 184, 203. 188, 460. 275, 19. cf. Hemigymnus melanopterus Gïnth.

Tautoga melapterus $C . V .11,15,16.12,48.51,58.119,13.126$, 344. 156, 216. 157, 234. 270, 37. cf. Hemigymnus melanopterus Gthr.

Tautoga notophthalmus Blkr. 143, 53. 170, 479. 184, 203. 275, I9.

Tautoga parila Rich. 119, I3.

Tautoga psittacula Rich. 119, I3.

Tautoga tetrica Rich. 119, I3.

Telescops Blkr. 468, 26 I.

Telestes Bp. 314, 212. 329, 264.

Tellia Gerv. 261, 439.

Temera Hardwickii Gray 51, 60. 91, 82. 263, 451. 288, 67.

Temnodon 1, 553 .

Temnodon inornatus $K . v$. $H$. cf. Stromateus niger $B l$.

Temnodon javanicus $K . v . H .57,74$. cf. Psenes javanicus $C$. $V$.

Temnodon saltator $C . V .266,64$. cf. Cheilodipterus saltator Blkr.

Tephraeops Gthr. 468, 295 .

Tephraeops Richardsoni Gthr. 468, 296.

Tephrinectes sinensis Gthr. 425, г30.

Tetrabranchus microphthalmus Blkr. 42, 69. 45, 197. 49, 42I. 88, 58 . cf. Symbranchus immaculatus $B l$.

Tetrades xanthosoma $B l k r$. cf. Tetradrachmum reticulatum 'Blkr.

Tetradrachmum arcuatum Cant., Blkr. 394, 286. 396, 299. 425, I35. $437,38.485,147.491,47.495$, I 5 . 
Tetradrachmum aruanum Cant., Blkt. 315, 23r. 316, 24t. 318, 250. $320,267.354$, I 45.358 , I $85.364,278.369,42.388,272.459$, 85. cf. Tetradrachmum arcuatum Cant.

'Tetradrachmum marginatum Day cf. Tetradrachmum reticulatum Blkr. 'Tetradrachmum melanurus Blkr. 315, 23I. 316, 24I. 318, 250. 354, I 45.358, I $85.364,278.388,272.485$, I $49.491,47$.

Tetradrachmum polyacanthus Blkr. 364, 278. cf. Acanthochromis polyacanthus Gill.

Tetradrachmum reticulatum Blkr. 425, I35. 483, 41. 485, I 45. 491, $47.495, x 5$.

Tetradrachmum trimaculatum Blkr. 354, I45. 358, I86. 364, 278. $369,42.459,85.483,4$ I. 485, I $44.491,47.495$, I 5.

Tetradrachmum xanthosoma Blkr. 354, I 45. 364, 278.425, I35.459, 85. cf. Tetradrachmum reticulatum Blkr.

Tetradrachmum xanthurus Blkr. 318, 250. 358, I86. 364, 278 . cf. Pomacentrus (Pomacentrus) violascens Blkr.

Tetragonopterus (Tetragonopterus) aureus Blkr. 497, 8.

Tetragonopterus auriga Blkr. 315, 234. 318, 251. 320, 269. 354, I48. 358, 189. 364, 286. 391, 276. cf. Tetragonopterus setifer Blkr.

Tetragonopterus baronessa Blkr. 315, 234. 316, 242. 318, 251. 320, 269. 354. I48. 358, I89. 364, $286.394,287$.

Tetragonopterus Bennetti Blkr. 364, 286.

Tetragonopterus biocellatus Blkr. 320, 269. 354, I48.

Tetragonopterus chrysozonus Blkr. 364, 286.

'Tetragonopterus citrinellus Blkr. 315, 234. 354, I48. 358, IS9. 364, 286. 397, 302 .

Tetragonopterus ephippium Swns., Blkr. 315, 234. 318, 251. 320, 270. 358, I89. 364, 286. 369, 42 .

Tetragonopterus falcula $B / \mathrm{kr} .315,234.364,286$.

Tetragonopterus fasciatus $B l k i .315,234.320,270.358$, I $89.364,286$.

Tetragonopterus Kleini Bl. Schn。 394, 287.

Tetragonopterus lineolatus Blkr. 318, $25 \mathrm{I}$.

Tetragonopterus melanopus Blkr. 358, I89. 364, 286.

'Tetragonopterus melanotus Blkr. 315, 234. 318, 251. 320, 269. 358, I $89.364,286$.

Tetragonopterus melastomus Bl. Schn., Blkr. 315, 234. 318, 252.320, 270. 354, I48. 358, 189. 364, 286.

Tetragonopterus Meyeri Blkr. 315, 234. 340, 365. 358, x So. 364, 2S6.

Tetragonopterus nesogallicus $B l k r .315,234.354$, I 48.358 , I 8 9. $364,286$.

Tetragonopterus octofasciatus Blkr. 358, I89. 364, 286.

Tetragonopterus oligacanthus $B l k r .315,234.346,3$ r. 354, r.4S. 358, I 89. 364, 286. 395, 292.

Tetragonopterus ornatissimus Blkr. 340, 365. 364, 286 .

'Tetragonopterus oxycephalus Blkr. 315, 234.

INDEX BLEEKER. 
Tetragonopterus polylepis Blkr. 320, 270. 364, 286.

Tetragonopterus punctatofasciatus Blkr. 316, 242. 354, I48. 364, 286. $394,287$.

Tetragonopterus Rafflesi Blkr. 315, 234. 320, 270. 358, 189. 364, 286. 369, 42 .

Tetragonopterus selene Blkr. 364, 286. 394, 287 .

Tetragonopterus setifer Blkr. 394, 287 .

Tetragonopterus speculum Blkr. 358, IS9. 364, 286.

Tetragonopterus strigangulus Blkr. 316, 242. 320, 270. 358, I89. 364, 286.

Tetragonopterus ulietensis Blkr. 315, 234. 364, 287.

Tetragonopterus unimaculatus Blkr. 315, 234. 320, 270. 354, 148 . 358, I89. 364, 287. 394, 288.

Tetragonopterus vagabundus Blkr. 315, 234. 320, 270. 354, I 48 . $358,189.364,287$.

Tetragonopterus vittatus Blkr. 315, 234. 316, 242. 318, 252. 320, 270.354, I $48.358,189.364,287.369,42.394,288$.

Tetragonopterus xanthurus Blkr. 364, 287 .

Tetragonopterus Klein 468, 306.

Tetragonopterus (Chaetodontops?) amplexicollis. 474, 3I8.

Tetragonoptrus ataeniatus 474,3 r 8.

Tetragonoptrus aureus Blkr. 425, I 40.

Tetragonoptrus (Chaetodontops) aureus Blkr. 474, 3i 8 .

Tetragonoptrus auriga Blkr. 425, I40. 459, 95. cf. Tetragonoptrus (Linophora) auriga Blkr.

Tetragonoptrus (Linophora) auriga Blkr. 474, 319. 481, 92. 491, 45 . 495, I 3. 497, 9.

Tetragonoptrus baronessa Blkr. cf. Tetragonoptrus (Gonochaetodon) triangulum Blkr.

Tetragonoptrus Bennetti Blkr. cf. Tetragonoptrus (Rabdophorus) Bennetti. Blkr.

Tetragonoptrus (Rabdophorus) Bennetti Blkr. 474, 316. 481, 6 o.

Tetragonoptrus bimaculatus Blkr. 459, 95 .

Tetragonoptrus biocellatus Blkr. 459, 95. cf. Tetragonoptrus (Chaetodontops) fasciatus Blkr.

Tetragonoptrus Blackburni Blkr. 459, 95.

Tetragonoptrus (Citharoedus) Blackburni Blkr. 474, 3 I6.

Tetragonoptrus (Rabdophorus) Blackburni Blkr. 495, I3.

Tetragonoptrus (Hemichaetodon) capistratus Blkr. 474, 3 I 8.

Tetragonoptrus chrysozonus Blkr. 425, I40. cf. Coradion chrysozonus $K p$.

Tetragonoptrus chrysurus Blkr. 459, 95. cf. Tetragonoptrus (Linophora) Mertensi Blkr.

Tetragonoptrus citrinellus Blkr. cf. Tetragonoptrus (Tetragonoptrus) miliaris Blkr. 
Tetragonoptrus collaris Blkr. 425, I fo. cf. 'Tetragunoptrus (Chaetodontops) collaris Blkr.

Tetragonoptrus (Chaetodontops) collaris Blkr. 474, 318. 481, 8 o.

Tetragonoptrus (Chaetodontops) Dayi Blkr.474, 3 r9.

Tetragonoptrus (Tetragonoptrus) dichrous Blkr. 474, 3 I 7.

Tetragonoptrus dizoster Blkr. 459, 95. cf. Tetragonoptrus (Oxychaetodon) falcula $B l k r$.

Tetragonoptrus dorsalis Blkr. 459, 95. cf. Tetragonoptrus (Chaetodontops) melanotus Blkr.

Tetragonoptrus ephippium Blkr. cf. Tetragonoptrus (Rabdophorus) ephippium Blkr.

Tetragonoptrus (Rabdophorus) ephippium Blkr. 474, 316. 481, 65 . 491,44 .

Tetragonoptrus falcula Blkr. cf. Tetragonoptrus (Oxychaetodon) falcula Blkr.

Tetragonoptrus (Oxychaetodon) falcula Blkr. 474, 320. 481, I04. 491, 45. 495, I 3 .

Tetragonoptrus fasciatus $B l k r .425$, I40. 459, 95. cf. Tetragonoptrus (Chaetodontops) fasciatus Blkr.

Tetragonoptrus (Chaetodontops) fasciatus Blkr. 474, 319. 481, 77. 491, 44. 495, I3.

Tetragonoptrus festivus Blkr. 459, 95. cf. Coradion merlangus Blkr.

Tetragonoptrus (Tetragonoptrus) flavirostris Blkr. 474, 3I 7.

Tetragonoptrus (Rabdophorus) Fremblii Blkr. 474, 3і6.

Tetragonoptrus ('Tetragonoptrus) humeralis Blkr. 474, 3 I 7 .

Tetragonoptrus Kleini Blkr. 425, I40. 459, 95. cf. 'l'etragonoptrus (Lepidochaetodon) Kleini Blkr.

Tetragonoptrus (Chaetodontops) Kleini Blkr. 474, 3 I9.

Tetragonoptrus (Lepiclochactodon) Kleini Blkr. 481, SS.491, +5. 495, 13.

Tetragonoptrus Klunzingeri Blkr. 474, 3 r8.

Tetragonoptrus Layardi 474, 3 I 8.

Tetragonoptrus (Rabdophorus) leucopleura Blkr. 474, 3 I6.

Tetragonoptrus lineolatus Blkr. 459, 95. cf. Tetragonoptrus (Oxy. chaetodon) lineolatus Blkr.

Tetragonoptrus (Oxychaetodon) lineolatus Blkr. 474, 320.481, I02. 495, I3.

Tetragonoptrus (Rabdophorus) luctuosus Blkr. 474, 3 I 7 .

Tetragonoptrus lunula Blkr. 459, 95. cf. Tetragonoptrus (Chaetodontops) fasciatus Blkr.

Tetragonoptrus lunulatus 474,3 I8.

Tetragonoptrus maculatus Blkr. 459, 95.

Tetragonoptrus (Tetragonoptrus) maculocinctus Blkr. 474, 3 I 7 .

'letragonoptrus (Chaetodontops) melanopoma Blkr. 474, 3 I9.

Tetragonoptrus melanopterus Blkr. 459, 95.

Tetragonoptrus (Rabdophorus) melanopterus Blkr. 474, 3r7. 
Tetragonoptrus melanopus Blkr. cf. Coradion melanopus Blkr.

Tetragonoptrus melanotus Blkr. cf. Tetragonoptrus (Chaetodontops) melanotus Blkr.

Tetragonoptrus (Chaetodontops) melanotus Blkr. 474, 319. 481, 82. 495, I 3 .

Tetragonoptrus melastomus Blkr. cf. Tetragonoptrus (Lepidochaetodon) Kleini Blkr.

Tetragonoptrus (Linophora) Mertensi Blkr. 474, 319. 495, г3.

Tetragonoptrus (Oxychaetodon) mesoleucus Blkr. 474, 320.

Tetragonoptrus Meyeri Blkr. cf. Tetragonoptrus (Citharoedus) Meyeri Blkr.

Tetragonoptrus (Citharoedus) Meyeri Blkr. 474, 3I6. 481, 55.491, 44.

Tetragonoptrus (Tetragonoptrus) miliaris Blkr. 474, 318. 481, 72. 495, 14.

Tetragonoptrus mitratus Blkr. 459, 95 .

Tetragonoptrus (Tetragonoptrus) mitratus Blkr. 474, 3r7. 495, 13.

Tetragonoptrus modestus Blkr. 425, I 40.

Tetragonoptrus (Rabdophorus) modestus Blkr. 497, 9.

Tetragonoptrus (Tetragonoptrus) modestus Blkr. 474, 3r7.

Tetragonoptrus nesogallicus Blkr. 425, I40. 459, 95. cf. 'Tetragonoptrus (Linophora) auriga Blkr.

Tetragonoptrus (Oxychaetodon) nigripinnis Blkr. 474, 320.

Tetragonoptrus (Chaetodontops) nigrirostris Blkr. 474, 318.

Tetragonoptrus (Chaetodontops) ocellatus Blkr. 474, $3 \mathrm{r} 8$.

Tetragonoptrus (Oxychaetodon) ocellicauda Blkr. 474, 320.

Tetragonoptrus octofasciatus Blkr. cf. Tetragonoptrus (Tetragonoptrus) octofasciatus Blkr.

Tetragonoptrus (Tetragonoptrus) octofasciatus Blkr. 474, 318.481, $70,491,44$.

Tetragonoptrus oligacanthus Blkr. 396, 300. cf. Parachaetodon ocellatus Blkr.

Tetragonoptrus ornatissimus Blkr. cf. Tetragonoptrus (Citharoedus) ornatissimus Blkr.

Tetragonoptrus (Citharoedus) ornatissimus Blkr. 474, 3I6. 481, 57. 491,44 .

Tetragonoptrus osseus 474,3 I 8 .

Tetragonoptrus oxycephalus Blkr. cf. Tetragonoptrus (Oxychaetodon) lineolatus Blkr.

Tetragonoptrus (Chaetodontops) pelewensis Blkr. 474, 3 I 8.

Tetragonoptrus (Rabdophorus) plebejus Blkr. 474, 3 I 7.

Tetragonoptrus polylepis Blkr. cf. Hemitaurichthys polylepis Blkr.

Tetragonoptrus (Chaetodontops) pulcher Blkr. 474, 319. 495, I3.

Tetragonoptrus (Tetragonoptrus) punctato-fasciatus Blkr. 474, 318. 481. 74. 491, 44.

Tetragonoptrus (Tetragonoptrus) quadrimaculatus Blkr. 474, 3 I 7 . 
Tetragonoptrus Rafflesi Blkr. 396, 300.437, 39. cf. Tetragonoptrus (Linophora) Rafflesi Blkr.

Tetragonoptrus (Linophora) Rafflesi Blkr. 474, 319. 481, 97. 491, 45. 'Tetragonoptrus (Tetragonoptrus) robustus Blkr. 425, I40. 474, 3 I 7 .

Tetragonoptrus (Tetragonoptrus) sanctae Helenae Blkr. 474, 3 I 7 .

Tetragonoptrus (Tetragonoptrus) sedentarius Blkr. 474, 3 I 7 .

Tetragonoptrus selene Blkr. cf. Tetragonoptrus (Chaetodontops) selene Blkr.

Tetragonoptrus (Chaetodontops) selene Blkr.474, 3r8.481, 84. 491, 45 .

Tetragonoptrus (Rabdophorus) semeion Blkr. 474, 3I6. 481, 67.

'Tetragonoptrus (Oxychaetodon) semilarvatus Blkr. 474, 320.

Tetragonoptrus speculum Blkr. cf. Tetragonoptrus (Rabdophorus) speculum Blkr.

Tetragonoptrus (Rapdophorus) speculum Blkr. 474, 3I6. 481, 62.

Tetragonoptrus (Tetragonoptrus) striatus Blkr. 474, 3 I 7 .

Tetragonoptrus strigangulus Bl., Blkr. 459, 95. cf. Megaprotodon strigangulus $B l k r$.

Tetragonoptrus (Rabdophorus) tau-nigrum Blkr. 474, 3 I 7 .

Tetragonoptrus (Gonochaetodon) triangulum Blkr.474, 320.481 , ro7. 491,45 .

Tetragonoptrus (Tetragonoptrus) trichrous Blkr.474, 3 7 .

Tetragonoptrus (Rabdophorus) trifasciatus Blkr. 474, 3i6. 481, 63 . 491, 44. 495, 13.

Tetragonoptrus ulietensis Blkr. cf. Tetragonoptrus (Oxychaetodon) falcula Blkr.

Tetragonoptrus unimaculatus Blkr. 459, 95. cf. Tetragonoptrus (Lepidochaetodon) unimaculatus Blkr.

Tetragonoptrus (Lepidochaetodon) unimaculatus Blkr. 474, 319. 481, 87. 491, 45. 495, I3.

Tetragonoptrus vagabundus Blkr. 437, 39. 459, 95. cf. Tetragonoptrus (Linophora) vagabundus Blkr.

Tetragonoptrus (Linophora) vagabundus Blkr. 474, 319. 481, 94. $491,45.495, \mathrm{r} 3$.

Tetragonoptrus vittatus Bl., Blkr. 396, 300. 437, 39. 459, 95. cf. Tetragonoptrus (Rabdophorus) trifasciatus Blkr.

Tetragonoptrus zanzibarensis Blkr. 459, 95. cf. Tetragonoptrus (Rabdophorus) speculum Blkr.

Tetragonoptrus zoster Blkr. 459, 95. cf. Hemitaurichthys zoster Blkr.

Tetragonoptrus (Tetragonoptrus) xanthocephalus Blkr.474, 3 I 7 .

Tetragonoptrus xanthurus Blkr. cf. Tetragonoptrus (Linophora) xanthurus Blkr.

Tetragonoptrus (Linophora) xanthurus Blkr. 474, 319. 481, 99.

Tetranematichthys Blkr. 306, ro8.

Tetranematichthys quadrifilis Blkr. 306, roS.

Tetraödon L. 1, 552. 2, 506. 367, IS. 
Tetraödon alboplumbeus Rich. 91, r6r. 368, 35. 425, I2 1. 497, 27.

Tetraödon argenteus Lac. 70, 737. 91, 78. 364, 27 1. 368, 35. 398, 306. cf. Tetraödon sceleratus $L$. Gm.

Tetraödon argenteus S. Mïll. 10, 68, 46, 2 I4.

Tetraödon aspilos Blkr. 50, 495. 62, 22. 91, 78 .

Tetraödon astrotaenia Blkr. 72, I29.

Tetraödon atratus Kich. cf. Tetraödon Honckenii $B l$.

Tetraödon bicolor Brev. cf. Tetraödon sceleratus Forst., L. Gm.

Tetraödon bimaculatus Rich. cf. Gastrophysus xanthopterus Blkr.,

Tetraödon ocellatus Osb., Rich.

Tetraödon bondarus Cant. 91, 78 .

Tetraödon brunneus Bre7! 497, 27.

Tetraödon calamara Russ., Cuv. 62, I 5. 91, 78.

Tetraödon calamaroides Blkr. 30, 96. 62, г6.

Tetraödon Commersonii Bl. Schn. cf. Tetraödon calamara Russ.

Tetraödon curvus Stor. cf. Gastrophysus laevigatus Blkr.

Tetraödon cutcutia Buch. 91, I60.

Tetraödon erythrotaenia Blkr. 81, I 74 .

Tetraodon fasciatus $M c C l$. cf. Gastrophysus xanthopterus Blkr., Tetraödon ocellatus Osb., Rich.

Tetraödon firmamentum $T$. Schl. cf. Arothron firmamentum Blkr., Crayracion firmamentum Blkr.

Tetraödon fluviatilis Buch. 16, г6. 91, 78.

Tetraödon grammatocephalus Schl. cf. Canthogaster grammatocephalus Blkr.

Tetraödon grammatocephalus $T$. Schl. 103, 503. cf. Tropidichthys striolatus Blkr.

Tetraödon Hamiltonii Rich. 54, $30 \mathrm{r}$.

Tetraödon Honckenii Bl. 10, 68. 46, 2 I 4. 91, I66. 368, 34.425, I 2 I.

Tetraödon hypselogeneion Blkr. 54, 300. 62, 24. 364, 272. 368, 34 .

Tetraödon immaculatus Lac. 91, 78.

Tetraödon immaculatus Blkr. cf. Crayracion immaculatus Blkr.

Tetraödon inermis Schl. 497, 27.

Tetraödon kappa Russ. 54, 301. 62, I6. 91, г60. 339, 362.

Tetraödon Kunhardtii Blkr. 30, 97. 50, 472. 51, 79. 62, 17, 23. 132, I I 2 .

Tetraödon lagocephalus $L .425$, I2 I.

Tetraödon laterna Rich. 54, 299. 62, 23. 320, 265. cf. Crayracion implutus Blkr.

Tetraödon leiopleura $G r$. cf. Tetraödon lunaris $C \iota v$.

Tetraödon leiurus Blkr. 30, 97. 55, 440. 62, 18, 22.

Tetraödon lineatus $T$. Schl. cf. Arothron lineatus Bl., Crayracion lineatus Blkr.

Tetraödon lunaris Bl. Schn., Cuv. 51, 60. 55, 4г5. 56, 446. 62, г2. 91, 78. 346, 31. 356, I72. 364, 272. 368, 35. 425, I 2 I. 497, 27. 
Tetraödon maculatus Lac. cf. Tetraödon calamara Russ.

Tetraödon mappa Less. 318, 249. cf. Crayracion mappa Blkr.

Tetraödon margaritatus Rüpp. 54, 302. 62, 25. cf. Canthogaster margaritatus Blkr., Crayracion margaritatus Blkr., Tropidichthys margaritatus $B l k r$.

Tetraödon melanorhynchus Blkr. 320, 265.

Tetraödon meleagris Sol. 80, 9I. cf. Crayracion meleagris Blkr.

Tetraödon modestus Blkr. 45, I97. 49, 42 I. 55, 440. 62, I9, 2 I.

Tetraödon (Arothron) modestus Blkr. 26, I6. 35, 262. 42, 60.

Tetraödon naritus Rich. 33, I62. 35, 262. 42, 60. 45, х97. 49, 42I.

55, 439. 62, 21. cf. Chonerhinus naritus Blkr.

Tetraödon niveatus Brcv. cf. Tetraödon oblongus $B l$.

Tetraödon oblongus $B l .50,472.55,4$ I 5. 56, 446. 62, г2. 91, I66. $368,35.395,290.425$, I 2 I. $497,27$.

Tetraödon ocellatus Osb., Rich., Gthr. 425, г 2т. 496, 4. 497, 27.

Tetraödon pachycephalus Ranz. cf. Gastrophysus laevigatus Blkr.

Tetraödon palembangensis Blkr. 62, 25.67, 605 .

Tetraödon papua Buch., Blkr. 9, 638. 54, 302. 62, г3. 91, 78. cf. Gastrophysus alboplumbeus $B l k r$., Tropidichthys margaritatus Blkr.

Tetraödon pardalis Schl. 497, 27.

Tetraödon patoca Buch.62, I I.

Tetraödon poecilonotus Schl. cf. Tetraödon oblongus Bl.

Tetraödon porphyrens Schl. 497, 27.

Tetraödon potamophilus Blkr. 16, I6. 25, I2. 26, 3. 45, I97. 49, 42 I. 55, 4I5. 62, I7.

Tetraödon (Chelonodon) potamophilus Blkr. 26, 3. 35, 262. 42. 60.

Tetraödon psittacus $B l$. Schn. 91, г66.

Tetraödon reticulatus $B l k r .16$, I6. 25, เ 2.62, r 8 .

Tetraödon Richei Fréminv. 368, 35.

Tetraödon rivulatus Schl. cf. Canthogaster rivulatus Blkr.

Tetraödon rostratus Bl. Schn. 54, 302. 91, I66.

Tetraödon rubripes $T$. Schl. 425, 121. 497, 27. cf. Gastrophysus rubripes Blkr.

Tetraödon scaber Eyd. \& Soul. cf. Crayracion immaculatus Blkr.

Tetraödon sceleratus Forst., L. Gm. 425, I2r. 437, 36. 497, 27.

Tetraödon simulans Cant. 51, 60.

Tetraödon spadiceus Rich. 364, 272. 368, 35. 425, x2I.

'Tetraödon Spengleri Bl. Schn.91, I66.

Tetraödon stictonotus T. Schl.497, 27. cf. Gastrophysus stictonotus Blkr.

Tetraödon striolatus Q. G. cf. Tropidichthys striolatus Blkr.

Tetraödon testudineus $B l .51,78.54,299.62$, I 4 .

Tetraödon trichoderma Blkr. 86, 532.

Tetraödon trichodermatoides Blkr. 99, 336. 318, 249. 320, 265.

Tetraödon Valentini Blkr. 72, I34.

Tetraödon Valentyni Blkr. cf. Canthogaster Valentyni Blkr. 
Tetraödon vermicularis $T$. Schl. cf. Gastrophysus vermicularis Blkr.

Tetraödon vermiculatus Schl. 497, 27.

Tetraödon virgatus Rich. 54, 299. 62, 24. 315, 229.

Tetraödon Waandersii Blkr. 82, I94.

Tetraödon xanthopterus $T$. Schl. cf. Gastrophysus xanthopterus Blkr.

Tetraödon xanthopterus Schl. cf. Tetraödon rubripes Schl.

Tetrapturus indicus C. $V$. 270, 28.

Tetraroge amblycephaloides Blkr. 354, I48. 364, 286.

Tetraroge amblycephalus Gthr. cf. Gymnapistus niger Blkr.

Tetraroge barbata Blkr. 364, 286.

Tetraroge barbata Gthr. cf. Gymnapistus barbatus Swons.

Tetraroge binotata Gthr. cf. Amblyapistus taenianotus Blkr.

Tetraroge crista-galli Gthr. cf. Amblyapistus taenianotus Blkr., Prosopodasys (Tetraroge) crista-galli Blkr.

Tetraroge dermacanthus Gthr. cf. Cocotropus dermacanthus Blkrr.

Tetraroge echinata Gthr. cf. Cocotropus echinatus $K p$.

'Tetraroge longispinis Blkr. 364, 286.

Tetraroge longispinis Gthr. cf. Paracentropogon longispinis Blkr.

Tetraroge macracanthus Gthr. 358, I89. cf. Amblyapistus macracanthus Blkr.

Tetraroge taenianotus Blkr. 364, 286.

Tetraroge taenianotus Gthr. 315, 234. 358, I89. cf. Amblyapistus taenianotus Gthr., Blkr.

Tetrodon albo-plumbeus Rich. cf. Gastrophysus alboplumbeus Blkr., Tetraödon patoca Buch.

Tetrodon argenteus Lac. cf. Tetrodon sceleratus $L$. Gm.

Tetrodon Basilewskianus Basil. cf. Crayracion immaculatus Blkr.

Tetrodon Bernieri $K p$. (Stenometopus) 459, 69.

Tetrodon calamara Rïpp. cf. Crayracion stellatus Blkr.

Tetrodon cinctus Sol. 62, r3. cf. Tetraödon papua Blkr., Tropidichthys margaritatus Blkr.

Tetrodon dissutidens Cant. cf. 'Tetraödon kappa_Russ.

Tetrodon hispidus Lac. cf. Crayracion hispidus Blkr.

Tetrodon Honckenii Bl. 495, 23. cf. Gastrophysus Honckenii Blkr. Tetrodon hypselogeneion Blkr. 459, 69.

Tetrodon immaculatus Lac. cf. Crayracion immaculatus Blkr.

Tetrodon insignitus Rich. cf. Canthogaster margaritatus Blkr.

Tetrodon laevigatus $L$. Gm. cf. Gastrophysus laevigatus Blkr.

Tetrodon lagocephalus $L .459,69.495,23$.

Tetrodon lineatus $B l$. cf. Arothron lineatus $B l k r$., Crayracion lineatus Blkr.

T'etrodon mathematicus Mitch. cf. Gastrophysus laevigatus Blkr.

Tetrodon nigropunctatus $B l$. Schn. cf. Crayracion nigropunctatus Blkr.

Tetrodon oblongus $B l .459,69$. 
Tetrodon ocellatus Bcnn. cf. Tetraödon papua Blkr., Tropidichthys Bennetti Blkr.

Tetrodon pantherinus Eyd. Soul. cf. Crayracion stellatus Blkr.

'Tetrodon patoca Buch. cf. Tetraödon kappa Russ.

Tetrodon Richei Fremenv. cf. Gastrophysus Richei Blkr.

Tetrodon sceleratus L. Gm. 459, 69 .

Tetrodon Solandri Rich. 62, I3. cf. Tetraödon papua Blkr., Tropidichthys margaritatus Blkr.

Tetrodon sordidus Rüpp. cf. Crayracion immaculatus Blkr.

Tetrodon spadiceus Rich. cf. Tetraödon lunaris Cuv.

Tetrosomus Stons. 367, I5.

Tetrosomus turritus Sivns. 354, I43. cf. Ostracion turritus Forsk.

Teuthis Abhortani Gthr. 459, 96. 495, I 9.

'Teuthis albopunctata Gthr., Blkr. 425, I34. 497, I6.

Teuthis aurantiaca Blkr. 497, I6.

Teuthis australis J. Gr. 105, 43. cf. Acanthurus velifer $B l$.

Teuthis concatenata Cant., Gthr. 354, 149. 364, 287.

Teuthis corallina Gthr. 364, 287. 394, 288. 459, 96.

Teuthis doliata Cant., Günth. 296, ro8. 315, 235. 320, 270. 354, I 49. 358, 190. 491, 5 I.

Teuthis dorsalis Cant. 296, Iо8. 315, 235. 320, 270. 358, 190. 364, 287. cf. Amphacanthus dorsalis C. $V$.

Teuthis fuscescens Gthr., Blkr. 346, 31. 459, 96. 491, 51. 497, 16.

Teuthis guttata Gthr. 315, 235. 316, 242. 354, I 49. 358, I90. 364, 288. 395, 292.

Teuthis hepatus $L$. cf. Acanthurus hepatus Bl. Schn.

Teuthis hexagonata Blkr. 364, 288.

Teuthis java L., Gthr. 364, 288. 395, 292.

Teuthis javus L., Cant. 346, 3I. cf. Amphacanthus javus C. $V$.

Teuthis Kopsi Blkr., Gthr. 364, 288. 395, 292. 491, 5 I.

Teuthis labyrinthodes $B l k \%$. 390, 275.

Teuthis lineata Giüth. 491, 51.

Teuthis lurida Gthr. 459, 96.

Teuthis margaritifera Gthr. 315, 235. 320, 270. 358, I 90. 364, 288. 425, I $34.459,96$.

Teuthis marmorata Blkr., Gthr. 315, 235. 320, 270. 354, I49. 358, I90. 364, 288. 369, 42. 391, 276. 437, 37. 491, 5I.

Teuthis nebulosa Gthr. 320, 270. 459, 96. 495, 19.

Teuthis puella Gthr. 309, I56. 315, 235. 318, 252. 358, r9o. 364, 288. 437,37 .

Teuthis sutor Gthr. 459, $9^{6 .}$

Teuthis tumifrons Gthr. 356, I74.

Teuthis vermiculata Gthr. 364, 288. 396, 300.459, 96. 491, $5^{\text {I. }}$. 495, I9.

Teuthis vermiculatus Cant. 308, I52. 320, 270. 
Teuthis virgata Gthr. 395, 292. 425, I34. 491, 51. 496, 2.

Teuthis vulpina Gthr. 315, 235. 364, 288. 491, 5 I.

Thaerodontis $M C C l .88,40.351, \mathrm{I} 2 \mathrm{I}$.

Thaerodontis ophis $M_{c} C l$. cf. Echidna variegata J. R. Forst.

Thaerodontis reticulata $M c C l .88,4 \mathrm{I}$. cf. Muraena Mac Clellandi Blkr.

Therapaina $K p .468,298$.

Therapaina strigata $K p .425$, I40. 468, 298.

Therapon C. V. 1, 553. 2, 524. 468, $267,33^{6}$.

Therapon angulatus Guich.459, 89.

Therapon argenteus Gthr. cf. Therapon (Datnia) argenteus Blkr.

Therapon (Datnia) argenteus Blkr. 435, 382.

Therapon brevispinis Pet. cf. Therapon (Datnia) brevispinis Blkr.

Therapon (Datnia) brevispinis Blkr. 435, 384 .

Therapon Bouzetianus Hombr. Jacq. cf. Therapon (Datnia) jarbua Blkr.

Therapon cancellatus Gthr. cf. Therapon (Datnia) cancellatus Blkr.

Therapon (Datnia) cancellatus Blkr. 435, 385 .

Therapon cinereus $C . V$. cf. 'Therapon (Datnia) theraps Blkr.

Therapon Cuvieri Blkr. 98, 2 I I. 119, 6. 156, 21 3. 275, 24. 287, 45. 296, Iо6. 315, 232. 316, 24I. 320, 268. 354, I 46. 364, 280. 395, 291. cf. Therapon (Pelates) quadrilineatus Blkt.

Therapon (Datnia) farna Blkr. 495, I I.

'Therapon ghebul (Ehr.) C. V. 13, 5I. 50, 47 I. cf. Therapon (Datnia) trivittatus Blkr.

Therapon jarbua Blkr.459, 89. 468, 267 .

Therapon jarbua Klunz. cf. Therapon (Datnia) jarbua Blkr.

Therapon (Datnia) jarbua Blkr. 435, 377. 491, 42.

Therapon (Datnia) micracanthus Blkr. 435, 388.

Therapon obscurus $C . V$. 2, 526. 13, 51. 288, 7 I. cf. Therapon (Datnia) theraps Blkr.

Therapon obtusirostris Guich.459, 89.

Therapon oxyrhynchus $T$. Schl. 118, 64. 425, 139. cf. Therapon (Datnia) oxyrhynchus Blkr.

Therapon (Datnia) oxyrhynchus Blkr. 497, 7 .

Therapon (Datnia) plumbeus Blkr. 435, $3^{8} 3$.

Therapon puta $C . V .2,526.13,50.51,55.56,444.91,32.275$, 24. cf. Therapon trivittatus Cant., Therapon (Datnia) trivittatus Blkr.

Therapon quadrilineatus $C . V .2,526.13$, Io, 51. 50, 47 I. 235, 364 . 270, I8. 275, 24 395, 29I. 425, I39. cf. Therapon (Pelates) quadrilineatus Blkr.

Therapon (Pelates) quadrilineatus $B l k r .435,389.437,38.497,7$.

Therapon (Datnia) Rosenbergii Blkr. 435, 387 .

Therapon rubricatus Rich. 119, 6. cf. Therapon (Datnia) theraps Blkr.

Therapon servus $C . V .2,526.9,632.10,66.13,49.16,4.25,7$. 46, 2гі. 53, г6о. 54, 234. 55, 4гі. 91, 32. 108, т64. 119, 6. 126, 344. 156, 213. 157, 232. 184, І 99. 185, 222. 194, 26. 200, 
48. 208, 239. 231, I 45. 234, 352. 235, 364. 236, 330. 270, I8. 272, 1о. 275, 24. 287, 45. 288, 71. 296, го6. 315, 232. 316, 241. 320, 268. 356, I 73. 358, I86. 364, 280. 425, 139. 468, 267. cf. Therapon (Datnia) farna Blkr., Therapon jarbua Blkr., 'Therarapon (Datnia) jarbua Blkr.

Therapon sexlineatus $Q . G$. cf. Helotes sexlineatus $C$. $V$., Therapon (Helotes) sexlineatus Blkr.

Therapon sexlineatus Stcind. cf. Therapon (Pelates) quadrilineatus Blkr. Therapon (Helotes) sexlineatus Blkr. 435, 392. 437, 38 .

Therapon squalidus $C . V$. cf. Therapon (Datnia) theraps Blkr.

Therapon theraps C. $V .2,526.13,50.16,4.25,7.46,211.51$, 55. 54, 234. 56, 444. 91, 32. 119, 6. 127, 345. 170, 478, 479. 191, I. 222, 316. 227, 407. 231, 145. 237, 436. 270, 18. 272, го. 275, 24. 288, 7x. 296, Iо6. 316, 241. 354, I4. 364, 280. 356, I 73. 394, 287. 425, I39. cf. Therapon (Datnia) theraps Blkr.

Therapon (Datnia) theraps Blkr. 435, 379. 491, 42.

Therapon timoriensis Q.G. cf. Therapon (Datnia) jarbua Blkr.

Therapon transversus C. V. 91, 32. cf. Therapon (Datnia) theraps Blkr. Therapon trivittatus Cant. 395, 29 . cf. Therapon (Datnia) jarbua Blkr., Therapon puta $C . V$.

Therapon trivittatus Gthr. cf. Therapon (Datnia) trivittatus Blkr.

Therapon (Datnia) trivittatus Blkr. 435, 375 .

Therapon unicolor var. Kner cf. Moronopsis rupestris Gill.

Therapon virgatus Gthr. cf. Therapon (Datnia) theraps Blkr.

Therapon xanthurus $C$. $V$. cf. Therapon quadrilineatus $C . V$., Therapon (Pelates) quadrilineatus Blkr.

Tholichthys Gthr. 468, $3 \circ 5$.

Tholichthys osseus Gthr., Day cf. Tetragonoptrus (Chaetodontops) Dayi Blkr., Tetragonoptrus osseus.

Threpterius Rich.468, 3 I 5 .

Threpterius maculosus Rich. 119, 9.

Thrissa Hamiltonii Gray cf. Engraulis Grayi Blkr.

Thryssa 2, 509.

Thryssa macrognathos Blkr. 16, I 4. 25, r2. cf. Engraulis setirostris $C . V$. Thryssa mystax Rich. cf. Engraulis poorawah Blkr.

Thryssa porava Blkr. 16, 14. 25, 12. cf. Engraulis mystacoïdes Blkr. 'Thryssa setirostris Cur'. cf. Engraulis setirostris $C . V$.

'Thynnichthys Blkr. 261, 433. 314, 201.

Thynnichthys polylepis Blkr. 270, 54. 272, 24.

Thynnichthys thynnoides Blkr. 270, 54. 272, 24.

Thynnus 1, 553 .

Thynnus affinis Cant. 288, 74 .

Thynnus macropterus T. Schl. 57, 37. 296, I09. cf. Pelamys macropterus Blkr.

Thynnus orientalis Schl. cf. Pelamys japonicus Blkr. 
Thynnis pelamys $C . V$. 142, 4I. 266, 53. 270, 29. 296, I09. cf. Pelamys pelamys Blkr.

Thynnus sibi Schl. cf. Pelamys sibi Blkr.

Thynnus thunnina $C . V .57,36.270,29.275,38.287,52.296$, I09. 356, I73. 459, I00. cf. Pelamys thunninus Blkr.

Thynnus thynnus White 459 , 100.

Thynnus tonggol Blkr. 57, 89. 287, 52. 371, 356.

Thynnus vagans Less. cf. Thynnus pelamys $C$. $V$.

Thynnus vulgaris Cuv. cf. Thynnus thynnus White.

Thyrsites atun $C . V$. 119, Iо. 266, 68.

Thyrsites lepidopoïdes $C . V$. 142, 43.

Thyrsites macrophthalmus 142, 43 .

Thyrsites prometheoïdes Blkr. 142, 42, 167, 372.

Thyrsites prometheus 142, 43.

Thyrsoidea $K p$. 351, I 21 .

Thyrsoidea arenata $K p$. cf. Gymnothorax thyrsoideus $B l k r$.

Thyrsoidea aterrima $K p$. cf. Gymnothorax aterrimus Blkr.

Thyrsoidea Blochii $K p$. cf. Gymnothorax borneensis Blkr.

Thyrsoidea Boschii $K p$. cf. Gymnothorax Boschi Blkr.

Thyrsoidea bullata $K p$. cf. Gymnothorax bullatus $B l k r$.

Thyrsoidea cancellata $K p$. cf. Gymnothorax cancellatus Blkr.

Thyrsoidea ceramensis $K p$. cf. Gymnothorax ceramensis Blkr.

Thyrsoidea chlorostigma $K p$. cf. Gymnothorax chlorostigma Blkr., Gymnothorax meleagris Blkr., Muraena chlorostigma Blkr.

Thyrsoidea colubrina $K p$. cf. Gymnothorax reticularis $B l$.

Thyrsoidea griseobadius $K p$. cf. Gymnothorax griseobadius $B l k r$.

Thyrsoidea isingleena $K p$. cf. Gymnothorax isingteena $B l k r$.

Thyrsoidea isingleenoides $K p$. cf. Gymnothorax isingleenoides Blkr.

Thyrsoidea longissima $K p$. cf. Thyrsoidea macrurus Blkr.

Thyrsoidea macrurus Blkr. 352, 135.

Thyrsoidea maculipinnis $K p$. cf. Gymnothorax maculaepinnis $B / k r$.

'Thyrsoidea meleagris $K p$. cf. Gymnothorax meleagris $B l k r$.

Thyrsoidea multifasciata $K p$. cf. Gymnothorax ceramensis Blkr.

Thyrsoidea prosopeion $K p$. cf. Gymnothorax prosopeion Blkr.

Thyrsoidea sathete $K p$. cf. Strophidon sathete Blkr.

Thyrsoidea tenelata $K p$. cf. Gymnothorax tenalatus Blkr.

Thyrsoidea tile $K p$. cf. Gymnothorax tile Blkr.

Tiaroga Gir. 261, 434. 314, 209.

Tigoma Gir. 261, 436. 314, 213. 329, 264.

Tilapia madagascariensis Blkr. 459, 84 .

Tilapia mossambica Blkr. 459, 84. 495, I5.

Tilapia nilotica Blkr. 459, 84 .

Tilapia oligacanthus Blkr. 399, 309. 459, I I, 84 .

Tilapia Sparmanni Smith 266, 54.

Tinca Rond. 261, 434. 314, 208. 
'Tinca marina Bont. cf. Notopterus kapirat Blkr.

Tor Hamiltonii Gray cf. Labeobarbus tor Blkr.

'Torpedo 1, $55^{2}$.

Torpedo (Astrape) japonica T. Schl. cf. Astrape japonica T. Schl.

Torpedo fuscomaculata Pet. cf. Narcacion fuscamaculatus Blkr.

Torpedo marmorata Rud. cf. Narcacion marmoratus Blkr.

Toxoteoidei 468, 3 I I.

Toxotes Cuv. 1, 553. 468, 3II.

Toxotes chatareus Blkr. 471, 160.

Toxotes jaculator C. V. 1, 553. 2, 52 I. 9, 633. 10, 66. 22, 31. 25,

8. 35,26 1. $42,59.45$, т95. 46, 212. 49, 419. 51, 57. 54, 235. 55, 412. 91, 42. 156, 2 I 4. 157, 233. 167, 37 I. 170, 479. 185, 223. 191,2 . $235,364.270,22.272,12.275,34.287,50.288$, 73. 296, го8. 315, 234. 320, 269. 354, т 48. 356, I 73. 358, 189. $364,285.369,42.395,292.468,3$ II. 471, I64. 491, 44. cf. Toxotes chatareus Blkr., Toxotes oligolepis Blkr.

Toxotes jaculator, var. malaccensis Cant. cf. Toxotes chatareus Blkr. Toxotes microlepis Gthr. 356, I 73. 471, I $5^{8 .}$

Toxotes oligolepis Blkr. 471, 162 .

Toxotes squamosus Hutton 468 , 3 I I.

'Trachelyopterichthys Blkr.300, 402, 306, III.

Trachelyopterichthys taeniatus Blkr. 306, ri

Trachelyopterus $\mathrm{Val} .300,402.306$, I Ir.

Trachelyopterus coriaceus $\mathrm{Val} .306$, I I I.

Trachelyopterus taeniatus Kner cf. Trachelyopterichthys taeniatus Blkr.

Trachicephalus elongatus Szons. cf. Polycaulus elongatus Gthr.

Trachichthys australis Shaw, Cv. 119, 6.

Trachinops Gthr. 468, 32 I.

Trachinops taeniatus Gthr. 468, 322.

Trachinotus 1, 553. cf. 'Trachynotus.

Trachinotus affinis $C . V .2,5$ 16. cf. Trachinotus mookalee $C . V$.

Trachinotus anomalus T. Schl. cf. Psenes anomalus Blkr., Psenopsis anomalus Gill.

Trachinotus auratus Rich. cf. Trachynotus ovatus Gthr.

Trachinotus Baillonii C. V. 54, 237. 57, 46. 147, 469. 156, 2 I 4. 170, 479. 231, 146. 271, 2. 275, 38. 276, 65. 288, 74 .

Trachinotus Blochii C. V. 287, 52. 288, 74. cf. Trachinotus mookalee C. $V$.

Trachinotus drepanis $C . V$. cf. Trachinotus mookalee $C . V$.

Trachinotus falciger $C . V$. cf. Trachinotus mookalee $C . V$.

Trachinotus melo Rich. cf. Psenes anomalus Blkr.

Trachinotus mookalee C. $V .2$, 516. 25, S. 55, 412. 56, 445. 57, 47.

$91,44.185,223.188,459.191,2.270,28.272,13.275,38$.

Trachinotus oblongus $C . V .2,5$ I6. 91, 44 .

Trachinotus quadripunctatus $C$. $V$. cf. 'Trachinotus Baillonii $C . V$. 
Trachinotus Russellii $C . V .91,44$.

Trachinus araneus $C . V .291$, II 7 .

Trachinus armatus Schl. 291, I I 7. 302, 94.

Trachinus draco $L .291$, II 7 .

Trachinus draco Gthr. cf. Trachinus armatus Schl.

Trachinus radiatus $C$. $V$. cf. Pseudotrachinus radiatus $B l k r$.

Trachinus vipera Cuv. cf. Echiichthys vipera Blkr.

Trachurus Rotleri $C . V$. 2, 5 I7.

Trachurus trachurus $C . V .90,35$.

Trachybrama Heck. 314, 2 ro.

Trachycorystes Blkr. 306, 88.

Trachycorystes galeatus Blkr. cf. Parauchenipterus galeatus $B l k r$.

Trachycorystes typus Blkr. 306, 88.

Trachynotus Baillonii $C$. $V$. 296, I09. 316, 242. 338, 300. 356, I 74 . 358, I91. 364, 289. 459, 98. 495, I 8.

Trachynotus Blochii $C$. $V$. cf. Trachynotus ovatus Gthr.

Trachynotus drepanis $C$. $V$. cf. Trachynotus ovatus Gthr.

Trachynotus gorëensis $C . V$. 302, 77 .

Trachynotus maxillosus $C . V .302,78$.

Trachynotus ovatus Gthr. 316, 242. 364, 289. 425, I32. 459, 98.

Trachynotus quadripunctatus $C . V$. cf. Trachynotus Bailioni $C . V$.

Trachypoma Gthr. 468, 254.

Trachypoma macracanthus Gthr. 468, 254.

Trachypterus semiophorus Blkr. 387, 279.

Trachyrhamphus intermedius $K p .497$, I3. cf. Syngnathus intermedius Gthr.

Trachyrhamphus longirostris $K p$. cf. Syngnathus longirostris Gthr.

Trachyrhamphus serratus $K p$. 270, 7 I. 497, I3. cf. Syngnathus serratus Schl.

Triacanthodes Blkr. 175, 37. 367, 9.

Triacanthodes anomalus $B / k r .175,37.497$, I 7.

Triacanthus Cuv. 2, 506. 367, 9.

Triacanthus anomalus $T$. Schl. cf. Triacanthodes anomalus Blkr.

Triacanthus biaculeatus Bl., Blkr. 10, 68. 46, 2 16. 91, 166. 287, 38 . $288,68$.

Triacanthus biaculeatus Cuv., Benn. 16, 6. 25, I2. 42, 60. 425, I 2 1. cf. Triacanthus Russellii Blkr.

Triacanthus Blochii Blkr. 51, 8r. 368, 20.

Triacanthus brachysoma Blkr. 72, х2S. 235, 374. 270, 65. 275, 10. 296, I02. 364, 273 .

Triacanthus brevirostris Schl. 368, 20.

Triacanthus brevirostris $V$ al. 170, 480. 235, 374. 270, 65. 272, 7 . 275 , 10. $287,38.296$, го2. 354, г 43. 364, 273. 393, 282. 425, I22. $491,52.497$, I 7. cf. Triacanthus Russellii Blkr.

Triacanthus longirostris Hollard cf. Triacanthus strigilifer Cant. 
Triacanthus macrurus Blkr. 368, 2 1. 371, 5 r.

Triacanthus Nieuhofii Blkr. 50, 472. 55, 415. 56, 459. 63, 26. 156, 2 I8. 270, 65. 272, 7. 287, 38. 296, Іо2. 354, 143. 358, I83. 364, 273. 368, 2 I. 382, 397. 395, 290.425, I22.

Triacanthus oxycephaius Blkr. 50, 496. 63, 27. 227, 408. 235, 374. $270,65.275$, II. 287, 38. 364, 273. 368, 20. 395, 290.

Triacanthus rhodopterus Blkr. 63, 25. 281, 108. 287, 39. 356, I 72. 393,282 . cf. Triacanthus brevirostris $\mathrm{Val}$.

Triacanthus Russellii Blkr. 45, I97. 46, 214, 216. 49, 42 I. 51, 60. 55,4 I $5.63,25.91,80.119,17$.

Triacanthus strigilifer Cant. 163, 97. 287, 39. 288, 68. 368, 20.425, 1 22.

Triaenodon obesus $M . H .91,80.459,67.495,8$.

Triaenodon Smithii $M . H .266,57$.

Triaenophorichthyini 453, 312.

Triaenophorichthys Gill 453, 3 I2.

Triaenophorichthys barbatus Gthr. 425, x29. 453, 3 I2.

Triaenophorichthys trigonocephalus Gill 425, I29. 453, 3 I 2.

Triaenophorus Gill 453, 312.

Triaenopogon Blkr. 453, 3 I2.

Triakis scyllium M. H. 497, 3 .

Trichidion Guichenoti Blkr. 356, I74.

Trichidion indicum $B l k r .395,293.396,300.495$, I7.

Trichidion kuru Blkr. 309, 156. 315, 235. 316, 242. 338, 360. 364, 290. 396,300 .

Trichidion lineatus Blkr. 364, 29r.

Trichidion microstoma Blkr. 358, I91. 364, 291. 491, 64.

Trichidion multifilis $B l k r .356, \mathrm{r} 74$.

Trichidion multiradiatum Blkr. 425, 143 .

Trichidion paradiseus Blkr. 459, 78, 495, I7.

Trichidion plebejus $B l k r .296$, I Iо. 320, 27 I. 356, I74. 364, 29 I. 459, 79. 495, 17. 497, I 7.

Trichidion quadrifilis Blkr. 302, 88.

Trichidion sexfilis Blkr. 459, 79. 495, I7.

Trichidion sextarium Blkr. 356, I 74. 425, I 43 .

Trichidion xanthonema Blkr. 425, I43.

Trichiurus L. 1, 553. 272, 29.

Trichiurus argenteus Shaw cf. Enchelyopus lepturus Blkr.

Trichiurus armatus $G r$. cf. Enchelyopus savala Blkr., Trichiurus savala $C . V$.

Trichiurus glossodon Blkr. 272, 38. 287, 52 .

Trichiurus haumela $C . V .2,5$ I6. 16, 4. 25, 8. 46, 212. 50, 47 . 51, 57. 55, 412. 57, 41. 91, 42. 127, 345. 156, 214. 191, 2. 227, 407. 235, 366. 270, 29. 272, 36. 275, 38. 288, 74. cf. Enchelyopus haumela $B l k r$.

Trichiurus intermedius $G r$, Rich. cf. Enchelyopus muticus Blkr. 
Trichiurus japonicus $T$. Schl., Blkr. 118, 98. 272, 34. 496, 2.497, I5. Trichiurus lajor Blkr. 109, 248. 272, 33. 275, 38.

Trichiurus lepturus $L$. cf. Enchelyopus lepturus $B l k r$.

Trichiurus lepturus Basil. cf. Enchelyopus savala Blkr.

Trichiurus lepturus Lac., Russ. cf. Trichiurus haumela $C . V$.

Trichiurus lepturus japonicus T. Schl. cf. Trichiurus japonicus T. Schl., Blkr.

Trichiurus muticus Gr., Rich. cf. Enchelyopus muticus Blkr.

Trichiurus Roelandti Blkr. 272, 35 .

Trichiurus savala $C . V$. 32, I6o. 50, 47 I. 51, 57. 56, 445. 57, 4r. $91,42.178,2.222,3$ I7. 231, I 47. 237, 437. 270, 29, 288, 74.

Trichiurus savala Cuv. 272, 32. cf. Enchelyopus savala Blkr., Lepturus savala Blkr.

Trichiurus savala $V a l$. cf. Trichiurus haumela $C u v$.

Trichoderma hystrix Szens. cf. Monacanthus (Amanses) hystrix Burt. Trichodiodon Blkr. 367, I8.

Trichogaster fasciatus $B l$. Schn. cf. Colisa vulgaris $C . V$.

Trichogaster trichopterus Bl. cf. Trichopodus trichopterus Lac., Trichopus trichopterus Lac.

Trichomycterus $V a l .306$, I 2 .

Trichomycterus inermis Guich. cf. Nematogenys inermis Gir.

Trichomycterus nigricans $V a l .306$, I 12.

Trichonotus polyophthalmus Blkr. 83, 243. cf. Trichonotus setiger Bl. Schn.

Trichonotus setifer Bl. Schn. 430, 233.

Trichonotus setiger $B l$. Schn. 109, 25 1. 231, I48. 275, 45. 340, 365. 358, 192. 364, 293.

Trichonotus setigerus $B l$. Schn. cf. Trichonotus setiger Bl. Schn.

Trichopleura $K p .473,299$.

Trichopleura mollis Gthr. 425, I4I.

Trichopodus bejeus Buch. cf. Colisa vulgaris $C$. $V$.

Trichopodus colisa Buch. cf. Colisa vulgaris $C . V$.

Trichopodus cotra Buch. cf. Colisa vulgaris C. $V$.

Trichopodus lalius Buch. cf. Colisa vulgaris $C . V$.

Trichopodus mentum Lac. 21, Iо. cf. Osphromenus olfax Comm.

Trichopodus trichopterus Lac. 498, 2 I. cf. Trichopus trichopterus Lac.

Trichopsis striata Kner cf. Ctenops vittatus Blkr.

Trichopterus Gron. 468, 3I5.

Trichopus gorami Shaw cf. Osphromenus olfax Comm.

Trichopus goramy satyrus Shaw cf. Osphromenus olfax Comm.

Trichopus Leerii Blkr. 67, 577. 270, 34. 272, Io. cf. Trichopodus trichopterus Lac.

'Trichopus maculatus Szons. cf. Trichopodus trichopterus Lac.

Trichopus Pallasii Shaze cf. Trichopodus trichopterus Lac., Trichopus trichopterus Lac. 
Trichopus satyrus Shaw 2, 519. cf. Osphromenus olfax Comm.

Trichopus sepat Blkr. 2, 520. cf. Trichopodus trichopterus Lac., Trichopus trichopterus $L a c$.

Trichopus striatus $B l ., B l k r .2,520.21$, I . 26, 3. 31, то6. 35, 26 т. 42, 59. 45, I95. 49, 4I9. 55, 4II. 170, 476, 477. 239, гог. 270, 34. 272, Iо. cf. Ctenops vittatus Blkr.

Trichopus trichopterus Lac. 21, Io.

Trichopus trichopterus $C . V .2,520.16,4.25,8.26,3.35,26$ I. 42, 59. 45, I95. 49, 4I9. 55, 4II. 170, 476, 477. 191, 2. 225, 357. 231, 146. 235, 368. 239, гог. 255, 241. 270, 34. 272, го. 279, 100. 288, 70. cf. Trichopodus trichopterus Lac.

Trichosomus Szens. 473, 298.

Trichosomus trachinoides Szens. cf. Prosopodasys trachinoides Cant.

Tridentiger Gill 453, 3 I3.

Tridentiger obscurus Gill 497, I9.

Trigla Art. 468, 334 .

Trigla Brandesii Blkr. 27, 24. 47, 226.

Trigla Bürgeri $T$. Schl. 118, 73. 253, 235. cf. Lepidotrigla Bürgeri Gthr.

Trigla capensis $C . V .266,53$.

Trigla hemisticta Schl. 425, I26. 497, I3.

Trigla kumu Less. 118, 74. 119, 7. 266, 53. 425, г27.497, г3.

Trigla papillionacea Park cf. Trigla kumu Less.

Trigla Peronii C. $V .266,64$.

Trigla pleuracantha Rich. 119, 7 .

Trigla polyommata Rich. 119, 7 .

Trigla rubicunda Hornst. cf. Pelor obscurum $C$. $V$., Pelor didactylus Gthr.

Trigla spinosa $M c C l$. cf. Trigla kuma Less.

Trigla vanessa Rich. 119, 7 .

Trigon Forskaolii Rüpp. cf. Hypolophus sephen M. H.

Triloburus Gill 468, 336.

Trinematichthys Blkr. 261, 433. 314, 204.

Triodon Rzudt. 367, I9.

Triodon bursarius Rudt. 47, 228. 62, 20. 274, 2. 368, 40. 369, 42. 459, 70. 495, 23 .

Triodon macropterus Less. cf. Triodon bursarius Rzudt.

Tripterodon Playf. 468, 297.

Tripterodon orbis Playf. 468, 297.

Tripterygion capito Jen. 119, r2.

Tripterygion elegans Peters 495, 2 I.

Tripterygion fenestratum $C v .119,12$.

Tripterygion nigripenne $C v, 119,12$.

Tripterygion trigloides Blkr. 185, 234.

Tripterygion varium $C v .119,12$. 
Tristius brasiliensis Gill, Gïnth. 495, $8^{1}$ ).

Trochocopus opercularis Gthr. 459, 82. 495, 9.

Tropidichthys Bennetti Blkr. 103, 504. cf. Anosmius Bennetti Blkr.

Tropidichthys janthinopterus Blkr. 128, 429.

Tropidichthys margaritatus Blkr. 103, 501. 126, 344.

Tropidichthys striolatus Blkr. 103, 503.

Tropidinius Gill 468, 276.

Trulla Cantori $K p .288,69$.

Trulla grandisquamis $K p .288,69$.

'Trygon 1, 552.

'Trygon akajei $M . H .175,44$. cf. Leiobatis akajei Blkr., Leiobatis (Trygon) pastinaca Blkr.

'Trygon Bennetti M.H. 307, I37.

Trygon carnea Rich. cf. Leiobatis (Trygon) Bennetti Blkr., Tıygon Bennetti $M$. $H$.

Trygon chindrakee Cuथ. 91, 82.

Trygon dadong Blkr. 144, 355. cf. Leiobatus dadong Blkr.

Trygon Gerrardi Gr. cf. Leiobatis Gerrardi Blkr.

Trygon Halgani Less. cf. Taeniura lymma $M$. $H$.

Trygon heterurus Blkr. 64, 67. 287, 37.

Trygon imbricata $M . H .16,6.25$, 13. 51, 6o. 91, 82. 288, 67.

Trygon Kuhli $M . H .25$, I3. 64, 73. 91, 82. 275, Iо. 287, 37. cf. Leiobatus Kuhli Blkr.

Trygon kunsua Cuv. cf. Pteroplatea micrurus $M$. $H$.

Trygon lymma Cuv. 2, 507. cf. Taeniura lymma $M$. $H$.

Trygon macrura Blkr. 25, I3.

Trygon macrurus Blkr. 64, 74. 67, 607. 270, 67. 287, 37. 305, 73. cf. Leiobatis Gerrardi Blkr.

'Trygon nuda Gïnth., cf. Leiobatis (Trygon) nuda Blkr.

Trygon ornatum $G r$. cf. Taeniura lymma $M$. $H$.

'Trygon pareh Blkr. 64, 71. 85, 461. 272, 6.

Trygon pastinaca $B p .266,58$. cf. Leiobatis (Trygon) pastinaca $B l k r$.

Trygon pastinacoïdes Blkr. 64, 75 .

Trygon poecilurus Benn. cf. Pteroplatea micrurus $M$. $H$.

Trygon polylepis Blkr. 64, 73. 287, 38. cf. Leiobatis polylepis Blkr. Trygon purpurea Smith 266, 58.

Trygon sephen Cuv. cf. Hypolophus sephen $M$. $H$.

Trygon uarnak Rïpp. 51, 60. 70, 738.|266, 58. 288, 67. 391, 276. cf. Leiobatis uarnak Blkr.

Trygon uarnak M. H. 64, 69. 91, 82. cf. Leiobatis (Himantura) uarnak Blkr.

Trygon uarnakoides Blkr. 25, I3. 64, 72. 70, 738 .

Trygon undulata Blkr. 25, 13. 64, 70. 121, 167. 272, 6.

I) Iristius brasiliensis Gill is a misprint by Bleeker for Isistins brasiliensis Gill. 
Trygon variegatus $M C C l .91,82$.

'Trygon walga $M$. $H .25$, x 3. 64, 67.91, 82. 267, x39. 287, 38.

Trygon zugei Bürg. 56, 446. 64, 68. 91, 82. 287, 38. 288, 67.

Trygon zugei M. H. 227, 409. cf. Cynocephalus (Scoliodon) macro-

rhynchus Blkr., Leiobatus zugei Blkr., Leiobatis (Trygon) zugei Blkr.

Trygonobatus ephippiatus Gr. 119, I9.

Trygonoptera testacea $M . H .119,19$.

Trygonorhina fasciata $M . H .119$, I9.

Trypauchen Val. 453, 33 .

Trypauchen chinensis Steind. 430. 233.

'Trypauchen microcephalus Blkr. 272, 62.

Trypauchen vagina $C . V$. 15, 37. 16, 5. 17, 3. 25, ro. 55, 41 2. 91, 50. $191,2.231,148.270,42.272,6$ o. $275,45.287,57.288$, 77. 358 , I $92.395,293.425$, г $29.453,330$.

Trypauchen vagina Stons. cf. Trypauchen vagina $C . V$.

Trypauchenichthys Blkr. 272, 63. 453, 33 r.

Trypauchenichthys typus Blkr. 272, 63. 453, 33r.

Trypauchenini 453, 330 .

Tylognathus Heck. 261, 423. 314, 194.

Tylognathus sinensis Kner cf. Pseudogobio rivularis $B l k r$.

Tylometopon Van Bemm. 468, 299.

Tylometopon Dussumieri Blkr. 425, I33.

Tyntlastes Gthr. 453, 329 .

Typhlus Goudotii Bibr. cf. Coelonotus liaspis Gthr.

Umbrina Cuv. 468, 326 .

Umbrina amblycephalus Blkr. 128, 412. 270, 23. cf. Sciaena Dussumieri Blkr.

Umbrina capensis Pappe 266. 52.

Umbrina Dussumieri $C . V .2,523.18$, I9. 25, 9. 91, 36. 231, I 45. 270, 23. 281, 108. 287, 50. cf. Sciaena Dussumieri Blkr.

Umbrina Kuhlii $C . V .2,523.10,66.16,4.18,19.25,8.46,212$. 227, 407. 235, 364. 275, 33. 287, 50. cf. Sciaena Kuhli Blkr., Sciaena Russelli Blkr.

Umbrina macropterus Blkr. 76, 254. 156, 2 14. 270, 23. cf. Sciaena macropterus Blkr.

Umbrina Russelli $C . V .51,56.91,36.288,73.425$, I 42. cf. Sciaena Dussumieri Blkr., Sciaena Russelli Blkr.

Unibranchapertura 88, 56 .

Unibranchapertura cuchia Buch. cf. Amphipnous cuchia $J$. Mïll.

Unibranchapertura immaculata Lac. cf. Synbranchus marmoratus Bl. Unibranchapertura laevis Lac. 88, 59. cf. Monopterus javenensis Lac., Synbranchus marmoratus $B l$.

Unibranchapertura marmorata Lac. cf. Synbranchus marmoratus Bl. Unibranchapterura laevis Lac. cf. Monopterus javanicus Cuv. 
Upeneichthys Blkr. 468, 333 .

Upeneichthys chrysopleuron Blkr. 497, ro.

Upeneichthys porosus Blkr. 119, 7. 468, 333 .

Upeneoïdes Blkr. 468, 333 .

Upeneoïdes bensasi Blkr. 90, 4. 118, 7I. 253, 235. cf. Upeneus bensasi Rich.

Upeneoïdes bivittatus Blkr. 13, 64. 16, 4. 25, 7. 53, х60. 54, 235. $56,444.91,34.128,4$ I I. cf. Upeneus sulphureus $C . V$., Upeneoïdes vittatus Blkr.

Upeneoïdes bivittatus $D a y$ cf. Upeneus vittatus $C . V$.

Upeneoïdes chrysopleuron Blkr. cf. Upeneus chrysopleuron $B l k r$.

Upeneoïdes fasciolatus Day cf. Upeneus moluccensis Blkr.

Ujeneoïdes moluccensis Blkr. 128, 409. 237, 436. 275, 33. cf. Upeneus moluccensis Blkr.

Upeneoïdes subvittatus Blkr. cf. Upeneus subvittatus Blkr.

Upeneoïdes sulphureus Blkr. 13, 63. 16, 4. 25, 7. 51, 56. 163, 45 . 227, 407. 231, I46. 235, 365. 237, 437. 270, 22. 275, 33. cf. Upeneus sulphureus $C$. $V$., Upeneoïdes sundaicus $B l k r$.

Upeneoïdes sundaicus Blkr. 128, 4I г. 156, 2 І3. 163, 47. 235, 365 . 270, 22. 275, 33. 287, 50. cf. Upeneus sundaicus Blkr.

Upeneoïdes taeniopterus Blkr. 91, 34.

Upeneoïdes tragula Gthr. 308, I52. cf. Upeneus tragula Rich.

Upeneoïdes variegatus Blkr. 13, 64. 46, 212.47, 226. 50, 471. 51, 56. 54, 235. 56, 444. 126, 344. 156, 2 г 3. 158, 273. 163, 48. 184, I99. 185, 223. 275, 33. 285, 245. cf. Upeneus tragula Rich.

Upeneoïdes vittatus Blkr. 13, 63. 16, 4. 25, 7. 46, 212. 51, 56. 56, 444. 91, 34. 127, 345. 128, 4 I I. 147, 469. 156, 2 г3. 163, 42. 167, 37 I. 170, 479. 231, I46. 234, 353. 236, 330. 237, 437. 270, 23. 275, 33. 296, го8. 309, г55. 468, 333. cf. Upeneus vittatus Cuv.

Upeneoides Vlamingii Blkr. 13, I2.

Upeneus $C . V .1,553.2,524,527.468,333$.

Upeneus barberinoides Blkr. 54, 263. 275, 33. cf. Parupeneus barberinoides Blkr.

Upeneus barberinus C. V. 44, I 72. 47, 226. 54, 237. 156, 213. 188, 459. 228, 424. 231, I 46. 270, 22. 275, 33. 285, 245. 309, I 55 . 4.68, 334. cf. Parupeneus barberinus Blkr.

Upeneus bensasi Rich., Schl. 425, 142. 497, 9.

Upeneus biaculeatus Gr. cf. Parupeneus chrysopleuron Blkr.

Upeneus bifasciatus $C$. $V$. cf. Parupeneus bifasciatus $B l k r$.

Upeneus bilineatus $C . V .13,4$. cf. Parupeneus bilineatus $B l k r$.

Upeneus bitaeniatus Benn. 128, $4 \mathrm{II}$. cf. Upeneus vittatus $C . V$., Upeneoïdes vittatus Blkr.

Upeneus bivittatus $C . V .13,64.128,4$ II. cf. Upeneus sulphureus $C$. $V$., Upeneö̈des bivittatus Blkr., Upeneoïdes sulphureus $B l k r$.

Upeneus Brandesi Blkr. 47, 236. cf. Parupeneus pleurostigma Blkr. 
Upeneus cherserydros C. $V$. cl. Parupeneus cherserydros Blkr.

Upeneus chryserydros $C . V .91,34$.

Upeneus chrysopleuron Blkr. 118, 70. 119, 7.

Upeneus cinnabarinus C. V.91, 34. 119, 7 .

Upeneus crassilabris $C . V .13,4$. cf. Parupeneus crassilabris Blkr.

Upeneus cyclostoma Gthr. cf. Parupeneus luteus Blkr.

Upereus cyclostomus $C$. $V$. cf. Parupeneus cyclostomus Blkr.

Upeneus cyprinoides $C$. $V$. cf. Parupeneus cyprinoides Blkr.

Upeneus dubius Rich., Gthr. 425, I42. 497, 9.

Upeneus dubius Kner cf. Upeneus moluccensis Blkr.

Upeneus flavolineatus $C . V .2,527.468,333$. cf. Mulloïdes flavolineatus Blkr.

Upeneus fraterculus $C \cdot V$. cf. Parupeneus fraterculus $B l k r$.

Upeneus immaculatus Benn. cf. Parupeneus cyclostomus Blkr.

Upeneus indicus Gthr. 308, I52. cf. Parupeneus indicus Blkr.

Upeneus Jansenii Blkr. 143, 44. 275, 33. cf. Parupeneus Janseni Blkr.

Upeneus japonicus $C$ r cf. Mulloides japonicus $B l k r$.

Upeneus lateristriata $\mathrm{Val}$. cf. Upeneus lateristriga $C . V$, Parupeneus macronema Blkr.

Upeneus lateristriga $C . V .109,242.275,33$. cf. Parupeneus macronema Blkr.

Upeneus luteus Guér. cf. Parupeneus cherserydros Blkr.

Upeneus luteus $C . V$., Blkr. 13,63.270, 22.cf. Parupeneus luteus Blkr. Upeneus macronema Blkr. C. $V .270,22$. cf. Parupeneus nacronema Blkr.

Upeneus malabaricus $C . V .91,34$. cf. Parupeneus indicus $B l k r$.

Upeneus mauritianus Benn. cf. Mulloides mauritianus $B l k r$.

Upeneus moluccensis Blkr. 364, 285. 425, I 42. 464, 8.

Upeneus oxycephalus Bl., Blkr. 143, 45. 167, 371. 184, 199. 275, 33. cf. Parupeneus cherserydros Blkr.

Upeneus pleurospilos Blkr. 72, г 10. 118, 69. 231, т46. cf. Parupeneus pleurospilus Blkr.

Upeneus pleurostigma Benn. cf. Parupeneus pleurostigma Blkr.

Upeneus porosus $C . V .468,333$.

Upeneus prayensis $C . V .297$, I33. 468, 333.

Upeneus Russelli $C . V$. Blkr. 13, 62. 91, 34, I65. 156, 213. 157, 232. 171, 245. 185, 223. 231, I 46. 236, 330, 333. 270, 22. 275, 33.

Upeneus spilurus Blkr. 102, 395. 118, 68. 331, 447. cf. Parupeneus spilurus Blkr.

Upeneus subvittatus Rich., Blkr. 425, г 42. 497, го.

Upeneus sulphureus C. V. 2, 527. 395, 292. 396, 299.425, 1 42.459,

8о. 464, 4. 495, 14. 497, го. cf. Upeneö̈des sulphureus Blkr.

Upeneus sulphureus $B l k r .364,285$. cf. Upeneus sulphureus $C . V$.

Upeneus sundaicus Blkr. 354, 147. 464, то.

Upeneus tetraspilus Gthr. 468, 334 . 
Upeneus tragula Rich. 315, 234. 358, r89. 364, 285. 395, 292. 396, 299. 425, I 42. 464, I I. 491, 46. 496, 2.

Upeneus trifasciatus $C . V .47,237.184$, I99. 275, 33. 296, 108. 309, I55. cf. Parupeneus multifasciatus Blkr.

Upeneus vanicolensis $C$. $V$. cf. Mulloïdes vanicolensis Blkr.

Upeneus variegatus $B l k r .2,528$.

Upeneus vittatus $C . V .2,528.10,66$ 128, 4II. 316, 242. 320, 269 . 338, 36о. 364, 285. 459, 8о. 464, 6. 468, 333. 495, 14. 497, го. cf. Upeneoïdes vittatus $B l k r$.

Upeneus Vlamingii C. $V .143,45$. cf. Upeneoïdes Vlamingii Blkr.

Upeneus waigiensis $C . V .13,62$. cf. Parupeneus indicus Blkr., Upeneus Russellii C. V., Blkr.

Upeneus zeylonicus $C$. $V$. cf. Mulloides zeylonicus Blkr.

Uranoscopus asper $T$. Schl. 90, 27. 118, 66. 253, 235. 267, 140. $287,55.364,29$ г. 425, г $26.493,53.497$, г 8.

Uranoscopus asper Blkr. cf. Uranoscopus oligolepis $B l k r$.

Uranoscopus bicinctus T. Schl. 163, 4I. 425, I26. 437, 37. 493, 5 I. 497, I 8 .

Uranoscopus cognatus Cant. 274, 3. 288, 76. 493, 49.

Uranoscopus elongatus Schl. cf. Guathagnus elongatus Gill.

Uranoscopus guttatus C. $V .91,34.425$, r 26.

Uranoscopus indicus $K . v$. $H$. cf. Synanceia elongata $C$. $V$., Polycaulus elongatus Gthr.

Uranoscopus inermis C. V.91, 34. cf. Ichthyscopus inermis Szens.

Uranoscopus laevis Bl. Schn. 119, 7. 163, 41. cf. Uranoscopus bicinctus Schl.

Uranoscopus macropygus Rich. 119, 7.

Uranoscopus maculatus J. R. Forst. 119, 7.

Uranoscopus malacopterus Benn. 270, 40.

Uranoscopus melanopterus $B c n n$. cf. Katethostoma malacopterus.

Uranoscopus occidentalis $A g .302,15$.

Uranoscopus oligolepis Blkr. 493, 55. 497, I8.

Uranoscopus scaber $L .90,28.425$, I 26.

Uranoscopus scaber Rich. cf. Uranoscopus oligolepis Blkr.

Uraspis Blkr. 128, 4I8. 297, I38.

Uraspis carangoïdes Blkr. 128, 418.

Uriphaeton Szons. 468, 256.

Urocampus nanus Gthr. 425, г 26.

Uroconger $K p .175,28.351$, I I 7 .

Uroconger lepturus $K p$. 352, I 25. 425, I 23.

Urogymnus asperrimus $A$. Dum. 459, 68.

Urolophus aurantiacus M. H. 331, 442. cf. Urolophus cruciatus Gthr.

Urolophus cruciatus Günth. 497, 4.

Uropterygius Riüp. 54, 298. 88, 39. 348, 54. 351, I22.

Uropterygius concolor Rüpp. cf. Gymnomuraena concolor Blkr. 
Uropterygius micropterus Blkr. 310, I60. cf. Gymnomuraena micropterus Blkr.

Uropterygius xanthopterus Blkr. 236, 350. cf. Gymnomuraena xanthoptera Blkr.

Valencienna strigata Blkr. cf. Valenciennesia strigata Blkr.

Valenciennea Blkr. 450, 372. 453, 307.

Valenciennea Hasseltii Blkr. 390, 275.

Valenciennesia Blkr. 450, 372. 453, 307. 460, 104, 106.

Valenciennesia elegans Blkr. 425, I28, 152.

Valenciennesia Hasselti Blkr. 396, 300.

Valenciennesia Helsdingenii Blkr. 478, 91.

Valenciennesia longipinnis Blkr. 478, 93.

Valenciennesia macropterus Blkr. 425, r28, I53.

Valenciennesia muralis Blkr.478, 96.

Valenciennesia notophthalmus Blkr. 425, I28, I 53.

Valenciennesia sexguttata $B l k r .437,37.478,98$.

Valenciennesia sinensis Blkr. 425, I28, I 52.

Valenciennesia strigata Blkr. 394, 288. 478, 89.

Vandellia $\mathrm{Val}$. 306, I I3.

Vandellia cirrhosa Val. 306, i I3.

Variola Stons. 468, 255.

Variola flavimarginata Blkr. 438, 45. 451, 9.

Variola longipinna Stons. 468, 255. cf. Variola louti Blkr.

Variola louti Blkr. 315, 232. 320, 268. 396, 296. 451, I I. 459, 88. $468,255.491,42.495$, I I.

Variola melanotaenia Blkr. 364, 2 Sr. cf. Variola flavimarginata Blkr.

Velifer Schl. 497, г6.

Velifer hypselopterus Blkr. 497, т6.

Verilus Pocy 468, 336.

Vincentia Cast. 468, 260.

Vincentia Waterhousii Cast. 468, 260.

Vomer Cuv. 297, I37.

Vomer Brownil Cuv. cf. Vomer setipinnis Ayr.

Vomer setipinnis Ayr. 302, 83 .

Vorax Poey 468, 336.

Vulsus dactylopus Gthr. 317, 246. 339, 362. 340, 365. 354, 149. 358, 191. 364, 292. 494, 105.

Wallago Blkr. 299, 394. 306, I I 4.

Wallago anostomus B?kr. 91, rog.

Wallago asotus Blkr. 91, 54.

Wallago attu Blkr.306, II4. 356, 175.

Wallago bimaculatus Blkr. 91, 54.

Wallago dinema Blkr. 45, 202. 49, 4r9. 55, 412. 
Wallago heterohynchus Blkr. 86, 5 I4.

Wallago Leerii Blkr. 49, 427. 55, 4r2. 235, 370. 270, 47. 272, I9.

Wallago leiacanthus Blkr. 82, I89.

Wallago malabaricus Blkr. 91, 54.

Wallago microcephalus $B l k r .91$, Ioo.

Wallago Mülleri Blkr. cf. Wallago Russellii Blkr.

Wallago pabda Blkr. 91, 54.

Wallagọ pabo Blkr. 91, 54.

Wallago Russellii Blkr. 91, 108. 239, 102.

Xanthurus indicus Will. cf. Caesio erythrogaster $K . v . H$.

Xenichthys Gill 468, 276.

Xenichthys Xanthi Gill 468, 276.

Xenocypris argentea Gthr. 415, 12. 425, I45.

Xenocypris Davidi Blkr. 411, 253. 415, 56. 418, 66. 425, I 45.

Xenocypris homospilotus Blkr. 415, I3. 425, I 45.

Xenocypris macrolepis Blkr. 411, 253. 415, 53. 418, 63. 425, I 45.

Xenocypris microlepis Blkr. 411, 253. 415, 58.418, 68.425, 145 .

Xenocypris plena Blkr. 415, I3. 425, 145.

Xenocypris Simoni Blkr. 415, I2. 425, I45.

Xenocypris tapeinosoma Blkr. 415, 55. 418, 64. 425, I45.

Xenodon niger Rüpp. 63, 37 .

Xenopterus auritus Gïnth. cf. Chonerhinos naritus Blkr.

Xiphasia madagascariensis Blkr. 459, то3.

Xiphasia setifer Stons. 91, 48.

Xiphasia trachypareia Blkr. 359, I96.

Xiphias gladius L. 266, 53 .

Xiphias velifer Gthr. 459, гоo.

Xiphiurus capensis Smith 266, 70.

Xiphocheilus Blkr. 156, 223. 292, 105.

Xiphocheilus typus Blkr. 156, 224. 293, 296.

Xiphochilus robustus Gthr. cf. Choerops robustus Blkr.

Xiphogadus madagascariensis Playf. cf. Xiphasia madagascariensis Blkr.

Xiphophorus Heck. 261, 440.

Xiphoramphus odoë M. Tr. 302, г 6.

Xyrichthys Cuv. 1, 552. 292, 103.

Xyrichthys cultratus $V$ al. cf. Novacula lineolata Blkr.

Xyrichthys cyanifrons $C . V .91,54$.

Xyrichthys dea $T$. Schl. cf. Novacula dea Blkr.

Xyrichthys macrolepidotus $C . V$. 11, 7. 12, 42. cf. Novaculichthys macrolepidotus Blkr.

Xyrichthys novacula Cuv. Val. cf. Novacula lineolata Blkr.

Xyrichthys novaculoïdes $B l k r .72$, I 22.

Xyrichthys pavo C. V. 292, 103. 293, 294. 315, 231. 459, 83. 491, 4I. 495, ro. cf. Novacula pavo Blkr. 
Xyrichthys pavoninus $C . V$. cf. Xyrichthys pavo $C . V$, Novacula pavo Blkr.

Xyrichthys puniceus Rich. cf. Novacula dea Blkr.

Xyrichthys taeniurus $C . V .91$, 165. cf. Novacula taeniurus Blkr., Novaculichthys taeniurus Blkr.

Xyrichthys tetrazona Blkr. 293, 294.

Xyrichthys tonsor $\mathrm{Val}$. cf. Novacula lineolata Blkr.

Xyrichthys virens $\mathrm{Val} .145 .382$.

Xyster Comm. 468, 293.

Zanclus Comm. 468, 309 .

Zanclus canescens Gthr. 481, I69.

Zanclus centrognathos $C$. $V$. cf. Zanclus canescens Gthr.

Zanclus cornutus $C . V .10,66.22,22.46,2$ 12. 47, 227.91, г66. 157, 233. 171, 245. 184, 200. 188, 459. 236, 331. 270, 26. 275, 35. 309, I $55.315,234.318,25$ I. 320, 270. 358, 190. 364, 287. $369,42.391,276.396,299.459,96.468,309.481,167.491$, 45. $495, \mathrm{I} 4$.

Zanclus Montrouzieri Thioll. cf. Zanclus canescens Gthr.

Zenarchopterus amblyurus Blkr. 375, I60. 430, 234.

Zenarchopterus Buffonis Blkr. 375, I62.

Zenarchopterus dispar Gill 375, 164. 459, 100.

Zenarchopterus microstoma Blkr. 425, I 49.

Zenopsis nebulosus Gill 497, I6.

Zeugopterus Gottsch.294, 424.

Zeugopterus hirtus Gottsch. 294, 424.

Zeus 1, 553 .

Zeus argentarius Forst. cf. Gazza minuta Blkr.

Zeus australis Rich. 119, II.

Zeus capensis $C . V .266,54$.

Zeus ciliaris $B l$. cf. Carangoïdes blepharis $B l k r$.

Zeus faber $L$. 266, 54 .

Zeus faber japonicus $T$. Schl. cf. Zeus japonicus $C . V$.

Zeus gallus Bl.cf. Carangoïdes gallichthys Blkr.

Zeus insidiator $B l$. cf. Equula insidiatrix $C . V$.

Zeus japonicus $C . V .118$, I05. 119, II. 425, I33. 497, I6.

Zeus luna L. Gm. cf. Lampris luna Risso.

Zeus maculatus $B l$. Schu. cf. Mene maculata C. $V$.

Zeus nebulosus Schl. cf. Zenopsis nebulosus Gill.

Zeus setapinnis Mitch.cf. Vomer setipinnis Ayr.

Zeus vomer Russ. cf. Carangoïdes gallichthys Blkr.

Zonogobius Blkr.453, 323 .

Zungaro Blkr.306, Iо .

Zungaro Humboldtii Blkr. 306, ror.

Zygaena 1, 55I. 
Zygaena Blochii Val. 270, 67. 272, 6. 275, 9. 287, 37. 288, 66. 305, 73. cf. Sphyrna Blochii $M$. $H$.

Zygaena indica v. Hass. cf. Sphyrna zygaena Rafin.

Zygaena laticeps Cant. cf. Sphyrna Blochii M. H.

Zygaena Lewisii Griff. cf. Sphyrna zygaena Rafin.

Zygaena malleus Risso 270, 67. 287, 37. 288, 66. cf. Cestracion malleus Gill, Sphyrna zygaena Rafin.

Zygaena malleus Sh. cf. Cestracion zygaena Gill.

Zygaena mokarran Rüpp. cf. Cestracion mokarran A. Dum.

Zygaena platycephala $v$. Hass. cf. Sphyrna Blochii $M . H$.

Zygaena tudes $V a l$. cf. Sphyrna zygaena Rafin.

Zygonectes $A g$. 261, 439.

\section{ADDENDA.}

For the species of Cichla (p. I3I) see also under Cychla (p. I49). 

A $u(w)$ 






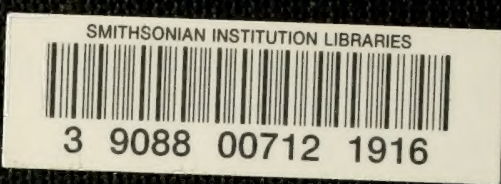

